\title{
Nasal High Flow Therapy in Chronic Obstructive Pulmonary Disease
}

\author{
By
}

Steven James McKinstry

\author{
A thesis submitted to the \\ Victoria University of Wellington \\ in fulfilment of the requirements for the degree of \\ Doctor of Philosophy
}

Victoria University of Wellington

2019 


\section{Table of Contents}

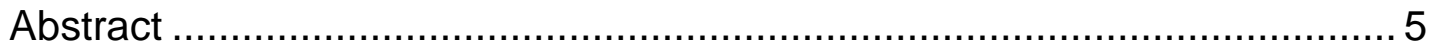

Table of figures .............................................................................. 7

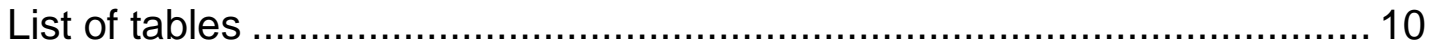

List of abbreviations and symbols .................................................... 13

Personal contribution and acknowledgements ..................................... 16

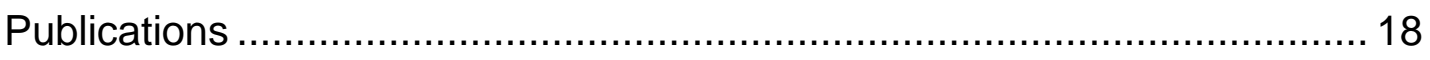

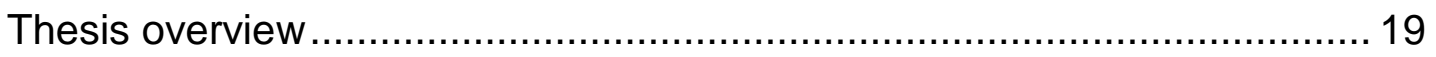

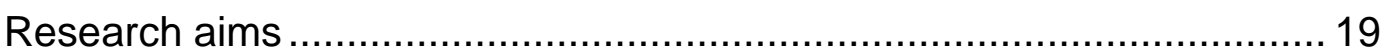

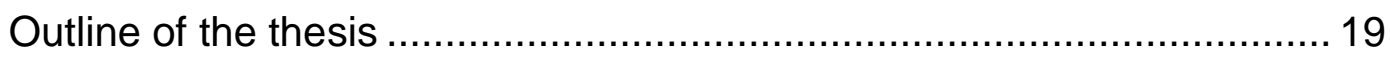

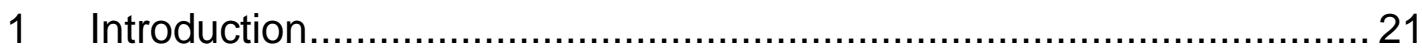

1.1 Overview of Chronic Obstructive Pulmonary Disease ..................2 21

1.2 Respiratory physiology and changes seen in COPD ....................23

1.3 Important clinical problems in COPD ..................................... 42

1.4 An overview of Nasal High Flow therapy ................................ 74

1.5 Summary of background information, knowledge gaps and aims of the thesis

2 Study 2 - Nasal High Flow therapy and $\mathrm{P}_{\mathrm{t}} \mathrm{CO}_{2}$ in stable COPD: a randomised controlled cross-over trial ............................................... 125

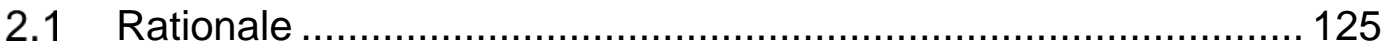

2.2 Aims and hypotheses .................................................... 125

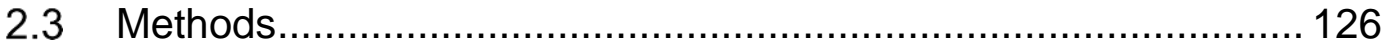

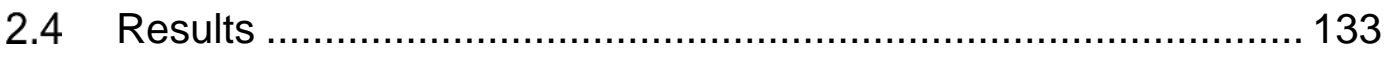

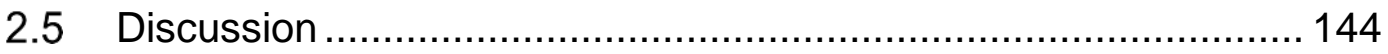

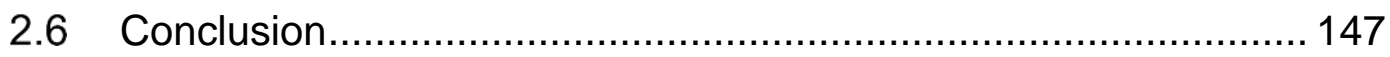

3 Study 3 - Nasal High Flow therapy use following hospitalisation for an exacerbation of COPD: a feasibility study ..................................... 149

3.1 Rationale ......................................................................... 149 


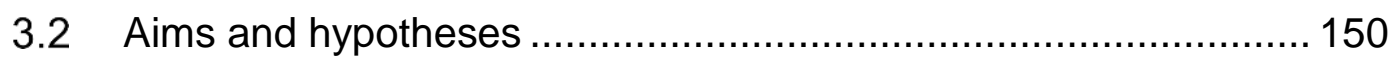

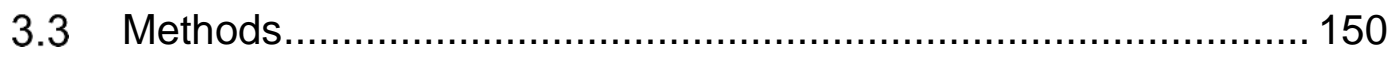

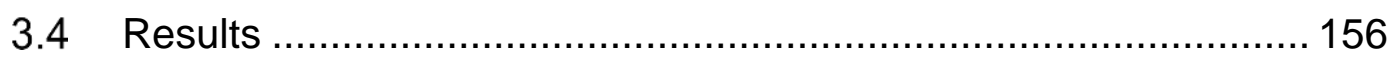

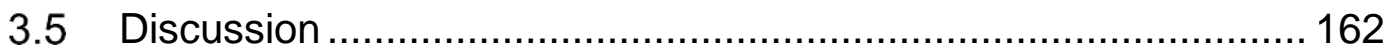

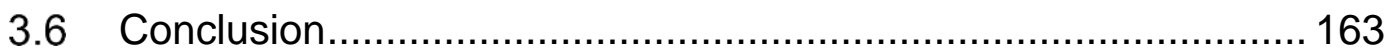

4 Study 4 - Nasal High flow therapy in acute hypercapnic respiratory failure with acidosis in patients presenting with an acute exacerbation of

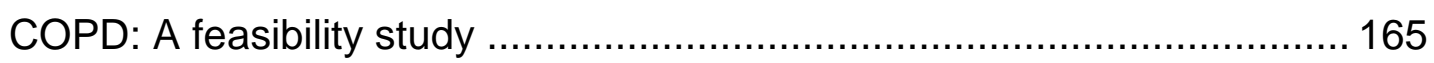

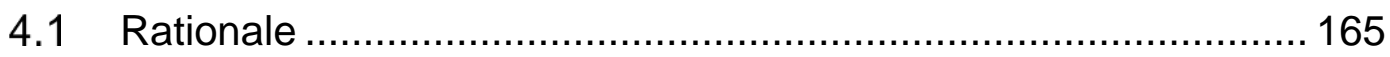

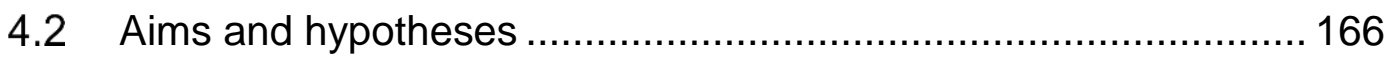

4.3 Methods - Study Part 1 .......................................................... 168

4.4 Methods - Study Part 2 ….................................................. 170

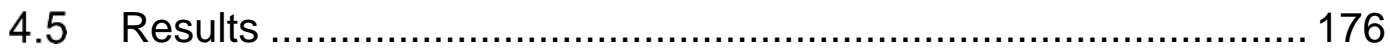

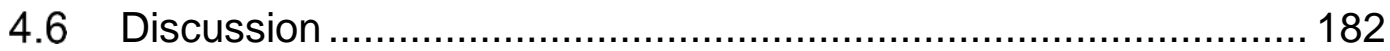

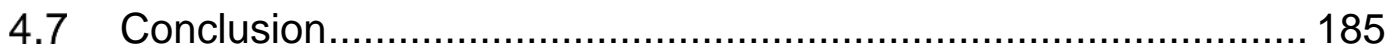

5 Study 5 - Nasal High Flow therapy compared with Non-invasive ventilation in COPD patients with chronic respiratory failure: a randomised controlled cross-over trial......................................................................... 186

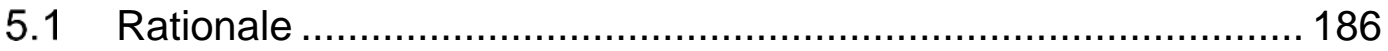

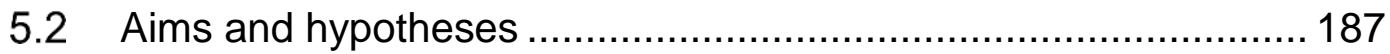

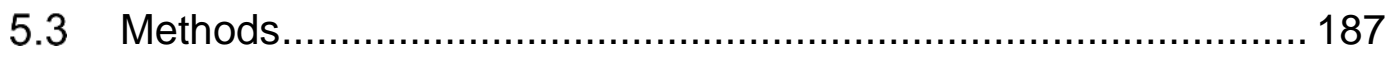

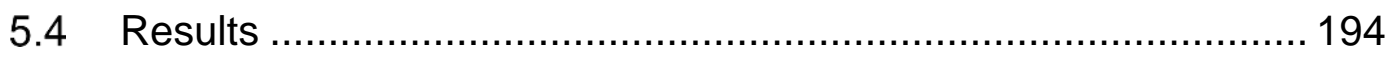

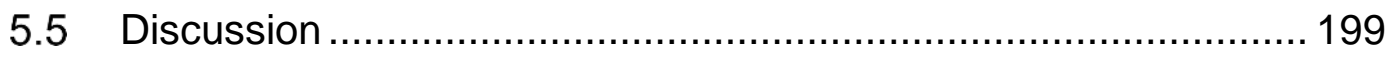

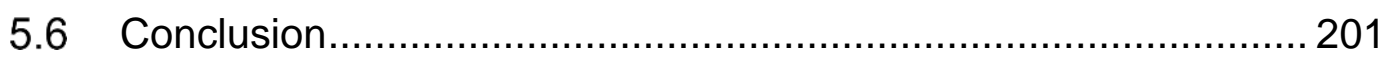

6 Study 6 - A dual NHF/NIV device compared to standard NIV in chronic respiratory disease: a randomised controlled cross-over trial..................... 203

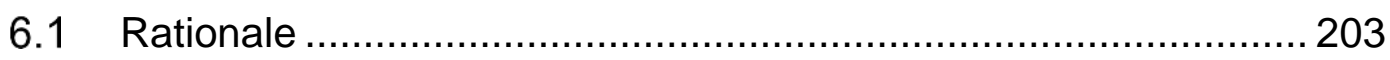

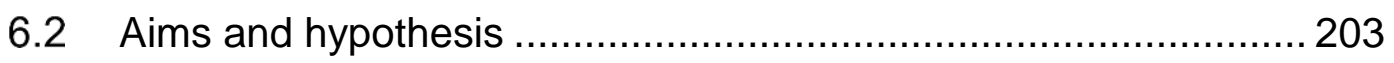

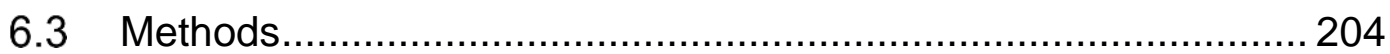




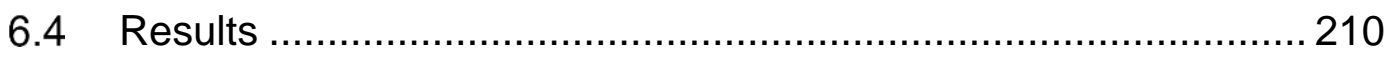

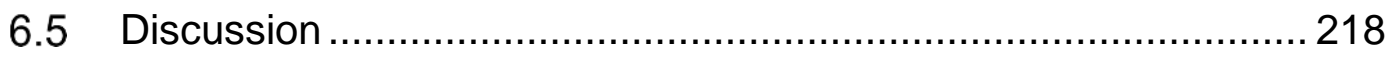

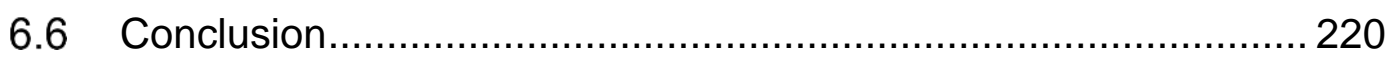

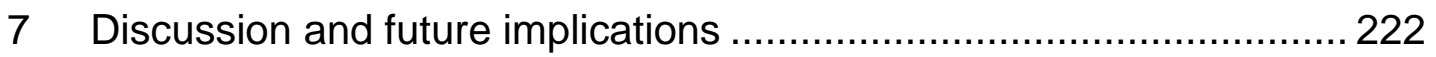

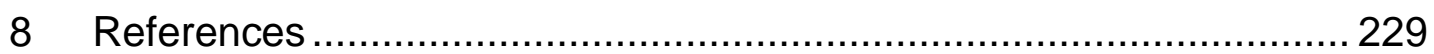

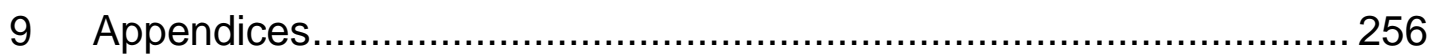

9.1 Appendix I - Full description of mechanism of actions studies.....256

9.2 Appendix II - Validation of methods: accuracy of transcutaneous monitoring - a prospective observational cohort study (Study 1a) and posthoc analyses from an RCT (Study 1b)............................................ 258

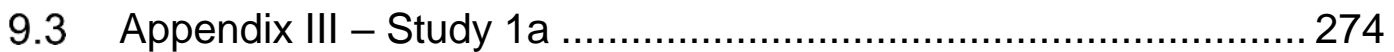

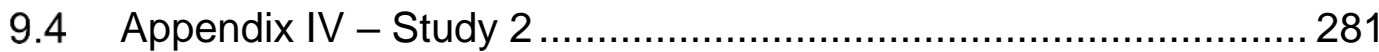

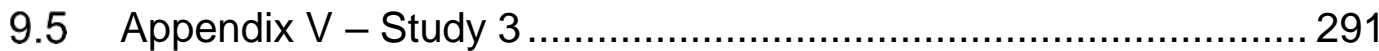

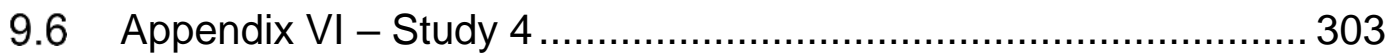

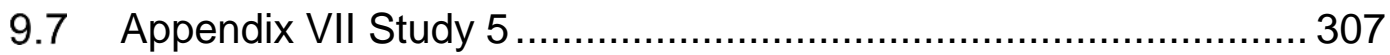

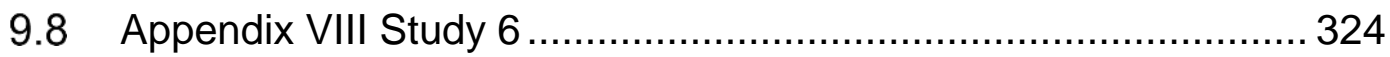




\section{Abstract}

\section{BACKGROUND}

In acute exacerbations of chronic obstructive pulmonary disease (AECOPD), hypercapnia (raised arterial partial pressure of carbon dioxide, $\mathrm{PaCO}_{2}$ ) is associated with worse clinical outcomes, including death. Nasal high flow (NHF) therapy is a common method of administering oxygen therapy in hypoxic respiratory failure, yet its effect on $\mathrm{P}_{\mathrm{a}} \mathrm{CO}_{2}$ in $\mathrm{COPD}$ are uncertain.

\section{AIMS}

To investigate the effects of NHF therapy in people with COPD.

\section{METHODS}

Two randomised controlled trials (RCTs) were undertaken to investigate the effect of NHF on transcutaneous partial pressure of carbon dioxide $\left(\mathrm{P}_{\mathrm{t}} \mathrm{CO}_{2}\right)$ in COPD: one comparing different flow rates (45 L/min, $30 \mathrm{~L} / \mathrm{min}$ and $15 \mathrm{~L} / \mathrm{min})$ to breathing room air as a control in 48 participants with stable COPD, and another comparing NHF against non-invasive ventilation (NIV) in 24 stable COPD patients with chronic hypercapnia. Two further studies tested the feasibility of undertaking multicentre RCTs utilising different applications of NHF in COPD: one in 100 patients (20 in New Zealand, 80 in the United States) using NHF at home for 30 days following hospital discharge to determine patterns of use and rates of hospital readmission, and another in the Emergency Department (ED) to test whether a pre-specified standard protocol for managing acute hypercapnic respiratory failure (AHRF) could be followed. Finally, another RCT compared the tolerability and change in $\mathrm{P}_{\mathrm{t}} \mathrm{CO}_{2}$ of a dual NHF/NIV device to a standard NIV device in people with chronic respiratory disorders including COPD.

\section{RESULTS}

In stable COPD, the mean $(95 \% \mathrm{Cl})$ change in $\mathrm{P}_{\mathrm{t}} \mathrm{CO}_{2}$ at 20 min was $-0.6 \mathrm{~mm}$ $\mathrm{Hg}(-1.1$ to 0.0$), \mathrm{P}=0.06 ;-1.3 \mathrm{~mm} \mathrm{Hg}(-1.9$ to 0.8$), \mathrm{P}<0.001$; and $-2.4 \mathrm{~mm}$ $\mathrm{Hg}(-2.9$ to -1.8$), \mathrm{P}<0.001$; for $\mathrm{NHF}$ at $15 \mathrm{~L} / \mathrm{min}, 30 \mathrm{~L} / \mathrm{min}$ and $45 \mathrm{~L} / \mathrm{min}$, respectively, compared with room air. In stable COPD with chronic 
hypercapnia, the mean (SD) reduction in $\mathrm{P}_{\mathrm{t}} \mathrm{CO}_{2}$ at $60 \mathrm{~min}$ from baseline with $\mathrm{NHF}$ was $-2.5 \mathrm{mmHg}$ (3.5) compared to $-5.3 \mathrm{~mm} \mathrm{Hg}$ (5.0) with NIV. The $\mathrm{P}_{\mathrm{t}} \mathrm{CO}_{2}$ change when analysed across all time points was lower using NIV than with NHF: $-2.5 \mathrm{mmHg}(95 \% \mathrm{Cl}-4.5$ to -0.5$), \mathrm{P}=0.016$.

In the international outpatient feasibility study, mean (SD) NHF use at home following hospital discharge was 1.6 (1.6) hours/day in the $20 \mathrm{NZ}$ participants. The 30-day hospital readmission rate was $10 \%(95 \% \mathrm{Cl} 1.8$ to 33.1$)$. Recruitment in the US was unsuccessful, despite a 12-month extension, and no data was available. In the second feasibility study, 120 patients with AECOPD were reviewed over a 4-month period in ED. All 3 patients with AHRF received the standard of care protocol and in one there was significant deviation from the agreed protocol.

There was no difference in tolerability between the dual NHF/NIV device and a standard NIV device on a $100 \mathrm{~mm}$ visual analogue scale: mean difference $1.3 \mathrm{~mm}(95 \% \mathrm{Cl}-7.9$ to $5.2, \mathrm{P}=0.69)$, and a lower $\mathrm{P}_{\mathrm{t}} \mathrm{CO}_{2}$ with the standard NIV device, mean difference $-0.61 \mathrm{~mm} \mathrm{Hg}(95 \% \mathrm{Cl}-1.05$ to $-0.17, \mathrm{P}=0.01)$.

\section{CONCLUSION}

The small, flow-dependent reduction in $\mathrm{P}_{\mathrm{t}} \mathrm{CO}_{2}$ observed with the currently available NHF device across the range of flows used in clinical practice indicates that it is safe to use in COPD from a physiological perspective. The reduction on $\mathrm{P}_{\mathrm{t}} \mathrm{CO}_{2}$ with $\mathrm{NHF}$ was smaller than with $\mathrm{NIV}$, however the difference was of uncertain clinical significance, suggesting NHF represents an alternative therapy for COPD patients with hypercapnia who cannot tolerate NIV, or during breaks from NIV. In NZ, it would be feasible to undertake an RCT using NHF in AECOPD patients discharged from hospital, but not those presenting to ED with AHRF. The recently developed dual NHF/NIV device had similar tolerability as a standard NIV device and there was no clinically significant difference in $\mathrm{P}_{\mathrm{t}} \mathrm{CO}_{2}$. These findings suggest that NHF represents a therapeutic option for patients with COPD, with the dual NHF/NIV device demonstrating potential as a modality for delivering NHF in COPD patients with AHRF. 


\section{Table of figures}

Figure 1-1 Schematic of human airways progression from the conducting to

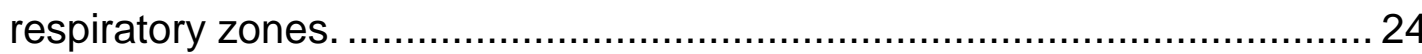

Figure 1-2 Oxygen-haemoglobin dissociation curve ................................ 26

Figure 1-3 Illustration of different scenarios for ventilation/perfusion ( $\mathrm{V} / \dot{Q})$

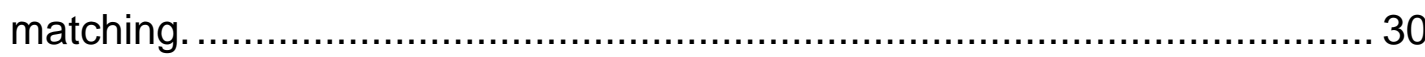

Figure 1-4 Relationship between changes in the ventilation/perfusion ( $\dot{V} / \dot{Q})$

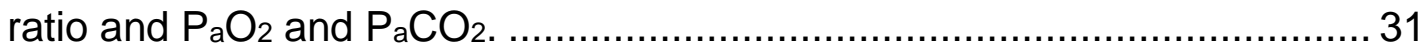

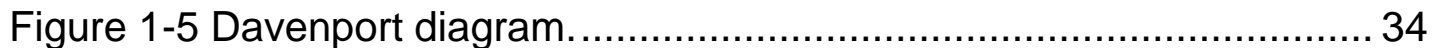

Figure 1-6 Illustration of mechanisms causing airflow limitation in COPD. .. 35

Figure 1-7 Indications for AECOPD hospitalisation. ................................... 43

Figure 1-8 Criteria for hospital discharge following an AECOPD................. 46

Figure 1-9 Kaplan-meier survival curves showing the influence of hypercapnia on 3 different categories of hypercapnic patients. ................... 55

Figure 1-10 Different types of Non-Invasive Ventilation interfaces. .............59 Figure 1-11 Schematic of the mechanical effects of AECOPD illustrated by pressure-volume plots.

Figure 1-12 - BTS/ICS guidelines - summary of process for initiating NIV in AHRF

Figure 1-13 Forest plot of NIV vs usual care (overall) - Mortality. 65

Figure 1-14 Forest plot of NIV vs usual care (overall) - Need for endotracheal intubation. 66

Figure 1-15 Forest plot of comparison between NIV and usual care for treatment intolerance. 70

Figure 1-16 Forest plot of NIV vs usual care complications of treatment..... 72

Figure 1-17 Photo of the Nasal High Flow device..................................... 74

Figure 1-18 Mucociliary "escalator" transport system. ............................... 79

Figure 1-19 Forest plot comparing intubation rates in patients with NHF compared to standard oxygen therapy and NIV.

Figure 1-20 Forest plot comparing in-hospital mortality in patients treated with NHF compared to standard oxygen therapy and NIV ........................... 90

Figure 1-21 Focussed clinical question using the PICO format 93 
Figure 1-22 Systematic review search strategy used in Medline and Embase.

Figure 1-23 PRISMA flowchart of the process used to select publications for the systematic review. 95

Figure 1-24. Illustration of the 4 NHF conditions tested in Braunlich et al,

BMC Pulmonary Medicine 2018. 108

Figure 2-1 A sample of a Labcharts recording of Respiratory Inductance

Plethysmography (RIP) 130

Figure 2-2 CONSORT summary of participant flow through Study 2 and the allocation of interventions. 134

Figure 2-3 Summary of how the technically acceptable 79 RIP band interventions were derived.

Figure 3-1 Histogram of daily hours (daily_hrs) NHF was used. 157

Figure 3-2 Q-Q Plot for the primary outcome variable (daily_hrs used)

indicating non-normal distribution. 158

Figure 3-3 Histogram for proportion of days used (X-axis labelled "proportion_days_used") adjusted for number of days NHF was available.

Figure 3-4 Box-plots of mean daily hours of NHF use by week. 160

Figure 4-1 Study part 1 - Usual care protocol agreed upon with ED staff. . 169 Figure 4-2 Participant flow through Study 4 and the input from study investigators. 173

Figure 4-3 Flow of participants through Study 4. 178

Figure 4-4 Bland-Altman plot of limits of agreement for arterial and venous $\mathrm{PCO}_{2}$. 181

Figure 4-5 Bland-Altman plot of limits of agreement for arterial and venous $\mathrm{pH}$. 181

Figure 5-1 Summary of Study 5 flow, demonstrating the cross-over design. 189

Figure 5-2 CONSORT diagram of participant flow through Study 5. 194

Figure 5-3 Mean $\mathrm{P}_{\mathrm{t}} \mathrm{CO}_{2}$ change over time. 197

Figure 6-1 Configuration of the NHF/NIV and ResMed device set-up. ...... 206 Figure 6-2 Example of $100 \mathrm{~mm}$ visual analogue scale with annotation depicting the levels of comfort. 208

Figure 6-3 Line plot of mean $\mathrm{P}_{\mathrm{t}} \mathrm{CO}_{2}$ over time for each intervention. 214 
Figure 9-1 Flow chart describing how the SenTec transcutaneous device measures $\mathrm{P}_{\mathrm{t}} \mathrm{CO}_{2}$ 260

Figure 9-2 Bland-Altman Plots of limits of agreement of $\mathrm{PCO}_{2}$ between $A B G$ and SenTec \#1.

Figure 9-3 Bland-Altman plots of limits of agreement of $\mathrm{PCO}_{2}$ between $A B G$ and SenTec \# 2

Figure 9-4 Bland-Altman plots of limits of agreement of $\mathrm{PCO}_{2}$ between $A B G$ and SenTec \# 1 and 2.

Figure 9-5 Bland-Altman plots of limits of agreement of $\mathrm{S}_{\mathrm{a}} \mathrm{O}_{2}$ between $A B G$ and SenTec \#1. 268

Figure 9-6 Bland-Altman plots of limits of agreement of $\mathrm{S}_{\mathrm{a}} \mathrm{O}_{2}$ between $A B G$ and SenTec \#2. 268

Figure 9-7 Bland-Altman plots of limits of agreement of $\mathrm{SaO} 2$ between ABG and SenTec \# 1 and \#2. 269

Figure 9-8 Bland altman plot of the mean $\mathrm{CBG}$ and $\mathrm{P}_{\mathrm{t}} \mathrm{CO}_{2}$ change over 35 min compared to the mean difference in $\mathrm{CBG}$ minus $\mathrm{P}_{\mathrm{t}} \mathrm{CO}_{2}$ change over 35 $\min$. 


\section{List of tables}

Table 1-1 An overview of hypoxaemic and hypercapnic respiratory failure. 37 Table 1-2 Management of acute hypercapnic (Type 2) respiratory failure... 44 Table 1-3 Management of acute hypoxaemic (Type 1) respiratory failure... 44 Table 1-4 Evidence-based interventions to reduce COPD readmissions. ... 51 Table 1-5 Odds of NIV failure with $\mathrm{PaCO}_{2}$ rise and $\mathrm{pH}$ fall......................... 53 Table 1-6 Summary of studies demonstrating a PEEP effect with NHF ...... 77 Table 1-7 Summary of publications pre-2015 investigating $\mathrm{PCO}_{2}$ change with NHF in COPD. 97

Table 1-8 Summary of publications post-2015 investigating PCO2 change with NHF 104

Table 1-9 Comfort of various oxygen-delivery devices compared to NHF. 114

Table 2-1 Study 2 baseline participant characteristics. 135

Table 2-2* $\mathrm{P}_{\mathrm{t}} \mathrm{CO}_{2}$ values and mixed linear models for the difference in $\mathrm{P}_{\mathrm{t}} \mathrm{CO}_{2}$ of NHF minus room air, adjusted for baseline (zero time-point). 138

Table 2-3* Mixed linear models for difference in respiratory rate of NHF minus room air adjusted for baseline (zero time-point) 139 Table 2-4* Mixed linear models for oxygen saturation difference in NHF minus room air adjusted for baseline (zero-time point) 140 Table 2-5* Mixed linear models for heart rate difference in NHF minus room air adjusted for baseline (zero-time point).

Table 2-6* Mixed linear models for difference in questionnaire responses between NHF interventions.

Table 3-1 Study 3 baseline participant characteristics. 156

Table 3-2 Study 3 primary outcome - data description of NHF use per day.

Table 3-3 NHF therapy questionnaire results - questions 1 to 4 . 160

Table 3-4 NHF therapy questionnaire results - questions 5 to 6 . 161

Table 3-5 NHF therapy questionnaire results - question 7 . 161

Table 3-6 Lung function change - day 31 compared to day 1 162

Table 4-1 Arterial $\mathrm{pH}$ agreement for proportion of patients with venous $\mathrm{pH}$ between 7.21 and 7.31 .

Table 4-2 Data description of $A B G$ and VBG values and their relationship to each other. 180 
Table 4-3 Agreement between venous and arterial for the proportion of patients with $\mathrm{pH}<7.35$. 182

Table 4-4 Agreement between venous and arterial for proportions of patients with $7.25 \leq \mathrm{pH}<7.35$. 182

Table 5-1 Study 5 baseline participant characteristics. 195

Table 5-2 $\mathrm{P}_{\mathrm{t}} \mathrm{CO}_{2}$ values and difference in $\mathrm{P}_{\mathrm{t}} \mathrm{CO}_{2}$ with NHF and NIV 196

Table 5-3 BORG score outcomes at baseline and difference postintervention. 198

Table 5-4 Mixed linear models for difference in questionnaire responses between NIV and NHF interventions.

Table 6-1 Definition of different bi-level triggering and cycling classification.

Table 6-2 Study 6 baseline participant characteristics.

Table 6-3 Data description of comfort score variables measured by visual

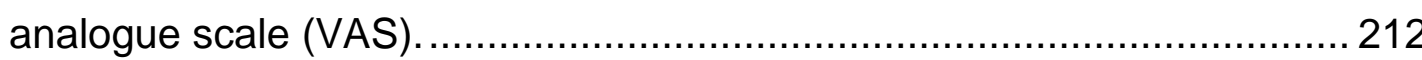

Table 6-4 Paired t-test comparisons for comfort score variables...............212 Table 6-5 Proportion of responses to preference questions. ................... 213 Table 6-6 $\mathrm{P}_{\mathrm{t}} \mathrm{CO}_{2}$ values and difference in $\mathrm{P}_{\mathrm{t}} \mathrm{CO}_{2}$ of ResMed minus NHF/NIV

Table 6-7 Mixed linear models for $\mathrm{P}_{\mathrm{t}} \mathrm{CO}_{2}$ change with zero time-point as a baseline co-variate. 215

Table 6-8 Mixed linear models of blood pressure change with zero time-point as a baseline covariate. 216

Table 6-9 Summary of device triggering. 216

Table 6-10 Summary of device cycling. 217

Table 6-11 Analyses of $\mathrm{P}_{\mathrm{t}} \mathrm{CO}_{2}$, respiratory rate and global comfort score in two participants with the highest auto-events. 217

Table 9-1 Study 1a baseline participant characteristics. 265

Table 9-2 Data description for the differences between each SenTec and ABG values, and between SenTec \#1 and \#2. 266

Table 9-3 Bias and limits of agreement between ABG and SenTec \#1 and SenTec \#2. 269

Table 9-4 Comparisons in CBG-measured $\mathrm{PCO}_{2}$ and SenTec-measured $\mathrm{PCO}_{2}$ 


\section{List of abbreviations and symbols}

ABG Arterial blood gas

AECOPD Acute exacerbation of chronic obstructive pulmonary disease

AHRF Acute hypercapnic respiratory failure

ANZCTR Australia and New Zealand Clinical Trials Registry

ATS American thoracic society

BiPAP Bi-level positive airways pressure

BTS British Thoracic Society

CBG Capillary blood gas

CHRF Chronic hypercapnic respiratory failure

$\mathrm{Cl}$ Confidence interval

COPD Chronic obstructive pulmonary disease

$\mathrm{CO}_{2}$ Carbon Dioxide

CPAP Continuous positive airways pressure

DH Dynamic hyperinflation

ED Emergency department

EELV End expiratory lung volume

EFL Expiratory flow limitation

EPAP Expiratory positive airways pressure

ERS European respiratory society

$f \quad$ Frequency [of respiration]

FEV 1 Forced expiratory volume in one second

$\mathrm{F}_{i} \mathrm{O}_{2}$ Fraction of inhaled oxygen

FPH Fisher and Paykel Healthcare

FVC Forced vital capacity 
Hb Haemoglobin

HDEC Health and Disability Ethics Committee (of New Zealand)

ICS Intensive care society

iPEEP Intrinsic positive end expiratory pressure

ICU Intensive care unit

LACE Length of stay, Acuity, Co-morbidities, ED visits in last $6 \mathrm{mth}$

L/min Litres per minute

LTOT Long term oxygen therapy

mcg Micrograms

mg Milligrams

MRINZ Medical Research Institute of New Zealand

$\mathrm{mmHg}$ Millimetres of mercury

NIV Non-invasive ventilation

NNT Numbers needed to treat

$\mathrm{O}_{2}$ Oxygen

$\mathrm{PaCO}_{2}$ Partial pressure of arterial carbon dioxide

$\mathrm{P}_{\mathrm{a}} \mathrm{O}_{2}$ Alveolar oxygen concentration

$\mathrm{P}_{\text {cap }} \mathrm{CO}_{2}$ Partial pressure of capillary carbon dioxide

PEEP Positive end expiratory pressure

$\mathrm{PvCO}_{2} \quad$ Partial pressure of venous carbon dioxide

$\mathrm{pH}$ Potential of hydrogen (negative log of $\mathrm{H}^{+}$concentration - a marker of acidity)

PS Pressure support (of NIV)

$\mathrm{P}_{\mathrm{t}} \mathrm{CO}_{2}$ Partial pressure of carbon dioxide measured transcutaneously

Q Perfusion 
QDC Qualitative Diagnostic Calibration

$\mathrm{R}$ Respiratory exchange ratio

RCT Randomised controlled trial

RIP Respiratory Inductance Plethysmography

RV Residual volume

$\mathrm{S}_{\mathrm{a}} \mathrm{O}_{2}$ Oxygen saturation measured by $\mathrm{ABG}$

SD Standard deviation

SGRQ St George's Respiratory Questionnaire

$\mathrm{S}_{\mathrm{p}} \mathrm{O}_{2}$ Oxygen saturations measured by pulse oximetry

$\mathrm{S}_{\mathrm{t}} \mathrm{O}_{2}$ Oxygen saturations measured transcutaneously

S/T Spontaneous/timed mode (of NIV)

TE Expiratory time

$\mathrm{T}_{\mathrm{i}} \quad$ Inspiratory time

TLC Total lung capacity

TSANZ Thoracic society of Australia and New Zealand

V Ventilation

$\mathrm{V}_{\mathrm{A}}$ Alveolar tidal volume

VBG Venous blood gas

$\dot{V}$ Minute ventilation

$\dot{V}_{A}$ Alveolar minute ventilation

$\mathrm{VCO}_{2}$ Carbon dioxide produced by metabolic activity of the body per unit time $V_{D}$ Physiological dead space

$\mathrm{V}_{\mathrm{T}}$ Tidal volume 


\section{Personal contribution and acknowledgements}

Team work and collaboration are vital ingredients to any research and I would not have been able to complete this body of work without such a strong surrounding network of fellow researchers, especially at MRINZ. I was a postgraduate year 3 doctor when I commenced this thesis. My interest in research stemmed from a clinical audit and while not being a respiratory medicine trainee, I was fortunate Prof Beasley gave me the opportunity to undertake this respiratory medicine research. Studies $1 \mathrm{a}$ and $1 \mathrm{~b}$ were collaborative efforts with Janine Pilcher and George Bardsley, who each have presented these studies in their respective theses. My role in these 2 studies was as a co-investigator, mainly involved in data collection and manuscript writing.

Studies 2-5 were my principal projects as a Health Research Council (HRC) of New Zealand clinical training fellow and I thank them for their financial support to enable me complete them. The HRC paid for a part of my salary at MRINZ and my academic fees at Victoria University. My role in these studies was from study conception right through to ethics application, recruitment, data collection, analysis and manuscript write-up. Studies 2, 4 and 5 were also sponsored by MRINZ who paid part of my salary also. Studies 3 and 6 were sponsored by Fisher and Paykel Healthcare (FPH) who provided the equipment and covered any research expenses, were involved in data monitoring and paid a fee to MRINZ to undertake the studies. I personally did not receive any financial support from $\mathrm{FPH}$. My involvement was to write the study protocols, ethics and regulatory applications, data collection and analyses of these studies.

Of course I could not have completed these studies without various MRINZ researchers lending a hand - especially Janine and George. Special thanks to Janine who moulded my early research ambitions to ensure data was collected with absolute integrity.

I am indebted to James Fingleton and Prof Beasley for the time and patience they have put into this thesis as well as John Miller for his expert review.

My colleagues at MRINZ have been super helpful in the day-to-day running of the trials, in particular Tony Mallon for his trouble-shooting abilities, Tanya Baker for any ethical dilemmas and Irene Braithwaite for her reliable advice. 
Christina Baggott proved to have an editor's eye and I appreciated her early reviews of this thesis. Thanks to the FPH Airvo team, especially James Revie, for their advice and input.

I have enormous gratitude to all the participants in my studies, some of whom are no longer with us, who selflessly volunteered their time and energy in the hope this body of work would improve the lives of others.

This thesis has been a big part of my life over the last 3 years, during which time l've been married to my lovely wife Anna and had a wonderful 2016 Christmas present in little Iris. I am incredibly fortunate to have such loving girls at home.

Finally, this thesis is dedicated to my late mother - she had insightful intelligence and was one of the smartest woman I've met - I miss her immensely and I hope this work has done her proud. 


\section{Publications}

The following is a list of manuscripts and abstracts directly related to this thesis which have been published:

1. McKinstry, S., Pilcher, J., Bardsley, G., Berry, J., Van de Hei, S., Braithwaite, I., Fingleton, J., Weatherall, M., Beasley, R. Nasal high flow therapy and $\mathrm{P}_{\mathrm{t}} \mathrm{CO}_{2}$ in stable COPD: A randomised controlled cross-over trial. Respirology, (2018) 23(4), 378-384. ('Thesis Study 2)

2. McKinstry, S., Singer, J., Baarsma, J.P., Weatherall, M., Beasley, R., Fingleton, $J$. Nasal high flow therapy compared to non-invasive ventilation in COPD patients with chronic respiratory failure: a randomised controlled cross-over trial. Respirology (2019), in press. ( ${ }^{*}$ Thesis Study 5)

3. Fingleton, J., McKinstry, S., Pilcher, J., Weatherall, M., Beasley, R., $G$ Bardsley, G. Accuracy of transcutaneous carbon dioxide measurement for change over time. Respirology (2017) 22 (Suppl. 2), 18-100 ( ${ }^{*}$ Thesis Study 1a)

4. Bardsley, G., Pilcher, J., McKinstry, S., Shirtcliffe, P., Berry, J., Fingleton, J., Weatherall, M., Beasley, R. Oxygen versus air-driven nebulisers for exacerbations of COPD: a randomised controlled trial. BMC Pulm Med, (2018) 18(1): 157. ("Thesis Study 1b)

5. Fingleton, J., McKinstry, S., Baarsma, J., Aldington S., Armstrong, P., Nguyen, M., Cromhout, A., Weatherall, M., Beasley, R. Feasibility of NHF for Acute Hypercapnic Respiratory Failure in COPD. Respirology (2018) 23 (Suppl. 1), 104-215 (Thesis Study 4) 


\section{Thesis overview}

\section{Research aims}

The aims of this thesis are to investigate the physiological and clinical effects of NHF in COPD. After validating a method of measuring transcutaneous partial pressure of carbon dioxide $\left(\mathrm{P}_{\mathrm{t}} \mathrm{CO}_{2}\right)$, the following questions will be addressed:

- Is NHF able to reduce to reduce $\mathrm{P}_{\mathrm{t}} \mathrm{CO}_{2}$ in COPD and if so, what is the optimal flow rate and how does it compare to non-invasive ventilation?

- Is it feasible to undertake a clinical trial investigating the efficacy of domiciliary NHF use after an AECOPD?

- Is it feasible to undertake a clinical trial of the efficacy of NHF in COPD patients presenting to ED with AHRF?

- How effective and tolerable is a dual NHF/Non-invasive ventilation (NIV) device compared to a standard NIV device in patients with chronic respiratory diseases?

The overall aim is to provide robust evidence on which the clinical guidelines for the use of NHF therapy in COPD can be based.

\section{Outline of the thesis}

The introduction chapter demonstrates where NHF therapy may fit into the continuum of COPD management. COPD will first be introduced to outline the magnitude of the disease and understand the causes. An overview of key normal respiratory physiology topics will lead in to the pathophysiology of COPD, introducing specific areas relevant to the research questions and methods. The two COPD clinical problems addressed in this thesis are then reviewed - 1) AECOPD hospitalisations and preventing hospital readmissions, and 2) hypercapnic respiratory failure and management with NIV, referring back to the COPD pathophysiology from earlier sections.

Limitations of current management strategies in these 2 scenarios is highlighted and NHF is introduced as one potential solution. Evidence for its mechanism of action is reviewed to demonstrate how it may produce a clinical effect. The clinical effects observed in its more established use in hypoxaemic respiratory failure are first reviewed, leading on to a systematic review of NHF 
therapy application in COPD, illustrating the scarcity of evidence available and highlighting the knowledge gaps in this area.

The first two clinical trials (Studies 1a and 1b) are to validate the method of $\mathrm{P}_{\mathrm{t}} \mathrm{CO}_{2}$ monitoring - the primary outcome for three of the following five trials, and are presented in the appendix (Appendix II). The five clinical trials are then reported, each discussion and conclusion flowing onto and building the hypothesis for the next trial, before a final summary and discussion about the clinical application of NHF in COPD. 


\section{Introduction}

\subsection{Overview of Chronic Obstructive Pulmonary Disease}

"COPD is a common, preventable and treatable disease that is characterised by persistent airway symptoms and airflow limitation that is due to airway and/or alveolar abnormalities usually caused by significant exposure to noxious particles or gases." (Vogelmeier et al., 2017)

The airflow limitation referred to above in the Global initiative for chronic Obstructive Lung Disease (GOLD) definition is due to destruction of different components of the lungs, which involves:

- narrowing of the small airways, sometimes referred to as "obstructive bronchiolitis", and

- damage to the main gas-exchanging surfaces called alveoli and respiratory bronchioles, collectively referred to as the lung parenchyma.

The damage to the small airways impairs the lungs ability to clear sputum also. People with COPD present with chronic progressive breathlessness, cough, wheeze and chest tightness impairing their function in daily life - clinical manifestations of these pathological processes.

Traditionally, COPD was known as emphysema and/or chronic bronchitis and these terms are still often interchangeably used in clinical practice today. Emphysema is a term to describe the destruction of the lung parenchyma. Chronic bronchitis describes symptoms of persistent cough and sputum production for at least 3 months in each of two consecutive years. These terms no longer form part of the formal diagnostic criteria for COPD, which is based on symptoms, risk factor assessment and lung function testing (Vogelmeier et al., 2017). The unifying term of COPD was first thought to be coined by William Briscoe in 1965 when describing the common features he noticed between "emphysematous" and "bronchitic" patients he had encountered (Briscoe \& Nash, 1965).

\subsubsection{Prevalence of COPD}

COPD is a leading cause of mortality worldwide. In 2015, an estimated 3 million people died of COPD - the third leading, age-standardised cause of 
death behind ischaemic heart disease and cerebrovascular disease (Wang et al., 2016). Up to a third of COPD patients die of cardiovascular diseases, suggesting that COPD mortality, directly or indirectly, is underestimated (Anthonisen et al., 2005; Calverley et al., 2007). Most (90\%) COPD deaths occur in low or middle income countries, demonstrating a clear socioeconomic inequality in COPD mortality (WHO, 2018).

Estimates of international COPD prevalence vary according to the diagnostic criteria used e.g. spirometry and/or COPD symptom surveys. Lung function naturally declines with age, and smoking is the main risk factor for COPD, therefore regional variations in population age and smoking rates affect prevalence estimates. The international Global Burden of Disease study in 2015 put the prevalence of COPD at 174 million cases (Soriano et al., 2017). Large systematic reviews and meta-analyses of spirometry-defined population-based studies report global COPD prevalence from 9.2\% (Halbert et al., 2006) to $11.7 \%$ (Adeloye et al., 2015).

In New Zealand, the estimated prevalence of COPD is higher at $14 \%$, highlighting the variation in the definition of COPD and heterogeneity in diagnosis and reporting (Shirtcliffe et al., 2007). Māori and Pacific people carry a higher burden of disease in New Zealand with increased hospitalisation rates (risk ratio $3.59,95 \% \mathrm{Cl} 3.46-3.74$ ) and are nearly 3 times more likely to die of COPD (risk ratio 2.94, 95\% Cl 2.68-3.22) (Robson, 2007; The Ministry of Health New Zealand., 2015).

COPD prevalence may well be higher than these global estimates as it is under-diagnosed in younger patients, those in low or middle income countries, those who have never smoked and those with a lower severity of symptoms (Buist et al., 2007; Duong et al., 2013; Lamprecht et al., 2015).

\subsubsection{Aetiology of COPD}

Development of COPD involves a complex interplay between genetic susceptibility and environmental influence (Rabe \& Watz, 2017). The main cause of COPD globally is tobacco smoking (Mannino \& Buist, 2007). Smoking is not the only explanation however, as fewer than $50 \%$ of heavy smokers will develop COPD during their lifetime, and COPD prevalence is as 
high as $16 \%$ in lifetime non-smokers (Rennard \& Vestbo, 2006). In the Burden of Obstructive Lung Disease study, 5.6\% of these non-smokers were classified as moderate to very severe COPD (Lamprecht et al., 2011).

The burning of biomass fuels indoors for cooking and heating in developing countries is another significant cause of COPD (Ezzati, 2005; Gan, et al, 2013; Zhou et al., 2014). An estimated 3 billion people worldwide burn substances such as wood, coal, animal dung and crop residues for heating and cooking, often in poorly ventilated areas, denoting this may become a central cause of increasing prevalence, as well as an area for potential huge gains as an intervention (Assad, et al, 2015). Inhalation of other noxious particles such as occupational dust and fume exposure and air pollution are other recognised risk factors.

Despite COPD trending towards a higher prevalence in males than females in the past (Adeloye et al., 2015), other factors more prominent in developing countries such as biomass fuel burning for heat and an increase in tobacco smoking in females may equalise the gender difference in the future. There is some evidence also that females are prone to a faster rate of lung damage from cigarette smoking (Foreman et al., 2011; Han et al., 2007; Lopez Varela et al., 2010; Sorheim et al., 2010).

Other risk factors for developing COPD include genetic factors such as alpha1 antitrypsin deficiency, (Silverman et al, 2009) the presence of asthma and airway hyper-reactivity (an exaggerated response of the airways to nonspecific stimuli resulting in airway obstruction), chronic bronchitis and recurrent chest infections, childhood lung insult or impairment in lung development, and socioeconomic status (Vogelmeier et al., 2017).

\subsection{Respiratory physiology and changes seen in COPD 1.2.1 Normal respiratory function and physiology}

The primary function of the lungs is to facilitate the exchange of oxygen and carbon dioxide between the atmosphere and the blood. Oxygen inhaled from the atmosphere diffuses from a high concentration in the alveoli to lower concentrations in the blood. The blood is contained in the surrounding capillary 
network and separated from the alveolar gas by a very thin alveolar membrane. The oxygenated blood returns to the left side of the heart and is pumped around the body to be used by tissues. Carbon dioxide is a by-product of this tissue metabolism, and de-oxygenated blood with increased carbon dioxide is returned to the right side of the heart and back to the lungs via the pulmonary arteries. Carbon dioxide then diffuses out of the capillary blood network surrounding the alveoli into the alveoli down a concentration gradient to be exhaled back into the atmosphere (Cloutier, 2007; West, 2012).

\subsubsection{Brief anatomy}

Figure 1-1 illustrates the progression from large to small airways in the lungs. The conducting airways, consisting of bronchi and bronchioles, transport air from the atmosphere into the gas exchanging parts of the lung - terminal bronchioles and alveoli. These parts contain the blood-gas interface and the pulmonary capillary bed.

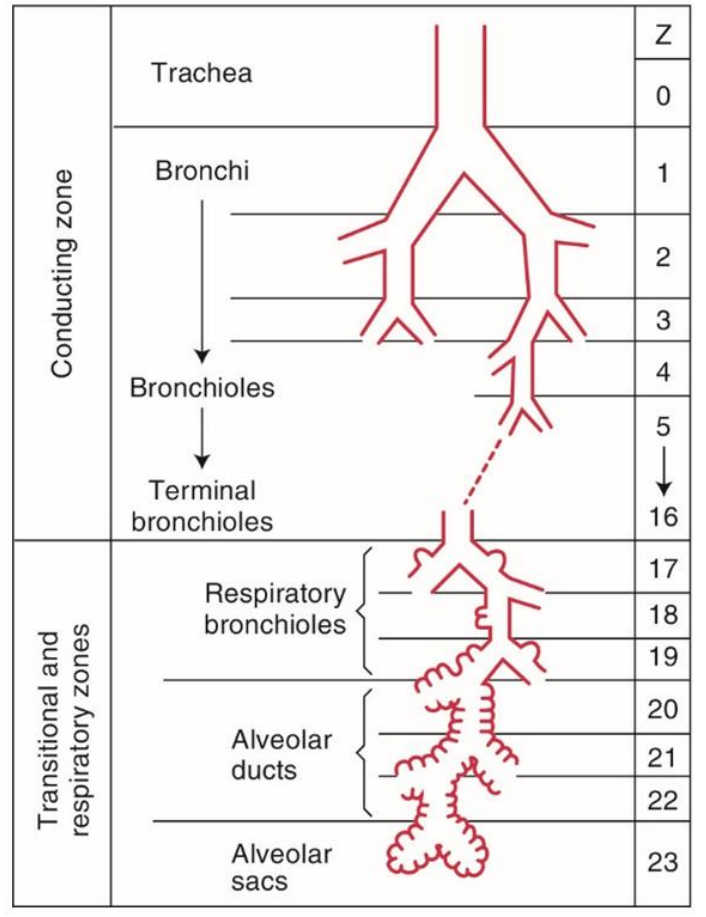

Figure 1-1 Schematic of human airways progression from the conducting to respiratory zones.

[Reproduced with permission from: Respiratory physiology: The essentials, John B West, $9^{\text {th }}$ edition, 2012, Wolters Kluwer. (WEST, 2012)]

The normal volume of inspired air is around $500 \mathrm{~mL}$ and is termed tidal volume $\left(\mathrm{V}_{\mathrm{T}}\right.$ ). A small amount of this, around $150 \mathrm{~mL}$ (or $2-3 \mathrm{~mL} / \mathrm{kg}$ body weight) will 
remain in the conducting airways, not contributing to gas exchange (John B. West, 2012). Collectively, the volume of the conducting airways such as the pharynx, trachea and bronchi down to the region where gas diffusion takes place is called the anatomical dead space and is largely fixed in size.

Alveolar dead space is the volume of air within alveoli that are well ventilated and should be contributing to gas exchange but are not because of reduced perfusion. Physiological dead space $\left(\mathrm{V}_{\mathrm{D}}\right)$ is the sum of anatomical and alveolar dead spaces. This variable dead space is highly relevant to this research as alveolar dead space often increases in COPD and other chronic lung diseases, compromising the efficiency of ventilation and gas exchange.

\subsubsection{Gas transport and exchange}

\subsection{OXYGEN}

Oxygen is primarily transported in the blood combined with haemoglobin in red blood cells, forming oxyhaemoglobin. A small amount of oxygen is dissolved in the blood also, around $0.3 \mathrm{~mL} / 100 \mathrm{~mL}$ of arterial blood (West, 2012). Oxygen moves from the alveoli to the blood then to the tissues by moving down a pressure gradient. The inhaled air gives alveoli a relatively high partial pressure of oxygen $\left(\mathrm{PAO}_{\mathrm{A}}\right)$ of around $100 \mathrm{mmHg}$, allowing it to diffuse into deoxygenated mixed venous blood in the capillary network surrounding the alveoli where the partial pressure is around $40 \mathrm{mmHg}$ (Cloutier, 2007). In the tissues, "unloading" of oxygen from oxyhaemoglobin is illustrated in the oxygen-haemoglobin dissociation curve below (Figure 1-2) which describes the relationship between the partial pressure of arterial oxygen $\left(\mathrm{P}_{\mathrm{a}} \mathrm{O}_{2}\right)$ and saturation of haemoglobin - the so-called "haemoglobin affinity for oxygen" (West, 2012). 


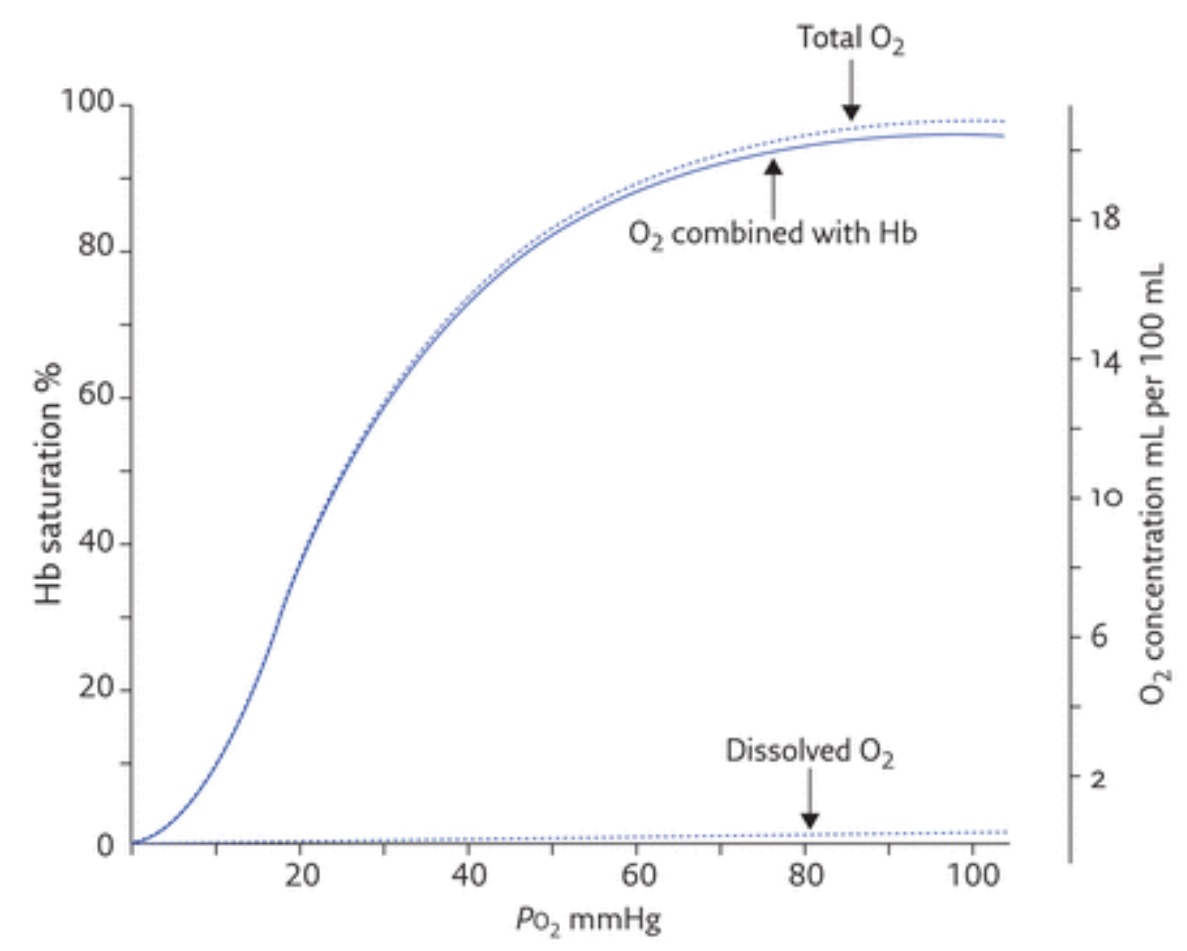

Figure 1-2 Oxygen-haemoglobin dissociation curve

[Reproduced with permission of the @ ERS 2018. European Respiratory Review Mar 2017, 26 (143) 160099; DOI: 10.1183/16000617.0099-2016. (Collins et al, 2015)]

The sigmoidal shape of the curve has the following favourable properties to allow transfer of oxygen:

a) Flat upper portion - a reduction in $\mathrm{PO}_{2}$ in the alveoli, to $50-60 \mathrm{mmHg}$, will not greatly affect the haemoglobin saturation with oxygen therefore maintaining its concentration in the blood delivered to the tissues. The $\mathrm{PO}_{2}$ remains high in the alveoli while the $\mathrm{O}_{2}$ is being transferred into the capillaries, maintaining the pressure gradient and rapid diffusion.

b) Steep middle portion - large amounts of oxygen are diffused into the tissues. A small drop in $\mathrm{PO}_{2}$ will cause a large drop in haemoglobin saturation, or "unloading" of oxygen.

The oxygen-haemoglobin dissociation curve shifts right or left depending on certain conditions. A shift to the right occurs in the tissues with a rise in $\mathrm{PCO}_{2}$, decrease in $\mathrm{pH}$, or rise in temperature. This causes less affinity of haemoglobin for oxygen, therefore releasing more oxygen into the tissues. For example, Figure 1-2 illustrates a $\mathrm{PO}_{2}$ of $60 \mathrm{mmHg}$ correlates 
approximately with $92 \%$ of haemoglobin being saturated, but with a right-shift, the haemoglobin saturation is reduced as oxygen is released.

\subsection{CARBON DIOXIDE}

Carbon dioxide is transported in the blood in 3 forms: dissolved, as bicarbonate, and combined with haemoglobin (as de-oxyhaemoglobin) (West, 2012). A pressure gradient drives its diffusion, this time in the reverse direction to oxygen. Carbon dioxide rapidly diffuses (20 times faster than oxygen as carbon dioxide is much more soluble) across the blood-gas membrane, meaning alveolar $\left(\mathrm{P}_{\mathrm{ACO}}\right)$ and arterial $\left(\mathrm{P}_{\mathrm{a}} \mathrm{CO}_{2}\right)$ partial pressures of carbon dioxide quickly equalise. This is also referred to as the steepness of the alveolar-arterial $(A-a)$ gradient. The removal of carbon dioxide is therefore highly dependent on alveolar ventilation which is described later.

The relationship between oxygen and carbon dioxide exchange is called the alveolar gas equation (John B. West, 2012):

$$
P_{A} O_{2}=P_{I} O_{2}-\frac{P_{A} \mathrm{CO}_{2}}{R}+F
$$

Where $\mathrm{PIO}_{2}$ is the partial pressure of inspired oxygen in $\mathrm{mmHg}, R$ is the "respiratory exchange" ratio determined by the $\mathrm{CO}_{2}$ production $/ \mathrm{O}_{2}$ consumption by the tissues, usually about 0.8 , and $F$ is a small correction factor (around $2 \mathrm{mmHg}$ ).

Hypoventilation is a reduction in respiratory rate and therefore will reduce $\mathrm{PaO}_{2}$ (and $\mathrm{P}_{\mathrm{A}} \mathrm{O}_{2}$ ) and increase $\mathrm{PaCO}_{2}$ (and $\mathrm{P}_{\mathrm{ACO}}$ ), as described by the alveolar gas equation. This is the process that occurs in alveolar hypoventilation.

\subsubsection{Ventilation and perfusion}

\subsection{ALVEOLAR VENTILATION}

The alveolar volume $\left(V_{A}\right)$ is the volume of inhaled air in a single breath which reaches the alveoli to be involved in gas-exchange. Accounting for the $V_{D}$ and $V_{T}$ described in 1.2.1.1, $V_{A}$ per inspiration in normal lungs is therefore $V_{T}$ $(500 \mathrm{~mL})$ minus $V_{D}(150 \mathrm{~mL})$ as per the equation:

$$
V_{A}=V_{T}-V_{D}
$$


When $\mathrm{V}_{\mathrm{T}}$ is multiplied by frequency $(f)$ of breathing (called respiratory rate) of, for example 15 breaths/minute, this gives a volume of $5250 \mathrm{ml} / \mathrm{min}$, and is termed the minute ventilation (MV):

$$
\mathrm{MV}=\mathrm{V}_{\mathrm{T}} \mathrm{x} f
$$

However, this includes the dead space volume. A more useful measure is to remove the dead space volume and is called the alveolar minute ventilation $\left(\dot{V}_{A}\right) . \dot{V}_{A}$ refers to the volume of atmospheric gas that enters the alveolar spaces per minute, therefore contributing to gas exchange, and equals:

$$
\begin{gathered}
\dot{\mathrm{V}}_{\mathrm{A}}=f \times \mathrm{V}_{\mathrm{A}} \\
\dot{\mathrm{V}}_{\mathrm{A}}=f \times\left(\mathrm{V}_{\mathrm{T}}-\mathrm{V}_{\mathrm{D}}\right) \\
\dot{\mathrm{V}}_{\mathrm{A}}=\dot{\mathrm{V}}_{\mathrm{E}} \times\left(1-\mathrm{V}_{\mathrm{D}} / \mathrm{V}_{T}\right)
\end{gathered}
$$

Where $\dot{V}_{E}$ is the volume of expired gas per unit time. Alveolar minute ventilation $\left(\dot{V}_{\mathrm{A}}\right)$ can therefore be increased by:

- Increasing respiratory rate $(f)$ and/or tidal volume $\left(\mathrm{V}_{\mathrm{T}}\right)$.

- Decreasing $V_{D}$ with an associated decrease in $V_{D} / V_{T}$ ratio.

\subsection{CARBON DIOXIDE IN ALVEOLAR VENTILATION}

Both $\dot{V}_{A}$ and $V_{A}$ are difficult to measure directly. However, as reported above, the steep A-a gradient for carbon dioxide causes rapid and complete diffusion of carbon dioxide between the alveoli and blood, therefore $\mathrm{P}_{\mathrm{ACO}} \mathrm{CO}_{2}$ and $\mathrm{PaCO}_{2}$ are nearly identical in healthy subjects.

$\mathrm{P}_{\mathrm{a}} \mathrm{CO}_{2}$ is simple to measure by sampling blood from an artery. In order to understand how $\mathrm{P}_{\mathrm{aCO}}$ is related to $\dot{\mathrm{V}}_{\mathrm{A}}$ and can be used as an indicator for $\dot{V}_{A}$, it must first be noted that $\mathrm{PaCO}_{2}$ is determined by the amount of $\mathrm{CO}_{2}$ produced during metabolism $\left(\mathrm{V}_{\mathrm{CO}}\right)$, the volume eliminated during ventilation $\left(\dot{\mathrm{V}}_{\mathrm{A}}\right)$ and a constant $\mathrm{K}(0.863)$ :

$$
P_{a} \mathrm{CO}_{2}=\frac{V C O 2 * K}{V_{A}}
$$

$\dot{\mathrm{V}} \mathrm{CO}_{2}$ is constant in a steady state, therefore the $\mathrm{PaCO}_{2}$ is inversely proportional to $\dot{\mathrm{V}}_{\mathrm{A}}$ : 


$$
\mathrm{PaCO}_{2} \propto \frac{1}{\dot{V}_{A}}
$$

The importance of this relationship is that if $\dot{\mathrm{V}}_{\mathrm{A}}$ is halved, $\mathrm{P}_{\mathrm{a}} \mathrm{CO}_{2}$ will double. Also, substituting back in the $\dot{V}_{A}$ derivatives from above gives:

$$
\mathrm{PaCO}_{2} \propto \frac{1}{f\left(V_{T}-V_{D}\right)}
$$

When rearranged to substitute in $\dot{\mathrm{V}}_{\mathrm{E}}$, the volume of expired gas per unit time, the dead space to tidal volume ratio $\left(\mathrm{V}_{\mathrm{D}} / \mathrm{V}_{\mathrm{T}}\right)$ emerges:

$$
\mathrm{PaCO}_{2} \propto \frac{1}{\dot{V}_{E}\left(1-\frac{V_{D}}{V_{T}}\right)}
$$

That is, the $\mathrm{PaCO}_{2}$ depends on the ratio of $\mathrm{V}_{\mathrm{D}}$ to $\mathrm{V}_{T}$. Because $\mathrm{P}_{\mathrm{a}} \mathrm{CO}_{2}$ and $\dot{\mathrm{V}}_{\mathrm{A}}$ are inversely related, $\mathrm{P}_{\mathrm{a}} \mathrm{CO}_{2}$ will rise if there is:

1. A decrease in $\dot{V}_{A}$ - which can be due to a decrease in respiratory rate (f) and/or $\mathrm{V}_{\mathrm{T}}$

2. An Increase in $V_{D}$, which increases the $V_{D} / V_{T}$ ratio.

Ventilation of the physiological dead space, $V_{D}$, is now discussed in more detail.

\subsection{PHYSIOLOGICAL DEAD SPACE VENTILATION}

Another method of relating $\mathrm{P}_{\mathrm{C}} \mathrm{CO}_{2}$ and the $\mathrm{V}_{\mathrm{D}} / \mathrm{V}_{T}$ ratio is by direct measurement. $V_{D}$ varies in COPD, compared to the fixed anatomical dead space volume, and helps to explain the pathophysiological changes in COPD. Bohr described a method to measure physiological dead space volume (Bohr, 1891) and the Bohr equation explains the relationship between $V_{D} / V_{T}$ and $\mathrm{PaCO}_{2}$, as follows:

$$
\frac{\mathrm{V}_{\mathrm{D}}}{\mathrm{V}_{\mathrm{T}}}=\frac{\mathrm{PaCO}_{2}-\mathrm{P}_{\mathrm{E}} \mathrm{CO}_{2}}{\mathrm{PaCO}_{2}}
$$

Where $\mathrm{P}_{\mathrm{E}} \mathrm{CO}_{2}$ is mixed expired gas. $A$ normal $\mathrm{V}_{\mathrm{D}} / \mathrm{V}_{\mathrm{T}}$ ratio is around 0.3 meaning the physiological dead space contributes about a third of the tidal volume and can rise to around 0.8 in COPD.

As mentioned earlier, $\mathrm{P}_{\mathrm{A}} \mathrm{CO}_{2}$ is difficult to measure and is near identical to $\mathrm{P}_{\mathrm{a}} \mathrm{CO}_{2}$ in healthy individuals. Ignoring the negligible amount of atmospheric 
carbon dioxide, all of the expired carbon dioxide comes from alveolar gas, rather than the dead space in a healthy individual. Bohr's equation was modified by Enghoff (Enghoff, 1938) to make the Bohr-Enghoff equation, explaining the relationship between $\mathrm{V}_{\mathrm{D}}$ and $\mathrm{P}_{\mathrm{a}} \mathrm{CO}_{2}$ :

$$
\frac{\mathrm{V}_{D}}{\mathrm{~V}_{T}}=\frac{\mathrm{P}_{a} \mathrm{CO}_{2}-\mathrm{P}_{E} \mathrm{CO}_{2}}{\mathrm{P}_{a} \mathrm{CO}_{2}}
$$

These relationships above and in 1.2.1.3.2 illustrate the key role of alveolar minute ventilation and physiological dead space volume in regulating $\mathrm{PaCO}_{2}$.

\subsection{VENTILATION-PERFUSION MATCHING}

In section 1.2.1.2, the movement of oxygen and carbon dioxide and their exchange in the lung was described. The "matching" of blood flow, or "perfusion", to ventilation is essential to understand abnormalities in gas exchange. "Mismatching" of ventilation $(\dot{V})$ and perfusion $(\dot{Q})$ is the most common cause of respiratory failure (O'Donnell \& Parker, 2006; Petersson \& Glenny, 2014; West \& Wagner, 1998). The ratio of ventilation to perfusion is denoted $\dot{V} / \mathbf{Q}$. To understand this ratio, it is helpful to consider a theoretical ideal alveolar-capillary unit having a ratio of 1 .

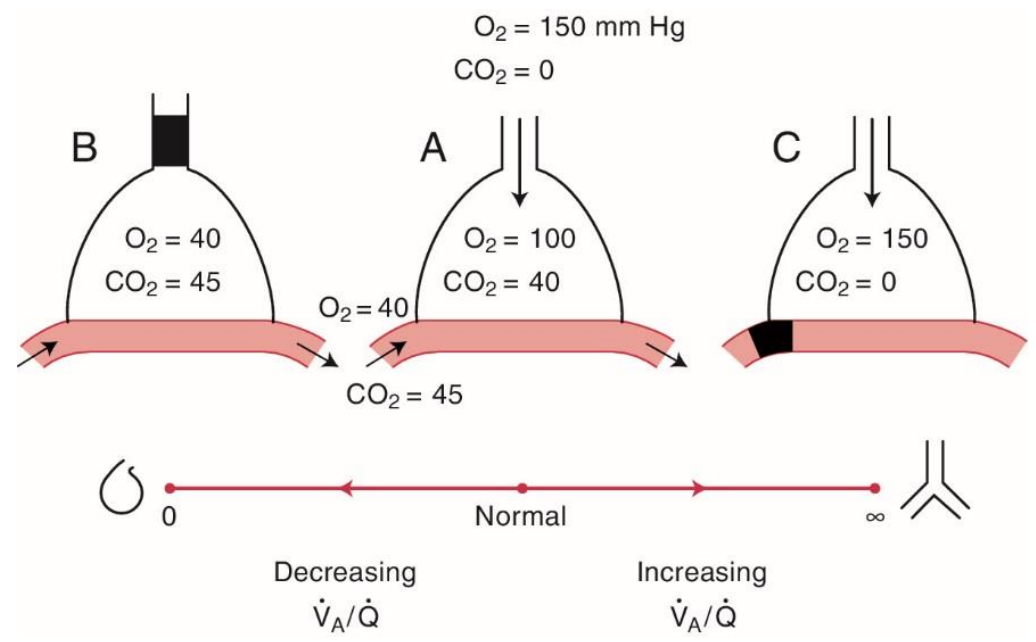

Figure 1-3 Illustration of different scenarios for ventilation/perfusion (V/Q) matching. Scenario $A: \dot{V} / \dot{Q}=1$; scenario $B: \dot{V} / \mathbf{Q}<1$; scenario $C: \dot{V} / \dot{Q}$ >1. V: ventilation, $Q$ : perfusion

[Reproduced with permission from: Respiratory physiology: The essentials, John B West, $9^{\text {th }}$ edition, 2012, Wolters Kluwer. (WEST, 2012)]

Figure 1-3 above illustrates different scenarios of both $\dot{V} / \dot{Q}$ matching $(A)$ and mismatching ( $\mathrm{B}$ and $\mathrm{C}$ ) in the alveolar-capillary unit: 
A) $\dot{V} / \dot{Q}=1$ : Ideal state - The alveolus is well ventilated so PO2 from the atmosphere can enter the alveolus, diffuse into blood via a well perfused capillary. Oxygen will travel down the pressure gradient from high $\mathrm{P}_{\mathrm{A}} \mathrm{O}_{2}=100$ to low $\mathrm{P}_{\mathrm{a}} \mathrm{O}_{2}=40$

B) $\dot{V} / \dot{Q}<1$ : Air is prevented from entering the alveoli therefore ventilation is impaired but perfusion remains the same. The $\mathrm{PAO}_{2}$ and $\mathrm{PACO}_{2}$ in the alveolar unit quickly become the same as in the capillary with $\mathrm{PaO}_{2}=40, \mathrm{PaCO}_{2}=45$. The extreme case of this is if the deoxygenated capillary blood doesn't enter the pulmonary circulation at all, travelling from the right side to left side of the heart - a so called "shunt".

C) $\dot{V} / \dot{Q}>1:$ In this case, there is a reduction in perfusion preventing adequate blood flow but ventilation remains the same. In the absence of carbon dioxide returning from the blood, $\mathrm{P}_{\mathrm{ACO}} \mathrm{CO}_{2}$ becomes 0 and $\mathrm{P}_{\mathrm{A}} \mathrm{O}_{2}$ continues to rise. This is the equivalent to the alveolar dead space described above.

The $\dot{V} / \dot{Q}$ ratio and the relationship with $\mathrm{PO}_{2}$ (x-axis) and $\mathrm{PCO}_{2}$ (y-axis) is illustrated in Figure 1-4 below.

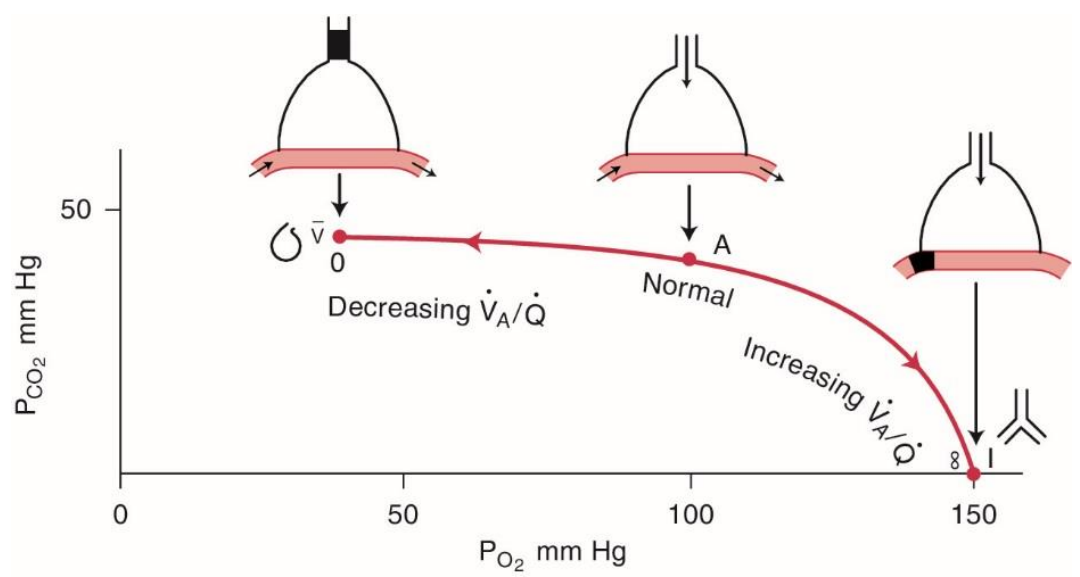

Figure 1-4 Relationship between changes in the ventilation/perfusion (V/Q̈) ratio and $\mathrm{PaO}_{2}$ and $\mathrm{PaCO}_{2}$.

$\dot{V}$ : ventilation, $\dot{Q}$ : perfusion, $P_{A} C \mathrm{O}_{2}$ : alveolar partial pressure of carbon dioxide, $P_{A} C_{2}$ : alveolar partial pressure of oxygen.

[Reproduced with permission from: Respiratory physiology: The essentials, John B West, $9^{\text {th }}$ edition, 2012, Wolters Kluwer. (WEST, 2012)] 


\subsubsection{5 $\mathrm{PaCO}_{2}$ AND RENAL ACID:BASE REGULATION}

The above described the relationships between volumes of air and dead space, partial pressures of carbon dioxide and oxygen, and blood supply.

There is further regulation of carbon dioxide, however, as changing alveolar ventilation to either remove or retain carbon dioxide tightly controls the $\mathrm{pH}$ of blood. $\mathrm{pH}$ is the negative logarithm of hydrogen ion $\left(\mathrm{H}^{+}\right)$concentration. A lowered pH (acidosis) can be caused by a "metabolic acidosis" from elevated levels of ketone bodies (ketoacidosis), for example, or a "respiratory acidosis" from a raised $\mathrm{PaCO}_{2}$ which will be the focus of this section.

Carbon dioxide affects $\mathrm{pH}$ as it easily dissolves in blood to form carbonic acid $\left(\mathrm{H}_{2} \mathrm{CO}_{3}\right)$ which dissociates into $\mathrm{H}_{+}$and bicarbonate $\left(\mathrm{HCO}^{-}\right)$as follows:

$$
\mathrm{CO}_{2}+\mathrm{H}_{2} \mathrm{O} \rightleftharpoons \mathrm{H}_{2} \mathrm{CO}_{3} \rightleftharpoons \mathrm{H}^{+}+\mathrm{HCO}_{3}{ }^{-}
$$

A rise in carbon dioxide will therefore cause a rise in carbonic acid, making the blood more acidic - a decrease in $\mathrm{pH}$ below the normal range of 7.35-7.45.

Peripheral chemoreceptors are located in the aortic and carotid bodies and detect changes in $\mathrm{PaO}_{2}, \mathrm{PaCO}_{2}$ and $\mathrm{pH}$. The brain is exquisitely sensitive to $\mathrm{pH}$ change which is detected by central chemoreceptors located in the medulla, a part of the brainstem. A pH in the range 7.35 to 7.45 is essential for normal organ function (Nattie \& $\mathrm{Li}, 2012$ ). A sustained high $\mathrm{PaCO}_{2}$ and respiratory acidosis will rapidly cause an intracellular acidosis in all cells in the body and has serious consequences for organ systems, primarily on the following:

- Cardiovascular system:

- Increased sympathetic tone causing tachycardia

- Peripheral vasodilation

- Increased cardiac output and blood pressure

- Arrhythmias e.g. Prolonged $Q T_{c}$ interval

- Neurological system:

- Cerebral vasodilation causing increased cerebral blood flow and raised intracranial pressure 
- Central nervous system depression and a decreased level of consciousness

- Lowered seizure threshold

These neurological effects will present clinically as disorientation, acute confusion, headache and possibly seizures and can be life-threatening (Davidson et al., 2016; Pilcher et al, 2013).

The response to a lowered $\mathrm{pH}$ from the peripheral and central chemoreceptors is to feedback to the lungs to change ventilation by increasing MV (increased respiratory rate and/or tidal volume) as described earlier, and increasing exhalation of carbon dioxide to return the $\mathrm{pH}$ to normal. $\mathrm{A}$ rising $\mathrm{PaCO}_{2}$ is therefore the primary stimulus to breathe.

Bicarbonate, a weak base, will also be increased in response to the lowered $\mathrm{pH}$ and acts as a "buffer" for acidic conditions. Bicarbonate concentration is regulated by the kidneys. The rising $\mathrm{P}_{2} \mathrm{CO}_{2}$ and reduced $\mathrm{pH}$ alters renal tubular function, leading to the excretion of more hydrogen ions in the urine and the generation of new bicarbonate normalising the $\mathrm{pH}$ despite the carbon dioxide retention. This process is called renal compensation and explains why people with chronic hypercapnia will usually have a blood $\mathrm{pH}$ in the normal range.

The Davenport diagram below (Figure 1-5) illustrates the relationship between $\mathrm{PaCO}_{2}, \mathrm{HCO}_{3}{ }^{-}$and $\mathrm{pH}$. It is termed a base excess if there is an increase in bicarbonate concentration and a base deficit if there is a reduction. 


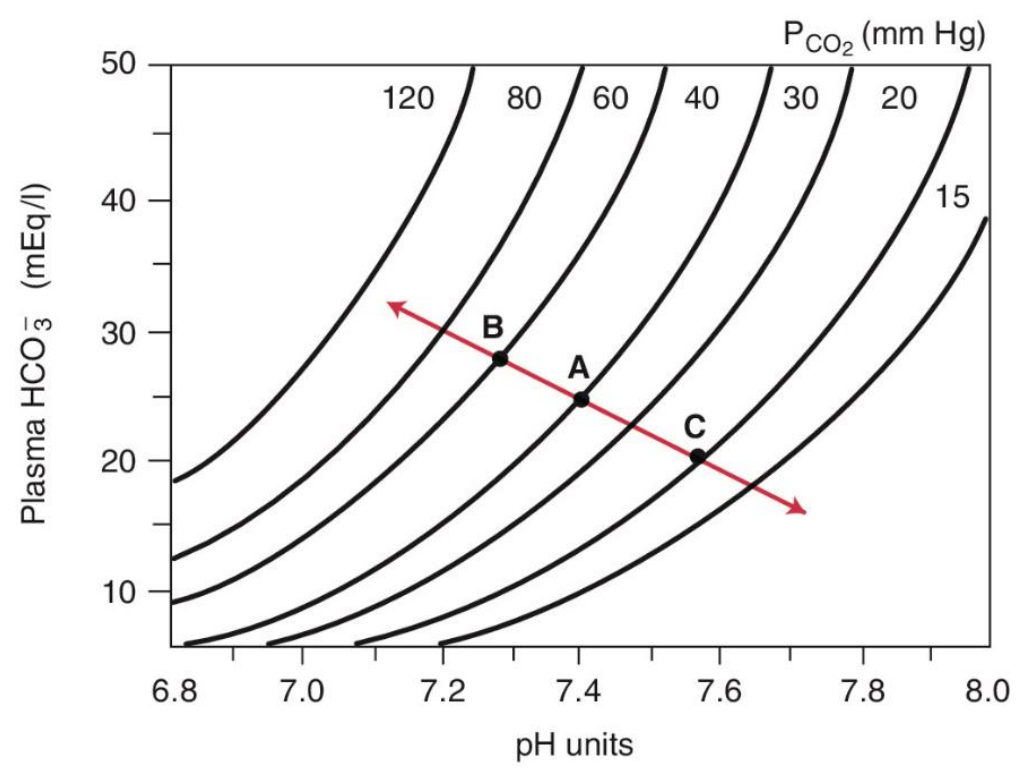

Figure 1-5 Davenport diagram. Plots of plasma bicarbonate concentration [HCO3-] versus blood $\mathrm{pH}$ at various $\mathrm{PaCO}_{2}$ values. Point $\mathrm{A}$ represents the normal state. A buffer line B-A-C shows the response to either an increase (point $\mathrm{B}$ ) or a decrease (point $\mathrm{C}$ ) in $\mathrm{PCO}_{2}$.

[Reproduced with permission from: Respiratory physiology: The essentials, John B West, $9^{\text {th }}$ edition, 2012, Wolters Kluwer. (WEST, 2012)]

\subsubsection{Pathophysiology of COPD and respiratory failure}

Structural changes occur in COPD due to inflammation of the lung parenchyma, larger airways and vasculature in response to irritation from noxious stimuli, leading to the hallmark functional change of incompletely reversible airflow limitation (Rabe \& Watz, 2017). The small airways remodel and narrow and some may even be completely destroyed, causing a reduction in the forced expiratory volume in 1 second (FEV1) (McDonough et al., 2011). Figure 1-6 illustrates the loss of elastin alveolar attachments, resulting in small airway collapse and inadequate expiration of alveolar gas. The nature of Airway-parenchymal forces of interdependence are weakened (Paré \& Mitzner, 2012). As a result, gas remains trapped in the small airways and the lungs remain hyperinflated in both the static phase (causing a reduction in inspiratory capacity at rest) and dynamic (during exercise) phase. The symptoms of dyspnoea and wheeze correlate with these structural changes (O'Donnell, 2006). 


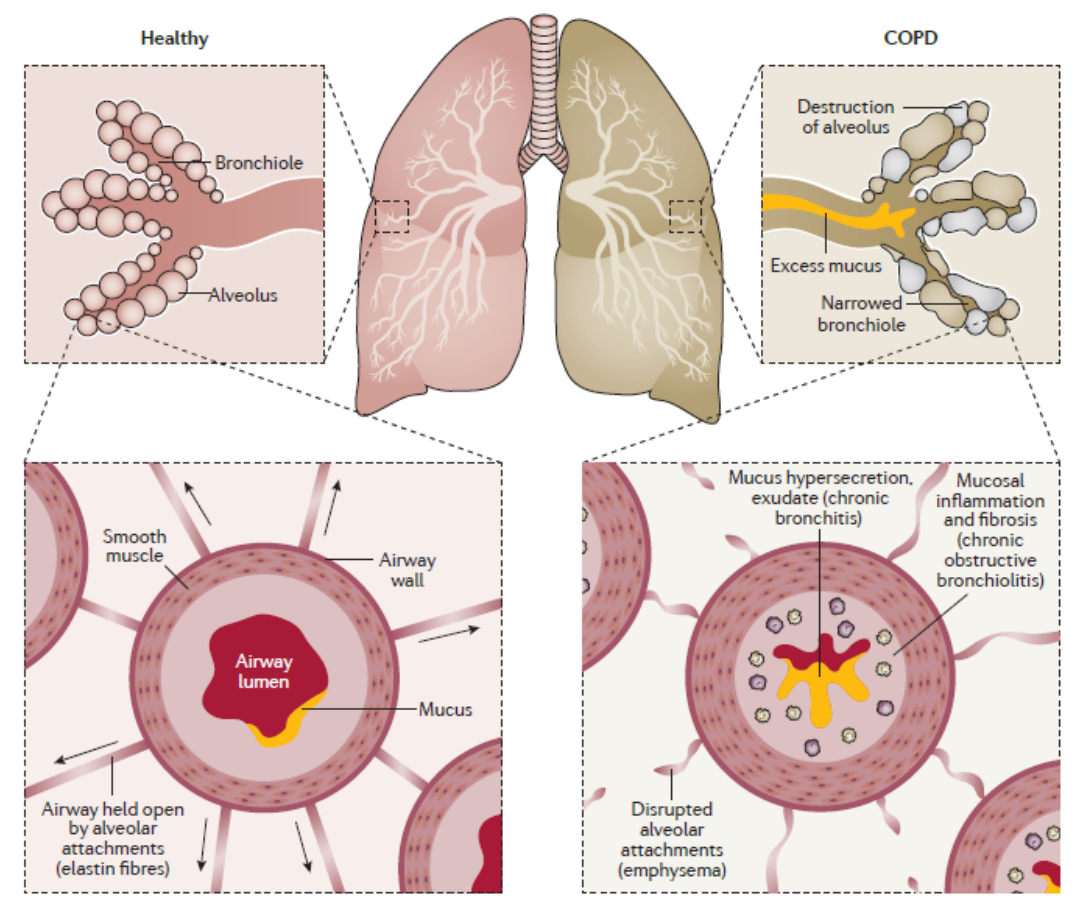

Figure 1-6 Illustration of mechanisms causing airflow limitation in COPD.

[Reprinted by permission from Springer Customer Service Centre GmbH: Springer Nature; Nature reviews disease primers; Chronic obstructive pulmonary disease (C) (BARNES ET AL., 2015)]

Injury to the airway epithelial cells sets off a cascading inflammatory response, characterised by: a) an increase in goblet cells and submucosal gland hyperplasia, both increasing mucous production, and b) altered repair of the small conducting airways, manifesting as chronic bronchitis (Rabe \& Watz, 2017). Cough is a common feature of chronic bronchitis and $30 \%$ of COPD patients at diagnosis have a productive cough (Vogelmeier et al., 2017).

Respiratory failure can develop as a consequence of these structural changes and signifies progression of the disease.

\subsubsection{Respiratory failure in COPD}

$\dot{V} / \dot{Q}$ mismatch is the most common cause of clinically important respiratory failure (Calverley et al, 2004; Petersson \& Glenny, 2014). Respiratory failure can be an abnormality of only $\mathrm{P}_{\mathrm{a}} \mathrm{O}_{2}$, or both $\mathrm{P}_{\mathrm{a}} \mathrm{O}_{2}$ and $\mathrm{P}_{\mathrm{a}} \mathrm{CO}_{2}$ as summarised in Table 1-1.Hypoxaemic (type 1) respiratory failure is caused by a $\dot{V} / \dot{Q}$ mismatch predominantly as a result of increased physiological shunting and 
distribution of blood to areas with lower $\dot{V} / \dot{Q}$ ratio (Calverley, 2003). Some examples are: a) an obstruction of a blood vessel in the lung e.g. pulmonary embolus or b) blood shunting away from areas of lung infection e.g. pneumonia.

Hypercapnic (type 2) respiratory failure is due to reduced ventilation (V) causing the $\dot{\mathrm{V}} / \mathrm{Q}$ mismatch. In COPD, there is obstruction of airflow into the alveoli causing a reduction in alveolar ventilation, called alveolar hypoventilation, leading to a reduced $\dot{V} / \dot{Q}$ ratio. Alveolar hypoventilation will decrease the $\mathrm{PaO}_{2}$ and increase $\mathrm{PaCO}_{2}$ according to the alveolar gas equation described earlier.

COPD patients may also have only hypoxaemic respiratory failure, as explained above by perfusion of under ventilated (low $\dot{V} / \dot{Q}$ ) regions (Petersson \& Glenny, 2014) however the focus of this thesis will be on hypercapnic respiratory failure, in order to address the critical treatment required. While hypoxaemic respiratory failure is relatively straightforward to treat, with supplemental oxygen, the tendency for COPD patients to develop hypercapnic respiratory failure leading to significant acidosis is a complex clinical scenario carrying an increased risk of death.

Acidosis may or may not be present in hypercapnic respiratory failure, depending on the degree of renal compensation (described in 1.2.1.3.5), the speed of $\mathrm{PaCO}_{2}$ rise and the adaptive ability of the ventilatory mechanisms, but is a life-threatening situation.

Hypercapnic respiratory failure is also seen in other medical conditions, such as obesity hypoventilation syndrome, obstructive sleep apnoea and neuromuscular disease. While the overall cause of hypercapnic respiratory failure in these conditions is predominantly due to the same mechanism of alveolar hypoventilation, the underlying mechanisms differ. 
Table 1-1 An overview of hypoxaemic and hypercapnic respiratory failure. Normal ABG values and the changes in ABG values are shown for each scenario.

\begin{tabular}{|c|c|c|c|}
\hline Respiratory status & $\mathrm{PaO}_{2}$ & $\mathrm{PaCO}_{2}$ & $\mathrm{HCO}_{3}^{-}$ \\
\hline Normal & $60-100 \mathrm{mmHg}$ & $35-45 \mathrm{mmHg}$ & $22-26 \mathrm{mmol} / \mathrm{L}$ \\
\hline $\begin{array}{l}\text { Hypoxaemic (Type 1) } \\
\text { respiratory failure }\end{array}$ & $\begin{array}{l}\text { Low } \\
\text { (hypoxaemia) }\end{array}$ & normal & Normal \\
\hline 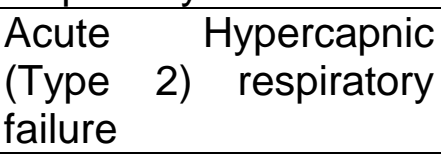 & $\begin{array}{l}\text { Low } \\
\text { (hypoxaemia) }\end{array}$ & $\begin{array}{l}\text { High } \\
\text { (hypercapnia) }\end{array}$ & $\begin{array}{l}\text { Normal } \\
\text { high }\end{array}$ \\
\hline $\begin{array}{lr}\text { Chronic } & \text { Hypercapnic } \\
\text { (Type } & 2 \text { ) } \\
\text { failure } & \end{array}$ & $\begin{array}{l}\text { Normal or } \\
\text { Low } \\
\text { (hypoxaemia) }\end{array}$ & $\begin{array}{l}\text { High } \\
\text { (hypercapnia) }\end{array}$ & High \\
\hline
\end{tabular}

$\mathrm{P}_{\mathrm{a}} \mathrm{O}_{2}$ : arterial partial pressure of oxygen, $\mathrm{P}_{\mathrm{a}} \mathrm{CO}_{2}$ : arterial partial pressure of carbon dioxide, $\mathrm{HCO}_{3}$ : bicarbonate concentration.

\subsubsection{Oxygen-induced hypercapnia}

Section 1.2.2 described respiratory failure due to the underlying COPD disease process; however hypercapnia can also occur as a result of supplemental oxygen therapy. This is known as oxygen-induced hypercapnia, and has been well demonstrated in patients with COPD (Cameron et al, 2012; Steer et al, 2010) and in a other conditions such as obesity hypoventilation syndrome and asthma (Wijesinghe et al, 2012; Wijesinghe et al, 2011).

Guidelines recommend oxygen therapy is administered to correct hypoxaemia in COPD and to carefully titrate flows to maintain oxygen saturations between $88-92 \%$, avoiding the risks of both hypoxaemia and oxygen induced hypercapnia (Cameron et al., 2012; O’Driscoll et al, 2017; Yang et al., 2017). These recommendations are reinforced by a landmark randomised controlled trial $(R C T)$ of patients with AECOPD, where high concentration oxygen therapy compared with titrated oxygen therapy resulted in a 2.4-fold increased risk of death (Austin et al, 2010).

Patients with more severe hypoxemia at presentation are at higher risk of $\mathrm{P}_{\mathrm{a}} \mathrm{CO}_{2}$ rise (O'Driscoll et al., 2017). The rise in $\mathrm{P}_{\mathrm{a}} \mathrm{CO}_{2}$ from high flow oxygen administration is postulated to come from the following mechanisms: 
- the release of hypoxic vasoconstriction

- an increased Haldane effect

- a reduction in hypoxic drive to breathe

- absorption atelectasis

There remains conflicting evidence for the relative contribution from all of these mechanisms and in fact, evidence for and against a mechanism such as a reduction in hypoxic drive to breathe. Overall, there is likely to be some contribution from each of these mechanisms, resulting in worsening $\dot{V} / \dot{Q}$ mismatch.

\subsection{RELEASE OF HYPOXIC VASOCONSTRICTION}

In response to hypoxaemia, pulmonary blood vessels contract (vasoconstrict) to move blood away from poorly ventilated lung to better ventilated lung, increasing the $\dot{V} / \dot{Q}$ ratio back towards normality - a process termed Hypoxic vasoconstriction, and in local lung areas is effective in normalising the low $\dot{V} / \dot{Q}$ ratio. If the lungs are then flooded with high concentrations of oxygen, the vasoconstriction effect is released with more blood now perfusing the same poorly ventilated lung tissue, lowering the $\dot{V} / \dot{Q}$ ratio even further. The $\dot{V} / \dot{Q}$ ratio remains low and carbon dioxide diffusing across the alveolar-capillary membrane is not matched by alveolar ventilation, causing $\mathrm{PaCO}_{2}$ to increase.

\subsection{INCREASED HALDANE EFFECT}

The Haldane effect describes the affinity haemoglobin has for carbon dioxide at different concentrations of oxygen. At low concentrations, deoxyhaemoglobin has a high affinity for carbon dioxide. At high oxygen concentrations, oxyhaemoglobin has a low affinity for carbon dioxide. Deoxyhaemoglobin is a better proton acceptor than oxyhaemoglobin and takes up more carbon dioxide at the level of the tissues, causing more $\mathrm{H}^{+}$to be produced according to the buffer equation in section 1.2.1.3.5 above. Carbon dioxide transport back to the lungs is improved, enabling efficient expiration. However, if high concentrations of oxygen are given in this scenario, more carbon dioxide will be released into the blood and $\mathrm{PaCO}_{2}$ will rise. The Haldane effect accounts for about $25 \%$ of the total $\mathrm{PaCO}_{2}$ increase due to oxygen administration (Abdo \& Heunks, 2012). 


\subsection{REDUCTION IN HYPOXIC DRIVE TO BREATHE}

As described above in 1.2.1.3.5, a rising $\mathrm{PaCO}_{2}$ is the usual physiological stimulus to drive ventilation. There is evidence to suggest people with COPD who have chronic hypercapnia become more reliant on peripheral chemoreceptors detecting hypoxia (decreased $\mathrm{PaO}_{2}$ ) to drive hyperventilation, rather than a rising $\mathrm{PaCO}_{2}$ - a so-called "hypoxic drive" (Robinson et al, 2000). Administering high concentrations of oxygen to correct the hypoxia in these patients would reduce this hypoxic drive therefore reduce the stimulus to breathe. Instead of expiring the carbon dioxide, the reduction in MV causes carbon dioxide retention and the $\mathrm{PaCO}_{2}$ rises. There is conflicting evidence for this mechanism however, with another study of high flow oxygen in COPD showing no change in $M V$, instead identifying an increased $V_{D} / V_{T}$ as the main contributor to hypercapnia (Aubier et al, 1980).

\subsection{ABSORPTION ATELECTASIS}

Atmospheric gas is $78 \%$ nitrogen, making it the dominant gas rather than oxygen $(21 \%)$. When atmospheric air is inhaled, the poorly blood-soluble nitrogen remains in high concentration in the alveoli. If high concentrations of oxygen are delivered, the nitrogen is "washed out" by the oxygen and oxygen moves down the concentration gradient into arterial blood, collapsing the volume of the alveoli - called absorption atelectasis. Ventilation is further impaired and the $\dot{V} / \dot{Q}$ ratio is reduced.

The key pathophysiological drivers in acute and chronic respiratory failure in COPD are 1) dynamic lung hyperinflation, and 2) expiratory flow limitation $(E F L)$. The influence of these two features differ slightly in the acute and chronic settings.

\subsubsection{Pathophysiology of AHRF}

Worsening EFL in AECOPD is a consequence of increased airways resistance from bronchospasm, mucosal oedema and thick sputum production (O'Donnell \& Parker, 2006). Expiration is prolonged (increased expiratory time, $T_{E}$ ) in order to overcome the EFL, meaning end-expiratory lung volume is increased. The relationship between end-expiratory lung volume and total lung capacity as a ratio is a marker of dynamic hyperinflation and has been 
shown to correlate with high $\mathrm{P}_{\mathrm{a}} \mathrm{CO}_{2}$ levels in severe COPD (O'Donnell et al, 2002) leading to AHRF.

The rapid, shallow breathing adopted in AECOPD reduces the $T_{E}$ and hyperinflates the lungs more (increased dynamic hyperinflation) - an adaptive response to lessen the fatigue on the already loaded respiratory muscles (Calverley, 2003).

Inspiratory muscle fatigue contributes to AHRF in AECOPD (O'Donnell \& Parker, 2006). The increased airways resistance requires a large increase in inspiratory pressures, up to $22 \mathrm{~cm} \mathrm{H}_{2} \mathrm{O}$ (mean $13.5 \mathrm{~cm} \mathrm{H}_{2} \mathrm{O}$ ), compared to 2$3 \mathrm{cmH}_{2} \mathrm{O}$ in a healthy individual, driven by muscles that are already loaded (Broseghini et al., 1988). Intrinsic positive end-expiratory pressure (iPEEP) refers to this increased pressure that a person must overcome before being able to produce inspiratory airflow (Calverley, 2003).

Overcoming a high iPEEP accounts for nearly a third of the increased work of breathing required during an exacerbation (O'Donnell \& Parker, 2006). In the stable condition of static hyperinflation, the accessory muscles are well conditioned but in the face of an exacerbation with reduced inspiratory time $\left(\mathrm{T}_{\mathrm{I}}\right)$ and high respiratory rates producing an elevated $\mathrm{P}_{\mathrm{a}} \mathrm{CO}_{2}$ and reduced $\mathrm{pH}$, inspiratory muscle fatigue worsens AHRF (O'Donnell \& Parker, 2006).

Hypoxaemia in AECOPD results from a small degree of shunting, worsening the $\dot{V} / \dot{Q}$ mismatch (Calverley, 2003). In response to hypoxaemia, oxygen uptake is optimised by hypoxic vasoconstriction as described earlier.

Another adaptive mechanism in COPD was seen in the earlier relationship between $\dot{\mathrm{V}}_{\mathrm{A}}$ and $\mathrm{P}_{\mathrm{a}} \mathrm{CO}_{2}$ where the response to alveolar hypoventilation was to increase respiratory rate and tidal volume, hence minute ventilation. Established dynamic hyperinflation mechanically restricts the ability to increase $\mathrm{V}_{\mathrm{T}}$ however. The increasing volume of carbon dioxide produced $\left(\mathrm{V}^{\circ} \mathrm{O}_{2}\right)$ from the rising metabolic demands of the respiratory muscles adds to the necessity to remove more carbon dioxide which the system is unable to cope with (O’Donnell \& Parker, 2006).

Physiological dead space is increased in response to increasing $\mathrm{PaCO}_{2}$ according to the Bohr-Enghoff equation. Carbon dioxide is further retained, 
elevating $\mathrm{PaCO}_{2}$ (Calverley, 2003;West \& Wagner, 1998) and leading to a respiratory acidosis, a blood $\mathrm{pH}<7.35$, due to the mechanisms described earlier in Section 1.2.1.3.5.

Some of these compensatory mechanisms may be effective in a stable state but when the respiratory system is put under stress by an insult such as a viral exacerbation, they are not able to respond sufficiently to continue effective gas exchange, worsening hypercapnia and hypoxaemia (O'Donnell \& Parker, 2006).

\subsubsection{Pathophysiology of chronic HRF}

Most of the pathophysiological mechanisms of AHRF described above apply to chronic hypercapnic respiratory failure (CHRF) also. The difference is the slower rate of deterioration and the gradual adaptation to changes in lung structure and capacity to manage the dynamic hyperinflation and EFL.

Multiple exacerbations punctuate the time course of CHRF, each time affecting lung structure and function (Seemungal et al, 2000). The main compensatory mechanism that drives chronic carbon dioxide retention is chronic alveolar hypoventilation in order to avoid needing to increase inspiratory pressures and $\mathrm{V}_{\mathrm{T}}$, which would further overload already fatigued inspiratory muscles. Chronic gas trapping dictates that at end-expiratory lung volume, the lung (intrapulmonary) pressure does not return to zero (see Figure 1-11). A constant requirement to overcome iPEEP then develops making an increase in VT difficult (O'Donnell \& Parker, 2006). The high resistive and elastic inspiratory loads associated with hyperinflation are also a mechanical impediment (Roussos \& Koutsoukou, 2003). Dysfunctional, shallow breathing patterns have been established as contributors to chronic hypercapnia (Begin \& Grassino, 1991; Gorini et al., 1996).

Compensation in COPD can occur to a certain degree by increasing minute ventilation but due to the severity of their underlying disease this is a delicate condition prone to decompensate during an exacerbation. The most serious clinical manifestation is AECOPD requiring hospitalisation, and the ensuing acute hypercapnic respiratory failure, described in more detail in Chapter 1.3. 


\subsection{Important clinical problems in COPD}

In this section, the two critically important clinical problems in COPD addressed by this thesis are reviewed. Firstly, an overview of hospitalisation for AECOPD, an introduction to AHRF and management, discharge planning and factors leading to readmission to hospital are covered. Section 1.3.2 will then review HRF in more detail in both acute and chronic phases, evidence for NIV efficacy and its limitations.

\subsubsection{Hospitalisation for AECOPD}

An AECOPD is defined as a persistent worsening of dyspnoea, cough or sputum production beyond day-to-day variation, requiring an increase in maintenance medications or additional medication treatment (GarciaAymerich et al., 2003; O'Donnell et al., 2008). Some patients may suffer recurrent exacerbations, resulting in a worsening of symptoms and reduction in lung function that may be irreversible (Garcia-Aymerich et al., 2003).

\subsubsection{Causes of AECOPD}

The pathophysiology of AECOPD was reviewed in section 1.2.2 above. The causes of a change from a chronic, stable state to an acute decompensation may be infective or non-infective (Bafadhel et al., 2011; O'Donnell \& Parker, 2006). Infective exacerbations are triggered by viral and/or bacterial pathology. Non-infective exacerbations can be further divided into 1) eosinophilic or 2) "pauci-inflammatory" (limited inflammatory change) and are caused by environmental pollutants, such as cigarette smoke, inter-patient or day-to-day variability or other unknown factors (Bafadhel et al., 2011; O'Donnell \& Parker, 2006). There may be an infective/non-infective overlap and in both cases there is a worsening of symptoms such as dyspnoea, cough, sputum production and wheeze.

\subsubsection{Hospitalisation for AECOPD}

Mild cases of AECOPD may be managed in the community but moderate and severe acute exacerbations of COPD are a frequent cause for hospitalisation. In New Zealand there are around 12000 annual AECOPD admissions, accounting for $1.2 \%$ of all New Zealand hospital admissions (Milne \& Beasley, 2015). Māori and Pacific people are 3-4 times as likely to be admitted for AECOPD as non-Maori and non-Pacific (Milne \& Beasley, 2015). The 
estimated annual financial impact of total hospital AECOPD admissions was \$NZ59.6 million in New Zealand (Milne \& Beasley, 2015). In the United States, hospitalisations due to AECOPD cost \$US13.2 billion per year, a significant part of the overall estimated COPD-related costs of $\$ U S 50$ billion (Shah et al, 2016).

\subsubsection{Indications for hospitalisation}

Indications for AECOPD hospitalisation from the Thoracic Society of Australia and New Zealand (TSANZ) guideline (Yang et al, 2018) is presented in Figure 1-7 below and similar guidelines exist from the National Institute of Clinical Excellence (Royal College of Physicians of London, 2010).

- Inadequate response to ambulatory management

- Inability to walk between rooms when previously mobile

- Inability to eat or sleep because of dyspnoea

- Cannot manage at home even with home-care resources

- High risk comorbidity condition - pulmonary (e.g., pneumonia) or nonpulmonary

- Altered mental status suggestive of hypercapnia

- Worsening hypoxaemia or cor pulmonale

- Newly occurring arrhythmia

Figure 1-7 Indications for AECOPD hospitalisation.

[Reproduced with permission from: Yang et al, The COPD-X Plan: Australian and New Zealand Guidelines for the management of Chronic Obstructive Pulmonary Disease 2018 Version 2.53]

The list of indications identifies high risk patients and signifies factors associated with poor prognosis, including death, such as: respiratory acidosis, significant co-morbidities eg ischaemic heart disease, and co-existing complications such as pneumonia (Steer et al, 2012; Yang et al, 2018).

\subsubsection{In-patient management of AECOPD}

The initial AECOPD management depends on the clinical severity, and the presence of respiratory failure, determined from an arterial blood gas ( $A B G)$. An $A B G$ is a small sample of blood taken from puncturing an artery with a needle, usually the radial artery, and allows analysis of the two important blood gases in respiratory failure $-\mathrm{PaO}_{2}$ and $\mathrm{P}_{\mathrm{a}} \mathrm{CO}_{2}$, as well as $\mathrm{pH}$ and several other 
metrics. ABG analysis alongside the clinical condition indicates the severity of respiratory failure (i.e. acidosis) and is defined and managed according to guidelines. A summary of the TSANZ and BTS/ICS guidelines for acute hypercapnic and hypoxaemic respiratory failure is presented in Table 1-2 and Table 1-3 respectively (Davidson et al., 2016; Yang et al., 2017).

Table 1-2 Management of acute hypercapnic (Type 2) respiratory failure.

\begin{tabular}{|c|c|}
\hline ABG result and clinical condition & Management \\
\hline $\begin{array}{l}\text { Severe: } \\
\mathrm{pH}<7.25 \mathrm{PaCO}_{2}>45 \mathrm{mmHg} \\
\text { and likely reduced consciousness (as } \\
\text { measured by Glasgow Coma Scale (GSC) }\end{array}$ & $\begin{array}{l}\text { Consideration of Invasive Mechanical } \\
\text { Ventilation (IMV- endotracheal intubation } \\
\text { and mechanical ventilation) and Intensive } \\
\text { Care Unit (ICU) admission. If not appropriate } \\
\text { i.e. advanced directive in place or clinically } \\
\text { futile, then maybe a trial of NIV, or for } \\
\text { palliative care. }\end{array}$ \\
\hline $\begin{array}{l}\text { Moderate: } \\
\text { pH 7.25-7.34 } \mathrm{PaCO}_{2}>45 \mathrm{mmHg} \\
\text { and, clinically a respiratory rate >23 } \\
\text { breaths/minute. }\end{array}$ & $\begin{array}{l}\text { Optimal medical treatment, including titrated } \\
\text { oxygen therapy, as described below. If a } \\
\text { repeat ABG shows persisting respiratory } \\
\text { acidosis after } 30-60 \text { mins then for NIV, or if } \\
\mathrm{pH}<7.25 \text { then consideration of IMV. The } \\
\text { indications and evidence for NIV in AHRF is } \\
\text { comprehensively reviewed in section } 1.3 .3 \\
\text { below. }\end{array}$ \\
\hline
\end{tabular}

ABG: arterial blood gas, $\mathrm{P}_{\mathrm{a}} \mathrm{O}_{2}$ : arterial partial pressure of oxygen, $\mathrm{P}_{\mathrm{a}} \mathrm{CO}_{2}$ : arterial partial pressure of carbon dioxide, NIV: non-invasive ventilation.

In both situations above, $\mathrm{P}_{\mathrm{a}} \mathrm{O}_{2}$ will likely be $<60 \mathrm{mmHg}$, therefore controlled oxygen therapy is administered and titrated to maintain oxygen saturations between 88-92\% according to international guidelines (O'Driscoll et al., 2017; Yang et al., 2017), improving $\mathrm{PaO}_{2}$ to above $60 \mathrm{mmHg}$.

Table 1-3 Management of acute hypoxaemic (Type 1) respiratory failure.

\begin{tabular}{ll}
\hline ABG result & Management \\
\hline $\boldsymbol{p H} \geq 7.35$ (assuming no concomitant & Controlled oxygen therapy is administered \\
metabolic acidosis), $\mathrm{P}_{\mathrm{C}} \mathrm{CO}_{2} \mathbf{3 5 - 4 5 m m H g}$, & and titrated to maintain oxygen \\
$\mathrm{PaO}_{2}<60 \mathrm{~mm} \mathbf{m g}$ & $\begin{array}{l}\text { saturations between 88-92\% }\left(\mathrm{P}_{\mathrm{a}} \mathrm{O}_{2}>60\right. \\
\mathrm{mmHg}) .\end{array}$
\end{tabular}

ABG: arterial blood gas, $\mathrm{P}_{2} \mathrm{O}_{2}$ : arterial partial pressure of oxygen, $\mathrm{P}_{2} \mathrm{CO}_{2}$ : arterial partial pressure of carbon dioxide.

Oxygen flows are started at 1 to $2 \mathrm{~L} / \mathrm{min}$ via nasal prongs. An oxygen flow rate of $4 \mathrm{~L} / \mathrm{min}$ is the upper limit of delivering oxygen via standard nasal prongs due to drying of nasal mucosa and discomfort, and if a patient requires higher flows they are switched to a Hudson face mask (O'Driscoll et al., 2017). 
A repeat $A B G$ should be checked within an hour to ensure improvement in $\mathrm{PaO}_{2}$ without worsening $\mathrm{PaCO}_{2}$ ie oxygen-induced hypercapnia, which may then require treatment for $\mathrm{AHRF}$ as above.

\subsubsection{Other standard management of AECOPD}

Optimal medical therapy for AECOPD is administered simultaneously and includes:

a. Short acting bronchodilators, which improve EFL by relaxing airway smooth muscle and dilating the airways.

- Salbutamol (short-acting beta agonist) and ipratropium (short acting muscarinic antagonist) via spacer or nebuliser. The dose administered and interval between doses are titrated to clinical response. If NIV is in place, nebulisers should be given during breaks in therapy to enhance efficacy (Davidson et al., 2016).

b. Glucocorticoids, which reduces local and systemic inflammation.

- Oral prednisone, or IV hydrocortisone if unable to take oral medication.

c. Antibiotics, if bacterial infection is suspected. Antibiotics have been shown to be effective if there is increased dyspnoea and increased, purulent sputum (Vogelmeier et al., 2017).

Once initiated, NIV is ideally administered semi-continuously for the first 24 hours with short breaks for feeding or taking oral medication and continued until there is normalisation of $\mathrm{pH}$ and $\mathrm{PaCO}_{2}$ and general improvement in a patient's condition, with the caveat that $\mathrm{PaCO}_{2}$ may not return to normal if there is underlying CHRF (Davidson et al., 2016). Weaning from NIV takes place with increasing breaks during the day and ongoing nocturnal use, usually over about 3 days.

\subsubsection{Discharge following hospital admission}

Discharge planning should be commenced within 24-48 hours of admission. Criteria for discharge, according to TSANZ guidelines (Yang et al, 2018), is shown in Figure 1-8. 
- The patient should be in a clinically stable condition and have had no parenteral therapy for 24 hours

- Inhaled bronchodilators are required less than four-hourly

- Oxygen delivery has ceased for 24 hours (unless home oxygen is indicated)

- If previously able, the patient is ambulating safely and independently, and performing activities of daily living

- The patient is able to eat and sleep without significant episodes of dyspnoea

- The patient or caregiver understands and is able to administer medications

- Follow-up and home care arrangements (eg, home oxygen, home-care, Meals on Wheels, community nurse, allied health, GP, specialist) have been completed.

Figure 1-8 Criteria for hospital discharge following an AECOPD.

[Reproduced with permission from: Yang et al, The COPD-X Plan: Australian and New Zealand Guidelines for the management of Chronic Obstructive Pulmonary Disease 2018 Version 2.53]

Effective discharge planning is essential to reduce the chances of requiring re-admission. A re-admission refers to a person who has been discharged from hospital after treatment for AECOPD but continues to experience symptoms at home and requires "re-admission" to hospital, implying failure to completely treat the index AECOPD. A readmission is a common occurrence in COPD, as well as some other chronic medical conditions (Elixhauser et al, 2006).

Readmission causes, risk factors and current interventions to reduce readmission rates are discussed in Section 1.3.1.10. The prevalence and costs of early readmissions is first addressed to outline the magnitude of the problem.

\subsubsection{Prevalence and costs of hospital readmissions}

A 30-day interval is commonly used to define an early "re-admission" to hospital, linking it with the index reason for admission, as opposed to a separate illness event.

New Zealand re-admission rates are between $6.7 \%$ and $8 \%$ (Milne \& Beasley, 2015; Pilcher et al., 2015). Approximately $14 \%$ are readmitted within 60 days of discharge (Pilcher et al., 2015). 
New Zealand's 30-day readmission rate is similar to the $7-9 \%$ rate in the United States (Elixhauser et al., 2006; Sharif et al, 2014), although other United States data have shown 30-day readmission rates as high as 18\% (with $59 \%$ of these inside the first 15 days) (Nguyen et al., 2014) and 22.6\% (Jencks, Williams, \& Coleman, 2009), possibly reflecting variation in data collection and hospital-record coding methods.

The readmission rate in a British cohort study was $13.4 \%$, including 34 patients who were responsible for 176 hospital admissions in the prior 3 years (Wilkinson et al, 2014). UK and European audits report the 90-day readmission rate at $31.4 \%$ and $35 \%$ respectively (Hartl et al., 2016; Price et al., 2006).

\subsubsection{Importance of readmissions}

The three important aspects of AECOPD readmissions are: 1) the large financial burden on healthcare systems, 2) some readmissions may be preventable and 3) patients who get readmitted have worse outcomes.

In the United States, readmission costs of the second hospital stay are estimated to be $18 \%$ higher for a primary COPD diagnosis and $50 \%$ higher for the readmission if COPD is a secondary diagnosis (Elixhauser et al., 2006). Preventing hospital readmissions, for any medical condition, in the USA has become a focus for the United States Patient Protection and Affordable Care Act (ACA or colloquially named "Obamacare") (Kocher \& Adashi, 2011).

The Medicare Payment Advisory Commission was set-up to streamline care for medicare patients. Medicare is the federal health insurance for people over 65 years of age and some younger people with disabilities. It comprises four main parts, with hospitalisation cover being the main part. The Commission declared in 2011 that hospital readmissions "remain prevalent, costly, and largely preventable" (Hackbarth \& Berenson, 2011). About 10-55\% of readmissions for AECOPD are thought to be preventable (Shah et al., 2016). The Medicare Hospital Readmission Reduction Program (HRRP) penalises hospitals in the US up to $3 \%$ on all Medicare revenues if they cannot meet targets to reduce all-cause 30-day readmission rates (Cms.gov/Medicare, 2016). Similar pecuniary penalties also exist in the United Kingdom and Germany (Kristensen et al, 2015). There has been some criticism of using 
high 30-day readmission rates as an indicator of poor hospital performance. Superior inpatient care resulting in a lower inpatient mortality rate, may lead to a higher proportion of readmissions (i.e. if the patient died in hospital then they can't be readmitted) therefore there exists a "competing risk of death" (Gorodeski et al, 2010; Krumholz et al., 2013).

Irrespective of the system's merits, reducing costly readmissions are a focus in a modern "value-based" healthcare system (Porter, 2010; Shah et al., 2016).

Readmissions contribute to increasing patient morbidity and are an indicator of disease severity (Shah et al., 2016). A priority is identifying why patients get readmitted, those at highest risk for readmission and then introducing interventions to reduce the risk of readmission.

\subsubsection{Why do COPD patients get readmitted to hospital?}

With clear criteria for hospital discharge after an AECOPD (Figure 1-8), it might be expected that patients leaving hospital are on the road to recovery but the question is why do between $10-20 \%$ of COPD patients get readmitted to hospital within 30 days?

Only half of readmissions are due to respiratory-related causes, and the remaining half of readmission causes varying greatly, from exacerbations of other co-morbidities eg congestive heart failure, to electrolyte disturbances (Shah et al, 2015).

Immediately post-discharge is a brittle period for AECOPD patients and it has been reported that $25 \%$ of patients had not returned to their pre-exacerbation peak expiratory flow rate by day 35 post-discharge (Seemungal et al., 2000). Alongside the precarious respiratory state, the general frailty that exists, possible social isolation and associated anxiety, any trigger which increases dyspnoea after discharge may set off a cascade of altered breathing patterns and worsening anxiety, the ensuing dynamic hyperinflation and expiratory flow limitation, which may lead to a further AECOPD as described in section 1.2.3.2. Seeking urgent medical attention is the first instinct in many cases and patients re-present to hospital. 
Patients are generally in a more pro-inflammatory state immediately postdischarge, and those who are more likely to further exacerbate will have persistent respiratory symptoms and raised inflammatory markers (Perera et al., 2007). A possible susceptibility phenotype of a COPD patient who frequently exacerbates exists which does not necessarily relate to the severity of disease, but includes a persistently elevated white cell count among other conditions (Hurst et al., 2010).

\subsubsection{Other factors contributing to COPD readmissions}

Other factors contributing to readmissions besides the respiratory-causes described above may be patient-related or hospital-related. Hospital-related factors imply a break-down in effective in-patient management including "premature" discharge from hospital and inadequate reconciliation of discharge medications (Auerbach et al., 2016; Benbassat \& Taragin, 2000). Between $10-55 \%$ of readmissions are reported to be preventable and are largely related to these hospital-related factors (Shah et al., 2016).

Patient-related factors can be called risk factors as they allow a stratification of risk based on individual conditions and can be divided into modifiable and non-modifiable categories. The distinction is vital as most of the factors are non-modifiable, making an intervention less likely to be successful.

Non-modifiable risk factors include male gender, non-Caucasian race, low socio-economic status, a longer length of hospital stay (Shah et al., 2015) and low FEV1.

Co-morbidities such as congestive heart failure, depression and anxiety, and general frailty (lyer et al., 2016; Singh et al, 2016) are associated with higher COPD readmission rates and while non-modifiable to some extent, care can be optimised to reduce their impact on the underlying respiratory function. Hypercapnia on discharge is potentially one risk factor associated with readmission that is modifiable (Bahadori et al., 2007) however the odds of hypercapnia causing further readmission are increased but to a small degree (odds ratio $1.04,95 \% \mathrm{Cl} 1.01$ to $1.08, \mathrm{P}=0.025$ ) (Garcia-Aymerich et al., 2001). 


\subsubsection{Current treatment options for reducing COPD hospital re-admission rates}

A variety of methods have been implemented in attempts to reduce hospital readmission rates and those with a reasonable evidence base are summarised below in Table 1-4.

Patient self-management plans are commonly used (including education such as smoking cessation, inhaler advice, awareness of disease, exercise and nutrition) and have been shown to reduce the index hospital AECOPD admission, but not re-admission (Zwerink et al., 2014).

This section has reviewed AECOPD hospitalisation, with a broad outline of management, and then focussed on the problem of hospital re-admissions, of which the causes can be modifiable or non-modifiable. With about half of readmissions due to respiratory causes, there is potential for an intervention to have a direct effect on reducing this rate. The clinical context of AHRF was also introduced in this section, following on from the earlier pathophysiology review. Section 1.3.2 will extend on the importance of AHRF and current treatment with NIV. 
Table 1-4 Evidence-based interventions to reduce COPD readmissions.

\begin{tabular}{|c|c|c|}
\hline Intervention & $\begin{array}{l}\text { Publications providing } \\
\text { evidence }\end{array}$ & Statistical summary \\
\hline $\begin{array}{l}\text { Inhaler device training } \\
\text { technique }\end{array}$ & $\begin{array}{l}\text { RCT of "Teach-to-goal" (TTG) } \\
\text { technique rather than "brief } \\
\text { instruction" } \quad(B I) \text { in } 120 \\
\text { patients. (Press et al., 2016) }\end{array}$ & $\begin{array}{l}\text { Reduction in ED visits } \\
\text { and/or hospitalisations } \\
\text { with TTG vs BI (9 } \\
\begin{array}{lll}{[17 \%]} & \text { vs. } 19 \quad[36 \%] \\
\text { respectively; } \mathrm{P}=0.03\end{array}\end{array}$ \\
\hline $\begin{array}{l}\text { Early outpatient follow- } \\
\text { up (within } 30 \text {-days of } \\
\text { discharge) }\end{array}$ & $\begin{array}{l}\text { (Gavish et al, 2015; Sharma et } \\
\text { al, 2010) }\end{array}$ & 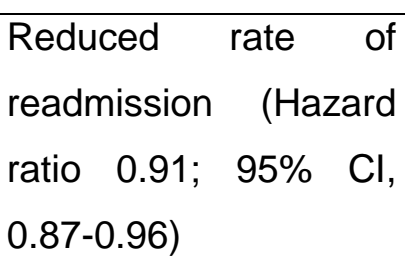 \\
\hline $\begin{array}{l}\text { Pulmonary } \\
\text { rehabilitation }\end{array}$ & $\begin{array}{l}\text { Systematic review and meta- } \\
\text { analysis of } 17 \text { studies ( } n=810) \\
\text { with pulmonary rehabilitation } \\
\text { commenced } 2 \text { days to } 2 \text { weeks } \\
\text { after exacerbation, } \\
\text { inpatient/outpatient/home care } \\
\text { duration between } 4 \text { days and } 6 \\
\text { months (Puhan et al, 2016). }\end{array}$ & $\begin{array}{l}\text { Reduced readmission } \\
\text { rate (odds ratio } 0.44 \text {, } \\
95 \% \mathrm{Cl} 0.21 \text { to } 0.91 \text { ). }\end{array}$ \\
\hline Discharge care bundles & $\begin{array}{l}\text { Systematic review and meta- } \\
\text { analysis of a combination of } \\
\text { ensuring inhaler technique, } \\
\text { education on self- } \\
\text { management, individually } \\
\text { tailored care plans for self- } \\
\text { management, pulmonary } \\
\text { rehab assessment/referral, } \\
\text { arranging outpatient follow-up } \\
\text { and smoking cessation } \\
\text { programme (Ospina et al., } \\
2017) \text {. }\end{array}$ & $\begin{array}{l}\text { Reduced readmission } \\
\text { rate (Risk Reduction } \\
0.8095 \% \text { Cl } 0.65 \text { to } \\
0.99 \text { ). }\end{array}$ \\
\hline
\end{tabular}

*Includes both asthma and COPD patients 


\subsubsection{Hypercapnic respiratory failure in COPD}

Hypercapnic respiratory failure predicts the overall severity of the COPD as well as the requirement for NIV (Davidson et al., 2016). Dividing COPD patients into the acuity of respiratory failure is useful because while the treatment is ultimately similar (with NIV), the clinical context is quite different as is the sequelae of the disease.

\subsubsection{Acute hypercapnic respiratory failure in COPD}

The British Thoracic Society (BTS)/Intensive Care Society (ICS) guideline for ventilatory management of AHRF in adults describes AHRF as:

"AHRF results from an inability of the respiratory pump, in concert with the lungs, to provide sufficient alveolar ventilation to maintain a normal $\mathrm{PaCO}_{2}$. Co-existent hypoxaemia is usually mild and easily corrected. Conventionally, a pH $<7.35$ and a $\mathrm{PaCO}_{2}>45 \mathrm{mmHg}(6.5 \mathrm{kPa})$ define acute respiratory acidosis and, when persisting after initial medical therapy, have been used as threshold values for considering the use of non-invasive ventilation. More severe degrees of acidosis, such as $\mathrm{pH}<7.25$, have been used as a threshold for considering provision of IMV" (Davidson et al., 2016).

The mechanism of physiological decompensation of AHRF in AECOPD was described earlier in Section 1.2. Around $20 \%$ of patients admitted with an AECOPD will have AHRF (Davidson et al., 2016; Hartl et al., 2016; Plant et al., 2001; Roberts et al., 2011).

\subsection{CLINICAL CONSEQUENCES}

Rapid rises in $\mathrm{P}_{\mathrm{a}} \mathrm{CO}_{2}$ lead to acidosis, and have serious clinical manifestations such as drowsiness, seizures and coma as described earlier. Respiratory acidosis is associated with an increased risk of death (Davidson et al., 2016; Pilcher et al, 2013). Outcomes in AHRF can be separated into short-term and long-term.

\subsection{SHORT-TERM OUTCOMES}

Hypercapnia and acidosis on admission is associated with an increased need for ventilation with NIV or IMV (Plant et al., 2001). Added to this, the degree 
of hypercapnia and acidosis are associated with increased odds of failing NIV treatment (Table 1-5).

Table 1-5 Odds of NIV failure with $\mathrm{PaCO}_{2}$ rise and pH fall. (Plant et al, 2001)

\begin{tabular}{llll}
\hline $\begin{array}{l}\boldsymbol{A B G} \\
\text { metric }\end{array}$ & Odds ratio of $\mathbf{N I V}$ failure & $95 \% \mathrm{Cl}$ & $\boldsymbol{P}$-value \\
\hline $\mathrm{PaCO}_{2}$ & 1.14 per $1 \mathrm{kPa}(7.5 \mathrm{mmHg}) \mathrm{P}_{\mathrm{a}} \mathrm{CO}_{2}$ rise above normal & 1.14 to 1.81 & $<0.01$ \\
\hline $\mathrm{pH}$ & 1.22 per $1 \mathrm{nmol} / \mathrm{L} \mathrm{H}^{+}$rise above normal & 1.09 to 1.37 & $<0.01$ \\
\hline
\end{tabular}

ABG: arterial blood gas, $\mathrm{P}_{\mathrm{a}} \mathrm{CO}_{2}$ : arterial partial pressure of carbon dioxide, NIV: non-invasive ventilation, $\mathrm{H}^{+}$hydrogen ion

The severity of hypercapnia and acidosis on admission was further investigated and related to failure with NIV, therefore requiring IMV (Confalonieri et al., 2005). Acidotic patients with a pH $<7.25$ on admission had twice the odds of needing IMV compared to normal pH (odds ratio 1.97, 9\%\% $\mathrm{Cl} 1.23$ to $3.15, \mathrm{P}=0.0046$ ). The odds are increased to over twenty times (odds ratio $21.02,95 \% \mathrm{Cl} 10.07$ to $43.87, \mathrm{p}<0.0001$ ) if the acidosis persisted at 2 hours after admission.

In-hospital mortality has been reported as 8-12\% (Groenewegen and Schols, 2003; Gunen et al., 2005; Roberts et al., 2011) of those with AHRF on presentation but range from as low as $2.5 \%$ (Patil et al., 2003) to as high as 24\% (Seneff et al, 1995) depending on the method of analyses (crosssectional versus prospective cohort respectively). In-hospital mortality rises to $33 \%$ in patients who develop AHRF during their admission, demonstrating a deterioration in their condition despite treatment and is an indicator of illness severity (Roberts et al., 2011).

Patients with AHRF who die in hospital, compared to those surviving, had a statistically significant higher mean $\mathrm{PaCO}_{2}$ of $55 \mathrm{mmHg}(\mathrm{SD}=5.6)$ versus 48.2 $(\mathrm{SD}=12.8)$, and lower mean $\mathrm{PaO}_{2}$ of $40.7(\mathrm{SD}=7.7)$ versus 48.3(SD=13.1)(Gunen et al., 2005).

\subsection{LONG-TERM OUTCOMES}

Hypercapnia on admission for AECOPD is a surrogate measure for disease severity and is linked clearly with long-term mortality once discharged from hospital (Almagro et al, 2002; Groenewegen and Schols, 2003; Gunen et al., 
2005). The relative risk for death at 1-year post-discharge after an AHRF episode was $1.18(95 \% \mathrm{Cl} 1.01-1.38, \mathrm{P}=0.04)$ in a large Dutch prospective study (Groenewegen and Schols, 2003) and an overall mortality rate at 1 year between $33-43 \%$ and 3 years of $49 \%$ (Connors et al., 1996; Gunen et al., 2005).

A higher $\mathrm{PaCO}_{2}$ at discharge was observed in those who died compared to those who were alive at 2 years (mean difference of $4.6 \mathrm{~mm} \mathrm{Hg}, \mathrm{SD}=1.6$ $\mathrm{mmHg}, \mathrm{P}<0.03$ ), illustrating an association between persistent hypercapnia and death after AHRF (Almagro et al, 2002).

Further support is provided for the association between persistent hypercapnia and death in a study by Costello and colleagues (Costello et al., 1997). After a $20 \%$ in-hospital AECOPD mortality rate, 68 patients were followed after hospital discharge for 5 years. Normocapnic (called 'type 1') patients were normocapnic on both admission and discharge. Type 2.1 patients were hypercapnic on admission but reverted to normocapnia on discharge, and those who were hypercapnic on admission and remained hypercapnic on discharge were termed type 2.2. Figure 1-9 shows type 2.2 patients had a significantly worse 5 year survival than type 2.1 , (11\% vs $28 \%$, $p<0.05)$ whereas type $1(33 \%)$ was similar to type 2.1 (not statistically significant). 


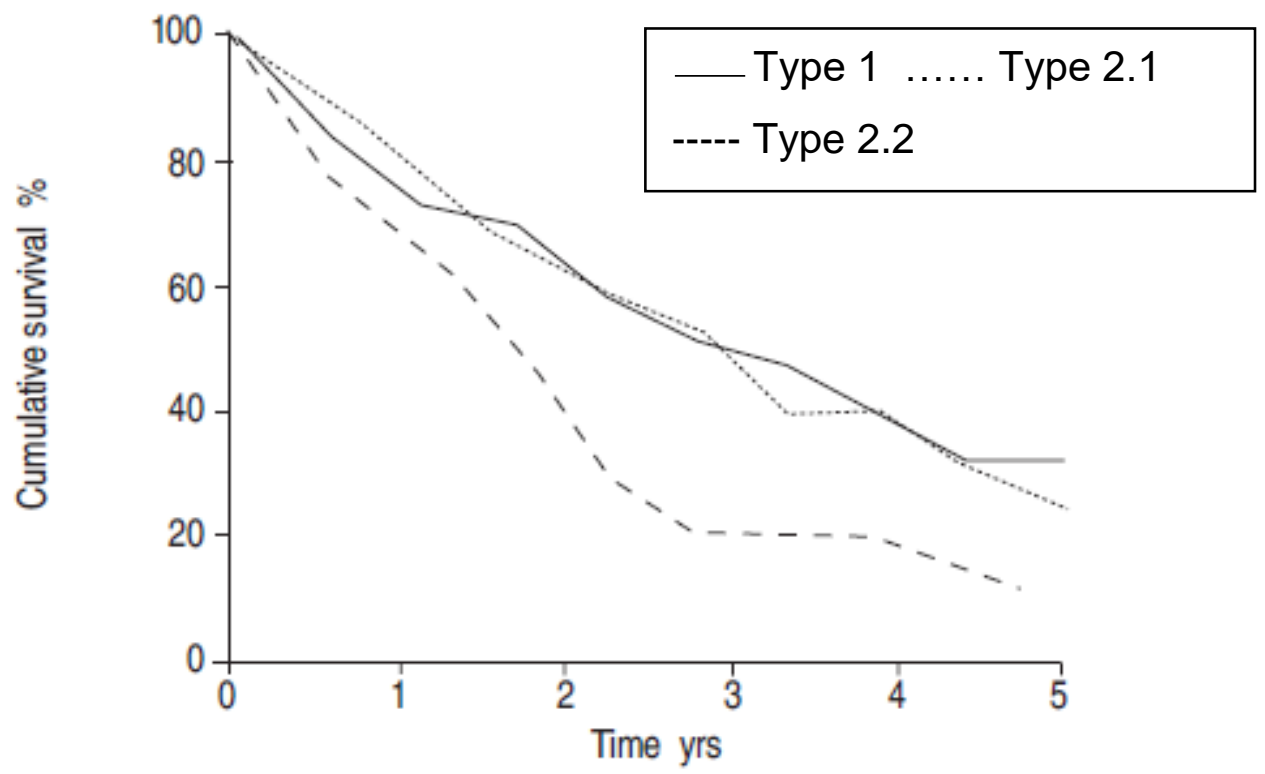

Figure 1-9 Kaplan-meier survival curves showing the influence of hypercapnia on 3 different categories of hypercapnic patients. Type 2.2 survival was significantly worse than types 1 and 2.1

[Reprinted from: The American Journal of Medicine, 102 (3), Richard Costello,Paul Deegan,Michael Fitzpatrick,Walter T. McNicholas, Reversible hypercapnia in chronic obstructive pulmonary disease: A distinct pattern of respiratory failure with a favorable prognosis, 239-244, @ 1997), with permission from Elsevier.]

Interestingly, only $24 \%$ of type 2.1 progressed to type 2.2 on long-term followup and a similar $18 \%$ reverted to type 1 normocapnia on subsequent admissions, demonstrating a distinct category of transient hypercapnia with an ability to avoid the deterioration to chronic hypercapnia, which has a worse prognosis. Not surprisingly, type 2.2 patients had worse lung function $(26.6 \%$ FEV1 percent predicted versus $32.7 \%$ in type 2.1 , although the difference was non-significant) and a higher percentage of cor pulmonale (right heart failure secondary to pulmonary disease). The type 2.2 group had progressed to chronic hypercapnic respiratory failure.

\subsubsection{Chronic hypercapnic respiratory failure in COPD}

There are no internationally recognised guidelines for the diagnosis and management of chronic hypercapnic respiratory failure (CHRF). The definition is similar to AHRF - that is, hypercapnia with or without hypoxaemia - but with the important difference being the progressive decline in gas exchange over time likely due to the mechanisms discussed in section 1.2.3.2. Chronic 
compensation for persistent hypercapnia is likely to have occurred, therefore acidosis will not normally be a feature of stable CHRF. The evolution of CHRF may be linked to one or more episode(s) of AECOPD with AHRF or follow a trajectory of slow deterioration without frequent acute exacerbations (Hurst et al., 2010).

Chronic hypoxaemia may be corrected with long-term oxygen therapy (LTOT), which involves people receiving low flows of supplementary oxygen for prolonged periods such as 16-20 hours/day, and has been shown to have a mortality benefit (Cranston et al,2005; Lacasse et al, 2018). TSANZ guidelines recommend LTOT use in COPD patients when: a) $\mathrm{PaO}_{2} \leq 55 \mathrm{mmHg}$, or b) $\mathrm{PaO}_{2}$ $\leq 59 \mathrm{mmHg}$ with evidence of pulmonary hypertension, right heart failure or polycythaemia (Yang et al., 2017). Caution must be taken with LTOT flow-rate prescription to correct hypoxaemia but to avoid oxygen-induced hypercapnia that has the serious consequences described earlier.

\subsection{PREVALENCE}

People with severe (GOLD stage 3) or very severe (GOLD stage 4 - the most severe stage) COPD are most likely to have chronic hypercapnia, therefore prevalence data from these cohorts are more useful than from the general COPD population. With no firm definition of CHRF, prevalence data vary and are indeed scarce, especially as trials with home NIV have focussed on patients that remain hypercapnic after discharge from hospital with AHRF, rather than patients who are chronically hypercapnic but in a stable state. Preliminary data from the "HOme Vent" registry set-up for this purpose - to measure the prevalence of $\mathrm{CHRF}$ in the outpatient setting - report 65 (28.1\%) of the 231 patients recruited had $\mathrm{PaCO}_{2} \geq 45 \mathrm{mmHg}$ - 26 patients in GOLD stage 3 and 39 in GOLD stage 4 (Dreher et al., 2018).

\subsection{CLINICAL CONSEQUENCES}

Mortality is the ultimate clinical consequence of CHRF. However, COPD is a progressive, incurable disease, and a proportion of people who develop CHRF may live in this state for an extended period of time. Quality of life (QOL) and symptom burden therefore become two other important clinical outcomes to assess, especially in the context of how effective a therapeutic intervention is. $\mathrm{QOL}$ is objectively measured in COPD with validated questionnaires such as 
the St Georges Respiratory Questionnaire, covering domains such as symptom burden, impact on activities and psycho-social impacts (Jones et al, 1992).

Symptoms can be measured with scores such as the BORG dyspnoea score - a self-reported discrete scale of breathlessness from 0 (no breathlessness) to 10 (maximal breathlessness)(Borg, 1982). If an intervention is effective but doesn't improve QOL or reduce symptoms, then adherence is less likely and real world efficacy will be limited.

Multivariate survival analyses from large prospective cohort studies have demonstrated hypercapnia as being an independent predictor for mortality in CHRF (Foucher, 1998; Soler-Cataluña et al., 2005; Steer et al., 2010).

In an observational study of 252 COPD patients receiving LTOT, survival analysis, adjusted for life expectancy, showed the mean observed 5-year survival was $41.5 \%$ (Foucher, 1998). Hypercapnia was the only independent predictor for mortality, having a relative risk of 1.97 (95\% Cl 1.16 to 3.34$)$ compared to patients without hypercapnia. Hypercapnia resulted in an adjusted hazard ratio of death of $1.07(95 \% \mathrm{Cl} 1.02$ to $1.12, \mathrm{P}=0.006)$ in 304 stable, male COPD patients followed for 5 years after AHRF (Soler-Cataluña et al., 2005). Age, hospitalization, co-morbidity and LTOT use were other notable predictors for mortality in this model.

While the mortality statistics may be difficult to improve, interventions targeted at improving symptom burden may be effective (Carone et al., 2007; Nishiyama et al., 2005).

\subsubsection{Current treatment options for hypercapnic respiratory failure in COPD}

Treatment of AHRF and CHRF share a common goal of providing positive pressure support to the airways to improve ventilation, clearance of $\mathrm{CO}_{2}$ and restoration of acid/base balance. Non-invasive ventilation, through the use of bi-level pressure support, is an effective treatment modality to achieve these goals and recommended in AHRF by TSANZ, BTS/ICS, ATS/ERS and GOLD guidelines (Davidson et al., 2016; Rochwerg et al., 2017; Vogelmeier et al., 2017; Yang et al., 2017). 
$\mathrm{NHF}$ is the central focus of this research and may also reduce hypercapnia and evidence for this is presented in Section 1.4.5. NIV already has a proven ability in reducing hypercapnia and improving outcomes in COPD. Study 4 in Chapter 1 directly compares NHF and NIV with respect to $\mathrm{P}_{\mathrm{a}} \mathrm{CO}_{2}$ change and the evidence for NIV use in HRF is now reviewed.

\subsubsection{What is non-invasive ventilation and how does it work?}

NIV is a form of positive pressure ventilation without an artificial airway, delivering assisted breaths through a mask interface to enable safe correction of the pathophysiological mechanisms of HRF (Antonelli et al., 2005; Jasmer et al,. 1997). Air is forced through a nasal and/or mouth interface and is connected to a positive pressure ventilator by tubing (Kelly et al., 2015). A variety of interfaces exist such as oro-nasal, full face, nasal mask or nasal pillows (Figure 1-10).

\subsection{BACKGROUND}

Section 1.3.2 has summarised the high morbidity and mortality associated with hypercapnic respiratory failure in COPD. Establishing non-invasive ventilation as an effective treatment modality to improve COPD outcomes was ground breaking in the 1990s and early 2000s, especially in the setting of AHRF. NIV was found to have many advantages over Invasive mechanical ventilation (IMV) which was the existing method of improving gas exchange. IMV involves endotracheal intubation, and NIV reduces the known risks of IMV such as tracheal injury causing stenosis (Torres et al., 1990), the need for sedation, inability of patients to swallow or talk while intubated and increased risks of infections such as nosocomial pneumonia (Nourdine et al., 1999) and sinusitis (McCulloch \& Bishop, 1991). NIV demonstrated a definite mortality benefit over IMV, producing significant improvements in dyspnoea and gas exchange in the setting of an acute exacerbation (Bott et al., 1993; Osadnik et al., 2017). 


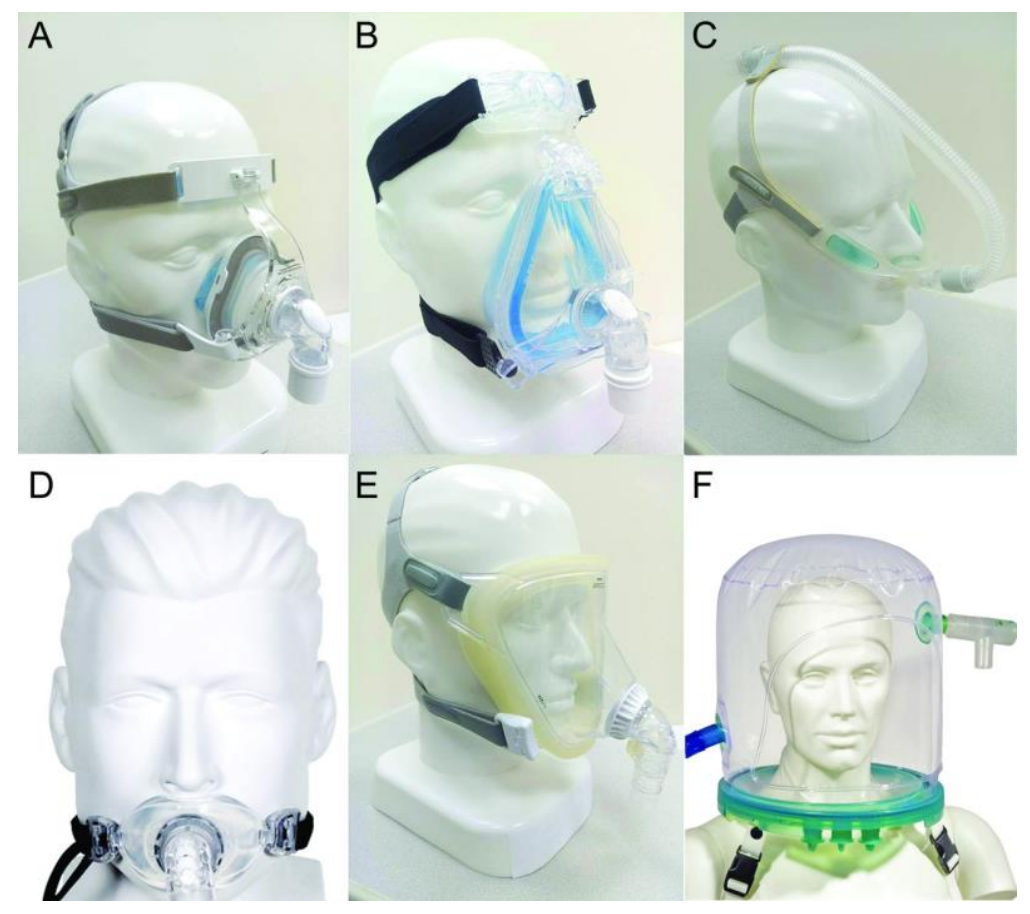

Figure 1-10 Different types of Non-Invasive Ventilation interfaces.

A: nasal mask, B: oro-nasal mask, C: nasal pillows, D: oral mask, E: total face mask, and F: helmet.

[Republished with permission of Daedalus Enterprises Inc, from "Choosing the Proper Interface for Positive Airway Pressure Therapy in Subjects With Acute Respiratory Failure",(BAHAMmAM ET AL, 2017), 63 (2), () 2018; permission conveyed through Copyright Clearance Center, Inc.]

\subsection{HOW DOES NIV WORK?}

"Bi-level" support is the form of NIV used in hypercapnic respiratory failure and is often referred to as BiPAP (Bi-level Positive Airways Pressure - a trade name for a Respironics bi-level device) or VPAP (variable positive airways pressure). "Bi-level" refers to the cycling of pressures between a higher inspiratory positive airway pressure (IPAP), and lower expiratory positive airway pressure (EPAP). Resistance is detected in the patient's breathing to trigger the delivery of pressurised air, and the pressure difference between IPAP and EPAP is the level of ventilator assistance or Pressure Support (PS).

There are different modes of bi-level support. In the acute setting, spontaneous/timed ( $\mathrm{S} / \mathrm{T})$ mode is most commonly used. In $\mathrm{S} / \mathrm{T}$ mode, the IPAP is triggered by an inspiratory effort however there is a "back-up" rate set to ensure the patient gets the required number of breaths/minute if they are not breathing spontaneously. Other modes are: a) Spontaneous mode, when 
the device triggers IPAP when an inspiratory effort is detected, and b) Timed Mode, when IPAP/EPAP cycling is purely device-triggered and the patient has no ability to set their own respiratory rate.

As well as being able to adjust IPAP and EPAP settings, other adjustable settings are $\mathrm{FiO}_{2}$, minimum respiratory rate, trigger sensitivity and inspiratory time (I-time) in order to target therapy to the patient's condition.

\subsection{PATHOPHYSIOLOGICAL MECHANISMS OF ACTION OF NIV}

NIV improves gas exchange through two main mechanisms that ultimately improve alveolar ventilation as a result of increased tidal volume and alveolar minute ventilation. $\mathrm{P}_{\mathrm{a}} \mathrm{O}_{2}$ is increased, the $\mathrm{P}_{\mathrm{A}} \mathrm{O}_{2}-\mathrm{P}_{\mathrm{a}} \mathrm{O}_{2}$ difference is decreased, and $\mathrm{P}_{\mathrm{a} C}$ is reduced. $\dot{\mathrm{V}} / \mathrm{Q}$ ratios are unlikely to be affected (Brochard et al., 1990; Kelly et al., 2015; O’Donnell \& Parker, 2006).

The first mechanism of action of NIV is offloading the fatigued inspiratory muscles during an exacerbation. As a consequence, work of breathing is significantly reduced, lowering the increased total volume of $\mathrm{CO}_{2}\left(\dot{\mathrm{V}} \mathrm{CO}_{2}\right)$ produced metabolically by the high breathing effort. The respiratory muscles are able to rest from their prolonged maximal loading (O'Donnell \& Parker, 2006). Measuring the reduction in trans-diaphragmatic pressure and muscle activity has confirmed a reduction in inspiratory effort required with NIV (Brochard et al., 1995).

Secondly, AECOPD creates a pathophysiological sequelae (Figure 1-11) of:

- worsening EFL $\rightarrow \mathrm{DH} \rightarrow$ increased EELV, increased residual volume (RV), reduction in inspiratory capacity and inspiratory reserve volume but an unchanged total lung capacity.

- Tidal breathing is shifted to the right, closer to TLC with an increased RV to maintain the same $\mathrm{V}_{\mathrm{T}}$.

- iPEEP is created as EELV is increased and the inspiratory pressure threshold loading is increased. 

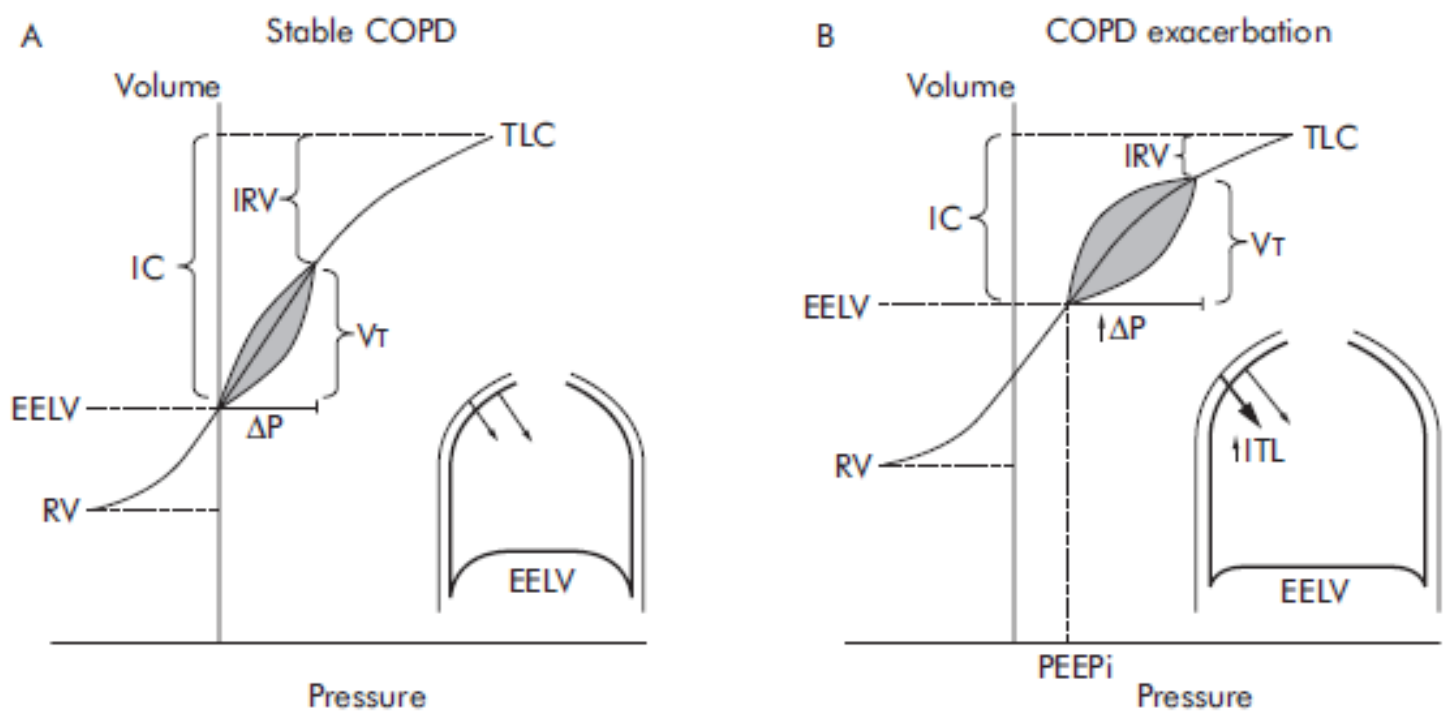

Figure 1-11 Schematic of the mechanical effects of AECOPD illustrated by pressure-volume plots. EELV and RV increase during AECOPD due to EFL and DH, resulting in a corresponding reduction in IC and IRV. Tidal breathing shifts to the right on the pressure-volume curve, closer to TLC. Increased pressures are required to be generated to produce the same $V_{T}$. Intra-pulmonary pressures do not return to zero at EELV, creating IPEEP and an increased ITL, which must be overcome to generate inspiratory flow.

Residual volume (RV); End expiratory lung volume (EELV); Inspiratory capacity (IC); Inspiratory reserve volume (IRV); Total lung capacity (TLC); intrinsic positive endexpiratory pressure (iPEEP); Inspiratory threshold loading (ITL)

[Reproduced from Thorax, "COPD exacerbations - 3: Pathophysiology", (O'Donnell \& Parker, 2006) 61(4),@ 2018, with permission from BMJ Publishing Group Ltd.]

The delivery of positive expiratory pressure (EPAP) with bi-level changes the pressure-volume curve of the lungs back towards scenario $A$ in Figure 1-11. EPAP (or sometimes called extrinsic PEEP to intuitively correspond with the iPEEP it overcomes) counteracts the increased iPEEP (Aldrich et al., 1993). As a person with COPD exhales, there is collapse of the small airways and expiratory flow limitation. Delivering EPAP splints these airways open, reducing expiratory flow limitation, limiting dynamic hyperinflation and improving alveolar ventilation. When ready for an inhalation, the pressure threshold to inhale (iPEEP) is lower. iPEEP has been reported to range from 4.6-13.6 $\mathrm{cm} \mathrm{H}_{2} \mathrm{O}$ in AECOPD, (Rossi et al, 1997) illustrating the importance of delivering EPAP as a counter-force.

EPAP also allows removal of expired air through vents in the NIV tubing, improved triggering of the NIV device and reduction in collapse of the upper airways during expiration (Davidson et al., 2016). 


\subsubsection{Evidence for NIV use in acute hypercapnic respiratory failure in COPD}

Clinical NIV use for AHRF secondary to AECOPD is well established in New Zealand and is based on TSANZ guidelines (Yang et al., 2017). The British Thoracic Society (BTS)/Intensive Care Society (ICS) guideline for the "Ventilatory management of acute hypercapnic respiratory failure in adults" provides extra detail and are established as an international standard (Davidson et al., 2016). European Respiratory Society/American Thoracic Society (ERS/ATS) guidelines for NIV use in acute respiratory failure support the BTS/ICS recommendations (Rochwerg et al., 2017). The following section will discuss two important recommendations from these guidelines which are relevant to my research: 1) optimising medical therapy prior to initiating NIV, as recommended in the BTS/ICS guidelines, and, 2) indications for NIV initiation, which are a collectively found in the TSANZ, BTS/ICS and ERS/ATS guidelines. Finally, the evidence for the efficacy of NIV will be reviewed with emphasis on a Cochrane systematic review and meta-analysis, which has contributed significantly to all three of the guidelines.

\subsection{OPTIMISING MEDICAL THERAPY PRIOR TO NIV INITIATION}

The BTS/ICS guideline recommends that patients with respiratory acidosis and $\mathrm{a} \mathrm{pH}$ of 7.25-7.34 have an initial 60-minute trial of optimising medical therapy and reviewing the clinical response before considering the initiation of NIV. Optimising medical therapy involves steroids, titrated oxygen and nebulised bronchodilators as outlined in section 1.3.1.5 (Davidson et al., 2016). The rationale behind delaying NIV in patients in this $\mathrm{pH}$ range is approximately $20 \%$ of patients will improve with this treatment alone (Davidson et al., 2016; Plant et al, 2000). The improvement is likely in part due to the reversal of the oxygen-induced hypercapnia by reducing the amount of oxygen given and carefully titrating the flow (Plant et al., 2000) as well as the effect of the other medical therapies such as inhaled bronchodilators.

\subsection{INDICATIONS FOR NIV INITIATION}

The main indication for NIV is a persisting respiratory acidosis with $\mathrm{pH}$ 7.257.34, $\mathrm{P}_{\mathrm{a}} \mathrm{CO}_{2}>45 \mathrm{mmHg}$ and respiratory rate $>23$ after the 60 minute trial of 
optimal medical therapy. Details of NIV contraindications, set-up and monitoring are presented below (Figure 1-12) from the BTS/ICS guidelines, with similar recommendations in TSANZ and ERS/ATS guidelines.

There are two other indications for initiating NIV. First, when IMV is indicated ( $\mathrm{pH}<7.25$ and increased respiratory distress), but a trial of NIV is thought to be appropriate with the intention to consider proceeding to IMV if NIV fails. NIV may be effective at this level and variable clinical scenarios make absolute values not useful, although it is important to recognise NIV failure rates are higher (Conti et al., 2002; Pierson, 2002). A pH of $<7.15$ is regarded as an absolute indication for IMV, if deemed clinically appropriate. Secondly, NIV may be initiated as a "ceiling of care" for patients who are thought to be inappropriate for IMV (Nava et al, 2006) such as those with end-stage disease and/or advanced care plans.

Studying the role of NIV in hypercapnic but non-acidotic patients and/or in the "early" phases of AECOPD to prevent deterioration to AHRF has generally shown no effect on mortality or rates of IMV, (Keenan et al, 2005; Wood at al, 1998) with the exception of a Chinese study showing a reduction in IMV rates (Collaborative Research Group of Noninvasive Mechanical Ventilation for COPD, 2005). Current guidelines do not recommend NIV use in this setting (Rochwerg et al., 2017). 


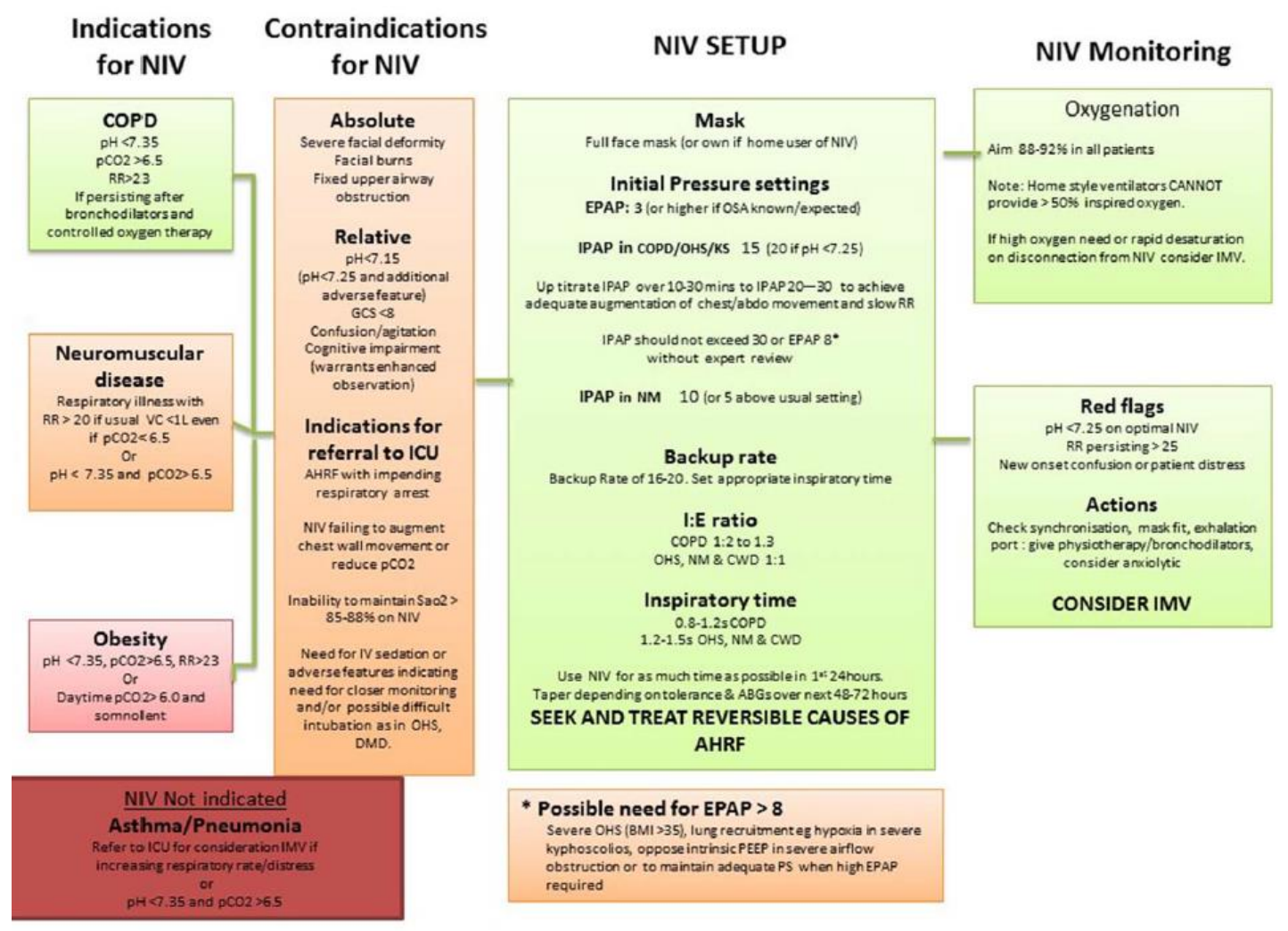

Figure 1-12 - BTS/ICS guidelines - summary of process for initiating NIV in AHRF.

IPAP: inspiratory positive airways pressure, EPAP: expiratory positive airways pressure, I:E ratio: inspiratory time:expiratory time ratio, RR: respiratory rate, VC: vital capacity, $\mathrm{PCO}_{2}$ : arterial partial pressure of carbon dioxide, SaO2: arterial oxygen saturation.

[Reproduced from Thorax, BTS/ICS guideline for the ventilatory management of acute hypercapnic respiratory failure in adults , (Davidson et al., 2016), 71, Suppl 2, ๔ 2018 with permission from BMJ Publishing Group Ltd.]

\subsection{EFFICACY OF NIV}

Several RCTs have demonstrated a reduction in mortality, rates of endotracheal intubation, length of hospital and ICU stay and improvement in acid/base balance in AHRF in COPD since the first clinical trial in 1991 (Meduri et al., 1991). A comprehensive systematic review and meta-analysis of the literature has recently been updated by the Cochrane Library (Osadnik et al., 2017) supporting international guidelines (Davidson et al., 2016; Rochwerg et al., 2017). The Cochrane meta-analysis reviewed key aspects of mortality, rates of endotracheal intubation, tolerability and $A B G$ measurements. 
The primary outcome measures used in the Cochrane review were: 1) inpatient mortality and 2) intubation rates. Secondary outcome measures relevant to this thesis were: 1) complications and tolerability (which are discussed later in "Limitations of current treatment options", Section 1.3.4) and 2) $A B G$ results one-hour post NIV commencement ( $\left.\mathrm{pH}, \mathrm{PaCO}_{2}, \mathrm{PaO}_{2}\right)$.

17 studies with a total of 1264 patients met the inclusion criteria of:

- acute respiratory failure with a $\mathrm{P}_{\mathrm{a}} \mathrm{CO}_{2}$ of $>45 \mathrm{mmHg}$ caused by AECOPD,

- randomised design and

- parallel-assignment of interventions

Twelve studies with a total of 854 patients were used for in-patient mortality analysis. Mortality was reduced with NIV, a $46 \%$ risk reduction (relative risk $0.54,95 \% \mathrm{Cl} 0.38-0.76$ ) (see Figure 1-13) giving a low NNT (the number of patients needed to treat for 1 successful treatment) of 12 (95\% $\mathrm{Cl} 9-23)$.

\begin{tabular}{|c|c|c|c|c|c|c|c|c|c|c|}
\hline Study or Subgroup & \multicolumn{2}{|l|}{ NIV } & \multicolumn{2}{|c|}{ Usual care } & Weight & $\begin{array}{c}\text { Risk Ratio } \\
\text { M-H, Fixed, } 95 \% \mathrm{CI} \\
\end{array}$ & & \multicolumn{2}{|c|}{$\begin{array}{c}\text { Risk Ratio } \\
\text { M-H, Fixed, } 95 \% \mathrm{Cl}\end{array}$} & \\
\hline Avdeev 1998 & 3 & 29 & 9 & 29 & $11.5 \%$ & $0.33[0.10,1.11]$ & & & & \\
\hline Barbe 1996 & 0 & 14 & 0 & 10 & & Not estimable & & & & \\
\hline Brochard 1995 & 4 & 43 & 12 & 42 & $15.6 \%$ & $0.33[0.11,0.93]$ & & & & \\
\hline Celikel 1998 & 0 & 15 & 1 & 15 & $1.9 \%$ & $0.33[0.01,7.58]$ & & & & \\
\hline Collaborative 2005 & 5 & 100 & 8 & 91 & $10.7 \%$ & $0.57[0.19,1.68]$ & & & - & \\
\hline Dikensoy 2002 & 1 & 17 & 2 & 17 & $2.6 \%$ & $0.50[0.05,5.01]$ & & & & \\
\hline Khilnani 2010 & 3 & 20 & 2 & 20 & $2.6 \%$ & $1.50[0.28,8.04]$ & & & & \\
\hline Liu 2005 & 1 & 18 & 3 & 18 & $3.8 \%$ & $0.33[0.04,2.91]$ & & & & \\
\hline Matuska 2006 & 7 & 30 & 7 & 30 & $9.0 \%$ & $1.00[0.40,2.50]$ & & & & \\
\hline Plant 2001 & 12 & 118 & 24 & 118 & $30.8 \%$ & $0.50[0.26,0.95]$ & & & & \\
\hline Samaria 2009 & 4 & 20 & 8 & 20 & $10.3 \%$ & $0.50[0.18,1.40]$ & & & & \\
\hline Thys 2002 & 2 & 10 & 1 & 10 & $1.3 \%$ & $2.00[0.21,18.69]$ & & & & \\
\hline Total $(95 \% \mathrm{CI})$ & & 434 & & 420 & $100.0 \%$ & $0.54[0.38,0.76]$ & & & & \\
\hline Total events & 42 & & 77 & & & & & & & \\
\hline $\begin{array}{l}\text { Heterogeneity: } \mathrm{Chi}^{2}= \\
\text { Test for overall effect }\end{array}$ & $\begin{array}{l}6.36, \mathrm{df} \\
\mathrm{Z}=3.49\end{array}$ & $\begin{array}{l}=10(P \\
(P=0\end{array}$ & $\begin{array}{l}=0.78) \\
.0005)\end{array}$ & $; I^{2}=$ & & & 0.01 & wer with NIV 1 & Lower with ${ }^{1}$ & usual care $^{100}$ \\
\hline
\end{tabular}

Figure 1-13 Forest plot of NIV vs usual care (overall) - Mortality.

[Republished with permission of John Wiley and Sons, from: Cochrane Database of Systematic Reviews, Non-invasive ventilation for the management of acute hypercapnic respiratory failure due to exacerbation of chronic obstructive pulmonary disease, (OSADNIK ET AL., 2017) (c) 2018; permission conveyed through Copyright Clearance Center, Inc.]

Seventeen studies with a total of 1105 patients were included in the analysis for intubation rates. NIV conferred a reduction in risk of intubation by $64 \%$ (RR $0.36,95 \% \mathrm{Cl} 0.28-0.46)$ (see Figure $1-14)$ with a NNT of $5(95 \% \mathrm{Cl} 5-6)$. 


\begin{tabular}{|c|c|c|c|c|c|c|c|c|c|c|}
\hline Study or Subgroup & NIV & Total & \multicolumn{2}{|c|}{ Usual care } & Weight & $\begin{array}{c}\text { Risk Ratio } \\
\text { M-H, Fixed, } 95 \% \mathrm{Cl}\end{array}$ & & \multicolumn{2}{|c|}{$\begin{array}{c}\text { Risk Ratio } \\
\text { M-H, Fixed, 95\% Cl }\end{array}$} & \\
\hline Avdeev 1998 & 3 & 29 & 8 & 29 & $4.2 \%$ & $0.38[0.11,1.27]$ & & & - & \\
\hline Barbe 1996 & 0 & 14 & 0 & 10 & & Not estimable & & & & \\
\hline Bott 1993 & 0 & 30 & 2 & 30 & $1.3 \%$ & $0.20[0.01,4.00]$ & & & & \\
\hline Brochard 1995 & 11 & 43 & 31 & 42 & $16.7 \%$ & $0.35[0.20,0.60]$ & & $\because$ & & \\
\hline Carrera 2009 & 5 & 37 & 13 & 38 & $6.8 \%$ & $0.40[0.16,1.00]$ & & & & \\
\hline Celikel 1998 & 1 & 15 & 2 & 15 & $1.1 \%$ & $0.50[0.05,4.94]$ & & & & \\
\hline Collaborative 2005 & 6 & 100 & 17 & 91 & $9.5 \%$ & $0.32[0.13,0.78]$ & & & & \\
\hline del Castillo 2003 & 1 & 20 & 3 & 21 & $1.6 \%$ & $0.35[0.04,3.09]$ & & & & \\
\hline Dikensoy 2002 & 2 & 17 & 7 & 17 & $3.7 \%$ & $0.29[0.07,1.18]$ & & & & \\
\hline Khilnani 2010 & 3 & 20 & 12 & 20 & $6.4 \%$ & $0.25[0.08,0.75]$ & & & & \\
\hline Kramer 1995 & 1 & 11 & 8 & 12 & $4.1 \%$ & $0.14[0.02,0.92]$ & & & & \\
\hline Liu 2005 & 2 & 18 & 8 & 18 & $4.2 \%$ & $0.25[0.06,1.02]$ & & & & \\
\hline Matuska 2006 & 3 & 30 & 10 & 30 & $5.3 \%$ & $0.30[0.09,0.98]$ & & & & \\
\hline Plant 2001 & 18 & 118 & 32 & 118 & $17.0 \%$ & $0.56[0.34,0.94]$ & & 了 & & \\
\hline Samaria 2009 & 4 & 20 & 11 & 20 & $5.8 \%$ & $0.36[0.14,0.95]$ & & & & \\
\hline Thys 2002 & 0 & 7 & 5 & 5 & $3.3 \%$ & $0.07[0.00,1.01]$ & & & & \\
\hline Zhou 2001 & 7 & 30 & 17 & 30 & $9.0 \%$ & $0.41[0.20,0.85]$ & & $\longrightarrow$ & & \\
\hline Total $(95 \% \mathrm{Cl})$ & & 559 & & 546 & $100.0 \%$ & $0.36[0.28,0.46]$ & & $\bullet$ & & \\
\hline Total events & 67 & & 186 & & & & & & & \\
\hline $\begin{array}{l}\text { Heterogeneity: } \mathrm{Chi}^{2}= \\
\text { Test for overall effect }\end{array}$ & $\begin{array}{l}6.68, \mathrm{df} \\
Z=8.22\end{array}$ & $\begin{array}{l}=15(P \\
(P<0\end{array}$ & $\begin{array}{l}=0.97) \\
.00001)\end{array}$ & $1^{2}=0$ & & & 0.005 & 0.1 ver with NIV & $\begin{array}{r}10 \\
\text { Lower with }\end{array}$ & $e^{200}$ \\
\hline
\end{tabular}

Figure 1-14 Forest plot of NIV vs usual care (overall) - Need for endotracheal intubation.

[Republished with permission of John Wiley and Sons, from: Cochrane Database of Systematic Reviews, Non-invasive ventilation for the management of acute hypercapnic respiratory failure due to exacerbation of chronic obstructive pulmonary disease, (OSADNIK ET AL., 2017) (c) 2018; permission conveyed through Copyright Clearance Center, Inc.]

$\mathrm{PaCO}_{2}$ and $\mathrm{pH}$ at 1 -hour post-NIV commencement was pooled for 8 studies totalling 585 patients. Two unbalanced studies were removed due to inconsistent statistical methodology causing a non-significant $\mathrm{PaCO}_{2}$ reduction. A post-hoc sensitivity analysis with these 2 studies removed meant $\mathrm{PaCO}_{2}$ reduction reached statistical significance (mean difference -8.35 $\mathrm{mmHg} 95 \% \mathrm{Cl} 14.84$ to -1.86$)$. $\mathrm{pH}$ difference was 0.05 units $(95 \% \mathrm{Cl} 0.02$ to 0.07). The difficulty with interpreting $\mathrm{pH}$ change is $\mathrm{pH}$ is the negative log of hydrogen ion concentration. A change in $\mathrm{pH}$ is therefore not linear and hydrogen ion concentration reporting may give a better sense of change although not common practice (see Section 4.2.4).

NIV has a high-level evidence base and is recommended to be available 24 hours/ day in hospitals that are treating COPD patients with AHRF (Davidson et al., 2016; Nava \& Hill, 2002).

\subsubsection{Evidence for NIV use in chronic hypercapnic respiratory failure in COPD}

Interest has been reignited in nocturnal domiciliary NIV care in COPD with chronic hypercapnia, although the evidence is more conflicting and less 
compelling than in the acute setting, and there are no current international guidelines. In 2009, the survival benefit of domiciliary NIV plus LTOT compared to LTOT alone in chronic hypercapnic COPD patients was demonstrated in a nocturnal NIV use study, with an adjusted hazard ratio of $0.63(95 \% \mathrm{Cl} 0.40$ to $0.99, \mathrm{P}=0.045)$, but not in the unadjusted analysis (McEvoy et al., 2009).

Less positive findings then came from a 2013 Cochrane systematic review and meta-analysis that included 7 studies investigating domiciliary NIV for at least 5 hours/day and for at least 3 consecutive weeks, involving 245 patients (Struik et al, 2013). Mortality was not included as an outcome measure and no clinically or statistically significant improvement in $\mathrm{PaO}_{2}$ or $\mathrm{P}_{a} \mathrm{CO}_{2}$ were found with domiciliary NIV compared to standard care.

However, a mortality benefit was shown in a subsequent trial. Hypothesizing the equivocal effect of NIV previously observed was due to inadequate pressure settings therefore minimal $\mathrm{PaCO}_{2}$ reduction, another trial used pressure settings aimed to reduce $\mathrm{P}_{\mathrm{aCO}}$ from baseline by $20 \%$ (Köhnlein et al., 2014). Mean pressure settings of IPAP/EPAP 22/5 cm H2O were applied to 102 COPD patients with CHRF, resulting in a significant $5.1 \%$ difference in change from baseline $\mathrm{P}_{\mathrm{a}} \mathrm{CO}_{2}$ with NIV compared to the control intervention of standard care at 1 year, an absolute reduction by $11.3 \mathrm{mmHg}$. One-year mortality favoured NIV also, with a hazard ratio of $0.24(95 \% \mathrm{Cl} 0.11-0.49$, $P=0.0004)$.

These results are tempered by further research around the same time that did not show a mortality benefit or increase in time to readmission despite a significant reduction in $\mathrm{P}_{\mathrm{aCO}}$ (Struik et al., 2014).

Recently there has been further support for domiciliary NIV as an adjunct to LTOT showing a mortality benefit and increase in time to readmission (composite absolute RR 17\%, 95\% Cl 0.1 to 34)(Murphy et al., 2017).

\subsubsection{Limitations of current treatment options}

Sections 1.3.1.11 and 1.3.3 reviewed current treatment options in COPD relevant to this thesis. Strategies such as the use of discharge care bundles 
in preventing COPD readmissions and the use of NIV in hypercapnic respiratory failure were discussed. The section below will highlight aspects of care that have potential for improvement.

\subsubsection{Limitations of treatment options to reduce AECOPD readmission rates}

Several risk factors were introduced in section 1.3.1.10 that may predict early AECOPD readmissions; however, most of them were non-modifiable. Interventions which may have a role in curbing high readmission rates were discussed in 1.3.1.11; however, there is no single therapy that is unequivocally effective, and the ideal strategy is likely a combination of multiple therapies. Also, interventions may show promise in reducing readmissions over any timepoint, however, not specifically early readmission, a critical metric for both patient and hospital outcomes. Patient self-management plans are effective in reducing overall admission rates however the benefit in preventing early readmissions is less certain. Early follow-up with a respiratory specialist may confer benefit to prevent readmissions but the effect size is small and practicality may prevent uptake in many centres due to limited resources. A model of multiple therapies, each bringing a degree of benefit is likely to be the most efficacious.

\subsubsection{Limitations of treatment options in hypercapnic respiratory failure in COPD}

Limitations of NIV relate to complications and low tolerability of the therapy. Actual treatment failure with NIV, defined as requiring IMV, occurs in between $8-40 \%$ of cases, with the degree of acidosis the most reliable predictor (Carron et al., 2013; Nava \& Ceriana, 2004). Many of these causes of NIV failure relate to delays in treatment, inappropriate settings, inexperience of the clinical team and inappropriate initiation when IMV would have been more suitable (Carron et al., 2013).

These factors do not represent a limitation of NIV as such, although some of these factors may be mitigated by an easier to implement therapy. The commonest causes of failure due to actual NIV use, are excessive mask leak, insufficient pressure support settings and/or patient/ventilator asynchrony (Davidson et al., 2016). NIV when initiated, delivered and monitored 
appropriately is very effective in hypercapnic respiratory failure as demonstrated in Section 1.3.3.2.3 earlier. However, NIV requires intensive monitoring, adjustment according to clinical response, a high level knowledge of NIV settings and a clear goal of treatment for each patient to maximise its benefits (Grainger, 2017).

There are certain clinical scenarios where AHRF treatment may be improved.

\subsubsection{Optimising treatment in the first hour of presentation}

Twenty percent of AHRF patients will improve without needing NIV (Plant et

al., 2000).The main explanation is by titrating oxygen therapy to maintain saturations of $88-92 \%$, over-oxygenation is avoided. While it is imperative to correct hypoxaemia, the risks of oxygen-induced hypercapnia may be lessened by delivering oxygen with a precise $\mathrm{FiO}_{2}$, minimising $\mathrm{P}_{2} \mathrm{CO}_{2}$ rise. Optimising oxygen therapy during the first hour of presentation may increase the proportion of AHRF patients who will improve without needing NIV.

\subsubsection{Continuity of NIV without breaks}

NIV should be administered semi-continuously for the first 24 hours in AHRF to maximise the effect and reverse the respiratory acidosis (Davidson et al., 2016).

Enduring therapy for longer periods of time is also essential for success (Soo Hoo et al, 1994). Despite this, the actual number of hours administered in RCTs has ranged from a median of only 6-8 hours (Bott et al., 1993; Brochard et al., 1995; Plant et al., 2000) to 20 hours (Kramer et al, 1995). Data of average hours of use in clinical practice may be variable and one nonpublished annual audit reported a mean (SD) of 15 (8) hours use in the first 24 hours (Davies, 2013). Frequent interruptions to NIV treatment occur for nutritional requirements, to expectorate sputum and to receive oral medication and nebuliser therapy, all of which will dilute the treatment effect.

\subsubsection{Tolerability of NIV}

\subsection{TOLERABILITY IN AHRF}

Intolerance of NIV is common and is a predictor of NIV failure (Ambrosino et al., 1995; Benhamou et al, 1992; Carlucci et al, 2001). Intolerance is usually related to discomfort of the mask interface, a feeling of claustrophobia due to 
the mask or inability to synchronise breathing with the ventilator. The Cochrane meta-analysis of 6 studies including 252 patients reported an $11 \%$ risk difference (95\% Cl 4 to 17\%) in intolerance of NIV compared to standard therapy (Osadnik et al., 2017) (see Figure 1-15).

\begin{tabular}{|c|c|c|c|c|c|}
\hline \multirow[t]{2}{*}{ Study or subgroup } & NIV & Usual care & $\begin{array}{r}\text { Rikk } \\
\text { Difference }\end{array}$ & Weihat & $\begin{array}{r}\text { Risk } \\
\text { Difference }\end{array}$ \\
\hline & $\mathrm{n} / \mathrm{N}$ & $\mathrm{r} / \mathrm{N}$ & MHFined,95\% Cl & & MH,Fined,95\% Cl \\
\hline Avdeer 1998 & $3 / 29$ & $0 / 29$ & $\mp$ & $23.1 \%$ & $0.10[-0.02,0.23]$ \\
\hline Barbe 1996 & $4 / 14$ & 0110 & $\longrightarrow$ & $9.3 \%$ & $0.29[0.02,0.55]$ \\
\hline Dikeresoy 2002 & $2 / 17$ & $0 / 17$ & $\longrightarrow$ & $135 \%$ & $0.12[-0.06,0.29]$ \\
\hline Khílharí 2010 & $1 / 20$ & $0 / 20$ & $\mp$ & $159 \%$ & $0.05[-0.08,0.18]$ \\
\hline Lin 2005 & $1 / 18$ & $0 / 18$ & $\longrightarrow$ & $14.3 \%$ & $0.06[-0.09,0.20]$ \\
\hline Matuska 2006 & $3 / 30$ & $0 / 30$ & $\mp$ & $239 \%$ & $0.10[-0.02,0.22]$ \\
\hline Total $(95 \% \mathrm{CI})$ & 128 & 124 & $\bullet$ & $100.0 \%$ & $0.11[0.04,0.17]$ \\
\hline \multicolumn{6}{|c|}{ Total everts 14 (NIV), 0 (Uaual care) } \\
\hline \multicolumn{6}{|c|}{ Heterogerety: $\left(\mathrm{Ch}^{2}=306, \mathrm{df}=5(\mathrm{P}=0.69), \mathrm{F}^{\mathrm{P}}=0.0 \%\right.$} \\
\hline \multicolumn{6}{|c|}{ Test for overal effect $Z=329(P=00010)$} \\
\hline \multicolumn{6}{|c|}{ Test for subproup differences Not applicable } \\
\hline
\end{tabular}

Figure 1-15 Forest plot of comparison between NIV and usual care for treatment intolerance.

NIV: Non-invasive ventilation

[Republished with permission of John Wiley and Sons, from: Cochrane Database of Systematic Reviews, Non-invasive ventilation for the management of acute hypercapnic respiratory failure due to exacerbation of chronic obstructive pulmonary disease, (C R OSADNIK ET AL., 2017) () 2018; permission conveyed through Copyright Clearance Center, Inc.]

Developing new patient interfaces, as illustrated earlier in Figure 1-10, has been a focus to improve tolerability but with limited success. One small trial in immunocompromised patients demonstrated a non-significant difference in treatment failure rate due to intolerance with a NIV helmet (14\%) compared to a standard face mask (44\%), $P=0.23$ (Rocco et al., 2004).

\subsection{TOLERABILITY IN CHRF}

Tolerance of NIV has not been an outcome measure in the RCTs reported in section 1.3.3.3. However, quality of life scores have been reported, and may infer a measure of tolerance or intolerance to the therapy. The Cochrane meta-analysis included 2 studies ( $n=103$ ) which used the SGRQ at 12 months and demonstrated no significant difference between domiciliary NIV use and 
usual therapy (mean difference 0.90 on a 100 -point score, $95 \% \mathrm{Cl}-19.21$ to 21.01)(Struik et al, 2013).

The 3 large RCTs described in section 1.3.3.3 have reported conflicting quality of life score results. General quality of life health status measured using the Short Form 36 (SF-36) questionnaire was significantly lower (equating to a poorer outcome) at 12 months in the NIV group for sub-sections such as general health $(P=0.002)$, and mental health $(P=0.009)$. There was no significant difference in the disease specific SGRQ scores however (McEvoy et al., 2009). Others reported a small improvement in SGRQ scores with NIV - adjusted between-group difference of $-4.85(95 \% \mathrm{Cl},-8.83$ to $-0.88 ; \mathrm{P}=$ 0.02 )(Murphy et al., 2017) and $-6.2(95 \% \mathrm{Cl}-11.8$ to $-7, \mathrm{P}=0.0289)$ (Köhnlein et al., 2014) or no significant difference between the NIV and usual care groups (Struik et al., 2014).

\subsubsection{Complications of NIV}

Complications have been reported with rates of up to $50 \%$ of cases (Nava \& Ceriana, 2004). Nasal-bridge rashes and ulceration are common complications (5-10\%), usually due to overtightening of the mask to address leaks (Carron et al., 2013). Ear or sinus pain and gastric distension causing nausea are also relatively common side-effects impacting on tolerability (Carron et al., 2013). Barotrauma causing pneumothorax and pneumocephalus are rare but serious complications (Carron et al., 2013).

It is not surprising that usual care (i.e. without NIV) in the Cochrane metaanalysis of NIV in AHRF (6 studies with 567 patients) carried a significantly lower risk of NIV-related complications in the standard care group compared to the NIV groups (risk ratio $29.695 \% \mathrm{Cl} 9.47$ to 92.51 )(Osadnik et al., 2017) (see Figure 1-16). 


\begin{tabular}{|c|c|c|c|c|c|}
\hline \multirow[t]{2}{*}{ Study or subgroup } & \multirow{2}{*}{$\begin{array}{l}\mathrm{NIV} \\
\mathrm{n} / \mathrm{N}\end{array}$} & \multirow{2}{*}{$\begin{array}{r}\text { Usual care } \\
\mathrm{n} / \mathrm{N}\end{array}$} & \multirow{2}{*}{$\begin{array}{c}\text { Risk Ratio } \\
\text { M-HFixed,95\% Cl }\end{array}$} & \multirow[t]{2}{*}{ Weight } & \multirow{2}{*}{$\begin{array}{r}\text { Risk Ratio } \\
\text { M-H,Fixed,95\% Cl }\end{array}$} \\
\hline & & & & & \\
\hline \multicolumn{6}{|l|}{ I NIV related } \\
\hline Brochard 1995 & $1 / 43$ & $0 / 42$ & & $16.8 \%$ & $293[0.12,70.00]$ \\
\hline Celikel 1998 & $8 / 15$ & $0 / 15$ & 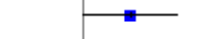 & $16.6 \%$ & $17.00[1.07,270.41]$ \\
\hline Collaborative 2005 & $66 / 171$ & a/17! & $\longrightarrow$ & $16.6 \%$ & $133.00[830,2131.37]$ \\
\hline Dikensoy 2002 & $7 / 17$ & $0 / 17$ & $\longrightarrow$ & $16.6 \%$ & $15.00[0.92,243.52]$ \\
\hline Khilhani 2010 & $3 / 20$ & $0 / 20$ & & $16.6 \%$ & $7.00[0.38,127.32]$ \\
\hline Liu 2005 & $1 / 18$ & $0 / 18$ & & $16.6 \%$ & $3.00[0.13,69.09]$ \\
\hline Subtotal $(95 \% \mathrm{CI})$ & 284 & 283 & - & $100.0 \%$ & $29.60[9.47,92.51]$ \\
\hline \multicolumn{6}{|c|}{ Total events: 86 (NIV), 0 (Usual care) } \\
\hline \multicolumn{6}{|c|}{ Heterogeneity. $C h i^{2}=6.55, d f=5(P=0.26) ; 1^{2}=24 \%$} \\
\hline \multicolumn{6}{|c|}{ Test for overall effect $Z=5.83(P<0.00001)$} \\
\hline \multicolumn{6}{|l|}{2 Non-NIV related } \\
\hline Brochard 1995 & $7 / 43$ & $20 / 42$ & 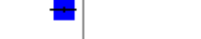 & $66.9 \%$ & $0.34[0.16,0.72]$ \\
\hline Khilhani 2010 & $1 / 20$ & $10 / 20$ & $\longrightarrow$ & $33.1 \%$ & $0.10[0.01,0.71]$ \\
\hline Subtotal $(95 \%$ CI $)$ & 63 & 62 & 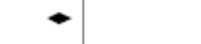 & $100.0 \%$ & $0.26[0.13,0.53]$ \\
\hline \multicolumn{6}{|c|}{ Total events: 8 (NIV), 30 (Usual care) } \\
\hline \multicolumn{6}{|c|}{ Heterogeneity. $C \mathrm{Ci}^{2}=1.41, \mathrm{df}=\mathrm{I}(\mathrm{P}=0.23) ; 1^{2}=29 \%$} \\
\hline Test for overall effect $Z=$ & $=0.0001$ & & & & \\
\hline
\end{tabular}

Figure 1-16 Forest plot of NIV vs usual care complications of treatment. NIV: Non-invasive ventilation

[Republished with permission of John Wiley and Sons, from: Cochrane Database of Systematic Reviews, Non-invasive ventilation for the management of acute hypercapnic respiratory failure due to exacerbation of chronic obstructive pulmonary disease, (OSADNIK ET AL., 2017) (c) 2018; permission conveyed through Copyright Clearance Center, Inc.]

\subsubsection{Resources and monitoring in NIV}

Regular ABG sampling, monitoring of progress and adjustments to NIV settings are critical to maximise safety and efficacy. Experienced nursing staff are required for adequate patient supervision and to address frequent treatment side-effects. Most NIV implementation occurs on dedicated High Dependency Unit (HDU) areas of general medical wards (Davidson et al., 2016). Some smaller hospitals may not have adequately experienced staff to support this ward-based treatment, therefore requiring mandatory admission to ICU at significant additional cost. The extra time demands on nursing staff to care for patients on NIV was no greater than delivering standard care in one trial, although RCTs generally involve experienced nursing staff which possibly masks the real time cost (Plant et al., 2000). There are also significant costs of both the NIV device and consumables, as well as regular 
maintenance and calibration that will be greater than usual care, although no greater than IMV (Bott et al., 1993; Kramer et al., 1995).

There is an unmet need for an alternative, easy to administer, well-tolerated, effective therapy in COPD to aid in reducing hospital readmissions and address the limitations of therapy in HRF. Nasal high flow may be one such therapy and is the focus of this clinical research, which will begin with a comprehensive review in Section 1.4 . 


\subsection{An overview of Nasal High Flow therapy}

NHF therapy has been established in the management of paediatric respiratory conditions for several years, and has a strong evidence base in adult hypoxaemic respiratory failure such that it is may be considered first-line treatment in many cases. Given the mechanisms of action of NHF, which will be reviewed in Section 1.4.3, it has the potential to be effective in COPD patients with HRF and has a growing evidence base in this condition. Chapter 1.4 introduces NHF therapy, provides a systematic review of the literature showing its efficacy in reducing hypercapnia in COPD, discusses the mechanisms of action of NHF and briefly reviews other clinical applications for NHF.

\subsubsection{What is NHF?}

A NHF device is a humidifier with an integrated flow generator that entrains room air and oxygen and humidifies these respiratory gases through a chamber. It then delivers these gases down a heated breathing tube, as depicted in Figure 1-17 below, to spontaneously breathing patients through wide-bore nasal cannulae. Other interfaces can be used rather than the nasal cannula. For example, it can be applied to patients with a tracheotomy but only the nasal high flow system will be discussed in this thesis.

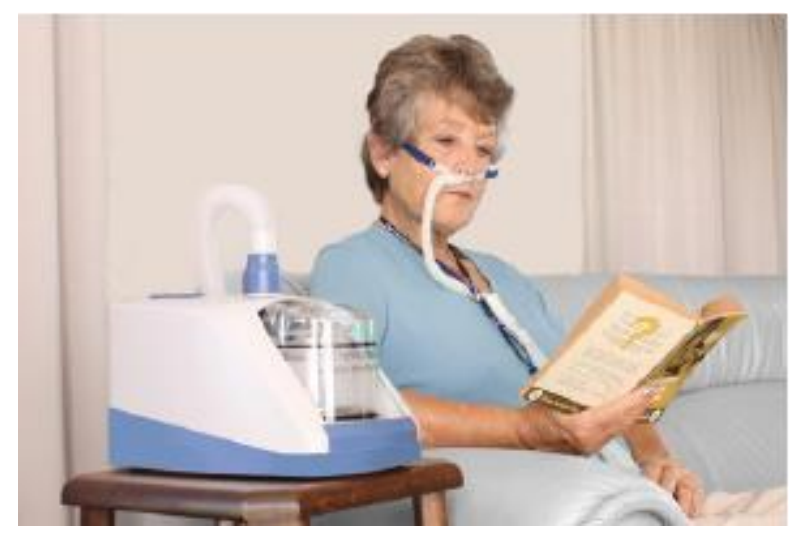

Figure 1-17 Photo of the Nasal High Flow device. The photo shows the device, heated breathing tube and nasal cannula interface.

(Reproduced with permission, () 2018 Fisher and Paykel healthcare).

Humidified air at body temperature pressure saturated (BTPS) is delivered to patients. BTPS air is $37^{\circ} \mathrm{C}$, has a relative humidity of $100 \%$ and an absolute humidity of $43.9 \mathrm{mg} \mathrm{H} \mathrm{H}_{2} \mathrm{O} / \mathrm{l}$ but $\mathrm{NHF}$ can also deliver respiratory gases at $34^{\circ} \mathrm{C}$ 
and $31^{\circ} \mathrm{C}$ dew point temperature settings. Flow rates up to $60 \mathrm{~L} / \mathrm{min}$ are available.

The NHF device is classified as low-medium risk (class Ila) in the Australian Regulatory Guidelines for medical devices by the Therapeutic Goods Administration (TGA)(Therapeutic Goods Adminstration, 2011). TGA regulates medicines and medical devices in Australia and while medical devices are unregulated in New Zealand, the low-risk classification is an appropriate guide in NZ also.

\subsubsection{Background of NHF therapy}

Delivering high flows of humidified air and/or oxygen intra-nasally originated in neonatology practice where Continuous Positive Airways Pressure (CPAP) delivered by face mask to babies may be more difficult. Pressure support through the nose was more practical and shown to be effective in many neonatal respiratory conditions, including the post-extubation period, weaning from CPAP, as well as treating respiratory distress syndrome, apnoea of prematurity and other causes of hypoxic respiratory failure in paediatric patients (Mayfield et al., 2014). High flow rates in neonatal patients is considered any flow over $1-2 \mathrm{~L} / \mathrm{min}$, generating an effect due to the small size of the nasal passages and airways.

The first report of NHF use undertaken in adults was a retrospective chart review in 2004, presented as a conference poster, and demonstrated NHF via a Vapotherm device compared to standard oxygen administration was able to reduce respiratory rate $(23 \mathrm{bpm} \vee 27 \mathrm{bpm} \mathrm{P}<0.001)$ and improve oxygen saturations $(96 \% \vee 90.9 \%, \mathrm{P}<0.001)$ in 33 patients with an unspecified indication for oxygen therapy (Lain and Candace, 2004).

Evidence has evolved since then and the first significant high-grade RCT for NHF use in adults came in 2015 for the treatment of acute hypoxaemic respiratory failure (Frat et al., 2015). Prior to discussing the efficacy of NHF in clinical trials, it is first important to review the evidence for its mechanisms of action to explain how the clinical effect manifests. 


\subsubsection{Mechanisms of action of NHF}

Mechanisms of action relevant to the clinical effects of NHF targeted in this thesis will be reviewed in the following section. That is, mechanisms which will improve alveolar ventilation, hence reduce $\mathrm{PCO}_{2}$ (PEEP effect, dead space washout, accurate $\mathrm{FiO} 2$ delivery, reduced metabolic cost of breathing) and mechanisms which may reduce COPD readmissions (improved mucociliary clearance, PEEP effect). The evidence for these mechanisms of action does not need to be specific to COPD as they are physiological effects, although predominantly extrapolated from model and bench studies rather than clinical trials. The bulk of the evidence is derived rather than directly demonstrating an effect on people. For example, direct measurement of dead space washout is difficult and the evidence is largely absent but can be assumed by changes to tidal volume. Overall, the evidence supporting an actual clinical effect possibly outweighs the evidence proving the mechanisms of action.

\subsubsection{Positive-end expiratory pressure (PEEP)}

The evidence for NHF providing PEEP (also called expiratory PEEP or ePEEP) has been well supported by several studies, summarised in Table 1-6 and is a critical mechanism of action for NHF to be an effective respiratory support therapy. A detailed description of the studies in Table 1-6 is in Appendix I (Section 9.1).

The PEEP effect is similar to that delivered by the EPAP component of bilevel, described in Section 1.3.3.1.3 (Mechanisms of action of NIV). Positive pressure at the end of expiration prevents alveoli collapse, reducing the already raised iPEEP requirement, improving overall alveoli recruitment and hence alveolar ventilation, and subsequently $\dot{V} / \mathbf{Q}$ ratio (Dysart et al, 2009; Lee et al, 2013).

Given the variety of methods of PEEP measurement used in clinical trials, the exact magnitude of the effect is not agreed upon in the literature. Reports have varied between $0-3 \mathrm{cmH} 2 \mathrm{O}$, with $30-50 \%$ leak in an in vitro model, (Lampland et al, 2009) to a maximum of $8 \mathrm{cmH} 20$ in people with their mouth closed providing a seal (Ritchie et al, 2011). 
Table 1-6 Summary of studies demonstrating a PEEP effect with NHF

\begin{tabular}{|c|c|c|c|c|c|c|c|c|c|}
\hline \multirow[t]{2}{*}{ Reference } & \multirow[t]{2}{*}{ Study type } & \multirow{2}{*}{$\begin{array}{l}\text { Sample } \\
\text { size }\end{array}$} & \multirow[t]{2}{*}{ Cohort } & \multirow[t]{2}{*}{ Control } & \multirow{2}{*}{$\begin{array}{l}\text { PEEP } \\
\text { measurement } \\
\text { method }\end{array}$} & \multirow{2}{*}{$\begin{array}{l}\text { NHF flow } \\
\text { rate } \\
\text { (max.) }\end{array}$} & \multicolumn{2}{|c|}{ PEEP effect (cm H2O) } & \multirow[t]{2}{*}{ Other conclusions } \\
\hline & & & & & & & $\begin{array}{l}\text { Mouth } \\
\text { closed }\end{array}$ & $\begin{array}{l}\text { Mouth } \\
\text { open }\end{array}$ & \\
\hline $\begin{array}{l}\text { (Groves \& Tobin, } \\
\text { 2007) }\end{array}$ & Prospective cohort & 10 & Healthy volunteers & Room air & Oro-pharyngeal & $60 \mathrm{~L} / \mathrm{min}$ & 7.4 & 2.7 & $\begin{array}{l}\text { PEEP increased linearly } \\
\text { with flow }\end{array}$ \\
\hline $\begin{array}{l}\begin{array}{l}\text { Ritchie et al., } \\
2011)\end{array} \\
\end{array}$ & $\begin{array}{l}\text { Randomised, cross- } \\
\text { over }\end{array}$ & 10 & Healthy volunteers & Nil & Oro-pharyngeal & $50 \mathrm{~L} / \mathrm{min}$ & 5 & - & $\begin{array}{l}\text { PEEP increased linearly } \\
\text { with flow. Accurate FiO2 } \\
\text { delivery }\end{array}$ \\
\hline $\begin{array}{l}\text { (Lampland et al., } \\
\text { 2009) }\end{array}$ & $\begin{array}{l}\text { Observational, cross- } \\
\text { over }\end{array}$ & 15 & Neonates & $\begin{array}{l}\text { Nasal CPAP at } \\
6 \mathrm{~cm} \mathrm{H} 2 \mathrm{O}\end{array}$ & Oro-pharyngeal & $6 \mathrm{~L} / \mathrm{min}$ & $\begin{array}{l}3 \text { (No sig diff } \\
\text { to nasal } \\
\text { CPAP) }\end{array}$ & - & - \\
\hline $\begin{array}{l}\text { (Kubicka, Limauro, } \\
\text { \& Darnall, 2008) }\end{array}$ & Prospective cohort & 27 & Neonates & $\begin{array}{l}\text { Nasal CPAP at } \\
\text { unreported } \\
\text { pressure }\end{array}$ & Oro-pharyngeal & $3 \mathrm{~L} / \mathrm{min}$ & 4.1 & - & - \\
\hline $\begin{array}{l}\text { (R. Parke, } \\
\text { McGuinness, \& } \\
\text { Eccleston, 2009) }\end{array}$ & Prospective cohort & 15 & Post-cardiac surgery & Hudson mask & Nasopharyngeal & $35 \mathrm{~L} / \mathrm{min}$ & 2.7 & 1.2 & $\begin{array}{l}\text { Significant difference } \\
\text { between mouth open and } \\
\text { closed. }\end{array}$ \\
\hline $\begin{array}{l}\text { Rachael L. Parke, } \\
\text { McGuinness, \& } \\
\text { Eccleston, 2011) }\end{array}$ & Prospective cohort & 15 & Post-cardiac surgery & Nil & Nasopharyngeal & $50 \mathrm{~L} / \mathrm{min}$ & 3.31 & 1.73 & $\begin{array}{l}\text { Significant difference } \\
\text { between mouth open and } \\
\text { closed. } 10 \mathrm{~L} / \mathrm{min} \text { increase } \\
\text { in NHF rate }=\text { increase } \\
\mathrm{PEEP} \text { by } 0.69 \mathrm{~cm} 2 \mathrm{H} \\
\text { with mouth closed and } \\
0.35 \mathrm{cmH} 2 \mathrm{O} \text { with mouth } \\
\text { open. }\end{array}$ \\
\hline $\begin{array}{l}\text { (R } \mathrm{L} \text { Larke \& } \\
\text { McGuinness, 2013) }\end{array}$ & Prospective cohort & 15 & Post-cardiac surgery & Nil & Nasopharyngeal & $50 \mathrm{~L} / \mathrm{min}$ & 3.1 & - & $\begin{array}{l}\text { Flow dependent pressure } \\
\text { effect seen throughout } \\
\text { respiratory cycle }\end{array}$ \\
\hline $\begin{array}{l}\text { (A Corley, Caruana, } \\
\text { Barnett, Tronstad, } \\
\text { \& Fraser, 2011) }\end{array}$ & Prospective cohort & 20 & Post-cardiac surgery & $\begin{array}{l}\text { Low-flow } \\
\text { oxygen }\end{array}$ & Oro-pharyngeal & $\begin{array}{l}35- \\
50 \mathrm{~L} / \mathrm{min}\end{array}$ & 2.7 & $\begin{array}{l}\text { No sig } \\
\text { difference } \\
\text { but no } \\
\text { values } \\
\text { reported }\end{array}$ & $\begin{array}{l}\text { Lung impedance, which } \\
\text { correlates with end } \\
\text { expiratory lung volumes } \\
\text { hence functional residual } \\
\text { capacity, was increased } \\
\text { by } 25.6 \%(95 \% \mathrm{Cl} 24.3- \\
26.9, \mathrm{p}<0.001) \text { with NHF, } \\
\text { and increased with } \\
\text { higher BMI. }\end{array}$ \\
\hline $\begin{array}{l}\text { (Bräunlich } \\
\text { Köhler, 2016) }\end{array}$ & Prospective cohort & 19 & COPD & $\begin{array}{l}\text { Nasal CPAP, } \\
\text { nasal bi-level }\end{array}$ & Nasopharyngeal & $50 \mathrm{~L} / \mathrm{min}$ & 3.01 & - & $\begin{array}{l}\text { Nasal CPAP and nasal } \\
\text { bi-level both significantly } \\
\text { higher PEEP than NHF }\end{array}$ \\
\hline
\end{tabular}


Given NHF is an open system and there is significant leak around the nares and mouth, cannula size and degree of occlusion of the nares is important (Adams et al, 2018) as is the mouth being open or closed (Groves \& Tobin, 2007). With such inconsistent delivery of pressure support with the mouth being open or closed, Lee and colleagues deduced that the PEEP effect is less likely to be a major contributor to relieving respiratory distress (Lee et al., 2013). Certainly the effect is far lower than the positive expiratory pressure delivered by CPAP, which is often above $10 \mathrm{cmH}_{2} \mathrm{O}$, but similar to the standard bi-level EPAP setting of around $3-5 \mathrm{cmH}_{2} \mathrm{O}$.

\subsubsection{Heating and humidification improving mucociliary clearance}

NHF delivers gas at $37^{\circ} \mathrm{C}$ with $100 \%$ relative humidity to the airways, essential to optimise mucosal function (Williams et al,1996).

Water vapour concentration in a gas mixture is expressed as absolute humidity, or relative humidity in relation to the temperature. Absolute humidity is the total water present in the gas (in $\mathrm{mg} \mathrm{H}_{2} \mathrm{O} / \mathrm{L}$ ), which is $44 \mathrm{mg} \mathrm{H}_{2} \mathrm{O} / \mathrm{L}$ for NHF. Relative humidity considers the maximum carrying capacity of the gas at a given temperature and is the amount of water present expressed a percentage of this (Shelly et al, 1988). The provision of gas at core temperature and $100 \%$ relative humidity at the alveolar surface optimises gas exchange and protects lung tissue (Williams et al., 1996). The water content of the mucous also affects viscosity and ease of its transport along the mucociliary escalator. Inadequate humidification of airway epithelial cells reduces sodium absorption and chloride elimination, damaging their structure as well as quality of mucous production (Williams et al., 1996).

Airway heating and humidification with NHF is proposed to confer two clinical benefits:

1. It improves the function of the mucociliary escalator, allowing easier removal of secretions (Gotera et al, 2013; Nishimura, 2016; Pisani \& Vega, 2017; Spoletini et al, 2015).

2. It reduces the metabolic cost of gas conditioning (Dysart et al., 2009; Pisani \& Vega, 2017). 
Providing direct evidence of these two benefits is difficult and is largely extrapolated from evidence demonstrating the detrimental effects of nonhumidification, such as reduced cilia-beat frequency and mucous transport speed when exposed to lower temperatures (Kilgour et al, 2004).

\subsection{THE MUCOCILIARY "ESCALATOR"}

The mucociliary "escalator" of the airways is so-called because of the coordinated, continuous fashion it moves mucus, containing trapped inhaled debris, along and out of the airways.

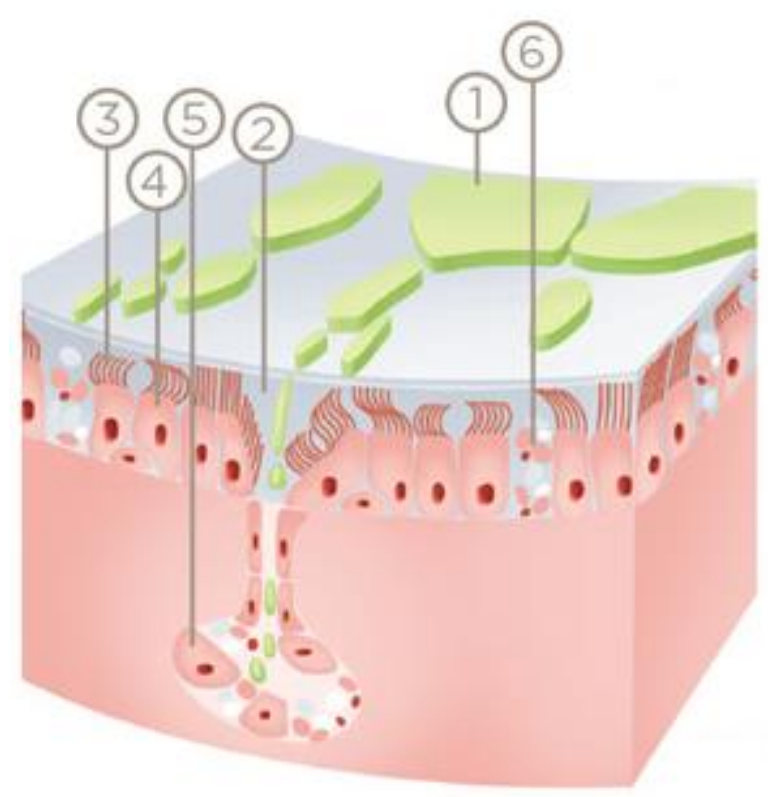

Figure 1-18 Mucociliary "escalator" transport system.

1 - mucus; 2 - aqueous layer; 3 - cilia; 4 - epithelial cell layer; 5 submucosal gland; 6 - goblet cell

[Reproduced with permission from Fisher and Paykel Healthcare $\odot$ 2018, adapted from Williams et al 1996]

Figure 1-18 illustrates the mucociliary transport system. Epithelial cells line the airways and constitute the first line of defence against invading irritants and pathogens, both as a physical barrier and with the production of mucus, immunoglobulins and defensins (Wanner et al, 1996; West, 2012). While there are several different epithelial cell types fastened to the basement membrane, the most relevant to the mucociliary escalator are 1) the ciliated cells, which make up $50-80 \%$ of the epithelial cells lining the airways, especially in the small airways (Harvey et al., 2007) and 2) Goblet cells, which secrete mucus. 
Ciliated epithelial cells extend from the proximal trachea down to the terminal bronchioles (Wanner et al., 1996). Cilia are tiny, hair-like, microtubule processes $6-7 \mu \mathrm{m}$ long and $0.2-0.3 \mu \mathrm{m}$ in diameter extending from the basal body, a centriole at the apical cell surface of the epithelial cell. Each epithelial cell possesses around 200 cilia (Wanner et al., 1996).

Cilia move in a cohesive, beating pattern at a certain frequency, somewhere between 600 and 850 beats per minute, moving debris along the overlying mucus (Yaghi \& Dolovich, 2016). The remainder of the mucociliary escalator consists of a thick protective mucus layer and an airway surface liquid layer (Mall, 2008) classically known as the 'gel' layer which traps the debris. The tips of the cilia just contact the gel layer to propel it towards the larynx.

Both cilia function (Yaghi \& Dolovich, 2016) and cilia-beat frequency are vital parts of the transport mechanism (Rutland et al, 1982; Wanner et al., 1996; Yager et al, 1980). Impaired CBF (reduced by 25\%) from inferior turbinate brushings was seen in a small COPD and pneumonia case-controlled trial (Piatti et al, 2005). Optimising this mucociliary transport system is paramount to improve mucus clearance, especially in chronic respiratory diseases such as COPD.

\subsection{IN-VIVO STUDIES DEMONSTRATING IMPROVED MUCOCILIARY CLEARANCE}

An in-vivo study exposing epithelial cell cultures to dry conditions $(<20 \%$ relative humidity) and NHF (90-100\%) demonstrated a reduction in transepithelial resistance (a measure of cell barrier integrity) and cell viability as well as an increase in inflammatory cytokines in the dry group compared to the NHF group (Chidekel et al., 2012). CBF and mucociliary transport velocity was reduced in vivo in sheep epithelial cells exposed to $30^{\circ} \mathrm{C}$ and $34^{\circ} \mathrm{C}$ despite having $100 \%$ relative humidity (Kilgour et al., 2004), demonstrating the importance of temperature also.

\subsection{HUMAN-STUDIES DEMONSTRATING IMPROVED MUCOCILIARY CLEARANCE}

Direct measurement of mucociliary clearance with NHF is limited to a single trial in 10 bronchiectasis patients (Hasani et al., 2008). Participants' received 3 hours per day of $37^{\circ} \mathrm{C} \mathrm{NHF}$ at $20-25 \mathrm{~L} / \mathrm{min}$ for seven days. A radio aerosol 
was inhaled prior to starting NHF therapy and then deposition measured after 24 hours, showing a significant increase in lung clearance of the radio aerosol with NHF compared to baseline.

Indirect evidence for clinically important mucociliary clearance was shown in a domiciliary NHF study by Rea et al in 108 COPD and bronchiectasis patients (Rea et al., 2010) with fewer exacerbation days and improved FEV1 (see Section 1.4.6 later for complete description).

NHF may also provide direct benefit to patients recovering from an AECOPD secondary to a respiratory virus by maintaining the nasal mucosa temperature at $37^{\circ} \mathrm{C}$, potentially reducing the risk of subsequent viral infection (Singh, 2006).

\subsection{REDUCTION IN METABOLIC COST OF GAS CONDITIONING}

Inhalation of cold, dry air has a bronchoconstricting effect on nasal mucosa receptors (Fontanari et al, 1996) and nasal muscarinic receptors in COPD (On et al., 2001). An energy requirement of about 156 calories/minute is required under normal conditions to warm and humidify air for breathing (Dysart et al., 2009) so theoretically will be far higher in someone with AECOPD who has a higher minute ventilation. NHF may therefore be able to reduce the effort expended with tachypnoea and accessory muscle use, which already requires high energy (Dysart et al., 2009), as does the effort of expectorating sputum. While there are confounding effects, indirect evidence of less energy use with NHF was seen in infants on NHF for lung diseases of prematurity who gained more weight than those on standard CPAP (Holleman-Duray et al, 2007).

\subsubsection{Dead-space washout}

The important relationship between physiological dead space and $\mathrm{PaCO}_{2}$ was described in Section 1.2.1.3.3. To recap, physiological dead space is the sum of anatomical and alveolar dead spaces and is related to alveolar ventilation or volume $\left(\mathrm{V}_{\mathrm{A}}\right)$, tidal volume $\left(\mathrm{V}_{\mathrm{T}}\right)$, respiratory rate (f) and $\mathrm{P}_{\mathrm{a}} \mathrm{CO}_{2}$ by the series of equations described earlier. Physiological dead space is increased in COPD ( $V_{D} / V_{T} \geq 0.8$ compared to the normal ratio of 0.3 ), especially alveolar dead space, and is sometimes termed "wasted ventilation" as it causes observed impairment of $\mathrm{CO}_{2}$ elimination (Petersson \& Glenny, 2014). 
$\mathrm{P}_{\mathrm{a}} \mathrm{CO}_{2}$ levels depend on alveolar ventilation which is the ratio of $\mathrm{V}_{\mathrm{D}}$ to $\mathrm{V}_{T}$ and will decrease if minute ventilation (consisting of $V_{T}$ multiplied by $f$ ) increases and/or physiological dead space decreases. Given this relationship between $\mathrm{PaCO}_{2}$ and dead space, the ability of $\mathrm{NHF}$ to washout dead space is critical to demonstrate its ability to reduce $\mathrm{PaCO}_{2}$.

Publications proposing the $\mathrm{PCO}_{2}$-lowering effect of $\mathrm{NHF}$ therapy comes from dead space washout become slightly confused when describing whether anatomical dead space alone and/or physiological dead space, which also incorporates alveolar dead space, is washed-out. There is reasonable evidence for a reduction in both types of dead space, more so for anatomical dead space, although each will clear $\mathrm{CO}_{2}$ but via a slightly different mechanism. There is no evidence of direct measurement of dead space volume with NHF, although imaging of changes in gas content of dead space has been visualised using spectroscopy (Möller et al., 2015).

The evidence for both anatomical and alveolar dead space washout can also be derived from changes in other ventilatory parameters, such as minute ventilation, tidal volume and respiratory rate, rather than direct measurement so will be described separately.

\subsection{EVIDENCE OF ANATOMICAL DEAD SPACE WASHOUT}

Anatomical dead space, especially more proximal in the nasopharynx, has reasonable evidence of being washed-out with NHF. The mechanism is likely two-fold. Firstly, the column of $\mathrm{CO}_{2}$-concentrated air that would otherwise be re-breathed, contributing to carbon dioxide retention further distal at the level of the gas-exchanging surfaces, is mechanically "washed-out" courtesy of the influx of high air-flow. Secondly, oxygen delivered into this anatomical dead space dilutes the high- $\mathrm{CO}_{2}$ concentrated air, allowing re-breathing of fresh oxygen-laden air, similar to the mechanisms of tracheal gas insufflation and trans-tracheal oxygen delivery described in Section 1.4.3.6 later.

Direct evidence of washout of anatomical dead space is lacking but indirect evidence is supported mainly by model and animal studies. The difficulty in measuring dead space volume in an awake person is due to its small volume, inter-personal variability in nasopharyngeal anatomy and inability to visualise and measure gas flow in the upper airways as well as the rapid process which 
changes with each breath (Möller et al., 2015). Attempting to quantify anatomical dead space in an intubated patient needs to take into account the significant added contribution of "prosthetic dead space" from the endotracheal tube and ventilator attachments.

\subsection{ANIMAL STUDIES SUPPORTING ANATOMICAL DEAD SPACE WASHOUT}

Thirteen piglets were anaesthetized and respiratory failure induced by administering IV oleic acid to reduce $\mathrm{P}_{\mathrm{a}} \mathrm{O}_{2}$ and respiratory compliance by $50 \%$ while maintaining their ability to spontaneously breathe (Frizzola et al., 2011). NHF at $8 \mathrm{~L} / \mathrm{min}$ with both high leak (using single nasal prong) and low leak (using 2 nasal prongs), significantly reduced $\mathrm{P}_{\mathrm{a}} \mathrm{CO}_{2}$ and increased $\mathrm{PaO}_{2}$ in a flow-dependent manner and was independent of the increase in tracheal pressure alone. The authors had previously reported CPAP with TGI being an effective method of nasopharyngeal $\mathrm{PCO}_{2}$ washout in an injured piglet model (Miller et al, 2004) and their conclusion from this NHF study highlighted the similar nasopharyngeal dead space washout as observed with TGI.

Measurement of carbon dioxide washout with NHF from the lower airways was shown in another animal model study (Bräunlich et al, 2017). The same effect was seen with airway pressures and both were flow dependent, less in peripheral airways than tracheal measurements.

\subsection{MODEL STUDIES SUPPORTING ANATOMICAL DEAD SPACE WASHOUT}

Realising the difficulties in directly measuring anatomical dead space volume change, a model study directly visualised gas flow in the upper airways in the only study directly demonstrating an anatomical dead space washout. Dynamic infrared $\mathrm{CO}_{2}$ spectroscopy and $\mathrm{Kr}$-gas radioactive gamma camera imaging was used in a CT-guided 3D model reconstruction of the upper airways (Möller et al., 2015). Complete gas tracer removal was seen within 1 second, and was faster still in the anterior compartments of the nasopharynx and speed of clearance had a positive linear dependency on NHF flow rate.

Measuring nasopharyngeal oxygen concentration with NHF compared to standard nasal prongs on an upper airway model showed that delivering higher flows of oxygen (10-30L/min) through nasal cannulae created a pool of oxygen in the nasopharynx (Tiep, 2002). They sampled this oxygen concentration and followed the delivery using ultrasonic techniques and 
concluded as there were higher oxygen concentrations, as well as a pool effect, the anatomical dead space must be washed-out and replaced with fresh gas. A similar model study published as an abstract demonstrated the same effect (Malinowski, 2002).

\subsection{HUMAN STUDIES SUPPORTING ANATOMICAL DEAD SPACE WASHOUT}

Sampling the fraction of expired carbon dioxide from the nasopharynx with a catheter showed no change with increasing flow rates in 10 healthy volunteers at rest or exercising (Ritchie et al., 2011). $\mathrm{FiO}_{2}$ was calculated from both the measured fraction of expired carbon dioxide and oxygen using the alveolar gas equation and did significantly increase with higher flow rates, also previously demonstrated in healthy subjects (Wettstein et al, 2005). The result offers conflicting evidence of nasopharyngeal washout and as the effect was more discernible with the mouth open, the oxygen-reservoir effect of the nasopharynx may be reduced with nasal breathing.

A case report of a single tracheotomised COPD patient measured carbon dioxide dead space washout (Fricke et al., 2016). Inspired carbon dioxide volume was derived using the modified Fowler method, from measuring endtidal carbon dioxide and tidal volume. The inspired carbon dioxide volume was shown to reduce instantly with $\mathrm{NHF}$ at $30 \mathrm{~L} / \mathrm{min}$, along with a reduction in $\mathrm{P}_{\mathrm{aCO}}$ and minute ventilation by $700 \mathrm{~mL}$. The authors conclude the only explanation is a reduction in dead space volume according to the mass balance equation described in section 1.2.1 (Normal respiratory physiology).

A study in 10 healthy subjects and 3 patients with tracheostomies confirmed findings from the same authors 2015 model study as well as the Ritchie et al 2011 study reported earlier in Section 1.4.3 (Möller et al., 2017). They used tracer gas scintigraphy to show a reduction in gas clearance half-time in healthy volunteers as the flow rates of NHF increased from 15 to 30 to 45L/min. Tracheal sampling from the 3 tracheotomised patients showed a reduction in inspired carbon dioxide concentration and an increase in oxygen concentration similar to a previous study (Ritchie et al., 2011) therefore a washout of the upper airway dead space. 


\subsection{EVIDENCE OF ALVEOLAR/PHYSIOLOGICAL DEAD SPACE WASHOUT}

Providing direct evidence for washout of alveolar dead space with NHF is difficult and studies claiming this as a mechanism of action mostly derive the reduction based on a decrease or increase in other ventilatory parameters. The known relationships between $\mathrm{PaCO}_{2}$, tidal volume, minute ventilation, respiratory rate and physiological dead space volume were described above in the respiratory physiology section (1.2.1.3.3).

\subsection{DECREASE IN RESPIRATORY RATE}

A significant reduction in respiratory rate has been shown in most of the trials reported later in the systematic review trials (Section 1.4.5), as well as other trials in non-COPD patients (Corley et al., 2011; Roca et al, 2010; Sztrymf et al., 2011). The decrease ranges from -1.8 breaths per minute (Nilius et al., 2013) to a maximum of -5.8 breaths per minute in COPD patients (Bräunlich \& Köhler, 2016; Pisani et al., 2017).

However, a Cochrane systematic review and meta-analyses of NHF in hypoxaemic respiratory failure patients in ICU showed no significant difference in respiratory rate, compared to standard oxygen or NIV, although the majority of these studies excluded COPD patients (Corley et al., 2017).

\subsection{INCREASE IN TIDAL VOLUME}

Four of the trials reported in the systematic review measured tidal volume. Three showed a significant increase while one showed a reduction, although this was while participants were sleeping. The increase ranged from $76.7 \mathrm{~mL}$ (Pisani et al., 2017) to $130 \mathrm{~mL}$ (Biselli et al., 2017).

\subsection{DECREASE IN MINUTE VOLUME}

Three of the trials reported in the systematic review to follow measured MV with two showing a reduction, of $1.7 \mathrm{~L} / \mathrm{min}$ (Bräunlich \& Köhler, 2016) and $0.9 \mathrm{~L} / \mathrm{min}$ (Biselli et al., 2017), and one showing no change compared to baseline.

The explanation for these changes in ventilatory parameters, along with the $\mathrm{PCO}_{2}$ reduction, means there must be an improvement in alveolar volume and/or reduction in metabolic carbon dioxide production. Given the proof for anatomical dead space wash out, the improvement in alveolar volume must 
have a contribution from an overall reduction in physiological dead space also. A reduction in metabolic carbon dioxide production is also likely a contributing factor but again is difficult to measure. One surrogate may be a "work of breathing" index and has been shown to be lowered with NHF (Biselli et al., 2017; Bräunlich \& Köhler, 2016; Pisani et al., 2017).

\subsubsection{Accurate delivery of $\mathrm{F}_{\mathrm{i}} \mathrm{O}_{2}$}

Delivering a precise $\mathrm{FiO}_{2}$ is critical to prevent oxygen-induced hypercapnia and the importance of titrating oxygen therapy in COPD patients to maintain oxygen saturations of $88-92 \%$ was discussed earlier. Precise $\mathrm{FiO}_{2}$ delivery is notoriously difficult to achieve and is generally much lower than thought. The amount of entrained air during inspiration and high inspiratory volumes when in respiratory distress will alter the $\mathrm{FiO}_{2}$ (Markovitz et al, 2010). Inter-personal variability exists, especially in patients with chronic lung disease and in one study, delivering 1-2L/min oxygen via standard nasal cannula resulted in an $\mathrm{FiO}_{2}$ (measured by deriving the value from expired oxygen and carbon dioxide concentrations) between $24-28 \%$ in healthy subjects but $23.7-34.9 \%$ in COPD patients (Bazuaye et al, 1992).

Matching ventilatory demands in a tachypnoeic patient with an altered respiratory pattern is essential to deliver the required $\mathrm{FiO}_{2}$. Venturi masks and non-rebreathing masks are perforated to prevent carbon dioxide rebreathing but will allow some rebreathing of air, diluting the oxygen delivered.

NHF is more likely to match peak inspiratory flow rates of patients although the problem of entraining some air when the mouth is open remains. NHF may still not be able to match peak inspiratory flow rates above 90/L as observed in a trial with NHF used during exercise to simulate respiratory distress where greater amounts of air was entrained with higher peak inspiratory flow rates (Ritchie et al., 2011).

Prior to purpose-made NHF devices becoming readily available, samples from the nasopharynx were taken with $15 \mathrm{~L} / \mathrm{min}$ oxygen piped through a bubblehumidifier and delivered via nasal cannula and showed a higher $\mathrm{F}_{\mathrm{i}} \mathrm{O}_{2}$ than $<6 \mathrm{~L} /$ min oxygen (Wettstein et al., 2005). Following on, NHF delivered a higher $\mathrm{FiO}_{2}$ compared to a standard Hudson face mask when a pattern of breathing seen in respiratory failure was induced in a model and healthy volunteers, but 
similar to a non-rebreathing mask and Venturi mask (Sim et al., 2008; Wagstaff \& Soni, 2007).

\subsubsection{Reduction in upper airway resistance}

The nasal cavity and nasopharynx present a large surface area to warm, humidify and filter air. Resistance from these parts of the upper airways contributes up to $50 \%$ of the total airway resistance (Lee et al., 2013). NHF may help to overcome this substantial resistance to airflow, manifesting as a reduction in clinical work of breathing. The mechanism may be due to: 1) providing an airflow that overcomes the patients peak inspiratory flow or 2) similar to CPAP, by mechanically "splinting" open the distensible nasopharynx (Dysart et al., 2009; Lee et al., 2013).

The splinting effect of CPAP has led it to be a mainstay of treatment for the conditions such as obstructive sleep apnoea where the narrowing of the nasopharynx significantly contributes to airway obstruction, and resistance to airflow, causing apnoea and hypoxaemia. Neonates have highly distensible nasopharyngeal regions and CPAP is commonly used to maintain the upper airways patent. CPAP has been reported to reduce supraglottic resistance by nearly two-thirds in neonates (Miller et al, 1990).

$\mathrm{NHF}$ at $5 \mathrm{~L} / \mathrm{min}$ provided a similar reduction in work of breathing as CPAP set at $6 \mathrm{~cm} \mathrm{H} \mathrm{H}_{2} \mathrm{O}$ in a neonatal study (Saslow et al., 2006). Work of breathing indices were derived using respiratory inductance plethysmography (RIP) bands and were similar between NHF and CPAP despite NHF showing significantly lower PEEP $(1.32+/-0.77$ vs $1.76+/-1.46 \mathrm{cmH} 2 \mathrm{O}, \mathrm{P}<0.05)$, suggesting NHF reduces work of breathing by another mechanism other than PEEP (Dysart et al., 2009).

Another measure of work of breathing, a "rapid shallow breathing index" (respiratory rate divided by tidal volume) showed a reduction but no significant difference in COPD patients using NHF, nasal CPAP or nasal bi-level (Bräunlich \& Köhler, 2016). 


\subsubsection{Other similar methods of ventilation supporting the mechanisms of action of NHF}

Tracheal gas insufflation (TGI) and trans-tracheal oxygenation (TTO) are similar, although far more invasive, methods of ventilation than NHF, providing useful evidence to support the ability of a device to washout anatomical dead space.

TGI is used in artificially ventilated patients, where fresh gas is flushed through a catheter in the airway or a special endotracheal tube to eliminate carbon dioxide sitting in the artificial dead space (Danan et al, 1996; Dassieu et al., 1998). Flushing gas in during expiration also allows a greater concentration of the inspired gas, for example oxygen. TGI improves carbon dioxide elimination in neonates who have a relatively large intrinsic dead space to tidal volume ratio, enabling lower tidal volume and ventilator pressure settings and reduction in carbon dioxide (Dassieu et al., 1998) (Claure et al, 2003). While theoretically effective, the technical demands of administering TGI, such as appropriate staff and specialised equipment, result in TGI not being common practice. A Cochrane Systematic Review in 2002 concluded that there was a paucity of evidence to show a difference in hard outcomes like mortality, length of hospital stay and length of time on the ventilator (Davies \& Woodgate, 2002).

Delivery of trans-tracheal oxygen via a catheter (TTO) was a form of supplemental oxygen delivery used during the 1990s, not only for chronic hypoxaemia but to improve exercise tolerance (Dewan \& Bell, 1994). It could almost be considered a precursor to modern NHF therapy. One small TTO study in people with COPD resembles the effect of NHF, comparing high flows (3-8L/min) against low flows $(0.25-2 \mathrm{~L} / \mathrm{min})$ of TTO against standard nasal prongs. A significant difference was found in mean distance exercised, as well as improved oxygen saturations and dyspnoea scores (Dewan \& Bell, 1994). The effect was probably due to oxygen-enrichment and washout of the anatomical dead space (Dysart et al, 2009), irrespective of delivery method but demonstrates the effect of high flow gas delivery. Another small COPD study found TTO reduced $\mathrm{PaCO}_{2}$ by up to $20 \%$ of baseline with an increase in tidal volume and minute ventilation as well as a reduction in the dead space to tidal volume ratio (Nakos et al., 1995). 


\subsubsection{Evidence for NHF efficacy in hypoxaemic respiratory failure}

A systematic review and meta-analyses of NHF compared to either/or standard oxygen therapy and NIV in acute hypoxaemic respiratory failure demonstrated a trend towards decreased intubation rates and in-hospital mortality but was not statistically significant (Lin et al,, 2017). Eight studies with a total of 1818 patients were analysed. Hypercapnic patients were excluded. The primary outcome of intubation rates favoured NHF with an odds ratio of 0.79 (95\% Cl 0.6 to 1.04$)$ but again did not reach statistical significance $(P=0.09)$ (Figure 1-19).

The secondary outcome measure of in-hospital mortality trended towards favouring NHF with an odds ratio of $0.89(95 \% \mathrm{Cl} 0.620127)$ but was not statistically significant $(P=0.051)$ (see Figure 1-20).

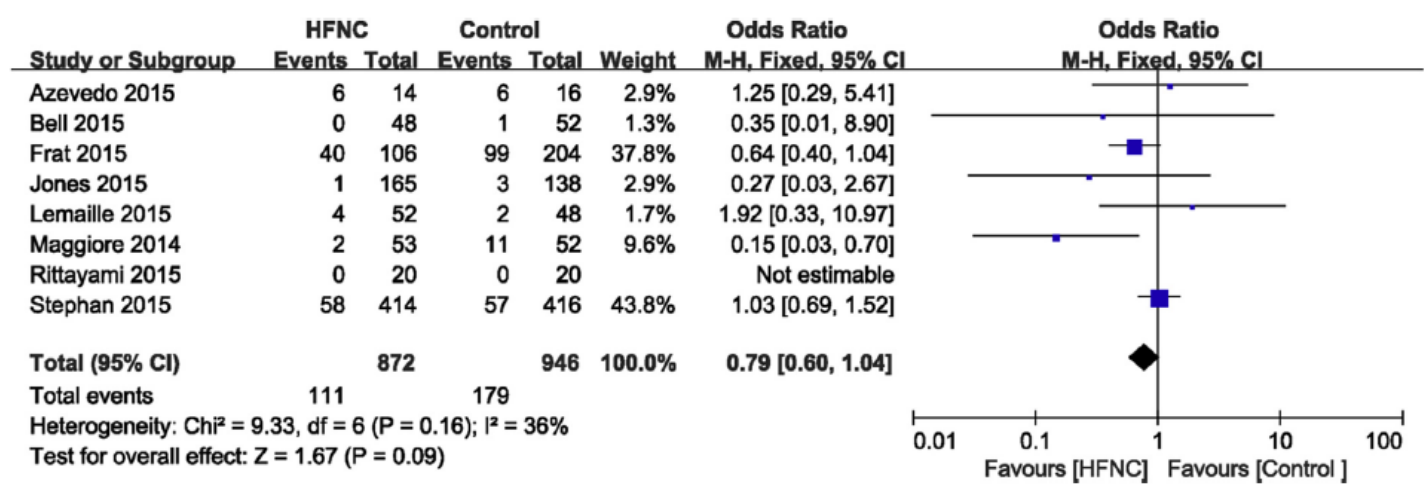

Figure 1-19 Forest plot comparing intubation rates in patients with NHF compared to standard oxygen therapy and NIV.

HFNC: high-flow nasal cannulae (also known as Nasal high flow).

[Reprinted from Respiratory Medicine, 131, Does high-flow nasal cannula oxygen improve outcome in acute hypoxemic respiratory failure? A systematic review and meta-analysis , (LIN ET AL., 2017) Pages 58-64., (c) 2018 with permission from Elsevier.] 


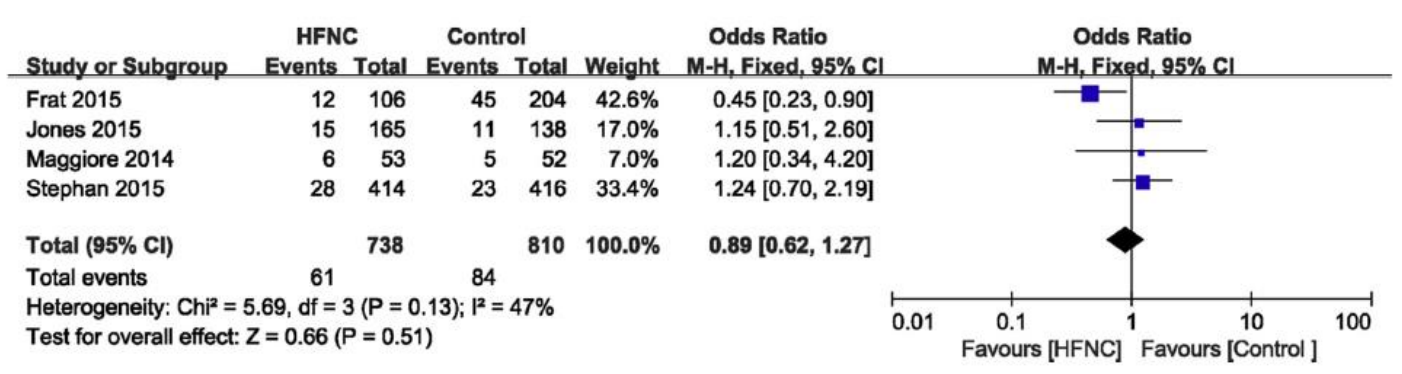

Figure 1-20 Forest plot comparing in-hospital mortality in patients treated with NHF compared to standard oxygen therapy and NIV.

HFNC: High-flow nasal cannulae (also known as Nasal high-flow).

[Reprinted from Respiratory Medicine, 131, Does high-flow nasal cannula oxygen improve outcome in acute hypoxemic respiratory failure? A systematic review and meta-analysis , (LIN ET AL., 2017) Pages 58-64., @ 2018 with permission from Elsevier.]

The author's conclusions from the systematic review and meta-analyses highlighted the limitations of statistical heterogeneity of included studies. Inclusion criteria were inconsistent, there were a variety of NHF flow rates used and therapy duration varied, as did oxygen flow rates. There was also uncertainty in the quality of the studies to limit bias and whether the sample size was sufficient to form a confident conclusion.

The original, small studies investigating NHF in acute hypoxaemic respiratory failure in 2010/11 in ICU showed an improvement in $\mathrm{PaO}_{2}$, reduction in respiratory rate and improved dyspnoea (Roca et al., 2010; Sztrymf et al., 2011). Since then, the evidence base has grown substantially. Most notably, a landmark 3-way RCT comparing oxygen delivered via $\mathrm{NHF}$ at $50 \mathrm{~L} / \mathrm{min}$ to standard face mask and NIV in 310 ICU patients with acute hypoxaemic respiratory failure demonstrated a survival benefit at 90 days with $\mathrm{NHF}$ compared to both standard face mask (hazard ratio of death $2.01(95 \% \mathrm{Cl} 1.01$ to 3.99 ), $\mathrm{P}=0.046$ ) and NIV (hazard ratio $2.50(95 \% \mathrm{Cl} 1.31$ to 4.78$), \mathrm{P}=0.006$ ) (Frat et al., 2015). Ventilator-free days by day 28 were also significantly higher in the NHF group. However, both 90-day mortality and day 28 ventilator-free days were secondary outcomes and the trial failed to show a significant difference by post-hoc analyses in the primary outcome which was rates of intubation at day 28 , although NHF did show a significant reduction in those who were most hypoxemic $\left(\mathrm{PaO}_{2}: \mathrm{FiO}_{2}\right.$ ratio $\left.\leq 200 \mathrm{mmHg}\right)$. There are other caveats to the conclusions from this study. Lower rates of septic shock in the 
NHF group $(17.9 \vee 30.9)$ and high tidal volumes required in the NIV group $(9 \mathrm{~mL} / \mathrm{kg})$ suggest the groups were not evenly matched in terms of degree of acute lung injury. Cross-over to NIV was allowed if treatment with NHF or standard face mask was failing and 40 patients received "rescue" NIV, 28 going on to require intubation.

NHF in post-cardiothoracic surgery patients with acute hypoxaemic respiratory failure showed a non-inferior rate of re-intubation compared to NIV (absolute difference, $0.9 \% ; 95 \% \mathrm{Cl},-4.9 \%$ to $6.6 \% ; \mathrm{P}=0.003$ )(Stéphan et al., 2015). ICU mortality was non-significant between the groups. COPD patients were again excluded.

There remain no international guidelines recommending NHF therapy for treatment of acute hypoxaemic respiratory failure, despite the affirmative results from the 2 large RCTs reviewed above and other clinical trials (Bell et al., 2015; Jones et al, 2016; Lemiale et al., 2017; Maggiore et al., 2014; Rittayamai et al, 2015).

Patients with COPD and HRF were excluded from these studies, likely due to the unproven ability of NHF to reduce hypercapnia and the existence of an already well-established standard therapy (NIV).

\subsubsection{Caveats for NHF use in hypoxaemic respiratory failure}

The main caveats when using a new therapy that has an evolving evidence base relate to generalizability of results from clinical trials which often include a narrow spectrum of patients with respiratory failure. Translating findings from one clinical scenario to another would require caution e.g. hypoxic respiratory failure post-extubation compared to pneumonia. Co-morbidities are often controlled for in clinical trials and the multi-morbid elderly critically ill patient may be excluded from clinical trials. Also, generalising the results of NHF superiority over standard nasal prongs, face masks or venturi masks should not be translated to mean it is superior over NIV without sound evidence. There has been some criticism in the literature about the comparisons to 
standard therapy regimens that are inadequate, such as the tidal volumes delivered with NIV in the Frat et al study already reported.

It would also be important to ensure there is a clear plan in place if NHF fails, as mandated in international NIV guidelines (Davidson et al., 2016). With that in mind, a retrospective audit looked at the risk of delayed intubation after NHF failure which resulted in increased mortality (Kang et al., 2015). One hundred and thirty of the 175 patients (74.3\%) intubated were done so within 48 hours of commencing NHF compared to $45(25.7 \%)$ after 48 hours. Patients who were intubated early, within 48 hours, had a lower ICU mortality rate $(39.2 \%$ vs $66.7 \%, P=0.001$ ) as well as more ventilator free days, higher extubation success rates and ventilator weaning. For the early intubation group, the propensity-adjusted and -matched analysis had a lower odds of dying in ICU (adjusted odds ratio $=0.317, \mathrm{P}=0.005$; matched odds ratio $=0.369, \mathrm{P}=$ $0.046)$ than delayed intubation. However, there was no significant difference in mortality rates at day 14 and 28 , suggesting more of the patients who had delayed intubation remained in ICU ventilated beyond 28 days.

With an evidence base for the mechanisms of action of NHF (discussed in section 1.4.3) evolving, an effect in COPD is also expected. It is apparent that robust clinical trials in hypercapnic respiratory failure are now required. Section 1.4.5 describes the small but encouraging evidence base for NHF use in reducing $\mathrm{PCO}_{2}$ in COPD and opens up the knowledge gap that the research from this thesis aims to fill.

\subsubsection{Evidence for NHF efficacy in COPD}

The efficacy of NHF in acute hypoxaemic respiratory failure was reviewed, in Section 1.4.4 above, and provides high-level evidence for its implementation in adults. While the evidence is still developing for NHF in this setting, its clinical use is increasing and sets the scene for the application of NHF in hypercapnic respiratory failure and COPD - the focus of this thesis. This chapter will review the limited evidence for NHF in AHRF that existed when the studies for this thesis were conceived and the variable evidence that has been published since. 


\subsubsection{Systematic review of NHF use reducing $\mathrm{PCO}_{2}$ in COPD}

NHF is a relatively new therapy and research in this field is dynamic so the systematic review that follows is divided into the literature available prior to conception of research trials for this thesis in 2015 and literature that has been published since.

\subsection{SYSTEMATIC REVIEW METHODOLOGY}

A variety of study types have been implemented to investigate the effect of NHF on $\mathrm{PCO}_{2}$ in COPD including $\mathrm{RCTs}$, prospective and retrospective observational cohort studies, case reports and case series. A systematic review was carried out in June 2018 with the aim of identifying all studies which could be used to answer a focussed clinical question formed using the PICO format, as below (Figure 1-21):

\begin{tabular}{|c|c|c|}
\hline$P$ & Population/Participants & People with COPD of any severity \\
\hline I & Intervention(s) & $\begin{array}{l}\text { NHF use with any flow, with or without } \\
\text { supplemental oxygen }\end{array}$ \\
\hline $\mathrm{C}$ & Control/comparison & $\begin{array}{l}\text { Either oxygen via standard nasal prongs, } \\
\text { face mask or NIV (with or without oxygen } \\
\text { and by any method), and/or room air alone. }\end{array}$ \\
\hline O & Outcome & $\begin{array}{l}\text { Change in } \mathrm{PCO}_{2} \text { measured by any method - } \\
\mathrm{P}_{\mathrm{a}} \mathrm{CO}_{2}, \mathrm{P}_{\mathrm{t}} \mathrm{CO}_{2}, \mathrm{P}_{\text {cap }} \mathrm{CO}_{2}, \mathrm{P}_{\mathrm{v}} \mathrm{CO}_{2} \text {. }\end{array}$ \\
\hline
\end{tabular}

Figure 1-21 Focussed clinical question using the PICO format

The research question to be answered by the systematic review of the literature is therefore:

"What is the effect of NHF at any flow rate, with or without oxygen, on the $\mathrm{PCO}_{2}$ in people with COPD compared to standard oxygen therapy and/or NIV?"

The literature search was carried out using appropriate search terms from the PICO question, in both Medline and Embase databases to answer the clinical research question (Figure 1-22) and selected according to Preferred 
Reporting Items for Systematic Reviews and Meta-Analyses

(PRISMA)(Moher et al., 2009) guidelines outlined in Figure 1-23 below.

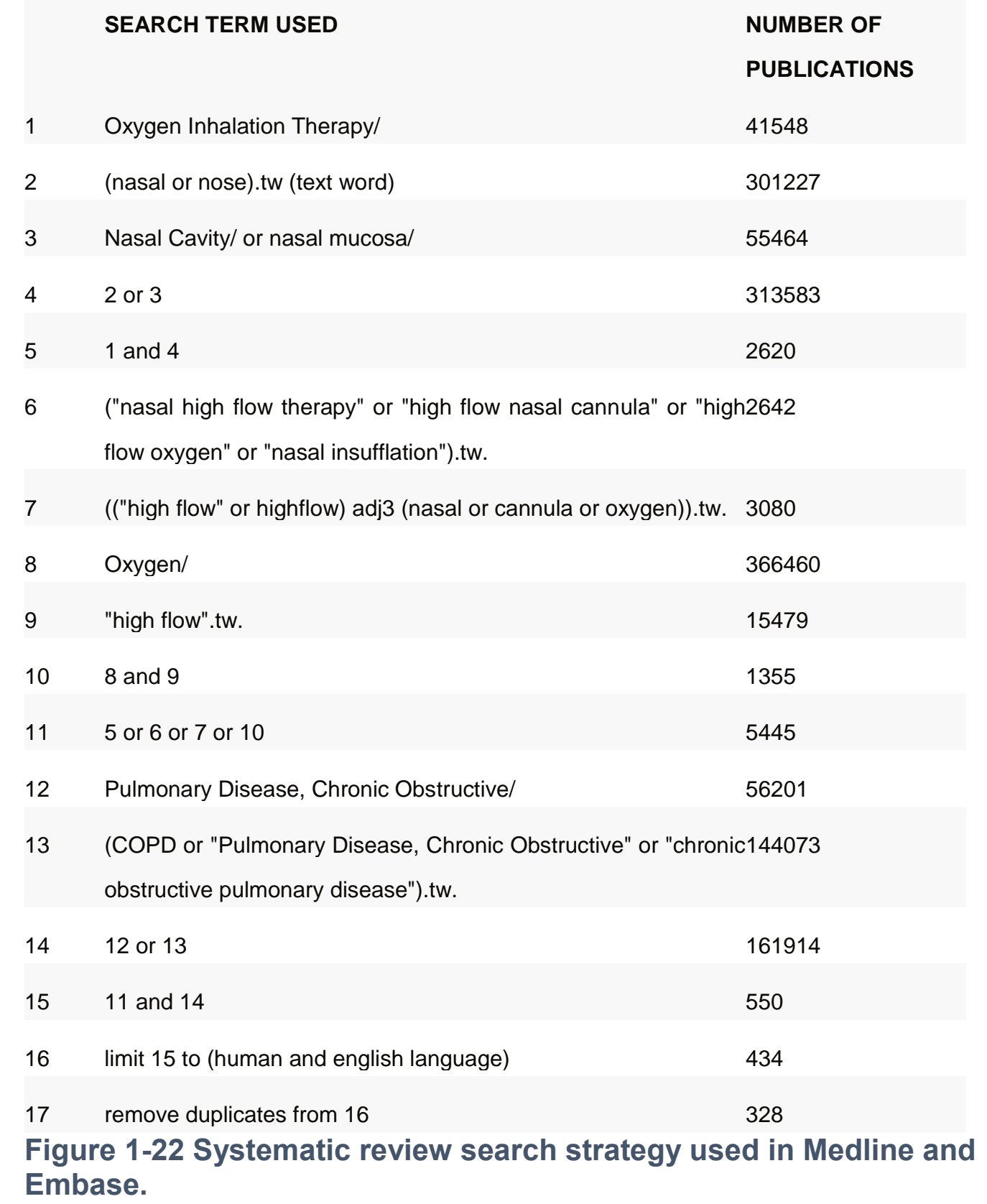




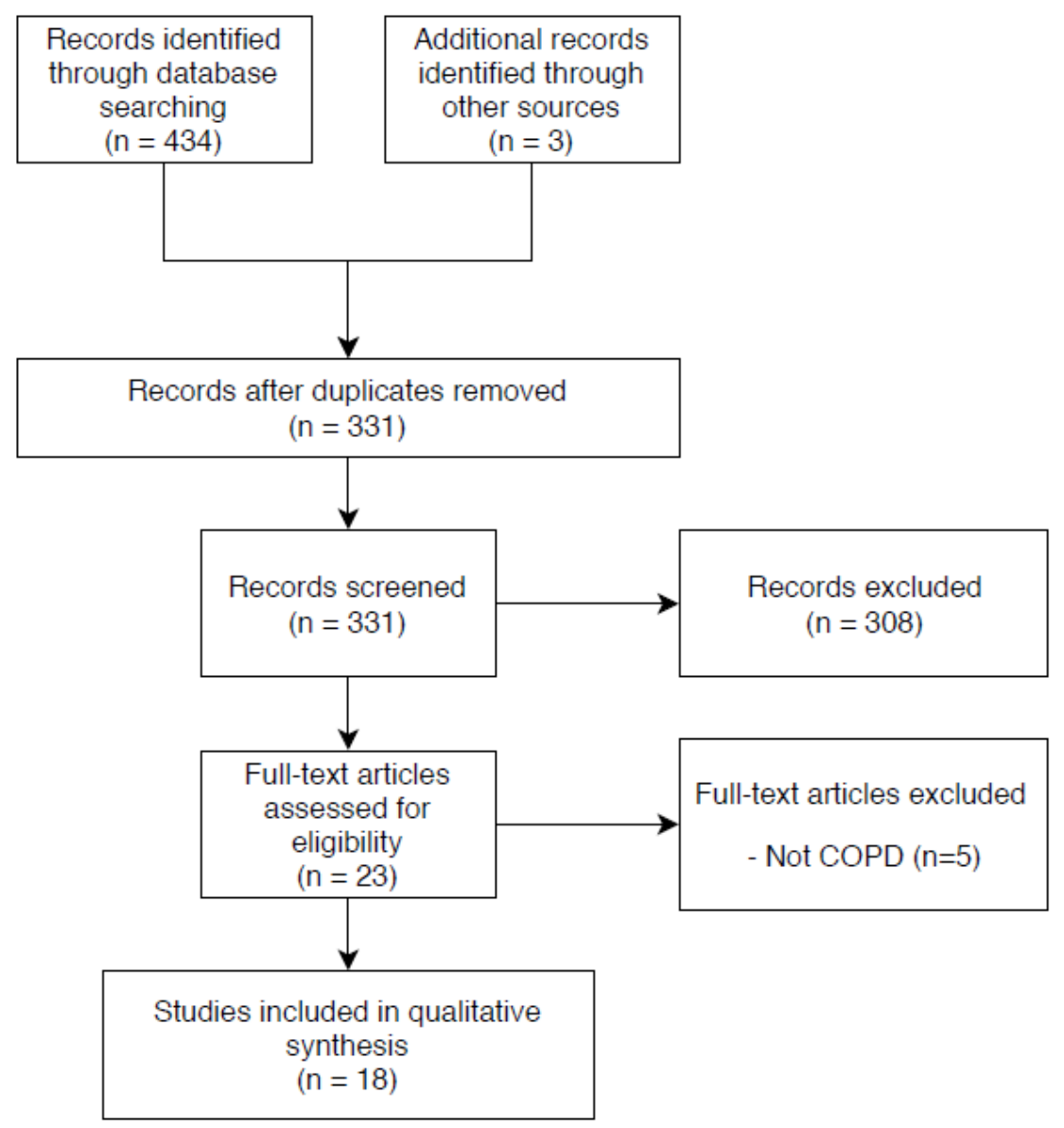

Figure 1-23 PRISMA flowchart of the process used to select publications for the systematic review.

Eighteen publications were identified from the systematic review. Publications available prior to this thesis commencing in 2015 are summarised in Table 1-7 and those becoming available during the thesis tenure are summarised in Table 1-8.

\subsection{DESCRIPTIVE REVIEW OF THE LITERATURE AVAILABLE PRIOR TO AND INCLUDING 2015}

Of the 18 publications selected according to the PRISMA guidelines only 7 were published prior to commencing the research trials for this thesis in 2015 (the Fraser et al study was first presented as an ATS conference abstract in 2013 before formal publication in Thorax in 2016). These 7 publications are summarised in Table 1-7.

Only 2 of these were RCTs, both with cross-over designs, and only 1 of the 7 trials was investigating the effects of NHF without concomitant oxygen, a known confounder for $\mathrm{PCO}_{2}$ change. 
Another one of the studies (Chatila et al, 2004) used a mouth-piece to deliver the humidified high-flow oxygen rather than nasal prongs but is included as an early "proof of concept" of high-flow therapy in COPD and is a frequently cited publication in the NHF literature.

Publications are classified according to the levels of evidence used by the Scottish Intercollegiate Guideline Network (SIGN), ranging from 1a (most robust) to 5 (least reliable) (Baird \& Lawrence, 2014). SIGN levels are used when establishing grades of recommendations for clinical guidelineconstruction by professional bodies. Organising the publications of NHF in COPD by SIGN level of evidence further illustrates the scarcity of quality research in this field. 
Table 1-7 Summary of publications pre-2015 investigating $\mathrm{PCO}_{2}$ change with NHF in COPD.

\begin{tabular}{|c|c|c|c|c|c|c|c|c|c|c|}
\hline \multirow[t]{2}{*}{ Reference } & \multirow{2}{*}{$\begin{array}{l}\text { SIGN } \\
\text { Level } \\
\text { of } \\
\text { evide } \\
\text { nce }\end{array}$} & \multirow{2}{*}{$\begin{array}{l}\text { Sample } \\
\text { size }\end{array}$} & \multirow[t]{2}{*}{ Design } & \multirow{2}{*}{$\begin{array}{l}\text { Cohort of } \\
\text { COPD } \\
\text { participants }\end{array}$} & \multirow{2}{*}{$\begin{array}{l}\text { Supplement } \\
\text { al Oxygen } \\
\text { used? }\end{array}$} & \multicolumn{3}{|c|}{ Interventions } & \multirow{2}{*}{$\begin{array}{l}\mathrm{PCO}_{2} \\
\text { measur } \\
\text { ement } \\
\text { techniq } \\
\text { ue }\end{array}$} & \multirow[t]{2}{*}{ Results of effect on $\mathrm{PCO}_{2}$} \\
\hline & & & & & & NHF flow-rate & Control & $\begin{array}{l}\text { Duration } \\
\text { (min/hour) }\end{array}$ & & \\
\hline $\begin{array}{l}\text { Millar et al, } 2014 \\
\text { (Ther Adv Resp } \\
\text { Med) }\end{array}$ & 3 & 1 & Case report & Hypercapnic & Yes & $50 \mathrm{~L} / \mathrm{min}$ & Nil & 6 hours & ABG & $\begin{array}{l}\mathrm{P}_{\mathrm{a}} \mathrm{CO}_{2} \text { reduced from } 69 \mathrm{mmHg} \\
\text { to } 62 \mathrm{mmHg} \text { after } 1 \text { hour and } \\
55 \mathrm{mmHg} \text { after 6hours. }\end{array}$ \\
\hline $\begin{array}{l}\text { Jeong et al, } 2015 \\
\text { (Am J Em Med) }\end{array}$ & $2-$ & 173 & $\begin{array}{l}\text { Retrospective } \\
\text { audit }\end{array}$ & Mixed* & Yes & $\begin{array}{l}\text { Variable flow rate with mean } \\
\text { of } 45 \mathrm{~L} / \mathrm{min}\end{array}$ & $\mathrm{Nil}$ & $\begin{array}{l}\text { Variable } \\
\text { time used } \\
\geq 1 \text { hour }\end{array}$ & ABG & $\begin{array}{l}\text { Significant reduction by } \\
3.4 \mathrm{mmHg} \text { overall and } 6 \mathrm{mmHg} \text { in } \\
\text { hypercapnic group }\end{array}$ \\
\hline $\begin{array}{l}\text { Chatila et al, } \\
2004 \text { (Chest) }\end{array}$ & $2-$ & 6 & $\begin{array}{l}\text { Prospective } \\
\text { cohort }\end{array}$ & Mixed* & Yes & $\begin{array}{l}\text { High-flow } 02 \text { via mouthpiece } \\
\text { at } 20 \mathrm{~L} / \mathrm{min}\end{array}$ & $\begin{array}{l}\text { Standard } \mathrm{O} 2 \text { at } 2.5-6 \mathrm{Lmin} \\
\text { while exercising }\end{array}$ & $12 \mathrm{~min}$ & ABG & $\begin{array}{l}\text { Non-significant difference } \\
\text { between means; NHF increased } \\
45-46 \mathrm{mmHg} \text {, LFO 41-44L/min }\end{array}$ \\
\hline $\begin{array}{l}\text { Braunlich et al, } \\
2013 \\
\text { (Respiration) }\end{array}$ & $2-$ & 8 & $\begin{array}{l}\text { Prospective } \\
\text { cohort }\end{array}$ & Hypercapnic & No & $20 \mathrm{~L} / \mathrm{min}$ & Unknown & 8 hours & CBG & $\begin{array}{lll}\text { Significant } & \text { reduction by } \\
5.7 \mathrm{mmHg} & \end{array}$ \\
\hline $\begin{array}{l}\text { Braunlich et al, } \\
2015 \\
\text { (Multidisciplnary } \\
\text { Resp Med) }\end{array}$ & $2-$ & 11 & $\begin{array}{l}\text { Prospective } \\
\text { cohort }\end{array}$ & Hypercapnic & Yes & $20 \mathrm{~L} / \mathrm{min}$ & $\begin{array}{lll}\text { NIV with } & \text { mean } & \text { IPAP } \\
16 \mathrm{cmH} 2 \mathrm{O} & \text { and } & \text { EPAP } \\
5.8 \mathrm{cmH} 2 \mathrm{O} & & \end{array}$ & $\begin{array}{l}5 \mathrm{hrs} / \text { day for } \\
6 \text { weeks }\end{array}$ & CBG & $\begin{array}{lll}\text { Significant } & \text { reduction by } \\
8.2 \mathrm{mmHg} & \end{array}$ \\
\hline $\begin{array}{l}\text { Nilius et al, } 2013 \\
\text { (Adv Exp Med) }\end{array}$ & $1-$ & 17 & $\begin{array}{l}\text { Randomised, } \\
\text { cross-over }\end{array}$ & Hypercpanic & Yes & $20 \mathrm{~L} / \mathrm{min}$ & Standard $\mathrm{O} 2$ at $2 \mathrm{~L} / \mathrm{min}$ & $45 \mathrm{~min}$ & CBG & $\begin{array}{l}\text { Non-significant reduction by } \\
3.1 \mathrm{mmHg}\end{array}$ \\
\hline $\begin{array}{l}\text { Fraser et al, } \\
2013 / 2016 \\
\text { (Thorax) }\end{array}$ & $1+$ & 30 & $\begin{array}{l}\text { Randomised, } \\
\text { cross-over }\end{array}$ & LTOT, mixed* & Yes & $\begin{array}{l}30 \mathrm{~L} / \mathrm{min} \text { for } \\
\begin{array}{l}20 \mathrm{mins} \\
\text { compared } \\
\text { oxygen alone }\end{array}\end{array}$ & Standard 02 at 2-4L/min & $20 \mathrm{~min}$ & $\begin{array}{l}\text { Transcu } \\
\text { taneous }\end{array}$ & $\begin{array}{lll}\text { Significant reduction } & \text { by } \\
3.4 \mathrm{mmHg} & \end{array}$ \\
\hline
\end{tabular}

SIGN: Scottish Intercollegiate Guideline Network, ABG: arterial blood gas, CBG: capillary blood gas; IPAP: inspiratory positive airways pressure, EPAP: expiratory positive airways pressure, NIV: non-invasive ventilation, $\mathrm{PCO}_{2}$ : partial pressure of carbon dioxide. ${ }^{*}$ Mixed refers to normocapnic and hypercapnic patients 
A description of the studies captured in the systematic review in Table 1-7 is presented below and in each case follows the level of evidence, from lowest (least reliable) to highest (most robust).

\subsection{CASE REPORT}

1. Millar, Lutton, \& O’Connor, 2014

(Journal: Ther Adv Resp Dis.)

A 57 yo female with mild AHRF secondary to AECOPD declined NIV due to previous poor experience but agreed to a trial of $\mathrm{NHF}$ at $50 \mathrm{~L} / \mathrm{min}$ with $\mathrm{FiO} 2$ set at $28 \%$. Symptoms and all ABG values improved after only 30 minutes, and $\mathrm{pH}$ normalised after 1 hour from the initial value of 7.31 .

CONCLUSION: Anecdote of successful reversal of mild AHRF with NHF plus oxygen at $50 \mathrm{~L} / \mathrm{min}$.

\subsection{RETROSPECTIVE COHORT STUDIES}

\section{Jeong et al., 2015}

(Journal: Am J Emerg Med.)

A retrospective audit of 81 patients with acute respiratory failure in ED, $85.7 \%$ with COPD and 46 (56.8\%) of whom were hypercapnic. Of the hypercapnic group and non-hypercapnic groups, $65.2 \%$ and $22.9 \%$ had AHRF secondary to AECOPD respectively. Although the audit was not exclusively COPD patients, the high proportion meant it was reasonable to include in this systematic review.

Varying clinician-set flows of NHF with oxygen were used for at least one hour and demonstrated a statistically significant reduction in $\mathrm{PaCO}_{2}$ in the hypercapnic group (73.2 \pm 20.0 to $67.2 \pm 23.4 ; \mathrm{P}=0.02)$, but not in the nonhypercapnic group. $\mathrm{PaO}_{2}$ and $\mathrm{S}_{\mathrm{p}} \mathrm{O}_{2}$ also showed significant increases in both groups. $\mathrm{pH}$ increased significantly in the hypercapnic group only. Despite this, similar rates of NIV or IMV-requirement and in-hospital mortality between the 2 groups were found.

STRENGTHS AND WEAKNESSES: As a retrospective audit, it is limited by adequate documentation, collection of some data points, and a strict criteria for initiating NHF. Also, there was significant inconsistency in the design, such as eligibility criteria, indications for NIV/IMV, varying flow rates and $\mathrm{FiO}_{2}$ set by the 20 different ED physicians. In addition, not all patients received the NHF for the full one hour. The cohort was certainly unwell with a third requiring ICU 
admission and the collection of ABG data provides an accurate measurement of blood gas values.

CONCLUSION: NHF demonstrated a trend towards blood gas improvement in hypercapnic COPD patients.

\subsection{PROSPECTIVE COHORT STUDIES}

1. Chatila et al, 2004

(Journal: Chest)

In an early NHF trial, an exercise protocol was used to test the oxygen-delivery performance of the Vapotherm device in 10 people with severe COPD. Vapotherm is a NHF device but in this study a mouthpiece was used to deliver oxygen at $20 \mathrm{~L} / \mathrm{min}$ for 12 mins while participants exercised on a stationary bicycle and then crossed-over to receive low-flow oxygen through standard nasal cannulae at $2.5-6 \mathrm{~L} / \mathrm{min}$ for $12 \mathrm{~min}$. $\mathrm{F}_{\mathrm{i}} \mathrm{O}_{2}$ was matched during interventions. Exercise was used to test the hypothesis that delivering highflow oxygen and air would match the inspiratory demands of participants, hence improving oxygenation, tidal volumes and exercise tolerance.

Only 5 participants were able to complete both 12 min exercise efforts with mean exercise time longer in the NHF group (10.0 \pm 2.4 minutes) than lowflow oxygen group $(8.2 \pm 4.3$ minutes $), P<0.05$. Blood gas data are only reported for 3 participants and showed a non-significant difference in mean $\mathrm{P}_{\mathrm{a}} \mathrm{CO}_{2}$ between the two groups. $\mathrm{PaCO}_{2}$ is only illustrated graphically and not clearly reported, but extrapolating from a line plot, $\mathrm{NHF}$ increased $\mathrm{P}_{\mathrm{a}} \mathrm{CO}_{2}$ from $41 \mathrm{mmHg}$ to $44 \mathrm{mmHg}$ and low-flow oxygen increased $\mathrm{PaCO}_{2}$ from $45 \mathrm{mmHg}$ to $46 \mathrm{mmHg}$. Similarly, $\mathrm{PaO}_{2}$ data are only illustrated graphically (change in NHF group was approximately $-15 \mathrm{mmHg}$ and $-28 \mathrm{mmHg}$ in LFO group, $\mathrm{P}<0.001$ ), indicating a significantly smaller decrease in $\mathrm{P}_{\mathrm{a}} \mathrm{O}_{2}$ with NHF than low-flow oxygen. Breathing parameters such as respiratory rate, respiratory rate to tidal volume ratio and inspiratory time to total respiration time were all significantly lower with NHF. This may indicate a trend towards matching inspiratory flow with the inspiratory demands of participants, although a missing metric here is end-expiratory lung volume. Dyspnoea scores were significantly lower with NHF; however, no difference was found with $M V, V_{T}$ and work of breathing. 
STRENGTHS AND WEAKNESSES: High-flow gas delivery was with a mouthpiece rather than nasal-prongs, making the study design inconsistent and a small sample size made the statistical conclusions uncertain, especially with only 3 sets of $A B G$ data reported. Added to this, the lack of randomisation limits interpretation. Strengths of the study were matching $\mathrm{FiO} 2$ between the two groups, and overall, useful proof of concept data is presented.

CONCLUSION: High-flows of humidified oxygen improved exercise tolerance in people with severe COPD and improved some breathing parameters.

\section{Braunlich et al., 2013}

(Journal: Respiration)

This was a pilot study of 8 patients with stable COPD and "global respiratory insufficiency" receiving NHF therapy with the Tnl Oxy NHF device at $20 \mathrm{~L} / \mathrm{min}$ for $8 \mathrm{hrs}$. It appears they were not receiving supplementary oxygen at the time although no baseline oxygen saturations are given to be certain. Extrapolating from the small line plot, 1 patient had a high baseline $\mathrm{P}_{\text {cap }} \mathrm{CO}_{2}$ of $66.8 \mathrm{mmHg}$, suggesting possible oxygen-induced hypercapnia, but as oxygensupplementation is not clearly described, I have concluded this is the only NHF study not using supplemental oxygen. All participants were hypercapnic at baseline and demonstrated a mean reduction in $\mathrm{P}_{\text {cap }} \mathrm{CO}_{2}$ of $5.7 \mathrm{mmHg}$ from baseline $(P<0.05)$, a reduction in respiratory rate by $2.4 \mathrm{bpm}$ and $V_{T}$ increase by $87.8 \mathrm{~mL}$, both with $\mathrm{P}<0.05$. $\mathrm{V}_{\mathrm{T}}$ is reported as " $87.8 \mathrm{~mL} \pm 88.6 \mathrm{~mL}$ ", illustrating the wide confidence bounds and statistical uncertainty of these results with such a small sample size.

STRENGTHS AND WEAKNESSES: Interpretation of the results are limited by a small sample size, lack of randomised design, inconsistency of design such that CBG results are reported for 8 COPD patients but different number of participants have respiratory rate, mean pharyngeal pressures and $\mathrm{V}_{T}$ measurements reported. It is not clear which participant had what measurement done. The absence of supplementary oxygen use with NHF adds some reliability to the $\mathrm{PCO}_{2}$ change, removing the confounding effect of oxygen-induced hypercapnia. A cross-over design appears to have been employed, with 8 hours of "spontaneous breathing" although the results of this control period are not reported, and it is unclear if the results presented are 
differences against the control period of spontaneous breathing or against NHF at baseline.

CONCLUSION: Small proof of concept study showing a moderate reduction in $\mathrm{P}_{\text {cap }} \mathrm{CO}_{2}$ and respiratory rate and increase in $\mathrm{V}_{\mathrm{T}}$ over a long time-period.

\section{Bräunlich, Seyfarth, \& Wirtz, 2015 (Journal: Multidisc Resp med)}

This was a preliminary report of 11 stable hypercapnic COPD patients who completed NHF at $20 \mathrm{~L} / \mathrm{min}$ for $5 \mathrm{hrs} /$ day for 6 weeks followed by NIV (unspecified pressure and volume settings) for 6 weeks. Supplemental oxygen was given, but flow rate and $\mathrm{F}_{\mathrm{i}} \mathrm{O}_{2}$ was not specified for either intervention. A dramatic reduction of $\mathrm{P}_{\text {cap }} \mathrm{CO}_{2}$ was found, from a baseline mean of $53.7 \mathrm{mmHg}$ down to $45.5 \mathrm{mmHg}$ for $\mathrm{NHF}$ and $46.4 \mathrm{mmHg}$ for $\mathrm{NIV}$, both with $\mathrm{P}<0.05$ for the change from baseline, but no significant difference was found between NHF and NIV.

STRENGTHS AND WEAKNESSES: It is difficult to draw conclusions from an 11 patient-study framed as a preliminary report, however the intended sample size is 105 on the clintrials.gov registration for this trial. The trial appears to have been completed in July 2017 but results have yet to be published. A description of the non-randomised design and more details about device settings and oxygen supplementation will be required in the final publication. Pisani et al (see 1.4.5.1.3.4) have since published an almost identical trial but with a randomised design. Also, there are no details of a washout period between interventions, and the NIV results appear to have been compared to the baseline at least 6 weeks earlier, making it difficult to interpret the results. CONCLUSION: Results of an interim report show a trend towards $\mathrm{PCO}_{2}$ reduction with long-term NHF use that was comparable to long-term NIV use.

\subsection{RANDOMISED CONTROLLED TRIALS}

1. Nilius et al., 2013

(Journal: Adv Exp Biology)

Seventeen patients hospitalised with AECOPD and hypercapnic respiratory failure were recruited. Baseline oxygen delivery through standard nasal prongs at $2 \mathrm{~L} / \mathrm{min}$ after $45 \mathrm{~min}$ was compared to NHF (using the TNIOxy device) at $20 \mathrm{~L} / \mathrm{min}$ with the same oxygen flow of $2 \mathrm{~L} / \mathrm{min}$. 
NHF was delivered through one nostril ("partial nasal insufflation") or both nostrils ("high flow nasal insufflation"). The change in $\mathrm{PcaPCO}_{2}$ from baseline was variable with an overall non-significant mean reduction of $3.1 \mathrm{mmHg}$ in both the partial and high flow nasal insufflation groups compared to baseline. Six participants had a reduction in $\mathrm{P}_{\text {cap }} \mathrm{CO}_{2}$ of $>8 \mathrm{mmHg}$ and a small but statistically significant reduction in respiratory rate was found of $19.8 \pm 4.2$ at baseline, compared to $18.0 \pm 4.7$ with high flow nasal insufflation, $\mathrm{P}<0.01$.

STRENGTHS AND WEAKNESSES: Because of a small sample size, this study probably lacked statistical power to detect the difference in $\mathrm{P}_{\text {cap }} \mathrm{CO}_{2}$. In addition, the $\mathrm{FiO}_{2}$ was not standardised, complicating the interpretation of any changes in $\mathrm{P}_{\text {cap }} \mathrm{CO}_{2}$.

CONCLUSION: NHF with oxygen at $20 \mathrm{~L} / \mathrm{min}$ resulted in a small reduction in $\mathrm{P}_{\text {cap }} \mathrm{CO}_{2}$, which is unlikely to be clinically significant.

\section{Fraser et al, 2016}

(Journal: Thorax)

This was a randomised controlled cross-over trial in 30 males with stable COPD on long-term oxygen therapy. NHF and oxygen at $30 \mathrm{~L} / \mathrm{min}$ was administered for 20 min at the same $\mathrm{FiO}_{2}$ from each participant's standard LTOT prescription. Each participant then crossed-over to receive their LTOT regimen at 2-4 L/min via standard nasal cannulae.

$\mathrm{P}_{\mathrm{t}} \mathrm{CO}_{2}$ decreased by a difference of $3.4 \mathrm{mmHg}$ between the 2 groups, a relative decrease of $9 \%, \mathrm{P}<0.001$. $A \mathrm{PCO}_{2}$ change of $\geq 4 \mathrm{mmHg}$ is considered physiologically significant, and these results fall marginally short of this threshold (K Perrin, Wijesinghe, Healy, et al., 2011; Meme Wijesinghe, Williams, Perrin, Weatherall, \& Beasley, 2011).

NHF improved the inspiratory:expiratory ratio, decreased respiratory rate by 3.8 breaths per minute $(P<0.001)$, increased $V_{T}$ by $100 \mathrm{~mL}(P=0.003)$ and increased end-expiratory lung volumes (measured with electrical impedance tomography) by $174 \%$ with NHF compared to $113 \%$ with standard LTOT, $P<0.001$. MV was no different between the groups, measured with RIP bands. On a scale from 0 (no dyspnoea/no discomfort) to 10 (maximum dyspnoea/maximum discomfort), dyspnoea and comfort scores favoured standard LTOT over NHF. For dyspnoea, the LTOT group median score was 
0.5 vs NHF 2.0, $\mathrm{P}<0.001$, and for comfort the LTOT group median score was 9 vs NHF group of $8, \mathrm{P}<0.02$.

STRENGTHS AND WEAKNESSES: This was a well-designed trial with meaningful results, albeit only in males, limiting its generalisability. Oxygen administration may have confounded the effect of $\mathrm{P}_{\mathrm{t}} \mathrm{CO}_{2}$ change and the difference observed between groups. Baseline oxygen saturations were not reported, and it is possible the $\mathrm{PtCO}_{2}$ difference resulted from unmatched oxygen saturations between groups, given the LTOT group had a higher mean $\mathrm{S}_{\mathrm{H}} \mathrm{O}_{2}(101.2 \mathrm{mmHg}$ vs $97.1 \mathrm{mmHg}, \mathrm{P}=0.01)$. The method of ventilatory data collection appears sound although the accuracy of RIP band data is questioned in a later chapter of this thesis (see Section 2.3.5.1.1).

CONCLUSION: NHF with oxygen at $30 \mathrm{~L} / \mathrm{min}$ resulted in a small reduction in $\mathrm{P}_{\mathrm{t}} \mathrm{CO}_{2}$, which is unlikely to be clinically significant. Comfort and dyspnoea scores favoured standard LTOT over NHF.

\subsection{DESCRIPTIVE REVIEW OF THE LITERATURE PUBLISHED SINCE THESIS COMMENCED}

Eleven further papers have been published while this thesis was being completed and are summarised in Table 1-8.They are organised according to the SIGN level of evidence and described in further detail below. 
Table 1-8 Summary of publications post-2015 investigating PCO2 change with NHF.

\begin{tabular}{|c|c|c|c|c|c|c|c|c|c|c|}
\hline \multirow[t]{2}{*}{ Reference } & \multirow{2}{*}{$\begin{array}{l}\text { SIGN } \\
\text { Level }\end{array}$} & \multirow{2}{*}{$\begin{array}{l}\text { Sam } \\
\text { ple } \\
\text { size }\end{array}$} & \multirow[t]{2}{*}{ Design } & \multirow{2}{*}{$\begin{array}{l}\text { Cohort of } \\
\text { COPD } \\
\text { participants }\end{array}$} & \multirow{2}{*}{$\begin{array}{l}\text { Oxy } \\
\text { gen } \\
\text { use } \\
\text { d? }\end{array}$} & \multicolumn{3}{|c|}{ Interventions } & \multirow{2}{*}{$\begin{array}{l}\mathrm{PCO}_{2} \\
\text { measurem } \\
\text { ent }\end{array}$} & \multirow[t]{2}{*}{ Results of effect on $\mathrm{PCO}_{2}$} \\
\hline & & & & & & NHF flow-rate & Control & $\begin{array}{l}\text { Durati } \\
\text { on }\end{array}$ & & \\
\hline $\begin{array}{l}\text { Lepere et al, } \\
2016 \text { (Am J } \\
\text { Em Med) }\end{array}$ & 3 & 1 & $\begin{array}{l}\text { Case } \\
\text { report }\end{array}$ & Hypercapnic & Yes & $\begin{array}{l}60 \mathrm{~L} / \mathrm{min} \text { with } \mathrm{FiO} 2 \\
0.6\end{array}$ & Nil & 4 days & $A B G$ & $\begin{array}{l}\mathrm{P}_{\mathrm{a}} \mathrm{CO}_{2} \text { reduced from } 75 \text { to } 70 \mathrm{mmHg} \text { over first } 24 \mathrm{hrs}, \mathrm{pH} \\
\text { normalized. }\end{array}$ \\
\hline $\begin{array}{l}\text { Fricke et al, } \\
2016 \text { a }\end{array}$ & 3 & 1 & $\begin{array}{l}\text { Case } \\
\text { report }\end{array}$ & Hypercapnic & Yes & $\begin{array}{l}30 \mathrm{~L} / \mathrm{min} \text { NHF with } \\
3 \mathrm{~L} / \mathrm{min} \mathrm{O} 2\end{array}$ & Nil & 25 mins & $\mathrm{P}_{\mathrm{t}} \mathrm{CO}_{2}$ & $\mathrm{P}_{\mathrm{t}} \mathrm{CO}_{2}$ reduced from 68 to $63 \mathrm{mmHg}$ \\
\hline $\begin{array}{l}\text { Pavlov et al, } \\
2017\end{array}$ & 3 & 4 & $\begin{array}{l}\text { Case } \\
\text { series }\end{array}$ & Hypercapnia & NK & $55 \mathrm{~L} / \mathrm{min}$ & Nil & $\begin{array}{l}3,4,16 \\
20 \text { hrs }\end{array}$ & VBG & $\begin{array}{l}n \text { too small for full stats analyses and endpoint was } \\
\text { "normalisation of } \mathrm{pH}-\mathrm{PvCO}_{2} \text { reduced by } 40,26,30 \text {, } \\
32 \mathrm{mmHg} \text { respectively }\end{array}$ \\
\hline $\begin{array}{l}\text { Braunlich et } \\
\text { al, 2016 (Int J } \\
\text { COPD) }\end{array}$ & $2-$ & 54 & $\begin{array}{l}\text { Prospec } \\
\text { tive } \\
\text { cohort }\end{array}$ & $\begin{array}{l}\text { Inpatients, } \\
\text { mixed }^{*}\end{array}$ & No & 20 and $30 \mathrm{~L} / \mathrm{min}$ & $\begin{array}{l}\text { Room air and nasal } \\
\text { CPAP and nasal bi- } \\
\text { level }\end{array}$ & 2 hours & CBG & $\begin{array}{l}\text { Significant reduction by } 5.2 \mathrm{mmHg} \text { at } 20 \mathrm{~L} / \mathrm{min} ; 7.3 \mathrm{mmHg} \\
\text { at } 30 \mathrm{~L} / \mathrm{min}\end{array}$ \\
\hline $\begin{array}{l}\text { Biselli et al, } \\
2017\end{array}$ & $2-$ & 12 & $\begin{array}{l}\text { Prospec } \\
\text { tive } \\
\text { cohort }\end{array}$ & $\begin{array}{l}\text { Normocapni } \\
\text { c }\end{array}$ & Both & $20 \mathrm{~L} / \mathrm{min} \mathrm{NHF}$ & $\begin{array}{l}\text { Standard } \mathrm{O} 2 \text { at } 2 \mathrm{~L} / \mathrm{min} \\
\text { and room air, cycled } \\
\text { through the night. }\end{array}$ & $10 \mathrm{~min}$ & $\mathrm{P}_{\mathrm{t}} \mathrm{CO}_{2}$ & $\begin{array}{l}\text { Raw numbers not presented. } \mathrm{P}_{1} \mathrm{CO}_{2} \text { increased by } 3 \% \\
\text { (approx. } 1.3 \mathrm{mmHg} \text { ) in standard } \mathrm{O} 2 \text { group, decreased by } \\
4 \% \text { (approx. } 1.7 \mathrm{mmHg} \text { ). Stat sig difference. }\end{array}$ \\
\hline $\begin{array}{l}\text { Vogelsinger et } \\
\text { al, } 2017 \text { (BMC } \\
\text { Pulm Med) }\end{array}$ & $2-$ & 77 & $\begin{array}{l}\text { Prospec } \\
\text { tive } \\
\text { cohort }\end{array}$ & Mixed $^{*}$ & Yes & 15L/min & $\begin{array}{l}\text { Standard oxygen at } \\
\text { mean rate } 2.1 \mathrm{~L} / \mathrm{min}\end{array}$ & $60 \mathrm{mins}$ & CBG & Significant reduction $-2.11 \mathrm{mmHg}$ compared to baseline \\
\hline $\begin{array}{l}\text { Braunlich et } \\
\text { al, } 2018 \text { (BMC } \\
\text { Pulm Med) }\end{array}$ & $2-$ & 36 & $\begin{array}{l}\text { Prospec } \\
\text { tive } \\
\text { cohort }\end{array}$ & Hypercapnic & Yes & $\begin{array}{l}20 \text { and } 40 \mathrm{~L} / \mathrm{min} \text { with } \\
1 \text { or } 2 \text { prongs }\end{array}$ & Nil & 1 hour & CBG & Significant reduction by $13.2 \%$ baseline \\
\hline $\begin{array}{l}\text { Pisani et al, } \\
2017 \text { (Thorax) }\end{array}$ & $1+$ & 14 & $\begin{array}{l}\text { RCT } \\
\text { cross- } \\
\text { over }\end{array}$ & Hypercapnic & Yes & $\begin{array}{l}20 \text { and } 30 \mathrm{~L} / \mathrm{min} \\
\text { mouth open and } \\
\text { closed }\end{array}$ & $\begin{array}{l}\text { Bi-level } \\
\text { pressure or volume } \\
\text { settings) }\end{array}$ & $30 \mathrm{~min}$ & ABG & Non-significant max reduction by $-5.5 \mathrm{mmHg}$ \\
\hline $\begin{array}{l}\text { Pilcher et al, } \\
2017 \\
\text { (Respirology) }\end{array}$ & $1+$ & 24 & $\begin{array}{l}\text { RCT, } \\
\text { cross- } \\
\text { over }\end{array}$ & Mixed $^{*}$ & Yes & $35 \mathrm{~L} / \mathrm{min}$ & $\begin{array}{l}\text { Standard } \mathrm{O} 2 \text { at } \\
\text { variable flows }\end{array}$ & $30 \mathrm{mins}$ & $\begin{array}{l}\text { Transcutan } \\
\text { eous }\end{array}$ & $\begin{array}{l}\text { Significant reduction by }-1.4 \mathrm{mmHg} \text { compared to control } \\
\text { group }\end{array}$ \\
\hline $\begin{array}{l}\text { Storgaard et } \\
\text { al, } 2018\end{array}$ & $1+$ & 200 & RCT & $\begin{array}{l}\text { Mixed }^{*} \\
\text { LTOT }\end{array}$ & Yes & $\begin{array}{l}20 \mathrm{~L} / \mathrm{min} \text { for mean } \\
6 \mathrm{hr} / \mathrm{day}\end{array}$ & Standard LTOT & 1 year & ABG & $\begin{array}{l}\text { Significant difference at 1yr compared to control group } \\
\text { but raw data not presented. Also, not controlled but } \\
\text { baseline mean } 48.8 \mathrm{mmHg} \text {, reduced to } 46.5 \mathrm{mmHg} \text { after } \\
\text { 30mins NHF }\end{array}$ \\
\hline $\begin{array}{l}\text { Nagata et al, } \\
2018 \text { (Annals } \\
\text { ATS) }\end{array}$ & $1+$ & 32 & $\begin{array}{l}\text { RCT } \\
\text { cross- } \\
\text { over }\end{array}$ & $\begin{array}{l}\text { Hypercapnic } \\
\text {, LTOT }\end{array}$ & Yes & $\begin{array}{l}\text { Mean } \sim 30 \mathrm{~L} / \mathrm{min} \text { for } \\
\sim 7-8 \mathrm{hrs} / \mathrm{night}\end{array}$ & Standard LTOT & $\begin{array}{l}6 \\
\text { weeks }\end{array}$ & ABG & $\begin{array}{l}\text { Significant reduction }-4.1 \mathrm{mmHg} \text { compared to control } \\
\text { group }\end{array}$ \\
\hline
\end{tabular}




\subsection{CASE REPORTS}

1. Fricke et al., 2016

(Journal: Respir Med Case Rep.)

A 62 yr old male with very severe COPD and a capped-tracheostomy received $30 \mathrm{~L} / \mathrm{min}$ NHF with $3 \mathrm{~L} / \mathrm{min}$ oxygen for 15 min intervals. Intra-tracheal pressure catheters recorded a reduction in tracheal pressures, $\mathrm{V}_{\mathrm{T}}$ and volume of inspired carbon dioxide. $\mathrm{P}_{\mathrm{t}} \mathrm{CO}_{2}$ decreased from 68 to $63 \mathrm{mmHg}$ and respiratory rate from 26 to 22 breaths/min over the single 15 min time-period presented.

CONCLUSION: Clearance of anatomical dead space may explain the decreased volume of inspired carbon dioxide and $\mathrm{P}_{\mathrm{t}} \mathrm{CO}_{2}$ reduction.

2. Lepere, Messika, \& Ricard, 2016

(Journal: Am J Emerg Med.)

A 67 yo male with AHRF secondary to AECOPD and influenza-induced pneumonia received NHF at $60 \mathrm{~L} /$ min with $\mathrm{FiO}_{2} 0.6$ after being unable to tolerate first-line NIV. On day 5, before NHF was initiated, his $\mathrm{P}_{\mathrm{a}} \mathrm{CO}_{2}$ had risen to $75 \mathrm{mmHg}$. $\mathrm{PaCO}_{2}$ decreased to $70 \mathrm{mmHg}$ over the following $24 \mathrm{hrs}$ on $\mathrm{NHF}$, with $\mathrm{pH}$ normalising and a return to his baseline conscious state.

CONCLUSION: Anecdotal evidence of successful AHRF reversal with NHF plus oxygen at $60 \mathrm{~L} / \mathrm{min}$.

\subsection{CASE SERIES}

1. Pavlov, Plamondon, \& Delisle, 2017 (Journal: Respir Med Case Rep.)

Four patients with severe AHRF secondary to AECOPD but contra-indicated to NIV, received NHF at $55 \mathrm{~L} / \mathrm{min}$ with oxygen adjusted to maintain saturations $\geq 90 \%$ for $3,4,16$ and $20 \mathrm{hrs}$ respectively to achieve the outcome reported of a normalised $\mathrm{pH}$ on venous blood gas (VBG - a sample of blood taken from puncturing a vein, rather than an artery). $\mathrm{PvCO}_{2}$ decreased by large amounts during these times on NHF - $40 \mathrm{mmHg}(3 \mathrm{hr}), 26 \mathrm{mmHg}(4 \mathrm{hr}), 30 \mathrm{mmHg}$ (16 hr) and $32 \mathrm{mmHg}(20 \mathrm{hr}) \mathrm{mmHg}$ respectively.

CONCLUSION: NHF may have a role in treating AHRF when NIV is contraindicated or intolerable or may be used as an alternative in specific cases. 


\subsection{PROSPECTIVE COHORT STUDIES}

1. Bräunlich \& Köhler, 2016 (Journal: Int J Chron Obstruct Pulmon Dis.) Sixty-seven hospitalised COPD patients received NHF at flows between 20 and $50 \mathrm{~L} / \mathrm{min}$ without supplemental oxygen for $2 \mathrm{hrs}$ and were compared against breathing room air, receiving nasal CPAP and nasal bi-level. Airway pressures were measured in 19 patients, $\mathrm{MV}$ in 18 patients and $\mathrm{P}_{\mathrm{cap}} \mathrm{CO}_{2}$ in 54 patients. The 54 patients who had $\mathrm{P}_{\text {cap }} \mathrm{CO}_{2}$ measured only received $\mathrm{NHF}$ at 20 and $30 \mathrm{~L} / \mathrm{min}$. A statistically significant, flow-dependent reduction in $\mathrm{P}_{\text {cap }} \mathrm{CO}_{2}$ with NHF of $5.2 \mathrm{mmHg}$ at $20 \mathrm{~L} / \mathrm{min}$ and $7.3 \mathrm{mmHg}$ at $30 \mathrm{~L} / \mathrm{min}$ was observed. There was a flow-dependent increase in mean airway pressures, reduction in respiratory rate and reduction in $\mathrm{MV}$ with all changes reaching statistical significance.

STRENGTHS AND WEAKNESSES: Similar to the 2013 study by the same authors reported earlier, the trial design was non-randomised, inconsistent and difficult to follow, with different patients receiving different interventions. No supplemental oxygen was again a strength to show $\mathrm{P}_{c a p} \mathrm{CO}_{2}$ reduction without the confounding effect of oxygen although it is surprising no patients were hypoxic, given the high mean $\mathrm{P}_{c a p} \mathrm{CO}_{2}$ of $58.2 \mathrm{mmHg}$ suggestive of chronic respiratory failure. No oxygen saturations are reported.

CONCLUSION: Another proof of concept study reaffirming findings from this group's 2013 trial, albeit with caveats around interpretation of the results, given the inconsistent study design.

\section{Biselli et al., 2017}

(Journal: J Appl Physiol.)

In a prospective observational study, 12 people with COPD were alternated between NHF at $20 \mathrm{~L} / \mathrm{min}$, low-flow oxygen at $2 \mathrm{~L} / \mathrm{min}$ via standard nasal cannulae and room air each for $10 \mathrm{~min}$, at night while sleeping. There was a control-group of 6 healthy volunteers. Polysomnography and ventilatory measurements were taken as well as $\mathrm{P}_{\mathrm{t}} \mathrm{CO}_{2}$. An oesophageal pressure monitor was inserted in 6 people ( 2 in the control group and 4 in the COPD group) to calculate work of breathing by integrating the flow volume signal from a pneumotachograph over the oesophageal pressure measured. 
Percentage change rather than raw numbers are presented in the results. $\mathrm{P}_{\mathrm{t}} \mathrm{CO}_{2}$ increased by $3 \%$ (approx. $1.3 \mathrm{mmHg}$ ) in the low-flow oxygen group, whereas it decreased by $4 \%$ (approx. $1.7 \mathrm{mmHg}$ ) with NHF which reached statistical significance compared to both room air and low-flow oxygen. Similarly, MV and $\mathrm{V}_{\mathrm{T}}$ and work of breathing decreased with NHF.

STRENGTHS AND WEAKENESSES: Non-randomised, small sample size and different parameters recorded in varying sub-groups amounts to an inconsistent design. $\mathrm{P}_{\mathrm{t}} \mathrm{CO}_{2}$ results are difficult to compare, given oxygen was was delivered through the standard nasal cannulae but not through NHF. The difference in $\mathrm{S}_{\mathrm{a}} \mathrm{O}_{2}$ of $97 \%$ (standard oxygen group) vs $93 \%$ (NHF with no oxygen group) reflects this discrepancy.

CONCLUSION: Interesting but complex, physiological study of uncertain significance.

\section{Vogelsinger et al., 2017}

(Journal: BMC Pulm Med.)

$\mathrm{P}_{\text {cap }} \mathrm{CO}_{2}$ reduced by $2.11 \mathrm{~mm} \mathrm{Hg}$ with $15 \mathrm{~L} / \mathrm{min}$ NHF compared to standard oxygen flow over 60 min in 77 COPD patients on LTOT. $\mathrm{P}_{\text {capO}} \mathrm{O}_{2}$ was reported to be higher in the NHF arm with a lower oxygen flow rate (a very small difference of $1.95 \mathrm{~L} / \mathrm{min}$ with $\mathrm{NHF}$ vs $2.1 \mathrm{~L} / \mathrm{min}$ with standard oxygen). NHF was "well tolerated" by all 77 patients, but no objective tolerability data is presented.

STRENGTHS AND WEAKENESSES: A non-randomised design, no regular carbon dioxide monitoring and imprecise oxygen supplementation limits the reliability of the results. Oxygen was titrated to maintain $\mathrm{P}_{c a p} \mathrm{O}_{2}>60 \mathrm{mmHg}$ but capillary oxygen sampling is an inaccurate surrogate measure for $\mathrm{P}_{a} \mathrm{O}_{2}(\mathrm{R}$. Murphy et al., 2006; Yildizdaş, Yapicioğlu, Yilmaz, \& Sertdemir, 2004). Oxygen was also increased if $\mathrm{S}_{\mathrm{p}} \mathrm{O}_{2}$ was $<90 \%$ which should have been $<88 \%$ according to international guidelines (O'Driscoll et al., 2017) and no upper $\mathrm{S}_{\mathrm{pO}} \mathrm{O}_{2}$ limit is reported. Six patients in the NHF did not require oxygen. It appears this was a sub-trial as part of a larger domiciliary NHF study, as the main purpose was probably to show less oxygen is required using NHF, therefore preserving oxygen-concentrators at home. 
CONCLUSION: No conclusions can be drawn about NHF improving oxygenation, and $\mathrm{PCO}_{2}$ results are also difficult to interpret in this context.

\section{Bräunlich, Mauersberger, \& Wirtz, 2018 (Journal: BMC Pulm Med.)}

$\mathrm{PCO}_{2}$ reduction and airways pressure were both flow and leakage dependent and correlated with the degree of hypercapnia in 36 COPD patients. NHF was applied under four different combinations of low (20 L/min)/high (40 L/min) flow and low/high leakage. Varying amounts of leakage were achieved by inserting one or both prongs inside the nostrils for $1 \mathrm{hr}$. Varying amounts of oxygen were applied, and interventions applied in a random order, although confusingly not all participants received all interventions. Twenty-two received $\mathrm{NHF}$ at $20 \mathrm{~L} / \mathrm{min}$ with both prongs inside the nares (termed 'low leak', condition A in Figure 1-24); 19 received NHF at $40 \mathrm{~L} /$ min with low leak (condition B), 20 received NHF at $40 \mathrm{~L} / \mathrm{min}$ but with 1 prong sitting outside the nare (termed 'high leak' or condition C) and 19 received $40 \mathrm{~L} / \mathrm{min}$ with 1 prong outside the nare but plugged closed (condition D). It is not clear which participants crossed over into which of the 4 interventions. Figure 1-24 illustrates the $4 \mathrm{NHF}$ conditions tested.

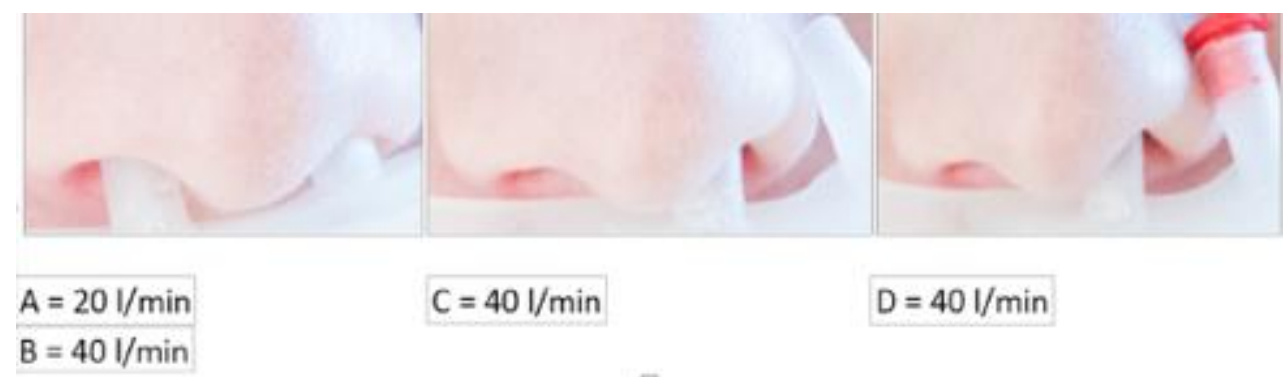

Figure 1-24. Illustration of the 4 NHF conditions tested in Braunlich et al, BMC Pulmonary Medicine 2018.

[Reproduced under the Creative commons 01.0 universal public domain dedication]

Ten people had nasopharyngeal airway pressures measured although it is obscure how many were from each of the $4 \mathrm{NHF}$ conditions tested.

Twenty-six of the 36 participants were hypercapnic at baseline, and this group showed the maximum reduction in $\mathrm{P}_{\mathrm{cap}} \mathrm{CO}_{2}$ of $13.2 \%$ from baseline under condition D. Unfortunately, baseline mean $\mathrm{P}_{\text {cap }} \mathrm{CO}_{2}$ for this group was not reported. The magnitude of $\mathrm{P}_{c a p} \mathrm{CO}_{2}$ reduction had a linear relationship with both flow-rate and leakage in the hypercapnic sub-group. 
STRENGTHS AND WEAKNESSES: Conclusions are limited by an inconsistent study design, unclear cross-over of participants and no prespecified primary outcome measures. A post-hoc analysis was reported as the main point of interest (participants with a baseline $\mathrm{P}_{\text {cap }} \mathrm{CO}_{2}>55$ ). $\mathrm{PO} 2$ from $\mathrm{CBG}$ samples was also presented which has been proven to be very inaccurate (Heidari et al., 2013; R. Murphy et al., 2006; Zavorsky, Cao, Mayo, Gabbay, \& Murias, 2007a). Conclusions were drawn between $\mathrm{PCO}_{2}$ change and airway pressure despite only 10 people having this measured. In fact, $\mathrm{PCO}_{2}$ clearance despite only a small increase in airway pressure could possibly have been a better conclusion to make.

CONCLUSION: A proof of concept study demonstrating a flow and leakagedependent reduction in $\mathrm{PCO}_{2}$ with NHF. While the authors do not comment on higher turbulent airflow in the nasopharynx with unilateral NHF, this may be the mechanism observed here to explain the increased effect with this condition.

\subsection{RANDOMISED CONTROLLED TRIALS}

1. Pisani et al., 2017

(Journal: Thorax)

This RCT compared NHF with oxygen in 4 different combinations $-20 \mathrm{~L} / \mathrm{min}$ and $30 \mathrm{~L} / \mathrm{min}$, each with mouth open and closed - and NIV, for $30 \mathrm{~min}$ in 14 people with stable hypercapnic COPD. A non-significant reduction from baseline $\mathrm{PaCO}_{2}$ was found with $\mathrm{NHF}$ at $20 \mathrm{~L} / \mathrm{min}$ (mouth closed minus 4 $\mathrm{mmHg}$ ), $30 \mathrm{~L} / \mathrm{min}$ (mouth closed minus $5.5 \mathrm{mmHg}$ ) and $6 \mathrm{mmHg}$ with NIV. $\mathrm{V}_{\mathrm{T}}$ significantly increased as NHF flow-rate increased and was higher again with NIV. Trans-diaphragmatic pressure (Pdi) was measured to derive inspiratory effort and iPEEP values. Expiratory time was increased in all NHF conditions and NIV, as were IPEEP and Pdi although this was significantly less with NIV compared to baseline and NHF.

STRENGTHS AND WEAKNESSES: Small sample size and oxygen supplementation limits the interpretation although $\mathrm{F}_{i} \mathrm{O}_{2}$ was maintained at a constant level. The accuracy of measuring inspiratory effort and other lung mechanics, such as iPEEP, by Pdi may be variable (Troyer \& Estenne, 1981). The use of $A B G$ s adds integrity to the blood gas results. There was no major differences in results between NHF with mouth open and closed, which makes 
the findings more generalisable, given it is unrealistic to expect people to keep their mouth closed to preserve a seal to maximise the effect, especially when acutely unwell.

CONCLUSION: Small physiological study showing NHF, with mouth open and closed, reduced $\mathrm{PaCO}_{2}$ by a similar magnitude to NIV, although not statistically significant. An improvement in breathing pattern and inspiratory effort was observed with both NHF and NIV.

2. Pilcher et al., 2017

(Journal: Respirology)

An inpatient randomised, cross-over trial in 24 patients with AECOPD found a small but statistically significant reduction in $\mathrm{P}_{\mathrm{t}} \mathrm{CO}_{2}$ of $1.4 \mathrm{mmHg}$ with $\mathrm{NHF}$ at $35 \mathrm{~L} / \mathrm{min}$ compared to standard oxygen alone, as well as a reduction in respiratory rate of 2 breaths per minute.

STRENGTHS AND WEAKNESSES: FiO2 was not controlled, instead oxygen was continued to match the $\mathrm{S}_{\mathrm{p}} \mathrm{O}_{2}$ at baseline, set by the in-patient medical team, which was often discordant with international guideline recommendations to maintain $\mathrm{S}_{\mathrm{p}} \mathrm{O}_{2}$ between 88-92\% (O'Driscoll et al., 2017). Applying NHF with oxygen to inpatients means the findings are generalisable and the treatment was well tolerated in this cohort.

CONCLUSION: Oxygen delivered with NHF at $35 \mathrm{~L} /$ min resulted in a small reduction in $\mathrm{P}_{\mathrm{t}} \mathrm{CO}_{2}$ and respiratory rate.

\section{Storgaard et al, 2018 (Journal: Int J Chron Obstruct Pulmon Dis.)}

The authors present a large $(n=200)$, multi-centre domiciliary trial in COPD patients equally randomised to LTOT via NHF at $20 \mathrm{~L} / \mathrm{min}$ for $6 \mathrm{hr} /$ day for 1 year, or to standard LTOT.

The NHF group had a baseline mean $\mathrm{PaCO}_{2}$ of $48.8 \mathrm{mmHg}$ which reduced to $46.5 \mathrm{mmHg}$ after an initial 30 mins of $\mathrm{NHF}$ and was then reported as a significant reduction of $2.0 \mathrm{mmHg}(P=0.005)$ at 12 months compared to the control group, although raw data were not presented nor was the change in $\mathrm{PaCO}_{2}$ for the standard LTOT group.

The AECOPD rate was lower in the NHF group (3.12 versus 4.95/patient/year, $P=0.001)$ and reduced further with increased NHF use, as well as increasing 
number of days of NHF use. "Modelled" hospital admission rates were lower in the NHF group - 0.79 versus 1.39/patient/year for 12- versus 1-month use of NHF, respectively $(P=0.001)$. The modelling refers to the inclusion of NHF use as a co-variate in the Poisson regression model. SGRQ improved at 6 and 12 months $(P=0.002, P=0.001)$, suggesting an improvement in quality of life and reduction in symptom burden. All-cause mortality showed no significant difference between groups - 15\% in the NHF group and $12 \%$ in the control group, $\mathrm{P}=0.636$.

STRENGTHS AND WEAKNESSES: Unfortunately the study was not registered with a trials registry prior to starting recruitment. The sample size allowed adequate power to show a difference in AECOPD rates, the primary outcome, based on a historical estimate of 3.8 per patient per year. While this estimate was not referenced in the publication, it was actually high compared to other estimates of 0.82 to 2.01 and will vary with severity of disease (Hoogendoorn, Feenstra, Hoogenveen, Al, \& Mölken, 2010; Hurst et al., 2010). The main short-coming of the study was no blinding of interventions which the authors report as being "unrealistic" but is in fact achievable with a sham device. A placebo arm may ameliorate the placebo-effect. Mean NHF usage of $6 \mathrm{hrs}$ per day is excellent, and the only reminder participants were given was a phone-call every 3 months, therefore reasonable generalisability of the findings to clinical practice. There was however no reporting of adherence to control therapy. Nocturnal NHF use was higher than day-time use (53\% vs $32 \%)$ which may affect results as the nocturnal NHF effect differs when sleeping, with a reduced MV (Mundel, Feng, Tatkov, \& Schneider, 2013). No sensitivity analyses were done to separate out the difference between day time and nocturnal use.

CONCLUSION: LTOT via NHF reduced exacerbation rates, hospitalisation rates and improved symptoms compared to standard LTOT.

\section{Nagata et al., 2017 (Journal: Ann Am Thorac Soc.)}

Thirty-two COPD patients on LTOT were randomised to receive NHF with oxygen titrated to $\mathrm{SpO} 2>88 \%$ for 6 weeks (Group A) and then crossed-over to receive their standard LTOT flow rate for 6 weeks. Group B received the interventions in the reverse order. The primary outcome was change in health- 
related quality of life scores (SGRQ) and $\mathrm{P}_{\mathrm{a}} \mathrm{CO}_{2}$ change was a secondary outcome. From a baseline $\mathrm{PaCO}_{2}$ of over $50 \mathrm{mmHg}$ in both groups, the adjusted within-subject effect of NHF compared with LTOT was $-4.1 \mathrm{mmHg}$ $(95 \% \mathrm{Cl}-6.5$ to $-1.7, \mathrm{P}<0.01)$. Overall and each individual SGRC scores were significantly lower with NHF also $(-7.8,95 \% \mathrm{Cl}-11.9$ to $-3.7, \mathrm{P}<0.01)$. Given no change in pulmonary function or exercise capacity, the authors conclude that the improved $\mathrm{QOL}$ is likely due to $\mathrm{PaCO}_{2}$ reduction and reduced work of breathing, supported by the greatest individual SGRQ component being "symptom" reduction. Zero participants on NHF were hospitalised with AECOPD while 3 on standard LTOT alone were.

STRENGTHS AND WEAKNESSES: A mean NHF use of $7.1 \mathrm{hrs}$ and $8.6 \mathrm{hrs}$ per night in each group demonstrated excellent compliance. Only 3 of the 29 participants who completed the trial were female. The authors compare their $\mathrm{PaCO}_{2}$ reduction with that reported in the large domiciliary-NIV trials reported earlier (Section 1.3.3.3, (Köhnlein et al., 2014; McEvoy et al., 2009; P. B. Murphy et al., 2017) adding significance to their conclusion that domiciliaryNHF has excellent potential.

CONCLUSION: Long-term oxygen therapy via NHF reduced $\mathrm{P}_{a} \mathrm{CO}_{2}$ and improved quality of life compared to standard LTOT alone.

\subsubsection{Summary of systematic review and answering the clinical question}

Very few publications were available prior to commencing this thesis and the trials published were limited by inconsistencies in interventions, such as differing amounts of supplemental oxygen, which itself can affect $\mathrm{P}_{2} \mathrm{CO}_{2}$ levels, non-randomised designs and small sample sizes. Critical gaps were identified in the pre-existing literature and used to create the hypotheses for the clinical trials in this thesis, and is further discussed in section 1.5 (Knowledge gaps and aims of the thesis). Trials published during the process of completing this thesis have complemented my research findings, as will be discussed in each study chapter to follow.

Results of the systematic review provide an answer to the focused clinical question proposed above: 
"What is the effect of NHF at any flow rate, with or without oxygen, on the $\mathrm{PCO}_{2}$ of people with COPD compared to standard oxygen therapy and/or NIV?"

The literature provides preliminary evidence to support the ability of NHF to reduce $\mathrm{PCO}_{2}$ in people with $\mathrm{COPD}$, but the $\mathrm{PCO}_{2}$ reduction appears to be of a modest magnitude and with inter-patient variability. There is no evidence it is more effective than NIV in reducing $\mathrm{PCO}_{2}$. Factors such as acuity of the clinical scenario, severity of COPD, length of therapy as well as flow-rates and $\mathrm{F}_{\mathrm{i}} \mathrm{O}_{2}$ may make it difficult to predict who will maximally respond to the therapy. There remains no landmark publication, as there is with Frat et al (N Engl J Med 2015) in acute hypoxaemic respiratory failure, confirming the efficacy of NHF in COPD.

\subsubsection{Evidence of NHF use reducing hospital readmission rates in COPD}

Prior to conception of the research trials for this thesis, evidence to support NHF use in reducing rates of COPD hospitalizations or readmissions was limited to a single RCT (Rea et al., 2010). Outpatients ( $n=108)$ with COPD $(58 \%)$ or bronchiectasis $(42 \%)$ were randomised to receive NHF at 20 to 25 $\mathrm{L} / \mathrm{min}$ for at least two hours per day, or standard care for 12 months. Compliance was reasonable with a mean (SD) NHF hourly use of $1.6(0.67)$ hours/day.

The NHF group had significantly fewer exacerbation days (18.2 versus 33.5 days; $P=0.045$ ) and increased time to first exacerbation (median 52 versus 27 days; $P=0.0495$ ). There was a non-significant trend towards a lower rate of exacerbations in the NHF group (2.97/patient/year versus 3.63/patient/year; $\mathrm{P}=0.067)$. There was also a trend towards a lower hospital admission rate for the NHF group during the 12 months (0.39/year) compared with the control group (0.47/year) but not statistically significant $(P=0.439)$.

Lung function significantly improved in the NHF group - a $0.12 \mathrm{~L}$ increase in FEV1 and $0.28 \mathrm{~L}$ increase in FVC, while the control group's lung function declined. An economic analysis of this study showed that the treatment was cost-effective at a willingness to pay threshold in the range for reimbursement of new medicines in NZ (Milne et al, 2014). 
Since the completion of research trials for this thesis, the Storgaard et al 2018 study discussed earlier (see Section 1.4.5.1.3.4) has provided additional evidence for the ability of NHF to reduce hospitalisation rates.

\subsubsection{Evidence of NHF improving comfort}

NHF therapy compared to various methods of oxygen delivery has generally been demonstrated to have superior comfort and tolerability as shown in Table 1-9 Comfort of various oxygen-delivery devices

Table 1-9 Comfort of various oxygen-delivery devices compared to NHF.

\begin{tabular}{lll}
\hline Oxygen delivery device & Comfort compared to NHF & References \\
\hline Standard Hudson face & Inferior & (Bell et al., 2015; \\
masks, venturi masks and & Cuquemelle et al., 2012; Frat \\
standard nasal prongs & et al., 2015; Maggiore et al., \\
& 2014; Rittayamai et al., 2015; \\
& Roca et al., 2010; Sztrymf et \\
& al., 2012)(Antonicelli et al., \\
& $2011)$
\end{tabular}

\begin{tabular}{lll} 
& No difference & (Lemiale et al., 2017) \\
\cline { 2 - 3 } & Superior & (Jones et al., 2016) \\
\hline Nasal CPAP & Marginally inferior & (Bräunlich \& Köhler, 2016) \\
\hline Oro-facial CPAP & Inferior & (Chanques et al., 2013; Frat \\
& & et al., 2015; Schwabbauer et \\
& & al., 2014) \\
\cline { 2 - 3 } & No difference & (Stéphan et al., 2015) \\
\hline Nasal bi-level & No difference & (Bräunlich \& Köhler, 2016) \\
\hline Oro-facial bi-level & Inferior & (Pisani et al., 2017) \\
\hline CPAP: Continuous positive airways pressure &
\end{tabular}

\subsubsection{Evidence for efficacy of NHF in non-COPD conditions}

\subsubsection{Other respiratory conditions}

NHF use for obesity hypoventilation syndrome is limited to a small sample conference abstract which reported similar ABG values and length of hospital stay compared to bi-level (Papachatzakis, 2017). Efficacy of NHF use in obstructive sleep apnoea is limited to case series and trials with small sample sizes in children (Hawkins et al, 2017; Joseph et al, 2015) and a small proof of concept study in adults (McGinley et al., 2007).

There are no published cystic fibrosis trials although there is a pilot study registered on the clinicaltrials.gov website investigating the role of NHF in improving quality of life. Evidence for use in neuromuscular disease is limited to a case report (Diaz-Lobato et al, 2013). 
Acute hypoxaemic respiratory failure in immunocompromised patients had mixed results using NHF with evidence of no difference in mortality or rates of intubation in a large non-randomised "propensity score based" study (Lemiale et al., 2017) but other evidence supporting its use to prevent escalation of ventilatory care when compared to NIV (Coudroy et al., 2016).

\subsubsection{Paediatrics}

Despite widespread use in paediatrics and neonatology, two Cochrane systematic reviews failed to show sufficient evidence of a benefit in either bronchiolitis (Beggs et al, 2014) or for general respiratory support in critically ill children (Mayfield et al., 2014). In both cases this was due to a lack of quality trials suitable for meta-analyses. However recent high-grade RCT evidence adds support for oxygen via NHF use in bronchiolitis.(Franklin et al., 2018) Rates of infants receiving escalation of ventilatory care was 12\% (87 of 739 infants) in the NHF group, as compared with 23\% (167 of 733) in the standardtherapy group (risk difference, - 11 percentage points; $95 \%$ confidence interval, -15 to $-7 ; P<0.001)$. Length of time on oxygen therapy and hospital stay showed no significant difference.

\subsubsection{Perioperative}

Evidence is conflicting around the benefits of oxygen via NHF compared to standard oxygen therapy and/or NIV in the perioperative and/or postextubation setting.

\subsection{EVIDENCE OF BENEFIT}

NHF use post-cardiac surgery with or without acute hypoxic respiratory failure had lower rates of treatment failure requiring NIV or IMV compared to standard oxygen therapy (Parke et al, 2013; Parke et al, 2011) and was non-inferior to bi-level (Stéphan et al., 2015). No difference in $\mathrm{PaO}_{2} / \mathrm{F}_{i} \mathrm{O}_{2}$ ratio was seen in a 340-patient post-cardiac surgery RCT (Parke et al., 2013) but fewer desaturations were seen in an earlier study (Parke et al., 2011) suggesting the oxygenation benefit of NHF in this setting is inconclusive.

Extubation onto NHF following acute hypoxemic respiratory failure in ICU conferred significant benefit compared to oxygen via venturi mask (Maggiore et al., 2014). $\mathrm{PaO}_{2} \mathrm{~F}_{\mathrm{i}} \mathrm{O}_{2}$ ratio was higher, desaturation rates were lower, 
discomfort scores were lower and rates of NIV/IMV were significantly lower with NHF.

\subsection{EVIDENCE OF NO BENEFIT}

Post-extubation prophylactic use of oxygen via NHF showed no difference in rates of respiratory complications after major abdominal surgery or length of hospital stay, compared to standard oxygen therapy (Futier et al., 2016). Atelectasis scores, $\mathrm{PaO}_{2} / \mathrm{FiO}_{2}$ ratio, length of $\mathrm{ICU}$ stay and respiratory rate were no different in 155 obese post-cardiac surgery patients randomised to NHF or standard oxygen therapy (Corley et al, 2015).

\subsubsection{Palliative care}

Patients with acute hypoxaemic respiratory failure from multiple causes but with "do-not-intubate" orders, were administered NHF and oxygen with $\mathrm{F}_{\mathrm{i}} \mathrm{O}_{2}$ of 0.67 (range $0.30-1.0$ ) and flow of $42.6 \mathrm{~L} / \mathrm{min}$ (range $30-60 \mathrm{~L} / \mathrm{min}$ ) to improve mean $\mathrm{S}_{\mathrm{p}} \mathrm{O}_{2}$ from $89.1 \%$ to $94.7 \%(\mathrm{P}<0.001)$, and reduce respiratory rate from 30.6 breaths/min to 24.7 breaths/min $(P<0.001)$ (Peters et al, 2013). Nine of the 50 participants (18\%) required escalation to NIV. The median duration of NHF was 30 hours (range 2-144 hr) Similar improvements in oxygenation as well as dyspnoea scores in the palliative care population have been documented (Epstein et al, 2011) but others have shown no difference in dyspnoea relief compared to bi-level (Hui et al., 2013). Overall there is probably a lack of evidence to confirm benefit in this entire population although NHF may be appropriate in select patients.

\subsubsection{Heart failure}

The data on whether NHF reduce $\mathrm{P}_{\mathrm{a}} \mathrm{CO}_{2}$ in patients with heart failure is limited. One non-randomised study in only 5 hospital in-patients demonstrated a nonsignificant reduction in $\mathrm{PaCO}_{2}$ after 24 hours of NHF use (Carratalá Perales et al., 2011). A physiological study in 10 patients with moderate heart failure (NYH class 3) investigated whether NHF could decrease right ventricular preload and improve haemodynamic and respiratory parameters (Oriol Roca et al., 2013). A reduction greater than $20 \%$ in the estimated inspiratory collapse of the inferior vena cava (a surrogate for right ventricular pre-load) from baseline was the primary end-point, measured with echocardiogram and showed a statistically significant reduction with NHF at $20 \mathrm{~L} / \mathrm{min}$ and $40 \mathrm{~L} / \mathrm{min}$. 
Respiratory rate was also significantly reduced suggesting some potential for NHF use in heart failure.

\subsection{Summary of background information, knowledge gaps and aims of the thesis}

COPD affects an estimated 174 million people worldwide and is associated with a poor quality of life, frequent exacerbations and causes the death of 3 million people per year. Hypercapnic respiratory failure in AECOPD is common and management is based primarily on NIV, which while being effective, has significant limitations. Readmission to hospital after discharge with AECOPD is a common occurrence, associated with worse outcomes and an enormous strain on the health dollar. Effective therapies to reduce this rate of readmission are scarce. There is an unmet need for an easy to administer, well-tolerated and effective therapy in COPD.

NHF therapy may provide an answer to this need. Chapter 1 introduced NHF as an alternative therapy in AHRF, supported by its mechanisms of actions,

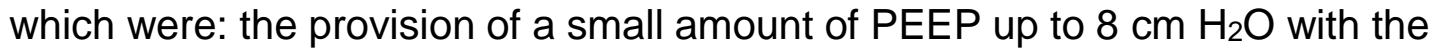
mouth closed, wash-out of the anatomical dead space, conditioning the respiratory gases to reduce the metabolic cost of breathing, accurate provision of oxygen to a set $\mathrm{F}_{\mathrm{i}} \mathrm{O}_{2}$ while meeting the patients inspiratory flow demands as well as improving the function of the mucociliary escalator.

While NHF has mainly been used in the critical care setting, with most evidence supporting its use in hypoxic respiratory failure, these mechanisms of action suggest an application in hypercapnic respiratory failure and COPD. The systematic review in Chapter 1 outlined the small number of NHF studies demonstrating $\mathrm{PCO}_{2}$ reduction, providing preliminary evidence of efficacy with an ability to reduce $\mathrm{PCO}_{2}$ by $3-6 \mathrm{mmHg}$ in the short-term and possibly up to $8 \mathrm{mmHg}$ over several weeks. However, there are no international clinical guidelines for the use of NHF therapy, and NHF is initiated at the discretion of clinicians and on a case-by-case basis. In an era of evidence-based medicine, well-designed clinical trials of NHF therapy in COPD are a priority.

The knowledge gaps identified in Chapter 1 were used to raise the questions to be answered by this thesis, as follows: 
- Does NHF reduce $\mathrm{PCO}_{2}$ in stable COPD and if so, what is the flowresponse relationship of the $\mathrm{PCO}_{2}$ reduction?

- Does NHF therapy reduce hospital re-admissions in COPD patients who have been recently discharged from hospital?

- Does NHF use in AHRF in ED reduce the requirement of subsequent NIV?

- What is the magnitude of NHF reduction of $\mathrm{PCO}_{2}$ compared to NIV?

- How can the tolerability of NIV therapy be improved in order to extend overall treatment time?

Each of these questions relating to the identified knowledge gaps is addressed separately below, alongside the corresponding aims of the thesis.

- Does NHF reduce $\mathrm{PCO}_{2}$ in stable COPD and if so, what is the flowresponse relationship of the $\mathrm{PCO}_{2}$ reduction?

The limited evidence for the ability of NHF to reduce $\mathrm{PCO}_{2}$ in COPD was reviewed in Section 1.4.5. Also, the optimum flow rate remains unknown as the studies presented above have all varied in their application of NHF flow rates and application of oxygen.

To address this knowledge gap, the following study was undertaken:

Nasal High Flow therapy and $\mathrm{P}_{\mathrm{t}} \mathrm{CO}_{2}$ in stable COPD: a randomised controlled cross-over trial (Study 2), to assess:

- The flow-response relationship of NHF therapy and $\mathrm{P}_{\mathrm{t}} \mathrm{CO}_{2}$ in participants with stable COPD.

- The change in oxygen saturations, respiratory rate, minute ventilation and heart rate during NHF therapy.

- Participant reported tolerability of NHF.

- Does NHF therapy reduce hospital re-admissions in COPD patients who have been recently discharged from hospital? 
Section 1.3.1.7 reviewed the significant problem of COPD re-admissions and the lack of effective interventions to reduce this problem. The mechanisms of action of NHF outlined in section 1.4.3 suggest it may offer effective respiratory support for home use to prevent re-admissions during the delicate post-discharge period. A large, well designed RCT is required to demonstrate a reduction in re-admission rates with NHF use. Before the RCT can be performed, a feasibility study is vital to: evaluate compliance with NHF use at home, optimum NHF therapy flow rate and temperature prescription, recruitment rates as well as hospital readmission rates. To address this knowledge gap, the following study was undertaken:

Nasal High Flow therapy use following hospitalisation for an exacerbation of COPD: A feasibility study (Study 3), to assess:

- The duration of use of NHF, including the mean hours of use per day and number of days use over 30 days, to inform a precise daily NHF prescription for the proposed RCT.

- The proportion of potential participants who could be eligible for the proposed RCT.

- Clinical outcomes such as 30-day readmission rates and requirement for GP/ED visits.

- The tolerability of the device and preferred flow-rate and temperature settings.

- Does NHF use in AHRF in ED reduce the requirement of subsequent NIV?

Given the limitations of NIV outlined in section 1.3.4.2 and the recommendations from BTS/ICS guidelines to optimise medical therapy in the first 1 hour of AHRF treatment, the mechanisms of action of NHF suggest it may be more effective during this critical time-period than standard therapy alone. A well-designed RCT comparing oxygen via NHF to standard oxygen therapy in the immediate presentation of AHRF is needed, but first I undertook a feasibility study to test the trial design by measuring adherence to a "usual care" protocol for treatment of AECOPD patients with AHRF.

To address this knowledge gap, the following study was undertaken: 
Nasal High flow therapy in acute hypercapnic respiratory failure with acidosis in patients presenting with an acute exacerbation of COPD: A feasibility study (Study 4), to establish:

- An estimate of the treatment failure rate (NIV requirement) in the usual care group.

- An estimate of the likely attrition rate in relation to clinician adherence to the usual care protocol.

- An estimate of the proportion of patients admitted with AECOPD who are in AHRF and are otherwise eligible for the proposed RCT.

- Other outcomes related to ABG sampling, uptake of a "deferred consent" model and rates of recruitment.

- What is the magnitude of $\mathrm{PCO}_{2}$ reduction with $\mathrm{NHF}$ compared to NIV?

Given NIV is already a well-established therapy in hypercapnic COPD but has limitations such as tolerability (Section 1.3.4.2), NHF may offer an alternative treatment option or be an adjunct therapy. There is scarce evidence comparing the $\mathrm{PCO}_{2}$-reducing ability of NHF directly against NIV.

To address this knowledge gap, the following study was completed:

Nasal High Flow therapy compared with Non-invasive ventilation in COPD patients with chronic respiratory failure: a randomised controlled crossover trial (Study 5), to assess:

- Whether NHF is able to reduce $\mathrm{PCO}_{2}$ by a similar magnitude to NIV over a 60 min time-period, in participants with hypercapnia at baseline.

- The change in oxygen saturations, respiratory rate, and heart rate during NHF and NIV therapy.

- Participant reported tolerability of NHF and NIV.

- The change in participant reported dyspnoea scores after NHF and NIV. 
- How can the tolerability of NIV therapy be improved in order to extend overall treatment time?

Continuous NIV therapy is recommended in AHRF (Section 1.3.4.4) in the first few days and is correlated with higher treatment success rates. Tolerability of NIV is a barrier to continuous use (Section 1.3.4.5). If NHF is proven to be effective at lowering $\mathrm{PCO}_{2}$ but is more tolerable than NIV, the NHF device may be modified to include an NIV function and be used for longer periods of time in hypercapnic respiratory failure.

To address this knowledge gap, the following study was completed:

The NIV mode of a dual NHF/NIV device compared to standard NIV in chronic respiratory disease: a randomised controlled cross-over trial (Study 6), to establish:

- How the comfort scores for the dual NHF/NIV device compare to a standard NIV device

- If the dual NHF/NIV device reduces $\mathrm{PCO}_{2}$ and triggers satisfactorily compared to a standard NIV device.

- The change in oxygen saturations, respiratory rate, blood pressure and heart rate during both NHF/NIV and standard NIV.

Overall, this thesis will provide evidence for future construction of clinical guidelines.

Transcutaneous partial pressure of carbon dioxide $\left(\mathrm{P}_{\mathrm{t}} \mathrm{CO}_{2}\right)$ change is an outcome measure for three of the five clinical trials in this thesis. Using $\mathrm{P}_{\mathrm{t}} \mathrm{CO}_{2}$ as a surrogate for $\mathrm{PaCO}_{2}$ avoided the painful procedure of arterial blood gas sampling, and the risks associated with this procedure, including potential ischaemia to the hand. It was imperative therefore to validate the method of measuring $\mathrm{P}_{\mathrm{t}} \mathrm{CO}_{2}$ change with a transcutaneous monitor prior to completing these 3 clinical trials. Studies $1 \mathrm{a}$ and $1 \mathrm{~b}$ are two methodological validation studies completed to confirm the accuracy of transcutaneous monitoring prior to undertaking Studies 2-6, and are reported in Appendix II. Study 2 is the first NHF clinical trial and is now presented. 




\section{Study 2 - Nasal High Flow therapy and $\mathrm{P}_{\mathrm{t}} \mathrm{CO}_{2}$ in stable COPD: a randomised controlled cross-over trial}

\subsection{Rationale}

In AECOPD, hypercapnia is associated with worse clinical outcomes including death (Steer et al., 2010). NIV is recommended to provide respiratory support to patients with AECOPD who have hypercapnic respiratory failure despite optimal medical therapy (Davidson et al., 2016). Tolerability of NIV may be a barrier to effective use (Nava \& Hill, 2002) and an alternative to NIV is a priority for the management of AECOPD.

The systematic review above (Section 1.4.5) concluded that NHF produces a modest reduction in $\mathrm{PaCO}_{2}$ in both stable and acute COPD. However, the interpretation of these NHF studies, and their applicability to clinical practice, remains variably limited by the confounding effect of concomitant oxygen therapy, absence of randomized controlled treatments and a lack of data on the dose-response relationship across the range of flows used in clinical practice.

The present study is a randomised, controlled, crossover trial of the effect of three different flow rates of NHF therapy compared with a control intervention of breathing room air, in stable COPD patients without the requirement for concomitant oxygen therapy.

\subsection{Aims and hypotheses}

The aims of the study were:

- To determine the flow-response relationship of $\mathrm{NHF}$ therapy and $\mathrm{P}_{\mathrm{t}} \mathrm{CO}_{2}$ in participants with stable COPD.

- To assess the change in oxygen saturations, respiratory rate, minute ventilation and heart rate during NHF therapy.

- To assess participant reported tolerability of the NHF device. 
The hypotheses were that NHF therapy in patients with stable COPD would reduce $\mathrm{P}_{\mathrm{t}} \mathrm{CO}_{2}$ levels, respiratory rate and minute ventilation, and that NHF therapy would be well tolerated.

\subsection{Methods}

\subsubsection{Trial design}

Single-blinded, randomised, controlled four-way cross-over design.

\subsubsection{Eligibility criteria}

\subsubsection{Inclusion criteria}

1. A doctor's diagnosis of COPD

2. Age $\geq 40$ years old

\subsubsection{Exclusion criteria}

1. Smoking pack year history $<10$ years

2. $\mathrm{FEV}_{1} / \mathrm{FVC}>70 \%$

3. Long term oxygen therapy

4. COPD not deemed to be 'stable':

a. Current exacerbation requiring acute treatment with short course antibiotic/oral steroid or oxygen therapy.

b. Hospital admission for an acute exacerbation of COPD in the last 6 weeks.

5. Nasal conditions such as deviated septum, chronic rhinitis, current cold/flu which, in the evaluation by the investigator, could impair nasal breathing.

6. Any other condition, which at the investigator's discretion, was believed may present a safety risk or impact the feasibility of the study or study results.

\subsubsection{Interventions}

Participants received all interventions for $20 \mathrm{~min}$ in a randomised order while seated. Each NHF flow setting was at a temperature of $37^{\circ} \mathrm{C}$ without oxygen: $15 \mathrm{~L} / \mathrm{min}, 30 \mathrm{~L} / \mathrm{min}$ or $45 \mathrm{~L} / \mathrm{min}$; or the control setting of breathing room air only without the NHF attached, using the AIRVO 2 device (PT101AZ, FPH, Auckland, New Zealand), a heated breathing tube and chamber (900PT551, 
FPH, Auckland, New Zealand), and Optiflow+ nasal cannula (OPT944, Fisher and Paykel Healthcare, Auckland, New Zealand).

Each of the four interventions was followed by a washout period breathing room air for at least $15 \mathrm{~min}$, allowing the $\mathrm{P}_{\mathrm{tCO}}$ to return to within $4 \mathrm{mmHg}$ of the baseline measurement for the particular intervention. The washout could be extended until this criterion was met.

$\mathrm{P}_{\mathrm{t}} \mathrm{CO}_{2}, \mathrm{~S}_{\mathrm{t}} \mathrm{O}_{2}$, heart rate and respiratory rate were recorded at the start of each intervention and then every 5 min until the end of each washout period.

Participant tolerability questionnaires were administered during the washout periods after each NHF intervention. Participants rated the ease of application, level of comfort, weight of the nasal interface, noisiness, amount of moisture in the nasal passages and likelihood of reusing the system on a continuous scale from most positive (0) to least positive (100).

The 20-min time period of each intervention reflects the minimum period of time NHF is likely to be used in clinical practice in order to obtain a clinical effect. Previous studies have demonstrated changes in $\mathrm{Pt}_{\mathrm{t}} \mathrm{CO}_{2}$ within 20 min of oxygen delivery, suggesting the length of intervention is sufficient to detect any changes in $\mathrm{P}_{\mathrm{t}} \mathrm{CO}_{2}$ and other measured variables as well as allow adequate time to identify any associated adverse events (Perrin et al., 2011; Wijesinghe et al., 2011, 2012).

Participants were able to stop the intervention at any time without giving a reason and without being withdrawn from subsequent portions of the study.

\subsubsection{Recruitment and Consent}

Participants were recruited from the MRINZ participant databases and were contacted by phone. If they were interested at the time of this call a Participant Information Sheet (PIS - See appendix 9.4.1) was sent out, with a follow up call arranged after they had time to read it and discuss with their family/whanau.

Participants were considered enrolled and part of the study population at the time the consent form was filled in by both the participant and study investigator (myself). 
The study was prospectively registered with ANZCTR (Trial ID: ACTRN12615000471583) and approved by the HDEC of New Zealand (Ref: 15/NTA/4). Full written informed consent was completed before any study specific procedures were carried out.

\subsubsection{Study procedures}

This involved one study visit to Wellington Regional Hospital in the respiratory physiology laboratory at the Medical Research Institute of New Zealand (MRINZ). The visit lasted around 4 hours in total.

After demographic and medical history data were collected, spirometry was performed in accordance with ATS/ERS criteria (Miller et al., 2005) using a Jaeger Master screen ${ }^{\mathrm{TM}}$ body volume constant plethysmography unit with pneumotachograph and diffusion unit (Erich-Jaegar, Wurzburg, Germany). Measurements of $\mathrm{PtCO}_{2}, \mathrm{StO}_{2}$, and heart rate were made using the SenTec transcutaneous monitor (SenTec digital monitor with V-Sign Sensor VSA/P/N, Therwill, Switzerland). The SenTec probe was kept on the patient for between 20 and 30 minutes before the subsequent study procedures to ensure a stable baseline measurement of $\mathrm{P}_{\mathrm{t}} \mathrm{CO}_{2} . \mathrm{P}_{\mathrm{t}} \mathrm{CO}_{2}$ was used as a surrogate measure of $\mathrm{PaCO}_{2}$ (see Appendix II for Studies 1a and 1bvalidation of transcutaneous monitoring of $\mathrm{PCO}_{2}$ ).

\subsubsection{Respiratory Inductance Plethysmography}

Minute ventilation was measured using Respiratory Inductance Plethysmography (RIP) bands (QDC-Pro device, CareFusion $\AA$, Yorba Linda, California, USA).

RIP bands are a non-invasive method of measuring ventilatory parameters such as $V_{T}, T_{I}$ and $T_{E}$, respiratory rate, end-expiratory lung volume and minute ventilation. Two specialised elastic bands are positioned - one around the upper rib-cage and the second around the abdomen, and stretch to generate an electrical signal when movement is detected during breathing. The electrical signal generated is proportional to the movements of the individual thoracic and abdominal components (Barbosa et al, 2012). The electrical voltage is then converted into useable ventilatory measures e.g. volume, by 
applying a conversion ratio (described below) through software such as Labcharts.

There are variable reports of RIP band accuracy in the ICU (Leino et al, 2001; Neumann \& Burchardi, 1998; Valta et al, 1992) and research (O'Reilly et al., 2014) settings. Reasons for inaccuracy have been reported to be due to movement artefacts or band slippage from patients coughing or excessive movement (O'Reilly et al., 2014), thermal instability and high baseline drift (Neumann \& Burchardi, 1998). It was therefore imperative to have a robust and reproducible method of validating the RIP recordings before deciding to report them.

\subsection{CALIBRATION OF RIP BANDS TO CHECK TECHNICAL ACCEPTABILITY}

A key component of RIP band use is the 2-step calibration of each individual participants breathing, enabling a robust method to demonstrate technical acceptability of the data (Barbosa et al., 2012; Neumann \& Burchardi, 1998). Firstly, once the RIP bands were attached, the small Qualitative Diagnostic Calibration (QDC) device unit attached to the 2 elastic bands was auto calibrated for at least 5 min before the study procedures commenced while the participant was sitting quietly, in order to capture a baseline breathing pattern. The QDC auto calibration only needed to be done once per participant and is a way of determining the volume motion co-efficient of the rib-cage and abdominal bands, which is then applied to subsequent RIP measurements (Neumann \& Burchardi, 1998).

The second step involved participants breathing in a relaxed manner through a pneumotachograph flow-meter for $30 \mathrm{sec}$, with the last 5 breaths used to obtain a quantitative volume calibration recording. Simultaneous RIP band voltages were recorded. If 5 breaths were unable to be used (e.g. there are only 4 breaths, or on visual inspection the breath is of a wave form which is out of keeping with a normal breath for that participant), then the number of breaths used was recorded.

A ratio of the calibrated volume and voltage recordings was calculated for 2 purposes -1 ) to check if the method was technically acceptable (as described below) and 2) if the intervention was valid with technically acceptable data, 
then the ratio was applied to the RIP voltages to convert them to volumes, volumes per unit time etc in order to give clinically useful metrics, such as $V_{T}$ and MV.

Calculation of the calibration ratio was done independently by myself and a clinical research scientist at Fisher and Paykel Healthcare, with the data then being reconciled. The ratio was calculated by analysing each of the last 5 breaths. The maximum voltage of the RIP band recording was divided by the corresponding maximum calibrated flow-meter volume recording to obtain a ratio. The 5 ratios were then added and divided by 5 to calculate the overall mean pre-intervention ratio ( $K$ pre).

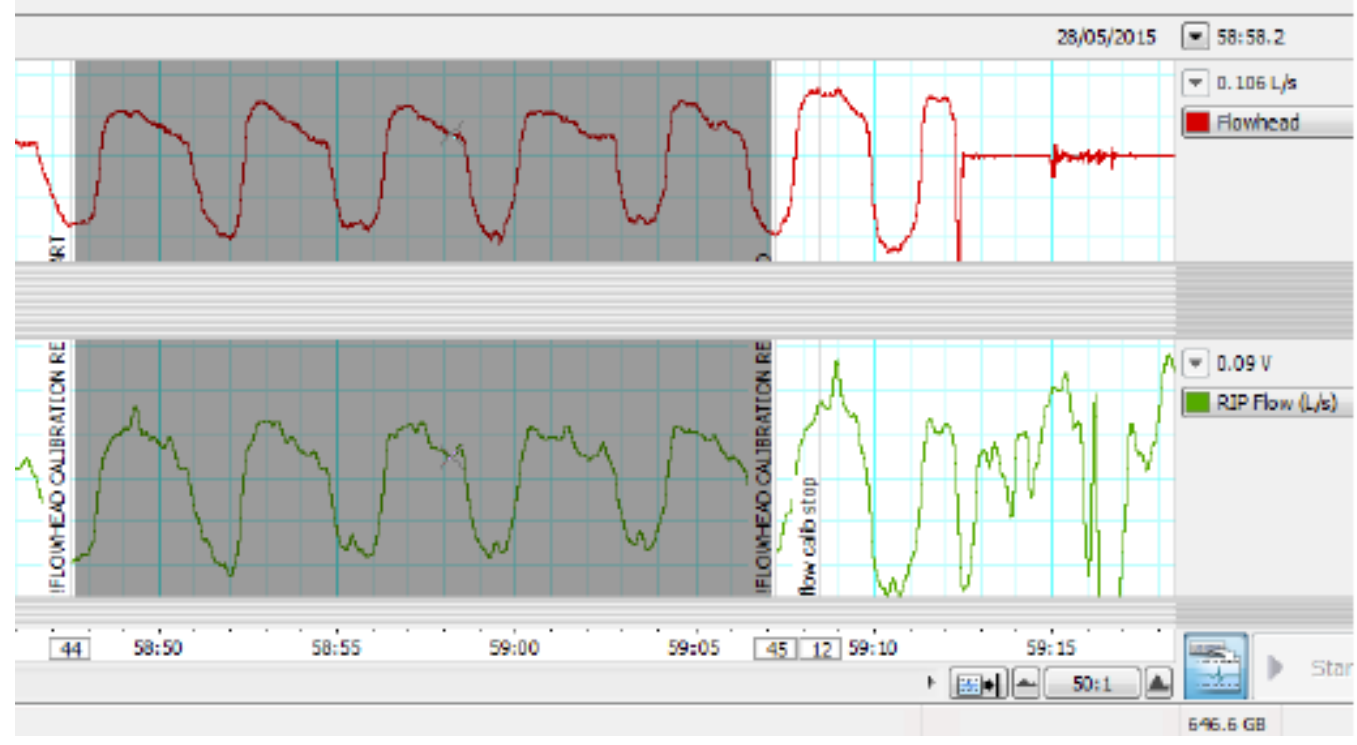

Figure 2-1 A sample of a Labcharts recording of Respiratory Inductance Plethysmography (RIP). The wave-forms are shown during calibrations of the volume (labelled "Flowhead", note the units L/s) and RIP (RIP flow, note the units of volts) for 5 breaths. The peak of the 5 breath waveforms were used to calculate a ratio used to check the RIP accuracy.

The process was repeated after each randomised intervention, during the washout period to obtain a post-intervention ratio ( $K$ post). To demine the accuracy of the RIP band readings for each intervention, the post-intervention ratio was required to be within $10 \%$ of the pre-intervention ratio for the recordings undertaken during the intervention to be considered technically acceptable, as shown in the equation below:

$$
K \text { post }=K \text { pre } \pm 10 \%
$$


This method of defining technically acceptable RIP measurements has been described previously (O'Reilly et al., 2014).

Also, because the trial was comparing NHF against the control room air intervention, a technically acceptable room air intervention was required if the measurements were going to be used to eventually report $\mathrm{V}_{\mathrm{T}}$ and $\mathrm{MV}$.

\subsubsection{Randomisation and blinding}

The randomisation was computer-generated by the study statistician, who had no role in the recruitment, study visits or data collection. Random allocations were sealed in sequentially numbered opaque envelopes before recruitment. After measurement of $\mathrm{P}_{\mathrm{t}} \mathrm{CO}_{2}, \mathrm{~S}_{\mathrm{t}} \mathrm{O}_{2}$ and heart rate, randomisation envelopes were opened by me (un-blinded investigator). The un-blinded investigator's role included manually counting the respiratory rate and controlling the settings on the NHF device. The blinded investigator recorded $\mathrm{P}_{\mathrm{t}} \mathrm{CO}_{2}$, heart rate and $\mathrm{S}_{\mathrm{t}} \mathrm{O}_{2}$ from the $\mathrm{SenTec}$ display while seated behind a screen so that they could not see the participant or NHF display and wearing ear plugs to avoid hearing changes to the NHF flow-rate. The participants were not told the order they received the different flow rates via the NHF although they were likely to notice the difference in flow rates in their nostrils, hence formally classified as single-blinded only. Participants were asked not to divulge any detected differences in flow to the blinded investigator, during a washout period for example, to avoid unblinding them.

\subsubsection{Outcome measures}

1. The primary outcome was $\mathrm{P}_{\mathrm{t}} \mathrm{CO}_{2}$ at 20 min, adjusted for baseline.*

2. Secondary outcomes were:

a. $\mathrm{P}_{\mathrm{t}} \mathrm{CO}_{2}$ at 5 min intervals during the intervention and at the end of the subsequent 15 min washout period, adjusted for baseline.

b. The proportion of participants that had a decrease in $\mathrm{P}_{\mathrm{t}} \mathrm{CO}_{2}$ by $\geq 4 \mathrm{mmHg}^{\wedge}$

C. Other physical measurements:

i. Oxygen saturation, heart rate, respiratory rate and minute ventilation at 5 min intervals during the intervention and at the end of the subsequent 15 min washout, adjusted for baseline*. 
d. Tolerability:

i. Proportion of participants who withdrew from the intervention before it was completed

ii. Tolerability questionnaire results

* Baseline refers to the measurements taken at the zero time-point at the start of each intervention.

^ $\mathrm{A} \mathrm{P}_{\mathrm{t}} \mathrm{CO}_{2}$ change of $\geq 4 \mathrm{mmHg}$ is considered to be physiologically significant and $\geq 8 \mathrm{mmHg}$ clinically significant based on previous research (Perrin et al, et al., 2011; Wijesinghe et al., 2011). Section 1.3.2.1.1 showed AHRF with a pH below 7.35 is associated with increased mortality and a greater need for intubation in patients with COPD (Ambrosino et al., 1995; Jeffrey et al, 1992). It is therefore important to relate $\mathrm{PCO}_{2}$ change with $\mathrm{pH}$ change, especially as the severity of an AECOPD is determined not by the absolute level of hypercapnia, but rather by the increase above the chronic stable $\mathrm{PaCO}_{2}$ value, as reflected by increased acidosis. A $3.8 \mathrm{mmHg}$ rise in $\mathrm{P}_{\text {cap }} \mathrm{CO}_{2}$ was observed in a study when high concentration oxygen with an $\mathrm{FiO}_{2}$ of 0.50 was administered to stable obesity hypoventilation syndrome patients (Hollier et al., 2014). This level of $\mathrm{P}_{\text {cap }} \mathrm{CO}_{2}$ increase induced a reduction in blood $\mathrm{pH}$ from 7.373 to 7.346 - a respiratory acidosis that as previously reported, is likely to be clinically significant.

\subsubsection{Sample size estimation}

The paired standard deviations of $\mathrm{P}_{\mathrm{t}} \mathrm{CO}_{2}$ in a previous study investigating oxygen administration to patients with stable COPD were between 1.8 and 4.4 $\mathrm{mmHg}$ (Edwards et al, 2012). Based on the highest $\mathrm{P}_{\mathrm{t}} \mathrm{CO}_{2}$ standard deviation of $4.4 \mathrm{mmHg}$, and an alpha value of 0.0083 (to take into account the potential for six possible comparisons for the four-way cross-over study) a sample size of $48 \mathrm{had} 90 \%$ power to detect a difference in $\mathrm{P}_{\mathrm{t}} \mathrm{CO}_{2}$ of $3.8 \mathrm{mmHg}$.

\subsubsection{Statistical analysis plan}

The main analysis for the comparison of each of the three NHF treatments compared to room air was by a mixed linear model with fixed effects for the randomisation sequence, the baseline measurement of the particular variable, and the randomised treatment, time, and their interaction; and a random effect 
for each participant, with an exponential time correlation structure for the repeated measurements. The comparison of paired proportions for those that had a decrease from baseline $\mathrm{P}_{\mathrm{t}} \mathrm{CO}_{2}$ of $\geq 4 \mathrm{mmHg}$ was by an exact McNemar's test and estimation of the confidence interval for the differences in paired proportions, NHF intervention minus room air, by an asymptotic method. The comparison of device questionnaire scores was by a mixed linear model with fixed effects for the randomisation sequence and treatment; and a random effect for the participant. SAS version 9.4 was used.

\subsection{Results}

\subsubsection{Participant characteristics}

There were 144 potentially eligible participants identified in the MRINZ database. Contact was made with 84 of these and 48 participants were randomised, completing a single study visit between May 2015 and February 2016 (Figure 2-2). 


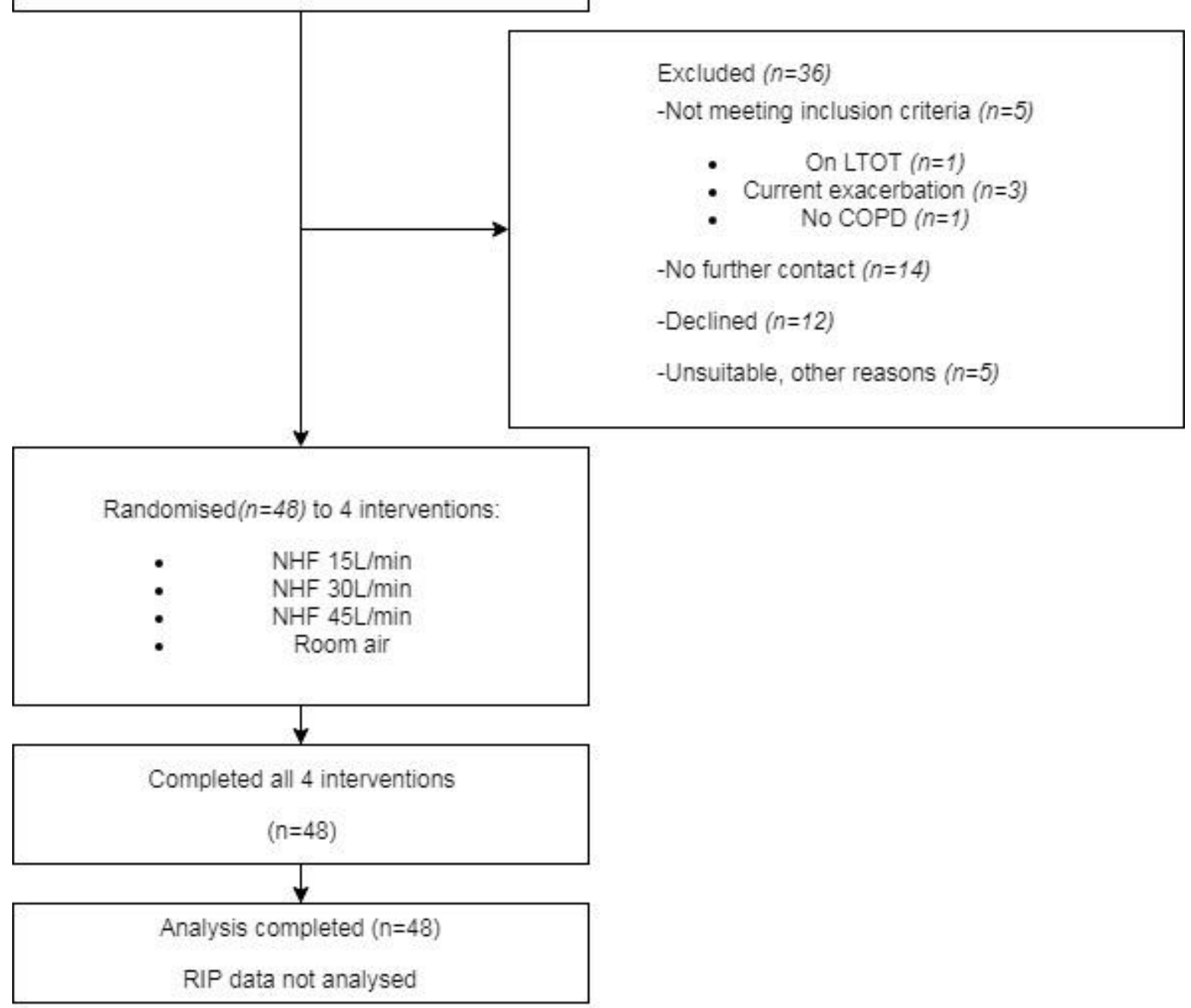

Figure 2-2 CONSORT summary of participant flow through Study 2 and the allocation of interventions.

LTOT, long-term oxygen therapy; NHF, nasal high flow; PIS, Participant Information Sheet; RIP, Respiratory Inductance Plethysmography.

[Republished with permission of John Wiley and Sons, from: Respirology, "Nasal high flow therapy and $\mathrm{P}_{\mathrm{t}} \mathrm{CO}_{2}$ in stable COPD: A randomized controlled cross-over trial", (McKinstry et al., 2017) @ 2018; permission conveyed through Copyright Clearance Center, Inc. ]

Participant characteristics are shown in Table 2-1. Twenty-nine of the participants were male and 6/48 (12.5\%) were hypercapnic, with $\mathrm{P}_{\mathrm{tCO}}$ $>45 \mathrm{mmHg}$, at randomisation. Twenty-four participants (50\%) had severe or very severe COPD according to the Global Initiative for Chronic Obstructive Lung Disease (GOLD) classification.(Vogelmeier et al., 2017) 
Table 2-1 Study 2 baseline participant characteristics.

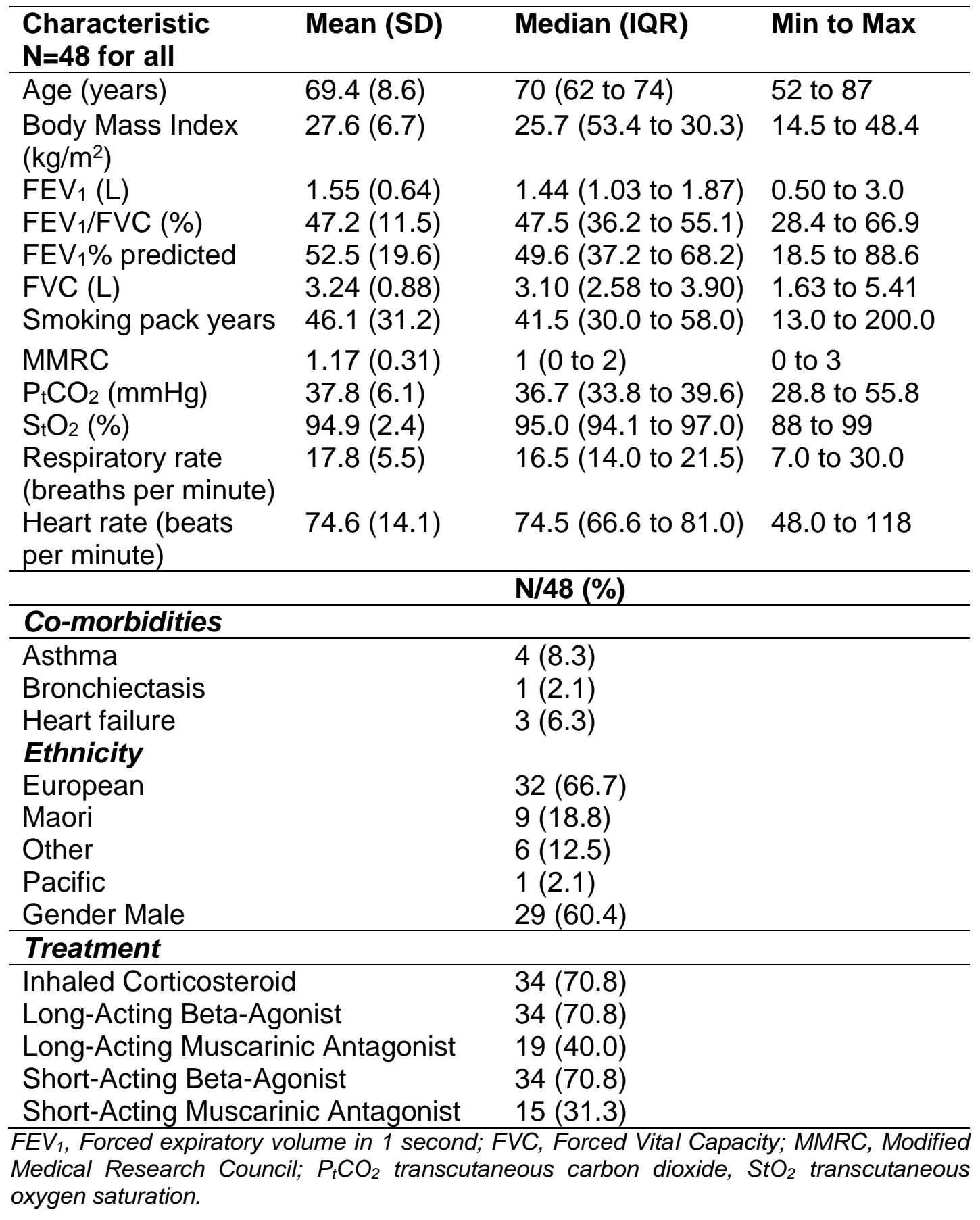

Three participants required an extended washout in at least one of the interventions past $15 \mathrm{~min}$ for the $\mathrm{P}_{\mathrm{t}} \mathrm{CO}_{2}$ to return to within $4 \mathrm{mmHg}$ of the zero time point reading, with the longest extension being $10 \mathrm{~min}$. 


\subsubsection{RIP band measurements}

There were 188 interventions with RIP measurements and 97 (52\%) were valid. Twenty-seven (28\%) out of the 97 overall valid interventions had a valid room air intervention. In those 27 participants, 18 had valid NHF at $15 \mathrm{~L} / \mathrm{min}$ interventions, 19 had valid NHF at 30L/min interventions and 15 had valid NHF at $45 \mathrm{~L} / \mathrm{min}$ interventions. Therefore, only 79 interventions (42\%) were valid (Figure 2-3), according to the calibration method described above which mandated a valid room air intervention. Overall, the method of calibration demonstrated significant unreliability in the RIP recordings and this data is not reported.

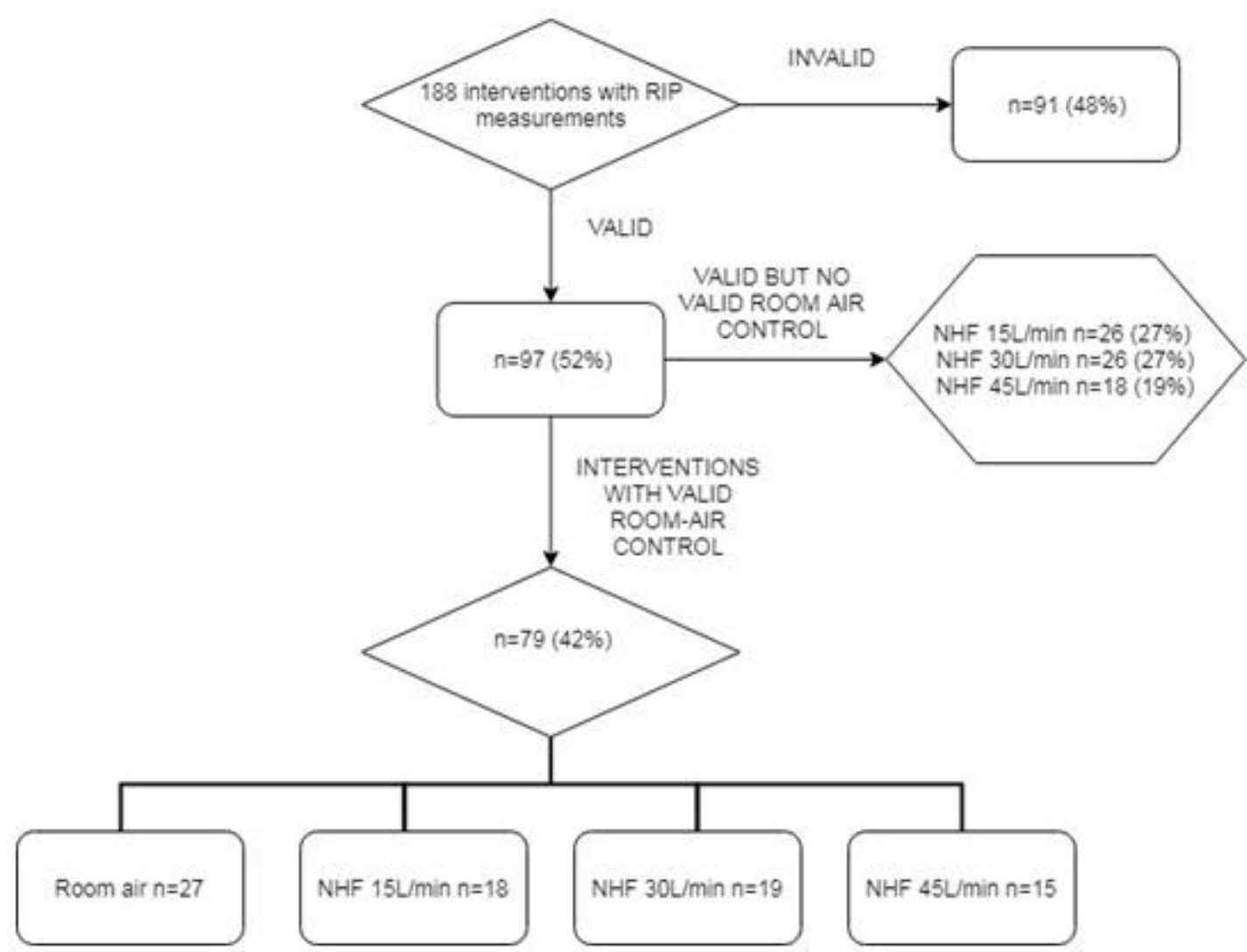

Figure 2-3 Summary of how the technically acceptable 79 RIP band interventions were derived.

RIP, Respiratory Inductance Plethysmography.

\subsection{3 $\mathrm{Pt}_{\mathrm{t}} \mathrm{CO}_{2}$}

The mean $\mathrm{P}_{\mathrm{t}} \mathrm{CO}_{2}$ adjusted for baseline after 20 minutes compared to room air was lower for NHF with a flow-dependent reduction in $\mathrm{P}_{\mathrm{t}} \mathrm{CO}_{2}$ (Table 2-2). The 
difference for $\mathrm{NHF}$ at $45 \mathrm{~L} / \mathrm{min}$ compared to room air was $-2.4 \mathrm{mmHg}(95 \% \mathrm{Cl}$ -2.9 to -1.8$), \mathrm{P}<0.001$, for $\mathrm{NHF}$ at $30 \mathrm{~L} / \mathrm{min}$ compared to room air it was $1.3 \mathrm{mmHg}(95 \% \mathrm{Cl}-1.9$ to -0.8$), \mathrm{P}<0.001$, and for $\mathrm{NHF}$ at $15 \mathrm{~L} / \mathrm{min}$ compared to room air it was $-0.6 \mathrm{mmHg}(95 \% \mathrm{Cl}-1.1$ to 0.0$), \mathrm{P}=0.06$.

The proportion of participants with at least one measurement of $\mathrm{P}_{\mathrm{t}} \mathrm{CO}_{2}$ which decreased from baseline greater than or equal to $4 \mathrm{mmHg}$, up to and including the 20 minute treatment period was $15 / 48(31.2 \%)$ for NHF at 45L/min, 8/48 (16.7\%) for NHF at $30 \mathrm{~L} / \mathrm{min}, 2 / 48(4.2 \%)$ for NHF at $15 \mathrm{~L} / \mathrm{min}$, and 1/48 (2.1\%) for room air. The paired proportions difference for $\mathrm{NHF}$ at $45 \mathrm{~L} /$ min minus room air was $29.7 \%(95 \% \mathrm{Cl} 16.3$ to 42.0$), \mathrm{P}<0.001,14.6 \%$ (95\% Cl 4.6 to 24.6$)$, $\mathrm{P}=0.016$, with NHF at $30 \mathrm{~L} / \mathrm{min}$, and $2.1 \%(95 \% \mathrm{Cl}-2.0$ to 6.1$), \mathrm{P}=0.99$, for $\mathrm{NHF}$ at $15 \mathrm{~L} / \mathrm{min}$. Four participants $(8.3 \%)$ had at least one $\mathrm{P}_{\mathrm{t}} \mathrm{CO}_{2}$ measurement of which decreased from baseline greater than or equal to $8 \mathrm{mmHg}$, up to and including the 20 minute treatment period for NHF at 45 L/min.

\subsubsection{Respiratory rate, oxygen saturations and heart rate}

There were statistically significant differences in respiratory rate between $\mathrm{NHF}$ compared to room air. The difference in respiratory rate for NHF at 45 $\mathrm{L} / \mathrm{min}$ at 20 minutes was -4.3 breaths per minute $(95 \% \mathrm{Cl}-5.5$ to -3.1$)$, $\mathrm{P}<0.001$; for NHF at $30 \mathrm{~L} / \mathrm{min},-4.1$ breaths per minute (95\% $\mathrm{Cl}-5.3$ to -2.9 ), $\mathrm{P}<0.001$, at $30 \mathrm{~L} / \mathrm{min}$, and for NHF at $15 \mathrm{~L} / \mathrm{min}-1.5$ breaths per minute $(95 \%$ $\mathrm{Cl}-2.7$ to -0.3 ), $\mathrm{P}=0.017$ (Table 2-3).

The maximum point estimate difference in respiratory rate was -5.0 breaths per minute $(95 \% \mathrm{Cl}-6.2$ to -3.8$), \mathrm{P}<0.001$, at 5 minutes with $\mathrm{NHF}$ at $45 \mathrm{~L} / \mathrm{min}$ compared to room air. 
Table 2-2* $\mathrm{P}_{\mathrm{t}} \mathrm{CO}_{2}$ values and mixed linear models for the difference in $\mathrm{P}_{\mathrm{t}} \mathrm{CO}_{2}$ of NHF minus room air, adjusted for baseline (zero time-point).

\begin{tabular}{|c|c|c|c|c|c|c|c|}
\hline & AIR & & HF 15 L/min & & NHF 30 L/min & & NHF 45 L/min \\
\hline $\begin{array}{l}\text { Time } \\
\text { point } \\
\text { (min) }\end{array}$ & $\begin{array}{c}\mathrm{P}_{\mathrm{t}} \mathrm{CO}_{2} \\
(\mathrm{mmHg})\end{array}$ & $\begin{array}{c}\mathrm{P}_{\mathrm{t}} \mathrm{CO}_{2} \\
(\mathrm{mmHg})\end{array}$ & $\begin{array}{l}\text { NHF-Air difference } \\
\text { from baseline } \\
(95 \% \mathrm{Cl}) \\
\text { P value }\end{array}$ & $\begin{array}{c}\mathrm{P}_{\mathrm{t}} \mathrm{CO}_{2} \\
(\mathrm{mmHg})\end{array}$ & $\begin{array}{l}\text { NHF-Air difference } \\
\text { from baseline } \\
(95 \% \mathrm{Cl}) \\
\text { P value }\end{array}$ & $\begin{array}{c}\mathrm{P}_{\mathrm{t}} \mathrm{CO}_{2} \\
(\mathrm{mmHg})\end{array}$ & $\begin{array}{c}\text { NHF-Air difference from } \\
\text { baseline }(95 \% \mathrm{Cl}), \\
\text { P value }\end{array}$ \\
\hline 0 & $38.4(5.5)$ & $37.9(5.5)$ & & $38.0(5.6)$ & & $38.2(5.1)$ & \\
\hline 5 & $38.6(5.3)$ & $37.4(5.3)$ & $\begin{array}{c}-0.95(-1.53 \text { to }-0.37) \\
P=0.001\end{array}$ & $37.0(5.9)$ & $\begin{array}{c}-1.43(-2.00 \text { to }-0.85) \\
P<0.001\end{array}$ & $36.3(6)$ & $\begin{array}{c}-2.21(-2.78 \text { to }-1.63) \\
P<0.001\end{array}$ \\
\hline 10 & $38.6(5.3)$ & $37.6(5.4)$ & $\begin{array}{c}-0.74(-1.31 \text { to }-0.16) \\
P=0.012\end{array}$ & $36.9(6.1)$ & $\begin{array}{c}-1.51(-2.09 \text { to }-0.94) \\
P<0.001\end{array}$ & $36.0(6.1)$ & $\begin{array}{c}-2.47(-3.05 \text { to }-1.90) \\
P<0.001\end{array}$ \\
\hline 15 & $38.7(5.2)$ & $37.9(5.3)$ & $\begin{array}{c}-0.50(-1.08 \text { to } 0.07) \\
P=0.087\end{array}$ & $37.1(6.0)$ & $\begin{array}{c}-1.44(-2.01 \text { to }-0.86) \\
P<0.001\end{array}$ & $36.4(5.9)$ & $\begin{array}{c}-2.15(-2.72 \text { to }-1.57) \\
P<0.001\end{array}$ \\
\hline 20 & $38.8(5.0)$ & 38 (5.3) & $\begin{array}{c}-0.55(-1.12 \text { to } 0.03) \\
P=0.063\end{array}$ & $37.3(6.0)$ & $\begin{array}{c}-1.32(-1.90 \text { to }-0.75) \\
P<0.001\end{array}$ & $36.3(5.6)$ & $\begin{array}{c}-2.37(-2.94 \text { to }-1.79) \\
P<0.001\end{array}$ \\
\hline
\end{tabular}

NHF: Nasal high flow; $\mathrm{P}_{t} \mathrm{CO}_{2}$ : transcutaneous partial pressure of $\mathrm{CO}_{2}$ 
Table 2-3* Mixed linear models for difference in respiratory rate of NHF minus room air adjusted for baseline (zero time-point)

\begin{tabular}{|c|c|c|c|c|c|c|c|}
\hline \multirow[b]{2}{*}{$\begin{array}{l}\text { Time } \\
\text { point } \\
\text { (min) }\end{array}$} & \multirow{2}{*}{$\begin{array}{c}\text { AIR } \\
\text { RR } \\
\text { (bpm) } \\
\text { Mean } \\
\text { (SD) }\end{array}$} & \multicolumn{2}{|c|}{ NHF 15 L/min } & \multicolumn{2}{|c|}{ NHF 30 L/min } & \multicolumn{2}{|c|}{ NHF 45 L/min } \\
\hline & & $\begin{array}{c}\text { RR } \\
\text { (bpm) } \\
\text { Mean } \\
\text { (SD) }\end{array}$ & $\begin{array}{l}\text { NHF-Air difference } \\
\text { from baseline } \\
\text { Mean }(95 \% \mathrm{Cl}) \\
\text { P value }\end{array}$ & $\begin{array}{c}\text { RR } \\
\text { (bpm) } \\
\text { Mean } \\
\text { (SD) }\end{array}$ & $\begin{array}{l}\text { NHF-Air difference } \\
\text { from baseline } \\
\text { Mean }(95 \% \mathrm{Cl}) \\
\text { P value }\end{array}$ & $\begin{array}{l}\text { RR } \\
\text { (bpm) } \\
\text { Mean } \\
\text { (SD) }\end{array}$ & $\begin{array}{c}\text { NHF-Air difference } \\
\text { from baseline } \\
\text { Mean }(95 \% \mathrm{Cl}) \\
\mathbf{P} \text { value }\end{array}$ \\
\hline 0 & $18.2(4.7)$ & $17.6(4.9)$ & & $18.1(5.7)$ & & $18.3(4.8)$ & \\
\hline 5 & $17.9(4.9)$ & $15.4(4.9)$ & $\begin{array}{c}-2.45(-3.65 \text { to }-1.24) \\
P<0.001\end{array}$ & $13.3(4.8)$ & $\begin{array}{c}-4.59(-5.79 \text { to }-3.39) \\
P<0.001\end{array}$ & $12.9(5.5)$ & $\begin{array}{c}-4.98(-6.19 \text { to }-3.78) \\
P<0.001\end{array}$ \\
\hline 10 & $17.1(4.6)$ & $15.0(5.0)$ & $\begin{array}{c}-1.99(-3.19 \text { to }-0.78) \\
P=0.001\end{array}$ & $12.6(4.4)$ & $\begin{array}{c}-4.42(-5.63 \text { to }-3.22) \\
P<0.001\end{array}$ & $12.9(5.7)$ & $\begin{array}{c}-4.13(-5.33 \text { to }-2.93) \\
P<0.001\end{array}$ \\
\hline 15 & $17.1(4.9)$ & $15.9(5.3)$ & $\begin{array}{c}-1.15(-2.36 \text { to } 0.05) \\
P=0.061\end{array}$ & $13.6(5.2)$ & $\begin{array}{c}-3.53(-4.73 \text { to }-2.32) \\
P<0.001\end{array}$ & $12.9(5.0)$ & $\begin{array}{c}-4.23(-5.44 \text { to }-3.03) \\
P<0.001\end{array}$ \\
\hline 20 & $17.5(4.8)$ & $16.0(5.7)$ & $\begin{array}{c}-1.47(-2.67 \text { to }-0.26) \\
P=0.017\end{array}$ & $13.4(5.2)$ & $\begin{array}{c}-4.13(-5.34 \text { to }-2.93) \\
P<0.001\end{array}$ & $13.3(4.8)$ & $\begin{array}{c}-4.25(-5.46 \text { to }-3.05) \\
P<0.001\end{array}$ \\
\hline
\end{tabular}


Table 2-4* Mixed linear models for oxygen saturation difference in NHF minus room air adjusted for baseline (zero-time point)

\begin{tabular}{|c|c|c|c|c|c|c|c|}
\hline \multirow[b]{2}{*}{$\begin{array}{l}\text { Time } \\
\text { point } \\
\text { (min) }\end{array}$} & \multirow{2}{*}{$\begin{array}{c}\text { AIR } \\
\begin{array}{c}\mathrm{StO}_{2} \\
(\%)\end{array}\end{array}$} & \multirow{2}{*}{\multicolumn{2}{|c|}{$\begin{array}{l}\text { NHF } 15 \text { L/min } \\
\text { NHF-air difference } \\
\text { from baseline (\%) } \\
\text { Mean ( } 95 \% \mathrm{Cl}) \\
\text { P value }\end{array}$}} & \multirow{2}{*}{\multicolumn{2}{|c|}{$\begin{array}{l}\text { NHF } 30 \text { L/min } \\
\text { NHF-air difference } \\
\text { from baseline }(\%) \\
\text { Mean }(95 \% \mathrm{Cl}) \\
\text { P value } \\
\end{array}$}} & \multirow{2}{*}{\multicolumn{2}{|c|}{$\begin{array}{l}\text { NHF } 45 \mathrm{~L} / \mathrm{min} \\
\text { NHF-air difference } \\
\text { from baseline }(\%) \\
\text { Mean }(95 \% \mathrm{Cl}) \\
\text { P value }\end{array}$}} \\
\hline & & & & & & & \\
\hline 0 & $94.8(2.1)$ & $94.9(2.4)$ & & $94.7(2.2)$ & & $94.9(2.4)$ & \\
\hline 5 & $94.5(2.3)$ & $94.5(2.3)$ & $\begin{array}{c}0.00 \\
(-0.43 \text { to } 0.44) \\
P=0.99\end{array}$ & $95.2(2.0)$ & $\begin{array}{c}0.70 \\
(0.26 \text { to } 1.13) \\
P=0.002\end{array}$ & $95.4(2.0)$ & $\begin{array}{c}0.84 \\
(0.41 \text { to } 1.28) \\
P<0.001\end{array}$ \\
\hline 10 & $94.7(2.3)$ & $94.6(2.4)$ & $\begin{array}{c}-0.21 \\
(-0.64 \text { to } 0.23) \\
P=0.36\end{array}$ & $95.1(2.0)$ & $\begin{array}{c}0.36 \\
(-0.07 \text { to } 0.80) \\
P=0.10\end{array}$ & $95.1(2.4)$ & $\begin{array}{c}0.32 \\
(-0.11 \text { to } 0.76) \\
P=0.15\end{array}$ \\
\hline 15 & $94.6(2.3)$ & $94.5(2.6)$ & $\begin{array}{c}-0.14 \\
(-0.58 \text { to } 0.29) \\
P=0.52\end{array}$ & $94.7(2.7)$ & $\begin{array}{c}0.18 \\
(-0.26 \text { to } 0.61) \\
P=0.43\end{array}$ & $95.0(2.3)$ & $\begin{array}{c}0.45 \\
(0.01 \text { to } 0.89) \\
P=0.044\end{array}$ \\
\hline 20 & $94.5(2.2)$ & $94.6(2.2)$ & $\begin{array}{c}0.02 \\
(-0.41 \text { to } 0.46) \\
P=0.92\end{array}$ & $94.8(2.5)$ & $\begin{array}{c}0.30 \\
(-0.14 \text { to } 0.74) \\
P=0.18\end{array}$ & $95.0(2.4)$ & $\begin{array}{c}0.47 \\
(0.03 \text { to } 0.91) \\
P=0.035\end{array}$ \\
\hline
\end{tabular}

$\mathrm{StO}_{2}:$ Transcutaneous oxygen saturation; NHF: Nasal high flow; SD: Standard deviation; Cl: Confidence interval 
Table 2-5* Mixed linear models for heart rate difference in NHF minus room air adjusted for baseline (zero-time point)

\begin{tabular}{|c|c|c|c|c|c|c|c|}
\hline \multirow[b]{2}{*}{$\begin{array}{l}\text { Time } \\
\text { point } \\
\text { (min) }\end{array}$} & \multirow{2}{*}{$\begin{array}{c}\text { AIR } \\
\text { Heart rate } \\
\text { (bpm) } \\
\text { Mean } \\
\text { (SD) }\end{array}$} & \multicolumn{2}{|c|}{ NHF 15 L/min } & \multicolumn{2}{|c|}{ NHF 30 L/min } & \multicolumn{2}{|c|}{ NHF 45 L/min } \\
\hline & & $\begin{array}{l}\text { Heart rate } \\
\text { (bpm) } \\
\text { Mean } \\
\text { (SD) }\end{array}$ & $\begin{array}{c}\text { NHF-Air difference } \\
\text { from baseline } \\
\text { (bpm) } \\
\text { Mean (95\%Cl) } \\
\text { P value } \\
\end{array}$ & $\begin{array}{l}\text { Heart rate } \\
\text { (bpm) } \\
\text { Mean } \\
\text { (SD) }\end{array}$ & $\begin{array}{c}\text { NHF-Air difference } \\
\text { from baseline } \\
\text { (bpm) } \\
\text { Mean (95\%Cl) } \\
\text { P value } \\
\end{array}$ & $\begin{array}{l}\text { Heart rate } \\
\text { (bpm) } \\
\text { Mean } \\
\text { (SD) }\end{array}$ & $\begin{array}{c}\text { NHF-Air difference } \\
\text { from baseline (bpm) } \\
\text { Mean }(95 \% \mathrm{Cl}) \\
\text { P value }\end{array}$ \\
\hline 0 & $72.8(11.9)$ & $73.1(13.0)$ & & $73.5(13.5)$ & & $71.7(12.4)$ & \\
\hline 5 & $72.4(13.0)$ & $73.5(12.8)$ & $\begin{array}{c}1.00 \\
(-0.77 \text { to } 2.77) \\
P=0.27\end{array}$ & 73.1 (13.4) & $\begin{array}{c}0.49 \\
(-1.28 \text { to } 2.27) \\
P=0.58\end{array}$ & $71.0(13.1)^{1}$ & $\begin{array}{c}-1.14 \\
(-2.92 \text { to } 0.64) \\
P=0.21\end{array}$ \\
\hline 10 & $72.1(11.8)$ & $72.5(13.4)$ & $\begin{array}{c}0.36 \\
(-1.41 \text { to } 2.13) \\
P=0.69\end{array}$ & $72.5(14.3)$ & $\begin{array}{c}0.18 \\
(-1.59 \text { to } 1.95) \\
P=0.84\end{array}$ & $71.6(13.1)$ & $\begin{array}{c}-0.15 \\
(-1.93 \text { to } 1.62) \\
P=0.87\end{array}$ \\
\hline 15 & $71.6(12.4)$ & $71.6(12.7)$ & $\begin{array}{c}-0.12 \\
(-1.89 \text { to } 1.65) \\
P=0.89\end{array}$ & $72.3(13.1)$ & $\begin{array}{c}0.47 \\
(-1.30 \text { to } 2.24) \\
P=0.60\end{array}$ & $71.2(12.9)$ & $\begin{array}{c}-0.07 \\
(-1.84 \text { to } 1.70) \\
P=0.94\end{array}$ \\
\hline 20 & $71.3(11.9)$ & $73.4(13.3)$ & $\begin{array}{c}2.02 \\
(0.25 \text { to } 3.80) \\
P=0.025\end{array}$ & 71.5 (13.2) & $\begin{array}{c}0.06 \\
(-1.71 \text { to } 1.83) \\
P=0.95\end{array}$ & $71.9(12.7)$ & $\begin{array}{c}0.99 \\
(-0.78 \text { to } 2.76) \\
P=0.27\end{array}$ \\
\hline
\end{tabular}

${ }^{1} \mathrm{~N}=47$, bpm: beats per minute; NHF: Nasal high flow; SD: Standard deviation; Cl: Confidence interval 
Oxygen saturations were higher for NHF at $45 \mathrm{~L} /$ min compared to room air at the 5, 15 and 20 minute time points. At 20 minutes the difference was an increase of $0.5 \%$ (95\% $\mathrm{Cl} 0.03$ to 0.91$), \mathrm{P}=0.035$, after 20 minutes with NHF. The maximum difference in oxygen saturation was $0.8 \%(95 \% \mathrm{Cl} 0.41$ to 1.28 , $\mathrm{P}<0.001$, observed after 5 minutes for $\mathrm{NHF}$ at $45 \mathrm{~L} / \mathrm{min}$ compared to room air (Table 2-4).

Heart rate remained largely constant throughout the interventions with no statistically significant differences between any of the NHF interventions and room air, with the exception of the 20 minute time point for the $15 \mathrm{~L} / \mathrm{min}$ where it was 2 beats per minute higher $(95 \% \mathrm{Cl} 0.25$ to 3.8), $\mathrm{P}=0.025$ (Table 2-5).

\subsubsection{Tolerability questionnaires}

For NHF at $45 \mathrm{~L} / \mathrm{min}$ compared to $15 \mathrm{~L} / \mathrm{min}$, the mean difference in comfort level was 20.2 units lower $(95 \% \mathrm{Cl} 13.8$ to 26.7$), \mathrm{P}<0.001$, mean difference in noisiness was 28.4 units $(95 \% \mathrm{Cl} 20.9$ to 35.9$), \mathrm{P}<0.001$, and slightly more moisture felt in the nasal cannula $(7.7 \%$ more moist, $95 \% \mathrm{Cl} 3.4$ to 11.9$)$, $\mathrm{P}<0.001)$. Mean difference in overall comfort scores for $\mathrm{NHF}$ at $30 \mathrm{~L} / \mathrm{min}$ compared to $15 \mathrm{~L} / \mathrm{min}$ was smaller than $45 \mathrm{~L} / \mathrm{min}$ compared to $15 \mathrm{~L} / \mathrm{min}(11.0$ ( $95 \% \mathrm{Cl} 4.5$ to 17.4 ) compared to 20.2 (13.8 to 26.7 ) respectively, both $\mathrm{P}<0.001$. There were no statistically significant differences in remaining questions relating to the weight of the nasal cannula, likelihood of using the $\mathrm{NHF}$ at that flow in the future, or ease of application (Table 2-6). 
Table 2-6* Mixed linear models for difference in questionnaire responses between NHF interventions.

\begin{tabular}{|c|c|c|c|}
\hline \multirow[t]{2}{*}{ Question } & \multicolumn{2}{|c|}{$\begin{array}{c}\text { Mean difference }(95 \% \mathrm{Cl}) \\
\text { P value for individual comparison }\end{array}$} & \multirow[t]{2}{*}{ P overall } \\
\hline & NHF 30L/min minus NHF 15L/min & $\begin{array}{l}\text { NHF } 45 \mathrm{~L} / \mathrm{min} \text { minus NHF } \\
15 \mathrm{~L} / \mathrm{min}\end{array}$ & \\
\hline Ease of application & 3.7 (0.7 to 6.7$) \mathrm{P}=0.017$ & $2.7(-0.3$ to 5.8$) P=0.076$ & 0.046 \\
\hline Overall Comfort & 11.0 (4.5 to 17.4) $\mathrm{P}=0.001$ & 20.2 (13.8 to 26.7) $\mathrm{P}<0.001$ & $<0.001$ \\
\hline Moisture in nasal passages & $-0.02(-4.3$ to 4.2$) \mathrm{P}=0.99$ & $-7.7(-11.9$ to -3.4$) \mathrm{P}<0.001$ & $<0.001$ \\
\hline Noisiness & 11.6 (4.1 to 19.1$) \mathrm{P}=0.003$ & 28.4 (20.9 to 35.9) $\mathrm{P}<0.001$ & $<0.001$ \\
\hline Likelihood of reusing NHF & $3.0(-2.9$ to 9.0$) P=0.31$ & 2.5 (-3.4 to 8.5$) \mathrm{P}=0.40$ & 0.55 \\
\hline Weight of nasal cannula & $1.5(-3.5$ to 6.4$) \mathrm{P}=0.56$ & $3.1(-1.8$ to 8.1$) \mathrm{P}=0.21$ & 0.46 \\
\hline
\end{tabular}

Values are on a continuous scale from most positive (0) to least positive (100). NHF: Nasal high flow.

[ Republished with permission of John Wiley and Sons, from: Respirology, "Nasal high flow therapy and $\mathrm{P}_{\mathrm{t}} \mathrm{CO}_{2}$ in stable COPD: A randomized controlled cross-over trial”, (McKinstry et al., 2017) @ 2018; permission conveyed through Copyright Clearance Center, Inc.] 


\subsection{Discussion}

NHF therapy resulted in a small, flow-dependent reduction in $\mathrm{P}_{\mathrm{t}} \mathrm{CO}_{2}$ in participants with stable COPD. There was a marked flow-dependent reduction in respiratory rate with the use of NHF. These findings suggest a favourable physiological effect with NHF in stable COPD.

There are a number of methodological issues relevant to the interpretation of the study findings. The study was single-blinded in that although participants were blinded to the actual flow rate they received, they could likely feel the difference between low, medium and high flows. The interventions were applied for 20 min periods, which was sufficient time to observe an effect on $\mathrm{P}_{\mathrm{t}} \mathrm{CO}_{2}$, with the maximum change usually observed at the 5 min time-point. There was a washout period which allowed each of the four intervention periods to begin within a similar baseline $\mathrm{P}_{\mathrm{t}} \mathrm{CO}_{2}$.

The external validity of the findings was limited to participants with stable COPD, rather than during a severe exacerbation, in which NHF administration is proposed to be more likely. However, this design enhanced the internal validity, allowing a cross-over design to be utilised with a single study visit, which importantly enabled a stable baseline $\mathrm{P}_{\mathrm{t}} \mathrm{CO}_{2}$ to be achieved before each intervention. It also avoided the confounding effect of supplemental oxygen use, a potential limiting factor in previous studies of NHF therapy in exacerbations of COPD in which lower inspired concentrations of oxygen with $\mathrm{NHF}$ may have contributed to the reductions in $\mathrm{P}_{\mathrm{a}} \mathrm{CO}_{2}$ observed (Fraser et al., 2016; Nilius et al., 2013). There was a broad cross-section of severity of COPD, with one in eight having baseline hypercapnia and one in two having an $\mathrm{FEV}_{1}<50 \%$ predicted. A post-hoc analysis showed no evidence that the change in $\mathrm{P}_{\mathrm{t}} \mathrm{CO}_{2}$ in response to treatment varied by whether the patient had chronic hypercapnic respiratory failure at baseline.

The transcutaneous SenTec monitor was used as a surrogate measure of $\mathrm{PaCO}_{2}$ change, allowing continuous monitoring and the avoidance of multiple arterial blood gas punctures (Domingo et al., 2006; Fingleton et al., 2017; Rodriguez et al., 2006; Roediger et al., 2011; Storre et al., 2011). 
The RIP measures were not valid for most interventions and so it was not possible to directly measure the effect of NHF on MV or $\mathrm{V}_{\mathrm{T}}$. The MV results utilising RIP bands proved to be an unreliable method of measurement. The measurements may have had sufficient statistical accuracy to measure MV but lacked statistical precision i.e. consecutive calibration ratios were insufficiently close to one another. Precision of RIP measurement was essential in this multiple-intervention trial design in order to draw reliable comparisons between the interventions. Some of the reasons for RIP unreliability described earlier are likely to have applied to this study. While "drift" of the bands was not quantified, thermal instability and slippage of the bands from postural changes or coughing during the interventions may have been contributing factors (Neumann \& Burchardi, 1998; O’Reilly et al., 2014). RIP band measurements of ventilation are published in the literature, and one method for improved reliability was to film each intervention and presumably exclude outlier measurements influenced by observed coughing or other movement (Fraser et al., 2016).

The observation that NHF reduces $\mathrm{P}_{\mathrm{t}} \mathrm{CO}_{2}$ in a flow-dependent manner complements the previous work discussed in Section 1.4.5.1 (Systematic review of NHF in COPD). The small reduction in $\mathrm{P}_{\mathrm{t}} \mathrm{CO}_{2}$ of $2.4 \mathrm{mmHg}$ at 45 $\mathrm{L} / \mathrm{min}$ is similar to the $3.4 \mathrm{mmHg}$ reduction with NHF at $30 \mathrm{~L} / \mathrm{min}$ for $20 \mathrm{~min}$ in COPD patients on LTOT (Fraser et al., 2016), the $3.1 \mathrm{mmHg}$ reduction with NHF at $20 \mathrm{~L} / \mathrm{min}$ for $45 \mathrm{~min}$ in COPD patients requiring supplemental oxygen at $2 \mathrm{~L} / \mathrm{min}$ in hospital (Nilius et al., 2013), and the reduction of $1.4 \mathrm{mmHg}$ observed in patients hospitalised with an AECOPD, where supplemental oxygen delivered with NHF was titrated to maintain patient $\mathrm{S}_{\mathrm{p}} \mathrm{O}_{2}$ at hospital pre-study levels (Pilcher et al., 2017). However, it is less than the $5.2 \mathrm{mmHg}$ and $7.3 \mathrm{mmHg}$ reduction in $\mathrm{P}_{\mathrm{t}} \mathrm{CO}_{2}$ observed with $2 \mathrm{hrs}$ of $\mathrm{NHF}$ treatment at 20 $\mathrm{L} / \mathrm{min}$ and $30 \mathrm{~L} / \mathrm{min}$, respectively, in the uncontrolled trial of hospitalised COPD patients (Bräunlich \& Köhler, 2016) and the non-significant $4.0 \mathrm{mmHg}$ and 5.5 $\mathrm{mmHg}$ reduction in $\mathrm{PaCO}_{2}$ in COPD patients with chronic hypercapnic respiratory failure receiving NHF therapy for $30 \mathrm{~min}$ at $20 \mathrm{~L} / \mathrm{min}$ and $30 \mathrm{~L} / \mathrm{min}$, respectively (Pisani et al., 2017). While the mean reduction in $\mathrm{P}_{\mathrm{t}} \mathrm{CO}_{2}$ of 2.4 $\mathrm{mmHg}$ found in our study is of uncertain clinical significance, the reduction in $\mathrm{P}_{\mathrm{t}} \mathrm{CO}_{2}$ from baseline of $\geq 8 \mathrm{mmHg}$ in $4 / 48$ participants on $\mathrm{NHF}$ at $45 \mathrm{~L} / \mathrm{min}$ 
suggests this therapy may have clinically important effects on $\mathrm{P}_{\mathrm{t}} \mathrm{CO}_{2}$ in a proportion of patients with COPD.

The observed reduction in respiratory rate with NHF has been reported in healthy volunteers (Ritchie et al., 2011), in COPD patients (Bräunlich et al., 2013; Chatila et al., 2004; Fraser et al., 2016; Nilius et al., 2013), and in other clinical situations such as pulmonary fibrosis and post-cardiac surgical patients (Bräunlich et al., 2013; Corley et al., 2011). The magnitude of the reduction in respiratory rate was marked with a maximum 5 breaths per min reduction after $5 \mathrm{~min}$ of $\mathrm{NHF}$ at $45 \mathrm{~L} / \mathrm{min}$, representing a greater than $25 \%$ reduction in respiratory rate. As reviewed earlier, patients with COPD and CHRF had a reduction in respiratory rate of this magnitude with NHF therapy, and is associated with reduced respiratory muscle load, a reduction in transdiaphragmatic pressures and an increase in expiratory time (Pisani et al., 2017)

The flow-dependent physiological effects on $\mathrm{P}_{\mathrm{t}} \mathrm{CO}_{2}$ and respiratory rate observed with NHF in COPD patients was consistent with the observed flowdependent increase in airway pressure, end expiratory pressures, end expiratory lung volume and inspiratory pressures observed in post-cardiac surgery patients (Corley et al., 2011; Parke \& McGuinness, 2013), and airway pressures in healthy volunteers (Ritchie et al., 2011) and COPD patients (Bräunlich \& Köhler, 2016). The modest reduction in $\mathrm{P}_{\mathrm{t}} \mathrm{CO}_{2}$ indicates alveolar ventilation was increased despite the marked reduction in respiratory rate. The relative contributions of increases in alveolar volume and/or reductions in physiological dead space were not assessed in this study. Other mechanisms discussed in Section 1.4.3 (Mechanism of action of NHF) which may play a role but were not assessed in the study include an increase in tidal volume, a small PEEP effect, reduction in upper airway resistance and improved mucociliary clearance from humidification of the airways.

Section 1.4.7 (Comfort of NHF) reviewed previous studies that have shown improved tolerability of NHF compared to both face masks and standard nasal prongs (Cuquemelle et al., 2012; Maggiore et al., 2014; Roca et al., 2010), and it was generally well tolerated in our study. Given NHF at $30 \mathrm{~L} / \mathrm{min}$ 
reduced $\mathrm{P}_{\mathrm{t}} \mathrm{CO}_{2}$ and respiratory rate by a similar amount to $\mathrm{NHF}$ at $45 \mathrm{~L} / \mathrm{min}$, but was more comfortable, this flow rate may be preferred in clinical practice.

\subsection{Conclusion}

In conclusion, NHF resulted in a small reduction in $\mathrm{P}_{\mathrm{t}} \mathrm{CO}_{2}$ and a marked reduction in respiratory rate in a flow-dependent manner with the higher the flow the greater the effect. Ultimately, these results follow onto a trial directly comparing NHF against the gold standard of NIV in COPD with hypercapnic respiratory failure (Study 5). However, these results also demonstrate potential applications for NHF in other clinical settings. The high tolerability alongside the ability to reduce work of breathing means NHF may have a role in patients recovering from an AECOPD (See Study 3 - Chapter 3 ). Prior to comparing NHF against NIV, the ability to arrest or reduce $\mathrm{PCO}_{2}$ in AHRF means NHF may be used in the initial stages of AHRF management to prevent requiring escalation to NIV (See Study 4 - Chapter 4). 



\section{Study 3 - Nasal High Flow therapy use following hospitalisation for an exacerbation of COPD: a feasibility study}

\subsection{Rationale}

Patients hospitalised with AECOPD are at high risk of early re-admission to hospital after discharge. Section 1.3.1.7 (Prevalence and costs of readmissions) reported 30-day re-admission rates of 6.7-8\% in New Zealand and $7-9 \%$ in the US, with high healthcare costs as well as worse outcomes for patients. Preventing AECOPD re-admissions is a high-priority in the US and Europe. Interventions to reduce re-admission rates have varying success and a limited evidence base.

Study 2 demonstrated NHF reduces the work of breathing and is well tolerated. Also, the NHF mechanisms of action described in Section 1.4.3 such as facilitating improved mucociliary clearance and offering a small amount of PEEP suggest NHF therapy use at home after hospital discharge with AECOPD may aid recovery and reduce readmission rates.

An RCT is required to investigate if patients discharged from hospital with an AECOPD will have a reduced risk of readmission with domiciliary NHF compared to standard care. The sample size required to show a difference in treatment failure ie re-admission with AECOPD within 30 days, is estimated to be 1,706 patients; $80 \%$ power with an alpha value of 0.05 to detect a $25 \%$ reduction in readmission for any cause at 30 days, based on a US all-cause readmission rate of $21 \%$ (Elixhauser et al., 2006).

A multi-national RCT would be required to recruit the large sample size, alongside MRINZ. Given preventing COPD re-admissions is a high priority in the US (as described in Section 1.3.1.8 Importance of readmissions), the much larger population size and corresponding large potential market for NHF use, this feasibility study was an international collaboration with Alana Healthcare through Crouse Hospital, New York, USA - a site selected by the study sponsor, Fisher and Paykel Healthcare who manufacturer the "myAIRVO 2" NHF device. 
A feasibility study was essential to inform the critical aspects of the RCT design. The important component to test in this feasibility study was participant compliance to the NHF therapy. Some of the therapy settings were modifiable by participants according to their preference, in order to better understand how much they would use NHF and with which settings.

\subsection{Aims and hypotheses}

The aims of the study, as related to the proposed RCT design, were:

1. To estimate the duration of use of NHF, including the mean hours of use per day and number of days use, to inform a precise daily NHF prescription for the proposed RCT.

2. To estimate the proportions of potential participants who could proceed to the proposed RCT.

3. To estimate the proportions of potential participants who would require oxygen delivery through NHF in the proposed RCT.

4. To estimate aspects of NHF use after discharge and in particular to estimate patient ratings of ease of use, and the proportion of those who required changes to initial settings of temperature and flow, and the magnitude of these changes. This information would inform prescription design for the proposed RCT.

5. To estimate particular clinical outcomes relevant to the planned RCT, including hospital readmission, Emergency Department (ED) visit rates and General Practitioner (GP) visit rates.

The hypothesis of this feasibility study was:

- Adequate feasibility data would be obtained to assess the processes, resources, and management required to inform the RCT design.

\subsection{Methods}

\subsubsection{Trial design}

Prospective cohort feasibility study. 


\subsubsection{Eligibility criteria}

\subsubsection{Inclusion criteria}

1. Participants 18 years of age or older

2. Participants admitted to the respiratory ward(s) of either recruiting hospitals with AECOPD as the primary diagnostic reason for admission.

\subsubsection{Exclusion criteria}

1. New domiciliary oxygen prescription during the current hospital admission

2. The investigator believed the participant or their care giver will be unable to safely use the NHF device following discharge

3. Any other condition which, at the investigator's discretion, was believed may present a safety risk or impact the feasibility of the study or the study results.

\subsubsection{Interventions}

Participants were divided into 2 groups - 1) those on a domiciliary oxygen prescription at the time of study entry and 2) those who were not. Participants on domiciliary oxygen received their oxygen supplementation via the NHF device but could revert to their usual nasal cannulae whenever they desired. Participants not on domiciliary oxygen were encouraged to use NHF as much as possible, day and night, whereas those on domiciliary oxygen were instructed to only use NHF during the day.

\subsubsection{Recruitment and consent}

Eligible participants were identified from Wellington Hospital, New Zealand and Crouse Hospital (New York, USA). Inpatient lists were reviewed and potential participants were approached on the ward when in a clinically stable condition, at least one day prior to expected discharge to ensure time to familiarise with NHF therapy prior to discharge. They were given a PIS and time to read it and discuss their participation with family/whanau and/or their attending medical staff.

The study was prospectively registered with ClinicalTrials.gov (Trial ID: NCT02552732) and approved by the HDEC of New Zealand (Ref: 15/NTB/92). 
Full written informed consent was completed before any study specific procedures were carried out.

\subsubsection{Study procedures}

Participants were visited in hospital, and once full written informed consent was obtained, demographic data were collected, including a LACE index score and whether domiciliary oxygen was prescribed. The LACE score is a 4-step tool to assess the risk of readmission based on factors such as acuity of admission and co-morbidities. Participants were allocated a NHF device, and a training session on how to use NHF was performed by the study investigator. The participants' next of kin were encouraged to be present at this session. Further familiarization to the device and the therapy was again conducted prior to leaving hospital. Where possible any issues regarding the device or therapy were addressed prior to discharge. The participant was also given the NHF user manual and 'Patient Summary Sheet' (See appendix 9.5.3) to read prior to the home visit and a phone number to contact the investigator with any questions during business hours. NHF was used until 30 days following discharge. Note the day of discharge was defined as Day 0.

\subsubsection{NHF therapy settings}

By default, NHF was set at $25 \mathrm{~L} / \mathrm{min}$ at $37^{\circ} \mathrm{C}$. However, all participants were able to lower the delivered temperature to $34^{\circ} \mathrm{C}$ if they felt that was more comfortable. For participants without a domiciliary oxygen prescription, flow rate could be increased/decreased (up to $30 \mathrm{~L} / \mathrm{min}$ or down to $20 \mathrm{~L} / \mathrm{min}$ ). For participants with domiciliary oxygen, flow rates were fixed in order to deliver a more consistent concentration of oxygen to maintain their prescribed oxygen saturation.

For participants on domiciliary oxygen only, oxygen saturation testing on their usual oxygen flow took place during the hospital admission. Participants on domiciliary oxygen had their oxygen saturations (Point $A$ ) recorded on standard nasal prongs according to their existing oxygen flow prescription (Flow A). NHF therapy was then administered to the participant via NHF set at a flow rate of $25 \mathrm{~L} /$ minute with supplemental oxygen delivered at Flow $A$ for at least 5 min whilst the participant was at rest. Oxygen was titrated via the 
device by $0.5 \mathrm{~L} / \mathrm{min}$ every $5 \mathrm{~min}$ until the oxygen saturation levels measured at Point A (i.e. with the portable oximetry probe after sitting at rest for at least 5 min using standard nasal prongs with oxygen administered at Flow A) were equivalent. This flow rate was then used for supplemental oxygen delivered during NHF therapy throughout the study period.

If the participant's oxygen saturation levels on their prescribed oxygen were $<88 \%$ or $>95 \%$, they were withdrawn from the study and referred for review of their domiciliary oxygen prescription. An explanation was given to the participant in respect to the risks and symptoms of hypoxia, and hypercapnia, how to use NHF with correct oxygen flow rates, and how to change the oxygen flow between NHF and their standard nasal prongs. This was important because it was expected participants would want to revert back to their usual nasal cannulae for some part of the day.

\subsubsection{Home visit}

A home visit was carried out by an investigator on the day of or day after discharge to set-up of the equipment and reinforce the previous training on NHF, perform pre-bronchodilator lung function tests using a spirometer (Jaegar pneumotac spirometer with $\mathrm{J}$-Lab software) according to ATS/ERS Taskforce standards, (M. R. Miller et al., 2005) and dispense the participant use diaries.

On day 3 post hospital discharge, the participants were contacted by phone to see if they were having any problems using NHF, encouraging future use and recording any adverse events. This phone call was repeated on day 7 and then weekly until day 28 . When study visits and phone calls fell outside of the working week, these were rescheduled to take place \pm 1 day of the specified date.

On day 31 post discharge, a final home visit was performed to administer the NHF therapy questionnaire (see appendix 9.5.2), perform pre-bronchodilator lung function tests using a spirometer (Jaegar pneumotac spirometer with $\mathrm{J}$ - 
Lab software) according to ATS/ERS Taskforce standards, (M. R. Miller et al., 2005) and collect the NHF device and associated equipment.

\subsubsection{Outcome measures}

\subsubsection{Primary outcome}

The primary outcome was NHF use for 30 days following discharge after AECOPD. Use was expressed as hours of NHF use per day over 30 days, as obtained by electronic monitoring in the NHF device and subsequent download.

\subsubsection{Secondary outcomes}

\subsection{OTHER NHF USE VARIABLES}

- Number of days of use, adjusted for number of days with NHF at home

- Average use per day of NHF during week 1, week 2, week 3 and week 4.

- Average use per day on days that NHF was used.

\subsection{WITHDRAWALS AND EXCLUSIONS}

- Proportion of participants screened that were excluded or withdrawn and reasons for either.

\subsection{PARTICIPANTS REQUIRING OXYGEN DELIVERY VIA NHF}

- Proportion of participants using domiciliary oxygen.

\subsection{VARIABLE ASPECTS OF NHF USE}

- Proportion of participants who reported having changed NHF flow settings and/or temperature from the initial settings and what flow/temperature was used.

- Other NHF therapy questionnaire results.

\subsection{CLINICAL OUTCOMES}

- Proportion of participants with at least one hospital readmission, ED visit* $^{*}$ and/or GP visit within 30 days of discharge and reasons for each (AECOPD, other respiratory cause or other cause).

- In those that had at least one hospital readmission, the number of readmissions

- Time to first hospital readmission and length of stay

- LACE Index for hospital readmissions 
- Lung function measurements (FEV1, Slow Vital Capacity in and FVC) at day 1 and 31 .

* An ED visit was counted if the patient went to ED for assessment and was not subsequently admitted to a hospital ward.

\subsubsection{Sample size estimation}

A participant number of 100 was planned (20 from NZ and 80 from USA sites). These values were based on an estimated drop-out rate of $20 \%$ and the requirement for a minimum of 80 patients in total to assess feasibility and provide robust information with respect to appropriate outcome measures.

\subsubsection{Statistical analysis plan}

Given the small sample size recruited (see Results below), the statistical analysis plan changed. Originally, with a sample size of 100 , the plan was for the primary outcome measure to be expressed as the mean number of hours per day used, with standard deviation. The mean would have been weighted for time in the study and allocated $95 \%$ confidence intervals for each by weighted regression. Time to first hospital readmission and first ED visit were to be analysed by Kaplan-Meier survival estimates and confidence interval for quantiles of survival. Those who didn't get admitted within the study time would become censored data.

However, with useable data from only 20 participants, the analysis was carried out as follows. Basic data summaries were shown for all continuous variables and counts and proportions for categorical variables. The primary outcome variable analysis was described and then a 1 -sample Student's $t$-test was used to calculate confidence intervals. Shapiro-Wilks test for normality was used and, when non-normal distribution was shown, square root data transformation was carried out. The histogram and Q-Q plot illustrates the non-normal nature of the sample followed by a repeat normality testing once data was transformed.

The days used, adjusted for days NHF was available (secondary outcome variable), was by proportion with confidence interval. Daily use by week was illustrated with box-plots. Remaining secondary outcome variables are described below, either using means and standard deviations or proportions 
where appropriate. RCommander 2.4-1 and RStudio 1.1.423 were used for analyses.

\subsection{Results}

Participants were recruited in NZ from January 2016 to October 2016. Unfortunately, I can present only the NZ data from 20 participants as no access to any of the USA data from the site at Crouse Hospital (Alana Healthcare) was accessible. The study was terminated by the study sponsor Fisher and Paykel Healthcare in late 2016 due to failure to recruit in the US site and listed as a "change in business of US site" on clinicaltrials.gov webpage. In the NZ cohort, the mean (SD) age was 68.7 (10.8) years and $45 \%$ were male. Baseline characteristics are shown below in Table 3-1.

Table 3-1 Study 3 baseline participant characteristics.

\begin{tabular}{|c|c|c|c|}
\hline Characteristic & Mean (SD) & Median (IQR) & Min to Max \\
\hline Age (years) & $68.7(10.8)$ & 70.6 (62.3 to 77.8$)$ & 46 to 83 \\
\hline Body Mass Index $\left(\mathrm{kg} / \mathrm{m}^{2}\right)$ & $24.3(5.4)$ & 23.9 (20.1 to 29.8$)$ & $17-33$ \\
\hline $\mathrm{FEV}_{1}(\mathrm{~L}) \mathrm{N}=16$ & $0.89(0.34)$ & $0.86(0.67$ to 1.17$)$ & 0.28 to 1.64 \\
\hline $\mathrm{FEV}_{1} / \mathrm{FV}$ C ratio & $0.40(0.14)$ & 0.34 (0.32 to 0.47$)$ & 0.19 to 0.77 \\
\hline $\mathrm{FEV}_{1} \%$ predicted & $30.9(12.4)$ & 30 (22 to 39$)$ & 9 to 53 \\
\hline Smoking pack years & $38.0(23.2)$ & $30(20-51)$ & 15 to 102 \\
\hline LACE (index admission) & $10.3(1.9)$ & 10.0 (9 to 11$)$ & 7 to 15 \\
\hline & & \multicolumn{2}{|l|}{$\mathrm{N} / 20(\%)$} \\
\hline Sex (Female) & & \multicolumn{2}{|l|}{$11(55)$} \\
\hline \multicolumn{4}{|l|}{ Co-morbidities } \\
\hline Bronchiectasis & & \multicolumn{2}{|l|}{$1(5)$} \\
\hline Heart failure & & \multicolumn{2}{|l|}{$3(15)$} \\
\hline Arrhythmias & & \multicolumn{2}{|l|}{$1(5)$} \\
\hline \multicolumn{4}{|l|}{ Ethnicity } \\
\hline Maori & & \multirow{3}{*}{\multicolumn{2}{|c|}{$\begin{array}{l}1(5.0) \\
16(80.0) \\
3(15.0)\end{array}$}} \\
\hline NZ European & & & \\
\hline Other & & & \\
\hline \multicolumn{4}{|l|}{ Inhaled Treatment } \\
\hline & \multicolumn{2}{|l|}{$15(75)$} \\
\hline Long-Acting Beta-Agoni & & \multicolumn{2}{|l|}{$15(75)$} \\
\hline \multicolumn{2}{|c|}{ Long-Acting Muscarinic Antagonist } & \multicolumn{2}{|l|}{$14(70)$} \\
\hline \multicolumn{2}{|c|}{ Short-Acting Beta-Agonist } & \multicolumn{2}{|l|}{$17(85)$} \\
\hline \multicolumn{2}{|c|}{ Short-Acting Muscarinic Antagonist } & \multicolumn{2}{|l|}{$2(10)$} \\
\hline \multicolumn{2}{|c|}{ Long-term oxygen therapy } & \multicolumn{2}{|l|}{$2(10)$} \\
\hline \multicolumn{4}{|c|}{ Smoking status } \\
\hline Current smoker & & \multirow{2}{*}{\multicolumn{2}{|c|}{$\begin{array}{l}3(15) \\
17(85)\end{array}$}} \\
\hline Ex-smoker & & & \\
\hline
\end{tabular}




\subsubsection{Primary outcome}

The mean (SD) NHF use for 30 days, expressed as hours of use per day over 30 days was 1.59 (1.59) hours/day. 19 participants were included in the analysis with 1 participant withdrawing on day 2 before registering any NHF use. The data summary and spread of data is illustrated in Table 3-2 and the histogram (Figure 3-1) below.

Table 3-2 Study 3 primary outcome - data description of NHF use per day.

\begin{tabular}{lll}
\hline Variable & Mean (SD) & $\mathbf{9 5 \%} \mathbf{C l}$ \\
\hline $\begin{array}{l}\text { Mean NHF daily use } \\
\text { (hr) }\end{array}$ & $1.59(1.59)$ & 0.83 to 2.36 \\
\hline
\end{tabular}

The mean daily NHF use was $1.59 \mathrm{hr}$, the maximum daily mean use was 6 $\mathrm{hr} /$ day and the minimum $0.1 \mathrm{hr} /$ day.

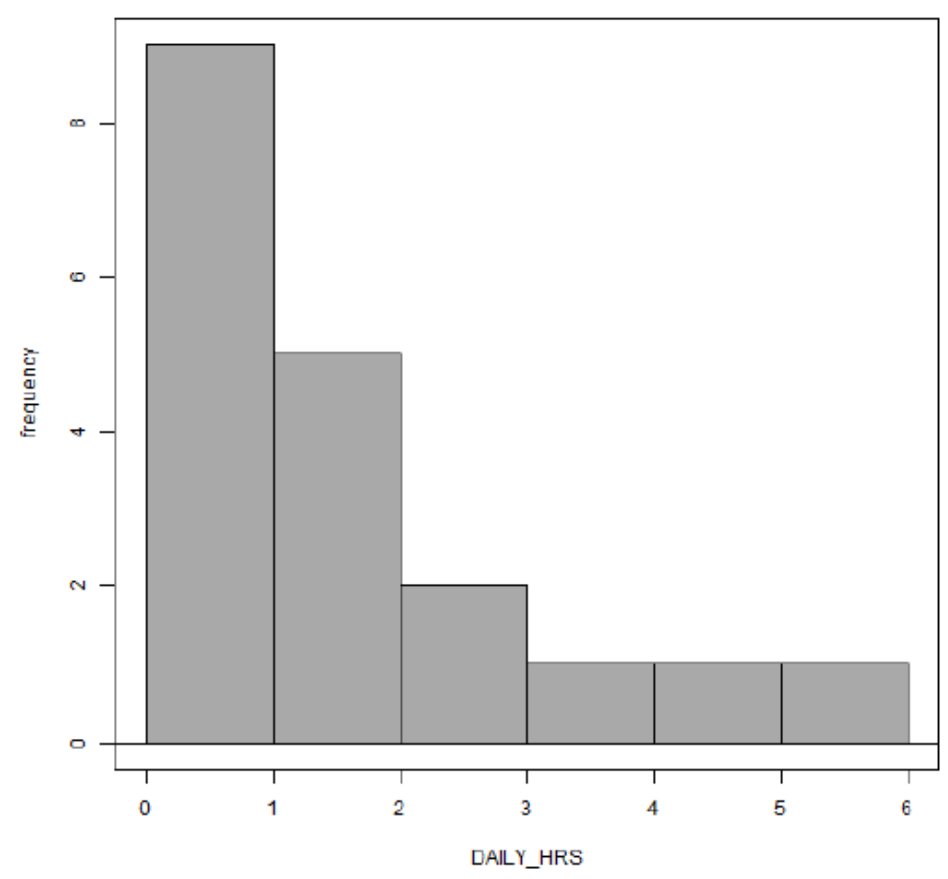

Figure 3-1 Histogram of daily hours (daily_hrs) NHF was used.

The primary outcome variable did not follow a normal distribution with a Shapiro-Wilks test for normality, $\mathrm{P}=0.005$, rejecting the null hypothesis that the 
data was from a normal distribution, and the $Q-Q$ plot shown in Figure 3-2 demonstrates data points away from the line of normality.

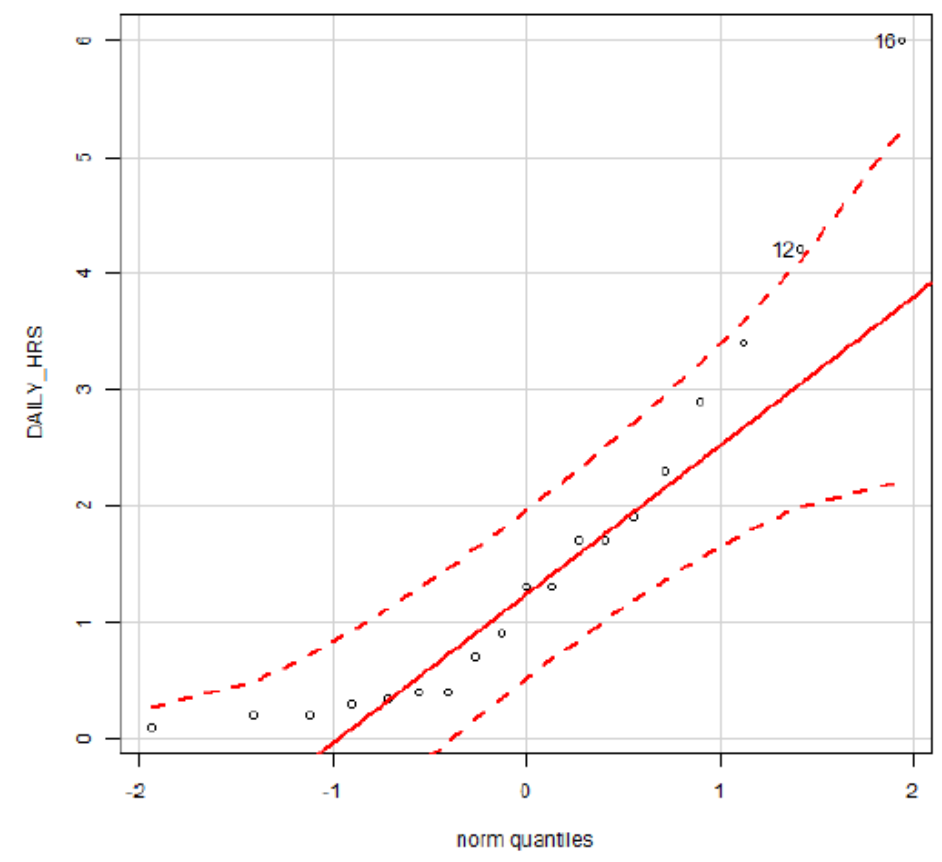

Figure 3-2 Q-Q Plot for the primary outcome variable (daily_hrs used) indicating non-normal distribution.

A square root transformation of the data was applied to obtain a normal distribution with a Shapiro-Wilks test of $P=0.304$ (failed to reject the null hypothesis) and a normal-appearing histogram. The transformed primary outcome of daily hours used had a mean of $1.12(95 \% \mathrm{Cl} 0.83$ to 1.41$)$ and when squared to re-transform the result, the mean was $1.56 \mathrm{hr}(95 \% \mathrm{Cl} 0.68$ to 1.98$)$.

\subsubsection{Secondary outcomes}

\subsubsection{Proportion of days NHF was used}

The number of days of use, adjusted for the number of days with the NHF device at home, was $59 \%(95 \%$ Cl 55 to $63 \%)$. A total of 538 days were available for use, allowing for the 2 withdrawals (one with 0 days use and one with 2 days use) and the 2 participants who were admitted to hospital, each 
unable to use the device during their 2 day inpatient stay. The histogram of the proportion of days used by participants is shown in Figure 3-3.

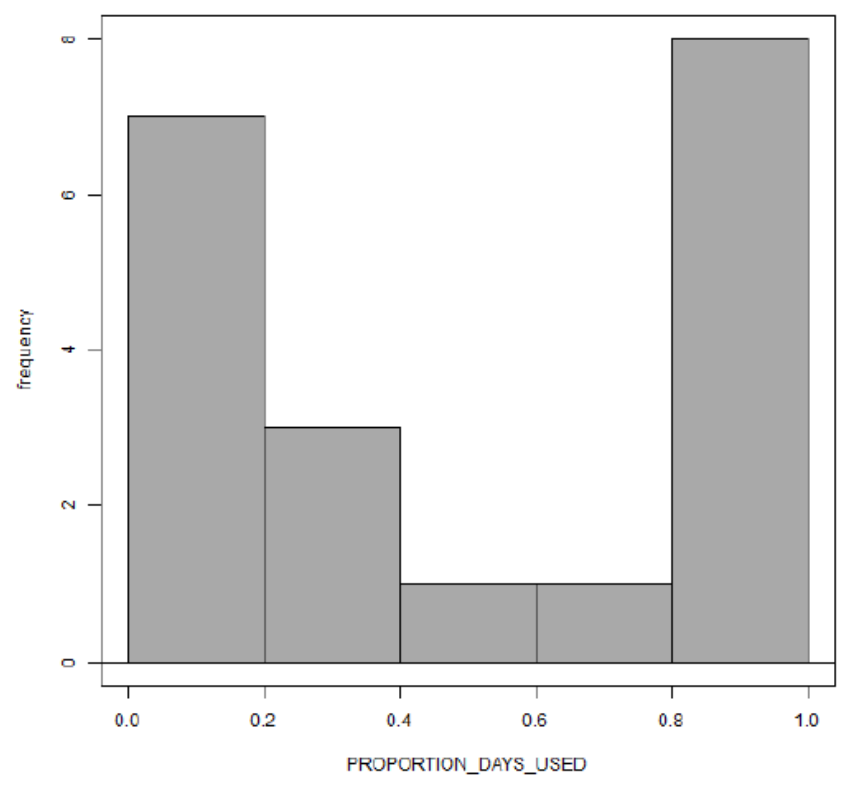

Figure 3-3 Histogram for proportion of days used (X-axis labelled "proportion_days_used") adjusted for number of days NHF was available.

Most participants did not use the device every day, although three (16\%) registered use of the NHF on all 30 days, $2(11 \%)$ on 29 days and $1(5 \%)$ on 28 days. Overall, the mean (SD) use per day on the days NHF was used was $2.0 \mathrm{hr}(0.9)$, slightly higher than the primary outcome of mean use averaged over all 30 days of $1.59 \mathrm{hr} /$ day.

\subsubsection{Variation of NHF use by week}

Seventeen participants' data were analysed to calculate the mean daily hours used per week. The 3 participants excluded from the analyses were the 2 who withdrew and 1 participant who was unable to use the device for periods of time during each of the weeks but had reasonable overall daily use so was included in the primary outcome analyses but not in this more specific secondary outcome analysis. Figure 3-4 demonstrates the reasonably even spread of daily hours used each week and some outliers, especially in week 1. 


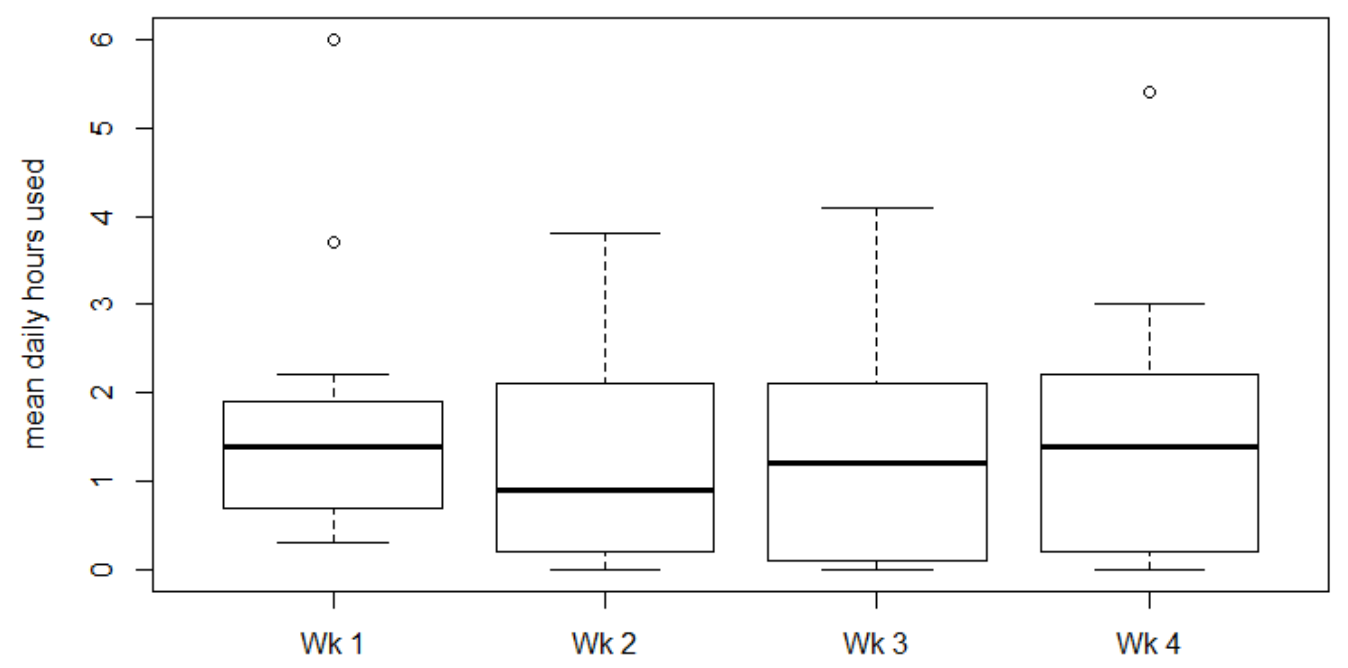

Figure 3-4 Box-plots of mean daily hours of NHF use by week.

\subsubsection{Various aspects of use from the NHF therapy Questionnaire results}

The NHF therapy questionnaire was completed by 17 participants and results are shown in Table 3-3. The discrete scores were from 1 (most positive) to 10 (most negative) indicating a strongly favourable rating by participants on the whole.

Table 3-3 NHF therapy questionnaire results - questions 1 to 4

\begin{tabular}{llll}
\hline Question & Mean (SD) & Median (IQR) & Min to Max \\
\hline Ease of use & $2.6(2.7)$ & $1(1$ to 4$)$ & 1 to 9 \\
Ease of set-up & $2.4(2.0)$ & $1(1$ to 3$)$ & 1 to 7 \\
Ease of cleaning & $2.5(2.4)$ & $1(1$ to 3.5$)$ & 1 to 8 \\
Overall comfort & $2.2(1.9)$ & $1(1$ to 3$)$ & 1 to 7 \\
\hline
\end{tabular}

Questions about specific NHF settings used by participants were asked because there were some setting choices and questions were designed to elicit any patterns of choice with the settings. 
Table 3-4 NHF therapy questionnaire results - questions 5 to 6 .

\begin{tabular}{|c|c|c|c|c|c|}
\hline Question & $\begin{array}{l}\text { No (N / } 17 \\
\%)\end{array}$ & $\begin{array}{l}\text { Yes (N / } \\
17 \%)\end{array}$ & Sub-question & & \\
\hline \multirow[t]{3}{*}{$\begin{array}{l}\text { Was flow rate } \\
\text { changed? }\end{array}$} & $12(70.5)$ & $5(29.4)$ & & & \\
\hline & & & $\begin{array}{l}\text { Most } \\
\text { comfortable } \\
\text { flow setting (if } \\
\text { changed) }\end{array}$ & $\begin{array}{l}25 \mathrm{~L} / \mathrm{min}(\mathrm{N} / 5 \\
\%)\end{array}$ & $\begin{array}{l}30 \mathrm{~L} / \mathrm{min}(\mathrm{N} / 5 \\
\%)\end{array}$ \\
\hline & & & & $4(80)$ & $1(20)$ \\
\hline \multirow[t]{3}{*}{$\begin{array}{l}\text { Was temperature } \\
\text { changed? }\end{array}$} & $8(47.0)$ & $9(52.9)$ & & & \\
\hline & & & $\begin{array}{l}\text { Most } \\
\text { comfortable } \\
\text { temperature } \\
\text { setting for } \\
\text { those that } \\
\text { changed it }\end{array}$ & $37^{\circ} \mathrm{C}(\mathrm{N} / 9 \%)$ & $34^{\circ} \mathrm{C}(\mathrm{N} / 9 \%)$ \\
\hline & & & & $5(55.56)$ & $4(44.44)$ \\
\hline
\end{tabular}

Table 3-5 shows over $50 \%$ of participants would continue to use the NHF device at home indefinitely if it was recommended for their future use.

Table 3-5 NHF therapy questionnaire results - question 7. Self-reported length of time participants would be prepared to use NHF in the future.

\begin{tabular}{ll}
\hline Length of time & $\mathbf{N} / \mathbf{1 7}(\%)$ \\
\hline Not at all & $6(35.3)$ \\
6 months & $1(5.9)$ \\
12 months & $1(5.9)$ \\
Indefinitely & $9(52.9)$ \\
\hline
\end{tabular}

\subsubsection{Withdrawals and screen failures}

All participants screened were included and 2 withdrew after enrolment. One participant had a change of personal circumstances and withdrew on Day 7 and one decided they did not like the high-flow air sensation of NHF once back at home and withdrew on Day 3.

\subsubsection{Clinical outcomes}

All 20 participants were included in the analyses of clinical outcomes as all participants enrolled had the potential to fit these secondary outcome measures. Two of the 20 (10\%) were re-admitted with AECOPD during the 30 day interval $(95 \% \mathrm{Cl} 1.8$ to $33.1 \%)$. Both participants were re-admitted on Day 26, and both had a 2 day inpatient stay. Their LACE indices of 10 and 11 
respectively were in keeping with the overall mean LACE of 10.3 for the index admission.

One (5\%) presented to ED with AECOPD on Day 30 and four (20\%) participants had a total of 5 GP presentations, 3 of which were for AECOPD. For participants who were able to complete spirometry at day 1 and day 31 , there was minimal lung function change during this period (Table 3-6).

Table 3-6 Lung function change - day 31 compared to day 1.

\begin{tabular}{|c|c|c|c|c|}
\hline Variable & $\begin{array}{l}\text { Measured } \\
\text { variable }\end{array}$ & Mean (SD) & Median (IQR) & Min to Max \\
\hline FEV1 & $\begin{array}{l}\text { Change } \\
\% \text { change }\end{array}$ & $\begin{array}{l}-0.05(0.17) \\
-2.53(15.58)\end{array}$ & $\begin{array}{l}0.01(-0.13 \text { to } 0.07) \\
0.86(-17.1 \text { to } 8.6)\end{array}$ & $\begin{array}{l}-0.42 \text { to } 0.11 \\
-25.61 \text { to } 21.95\end{array}$ \\
\hline FVC & $\begin{array}{l}\text { Change } \\
\% \text { change }\end{array}$ & $\begin{array}{l}0.001(0.286) \\
1.57(17.08)\end{array}$ & $\begin{array}{l}0.01(-0.3 \text { to } 0.2) \\
0.24(-12.9 \text { to } 11.2)\end{array}$ & $\begin{array}{l}-0.44 \text { to } 0.47 \\
-21.26 \text { to } 39.17\end{array}$ \\
\hline $\begin{array}{l}\text { FEV1/FVC } \\
\text { ratio }\end{array}$ & $\begin{array}{l}\text { Change } \\
\% \text { change }\end{array}$ & $\begin{array}{l}-0.02(0.04) \\
-3.50(10.29)\end{array}$ & $\begin{array}{l}-0.02(-0.04 \text { to } 0.03) \\
-2.68(-10.89 \text { to } 5.29)\end{array}$ & $\begin{array}{l}-0.11 \text { to } 0.03 \\
-23.20 \text { to } 9.44\end{array}$ \\
\hline $\operatorname{SVC}(\mathrm{N}=9)$ & $\begin{array}{l}\text { Change } \\
\% \text { change }\end{array}$ & $\begin{array}{l}0.05(0.41) \\
2.47(14.40)\end{array}$ & $\begin{array}{l}0.04(-0.15 \text { to } 0.41) \\
1.72(-8.9 \text { to } 13.9)\end{array}$ & $\begin{array}{l}-0.78 \text { to } 0.49 \\
-21.85 \text { to } 20.81\end{array}$ \\
\hline
\end{tabular}

$\mathrm{N}=10$, except where marked. Change is measured in litres.

\subsection{Discussion}

A clinical trial of NHF use following hospitalisation for AECOPD was demonstrated to be feasible in NZ but was not feasible in the USA. The reasons for the failure to continue recruiting in the US are documented as a "change in business of the US site". I was not privy to any further explanation from Fisher and Paykel, the study sponsor. Caution must be taken when interpreting the NZ results due to the small sample size of 20 , given the original power calculation was for a feasibility study with a sample size of 100 .

As reviewed in section 1.3.1.11 (current treatment options for reducing hospital readmissions), there is limited evidence to support interventions to reduce readmission rates. Domiciliary NHF use in COPD patients leaving hospital could be one of the most important roles for NHF as the mechanisms of action correlate with treating the causes of a further exacerbation during the fragile post-discharge period i.e. increased mucous production and increased breathlessness. Having a supportive aid like NHF at home for these people may prove sufficient to prevent readmission, with the caveat that they are able 
to recognise a serious exacerbation and not remain at home with $100 \%$ reliance on the NHF in this case.

A repeat feasibility study with a very similar design to the present study is currently underway in Melbourne (A/Prof Mansfield) and is also sponsored by $\mathrm{FPH}$. Compliance should be improved due to having daily home visits by a nurse for the first week and twice weekly thereafter, although this may limit the external validity of their findings. They have also set a minimum use of $6 \mathrm{hrs}$ per day, targeting nocturnal use, which is an ambitious target. The recently published domiciliary NHF study by Storgaard et al 2018 reviewed earlier in section 1.4.5.1.3.4 (systematic review of NHF in COPD) had a mean use of 6 hours/day showing it is possible to obtain this level of compliance. The Melbourne study is powered to show a $25 \%$ reduction in 60-day readmission rates from the current high rate of $25 \%$ as well as halving the 6 -day inpatient length of stay.

\subsection{Conclusion}

While NHF therapy may offer a benefit in recovering from an AECOPD hospitalisation in the home setting, overall this trial does not give adequate feasibility data to support a large RCT powered to detect a reduction in readmission rates. 



\section{Study 4 - Nasal High flow therapy in acute hypercapnic respiratory failure with acidosis in patients presenting with an acute exacerbation of COPD: A feasibility study}

\subsection{Rationale}

Section 1.3.1.4 reviewed inpatient management of AECOPD and the recommendation from the BTS/ICS guidelines that AECOPD patients with mild-moderate AHRF should be treated with an initial 1 hour of optimal medical therapy (Davidson et al., 2016). Medical optimisation includes titrated oxygen therapy to maintain oxygen saturations between $88-92 \%$ as well as the standard pharmacological therapy reviewed earlier. NIV should then be commenced only if treatment has failed after this initial 1 hour. The rationale for this initial 1 hour optimisation period was $20 \%$ of AHRF cases would normalise during this period, avoiding escalation to NIV (Plant et al., 2000) and the associated side-effects and logistical demands.

Study 2 demonstrated that NHF reduces $\mathrm{PCO}_{2}$ and respiratory rate in COPD patients, confirming preliminary findings from publications in the Section 1.4.5 systematic review. Another application for NHF therefore is to improve outcomes in this initial 1 hour optimisation period by reducing $\mathrm{PCO}_{2}$ and respiratory rate, improving on the current rate of $20 \%$.

To demonstrate efficacy, an RCT is required to investigate NHF in AECOPD patients with AHRF during the initial 1 hour period of optimisation - directly comparing: 1) usual care plus NHF, with 2) usual care plus standard oxygen therapy. Treatment failure would then be the measured as the proportion of patients who remained in AHRF and required escalation to NIV.

Similar to Study 3, the sample size for the proposed RCT is large - in this case, over 700 patients. A feasibility study was required to test several key design aspects of the proposed large RCT required to show a difference in treatment failure rates. 
Study 4 had two parts. Part 1 was an agreement with ED staff on a "usual care" protocol for management of AECOPD with AHRF. Once this had been achieved, Part 2 was a 40 patient prospective cohort feasibility study of AECOPD patients with AHRF to be undertaken in Wellington, Waikato and Christchurch Hospital EDs investigating key aspects of the proposed RCT design.

\subsection{Aims and hypotheses}

Six feasibility study (FS) issues were identified that needed to be addressed in order to facilitate the proposed RCT design. The 6 aims of the feasibility study were:

\subsubsection{FS1: Estimation of the treatment failure rate in the usual care group.}

There is no published New Zealand data on the treatment failure rate in AHRF patients with a $\mathrm{pH}$ of 7.25 to 7.34 .

The aim was to detect an absolute difference of $10 \%$ in the treatment failure rate. If the treatment failure rate in the usual care group was less than $60 \%$ then the total sample size needed to detect a change from $60 \%$ to $50 \%$ is substantially larger (achieved sample size per arm 388) which would require more centres and a longer recruitment time-frame.

\subsubsection{FS2: Estimate the likely attrition rate in relation to clinician adherence to the usual care protocol.}

The failure to adhere to the usual care protocol could result in cross-over to active treatment in the RCT and could result in substantial dilution of any treatment effect that in fact exists. A 90\% adherence was anticipated for the feasibility study. However, should this be less than $75 \%$ then it would need to be understood why adherence wasn't happening and be able to implement measures, including renegotiating the usual care protocol, to achieve this. 


\subsubsection{FS3: Estimate the proportion of patients admitted with AECOPD who are in AHRF and are otherwise eligible for the proposed RCT.}

The recruitment rate will allow an estimate of how long study recruitment for the proposed RCT would take. Although we have reasonable New Zealand data on the admission numbers for AECOPD we could not identify data on the proportion with AHRF in New Zealand; and have based the estimates of sample size on international data (Plant et al., 2000).

A prospective cohort log would be required to achieve this. If, as anticipated, $10 \%$ of patients with $\mathrm{AECOPD}$ have AHRF with a $\mathrm{pH}$ in the required range, then about 400 patients with AECOPD would need to be screened to achieve the sample size of 40 for the feasibility study. This gives a margin of error for estimating a proportion of about plus or minus 3\% so a reasonably precise estimate suitable for confirming the planning of the proposed RCT in terms of time and the number of centres likely to be needed to recruit sufficient participants

\subsubsection{FS4: Estimate the SD of the hydrogen ion concentration by ABG measurement.}

The Cochrane review of NIV in AECOPD reported that hydrogen ion concentration may be more preferable to $\mathrm{pH}$ as an outcome measure due to changes being linear in nature (Osadnik et al., 2017). Although international studies commonly report the $\mathrm{pH}$ it is not possible to estimate the SD of the actual hydrogen ion concentration from the $\mathrm{SD}$ of the $\mathrm{pH}$ (the negative logarithm of the hydrogen ion concentration). The SD from the hydrogen ion concentration would add to the overall sample size calculation.

\subsubsection{FS5: Confirm the acceptability and uptake of deferred consent.}

The potential participants are patients who are acutely unwell and, although deferred consent is approved in ICU studies in unconscious patients, it is less common to use deferred consent in very sick, but not unconscious, patients.

The use of deferred consent is a unique part of both the proposed RCT and the feasibility study in this patient group. The feasibility study was not powered 
on this feasibility issue, but should 40 participants be initially entered into the study, this sample size gives a margin of error for a proportion of about $\pm 15 \%$, a good indicator if deferred consent is practical. Should deferred consent only achieve $50 \%$ recruitment, for example, this would require double the time or number of centres for the RCT.

\subsubsection{FS6: Recruitment rates}

Estimate recruitment rates for the full study at each of the 3 centres based on consent to participate in the feasibility study.

The hypotheses were:

- feasibility data would be obtained to assess the processes of managing patients with AECOPD and AHRF with acidosis as they present to ED to inform the RCT design and ensure methodological robustness.

- Part 1: A usual care protocol for management of AECOPD with AHRF would be agreed upon between Wellington, Waikato and Christchurch Hospitals EDs.

- Part 2: Participants would be enrolled with a deferred consent model according to the eligibility criteria for the full proposed RCT and the protocol devised in Part 1 would be adhered to in $90 \%$ of cases, or at least $75 \%$ of cases.

\subsection{Methods - Study Part 1}

\subsubsection{Agreement on a usual care protocol}

Figure 4-1 shows the usual care protocol agreed upon with the ED Head of Department. The key aspects were mandatory serial VBGs and ABGs, and strict adherence to a standard guideline for management of AECOPD. 


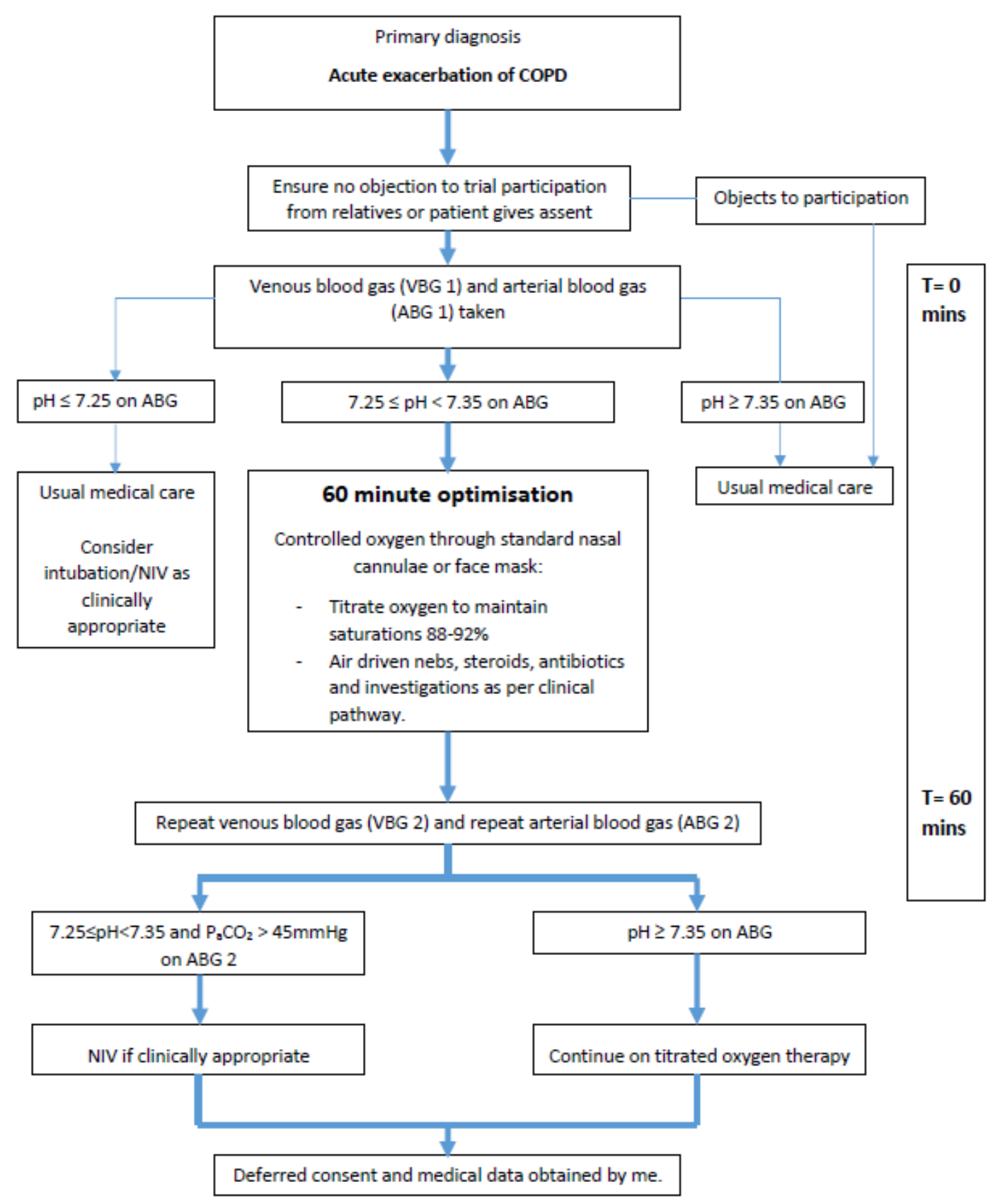

Figure 4-1 Study part 1 - Usual care protocol agreed upon with ED staff.

A full description of CCDHB management of AECOPD guidelines is in Section 9.6.2, appendix IV. 


\subsection{Methods - Study Part 2}

\subsubsection{Trial design}

Prospective cohort feasibility study

\subsubsection{Eligibility criteria}

The feasibility study used the same eligibility criteria as the proposed RCT.

\subsubsection{Inclusion criteria}

1. Diagnosis of AECOPD causing AHRF with acidosis ( $\mathrm{pH}$ of $7.25-7.34$ on baseline $A B G$ )

2. Age $>40$ years.

\subsubsection{Exclusion criteria}

1. Patients requiring immediate NIV or intubation;

2. Patients in whom NIV would never be considered clinically appropriate, e.g. advance care plan, refusing NIV;

3. Agitation sufficient to preclude study procedures;

4. Specific contra-indications to NIV according to BTS/ICS guidelines (Davidson et al., 2016), e.g: Life-threatening hypoxaemia; Inability to protect airway; facial burns/trauma/recent facial or upper airway surgery; significant nasal/septal pathology; vomiting; fixed upper airway obstruction; undrained pneumothorax; copious respiratory secretions; haemodynamic instability requiring inotropes/vasopressors; pregnancy or breastfeeding.

\subsubsection{Recruitment}

Recruitment was from Hospital Emergency Departments and was carried out by a triage nurse, who identified potentially suitable participants, and then an ED doctor who would complete the clinical part of the study protocol, and myself who would collect deferred consent and other duties described in Figure 4-2.

\subsubsection{Deferred consent}

Enrolment in the study and collection of data was completed before consent was obtained - a process called deferred consent. Deferred consent allowed 
for patients not having capacity to consent earlier, due to the cognitive effects of AHRF, and was the intended consent model for the proposed RCT.

Deferred consent was a 2 step process - firstly, it was assumed, by definition of the patients hypercapnia they were not competent to volunteer consent therefore "assent" was obtained by ED staff from a family member/interested party. Deferred Consent was then obtained by me at a later time when the patient was felt to be sufficiently recovered and competent to decide whether the data already collected could be used for research purposes or withdrawn. So there were 2 stages where patients could be withdrawn. This was the only way for the study to be performed in this patient population and the risks of participation were felt to be low. The approach to providing treatment to nonconsenting participants is in line with Intensive Care Unit clinical trials and the approach suggested in Right 7(4) of the Health and Disability Commission's Code of Rights ("Code of Health and Disability Services Consumers' Rights (2009): Right 7 (4) of the code ,").

While prospective consent is the preferred process in clinical trials, this is not possible when participants have impaired capacity to make decisions. ICU based trials commonly confront this issue but is also pertinent in this setting as all patients with AHRF will have a degree of cognitive impairment and marked confusion is common. While some participants will be semi-lucid and may appear to display features of having capacity to provide consent, we know that when they are in an acidotic state, their capacity is diminished and it would be unethical to seek their direct consent (Fields \& Calvert, 2015). There is a well-established relationship between the low-oxygen state found in acute exacerbations of COPD and cognitive impairment (Ambrosino et al, 2002; Dodd et al, 2010; Kirkil et al., 2007). Further evidence reiterates this link of global cerebral impairment in the outpatient setting (Grant et al, Heaton, 1982; Incalzi et al., 1993), comparing the deficit from COPD to that of chronic alcoholism (Fix et al., 1982).

The aim was to make every effort to discuss the study with participants as soon as possible when their clinical condition improved, normally within 12-24 hours if they improved, or if they remained acidotic and required NIV, their 
progress would be monitored and the study discussed with them later in the admission.

The study was prospectively registered with ANZCTR (Trial ID: ACTRN12617000301369) and approved by the HDEC of New Zealand (Ref: 16/NTA/227).

\subsubsection{Study procedures}

Figure 4-2 illustrates the order of study procedures and the role of various investigators. ABG measurements were recorded at baseline and after 60 90 min. VBG samples were taken immediately after the ABGs also, in order to have paired samples (see below). A pulse oximeter (Masimo Rad-8 Pulse Oximeter with Profox oximetry software) with data storage capabilities was kept in ED. Data was later downloaded to assess adherence to controlled oxygen titration between $\mathrm{S}_{p} \mathrm{O}_{2}$ of $88-92 \%$, as per the agreed protocol in Study Part 1.

For those potential participants who did not consent to follow-up, attempts were made to establish why they were not comfortable with further participation by simple discussion with each potential participant. Data was uploaded using REDCap - a secure, HIPAA/Part 11 (United States Health Insurance Portability and Accountability Act 1996) compliant web-based application designed to support data capture for research studies. All investigators had access to the REDCap portal and while the aim was to avoid paper copies and the need for later duplicated data entry, a paper worksheet was available in ED. 


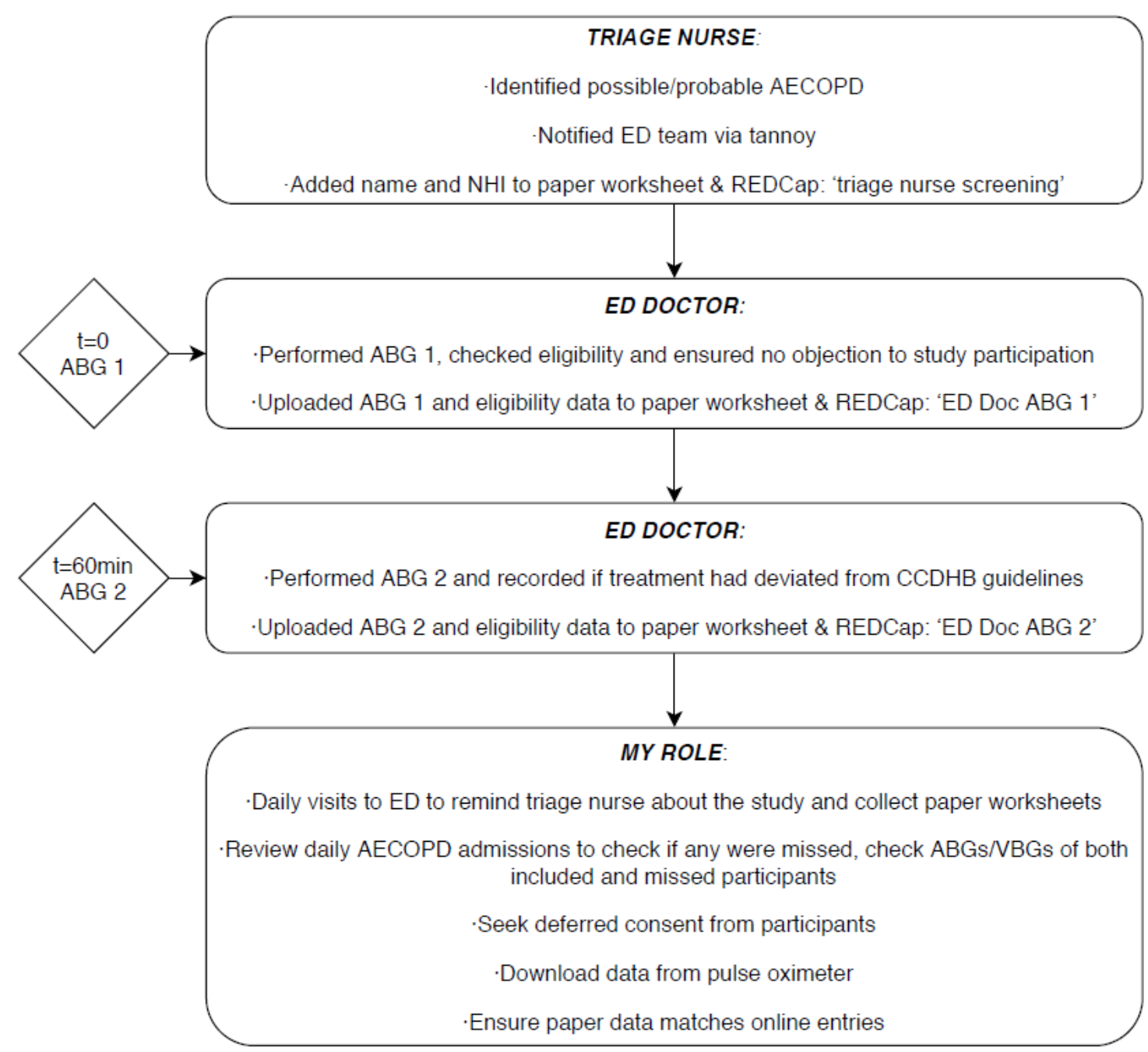

Figure 4-2 Participant flow through Study 4 and the input from study investigators. REDcap refers to the web-based database used.

\subsubsection{Serial Venous and arterial blood gas sampling}

As described above, ABGs and VBGs were taken the zero time-point and after 60-90 min. ABG sampling is the reference standard in AHRF to obtain $\mathrm{pH}, \mathrm{P}_{\mathrm{a}} \mathrm{CO}_{2}$ and $\mathrm{P}_{\mathrm{a}} \mathrm{O}_{2}$ to guide clinical management so was an important part of the usual care protocol (Davidson et al., 2016; Yang et al., 2017). However, Studies $1 \mathrm{a}$ and $1 \mathrm{~b}$ (see Appendix II) reviewed the side effects and risks of $A B G$ sampling and suggested transcutaneous monitoring may be an alternative in certain situations. Another surrogate method for ABG is with a VBG, where venous blood is sampled from a peripheral vein and many of the same blood gas parameters tested as an ABG. 
VBGs have become increasingly used to diagnose respiratory failure, especially in ED (Arnold et al., 2011; Rang et al, 2002). Often in ED an IV cannula is quickly placed to draw a venous sample for other blood tests, therefore no additional $A B G$ test is done, avoiding the potentially painful and higher risk $A B G$ procedure.

While it is accepted VBG will not accurately measure $\mathrm{PaO}_{2}$, the rationale for diagnosing respiratory failure from a VBG is that combined with pulse oximetry, which will measure $\mathrm{S}_{\mathrm{p}} \mathrm{O}_{2}$ to indicate the level of oxygenation, the VBG offers reasonable accuracy for $\mathrm{pH}$ and can be used to screen for elevated $\mathrm{PCO}_{2}$ (Kelly, 2010).

However, the evidence for reliability of VBG to diagnose respiratory failure is conflicting. Cohort studies report that venous $\mathrm{pH}$ has small difference and narrow limits of agreement with arterial $\mathrm{pH}$ and that a $\mathrm{PvCO}_{2}>45 \mathrm{mmHg}$ has high sensitivity to detect arterial hypercapnia (Lim \& Kelly, 2010; McCanny et al, 2012). Normocapnia on VBG ruled out hypercapnia on ABG so VBG could be used as an initial screening for hypercapnia and followed up with an ABG if required (Kelly et al, 2002; McCanny et al., 2012). However, although the overall mean difference in $\mathrm{PvCO}_{2}$ and $\mathrm{P}_{\mathrm{aCO}}$ was $4.1 \mathrm{mmHg}$ higher with $\mathrm{PvCO}_{2}$ in a meta-analyses of 18 studies, the limits of agreement were wide, from $-10.7 \mathrm{~mm} \mathrm{Hg}$ to $+2.4 \mathrm{~mm} \mathrm{Hg}$ meaning $\mathrm{PvCO}_{2}>55 \mathrm{mmHg}$ would be required to ensure arterial hypercapnia (Byrne et al., 2014). There was little difference between the $\mathrm{pH}$ obtained from the $\mathrm{VBG}$ and the $A B G$, with the arterial $\mathrm{pH}$ typically 0.03 higher than the venous $\mathrm{pH}(95 \% \mathrm{Cl} 0.029-0.038)$. It is reported that other factors such as haemodynamic status and peripheral sampling site may influence VBG reliability (Byrne et al., 2014).

Evidence for the agreement of serial $A B G$ and VBG sampling was lacking but if support for VBG accuracy was demonstrated, it may reduce the need for $A B G$ s in the proposed RCT. Given both an $A B G$ and VBG were taken at the zero time-point and a further $A B G$ at 60-90 min, a VBG sample at 60-90 min was the only extra test to investigate agreement between serial $A B G$ s and VBGs. The serial ABG/VBG analysis was not a specified outcome measure. 


\subsubsection{Outcome measures}

\subsubsection{Primary outcome}

The proportion of patients with treatment failure after 60 to 90 minutes as defined for the proposed RCT [related to FS1].

\subsubsection{Secondary outcomes}

1. Proportion of participants in whom care deviated from the agreed protocol [related to FS2]

2. Proportion of patients admitted with AECOPD who meet the inclusion criteria for the proposed RCT [related to FS3]

3. SD of the Hydrogen ion concentrations in the $A B G$ s of eligible participants [related to FS4]

4. Proportion of participants in whom deferred consent could be obtained [related to FS5]

5. Recruitment rate per month [related to FS6]

\subsubsection{Sample size estimation}

The proposed sample size of 40 for the feasibility study had $80 \%$ power, alpha 0.05 , to rule out a baseline treatment failure rate of $<60 \%$ assuming this was $80 \%$ [related to FS1]. The sample size would also give reasonable precision for estimates of the SD of the Hydrogen ion concentration from ABGs [related to FS4].

\subsubsection{Statistical analysis plan}

The statistical analysis plan with a sample size of 40 was for estimates of proportions by small sample technics e.g. Clopper-Pearson confidence intervals. The SD for the Hydrogen ion concentration would have a confidence interval estimated using the Chi-square distribution.

However, given the small sample size recruited (see Results below), the statistical analysis plan changed. Data from only 3 participants was available for the primary outcome variable and some secondary outcomes which was analysed by simple proportions. 
For the blood gas data collected, summaries are by simple data descriptions for continuous variables and tabulations and cross-tabulations for categorical variables.

Bland-Altman limits of agreements plots are shown for the comparison of arterial and venous $\mathrm{CO}_{2}$ and $\mathrm{pH}$ with the difference arterial minus venous. Paired t-tests estimate the mean bias. McNemar's test and an appropriate method for comparison of paired proportions are used to compare the proportion of patients with a $\mathrm{pH}$ less than 7.35 and for $\mathrm{pH}$ in the range 7.25 to 7.34 inclusive. SAS version 9.4 was used

\subsection{Results}

\subsubsection{Study Part 1}

The agreed usual care protocol presented above in Figure 4-1 constitutes the successful result for Study Part 1.

\subsubsection{Study Part 2}

The feasibility study was originally intended to be undertaken at three Emergency Departments of Wellington, Hamilton and Christchurch hospitals respectively, to achieve the required sample size of 40 . An HRC Programme grant application was submitted to facilitate funding for this multi-centre design. Recruitment in Wellington was started prior to the HRC Programme grant decision but unfortunately the application was declined. Insufficient funding therefore prevented additional recruitment at Christchurch and Waikato Hospitals. At Wellington Hospital, the original sample size of 40 was reduced to a more realistic 15, which if achieved, may have given sufficient preliminary data to bolster chances for a successful re-application for HRC funding at the next round.

Study enrolment was between March 2017 and July 2017. Participant flow through the study is shown in Figure 4-3. During this period, 120 patients presented to ED and had a primary diagnosis of AECOPD and $78(65 \%)$ were flagged by a triage nurse as potentially eligible for the trial. AECOPD admissions were monitored by me every week day, and 42 were noted to be missed and not flagged. Of those 78 who were flagged, 61 patients got picked 
up by an ED doctor to check for eligibility and all 61 received an initial VBG and 56 an $A B G$, with the difference of 5 reflecting failed $A B G$ attempts.

Three of the 56 patients had an ABG in the $\mathrm{pH}$ range for study eligibility. All 3 were initially included in the study, and received a second paired $A B G$ and VBG after 60-90 minutes and were monitored by the study pulse oximeter during the usual care period. Of those 3 patients, deferred consent was obtained from only 2 participants and 1 of those received the full 60 -minute optimisation period; the other one received NIV after 25 minutes, therefore not completing the agreed, usual care protocol.

The feasibility aims were achieved after 3.5 months so the study was concluded. Primarily, the low recruitment rate (FS6) was the limiting step to allow a sufficient sample size for the remaining feasibility study aims but it was felt unethical to continue the study given the low recruitment rate.

\subsubsection{Primary outcome}

This answered the FS1 aim. One (50\%) participant had treatment failure, receiving NIV after 25 minutes of optimisation.

\subsubsection{Secondary outcomes}

\subsection{PROPORTION OF PARTICIPANTS IN WHOM CARE DEVIATED FROM THE AGREED PROTOCOL}

This answered the FS2 aim. Care deviated from the agreed usual care protocol in 1 (50\%) participant whom received NIV after 25 minutes of optimisation instead of the full 60 minutes. Adherence to the usual care protocol (50\%) is therefore less than the anticipated $90 \%$.

\subsection{PROPORTION OF PARTICIPANTS IN WHO DEFERRED CONSENT COULD BE OBTAINED}

This answered the FS3 aim. Deferred consent was obtained in 2 (66.7\%) of the 3 otherwise eligible participants. The third participant was later found to have baseline cognitive impairment, therefore deferred consent was deemed inappropriate and data was discarded. 


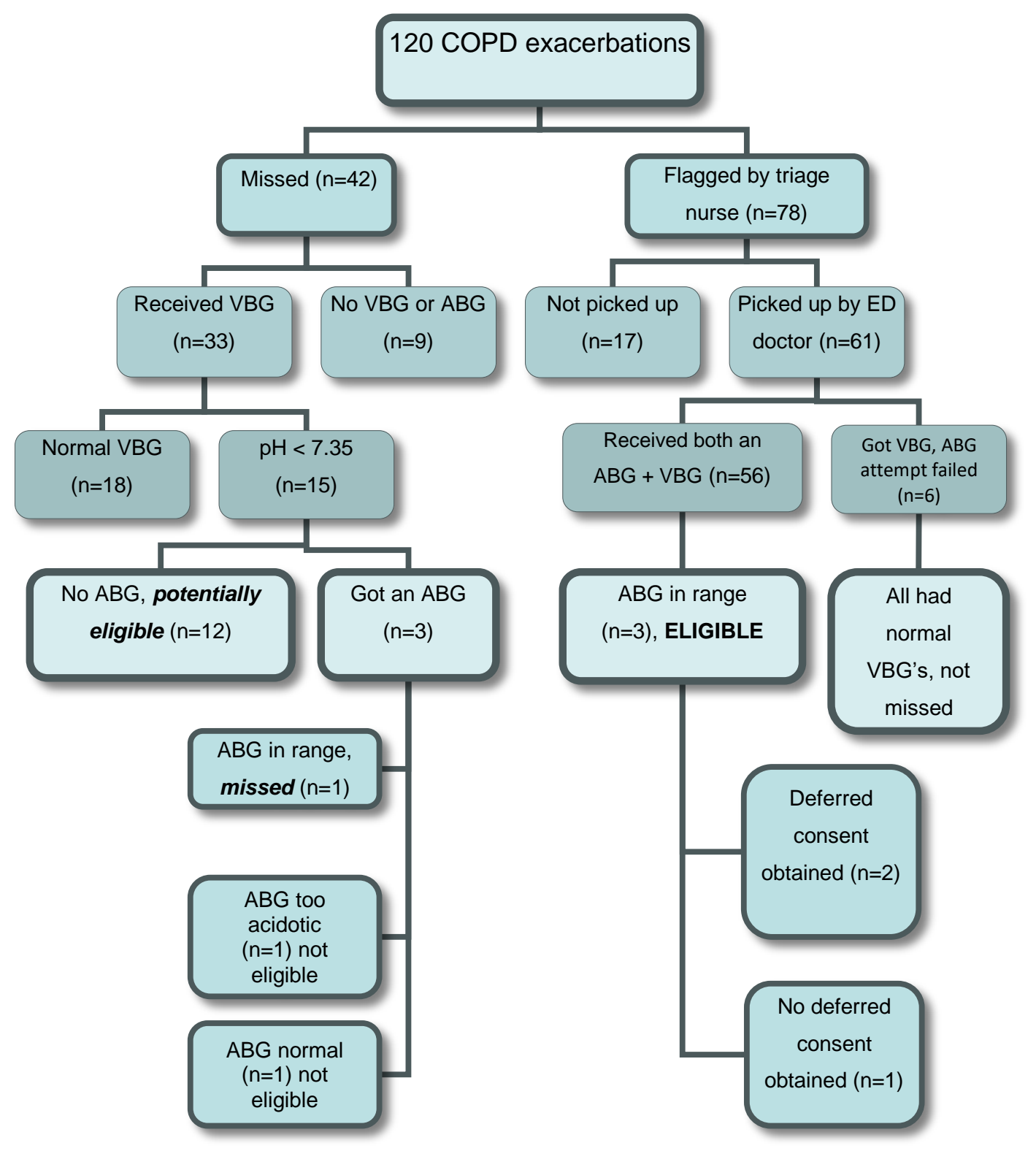

Figure 4-3 Flow of participants through Study 4.

ABG: Arterial blood gas, VBG: Venous blood gas, ED: Emergency department 


\subsection{PROPORTION OF PATIENTS ADMITTED WITH AECOPD WHO MET THE INCLUSION CRITERIA FOR THE PROPOSED RCT}

This answered the FS4 aim. Three participants meeting the $\mathrm{pH}$ criteria in 3.5 months equates to a recruitment rate of less than 1 per month which falls well below recruitment rates required to achieve a large sample size. While 3 patients were flagged and met eligibility, it was also possible to calculate if any of the 42 missed cases may have been eligible to give an estimate overall of the proportion of the 120 patients who were eligible. One of the missed cases had an $A B G$ within range and was clearly missed so would have been included. There were 12 missed cases with no ABG but an acidotic VBG and thus were potentially eligible, pending a method to reasonably correlate venous and arterial $\mathrm{pH}$. Contingency tables were used to compare proportions of different ranges of venous $\mathrm{pH}$ against arterial $\mathrm{pH} 7.25$ to 7.34. A venous $\mathrm{pH}$ range of 7.21 to 7.31 had the lowest false positive rate for the arterial $\mathrm{pH}$ range 7.25 to 7.34 (Table 4-1). Applying this venous $\mathrm{pH}$ range to the 12 missed patients' VBGs equated to 4 having a (derived) arterial $\mathrm{pH}$ in range for eligibility. This method of changing the eligible venous $\mathrm{pH}$ range to 7.21 to 7.31 dismisses the upper, borderline acidotic VBGs but in most of these cases, the paired $A B G \mathrm{pH}$ was normal.

Table 4-1 Arterial pH agreement for proportion of patients with venous pH between 7.21 and 7.31.

\begin{tabular}{llll}
\hline & \multicolumn{2}{l}{ Venous pH 7.21 to 7.31 } & Total \\
\cline { 2 - 4 } & Yes & No & \\
\hline $\begin{array}{llll}\text { Arterial } \\
\text { to 7.34 }\end{array}$ & & \\
\hline Yes & 3.25 (True positive) & 1 (False negative) & $4(7.1)$ \\
No & 2 (False positive) & 50 (True negative) & $52(92.9)$ \\
Total N/56 (\%) & $5(8.9)$ & $51(91.1)$ & 56 \\
\hline
\end{tabular}

Overall, 8 (6.67\%) of the 120 AECOPD patients had a mild respiratory acidosis in $\mathrm{pH}$ range 7.25 to 7.34 and met, or were likely to have met, eligibility for the proposed RCT. This is slightly lower than the anticipated $10 \%$ based on previous audits. Also, six patients were too acidotic and were not eligible, meaning a total of 14 (11.67\%) of 120 AECOPD patients had AHRF which is also lower than the rate of $20 \%$ reported earlier (A C Davidson et al., 2016). 


\subsection{ESTIMATION OF THE SD OF THE HYDROGEN ION CONCENTRATION BY ABG MEASUREMENT.}

This attempted to answer the FS5 aim. However, there was insufficient data to make a meaningful conclusion about the hydrogen ion concentration.

\subsection{RECRUITMENT RATE PER MONTH}

This answered the FS6 aim. With only 1 centre being used, three participants meeting the $\mathrm{pH}$ criteria in 3.5 months equates to a recruitment rate of less than 1 per month which falls well below recruitment rates required to achieve a large sample size.

\subsection{LIMITS OF AGREEMENT FOR SERIAL PAIRED ABG AND VBG.}

Only 3 participants had serial ABGs and VBGs which was not useful for conclusions to be made. Instead, a post-hoc analysis of the limits of agreement for the initial 56 paired $A B G$ and VBG samples was done.

Arterial $\mathrm{CO}_{2}$ was less than Venous $\mathrm{CO}_{2}$ by a mean (SD) of -7.9 (6.4) $\mathrm{mmHg}$ $(95 \% \mathrm{Cl}-9.6$ to $6.2 \mathrm{mmHg}), \mathrm{P}<0.001$ (Table $4-2)$. The limits of agreement around this bias were $\pm 12.8 \mathrm{mmHg}$ (Figure 4-4).

Arterial $\mathrm{pH}$ was greater than venous $\mathrm{pH}$ by +0.04 units $(95 \% \mathrm{Cl} 0.032$ to $0.049), P=0.039$. The limits of agreement around this bias were \pm 0.06 units (Figure 4-5).

Table 4-2 Data description of ABG and VBG values and their relationship to each other

\begin{tabular}{lllll}
\hline $\begin{array}{l}\text { Blood gas } \\
\text { parameter }\end{array}$ & Variable & Mean (SD) & Median (IQR) & $\begin{array}{l}\text { Minimum to } \\
\text { maximum }\end{array}$ \\
\hline $\mathrm{CO}_{2}(\mathrm{mmHg})$ & Arterial & $44.8(13.2)$ & $40.7(37.4$ to 47$)$ & 27.4 to 97.4 \\
& Venous & $52.7(13.5)$ & $50.1(43.8$ to 55.9$)$ & 33.2 to 108 \\
& Arterial minus & $-7.9(6.4)$ & $-7.9(-12.3$ to -3.4$)$ & -21 to 9.2 \\
& venous & & & \\
\hline $\mathrm{pH}$ (units) & Arterial & $7.41(0.07)$ & $7.42(7.39$ to 7.44$)$ & 7.14 to 7.52 \\
& Venous & $7.37(0.07)$ & $7.38(7.34$ to 7.41$)$ & 7.13 to 7.48 \\
& Arterial minus & $0.04(0.03)$ & $0.04(0.02$ to 0.06$)$ & -0.04 to 0.15 \\
& venous & & & \\
\hline
\end{tabular}




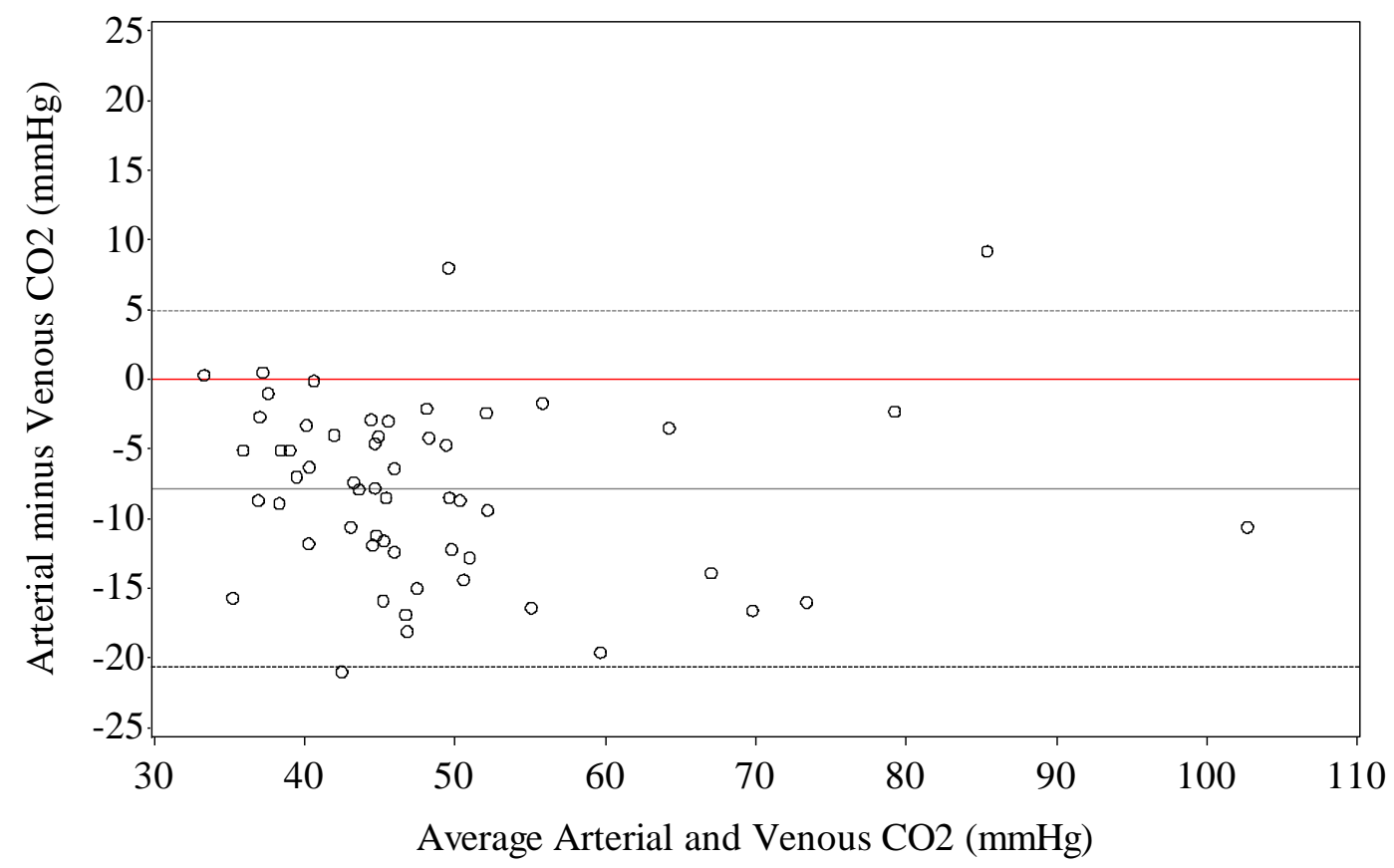

Figure 4-4 Bland-Altman plot of limits of agreement for arterial and venous $\mathrm{PCO}_{2}$.

For both Fig 5-4 and Fig 5-5: The red line represents the zero difference reference; the solid black line the bias; the dotted black lines the bias plus or minus two standard deviations (the limits of agreement with respect to the bias).

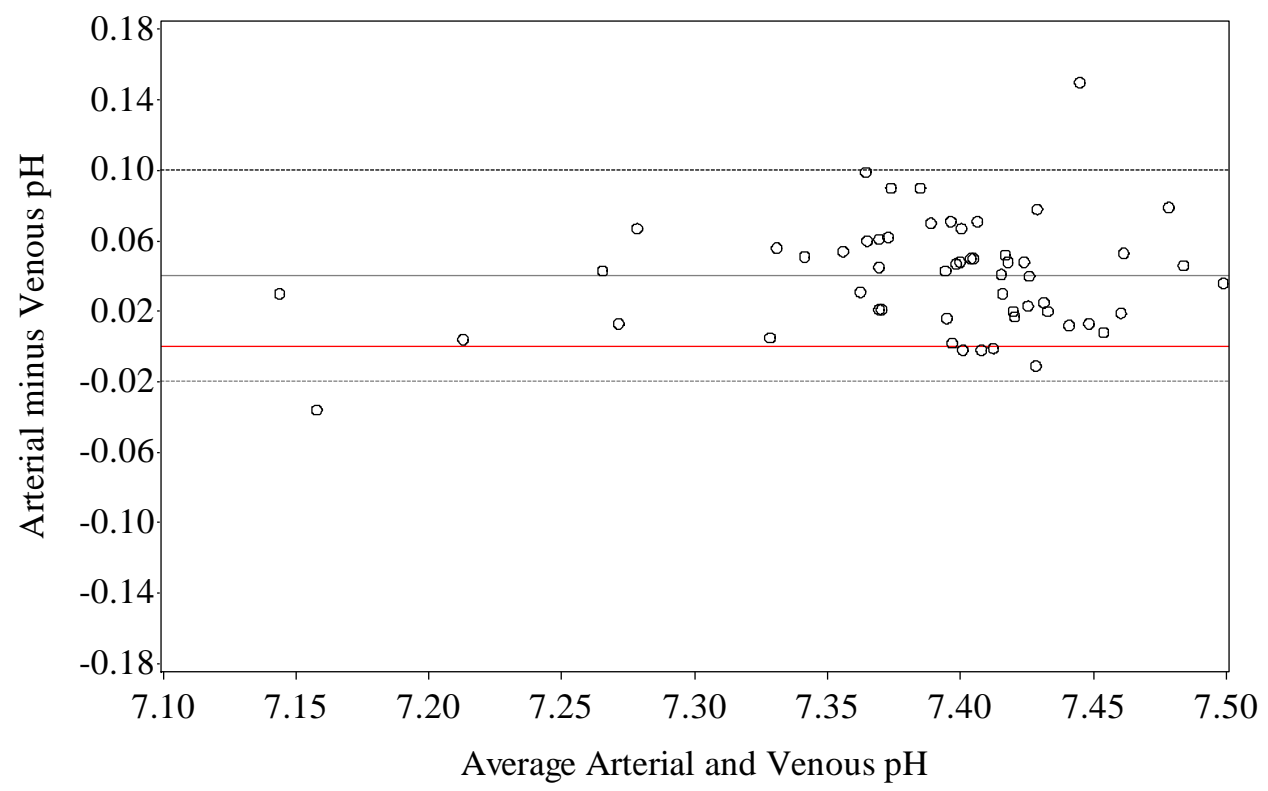

Figure 4-5 Bland-Altman plot of limits of agreement for arterial and venous $\mathrm{pH}$. 
Venous $\mathrm{pH}$ had a significantly greater proportion of patients with a $\mathrm{pH}<7.35$ : $32.1 \%$ compared to $12.5 \%$ (Table $4-3$ ) - a difference of $19.6 \%$ (95\% Cl 9.2 to $30.1 \%), P=0.001$.

There was no strong evidence of a difference in the proportions with $\mathrm{pH}$ in the band between 7.25 to 7.34 , with venous $\mathrm{pH}$ having $17.9 \%$ compared to arterial $\mathrm{pH}$ of $7.1 \%$ respectively (Table $4-4)$ - a difference of $10.7 \%(95 \% \mathrm{Cl} 10$ to $21.4 \%), P=0.11$. For both these comparisons of $\mathrm{pH}$, the confidence intervals were wide.

Table 4-3 Agreement between venous and arterial for the proportion of patients with $\mathrm{pH}<7.35$.

\begin{tabular}{llll}
\hline & \multicolumn{2}{l}{ Venous pH less than 7.35 } & Total \\
\hline & Yes & No & N/56 (\%) \\
\hline $\begin{array}{l}\text { Arterial pH } \\
\text { than 7.35 }\end{array}$ & less & & \\
Yes & 7 & 0 & $7(12.5)$ \\
No & 11 & 38 & $49(87.5)$ \\
\hline Total N/56 (\%) & $18(32.1)$ & $38(67.9)$ & $56(100)$ \\
\hline
\end{tabular}

Table 4-4 Agreement between venous and arterial for proportions of patients with $7.25 \leq \mathrm{pH}<7.35$.

\begin{tabular}{llll}
\hline & \multicolumn{2}{l}{ Venous 7.25 $\leq \mathrm{pH}<7.35$} & Total \\
\hline & Yes & No & $\mathrm{N} / 56(\%)$ \\
\hline $\begin{array}{l}\text { Arterial } 7.25 \leq \mathrm{pH} \\
<7.35\end{array}$ & & & \\
Yes & 2 & 2 & $4(7.1)$ \\
No & 8 & 44 & $52(92.9)$ \\
\hline Total N/56 (\%) & $10(17.9)$ & $46(82.1)$ & $56(100)$ \\
\hline
\end{tabular}

\subsection{Discussion}

The aim of this feasibility study was to collect essential data to facilitate the design of a proposed large-scale multi-centre RCT comparing usual care plus NHF, against usual care plus standard oxygen therapy. The results were that it is not feasible to conduct the proposed RCT using aspects of the design from this feasibility study.

The value of conducting a robust feasibility study before embarking on a large RCT is highlighted in this case. The study was designed to address 6 specific feasibility study aims. Recruiting at all 3 original centres was essential to 
ensure an adequate recruitment rate (as per FS3 and FS6) to achieve the required sample size to address the other feasibility study aims. The number recruited at a single-centre may have limited conclusions to be drawn about these other feasibility study aims, with only 2 participants fully enrolled. However, this is the nature of a feasibility study and indeed, similar results may have eventuated if the full 40-patient sample size was recruited. The incidence of both AECOPD and AHRF were lower in this feasibility study compared to international data (Plant et al., 2000; Price et al., 2006). Applying the low recruitment rate to the proposed RCT would mandate a multi-centre design, and over an extended period of time to achieve a sufficient sample size.

The feasibility study design tested many key logistical issues and much can be learnt from it. The aim was for the study protocol to be built into a standard ED pathway and be implemented predominantly by ED staff, as patients with AHRF present in an emergency with high-acuity and at any time of the day or night. However, one-third of all AECOPD presentations were not flagged by the triage nurse and missed completely. Twenty-two percent were flagged by the triage nurse but missed by ED doctors to assess for eligibility, meaning there were two critical steps where recruitment was lost. While this drop-off was less than what was hoped for and unable to be fully explained, it highlighted a critical feasibility issue.

It was not possible for me to be physically present in ED around the clock to improve recruitment. It was imperative all ED staff were aware of the study, accepting of the protocol and agreeable to its implementation. While the usual care protocol was agreed upon at a Head of Department-level, ED staff work various shifts with multiple different combinations of staff members and may have contributed to the missed recruitment. Measures taken to optimise study uptake were: 1) an ED Senior Medical Officer and Registrar were the coordinators on the ED floor to answer any queries and liaise with me, 2) An initial presentation of the study at an ED staff meeting was followed up with week-day visits to ED to check-in with the triage nurses and answer any questions.

Many of the missed patients presented at night and/or outside of usual daytime work hours. The solution would be to employ full-time research staff to roster the study 24 hours/day and 7 days/week. They could identify and 
enrol the patients then assist in the protocol procedures, such as $A B G$ sampling. Adequate funding would be required to make this possible.

A similar trial design was used in an RCT comparing NHF against standard oxygen therapy in ED for acute hypoxaemic respiratory failure patients and found no difference in the requirement for NIV or IMV (Jones et al., 2016). The researchers shared similar difficulties enforcing a study protocol across all treating clinicians in ED. The study was stopped with 322 patients recruited over nearly 2 years, rather than the 390 patients per RCT arm (780 total) required to detect their primary aim of a $33 \%$ reduction in NIV requirement. 27 patients were placed on NIV immediately on arrival rather than being recruited into the study and they found a lower NIV escalation rate than their own pilot study where NIV was used at "clinician-discretion". Conducting robust RCTs in the emergency setting faces many logistical difficulties.

A key aspect to the usual care protocol was the use of $A B G$ sampling as it would be difficult to justify a study on respiratory failure without using ABGs, given the international recommendations for their use. Overall, $A B G$ sampling was well adhered to and was not a limiting step in recruitment, with $A B G$ attempts made on all patients assessed by ED doctors. Whether aversion to potential $A B G$ sampling prevented uptake of the study by other ED doctors is unknown.

Data capture on REDCap was trialled to investigate feasibility, as it would significantly improve efficiency of data capture in the proposed RCT. Uptake of data capture in REDCap by ED staff was sub-optimal and their feedback was it was too time-consuming in such a busy, time-limited environment. Again, whether a reluctance to complete data entry in REDCap or the worksheets reduced recruitment is unknown.

The close agreement between VBG and $A B G$ pH values but higher $\mathrm{PvCO}_{2}$ than $\mathrm{PaCO}_{2}$ values found in this study were consistent with other publications (Byrne et al., 2014; Kelly, 2010; Kelly et al., 2002; McCanny et al., 2012).

Further trials with different designs to this feasibility study are required to investigate if $\mathrm{NHF}$ can reduce treatment failure during the initial 1 hour of medical optimisation in AHRF. To my knowledge, no further studies have been published answering this question. A modified trial design incorporating the suggestions above may improve feasibility and provide the answer. 


\subsection{Conclusion}

The proposed RCT comparing usual care plus NHF, with usual care plus standard oxygen therapy is not feasible based on the design and results of this feasibility study. VBGs may be reasonable used instead of ABGs for $\mathrm{pH}$ but $\mathrm{P}_{\mathrm{v}} \mathrm{CO}_{2}$ is less accurate than $\mathrm{PaCO}_{2}$. 


\section{Study 5 - Nasal High Flow therapy compared with Non-invasive ventilation in COPD patients with chronic respiratory failure: a randomised controlled cross-over trial}

\subsection{Rationale}

Study 2 demonstrated NHF reducing $\mathrm{P}_{\mathrm{t}} \mathrm{CO}_{2}$ and respiratory rate in a flowdependent manner and was well tolerated, a critical result to demonstrate NHF efficacy in stable COPD. However, most participants were not hypercapnic. A sensitivity analysis showed no difference in $\mathrm{P}_{\mathrm{t}} \mathrm{CO}_{2}$ reduction whether hypercapnic or eucapnic, likely due to the small sample size. The effect of NHF in hypercapnic respiratory failure remained unknown but is the setting for which NHF may offer an alternative to NIV, the current standard of care in AHRF (Davidson et al., 2016; Rochwerg et al., 2017; Yang et al., 2017). A direct comparison between NHF and NIV was required. There remained insufficient evidence of NHF efficacy for this comparison to be safely investigated in the setting of AHRF so a trial in the stable setting was most appropriate, before contemplating a trial in the acute setting.

Around $20-25 \%$ of people admitted to hospital with AECOPD either present with or develop AHRF during the admission, necessitating NIV (Davidson et al., 2016; Plant et al., 2000; Roberts et al., 2011). Section 1.3.3 (NIV in HRF) reviewed the benefits of NIV, including a reduction in the need for subsequent endotracheal intubation, hospital length of stay, and mortality in this setting (Osadnik et al., 2017; Plant et al., 2000).

However, even with NIV treatment for AHRF, in-hospital mortality remains between 14 and 25\%. (Alleway et al., 2017; Cabrini et al., 2016; Roberts et al., 2011) Correct administration of NIV requires staff training, close patient monitoring, and regular $A B G$ testing to monitor progress and ensure appropriate adjustments to NIV device settings.(Alleway et al., 2017; Davidson et al., 2016; Rochwerg et al., 2017) In addition, NIV may be difficult to tolerate in some patients with reported rates of intolerance around $20 \%$ (Benhamou et al., 1992; Carlucci et al., 2001). Intolerance of the mask interface significantly 
increases the odds of NIV failure (Carlucci et al., 2001) and different interfaces may allow longer duration of therapy and better tolerance (Rochwerg et al., 2017).

NHF may meet the need for a treatment that is both well-tolerated and easy to administer, while reducing $\mathrm{PCO}_{2}$ sufficiently, therefore either reducing the need for NIV and/or providing another treatment option for those patients who cannot tolerate NIV.

Study 2 demonstrated that NHF is well-tolerated, reduces $\mathrm{PtCO}_{2}$, and respiratory rate and is supported by trials in patients with AECOPD requiring supplemental oxygen (Fraser et al., 2016; Pilcher et al., 2017), and in people with stable COPD (Bräunlich et al., 2013; Bräunlich \& Köhler, 2016). However, there is limited data directly comparing NHF with NIV in people with COPD.

\subsection{Aims and hypotheses}

The aim of the study was to directly compare NHF therapy to NIV in people with stable COPD and chronic hypercapnic respiratory failure. While the proposed clinical application of NHF is in the acute setting, it was first necessary to investigate NHF efficacy in the safer, stable outpatient setting. The main aim was to compare the effect of NHF therapy and NIV on $\mathrm{PaCO}_{2}$, as measured by $\mathrm{P}_{\mathrm{t}} \mathrm{CO}_{2}$.

The hypotheses were that NHF therapy in people with stable hypercapnic COPD would cause a similar change in $\mathrm{P}_{\mathrm{t}} \mathrm{CO}_{2}, \mathrm{~S}_{\mathrm{t}} \mathrm{O} 2$, and respiratory rate to NIV, but with improved tolerability.

\subsection{Methods}

\subsubsection{Trial design}

Single centre, single blind, randomised active-controlled two-way crossover trial.

\subsubsection{Eligibility criteria}

\subsubsection{Inclusion criteria}

1. A doctor's diagnosis of COPD

2. $\mathrm{P}_{\mathrm{cap}} \mathrm{CO}_{2}>45 \mathrm{mmHg}$ on capillary blood gas, at point of randomisation

3. Age $\geq 40$ years 


\subsubsection{Exclusion criteria}

1. Smoking pack year history $<10$ years

2. $\mathrm{FEV}_{1} / \mathrm{FVC} \geq 70 \%$

3. Body mass index $>35$

4. Hypercapnia believed to be primarily due to Obesity Hypoventilation syndrome and/or Obstructive Sleep Apnoea

5. Any condition which makes NIV contra-indicated as per BTS/ICS guidelines.(Davidson et al., 2016)

6. Requiring $\geq 4 \mathrm{~L} / \mathrm{min}$ through standard nasal cannulae during the washin period to maintain oxygen saturations of $88-92 \%$.

7. COPD not deemed to be 'stable':

a. Current exacerbation requiring acute treatment with a short course of antibiotics/oral steroids within the last 2 weeks.

b. Hospital admission for an acute exacerbation of COPD in the last 6 weeks.

8. Nasal conditions such as deviated septum, chronic rhinitis, current cold/flu which, in the evaluation by the investigator, could impair nasal breathing.

9. Any other condition, which at the investigator's discretion, was believed may present a safety risk or impact the feasibility of the study or study results.

\subsubsection{Interventions}

Participants received both NHF and NIV for 60 min, each while seated. All participants received both interventions with the order of administration randomised i.e. an AB/BA cross-over design (Figure 5-1). NHF therapy was delivered at a flow rate of $45 \mathrm{~L} / \mathrm{min}$ and a temperature of $37^{\circ} \mathrm{C}$ with additional oxygen as required, using the AIRVO 2 device (PT101AZ, FPH). NIV was delivered at an IPAP of $15 \mathrm{~cm} \mathrm{H}_{2} \mathrm{O}$ and EPAP of $4 \mathrm{~cm} \mathrm{H} \mathrm{H}_{2} \mathrm{O}$, with additional oxygen as required, using the Philips Respironics BiPAP AVAPS-ST 60 Series (Philips Respironics).

If participants were unable to tolerate NIV at $15 / 4 \mathrm{~cm} \mathrm{H} \mathrm{H}_{2} \mathrm{O}$, the IPAP was reduced to $10 \mathrm{~cm} \mathrm{H}_{2} \mathrm{O}$ and up-titrated slowly over the following $10 \mathrm{~min}$ to 15 $\mathrm{cm} \mathrm{H}_{2} \mathrm{O}$. If the subject still could not tolerate NIV at $15 \mathrm{~cm} \mathrm{H}_{2} \mathrm{O}$ then the 
pressure settings were adjusted for optimal participant comfort for the remainder of the intervention.

Each of the two interventions was followed by a washout period of at least 15 min, during which participants breathed room air plus supplementary oxygen as required. The washout period allowed the $\mathrm{P}_{\mathrm{t}} \mathrm{CO}_{2}$ to return to within $2 \mathrm{mmHg}$ of the baseline measurement for the particular intervention. 15 min has been shown to be an effective period of time to return the $\mathrm{P}_{\mathrm{t}} \mathrm{CO}_{2}$ to near baseline (Edwards et al., 2012; Rudolf et al, 1979).

Where required, the wash-out was extended for a further 15 minutes until this criterion was met. During the wash-out titrated oxygen was again provided via nasal cannulae, initially set to the flow rate used at the end of the wash-in period, and titrated to maintain oxygen saturations between $88 \%$ and $92 \%$.

If participants had resting oxygen saturations of less than $88 \%$, supplemental oxygen was provided via nasal cannulae at up to four litres per minute and was titrated to maintain oxygen saturations between $88 \%$ and $92 \%$. Participants requiring oxygen at the start of the intervention continued to receive titrated supplemental oxygen via the NHF and NIV devices if required.

$\mathrm{P}_{\mathrm{t}} \mathrm{CO}_{2}$, oxygen saturation, heart rate, and respiratory rate were manually recorded at the start of each intervention and then every 5 min until the end of each washout period.

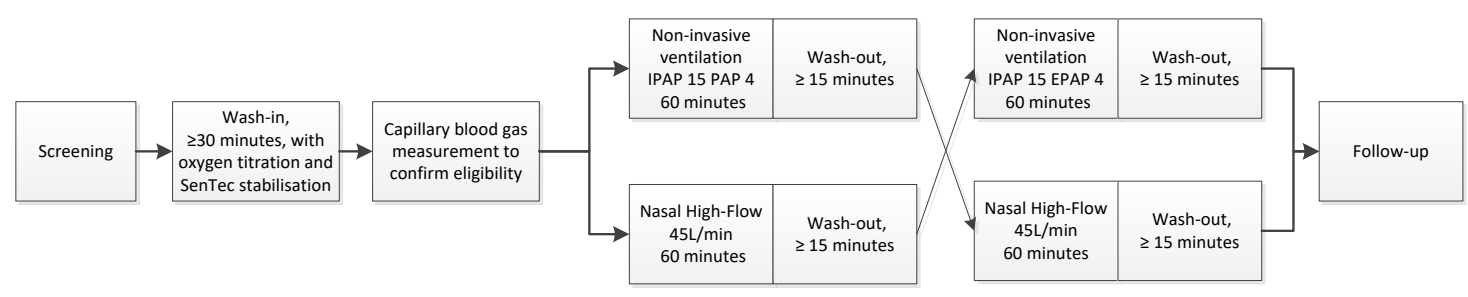

Figure 5-1 Summary of Study 5 flow, demonstrating the cross-over design.

\subsubsection{Recruitment and Consent}

Participants were recruited from CCDHB, Hutt Valley District Health Board (HVDHB), MRINZ and GP clinics. Clinic and ward lists and participant databases were screened to identify suitable participants. This involved 
accessing the previous 12 months' AECOPD admissions at Wellington and Hutt Valley Hospitals, collated by the respective hospital data "Decision Support" units. Patient arterial or venous blood gas results and admissions were reviewed to assess the likelihood of having chronic hypercapnic respiratory failure, therefore potentially eligible for the study. Approximately 800 blood gas results and clinical notes were reviewed.

Potential participants identified were sent an introductory letter, from their treating clinician, inviting them to contact me if they would like to participate. I would then contact them and send a participant information sheet (PIS). This method of recruitment was approved during the ethics application process and was to ensure there was a clear boundary between clinician access to patient data and researcher access, an important privacy protection issue. However, the method was very inefficient and there was never certainty potential participants ever received introductory letters or were fully made aware of the study.

Patients admitted with AECOPD were met on the ward to discuss the study and dispense a PIS. Potential participants identified from MRINZ databases were contacted directly by phone, because they had already consented to allow access to their data.

The study was prospectively registered with the ANZCTR (Trial ID: ACTRN12616001701415) and approved by the HDEC of New Zealand (Ref: 16/NTB/207). Full written informed consent was completed before any study specific procedures were begun.

\subsubsection{Study procedures}

Participants attended a single study visit at the MRINZ Respiratory Physiology Laboratory at Wellington Regional Hospital. After demographic and past medical history data were collected, height and weight were recorded and spirometry performed in accordance with ATS/ERS criteria,(Miller et al., 2005) using a Jaeger Masterscreen body volume constant plethysmography unit with pneumotachograph and diffusion unit (Erich-Jaegar, Wurzburg). 


\subsubsection{Wash-in period}

Measurements of $\mathrm{P}_{\mathrm{tCO}}$, transcutaneous oxygen saturation, and heart rate were made using the SenTec ${ }^{\mathrm{TM}}$ transcutaneous monitor (SenTec digital monitor with V-Sign Sensor VS-A/P/N) (see Appendix II). Once attached, there was a $30 \mathrm{~min}$ wash-in period to stabilise the SenTec and ensure participant oxygenation. If participants had resting oxygen saturations of less than $88 \%$, supplemental oxygen was provided via nasal cannulae at up to $4 \mathrm{~L} / \mathrm{min}$. Supplemental oxygen was titrated to maintain oxygen saturations between $88 \%$ and $92 \%$. Participants requiring oxygen at the start of the intervention continued to receive titrated supplemental oxygen via the NHF and NIV interventions if required.

\subsubsection{Capillary blood gas sampling}

At the conclusion of the wash-in period a capillary blood gas sample was taken from the finger-tip to measure $\mathrm{P}_{\text {cap }} \mathrm{CO}_{2}$, which was used to confirm hypercapnia, hence eligibility. $\mathrm{P}_{\text {cap }} \mathrm{CO}_{2}$ was used to confirm eligibility for this study rather than $\mathrm{P}_{\mathrm{t}} \mathrm{CO}_{2}$ because Study 1a demonstrated $\mathrm{P}_{\mathrm{t}} \mathrm{CO}_{2}$ had an unacceptably wide limit of agreement with $\mathrm{PaCO}_{2}$ for a single, absolute value and the accuracy of $\mathrm{P}_{\mathrm{cap}} \mathrm{CO}_{2}$ as a surrogate for $\mathrm{P}_{a} \mathrm{CO}_{2}$ is described in Study 1b (Appendix II). CBG sampling is a less painful alternative to $A B G$ sampling with fewer risks.

CBGs were taken using lancets and capillary tubes. I carried out the analyses in the Wellington ICU (Radiometer ABL800 FLEX) in order to get immediate results to confirm eligibility.

Continuous $\mathrm{P}_{\mathrm{t}} \mathrm{CO}_{2}$ measurement using the SenTec monitor was then used during the interventions to avoid multiple CBG sampling. Study 1b (Appendix II) demonstrated that $\mathrm{P}_{\mathrm{t}} \mathrm{CO}_{2}$ accurately reflects change in $\mathrm{P}_{\mathrm{cap}} \mathrm{CO}_{2}$ over time, mean bias $-0.03 \mathrm{mmHg}$ with limits of agreement of -0.44 to 0.38 and was appropriate for this trial.

\subsubsection{Participant questionnaires}

\subsection{BORG DYSPNOEA SCORES}

Borg dyspnoea scores were completed immediately prior to and immediately after each intervention (Borg, 1982). Participants scored their level of 
perceived breathlessness from 0 (no breathlessness) to 10 (maximal breathlessness).

\subsection{TOLERABILITY QUESTIONNAIRES}

Participant tolerability questionnaires (as used in Study 2) were administered during the wash-out periods after each intervention. Participants rated the ease of application, level of comfort, weight of the nasal/face mask interface, noisiness, amount of moisture in the nasal passages, and likelihood of reusing the system on a continuous scale from most positive (0) to least positive (100) in accordance with the appendix VI (Section 9.7.2).

\subsubsection{Randomisation and Blinding}

Randomisation was administered by pre-sealed opaque envelopes which were opened at the point of randomisation by me (un-blinded investigator). The schedule was computer-generated prior to the start of the study by the study statistician, who was not involved in study recruitment, procedures, or data collection. My role as the un-blinded investigator included manually counting the respiratory rate and controlling the settings on the NHF and NIV devices. Due to the nature of the intervention, participants and the un-blinded investigator could not be masked to treatment allocation. A blinded investigator recorded $\mathrm{P}_{\mathrm{t}} \mathrm{CO}_{2}$, heart rate, and oxygen saturation from the SenTec display while seated behind a screen and wearing ear plugs, so that they could neither see nor hear the participant or equipment.

\subsubsection{Outcome measures}

1. The primary outcome was $\mathrm{P}_{\mathrm{t}} \mathrm{CO}_{2}$ at 60 min, adjusted for baseline. *

2. Secondary outcomes was:

a. $\mathrm{PtCO}_{2}$ at 5 min intervals during the intervention, adjusted for baseline*.

b. The proportion of participants that have a decrease in $\mathrm{P}_{\mathrm{t}} \mathrm{CO}_{2}$ by $\geq 4 \mathrm{mmHg}$ and $\geq 8 \mathrm{mmHg}$ compared to baseline.

c. Other physical measurements:

i. Oxygen saturation, heart rate, respiratory rate at $5 \mathrm{~min}$ intervals during the intervention, adjusted for baseline*.

d. Tolerability: 
i. Proportion of participants who withdrew from the intervention before it was completed

ii. Tolerability questionnaire results

iii. BORG dyspnoea score results

* Baseline was the measurement taken at the zero time-point at the start of each intervention. Intervention 2 required a baseline of \pm 2 $\mathrm{mmHg}$ from the baseline for Intervention 1.

\subsubsection{Sample size estimation}

A change in $\mathrm{P}_{\mathrm{t}} \mathrm{CO}_{2}$ of $4 \mathrm{mmHg}$ is considered physiologically significant (Perrin, et al., 2011; Wijesinghe et al., 2011). The sample size of 24 had $80 \%$ power, type I error rate $5 \%$, to detect a difference of $2.4 \mathrm{mmHg}$. This is half the difference found in a study of participants with obesity hypoventilation syndrome which reported a mean (SD) paired difference of 5 (4) $\mathrm{mmHg}$ (Wijesinghe et al., 2011).

\subsubsection{Statistical analysis plan}

Analysis of the primary outcome variable utilised mixed linear modelling to compare mean values of the outcome variables between randomised treatment arms, with baseline $\mathrm{P}_{\mathrm{t}} \mathrm{CO}_{2}$ as a continuous co-variate and treatment order, time of measurement, and randomised treatment as fixed effects. A time by treatment interaction term was also assessed. The repeated measures design was accounted for using a co-variance model with an exponential correlation in time structure and with different parameter estimates for each of the two treatment arms to allow for correlated measurements within individuals and treatments. The analysis plan was to report the estimates of the individual time-wise comparisons between treatment arms if the time by treatment interaction was not statistically significant $(P<0.05)$, with the main interest in the difference after $60 \mathrm{~min}$, and to otherwise report the overall difference between treatment arms.

For the derived variables of a change in $\mathrm{P}_{\mathrm{t}} \mathrm{CO}_{2}$ of $\geq 4 \mathrm{mmHg} / 8 \mathrm{mmHg}$ from baseline, McNemar's exact test was used to compare the paired proportions and an appropriate estimate of the paired difference in proportions was used. SAS version 9.4 was used. 


\subsection{Results}

\subsubsection{Participant characteristics}

A CONSORT diagram of participant flow through the study is provided in Figure 5-2 and participant characteristics are shown in Table 5-1. Eleven $(45.8 \%)$ of the participants were female and the mean (SD) $\mathrm{P}_{\mathrm{cap}} \mathrm{CO}_{2}$ at randomisation was $50.6 \mathrm{mmHg}$ (4.8). All but one participant had severe or very severe airflow obstruction according to the Global Initiative for Chronic Obstructive Lung Disease (GOLD) classification (Vogelmeier et al., 2017). Nine $(37.5 \%)$ participants had documented previous experience with NIV for AHRF, however none had a domiciliary NIV prescription. Details of supplemental oxygen administration are provided in the appendix 9.7.4.1. Three participants required an extended washout to return to within $2 \mathrm{mmHg}$ of the time point zero reading prior to the second intervention, and one participant did not return to within $2 \mathrm{mmHg}$ within the 30 minute washout period and had a baseline reading at the start of the second intervention of $-4.7 \mathrm{mmHg}$ relative to the time-point zero reading.

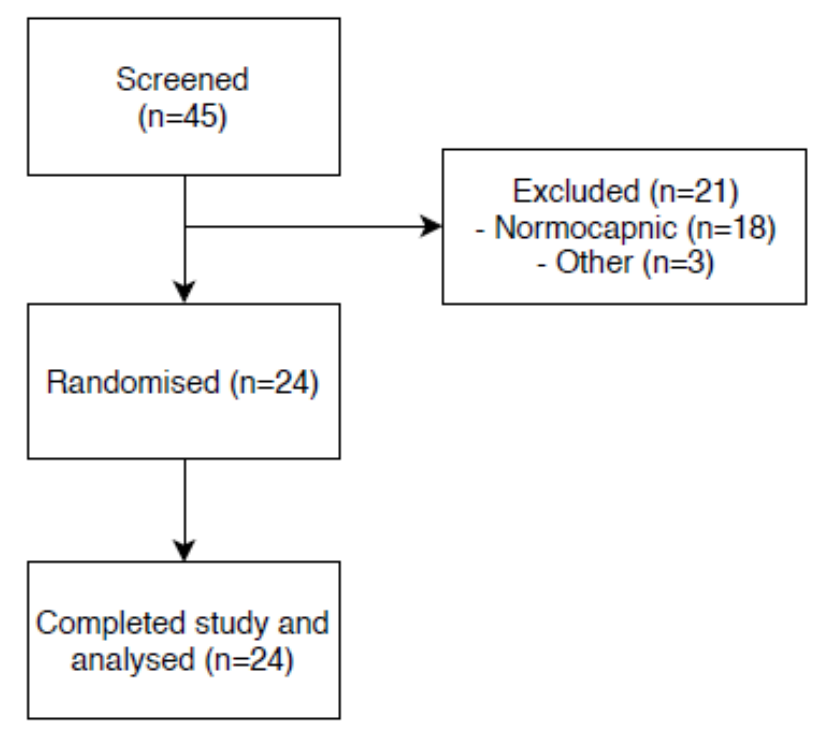

Figure 5-2 CONSORT diagram of participant flow through Study 5. 
Table 5-1 Study 5 baseline participant characteristics.

\begin{tabular}{|c|c|c|c|}
\hline Characteristic & Mean (SD) & Median (IQR) & Min to Max \\
\hline Age (years) & $68(9.0)$ & $70(63$ to 73.5$)$ & 46 to 83 \\
\hline Body Mass Index (kg/m²) & $26.3(5.2)$ & $25(22.7$ to 30.5$)$ & 18.4 to 34.9 \\
\hline $\mathrm{FEV}_{1}(\mathrm{~L})$ & $0.75(0.24)$ & $0.72(0.57$ to 0.95$)$ & 0.34 to 1.32 \\
\hline $\mathrm{FEV}_{1} / \mathrm{FVC}$ ratio & $0.36(0.09)$ & $0.33(0.31$ to 0.39$)$ & 0.25 to 0.62 \\
\hline $\mathrm{FEV}_{1} \%$ predicted & $27.0(10.56)$ & 25.0 (19.5 to 32.5$)$ & 11 to 61 \\
\hline Smoking pack years & $41.2(19.5)$ & 41.5 (26 to 50$)$ & 13 to 90 \\
\hline MMRC & $2.2(0.8)$ & $2(2$ to 3$)$ & 1 to 4 \\
\hline \multirow[t]{2}{*}{$\mathrm{P}_{\mathrm{t}} \mathrm{CO}_{2}(\mathrm{mmHg})$} & $47.2(5.8)$ & 45.9 (42.4 to 51.6$)$ & 40.1 to 59.2 \\
\hline & & $\mathrm{N} / 24(\%)$ & \\
\hline Sex (Female) & & $11(45.8)$ & \\
\hline \multicolumn{4}{|l|}{ Co-morbidities } \\
\hline Asthma & & $3(12.5)$ & \\
\hline Bronchiectasis & & $1(4.2)$ & \\
\hline Heart failure & & $1(4.2)$ & \\
\hline Arrhythmias & & $3(12.5)$ & \\
\hline \multicolumn{4}{|l|}{ Ethnicity } \\
\hline NZ European & & $15(62.5)$ & \\
\hline Maori & & $6(25)$ & \\
\hline Pacific & & $1(4.2)$ & \\
\hline Other & & $3(12.5)$ & \\
\hline \multicolumn{4}{|l|}{ Inhaled Treatment } \\
\hline \multicolumn{2}{|l|}{ Inhaled Corticosteroid } & $22(91.7)$ & \\
\hline \multicolumn{2}{|c|}{ Long-Acting Beta-Agonist } & $21(91.7)$ & \\
\hline \multicolumn{2}{|c|}{ Long-Acting Muscarinic Antagonist } & $21(87.5)$ & \\
\hline \multicolumn{2}{|c|}{ Short-Acting Beta-Agonist } & $19(79.2)$ & \\
\hline \multicolumn{2}{|c|}{ Short-Acting Muscarinic Antagonist } & $2(8.3)$ & \\
\hline \multicolumn{2}{|c|}{ Long-term oxygen therapy } & $8(33.3)$ & \\
\hline $\begin{array}{l}\text { ledical Research Council; } P_{t} C \\
\text { xygen saturation. }\end{array}$ & & Forced Vitc & ied \\
\hline
\end{tabular}

\subsubsection{Primary Outcome}

Data summaries for $\mathrm{P}_{\mathrm{t}} \mathrm{CO}_{2}$ values at each time-point are shown in and Figure 5-3. The time by treatment interaction was not significant $(P=0.21)$, consistent with the difference between NIV and NHF being the same at every measurement time point. This is also consistent with the appearance of Figure 5-3. NIV reduced the $\mathrm{P}_{\mathrm{t}} \mathrm{CO}_{2}$ more than NHF, mean (SD) at 60 min -5.3 (5.0) versus -2.5 (3.5) $\mathrm{mmHg}$; difference averaged across all time points $-2.5 \mathrm{mmHg}$ $(95 \% \mathrm{Cl}-4.5$ to -0.5$), \mathrm{P}=0.016$. 


\subsubsection{1 $\mathrm{P}_{\mathrm{t}} \mathrm{CO}_{2}$ change from baseline}

The maximum change from baseline after 60 min with NIV was $-15.2 \mathrm{mmHg}$ and for NHF was $-13.4 \mathrm{mmHg}$. There was no evidence of a difference in the proportion of participants with a reduction of $\mathrm{PtCO}_{2} \geq 4 \mathrm{mmHg}$ or $\geq 8 \mathrm{mmHg}$ for NIV compared to NHF after $60 \mathrm{~min}$. The proportion in NIV with a reduction of $\geq 4 \mathrm{mmHg}$ was $11 / 24(45.8 \%)$ and for NHF 6/24 (25\%), absolute difference ( $95 \% \mathrm{Cl}$ ) $20.8 \%$ ( 0.9 to 40.8$), \mathrm{P}=0.13$. Note that for this estimate, the $95 \% \mathrm{Cl}$ was estimated by asymptotic methods but the $\mathrm{P}$ value by an exact method. The proportion in NIV with a reduction $\geq 8 \mathrm{mmHg}$ was $6 / 24(25.0 \%)$ and for NHF 2/24 (8.3\%), absolute difference $16.7 \%$ (-5.5 to 38.8$), P=0.2$.

Table 5-2 $\mathrm{P}_{\mathrm{t}} \mathrm{CO}_{2}$ values and difference in $\mathrm{P}_{\mathrm{t}} \mathrm{CO}_{2}$ with NHF and NIV

\begin{tabular}{lccccc}
\hline Time \\
$\begin{array}{c}\text { point } \\
(\mathbf{m i n})\end{array}$ & $\begin{array}{c}\mathbf{P}_{\mathbf{t}} \mathbf{C O}_{2} \\
\mathbf{m m H g}) \\
\mathbf{M e a n} \\
\mathbf{( S D )}\end{array}$ & $\begin{array}{c}\text { Difference from } \\
\text { baseline } \\
\text { Mean (SD) }\end{array}$ & $\begin{array}{c}\mathbf{P}_{\mathbf{t}} \mathbf{C O}_{2} \\
\mathbf{m m H g}) \\
\mathbf{M e a n} \\
\mathbf{( S D})\end{array}$ & $\begin{array}{c}\text { Difference from } \\
\text { baseline } \\
\text { Mean (SD) }\end{array}$ & $\begin{array}{c}\text { NIV - NHF } \\
\text { change } \\
\text { from } \\
\text { baseline } \\
\text { Mean (SD) }\end{array}$ \\
\hline 0 & $47.1(5.5)$ & & $47.1(5.7)$ & & \\
5 & $45.1(5.2)$ & $-1.9(1.4)$ & $43.2(6.0)$ & $-3.9(2.9)$ & $-2.0(2.7)$ \\
10 & $45.2(5.1)$ & $-1.9(1.7)$ & $42.0(6.4)$ & $-5.1(4.1)$ & $-3.2(3.8)$ \\
15 & $45.2(5.1)$ & $-1.9(1.9)$ & $42.3(6.7)$ & $-4.8(4.4)$ & $-2.9(4.3)$ \\
20 & $45.3(5.1)$ & $-1.8(2.0)$ & $42.2(7.0)$ & $-4.9(4.8)$ & $-3.2(4.5)$ \\
25 & $45.4(5.2)$ & $-1.7(2.0)$ & $42.6(7.0)$ & $-4.6(4.8)$ & $-2.9(4.9)$ \\
30 & $45.6(5.2)$ & $-1.5(2.3)$ & $43.1(6.8)$ & $-4.0(4.7)$ & $-2.5(5.1)$ \\
35 & $45.2(5.2)$ & $-1.9(2.4)$ & $42.9(7.0)$ & $-4.2(4.8)$ & $-2.3(5.2)$ \\
40 & $45.0(5.2)$ & $-2.1(2.9)$ & $43.0(7.2)$ & $-4.2(4.9)$ & $-2.1(5.6)$ \\
45 & $44.9(5.0)$ & $-2.2(2.9)$ & $42.7(7.2)$ & $-4.4(5.0)$ & $-2.2(5.5)$ \\
50 & $44.6(5.1)$ & $-2.5(3.4)$ & $42.7(7.1)$ & $-4.4(5.1)$ & $-1.9(5.6)$ \\
55 & $44.7(4.9)$ & $-2.4(3.3)$ & $42.4(7.1)$ & $-4.8(5.0)$ & $-2.4(5.7)$ \\
60 & $44.6(5.1)$ & $-2.5(3.5)$ & $41.9(7.0)$ & $-5.3(5.0)$ & $-2.8(5.5)$ \\
\hline
\end{tabular}




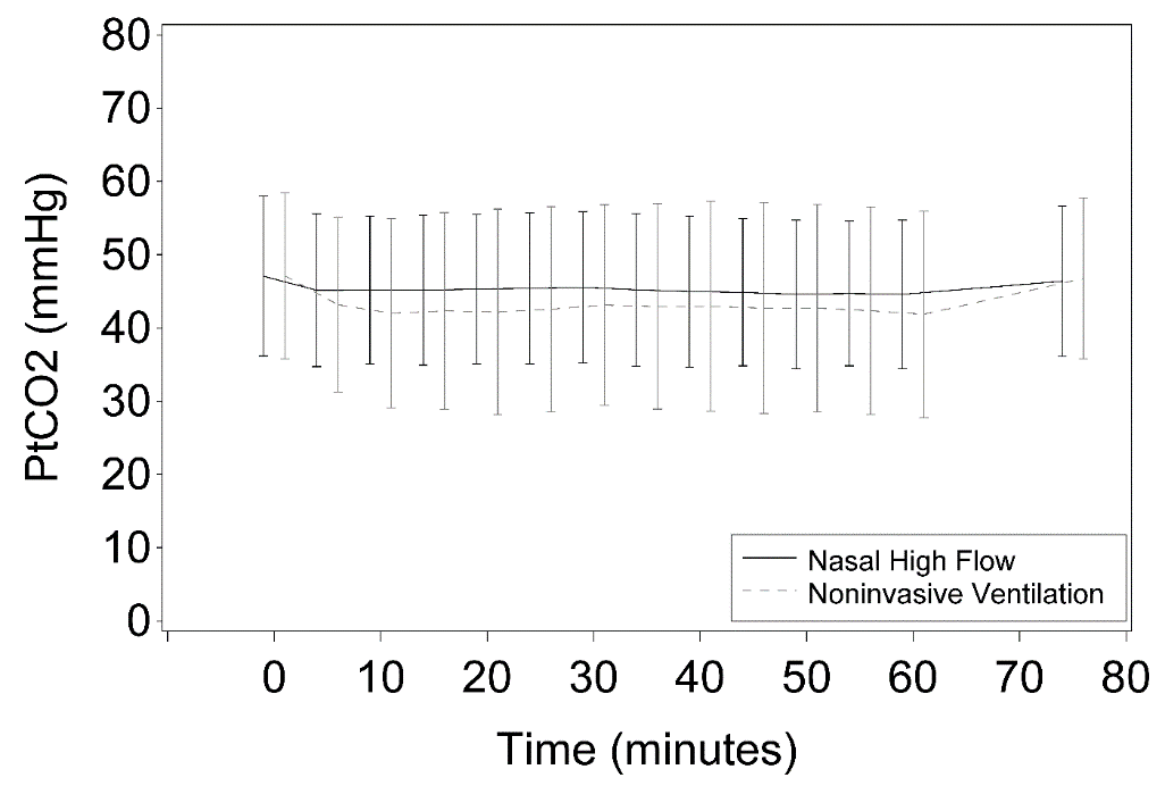

Figure 5-3 Mean $\mathrm{PtCO}_{2}$ change over time. The plots are means and the error bars are plus and minus one standard deviation.

\subsubsection{Respiratory rate}

Although the time by treatment interaction was of modest significance $(P=0.038)$, it was consistent with some differences in the difference between NIV and NHF at the different time points. Both NIV and NHF slightly reduced the respiratory rate but there was no evidence of a difference in respiratory rate between NIV and NHF at each individual time point (See appendix 9.7). Mean (SD) respiratory rate at $60 \mathrm{~min}$ for NIV and NHF, respectively: 17 (4.1) versus 14.8 (4.5); difference 1.3 breaths per minute (-0.7 to 3.4), $P=0.20$.

\subsubsection{Oxygen saturation and heart rate}

The time by treatment interaction for oxygen saturation was not significant $(P=0.90)$, consistent with no evidence that there was a difference between the difference between NIV and NHF at any of the time points. The oxygen saturations were higher for NIV, with the estimated difference (95\% CI) for NIV minus NHF of mean (SD) oxygen saturations at $60 \mathrm{~min}$ for NIV and NHF, respectively: $94.1 \%$ (3) versus $92.5 \%$ (2.8); difference across all time points $2.1 \%(1.1$ to 3.1$), P<0.001$.

Similarly, with heart rate change, the time by treatment interaction was not significant; $P=0.22$. There was no evidence of a difference in overall heart rate 
between NIV and NHF; difference $(95 \% \mathrm{CI})$ for NIV minus NHF 0.3 beats per $\min (-1.5$ to 2.1$), P=0.74$.

\subsubsection{Borg dyspnoea scores}

Borg dyspnoea scores are shown in Table 5-3. There was a very limited range of responses ( 0 to 3 on an eleven point scale) and the median differences were all zero or close to this, so no meaningful analyses were undertaken.

Table 5-3 BORG score outcomes at baseline and difference postintervention.

\begin{tabular}{llll}
\hline Variable & Mean (SD) & Median (IQR) & Min to Max \\
\hline NHF at baseline & $0.88(1.11)$ & $0.25(0$ to 2$)$ & 0 to 3 \\
NIV at baseline & $0.9(1.41)$ & $0(0$ to 1$)$ & 0 to 5 \\
NHF change & $-0.17(0.89)$ & $0(-0.25$ to 0$)$ & -3 to 2 \\
NIV change & $-0.1(1.03)$ & $0(-0.75$ to 0$)$ & -2 to 3 \\
\hline
\end{tabular}

\subsubsection{Tolerability questionnaires}

Full data description for the patient reported outcome measures are presented in the appendix 9.7. Participants rated NHF significantly better for ease of application, fit, and overall comfort, with $\mathrm{P} \leq 0.004$ for each comparison (Table 5-4). There was no difference in participant experience with regard to moisture. There was no significant difference in noisiness or the likelihood of reusing the device, with mean (NIV minus NHF) 15.0 (-0.5 to 30.5$), P=0.06$.

Table 5-4 Mixed linear models for difference in questionnaire responses between NIV and NHF interventions.

\begin{tabular}{|c|c|c|c|c|}
\hline Question & NIV $^{*}$ & NHF* $^{*}$ & $\begin{array}{l}\text { Difference }(95 \% \mathrm{Cl}) \\
\text { NIV minus NHF }\end{array}$ & $\mathbf{P}$ \\
\hline $\begin{array}{l}\text { 1. Ease of } \\
\text { application } \\
\text { 2. Nasal }\end{array}$ & $33.1(31.7)$ & $13.8(18.8)$ & $19.3(6.7$ to 31.9$)$ & 0.004 \\
\hline $\begin{array}{l}\text { passage/mouth } \\
\text { moisture }\end{array}$ & $21.1(22.7)$ & $23(25.2)$ & $-1.9(-13.7$ to 9.9$)$ & 0.75 \\
\hline $\begin{array}{l}\text { 3. Comfort of fit } \\
\text { on head/face }\end{array}$ & $44.5(33.0)$ & $18.3(22.0)$ & 26.3 (16.2 to 36.4$)$ & $<0.001$ \\
\hline $\begin{array}{l}\text { 4. Overall } \\
\text { Comfort }\end{array}$ & $41.3(33.0)$ & $16.3(21.9)$ & 25.1 (11.7 to 31.7 ) & $<0.001$ \\
\hline 5. Noisiness & $38.6(29.7)$ & $30.4(26.1)$ & $8.2(-6.4$ to 22.7$)$ & 0.26 \\
\hline $\begin{array}{l}\text { 6. Likelihood of } \\
\text { reusing device }\end{array}$ & $30(38.4)$ & $15.1(23.7)$ & $15.0(-0.5$ to 30.5$)$ & 0.058 \\
\hline
\end{tabular}




\subsection{Discussion}

In this randomised controlled cross-over study in patients with stable COPD and chronic hypercapnic respiratory failure, treatment with NIV led to a greater reduction in $\mathrm{P}_{\mathrm{tCO}}$ than treatment with $\mathrm{NHF}$. However, the $2.5 \mathrm{mmHg}$ difference between the two interventions is of uncertain clinical significance. NHF was superior across a range of tolerability aspects. These findings suggest that there may be a role for NHF in people with hypercapnic respiratory failure who are unable to tolerate NIV.

There are methodological issues pertinent to the interpretation of the study findings. Due to the nature of the interventions, it was impossible to blind participants to the allocated intervention. The use of a blinded investigator to record $\mathrm{P}_{\mathrm{t}} \mathrm{CO}_{2}$ and oxygen saturations ensured that single-blinding was achieved for key primary and secondary outcome measurements. The close correlation between $\mathrm{PaCO}_{2}$ and $\mathrm{PCapCO}_{2}$ as presented in Appendix II (Validation of accuracy of transcutaneous $\mathrm{PCO}_{2}$ monitoring) was justification for $\mathrm{P}_{\mathrm{t}} \mathrm{CO}_{2}$ use for continuous monitoring rather than multiple $\mathrm{CBG}$ or $\mathrm{ABG}$ samples.

Supplemental oxygen, if required, was carefully titrated to maintain oxygen saturations of $88-92 \%$, off-setting the potential confounding effect of oxygeninduced $\mathrm{PCO}_{2}$ change. Supplemental oxygen use has been a potential confounding factor in $\mathrm{P}_{\mathrm{t}} \mathrm{CO}_{2}$ change in previous studies of $\mathrm{NHF}$ therapy in exacerbations of COPD (Fraser et al., 2016; Nilius et al., 2013).

A 60-min duration for each intervention was selected as this corresponds to the point at which the response to NIV therapy is commonly assessed in clinical practice. The mean change from baseline in $\mathrm{P}_{\mathrm{t}} \mathrm{CO}_{2}$ was highest at the 60-min time point for both NHF and NIV, although this reduction began to occur within $5 \mathrm{~min}$ for both interventions. As with NIV in the acute setting, the difference in $\mathrm{P}_{\mathrm{t}} \mathrm{CO}_{2}$ reduction at the 60-min time-point is unlikely to represent the maximum response to treatment (Bräunlich \& Wirtz, 2017), and it is possible that the difference in carbon dioxide reduction between the interventions may have passed the thresholds for physiological or clinical significance, i.e. $4 \mathrm{mmHg}$ (Bräunlich et al., 2013; Sztrymf et al., 2012) and 8 
$\mathrm{mmHg}$ (Perrin et al., 2011), respectively, with prolonged treatment. In this study both NIV and NHF were administered with fixed settings, whereas in clinical practice both the inspiratory pressure and flow rate can be titrated to response and tolerability. It is also possible the difference between NIV and NHF may vary with changes to inspiratory pressure or flow rate.

Participants in the study had stable COPD and chronic hypercapnic respiratory failure and therefore, although the physiological mechanisms for carbon dioxide clearance are the same, the results cannot be assumed to be generalisable to people with AECOPD and acute hypercapnic respiratory failure or to people with hypercapnic respiratory failure from other causes. However, this patient selection allowed the internal validity to be improved, with a crossover design being utilised at a single study visit, enabling both interventions to be tested with a similar baseline $\mathrm{P}_{t} \mathrm{CO}_{2}$.

The finding that both NHF and NIV lowered $\mathrm{P}_{\mathrm{t}} \mathrm{CO}_{2}$ is in keeping with previously published studies, reviewed in Sections 1.3.3.2.3 (Efficacy of NIV) and 1.4.5 (Systematic review of NHF in COPD). While this trial did not directly measure any mechanisms of action of NHF, the ability to reduce upper airway resistance, improve alveolar recruitment, increase $\mathrm{V}_{\mathrm{T}}$, and produce a flowdependant increase in airway pressure are all likely contributors to the effect observed (Dysart et al., 2009; Fraser et al., 2016; Lee et al, 2013; Nishimura, 2015). However, the dominant mechanism for $\mathrm{PCO}_{2}$ reduction may be the nasopharyngeal dead space washout and reduction of functional dead space (Bräunlich et al., 2018).

Only one other RCT has compared the reduction in $\mathrm{PaCO}_{2}$ between NHF and NIV in people with stable hypercapnic COPD. Pisani et al. performed a randomised cross-over trial during which the 14 participants received 30 min periods of NIV, or NHF, with four different conditions according to flow rate (20 $\mathrm{L} / \mathrm{min}$ versus $30 \mathrm{~L} / \mathrm{min}$ ) and mouth open or closed (Pisani et al., 2017 - see 1.4.5.1.3.4). Both NIV and NHF, at $30 \mathrm{~L} / \mathrm{min}$ with the mouth closed lowered $\mathrm{P}_{\mathrm{a}} \mathrm{CO}_{2}$ by around $5 \mathrm{mmHg}$ relative to baseline.

Section 1.3.3 demonstrated that NIV is the preferred treatment modality for both acute and chronic hypercapnia and has been shown to reduce mortality in both settings (Köhnlein et al., 2014; Murphy et al., 2017; Osadnik et al., 
2017). However, subject intolerance of NIV is common and is a predictor of NIV failure (Ambrosino et al., 1995; Benhamou et al., 1992; Carlucci et al., 2001). The superior tolerability of NHF means that its use should be considered in people who are unable to tolerate NIV, provided intubation is not currently indicated or is not clinically appropriate. As patients receiving NIV have frequent interruptions of therapy to allow them to eat and drink, it may be appropriate to consider offering NHF during breaks off from NIV which would allow patients to continue to receive a therapy that reduces $\mathrm{PaCO}_{2}$ during these periods.

Parallel-group randomised controlled trials are now required to assess both the efficacy and effectiveness (Woodcock et al., 2018) of NHF in the setting of AHRF due to AECOPD. A focus on effectiveness as well as efficacy will be important as the real-world outcomes of NIV have been reported to be considerably poorer than the efficacy seen in clinical trials (Roberts et al., 2011). It is reasonable to consider the possibility that the differences in the reduction in $\mathrm{PaCO}_{2}$ between NIV and NHF that are seen in an ideal setting in highly standardised, short duration, clinical trials may actually be considerably smaller in an effectiveness study. In the real world setting the superior tolerability of NHF and the fact that it does not need to be interrupted for provision of nutrition may mean that patients receiving NHF do so for a greater proportion of the day than those receiving NIV. A quarter of participants in this study had a reduction in $\mathrm{P}_{\mathrm{t}} \mathrm{CO}_{2}$ of $\geq 4 \mathrm{mmHg}$ after 60 min of NHF therapy. It is therefore possible that uninterrupted use of NHF over the first $24 \mathrm{hrs}$ of an admission may be sufficient to normalise acid-base balance in many people with mild to moderate acidosis $(7.25 \leq \mathrm{pH}<7.35)$ at presentation.

\subsection{Conclusion}

In people with stable COPD and chronic hypercapnia, NIV resulted in a greater reduction in $\mathrm{P}_{\mathrm{tCO}}$ compared with NHF. The minor difference in $\mathrm{P}_{\mathrm{t}} \mathrm{CO}_{2}$ recorded is of uncertain clinical significance. NHF was better tolerated than NIV and may represent a current therapeutic option for some people with hypercapnic respiratory failure and poor toleration of NIV. Future RCTs comparing NIV and NHF in people with AECOPD and AHRF with mild to 
moderate acidosis may now be warranted, but the application for NHF may also solely be as an adjunctive therapy to NIV. 


\section{Study 6 - A dual NHF/NIV device compared to standard NIV in chronic respiratory disease: a randomised controlled cross-over trial}

\subsection{Rationale}

Study 2 demonstrated NHF has a modest effect in reducing $\mathrm{P}_{\mathrm{t}} \mathrm{CO}_{2}$ in COPD and Study 5 replicated these findings in people with COPD and hypercapnic respiratory failure. When directly compared to NIV, NHF was marginally inferior with respect to $\mathrm{PCO}_{2}$ reduction but had superior tolerability. The conclusions from Study 5 were that the role of NHF in HRF may be as an intermittent therapy to complement NIV use, hence extending the overall treatment period, or for those who are unable to tolerate NIV.

Overall, the thesis study results so far suggest that the ideal ventilatory support device in HRF may be one that can deliver both NIV and NHF and be easily switched between the 2 modes, depending on the clinical scenario and patient tolerance/preference. Study 6 was a preliminary investigation of an NHF device that is also capable of using the internal flow generator to provide NIV through bi-level pressure support. This dual NHF/NIV device can easily switch between the two therapy modes - possibly the ideal application for NHF therapy, making it a practical application for hospital ward-based therapy.

\subsection{Aims and hypothesis}

This study was the first time the FPH NHF/NIV device had been tested in a clinical trial. The aim was to establish whether participants with a chronic respiratory disease at risk of potential hypercapnic respiratory failure could determine a comfort preference between the NHF/NIV device and a commercially available ResMed bi-level device. Studies 2, 3 and 5 have demonstrated adequate comfort of the NHF mode, and standard internal calibration testing by FPH confirmed the device was able to deliver accurate NHF therapy flow-rates. The aim of this study therefore was to measure the comfort of the bi-level function, rather than the NHF function, of the NHF/NIV device. Other aims were to assess patient-ventilator synchrony and physiological effects of the NHF/NIV device such as $\mathrm{P}_{t} \mathrm{CO}_{2}, \mathrm{~S}_{\mathrm{t}} \mathrm{O}_{2}$, heart rate and respiratory rate. 
The hypothesis for this study was that patients with a chronic respiratory disease at risk of AHRF will not consistently prefer one bi-level device over the other with respect to comfort of the delivered pressure and the NHF/NIV device will reduce $\mathrm{P}_{\mathrm{t}} \mathrm{CO}_{2}$ in the same magnitude compared to the standard ResMed bi-level device.

\subsection{Methods}

\subsubsection{Trial design}

Randomised, controlled 2-way crossover non-inferiority trial.

\subsubsection{Eligibility criteria}

\subsubsection{Inclusion criteria}

- $\quad$ Aged $\geq 18$ years.

- Participants with a doctors diagnosis of a chronic respiratory disease associated with potential hypercapnic respiratory failure

\subsubsection{Exclusion criteria}

- Significant hypoxia

- $\mathrm{S}_{\mathrm{t}} \mathrm{O}_{2}<88 \%$ after 20-30 min of the initial SenTec stabilisation period, or $\mathrm{StO} 2<88 \%$ for a sustained time-period during the intervention (according to investigators clinical discretion)

- Use of supplemental oxygen

- Chronic respiratory disease that is not deemed to be stable

- Current exacerbation requiring acute treatment with a short course of antibiotics/oral steroids within the last 1 week

- Hospital admission for an acute exacerbation in the last 2 weeks

- Recent cardiac or respiratory arrest

- Gastrointestinal bleeding, ileus or recent gastrointestinal surgery

- Coma, decreased level of consciousness or agitation

- Anatomical or subjective difficulty with airway access e.g. facial surgery, trauma, vomiting as a result of upper airway obstruction

- Cerebrospinal fluid leak, or abnormalities of the cribriform plate or prior history of head trauma.

- Fluid depletion, poor left ventricular function or cardiac disease without adequate pharmacological therapy 
- Pneumothorax, or at high clinical risk of pneumothorax or barotrauma due to, for example, previous pneumothorax, pneumomediastinum, severe bullous lung disease, acute lung injury secondary to pneumonia, interstitial lung diseases, cystic fibrosis and neuromuscular disease

- Diagnosis of a notifiable disease

- Use of an implantable medical device

- Presence of any other condition which, at the investigator's discretion, was believed may have presented a safety risk or impact the feasibility of the study or the study results.

\subsubsection{Interventions}

Participants received the NIV mode of the NHF/NIV device and ResMed NIV therapy, each for $30 \mathrm{~min}$ with at least $15 \mathrm{~min}$ washout in between to allow $\mathrm{P}_{\mathrm{t}} \mathrm{CO}_{2}$ recordings to return to $\pm 4 \mathrm{mmHg}$ of the previous intervention. Treatments were assigned in a 1:1 ratio in accordance with the computergenerated randomisation schedule, with the sequence being $A B$ or $B A$, where A is NHF/NIV and B is ResMed NIV.

\subsubsection{Recruitment and consent}

Adults with a doctor's diagnosis of a chronic respiratory disease were identified in a similar manner to Studies 2 and 5 above, through a combination of the MRINZ participant database and CCDHB clinics.

This study was prospectively registered with the ANZCTR (Trial ID: ACTRN12617001600336) and approved by the Central HDEC of New Zealand (17/CEN/225). Full written informed consent was completed before any study specific procedures.

\subsubsection{Study procedures}

Participants attended a single study visit at the MRINZ Respiratory Physiology Laboratory at Wellington Regional Hospital. Demographic and past medical history data were collected, and spirometry performed in accordance with ATS/ERS criteria using a Jaeger Masterscreen ${ }^{\text {TM }}$ body volume constant plethysmography unit with pneumotachograph and diffusion unit (ErichJaegar, Wurzburg). 
The SenTec transcutaneous monitor (SenTec digital monitor with V-Sign Sensor VS-A/P/N) was again used rather than $A B G$ s and was stabilised for 20 min before interventions were started.

The NHF/NIV device used the flow signal to detect inspiration and expiration in order to provide different inspiratory and expiratory pressures (IPAP and EPAP) (Figure 6-1).

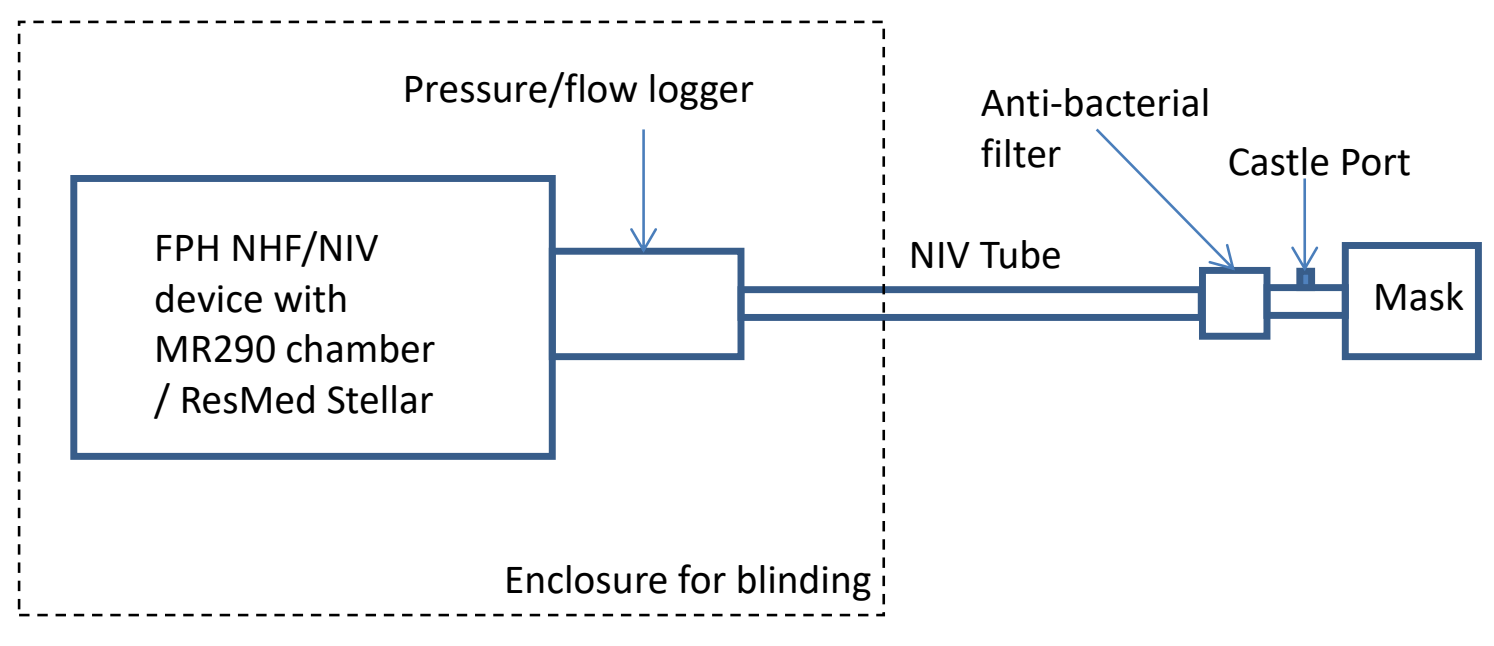

Figure 6-1 Configuration of the NHF/NIV and ResMed device set-up.

Both devices were set to the following:

- Bi-level ST mode without humidity

- IPAP $15 \mathrm{~cm} \mathrm{H}_{2} \mathrm{O}$

- EPAP $4 \mathrm{~cm} \mathrm{H} \mathrm{H}_{2} \mathrm{O}$

- Rise time and fall time $200 \mathrm{~ms}$

- Triggering sensitivity $=$ medium $(6.0 \mathrm{lpm})$

- No ramp function

Similar to Study 4, although BTS/ICS guidelines recommend a starting EPAP

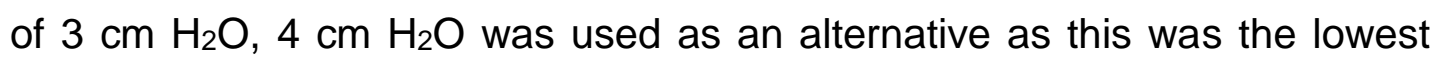
pressure the machine could provide. No supplemental oxygen was able to be used with the NHF/NIV device due to the prototype not having had adequate safety testing. The mask was sized according to the manufacturer's instructions and adjusted to minimise any leaks.

\subsubsection{Patient ventilator synchrony measurements}

A pressure/flow logger unit was attached to each device outlet (Figure 6-1) and breathing tube to record the delivered pressure and flow waveforms. The 
data was then analysed afterwards to collate a patient-ventilator synchrony score and to detect any instances of asynchrony.

Patient Ventilator synchrony metrics for the devices were determined from the circuit pressure and flow measurements made using the pressure flow loggers. Trigger and cycle points were determined from the circuit pressure waveform. The start of inspiration (SOI), end of inspiration (EOI) and end of breath $(\mathrm{EOB})$ were determined from the measured flow waveform. The ventilator synchrony metrics were calculated by comparing the temporal location of the trigger and cycle points relative to the breath markers. Definitions of the different types of the trigger and cycles are listed in Table 6-1.

Table 6-1 Definition of different bi-level triggering and cycling classification.

\begin{tabular}{lll}
\hline Classification & \multicolumn{1}{c}{ Description } & \multicolumn{1}{c}{$\begin{array}{c}\text { Binary } \\
\text { Class. }\end{array}$} \\
\hline Normal Trigger & $\begin{array}{l}\text { Trigger associated with SOI. i.e. it occurs within } \\
\text { search range, [max(0, SOI - (EOI - SOI) / 2), EOI] } \\
\text { "inspiration present AND trigger present" }\end{array}$ & TP \\
\hline Auto Trigger & $\begin{array}{l}\text { Trigger not associated with SOI } \\
\text { "no inspiration BUT trigger present" }\end{array}$ & FP \\
\hline Missed Trigger & $\begin{array}{l}\text { No trigger associated with SOI } \\
\text { "inspiration present BUT no trigger" }\end{array}$ & FN \\
\hline Double Trigger & Multiple triggers associated with a single SOI & Fycle associated with EOI. i.e. it Occurs within \\
\hline Normal Cycle & $\begin{array}{l}\text { Cyarch range, [SOI, min(EOI + (EOI - SOI) / 2, EOB)] } \\
\text { "inspiration present AND cycle present" }\end{array}$ & TP \\
\hline Auto Cycle & $\begin{array}{l}\text { Cycle not associated with EOI } \\
\text { "no inspiration BUT cycle present" }\end{array}$ & FN \\
\hline Missed Cycle & $\begin{array}{l}\text { No cycle associated with EOI } \\
\text { "inspiration present BUT no cycle" }\end{array}$ & \\
\hline SOl: start of inspiration, EOI: end of inspiration, EOB: end of breath, TP: True Positive, FP; \\
False Positive, FN; False Negative.
\end{tabular}

Auto triggers and auto cycles are caused by artefacts in the flow which can also be due to movement, variable leaks, variable breathing patterns, talking, coughing etc.

\subsubsection{Comfort score questionnaires}

Comfort score questionnaires were administered to participants after each intervention. Four questions relating to comfort were asked and the response was measured via the $100 \mathrm{~mm}$ Visual Analogue Scale (VAS) shown in Figure 6-2. The four comfort questions for the primary outcome related to: 1 ) overall 
breathing comfort, 2) how well changes in device pressure coordinated with participants breathing, 3) how comfortable the pressure was when breathing in, and 4) when breathing out. Other questions related to secondary outcomes such as the participants: 1) perception of delivered temperature and device noise and if these affected their comfort and 2) overall preference between the 2 devices. The complete comfort score questionnaire is in appendix VII (Section 9.8.2).

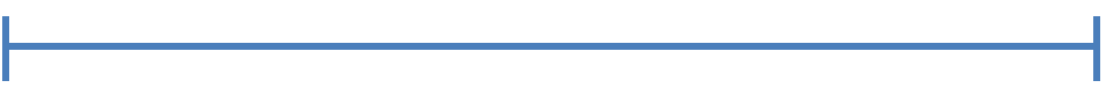

Very uncomfortable

Very comfortable

Figure 6-2 Example of $100 \mathrm{~mm}$ visual analogue scale with annotation depicting the levels of comfort.

\subsubsection{Outcome measures}

\subsubsection{Primary outcome}

Global comfort scores as assessed by the participants. The global comfort scores were calculated by averaging VAS scores for the 4 specific comfort questions. The non-inferiority margin was set at $25 \mathrm{~mm}$ as this corresponds to a single increment on the comfort questionnaire (e.g. 'slightly comfortable' to 'very comfortable', or 'neutral' to 'slightly uncomfortable') used in an FPH internal test. A difference of less than one increment in the comfort questionnaire was considered clinically insignificant, justifying a difference of $25 \mathrm{~mm}$ for the non-inferiority margin in this study.

\subsubsection{Secondary outcomes}

1.Mean of each of the 4 comfort questionnaire scores on the $100 \mathrm{~mm}$ VAS:

a. Breathing comfort of the device

b. Synchrony with breathing

c. Comfort during inspiration

d. Comfort during expiration

2.Participant perception of delivered temperature and device noise

3.Overall preference between the 2 devices

4.Measures of patient-ventilator synchrony as scored from pressure flow sensor readings 
5. Other physical measurements:

- $\mathrm{P}_{\mathrm{t}} \mathrm{CO}_{2}$, Oxygen saturation, heart rate and respiratory rate at zero-time point and then at $5 \mathrm{~min}$ intervals during the intervention and at the end of the subsequent 15 min washout, adjusted for baseline*.

- The proportion of participants that have a decrease in $\mathrm{P}_{\mathrm{t}} \mathrm{CO}_{2}$ by $\geq 4 \mathrm{mmHg}$ and $\geq 8 \mathrm{mmHg}$ compared to baseline.

6 . Arterial blood pressure (measured at zero time-point and 30 min timepoint only)

- Mean (MAP), Systolic (SBP) and Diastolic (DBP) ( $\mathrm{mm} \mathrm{Hg}$ )

7.Observed moisture in mask or castle port at the end of each intervention (Yes or No).

* Baseline refers to measurements taken at the zero time-point for intervention 1.

\subsubsection{Sample size estimation}

A sample size of 25 participants was required to demonstrate non-inferiority of the primary outcome (global comfort score), assuming the devices were equally comfortable, with a significance level of $0.05,90 \%$ power, an SD of 43 $\mathrm{mm}$ and a non-inferiority margin of $25 \mathrm{~mm}$. The assumed SD was based on a similar internal study conducted by FPH. To minimise the likelihood of the study being underpowered and to allow for drop-outs, 30 participants were recruited.

\subsubsection{Statistical analysis plan}

Simple data descriptions were shown for continuous variables and counts, and proportions for categorical variables. A paired t-test was used to compare $\mathrm{NHF/NIV}$ and ResMed use comfort variables. McNemar's test and a method for estimating differences in paired proportions were used for the moisture variable as well as the proportions of participants changing by more than 4 or $8 \mathrm{mmHg} \mathrm{P}_{\mathrm{t}} \mathrm{CO}_{2}$ with the treatments.

Mixed linear models with the baseline (zero time-point) variable were used to estimate differences in the blood pressure variables after 30 minutes. A simple correlation structure was used to model the repeated measurements on the 
same participants. Mixed linear models with the baseline (T0) variable were used to estimate differences in heart rate, $\mathrm{P}_{\mathrm{t}} \mathrm{CO}_{2}, \mathrm{~S}_{\mathrm{p}} \mathrm{O}_{2}$, and respiratory rate between treatments. A simple correlation structure was used to model the repeated measures. In the first set of estimates a time by treatment interaction term was tested (and estimates of the difference between treatments at each time point). If this interaction term was not statistically significant, ie there was no evidence that the treatment differences varied with time, the overall averaged estimate is the appropriate estimate of treatment difference. SAS version 9.4 was used.

\subsection{Results}

\subsubsection{Participant characteristics}

Baseline participant characteristics are shown in Table 6-2. Participants were recruited between December 2017 and March 2018. Nineteen (63.3\%) of the participants were male and six (20\%) were Maori and Pacific Islanders. Most participants had COPD (86.7\%) and around 1 in 5 participants had documented previous experience with NIV. One participant required an extended washout period of $12 \mathrm{~min}$ to get within $4 \mathrm{mmHg}$ of the baseline reading. 
Table 6-2 Study 6 baseline participant characteristics.

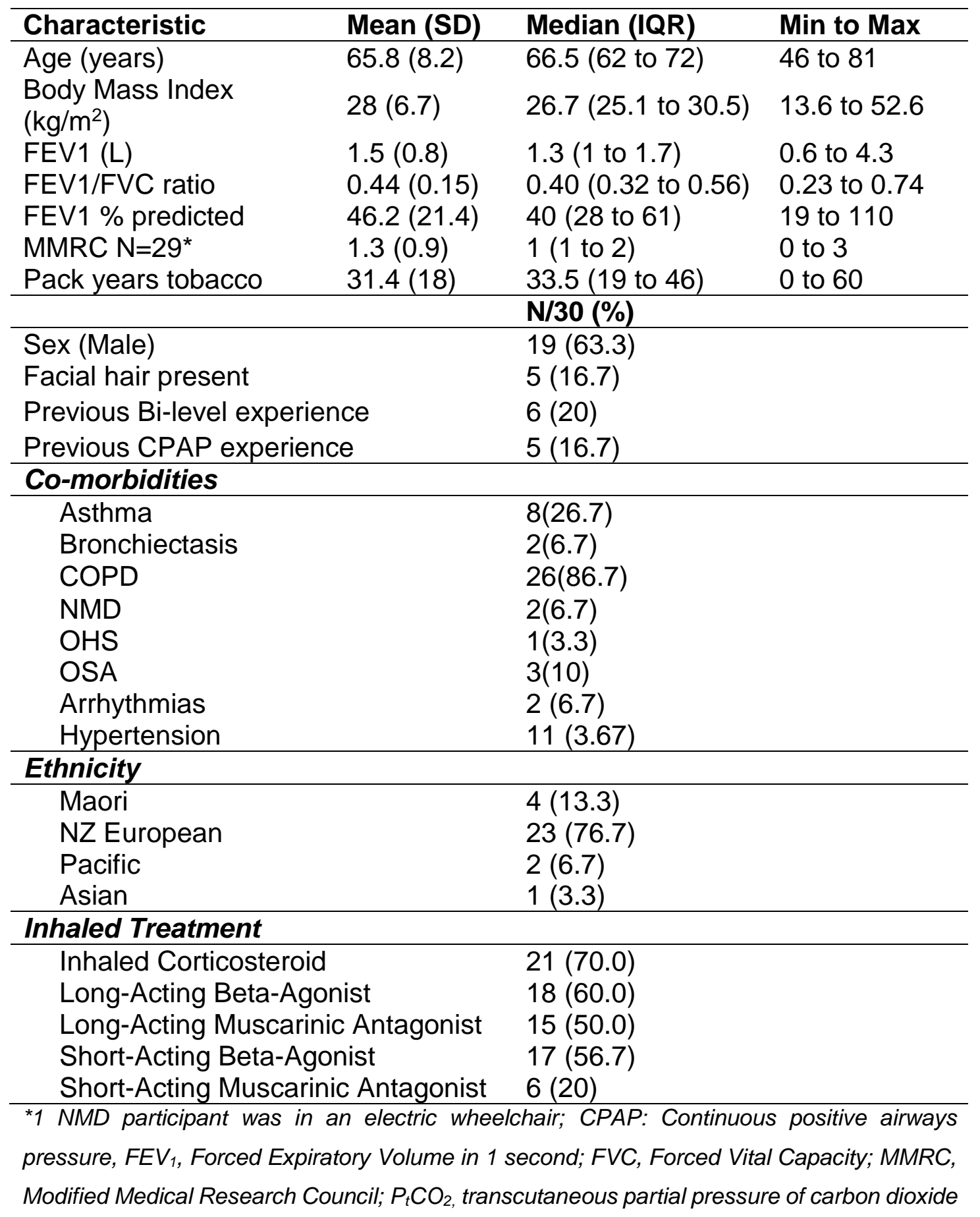

\subsubsection{Primary Outcome}

Data summaries for the four VAS scores and global comfort score (mean of the four VAS scores) is shown in Table 6-3. The paired t-test comparison of global comfort scores of ResMed minus NHF/NIV was $-1.3 \mathrm{~mm}(95 \% \mathrm{Cl}-7.9$ to 5.2), $P<0.001$. Therefore, on the scale of measurement of $100 \mathrm{~mm}$ and a non-inferiority bound of $25 \mathrm{~mm}$, the test for a lower confidence limit of less than $25 \mathrm{~mm}$ was $<0.001$; so that there was no evidence the mean difference was 
greater than $25 \mathrm{~mm}$ (one VAS unit difference) ie NHF/NIV was not inferior to ResMed on the global comfort score.

\subsubsection{Secondary outcomes}

\subsubsection{Individual comfort scores}

Table 6-3 shows similar mean individual comfort and global scores with NHF/NIV and ResMed and minimal mean differences for each. Paired t-tests demonstrated no difference in means between for any of the individual comfort scores. ( $P>0.05$ for all scores) (Table 6-4).

Table 6-3 Data description of comfort score variables measured by visual analogue scale (VAS).

\begin{tabular}{lccc}
\hline \multirow{2}{*}{$\begin{array}{l}\text { Questionnaire } \\
\text { variable }\end{array}$} & \multicolumn{2}{c}{ VAS } & \multirow{2}{*}{$\begin{array}{c}\text { VAS difference ResMed } \\
\text { minus NHF/NIV } \\
\text { Mean (SD) }\end{array}$} \\
\cline { 2 - 3 } & NHF/NIV & ResMed & \multirow{2}{\text{Mean(SD)}}{} \\
\hline Comfort & $75.5(20.7)$ & $72.2(24.8)$ & $-3.3(21.6)$ \\
Coordination & $79.5(18.2)$ & $80.8(20.3)$ & $1.3(20.1)$ \\
Exhalation & $82.9(15)$ & $79.3(17.4)$ & $-3.6(18.2)$ \\
Inhalation & $75.5(22.9)$ & $75.8(18.4)$ & $0.3(23.9)$ \\
Global score & $78.3(16.6)$ & $77.0(16.2)$ & $-1.3(17.5)$ \\
\hline
\end{tabular}

Table 6-4 Paired t-test comparisons for comfort score variables.

\begin{tabular}{lcc}
\hline \multirow{2}{*}{$\begin{array}{l}\text { Questionnaire } \\
\text { variable }\end{array}$} & \multicolumn{2}{c}{ VAS difference ResMed minus NHF/NIV } \\
\cline { 2 - 3 } Comfort & $-3.3(-11.4$ to 4.8$)$ & P-value \\
Coordination & $1.3(-6.3$ to 8.8$)$ & 0.41 \\
Exhalation & $-3.6(-10.4$ to 3.2$)$ & 0.73 \\
Inhalation & $0.3(-8.6$ to 9.2$)$ & 0.29 \\
\hline
\end{tabular}

\subsubsection{Temperature, noise and overall preference}

Table 6-5 shows a similar preference for both devices for those that noticed a difference in noise. A preference for NHF/NIV was observed in those who thought there was a difference in temperature. Overall, there was a higher proportion with a preference for NHF/NIV (18/30, 60\%) compared to ResMed $(5 / 30,16.7 \%)$ with $7 / 30(23.3 \%)$ having no preference. 
Table 6-5 Proportion of responses to preference questions.

\begin{tabular}{lrl}
\hline Questionnaire Variable & N/30 (\%) \\
\hline Preference overall? & NHF/NIV $18(60)$ \\
& ResMed $5(16.7)$ \\
& Neither $7(23.3)$ \\
\hline Did you notice a difference in Noise- Yes & $14(46.7)$ \\
Which did you prefer with respect to noise? & NHF/NIV & $7 / 14(50.0)$ \\
& ResMed & $6 / 14(42.9)$ \\
& Neither & $1(7.0)$ \\
\hline Did you notice a temperature difference? - Yes & & $9(30)$ \\
Did if affect comfort overall? - Yes & $3 / 9(33.3)$ \\
Which device did you prefer with respect to temperature? & \\
(N=5, N=4 missing) & NHF/NIV & $4 / 5(80)$ \\
& ResMed & $1 / 5(20)$ \\
\hline
\end{tabular}

\subsubsection{3 $\mathrm{Pt}_{\mathrm{t}} \mathrm{CO}_{2}$ change}

The time by treatment interaction was not significant; $P=0.97$, consistent with no evidence that the difference between NHF/NIV and ResMed varied with time (Figure 6-3). The mean (SD) $\mathrm{P}_{\mathrm{t}} \mathrm{CO}_{2}$ difference between ResMed and $\mathrm{NHF} / \mathrm{NIV}$ at $30 \mathrm{~min}$ was 0.2 (3.8) $\mathrm{mm} \mathrm{Hg}$ and when adjusted for baseline $\mathrm{P}_{\mathrm{t}} \mathrm{CO}_{2}$, the mean difference across all time points was $-0.61(-1.05$ to -0.17$)$ $\mathrm{mmHg}, \mathrm{P}=0.01$, indicating a greater $\mathrm{P}_{\mathrm{t}} \mathrm{CO}_{2}$ reduction with ResMed than NHF/NIV (Table 6-7). 


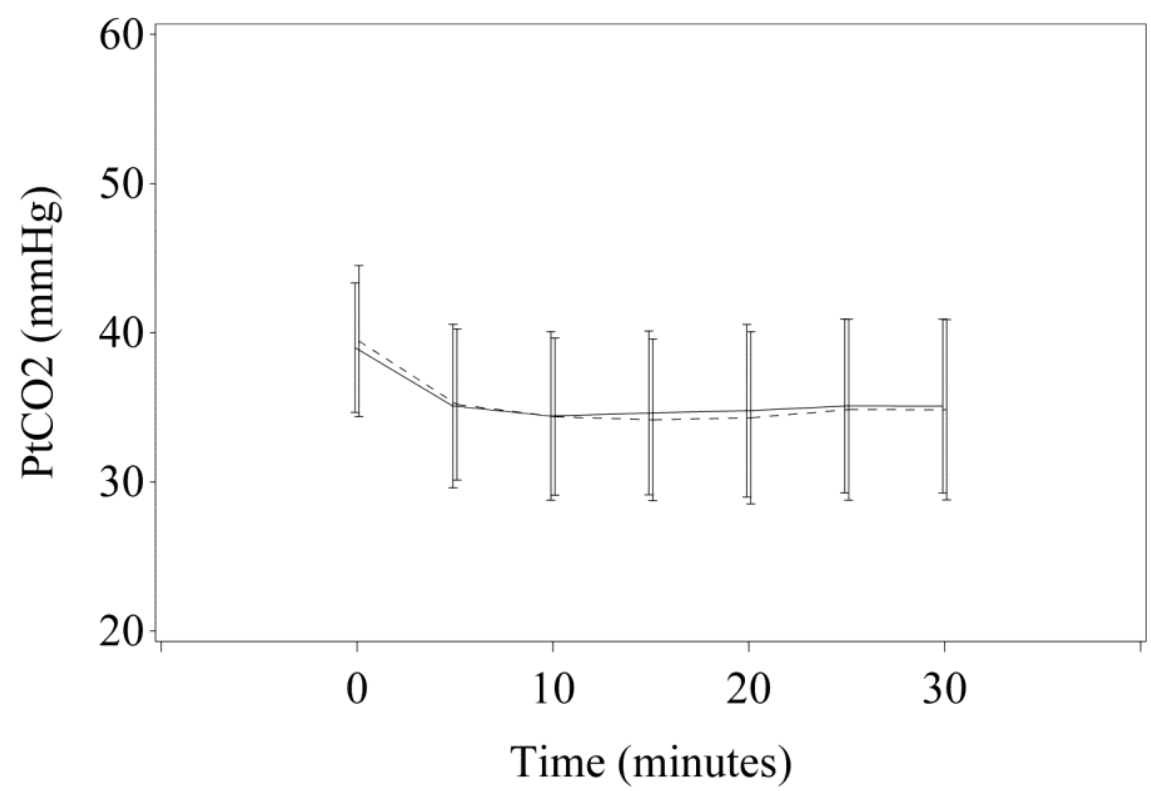

Figure 6-3 Line plot of mean $\mathrm{PtCO}_{2}$ over time for each intervention.

Error bars are plus and minus one standard deviation; solid line= NHF/NIV, dashed line=ResMed.

There was no evidence of a difference in the proportion of participants with a reduction of $\mathrm{P}_{\mathrm{t}} \mathrm{CO}_{2} \geq 4 \mathrm{mmHg}$ or $\geq 8 \mathrm{mmHg}$ for ResMed compared to NHF/NIV after 30 minutes. The proportion in ResMed with a reduction of $\geq 4 \mathrm{mmHg}$ was 14/30 (48.3\%) and for NHF/NIV 11/30 (37.9\%); absolute difference (95\% Cl) $10.3 \%(-7.1$ to 27.8$), \mathrm{P}=0.26$. Note that for this estimate the $95 \% \mathrm{Cl}$ was estimated by asymptotic methods but the P-value by an exact method. The proportion in ResMed with a reduction $\geq 8 \mathrm{mmHg}$ was $4 / 30$ (13.8\%) and for NHF/NIV 6/30 (20.7\%); absolute difference $-6.3 \%$ (-18.3 to 5.8), $P=0.32$.

Table 6-6 $\mathrm{PtCO}_{2}$ values and difference in $\mathrm{Pt}_{\mathrm{t}} \mathrm{CO}_{2}$ of ResMed minus NHF/NIV

\begin{tabular}{|c|c|c|c|c|c|}
\hline \multirow{2}{*}{$\begin{array}{l}\text { Time } \\
\text { point } \\
\text { (min) }\end{array}$} & \multicolumn{2}{|c|}{$\begin{array}{l}\mathrm{PtCO}_{2}(\mathrm{mmHg}) \\
\text { Mean (SD) }\end{array}$} & \multicolumn{3}{|c|}{$\begin{array}{c}\mathrm{PtCO}_{2}(\mathrm{mmHg}) \text { difference ResMed } \\
\text { minus NHF/NIV }\end{array}$} \\
\hline & NHF/NIV & ResMed & $\begin{array}{l}\text { Mean } \\
\text { (SD) }\end{array}$ & Median (IQR) & $\begin{array}{l}\text { Min } \\
\text { Max }\end{array}$ \\
\hline 0 & & & & & -2.2 to 3.2 \\
\hline 5 & & & 0.1 & & \\
\hline 10 & & & -0.1 & & \\
\hline 15 & $34.6(5.5)$ & $34.2(5.4)$ & $-0.5(2$ & $-0.2(-2.1$ & to 4 \\
\hline 20 & $18(58)$ & $34.3(5.8)$ & $-0.4(3.2)$ & $-0.3(-1.5$ & -7.5 to 5.4 \\
\hline 25 & $5.1(5.8)$ & $34.8(6.1)$ & $-0.2(3$ & $-0.2(-2.6$ to 1$)$ & -7.5 to 7.8 \\
\hline 30 & 35.1 (5.8) & $34.8(6)$ & $-0.2(3.8)$ & $0.2(-2.4$ to 2$)$ & -8 to 6.9 \\
\hline
\end{tabular}


Table 6-7 Mixed linear models for $\mathrm{P}_{\mathrm{t}} \mathrm{CO}_{2}$ change with zero time-point as a baseline co-variate.

\begin{tabular}{ccc}
\hline $\begin{array}{l}\text { Time point } \\
\text { (min) }\end{array}$ & $\mathbf{P}_{\mathbf{t}} \mathbf{C O}_{2}(\mathbf{m m H g})$ difference ResMed minus NHF/NIV \\
\cline { 2 - 3 } & Mean (95\% Cl) & P-value \\
\hline 0 & - & - \\
5 & $-0.27(-1.32$ to 0.78$)$ & 0.61 \\
10 & $-0.42(-1.48$ to 0.63$)$ & 0.43 \\
15 & $-0.84(-1.89$ to 0.22$)$ & 0.12 \\
20 & $-0.86(-1.92$ to 0.2$)$ & 0.11 \\
25 & $-0.64(-1.7$ to 0.43$)$ & 0.24 \\
30 & $-0.63(-1.69$ to 0.43$)$ & 0.24 \\
\hline Average over all & $-0.61(-1.05$ to -0.17$)$ & 0.01 \\
time points & \\
\hline
\end{tabular}

NHF: Nasal high flow; NIV: Non-invasive ventilation; $\mathrm{P}_{t} \mathrm{CO}_{2}$ : Transcutaneous partial pressure of carbon dioxide; SD: Standard deviation. * Main effects model.

\subsubsection{Respiratory rate}

The time by treatment interaction was not significance, $P=0.19$; consistent with no evidence of a difference in the difference between ResMed and NHF/NIV at any time point. Averaged across all time points, there was evidence of a higher respiratory rate with ResMed compared to NHF/NIV - a difference of 1.79 breaths per min $(95 \% \mathrm{Cl} 1.23$ to 2.36$), \mathrm{P}<0.001$. Mean (SD) respiratory rate at 30 minutes for ResMed and NHF/NIV respectively: 17.7 (5.4) versus 15.1 (4.0); difference $2.69(95 \% \mathrm{Cl} 1.32$ to 4.07$)$ breaths per minute, $\mathrm{P}<0.001$.

\subsubsection{Oxygen saturation and heart rate}

The time by treatment interaction was not significant for oxygen saturation or heart rate; $P=0.40$ and $P=0.29$ respectively. There was no difference in oxygen saturations between ResMed and NHF/NIV - Mean (SD) oxygen saturations at 30 minutes for ResMed and NHF/NIV respectively: 96.6\% (1.6) versus $97.0 \%$ (1.4); difference across all time points $-0.05 \%(-0.19$ to 0.09$)$ $\mathrm{P}=0.5$.

There was no evidence of a difference in overall heart rate between ResMed and NHF/NIV; mean difference $(95 \% \mathrm{Cl}$ ) for ResMed minus NHF/NIV -0.27 beats per minute $(95 \% \mathrm{Cl}-1.13$ to $0.59, \mathrm{P}=0.53)$. 


\subsubsection{Blood pressure}

Table 6-8 shows no difference in systolic, diastolic or mean arterial blood pressure between ResMed and NHF/NIV ( $P>0.05$ for each variable).

Table 6-8 Mixed linear models of blood pressure change with zero timepoint as a baseline covariate.

\begin{tabular}{lcc}
\hline Variable & ResMed minus NHF/NIV $(\mathbf{9 5 \%}$ Cl) & P \\
\hline Mean arterial pressure & $1.0(-2.5$ to 4.5$)$ & 0.56 \\
Diastolic blood pressure & $1.5(-2.8$ to 5.7$)$ & 0.49 \\
Systolic blood pressure & $0.2(-4.2$ to 4.5$)$ & 0.94 \\
\hline
\end{tabular}

\subsubsection{Patient Ventilator Synchrony}

A summary of device triggering (IPAP initiated) and cycling (IPAP changed to EPAP) are shown below. (Table 6-9 and Table 6-10). The true positives (TP), false positives (FP), and false negatives (FN) were used to quantify precisions and sensitivity results for each device which were closely matched between the 2 devices.

There was a difference in the proportion of auto-triggers $(0.9 \%$ for NHF/NIV vs $2.1 \%$ for ResMed) and auto-cycles $(0.7 \%$ for NHF/NIV vs $2.0 \%$ for ResMed). It was discovered after the study concluded that the ResMed default settings had reverted back to the default restricted inspiratory times of $T_{i}$ min $0.5 \mathrm{sec}$ and $T_{i} \max 1.5 \mathrm{sec}$, compared to the NHF/NIV device default settings which were unrestricted.

Table 6-9 Summary of device triggering.

\begin{tabular}{clllllll}
\hline Device & $\begin{array}{l}\text { Total } \\
\text { Breaths }\end{array}$ & $\begin{array}{l}\text { Normal } \\
\text { Triggers } \\
\text { (TP) }\end{array}$ & $\begin{array}{l}\text { Missed } \\
\text { Triggers } \\
\text { (FN) }\end{array}$ & $\begin{array}{l}\text { Auto } \\
\text { Trigger } \\
\text { (FP) }\end{array}$ & $\begin{array}{l}\text { Double } \\
\text { Trigger }\end{array}$ & $\begin{array}{l}\text { Precision } \\
\text { TP/(TP+FP) }\end{array}$ & $\begin{array}{l}\text { Sen } \\
\text { sitiv } \\
\text { ity } \\
\text { TP/( } \\
\text { TP+ } \\
\text { FN) }\end{array}$ \\
\hline NHF/NIV & 13267 & 13238 & 16 & 122 & 15 & 0.99 & 1.00 \\
ResMed & 14684 & 14617 & 4 & 304 & 63 & 0.98 & 1.00 \\
\hline TP: True Positive, FP; False Positive, FN; False Negative.
\end{tabular}


Table 6-10 Summary of device cycling.

\begin{tabular}{lllllll}
\hline Device & $\begin{array}{l}\text { Total } \\
\text { Breaths }\end{array}$ & $\begin{array}{l}\text { Normal } \\
\text { Cycles } \\
\text { (TP) }\end{array}$ & $\begin{array}{l}\text { Missed } \\
\text { Cycles } \\
\text { (FN) }\end{array}$ & $\begin{array}{l}\text { Auto } \\
\text { Cycles } \\
\text { (FP) }\end{array}$ & $\begin{array}{l}\text { Precision } \\
\text { TP/(TP+FP) }\end{array}$ & $\begin{array}{l}\text { Sensitivity } \\
\text { TP/(TP+FN) }\end{array}$ \\
\hline NHF/NIV & 13267 & 13292 & 3 & 98 & 0.99 & 1.00 \\
ResMed & 14684 & 14762 & 0 & 292 & 0.98 & 1.00 \\
\hline
\end{tabular}

TP: True Positive, FP; False Positive, FN; False Negative.

Further analyses were carried out to investigate the cause of the differences in auto-events (auto-triggering and/or auto-cycling) and if it was due to the different inspiratory time settings on the 2 devices. All participants had some breaths that were affected by a false trigger - either missed triggers, autotriggers or double-triggers, irrespective of the device used and would be expected with the variation in participant breathing patterns and within the limits of the devices. Given the low proportion of overall breaths affected by auto-events, a threshold of $5 \%$ breaths affected was selected to check if there was some explanation for the auto-events. Four participants had over $5 \%$ of their total breaths as false trigger breaths when using the ResMed device. Two of these 4 participants also had over $5 \%$ false cycle breaths.

Auto-cycling is likely to have a more noticeable influence on comfort. Analyses of these 2 participants' comfort scores, $\mathrm{P}_{\mathrm{t}} \mathrm{CO}_{2}$ and respiratory rate data at 30 min is shown in Table 6-11 to delineate if there was a pattern favouring NHF/NIV or ResMed. For the first participant, despite the NHF/NIV having an unrestricted inspiratory time, the respiratory rate change was actually -6 breaths per minute and corresponded with a $\mathrm{P}_{\mathrm{tCO}} \mathrm{CO}_{2}$ change of $7.2 \mathrm{mmHg}$ which is not what would be expected i.e. a reduced respiratory rate should result in a $\mathrm{P}_{\mathrm{t}} \mathrm{CO}_{2}$ rise. There was minimal difference in comfort scores, $\mathrm{P}_{\mathrm{t}} \mathrm{CO}_{2}$ or respiratory rate change in the other participant.

Table 6-11 Analyses of $\mathrm{P}_{\mathrm{t}} \mathrm{CO}_{2}$, respiratory rate and global comfort score in two participants with the highest auto-events.

\begin{tabular}{lcccccc}
\hline Participant & \multicolumn{2}{c}{$\begin{array}{c}\mathbf{P t C O}_{2} \text { change } \\
(\mathbf{m m H g})\end{array}$} & \multicolumn{2}{c}{$\begin{array}{c}\text { Resp rate } \\
\text { change } \\
\text { (breaths/min) }\end{array}$} & \multicolumn{3}{c}{$\begin{array}{c}\text { Global } \\
\text { comfort } \\
\text { score }\end{array}$} \\
\hline & ResMed & NHF/NIV & ResMed & NHF/NIV & ResMed & NHF/NIV \\
\hline $\begin{array}{l}\text { Participant } \\
\text { X }\end{array}$ & -1.9 & -7.2 & 1 & -6 & 48.5 & 99.25 \\
\hline $\begin{array}{l}\text { Participant } \\
\text { Y }\end{array}$ & -5.4 & -8.4 & 6 & -3 & 73 & 67.75 \\
\hline
\end{tabular}




\subsubsection{Mask moisture}

There was no evidence of a difference in the proportion of cases with moisture observed in the mask for ResMed compared to NHF/NIV. The proportion in ResMed with moisture observed was 14/30 (46.7\%) minus NHF/NIV 13/30 (43.3\%) - absolute difference $3.3 \%(95 \% \mathrm{Cl}-13.9$ to 20.6$), \mathrm{P}=0.71$.

\subsection{Discussion}

Comfort scores showed no significant difference between the ResMed and NHF/NIV devices in this randomised controlled cross-over study in patients with chronic respiratory diseases at risk of hypercapnic respiratory failure. The NHF/NIV device has been shown to trigger satisfactorily and produced similar clinical effects to the ResMed device while overall more patients preferred its use.

Limitations of the study relate to the generalisability of the findings. Patients with chronic respiratory diseases who would be treated with NIV if they were hypercapnic were sampled. This improved the internal validity of the study enabling patients to be in a stable condition and complete the study in a single visit with no drop-outs, however they were not hypercapnic or acutely unwell at the time which would be the usual clinical scenario for using NIV. Including some patients with previous experience of NIV $(20 \%)$ may have made their comfort scores more sensitive or may have prejudiced their scoring due to previous adverse experiences. The cross-over design of the trial will have mitigated this effect to some degree and their inclusion offered useful feedback, relating their present clinical trial experience to previous NIV experiences.

Similar methodological issues apply to the interpretation of the study findings as studies 2 and 5 with the SenTec monitor, rather than ABGs, providing continuous $\mathrm{P}_{\mathrm{t}} \mathrm{CO}_{2}$ measurement.

Supplemental oxygen was unable to be used in the prototype NHF/NIV device and patients with an oxygen requirement were excluded. While this conferred some degree of selection bias with the severity of patients, it was unavoidable due to the safety testing profile of the NHF/NIV device being incomplete. But, 
the lack of oxygen use did allow the $\mathrm{P}_{\mathrm{t}} \mathrm{CO}_{2}$ change to be unaffected by the confounding influence of oxygen.

Both devices were administered with default settings which while reflecting clinical practice, they differed slightly between devices and this may have influenced ventilation and comfort. While it is less likely to have been an issue in this cohort, participants who are acutely unwell may adapt differently to a restricted inspiratory time due to their altered breathing pattern and a future study in an acutely unwell group would require careful consideration of device settings and possibly the ability to change settings according to patient response.

Participants in the study had stable chronic respiratory diseases, as did participants in Study 5 and the $\mathrm{P}_{\mathrm{t}} \mathrm{CO}_{2}$ reduction of $4-5 \mathrm{mmHg}$ was very similar to the $\mathrm{P}_{\mathrm{t}} \mathrm{CO}_{2}$ reduction with NIV from Study 5, as was the maximum reduction occurring in the first $5 \mathrm{~min}$. Similar to Study 2 where a 20 min intervention was used, the $30 \mathrm{~min}$ in this trial was effective in demonstrating physiological changes with the devices but may not reflect the comfort and ventilation changes seen over a prolonged period. Generalising these findings therefore to acute disease management may produce different magnitudes of comfort score and physiological change although it would be expected to change similarly with both devices.

While most participants had COPD as their primary cause for potential HRF, obesity related respiratory diseases (OSA and OHS) and NMD were also included. Small sample sizes precluded sensitivity analyses to be undertaken to investigate the generalisability of the results according to each condition. Studies targeting each individual condition e.g. COPD, OSA etc would be required to draw conclusions about the comfort and efficacy of the NHF/NIV in these individual conditions. More confidence can be gained from the application of the results to COPD patients given they were the predominant group tested.

The comfort score used as the primary outcome measure was a simple, continuous scale that extrapolated the exact information required to interrogate the comfort of the device. While it had previously been used for 
internal testing by, it is difficult to compare scores against previous device comfort scores in the literature, although a continuous VAS is an accurate measure of subjective feelings towards an experience (Luria, 1975).

The application of the NHF/NIV device to clinical practice follows on from the results of Study 5. A possible clinical scenario ideally suited to the NHF/NIV device is a patient with AHRF who would initially receive bi-level therapy with the NHF/NIV device, but during the frequent therapy interruptions for nutrition, nebulisers and sputum expectoration, the mode could be switched to NHF. Likewise, during patient-requested breaks off NIV, the NHF mode could be utilised. Patients are therefore able to continually receive a $\mathrm{PCO}_{2}$-lowering therapy during these periods off NIV while extending the total time therapy is able to be tolerated. A clinical trial has recently been registered using NHF during breaks from NIV to investigate if total therapy time can be extended and outcomes such as NIV-free days and mortality improved (Ricard et al, 2018).

\subsection{Conclusion}

Bi-level therapy with both a dual NHF/NIV device and a standard ResMed device demonstrated no difference in comfort scores in people with stable chronic respiratory diseases. The ResMed device resulted in a slightly greater reduction in $\mathrm{P}_{\mathrm{t}} \mathrm{CO}_{2}$ and increase in respiratory rate compared with NHF/NIV that is unlikely to have clinical significance. The NHF/NIV device offers a novel, dual therapeutic option in hypercapnic respiratory failure. RCTs comparing the dual NHF/NIV device with a standard NIV device in people with AECOPD and acute hypercapnic respiratory failure over the course of 2-3 days are now required to investigate outcomes such as treatment failure, $\mathrm{PCO}_{2}$ change, hospital/ICU length of stay and mortality. 


\section{Discussion and future implications}

The central theme of this research has been assessing the efficacy of NHF in a variety of COPD clinical states. While there has been an increasing use of $\mathrm{NHF}$ in acute hypoxic respiratory failure, the evidence for NHF use in COPD was limited to a few studies, each with limitations to draw conclusions such as the use of supplementary oxygen, variability in flow rates and methodological inconsistencies.

Several knowledge gaps of NHF therapy in COPD were identified from the Chapter 1 introduction (Section 1.5), and were addressed as follows:

- Does NHF reduce $\mathrm{PCO}_{2}$ in stable COPD and if so, what is the flowresponse relationship of the $\mathrm{PCO}_{2}$ reduction?

To answer this question, I undertook Study 2 - a four-way cross-over RCT comparing NHF at $15 \mathrm{~L} / \mathrm{min}, 30 \mathrm{~L} / \mathrm{min}$ and $45 \mathrm{~L} / \mathrm{min}$ against a control of breathing room air in a controlled, stable COPD cohort. The findings of a modest, flow-dependent reduction in $\mathrm{P}_{\mathrm{t}} \mathrm{CO}_{2}$, up to $2.4 \mathrm{mmHg}$ with $\mathrm{NHF}$ at $45 \mathrm{~L} / \mathrm{min}$, were accompanied by a significant reduction in respiratory rate up to 5 breaths per minute and a well-tolerated therapy.

The small, flow-dependent reduction in $\mathrm{P}_{\mathrm{t}} \mathrm{CO}_{2}$ observed with the currently available NHF device across the range of flows used in clinical practice indicates that it is safe to use in COPD from a physiological perspective. This was important, both confirming previous research results and opening up further applications for NHF in COPD. With a marked reduction in respiratory rate and reported high level of tolerability, as well as the other mechanisms of action reported earlier such as improved mucociliary clearance, NHF has the potential to address the substantial clinical problem of AECOPD readmissions after hospital discharge, which was the next knowledge gap outlined in section 1.5:

- Does NHF therapy reduce hospital re-admissions in COPD patients who have been recently discharged from hospital?

To answer this question, I first needed to undertake Study 3, a feasibility study in AECOPD patients discharged from hospital who used NHF at home for 30 days. Testing the study design was important to aid planning of a future large, 
multi-centre RCT. While the study design proved feasible in New Zealand, it was not feasible in the US site and conclusions were limited to the New Zealand cohort. In NZ, the mean (SD) use of NHF at home over 30 days postdischarge was 1.6 (1.6) hrs and over half of participants reported they would use NHF at home indefinitely. NHF use at home following hospital discharge is a promising application of NHF, potentially addressing the vicious cycle of breathlessness-provoked anxiety and altered breathing patterns triggering a further AECOPD. The design of Study 3 was feasible in New Zealand and it is encouraging to see a very similar clinical trial currently underway in Melbourne, as well as the conclusions from Storgaard et al in LTOT patients of reduced exacerbation rates and improved quality of life. As domiciliary ventilatory support gains more traction in COPD with CHRF, I would expect further studies applying NHF in this setting to emerge.

The next application for NHF was translating the findings into an acute clinical setting. While the $\mathrm{P}_{\mathrm{t}} \mathrm{CO}_{2}$ reduction demonstrated in Study 2 was modest and lacked the magnitude for a safe, direct comparison to NIV in AHRF, it demonstrated a satisfactory safety signal to address the next knowledge gap outlined in section 1.5:

- Does NHF use in AHRF in ED reduce the requirement for subsequent NIV?

To answer this question, I first needed to undertake Study 4 - a feasibility study to determine if COPD with AHRF and a mild to moderate acidosis could be managed in the ED in a standardised manner. The results would feed the design of a large multi-centre RCT addressing the knowledge gap identified in section 1.5 of an effective intervention to optimise titrated oxygen therapy in the first hour of AHRF presentation. Logistical issues proved the study design to not be feasible, with difficulty in adherence to a standard of care protocol in the high pressure environment of ED. Factors such as multiple ED personnel contributed to difficulty adhering to an agreed usual care protocol, with 3 patients being eligible over a 5 month period and only 1 participant completing the $60 \mathrm{~min}$ optimisation period. The results were similar to findings from a previous RCT investigating NHF in acute hypoxic respiratory in the ED, demonstrating difficulty in recruiting patients in the ED environment (P. G. 
Jones et al., 2016). The application of NHF in the early stages of AHRF remains unknown. While the design of Study 4 was not feasible in Wellington, it may be feasible in another centre and I think it warrants further attempts, with my suggestions provided earlier of embedding research staff in the ED. Reducing the requirement for NIV, will improve clinical outcomes for these patients as well as significant cost savings for hospitals. An argument could be made for simply paying closer attention to titrating oxygen therapy in this first 1 hour of presentation could prevent the harms of oxygen-induced hypercapnia, hence reduce NIV requirement. However, with the emerging evidence of NHF being both a well-tolerated method of oxygen delivery, simple to set-up as well as being an effective $\mathrm{PCO}_{2}$ lowering therapy, future studies in this area deserve attention.

While developing a protocol for optimising therapy in COPD with AHRF proved to not be feasible in Study 4, the next application of NHF was to directly compare NHF with NIV, a knowledge gap identified in section 1.5:

- What is the magnitude of $\mathrm{PCO}_{2}$ reduction with $\mathrm{NHF}$ compared to NIV?

To answer this question, I undertook Study 5 - a two-way cross-over RCT of Nasal High Flow therapy compared with Non-invasive ventilation in COPD patients with chronic respiratory failure. Study 5 was undertaken in the controlled, stable, outpatient setting in participants with chronic HRF rather than the acute HRF setting which was inappropriately risky given the available evidence for lowering $\mathrm{PCO}_{2}$ with NHF. A similar mean (SD) $\mathrm{P}_{\mathrm{t}} \mathrm{CO}_{2}$ reduction to Study 2 was seen with NHF at $45 \mathrm{~L} / \mathrm{min}$ of -2.5 (3.5) $\mathrm{mmHg}$, and while this was less than the $\mathrm{P}_{\mathrm{t}} \mathrm{CO}_{2}$ reduction seen with NIV of $-5.3(5.0) \mathrm{mmHg}$ $(P=0.016)$, NHF demonstrated superior tolerability. The small difference in $\mathrm{P}_{\mathrm{t}} \mathrm{CO}_{2}$ reduction between the 2 devices is of uncertain clinical significance and demonstrates a potential role for NHF in AHRF for those patients who cannot tolerate NIV or during breaks in NIV therapy. Also, real-world outcomes (effectiveness) of NHF compared to NIV may be different to the efficacy results seen in a clinical trial. The overriding conclusion from Study 5 was that the efficacy of $\mathrm{NHF}$ in reducing $\mathrm{PCO}_{2}$ in COPD suggests it being used as an adjunct to NIV, rather than as first-line treatment in AHRF. 
Following on from this conclusion then relates to the final knowledge gap identified in Chapter 1 - identifying an AHRF therapy that is efficacious and tolerable so that continuous $\mathrm{PCO}_{2}$-lowering therapy can be applied, extending the overall AHRF treatment time. The question to be answered was:

- How can the tolerability of NIV therapy be improved in order to extend overall treatment time in HRF?

To answer this question, I completed Study 6 - a two-way cross-over RCT comparing the NIV mode of a dual NHF/NIV device with a standard NIV device in participants with stable chronic respiratory diseases at risk of AHRF. A dual NHF/NIV device would utilise the strengths of both NHF and NIV functions NHF providing a modest $\mathrm{P}_{\mathrm{t}} \mathrm{CO}_{2}$ reducing ability but being well-tolerated, and NIV providing proven $\mathrm{P}_{\mathrm{t}} \mathrm{CO}_{2}$ lowering ability but limited tolerability. Study 6 provided preliminary data to confirm similar comfort scores of the NIV mode of a dual NHF/NIV device to a standard NIV device (mean difference of $1.3 \mathrm{~mm}$ on a $100 \mathrm{~mm}$ VAS, $95 \% \mathrm{Cl}-7.9$ to $5.2, \mathrm{P}$ value for non-inferiority $<0.001$, therefore no evidence the mean comfort score was greater than $25 \mathrm{~mm}$ different). With proven comfort of the NIV mode of the dual NHF/NIV device, the future direction of research will need to compare it directly with NIV in the AHRF setting. The clinical trial (Ricard et al., 2018) currently recruiting, discussed in Chapter 7, may provide evidence of NHF efficacy when used as an adjunct with NIV in AHRF.

Interpreting all of the thesis results, especially translating the findings from stable, COPD outpatients to the acute clinical setting is essential to understand the context of this body of research. The study design of carrying out 3 trials in outpatients in this thesis was to ensure participant safety given NHF was untested in clinical trials involving AHRF patients, but the future direction of NHF in COPD will rely on translation of these research findings into acute care. Also, more research emphasis may need to be placed on NHF in "effectiveness" rather than "efficacy" trials because the clinical scenario where NIV has failed due to tolerability and patient agitation for example, is challenging to incorporate into a clinical trial.

The key components of designing future NHF clinical trials will require outlining a research question, defining a study population, creating a study 
aim/hypothesis and then defining a primary outcome measure. This thesis proposes NHF has a broad application in COPD treatment - both in the acute and domiciliary setting. While the results from this thesis do not provide sufficient evidence to justify NHF as first-line treatment in AHRF, further research could take 3 directions.

Firstly, for the dual NHF/NIV device to be investigated further, it needs to be compared to standard NIV in the management of AECOPD with AHRF (study population) over 2-3 days. The research question would be "does the dual NHF/NIV device improve overall outcomes in AHRF?" Outcome measures would include length of HDU/ICU stay, rates of IMV (treatment failure), and mortality, as well as tolerability scores, length of time using each device and $\mathrm{P}_{\mathrm{a}} \mathrm{CO}_{2}$ and $\mathrm{pH}$ change.

Secondly, NHF therapy alone could be compared directly to NIV in AECOPD with AHRF but there would need to be strict safety backstops. One such clinical trial may be to use NIV for the first 4-6 hours after presentation and then randomised to continue NIV or change to NHF. Another way to structure the study would be for all AHRF patients to receive NIV but during set rest periods, then randomised to receive NHF or standard therapy. The primary outcome measure would be the rates of IMV i.e. treatment failure, and other outcome measures would be the total length of time the therapy is used, essential to overall success, and may favour those interventions involving NHF due to its superior tolerability.

Thirdly, the application of NHF in COPD patients on LTOT with CHRF was gaining more attention in 2017/18 with the publication of Nagata et al and Storgaard et al, in the context of increasing attention for domiciliary use of NIV in CHRF. However, there is only 1 published trial comparing long-term home use of NHF against NIV (Bräunlich et al., 2015) and 2 proof of concept shortterm studies (Bräunlich \& Köhler, 2016; Pisani et al., 2017). The most recent home NIV study (P. B. Murphy et al., 2017) showed a mortality benefit with NIV and improved QOL scores despite a modest non-significant $\mathrm{PaCO}_{2}$ difference of $3.0 \mathrm{mmHg}$. A well-designed, long-term NHF domiciliary study may show equivalence to home NIV with respect to $\mathrm{PaCO}_{2}$ change, mortality, hospitalisation rates as well as the critical measure of QOL scores. It would 
stand to reason that if NHF is more tolerable than NIV in the domiciliary setting, it is more likely to be used and produce superior outcomes due to prolonged use. Close attention to measure compliance and methods to overcome noncompliance is essential to maximise uptake of the therapy.

In conclusion, this thesis has demonstrated NHF therapy reduces $\mathrm{PCO}_{2}$ by a modest amount and in a flow-dependent manner in stable COPD. There was some evidence of a reduction in respiratory rate also, suggesting promise in the acute setting with the mechanisms of action NHF capable of combatting the dynamic hyperinflation and expiratory flow limitation of AECOPD. Assisting recovery post-discharge from hospital with AECOPD is another application of NHF which I believe has excellent capability. However, there is insufficient clinical trial evidence for NHF to be used as first line treatment in AECOPD with AHRF. The explanation for this is most probably due to an inability to match the superior pressure-support of NIV. The emergence of a dual device which can deliver both NIV and NHF has the potential to be the ultimate therapy in AHRF by providing the strengths of both NHF and NIV. 


\section{References}

Abdo, W. F., \& Heunks, L. M. A. (2012). Oxygen-induced hypercapnia in COPD: myths and facts. Critical Care, 16(5), 323.

Adams, C. F., Geoghegan, P. H., Spence, C. J., \& Jermy, M. C. (2018). Modelling nasal high flow therapy effects on upper airway resistance and resistive work of breathing. Respiratory Physiology and Neurobiology, 254, 23-29.

Adeloye, D., Chua, S., Lee, C., Basquill, C., Papana, A., Theodoratou, E., ... Rudan, I. (2015). Global and regional estimates of COPD prevalence: Systematic review and meta-analysis. Journal of Global Health, 5(2): 020415.

Aldrich, T. K., Hendler, J. M., Vizioli, L. D., Park, M., Multz, A. S., \& Shapiro, S. M. (1993). Intrinsic Positive End-Expiratory Pressure in Ambulatory Patients with Airways Obstruction. American Review of Respiratory Disease, 147(4), 845-849.

Alleway, R., Butt, A., Ellis, D., Freeth, H., Gomez, R., Jarman, D., ... Warsame, A. (2017). Inspiring Change. A review of the quality of care provided to patients receiving acute non-invasive ventilation. National Confidential Enquiry into Patient Outcome and Death (NCEPOD).

Almagro P, Calbo E, Ochoa de Echaguen A, Barreiro B, Quinatana S, Heredia JL, et al. (2002). Mortality after hospitalization for COPD. Chest, 121, 1441-1448.

Ambrosino, N., Bruletti, G., Scala, V., Porta, R., \& Vitacca, M. (2002). Cognitive and perceived health status in patient with chronic obstructive pulmonary disease surviving acute on chronic respiratory failure: a controlled study. Intensive Care Med, 28(2), 170-177.

Ambrosino, N., Foglio, K., Rubini, F., Clini, E., Nava, S., \& Vitacca, M. (1995). Non-invasive mechanical ventilation in acute respiratory failure due to chronic obstructive pulmonary disease: correlates for success. Thorax, 50, 755-757.

Anthonisen, N. R., Skeans, M. a, Wise, R. a, Manfreda, J., Kanner, R. E., \& Connett, J. E. (2005). The effects of a smoking cessation intervention on 14.5-year mortality. Annals of Internal Medicine, 143(8), 615.

Antonelli, M., Pennisi, M. A., \& Montini, L. (2005). Clinical review: Noninvasive ventilation in the clinical setting--experience from the past 10 years. Critical Care, 9(1), 98-103.

Antonicelli, F., Cataldo, A., Festa Fldone, R., Moccaldo, A., Antonelli, M., \& Maggiore, S. M. (2011). High-flow oxygen therapy through nasal cannulae versus low-flow oxygen therapy via Venturi mask after extubation in adult, critically ill patients. Critical Care., 15(Suppl 1), P165.

Arnold, T. D. W., Miller, M., Van Wessem, K. P., Evans, J. A., \& Balogh, Z. J. (2011). Base deficit from the first peripheral venous sample: A surrogate for arterial base deficit in the trauma bay. Journal of Trauma - Injury, Infection and Critical Care, 71(4), 793-797. 
Assad, N. A., Balmes, J., Mehta, S., Cheema, U., \& Sood, A. (2015). Chronic obstructive pulmonary disease secondary to household air pollution. Seminars in Respiratory and Critical Care Medicine, 36(3), 408-421.

Aubier M, Murciano D, Milic-Emili J, Touaty E, Daghfous J, Pariente R, D. J. (1980). Effects of the administration of O2 on ventilation and blood gases in patients with chronic obstructive pulmonary disease during acute respiratory failure. American Review of Respiratory Disease, 122(5), 747-754.

Auerbach, A. D., Kripalani, S., Vasilevskis, E. E., Sehgal, N., Lindenauer, P. K., Metlay, J. P., ... Schnipper, J. L. (2016). Preventability and causes of readmissions in a national cohort of general medicine patients. JAMA Internal Medicine, 176(4), 484-493.

Austin, M. A., Wills, K. E., Blizzard, L., Walters, E. H., \& Wood-Baker, R. (2010). Effect of high flow oxygen on mortality in chronic obstructive pulmonary disease patients in prehospital setting: randomised controlled trial. BMJ, 341, c5462.

Bafadhel, M., McKenna, S., Terry, S., Mistry, V., Reid, C., Haldar, P., ... Brightling, C. E. (2011). Acute exacerbations of chronic obstructive pulmonary disease: Identification of biologic clusters and their biomarkers. American Journal of Respiratory and Critical Care Medicine, 184(6), 662-671.

Bahadori, K., Fitzgerald, J. M., \& Fitzgerald, M. (2007). Risk factors of hospitalization and readmission of patients with COPD exacerbation systematic review. International Journal of COPD, 2(3), 241-251.

BaHammam, A. S., Singh, T. D., Gupta, R., \& Pandi-Perumal, S. R. (2017). Choosing the Proper Interface for Positive Airway Pressure Therapy in Subjects With Acute Respiratory Failure. Respiratory Care, 63(2), 227237.

Baird, A. G., \& Lawrence, J. R. (2014). Guidelines: Is bigger better? A review of SIGN guidelines. BMJ Open, 4(e004278).

Barbosa, R. C. C., Carvalho, C. R. F. de, \& Moriya, H. T. (2012). Respiratory inductive plethysmography: a comparative study between isovolume maneuver calibration and qualitative diagnostic calibration in healthy volunteers assessed in different positions. Jornal Brasileiro de Pneumologia : Publicaçäo Oficial Da Sociedade Brasileira de Pneumologia e Tisilogia, (In English) 38(2), 194-201.

Barnes, P. J., Burney, P. G. J., Silverman, E. K., Celli, B. R., Vestbo, J., Wedzicha, J. A., \& Wouters, E. F. M. (2015). Chronic obstructive pulmonary disease. Nature Reviews Disease Primers, (December), 15076.

Bazuaye, E. A., Stone, T. N., Corris, P. A., \& Gibson, G. J. (1992). Variability of inspired oxygen concentration with nasal cannulas. Thorax, 47(8), 609-611.

Beggs, S., Zh, W., Kaul, S., Kj, O., \& Jae, W. (2014). High-flow nasal cannula therapy for infants with bronchiolitis ( Review ). Cochrane Database of Systematic Reviews, 1, 1-11. 
Begin, P., \& Grassino, A. (1991). Inspiratory muscle dysfunction and chronic hypercapnia in chronic obstructive pulmonary disease. Am Rev Respir Dis, 143(5 Pt 1), 905-912.

Bell, N., Hutchinson, C. L., Green, T. C., Rogan, E., Bein, K. J., \& Dinh, M. M. (2015). Randomised control trial of humidified high flow nasal cannulae versus standard oxygen in the emergency department. EMA Emergency Medicine Australasia, 27(6), 537-541.

Benbassat, J., \& Taragin, M. (2000). Hospital readmissions as a measure of quality of health care: Advantages and limitations. Archives of Internal Medicine, 160(8), 1074-1081.

Bendjelid, K., Schutz, N., Stotz, M., Gerard, I., Suter, P. M., \& Romand, J. A. (2005). Transcutaneous PCO2 monitoring in critically ill adults: clinical evaluation of a new sensor. Crit Care Med, 33, 2203-2206.

Benhamou, D., Girault, C., Faure, C., Portier, F., \& Muir, J. F. (1992). Nasal mask ventilation in acute respiratory failure; Experience in elderly patients. Chest, 102(3), 912-917.

Biselli, P. J. C., Kirkness, J. P., Grote, L., Fricke, K., Schwartz, A. R., Smith, P., \& Schneider, H. (2017). Nasal high-flow therapy reduces work of breathing compared with oxygen during sleep in COPD and smoking controls: a prospective observational study. Journal of Applied Physiology, 122(1), 82-88.

Bohr, C. (1891). Ueber die Lungenathmung. Skandinavisches Archiv Physiologie, 2(1), 236-268.

Borg, G. A. V. (1982). Psychophysical bases of percieved exertion. Medicine and Science in Sports and Exercise, 14(5), 377-381.

Bott, J., Carroll, M. P., Conway, J. H., Keilty, S. E., Ward, E. M., Brown, A. M., ... et al. (1993). Randomised controlled trial of nasal ventilation in acute ventilatory failure due to chronic obstructive airways disease. Lancet, 341(8860), 1555-1557.

Braunlich, J., Beyer, D., Mai, D., Hammerschmidt, S., Seyfarth, H. J., \& Wirtz, H. (2013). Effects of nasal high flow on ventilation in volunteers, COPD and idiopathic pulmonary fibrosis patients. Respiration, 85(4), 319-325.

Bräunlich, J., Goldner, F., \& Wirtz, H. (2017). Nasal high-flow eliminates $\mathrm{CO} 2$ from lower airways. Respiratory Physiology and Neurobiology, 242, 86-88.

Bräunlich, J., \& Köhler, M. (2016). Nasal highflow improves ventilation in patients with COPD. Int J Chron Ob Pulm Dis. (11);1077-1085.

Bräunlich, J., Mauersberger, F., \& Wirtz, H. (2018). Effectiveness of nasal highflow in hypercapnic COPD patients is flow and leakage dependent. BMC Pulmonary Medicine, 18(1), 14.

Bräunlich, J., Seyfarth, H.-J., \& Wirtz, H. (2015). Nasal High-flow versus noninvasive ventilation in stable hypercapnic COPD: a preliminary report. Multidisciplinary Respiratory Medicine, 10(1), 27. 
Bräunlich, J., \& Wirtz, H. (2017). NHF and hypercapnia: How brief can you look? Respirology, 22(6), 1049-1050.

Briscoe, W. A., \& Nash, E. S. (1965). The Slow Space in Chronic Obstructive Pulmonary Diseases. Annals of the New York Academy of Sciences, 121, 706-722.

Brochard, L., Isabey, D., Piquet, J., Amaro, P., Mancebo, J., Messadi, A. A., ... Harf, A. (1990). Reversal of acute exacerbations of chronic obstructive lung disease by inspiratory assistance with a face mask. The New England Journal of Medicine, 323(22), 1523-1530.

Brochard, L., Mancebo, J., Wysocki, M., Lofaso, F., Conti, G., Rauss, A., ... et al. (1995). Noninvasive ventilation for acute exacerbations of chronic obstructive pulmonary disease. N Engl J Med, 333(13), 817-822.

Broseghini, C., Brandolese, R., Poggi, R., Polese, G., Manzin, E., Milic-Emili, J., \& Rossi, A. (1988). Respiratory mechanics during the first day of mechanical ventilation in patients with pulmonary edema and chronic airway obstruction. American Review of Respiratory Disease, 138(2), 355-361.

Buist, A. S., McBurnie, M. A., Vollmer, W. M., Gillespie, S., Burney, P., Mannino, D. M., ... Nizankowska-Mogilnicka, E. (2007). International variation in the prevalence of COPD (The BOLD Study): a populationbased prevalence study. Lancet, 370(9589), 741-750.

Byrne, A. L., Bennett, M., Chatterji, R., Symons, R., Pace, N. L., \& Thomas, P. S. (2014). Peripheral venous and arterial blood gas analysis in adults: Are they comparable? A systematic review and meta-analysis. Respirology, 19(2), 168-175.

Cabrini, L., Landoni, G., Bocchino, S., Lembo, R., Monti, G., Greco, M., ... Zangrillo, A. (2016). Long-Term Survival Rate in Patients with Acute Respiratory Failure Treated with Noninvasive Ventilation in Ordinary Wards. Critical Care Medicine, 44(12), 2139-2144.

Calverley, P. M. A. (2003). Respiratory failure in chronic obstructive pulmonary disease. European Respiratory Journal, 22(Supplement 47), $26 s-30 s$.

Calverley, P. M. A., Anderson, J. A., Celli, B., Ferguson, G. T., Jenkins, C., Jones, P. W., ... TORCH investigators. (2007). Salmeterol and fluticasone propionate and survival in chronic obstructive pulmonary disease. The New England Journal of Medicine, 356(8), 775-789.

Cameron, L., Pilcher, J., Weatherall, M., Beasley, R., \& Perrin, K. (2012). The risk of serious adverse outcomes associated with hypoxaemia and hyperoxaemia in acute exacerbations of COPD. Postgrad Med J, 88(1046), 684-689.

Carlucci, A., Richard, J. C., Wysocki, M., Lepage, E., \& Brochard, L. (2001). Noninvasive versus conventional mechanical ventilation: An epidemiologic survey. American Journal of Respiratory and Critical Care Medicine, 163(4), 874-880.

Carone, M., Patessio, A., Ambrosino, N., Baiardi, P., Balbi, B., Balzano, G., 
... Spanevello, A. (2007). Efficacy of pulmonary rehabilitation in chronic respiratory failure (CRF) due to chronic obstructive pulmonary disease (COPD): The Maugeri Study. Respiratory Medicine, 101(12), 24472453.

Carratalá Perales, J. M., Llorens, P., Brouzet, B., Albert Jiménez, A. R., Fernández-Cañadas, J. M., Carbajosa Dalmau, J., ... Ramos Forner, S. (2011). High-Flow Therapy via Nasal Cannula in Acute Heart Failure. Revista Española de Cardiología (English Edition), 64(8), 723-725.

Carron, M., Freo, U., Bahammam, A. S., Dellweg, D., Guarracino, F., Cosentini, R., ... Esquinas, A. (2013). Complications of non-invasive ventilation techniques: A comprehensive qualitative review of randomized trials. British Journal of Anaesthesia, 110(6), 896-914.

Chanques, G., Riboulet, F., Molinari, N., Carr, J., Jung, B., Prades, A., ... Jaber, S. (2013). Comparison of three high flow oxygen therapy delivery devices: A clinical physiological cross-over study. Minerva Anestesiologica, 79(12), 1344-1355.

Chatila, W., Nugent, T., Vance, G., Gaughan, J., \& Criner, G. J. (2004). The effects of high-flow vs low-flow oxygen on exercise in advanced obstructive airways disease. Chest, 126(4), 1108-1115.

Chidekel, A., Zhu, Y., Wang, J., Mosko, J. J., Rodriguez, E., \& Shaffer, T. H. (2012). The effects of gas humidification with High-flow nasal cannula on cultured human airway epithelial cells. Pulmonary Medicine, ePub Sept (380686).

Claure, N., D'Ugard, C., \& Bancalari, E. (2003). Elimination of ventilator dead space during synchronized ventilation in premature infants. Journal of Pediatrics, 143(3), 315-320.

Cloutier, M. M. (2007). Respiratory physiology. Philadelphia, PA : Mosby Elsevier.

Cms.gov/Medicare. (2016). Readmissions reduction program.

Code of Health and Disability Services Consumers' Rights (2009): Right 7 (4) of the code . (n.d.).

Collaborative Research Group of Noninvasive Mechanical Ventilation for COPD. (2005). Early use of non-invasive positive pressure ventilation for acute exacerbations of chronic obstructive pulmonary disease: a multicentre randomized controlled trial. Chinese Medical Journal, 118(24), 2034.

Collins, J. A., Rudenski, A., Gibson, J., Howard, L., \& O’Driscoll, R. (2015). Relating oxygen partial pressure, saturation and content: The haemoglobin-oxygen dissociation curve. Breathe, 11(3), 194-201.

Confalonieri, M., Garuti, G., Cattaruzza, M. S., Osborn, J. F., Antonelli, M., Conti, G., ... Vitacca, M. (2005). A chart of failure risk for noninvasive ventilation in patients with COPD exacerbation. European Respiratory Journal, 25(2), 348-355.

Connors, A. F., Dawson, N. V, Thomas, C., Harrell, F. E., Desbiens, N., Fulkerson, W. J., ... Knaus, W. A. (1996). Outcomes following acute 
exacerbation of severe chronic obstructive lung disease. American Journal of Respiratory and Critical Care Medicine, 154(4), 959-967.

Conti, G., Antonelli, M., Navalesi, P., Rocco, M., Bufi, M., Spadetta, G., \& Meduri, G. U. (2002). Noninvasive vs. conventional mechanical ventilation in patients with chronic obstructive pulmonary disease after failure of medical treatment in the ward: A randomized trial. Intensive Care Medicine, 28(12), 1701-1707.

Conway A, Tipton E, Liu W, et al. (2018). Accuracy and precision of transcutaneous carbon dioxide monitoring: a systematic review and meta-analysis. Thorax, Feb 74(2), 157-163.

Corley, A., Bull, T., Spooner, A. J., Barnett, A. G., \& Fraser, J. F. (2015). Direct extubation onto high-flow nasal cannulae post-cardiac surgery versus standard treatment in patients with a $\mathrm{BMI} \geq 30$ : a randomised controlled trial. Intensive Care Medicine, 41(5), 887-894.

Corley, A., Caruana, L. R., Barnett, A. G., Tronstad, O., \& Fraser, J. F. (2011). Oxygen delivery through high-flow nasal cannulae increase endexpiratory lung volume and reduce respiratory rate in post-cardiac surgical patients. Br J Anaesth, 107(6), 998-1004.

Corley, A., Cm, R., Lm, A., Johnston, A., Barnett, A., Jf, F., ... Af, S. (2017). High-flow nasal cannulae for respiratory support in adult intensive care patients (Review). Cochrane Database of Systematic Reviews, (5), 188.

Costello, R., Deegan, P., Fitzpatrick, M., \& McNicholas, W. T. (1997). Reversible hypercapnia in chronic obstructive pulmonary disease: A distinct pattern of respiratory failure with a favorable prognosis. American Journal of Medicine, 102(3), 239-244.

Coudroy, R., Jamet, A., Petua, P., Robert, R., Frat, J.-P., \& Thille, A. W. (2016). High-flow nasal cannula oxygen therapy versus noninvasive ventilation in immunocompromised patients with acute respiratory failure: an observational cohort study. Annals of Intensive Care, 6(1), 45.

Cranston, J. M., Crockett, A., Moss, J., \& Alpers, J. H. (2005). Domiciliary oxygen for chronic obstructive pulmonary disease. In Cochrane Database of Systematic Reviews, 19(4), CD001744.

Cuquemelle, E., Pham, T., Papon, J.-F., Louis, B., Danin, P.-E., \& Brochard, L. (2012). Heated and Humidified High-Flow Oxygen Therapy Reduces Discomfort During Hypoxemic Respiratory Failure. Respiratory Care, 57(10), 1571-1577.

Danan, C., Dassieu, G., Janaud, J. C., \& Brochard, L. (1996). Efficacy of dead-space washout in mechanically ventilated premature newborns. American Journal of Respiratory and Critical Care Medicine, 153(5), 1571-1576.

Dassieu, G., Brochard, L., Agudze, E., Patkaï, J., Janaud, J. C., \& Danan, C. (1998). Continuous tracheal gas insufflation enables a volume reduction strategy in hyaline membrane disease: Technical aspects and clinical results. Intensive Care Medicine, 24(10), 1076-1082. 
Davidson, A. C., Banham, S., Elliott, M., Kennedy, D., Gelder, C., Glossop, A., ... Thomas, L. (2016). BTS/ICS guideline for the ventilatory management of acute hypercapnic respiratory failure in adults. Thorax, 71(Suppl 2), ii1-ii35.

Davies, M. (2013). British Thoracic Society NIV Audit 2013, (March), 34-35.

Davies, M. W., \& Woodgate, P. G. (2002). Tracheal gas insufflation for the prevention of morbidity and mortality in mechanically ventilated newborn infants. Cochrane Database of Systematic Reviews, (2), CD002973.

Dewan, N. A., \& Bell, C. W. (1994). Effect of low flow and high flow oxygen delivery on exercise tolerance and sensation of dyspnea: A study comparing the transtracheal catheter and nasal prongs. Chest, 105(4), 1061-1065.

Diaz-Lobato, S., Folgado, M. A., Chapa, A., \& Mayoralas Alises, S. (2013). Efficacy of High-Flow Oxygen by Nasal Cannula With Active Humidification in a Patient With Acute Respiratory Failure of Neuromuscular Origin. Respiratory Care, 58(12), e164-e167.

Dodd, J. W., Getov, S. V., \& Jones, P. W. (2010). Cognitive function in COPD. European Respiratory Journal, 35(4), 913-922.

Domingo, C., Canturri, E., Lujan, M., Moreno, A., Espuelas, H., \& Marin, A. (2006). Transcutaneous measurement of partial pressure of carbon dioxide and oxygen saturation: validation of the SenTec monitor. Arch Bronconeumol, 42(5), 246-251.

Domingo, C., Canturri, E., Moreno, A., Espuelas, H., Vigil, L., \& Luján, M. (2010). Optimal clinical time for reliable measurement of transcutaneous $\mathrm{CO} 2$ with ear probes: Counterbalancing overshoot and the vasodilatation effect. Sensors, 10(1), 491-500.

Dreher, M., Neuzeret, P., Windisch, W., Busam, D., Hoheisel, G. B., Gröschel, A., \& Woehrle, H. (2018). Prevalence of Stable Hypercapnic Chronic Obstructive Pulmonary Disease ( COPD ) - Preliminary Data from the Homevent Registry. American Journal of Respiratory and Critical Care Medicine, B4O(197), 3265.

Duong, M. L., Islam, S., Rangarajan, S., Teo, K., O'Byrne, P. M., Schünemann, H. J., ... Yusuf, S. (2013). Global differences in lung function by region (PURE): An international, community-based prospective study. The Lancet Respiratory Medicine, 1(8), 599-609.

Dysart, K., Miller, T. L., Wolfson, M. R., \& Shaffer, T. H. (2009). Research in high flow therapy: mechanisms of action. Respir Med, 103(10), 14001405.

Edwards, L., Perrin, K., Williams, M., Weatherall, M., \& Beasley, R. (2012). Randomised controlled crossover trial of the effect on PtCO2 of oxygendriven versus air-driven nebulisers in severe chronic obstructive pulmonary disease. Emergency Medicine Journal : EMJ, 29(11), 894898.

Elixhauser, A., Au, D. H., \& Podulka, J. (2006). Readmissions for Chronic Obstructive Pulmonary Disease, 2008: Statistical Brief \#121. Healthcare 
Cost and Utilization Project (HCUP) Statistical Briefs.

Enghoff, H. (1938). Volumen inefficax. Uppsala Lakaref. Forhand, 44(191).

Epstein, A. S., Hartridge-Lambert, S. K., Ramaker, J. S., Voigt, L. P., \& Portlock, C. S. (2011). Humidified High-Flow Nasal Oxygen Utilization in Patients with Cancer at Memorial Sloan-Kettering Cancer Center. Journal of Palliative Medicine, 4(7), 835-838.

Ezzati, M. (2005). Indoor air pollution and health in developing countries. Lancet, 366(9480), 104-106.

Fields, L. M., \& Calvert, J. D. (2015). Informed consent procedures with cognitively impaired patients: A review of ethics and best practices. Psychiatry and Clinical Neurosciences, 69(8), 462-471.

Fingleton, J., McKinstry, S., Pilcher, J., Weatherall, M., Beasley, R., \& Bardsley, G. (2017). Accuracy of transcutaneous carbon dioxide measurement for change over time, in TSANZ Oral Presentations. Respirology, 22(S2), 98.

Fix, A. J., Golden, C. J., Daughton, D., Kass, I., Bell, C. W., \& Fix, J. (1982). Neuropsychological deficits among patients with chronic obstructive pulmonary disease. The International Journal of Neuroscience, 16(2), 99-105.

Fontanari, P., Burnet, H., Zattara-Hartmann, M. C., \& Jammes, Y. (1996). Changes in airway resistance induced by nasal inhalation of cold dry, dry, or moist air in normal individuals. Journal of Applied Physiology, 81(4), 1739-1743.

Foreman, M. G., Zhang, L., Murphy, J., Hansel, N. N., Make, B., Hokanson, J. E., ... DeMeo, D. L. (2011). Early-onset chronic obstructive pulmonary disease is associated with female sex, maternal factors, and African American race in the COPDGene study. American Journal of Respiratory and Critical Care Medicine, 184(4), 414-420.

Foucher, P. (1998). Relative Survival Analysis of 252 Patients With COPD Receiving Long-term Oxygen Therapy. Chest, 113(6), 1580.

Franklin, D., Babl, F. E., Schlapbach, L. J., Oakley, E., Craig, S., Neutze, J., ... Schibler, A. (2018). A Randomized Trial of High-Flow Oxygen Therapy in Infants with Bronchiolitis. New England Journal of Medicine, 378(12), 1121-1131.

Fraser, J. F., Spooner, A. J., Dunster, K. R., Anstey, C. M., \& Corley, A. (2016). Nasal high flow oxygen therapy in patients with COPD reduces respiratory rate and tissue carbon dioxide while increasing tidal and end-expiratory lung volumes: A randomised crossover trial. Thorax, 71(8), 759-761.

Frat, J.-P., Brugiere, B., Ragot, S., Chatellier, D., Veinstein, A., Goudet, V., ... Girault, C. (2015). Sequential Application of Oxygen Therapy Via High-Flow Nasal Cannula and Noninvasive Ventilation in Acute Respiratory Failure: An Observational Pilot Study. Respiratory Care, 60(2), 170-178.

Frat, J. P., Thille, A. W., Mercat, A., Girault, C., Ragot, S., Perbet, S., ... 
Network, R. (2015). High-flow oxygen through nasal cannula in acute hypoxemic respiratory failure. N Engl J Med, 372(23), 2185-2196.

Fricke, K., Tatkov, S., Domanski, U., Franke, K. J., Nilius, G., \& Schneider, H. (2016). Nasal high flow reduces hypercapnia by clearance of anatomical dead space in a COPD patient. Respiratory Medicine Case Reports, 19, 115-117.

Frizzola, M., Miller, T. L., Rodriguez, M. E., Zhu, Y., Rojas, J., Hesek, A., ... Dysart, K. (2011). High-flow nasal cannula: Impact on oxygenation and ventilation in an acute lung injury model. Pediatric Pulmonology, 46(1), 67-74.

Fruchter, O., Carmi, U., Ingenito, E. P., Refaeli, Y., \& Kramer, M. R. (2011). Transcutaneous carbon dioxide in severe COPD patients during bronchoscopic lung volume reduction. Respiratory Medicine, 105(4), 602-607.

Fuke, S., Miyamoto, K., Ohira, H., Ohira, M., Odajima, N., \& Nishimura, M. (2009). Evaluation of transcutaneous CO2 responses following acute changes in PaCO2 in healthy subjects. Respirology, 14(3), 436-442.

Futier, E., Paugam-Burtz, C., Godet, T., Khoy-Ear, L., Rozencwajg, S., Delay, J. M., ... OPERA study investigators. (2016). Effect of early postextubation high-flow nasal cannula vs conventional oxygen therapy on hypoxaemia in patients after major abdominal surgery: a French multicentre randomised controlled trial (OPERA). Intensive Care Medicine, 42(12), 1888-1898.

Gan, W. Q., FitzGerald, J. M., Carlsten, C., Sadatsafavi, M., \& Brauer, M. (2013). Associations of ambient air pollution with chronic obstructive pulmonary disease hospitalization and mortality. American Journal of Respiratory and Critical Care Medicine, 187(7), 721-727.

Garcia-Aymerich, J., Farrero, E., Felez, M. A., Izquierdo, J., Marrades, R. M., Anto, J. M., \& Estudi del Factors de Risc d'Aguditzacio de la, M. investigators. (2003). Risk factors of readmission to hospital for a COPD exacerbation: a prospective study. Thorax, 58(2), 100-105.

Garcia-Aymerich, J., Monsó, E., Marrades, R. M., Escarrabill, J., Félez, M. A., Sunyer, J., ... Morera, J. (2001). Risk factors for hospitalization for a chronic obstructive pulmonary disease exacerbation: Efram Study. American Journal of Respiratory and Critical Care Medicine, 164(6), 1002-1007.

Gavish, R., Levy, A., Dekel, O. K., Karp, E., \& Maimon, N. (2015). The association between hospital readmission and pulmonologist follow-up visits in patients with COPD. Chest, 148(2), 375-381.

Gorini, M., Misuri, G., Corrado, a, Duranti, R., landelli, I., De Paola, E., \& Scano, G. (1996). Breathing pattern and carbon dioxide retention in severe chronic obstructive pulmonary disease. Thorax, 51(7), 677-683.

Gorodeski, E. Z., Starling, R. C., \& Blackstone, E. H. (2010). Are All Readmissions Bad Readmissions? New England Journal of Medicine, 363(3), 297-298. 
Gotera, C., Diaz Lobato, S., Pinto, T., \& Winck, J. C. (2013). Clinical evidence on high flow oxygen therapy and active humidification in adults. Rev Port Pneumol, 19(5), 217-227.

Grainger, E. (Editor). (2017). Raising standards in the delivery of acute NIV. The Lancet Respiratory Medicine, 5(10), 761.

Grant, I., Heaton, R. K., McSweeny, a J., Adams, K. M., \& Timms, R. M. (1982). Neuropsychologic findings in hypoxemic chronic obstructive pulmonary disease. Archives of Internal Medicine, 142(8), 1470-1476.

Groenewegen KH, Schols AM, W. E. (2003). Mortality and mortality-related factors after hospitalization for acute exacerbation of COPD. Chest, 124 , 459-467.

Groves, N., \& Tobin, A. (2007). High flow nasal oxygen generates positive airway pressure in adult volunteers. Aust Crit Care, 20(4), 126-131.

Gunen, H., Hacievliyagil, S. S., Kosar, F., Mutlu, L. C., Gulbas, G., Pehlivan, E., ... Kizkin, O. (2005). Factors affecting survival of hospitalized patients with COPD. European Respiratory Journal, 26(2), 234-241.

Hackbarth, G., \& Berenson, R. (2011). Medicare and the Health Care Delivery System. Medicare Payment Advisory Commission Reports, 1208.

Halbert, R. J., Natoli, J. L., Gano, A., Badamgarav, E., Buist, A. S., \& Mannino, D. M. (2006). Global burden of COPD: Systematic review and meta-analysis. European Respiratory Journal. 28(3), 523-532.

Han, M. K., Postma, D., Mannino, D. M., Giardino, N. D., Buist, S., Curtis, J. L., \& Martinez, F. J. (2007). Gender and chronic obstructive pulmonary disease: why it matters. American Journal of Respiratory and Critical Care Medicine, 176(12), 1179-1184.

Hartl, S., Lopez-Campos, J. L., Pozo-Rodriguez, F., Castro-Acosta, A., Studnicka, M., Kaiser, B., \& Roberts, C. M. (2016). Risk of death and readmission of hospital-admitted COPD exacerbations: European COPD Audit. European Respiratory Journal, 47(1), 113-121.

Harvey, B.-G., Heguy, A., Leopold, P. L., Carolan, B. J., Ferris, B., \& Crystal, R. G. (2007). Modification of gene expression of the small airway epithelium in response to cigarette smoking. Journal of Molecular Medicine, 85(1), 39-53.

Hasani, A., Chapman, T. H., McCool, D., Smith, R. E., Dilworth, J. P., \& Agnew, J. E. (2008). Domiciliary humidification improves lung mucociliary clearance in patients with bronchiectasis. Chronic Respiratory Disease, 5(2), 81-86.

Hawkins, S., Huston, S., Campbell, K., \& Halbower, A. (2017). High-flow, heated, humidified air via nasal cannula treats CPAP-intolerant children with obstructive sleep apnea. Journal of Clinical Sleep Medicine, 13(8), 981-989.

Heidari, K., Hatamabadi, H., Ansarian, N., Alavi-Moghaddam, M., Amini, A., Safari, S., ... Vafaee, A. (2013). Correlation between capillary and arterial blood gas parameters in an ED. American Journal of Emergency 
Medicine, 31(2), 326-329.

Holleman-Duray, D., Kaupie, D., \& Weiss, M. G. (2007). Heated humidified high-flow nasal cannula: Use and a neonatal early extubation protocol. Journal of Perinatology, 27(12), 776-781.

Hollier, C. A., Harmer, A. R., Maxwell, L. J., Menadue, C., Willson, G. N., Unger, G., ... Piper, A. J. (2014). Moderate concentrations of supplemental oxygen worsen hypercapnia in obesity hypoventilation syndrome: a randomised crossover study. Thorax, 69(4), 346-353.

Hoogendoorn, M., Feenstra, T. L., Hoogenveen, R. T., Al, M., \& Mölken, M. R. van. (2010). Association between lung function and exacerbation frequency in patients with COPD. International Journal of Chronic Obstructive Pulmonary Disease, 5, 435-444.

Hornik, C. P., \& Turner, D. A. (2011). High-flow nasal cannula for neonatal respiratory distress: is it enough? Respir Care, 56(12), 1972-1974.

Hui, D., Morgado, M., Chisholm, G., Withers, L., Nguyen, Q., Finch, C., ... Bruera, E. (2013). High-flow oxygen and bilevel positive airway pressure for persistent dyspnea in patients with advanced cancer: A phase II randomized trial. Journal of Pain and Symptom Management, 46(4), 463-473.

Hurst, J. R., Vestbo, J., Anzueto, A., Locantore, N., Müllerova, H., TalSinger, R., ... Evaluation of COPD Longitudinally to Identify Predictive Surrogate Endpoints (ECLIPSE) Investigators. (2010). Susceptibility to exacerbation in chronic obstructive pulmonary disease. The New England Journal of Medicine, 63(12), 1128-1138.

Incalzi, R. a, Gemma, a, Marra, C., Muzzolon, R., Capparella, O., \& Carbonin, P. (1993). Chronic obstructive pulmonary disease. An original model of cognitive decline. The American Review of Respiratory Disease, 148(8), 418-424.

lyer, A. S., Bhatt, S. P., Garner, J. J., Wells, J. M., Trevor, J. L., Patel, N. M., ... Dransfield, M. T. (2016). Depression is associated with readmission for acute exacerbation of chronic obstructive pulmonary disease. Annals of the American Thoracic Society, 13(2), 197-203.

J., P., \& M.A., Chalaby. (2004). Acute respiratory failure, part 1: Establishing the diagnosis. Journal of Respiratory Diseases, 25(7), 294-297.

Janssens, J.-P., Howarth-Frey, C., Chevrolet, J.-C., Abajo, B., \& Rochat, T. (1998). Transcutaneous Pco2 to Monitor Noninvasive Mechanical Ventilation in Adults. Chest, 113(3), 768-773.

Jasmer, R. M., Luce, J. M., \& Matthay, M. A. (1997). Noninvasive positive pressure ventilation for acute respiratory failure. Chest, 111(6), 16721678.

Jeffrey, A. A., Warren, P. M., \& Flenley, D. C. (1992). Acute hypercapnic respiratory failure in patients with chronic obstructive lung disease: Risk factors and use of guidelines for management. Thorax, 47(1), 34-40.

Jencks, S. F., Williams, M. V., \& Coleman, E. A. (2009). Rehospitalizations among Patients in the Medicare Fee-for-Service Program. New England 
Journal of Medicine, 360(14), 1418-1428.

Jeong, J. H., Kim, D. H., Kim, S. C., Kang, C., Lee, S. H., Kang, T. S., ... Kim, D. S. (2015). Changes in arterial blood gases after use of high-flow nasal cannula therapy in the ED. American Journal of Emergency Medicine, 33(10), 1344-1349.

Jones, P. G., Kamona, S., Doran, O., Sawtell, F., \& Wilsher, M. (2016). Randomized Controlled Trial of Humidified High-Flow Nasal Oxygen for Acute Respiratory Distress in the Emergency Department: The HOT-ER Study. Respiratory Care, 61(3), 291-299.

Jones, P. W., Quirk, F. H., Baveystock, C. M., \& Littlejohns, P. (1992). A Self-complete Measure of Health Status for Chronic Airflow Limitation: The St. George's Respiratory Questionnaire. American Review of Respiratory Disease, 145(6), 1321-1327.

Joseph, L., Goldberg, S., Shitrit, M., \& Picard, E. (2015). High-flow nasal cannula therapy for obstructive sleep apnea in children. Journal of Clinical Sleep Medicine, 11(9), 1007-1010.

Kagawa, S., Otani, N., Kamide, M., Gisiger, P. A., Eberhard, P., \& Severinghaus, J. W. (2004). Initial transcutaneous PCO2 overshoot with ear probe at $42^{\circ} \mathrm{C}$. Journal of Clinical Monitoring and Computing, 18(56), 343-345.

Kang, B. J., Koh, Y., Lim, C. M., Huh, J. W., Baek, S., Han, M., ... Hong, S. B. (2015). Failure of high-flow nasal cannula therapy may delay intubation and increase mortality. Intensive Care Medicine, 41(4), 623632.

Keenan, S. P., Powers, C. E., \& McCormack, D. G. (2005). Noninvasive positive-pressure ventilation in patients with milder chronic obstructive pulmonary disease exacerbations: a randomized controlled trial. Respiratory Care, 50, 610-616.

Kelly, A.-M. (2010). Can venous blood gas analysis replace arterial in emergency medical care. Emergency Medicine Australasia, 22(6), 493498.

Kelly, A. A.-M., Kyle, E., \& McAlpine, R. (2002). Venous pCO2 and pH can be used to screen for significant hypercarbia in emergency patients with acute respiratory disease. Journal of Emergency Medicine, 22(1), 1519.

Kelly, C. R., Higgins, A. R., \& Chandra, S. (2015). Noninvasive PositivePressure Ventilation. New England Journal of Medicine, 372(23), e30.

Kilgour, E., Rankin, N., Ryan, S., \& Pack, R. (2004). Mucociliary function deteriorates in the clinical range of inspired air temperature and humidity. Intensive Care Medicine, 30(7), 1491-1494.

Kirkil, G., Tug, T., Ozel, E., Bulut, S., Tekatas, A., \& Muz, M. H. (2007). The evaluation of cognitive functions with P300 test for chronic obstructive pulmonary disease patients in attack and stable period. Clinical Neurology and Neurosurgery, 109(7), 553-560.

Kocher, R. P., \& Adashi, E. Y. (2011). Hospital readmissions and the 
affordable care act: Paying for coordinated quality care. Journal of the American Medical Association, 306(16), 1794-1795.

Köhnlein, T., Windisch, W., Köhler, D., Drabik, A., Geiseler, J., Hartl, S., ... Welte, T. (2014). Non-invasive positive pressure ventilation for the treatment of severe stable chronic obstructive pulmonary disease: A prospective, multicentre, randomised, controlled clinical trial. The Lancet Respiratory Medicine, 2(9), 698-705.

Kramer, N., Meyer, T. J., Meharg, J., Cece, R. D., \& Hill, N. S. (1995). Randomized, prospective trial of noninvasive positive pressure ventilation in acute respiratory failure. American Journal of Respiratory and Critical Care Medicine, 151(6), 1799-1806.

Kristensen, S. R., Bech, M., \& Quentin, W. (2015). A roadmap for comparing readmission policies with application to Denmark, England, Germany and the United States. Health Policy, 119(3), 264-273.

Krumholz, H. M., Lin, Z., Keenan, P. S., Chen, J., Ross, J. S., Drye, E. E., ... Normand, S.-L. T. (2013). Relationship Between Hospital Readmission and Mortality Rates for Patients Hospitalized With Acute Myocardial Infarction, Heart Failure, or Pneumonia. JAMA, 309(6), 587.

Kubicka, Z. J., Limauro, J., \& Darnall, R. a. (2008). Heated, humidified highflow nasal cannula therapy: yet another way to deliver continuous positive airway pressure? Pediatrics, 121(1), 82-88.

Lacasse, Y., Tan, A.-Y. M., Maltais, F., \& Jerry A. Krishnan. (2018). Home oxygen in chronic obstructive pulmonary disease. American Journal of Respiratory and Critical Care Medicine, 197(10).

Lain DC, Candace L, W. J. (2004). Average temperature and flow using the Vapotherm in an adult population. Chest, 126(4), 899 supplement.

Lampland, A. L., Plumm, B., Meyers, P. a, Worwa, C. T., \& Mammel, M. C. (2009). Observational study of humidified high-flow nasal cannula compared with nasal continuous positive airway pressure. The Journal of Pediatrics, 154(2), 177-182.

Lamprecht, B., McBurnie, M. A., Vollmer, W. M., Gudmundsson, G., Welte, T., Nizankowska-Mogilnicka, E., ... Buist, S. A. (2011). COPD in never smokers: Results from the population-based burden of obstructive lung disease study. Chest, 139(4), 752-763.

Lamprecht, B., Soriano, J. B., Studnicka, M., Kaiser, B., Vanfleteren, L. E., Gnatiuc, L., ... Jardim, J. R. B. (2015). Determinants of underdiagnosis of COPD in national and international surveys. Chest, 148(4), 971-985.

Lee, J. H., Rehder, K. J., Williford, L., Cheifetz, I. M., \& Turner, D. A. (2013). Use of high flow nasal cannula in critically ill infants, children, and adults: a critical review of the literature. Intensive Care Med, 39(2), 247257.

Leino, K., Nunes, S., Valta, P., \& Takala, J. (2001). Validation of a new respiratory inductive plethysmograph. Acta Anaesthesiologica Scandinavica, 45(1), 104-111.

Lemiale, V., Resche-Rigon, M., Mokart, D., Pene, F., Argaud, L., Mayaux, J., 
... Azoulay, E. (2017). High-Flow Nasal Cannula Oxygenation in Immunocompromised Patients With Acute Hypoxemic Respiratory Failure: A Groupe de Recherche Respiratoire en Reanimation OncoHematologique Study. Critical Care Medicine. 45(3), e274-280.

Lepere, V., Messika, J., La Combe, B., \& Ricard, J. D. (2016). High-flow nasal cannula oxygen supply as treatment in hypercapnic respiratory failure. American Journal of Emergency Medicine, 34(9), 1914.e11914.e2.

Lim, B. L., \& Kelly, A.-M. (2010). A meta-analysis on the utility of peripheral venous blood gas analyses in exacerbations of chronic obstructive pulmonary disease in the emergency department. European Journal of Emergency Medicine, 17(5), 246-248.

Lin, S. ming, Liu, K. xiong, Lin, Z. hong, \& Lin, P. hong. (2017). Does highflow nasal cannula oxygen improve outcome in acute hypoxemic respiratory failure? A systematic review and meta-analysis. Respiratory Medicine, 131, 58-64.

Lopez Varela, M. V., Montes De Oca, M., Halbert, R. J., Muiño, A., PerezPadilla, R., Tálamo, C., ... Menezes, A. M. B. (2010). Sex-related differences in COPD in five Latin American cities: The PLATINO study. European Respiratory Journal, 36(5), 1034-1041.

Luria, R. E. (1975). The validity and reliability of the visual analogue mood scale. Journal of Psychiatric Research, 12(1), 51-57.

M Cox, R Kemp, S Anwar, V Athey, T. A., \& Moloney, E. D. (2006). Noninvasive monitoring of $\mathrm{CO} 2$ levels in patients using NIV for AECOPD. Thorax, 61(4), 362-363.

Maggiore, S. M., Idone, F. A., Vaschetto, R., Festa, R., Cataldo, A., Antonicelli, F., ... Antonelli, M. (2014). Nasal high-flow versus Venturi mask oxygen therapy after extubation. Effects on oxygenation, comfort, and clinical outcome. Am J Respir Crit Care Med, 190(3), 282-288.

Malinowski T, L. J. (2002). Oxygen concentrations via nasal cannula at high flowrates. Respir Care, 47(9), 1079.

Mall, M. A. (2008). Role of Cilia, Mucus, and Airway Surface Liquid in Mucociliary Dysfunction: Lessons from Mouse Models. Journal of Aerosol Medicine and Pulmonary Drug Delivery, 21(1), 13-24.

Mannino, D. M., \& Buist, A. S. (2007). Global burden of COPD: risk factors, prevalence, and future trends. Lancet. 370(9589), 763-773.

Markovitz, G. H., Colthurst, J., Storer, T. W., \& Cooper, C. B. (2010). Effective inspired oxygen concentration measured via transtracheal and oral gas analysis. Respir Care, 55(4), 453-459.

Mayfield, S., Jauncey-Cooke, J., Hough, J. L., Schibler, A., Gibbons, K., \& Bogossian, F. (2014). High-flow nasal cannula therapy for respiratory support in children. The Cochrane Database of Systematic Reviews.

McCanny, P., Bennett, K., Staunton, P., \& McMahon, G. (2012). Venous vs arterial blood gases in the assessment of patients presenting with an exacerbation of chronic obstructive pulmonary disease. American 
Journal of Emergency Medicine, 30(6), 896-900.

McCulloch, T. M., \& Bishop, M. J. (1991). Complications of translaryngeal intubation. Clinics in Chest Medicine, 12, 507-521.

McDonough, J. E., Yuan, R., Suzuki, M., Seyednejad, N., Elliott, W. M., Sanchez, P. G., ... Hogg, J. C. (2011). Small-Airway Obstruction and Emphysema in Chronic Obstructive Pulmonary Disease. New England Journal of Medicine, 365(17), 1567-1575.

McEvoy, R. D., Pierce, R. J., Hillman, D., Esterman, A., Ellis, E. E., Catcheside, P. G., ... Grunstein, R. R. (2009). Nocturnal non-invasive nasal ventilation in stable hypercapnic COPD: A randomised controlled trial. Thorax, 64(7), 561-566.

McGinley, B. M., Patil, S. P., Kirkness, J. P., Smith, P. L., Schwartz, A. R., \& Schneider, H. (2007). A nasal cannula can be used to treat obstructive sleep apnea. American Journal of Respiratory and Critical Care Medicine, 176(2), 194-200.

McKinstry, S., Pilcher, J., Bardsley, G., Berry, J., Van de Hei, S., Braithwaite, I., ... Beasley, R. (2018). Nasal high flow therapy and PtCO2 in stable COPD: A randomized controlled cross-over trial. Respirology, 23(4), 378-384.

McVicar, J., \& Eager, R. (2009). Validation study of a transcutaneous carbon dioxide monitor in patients in the emergency department. Emergency Medicine Journal, 26(5), 344-346.

Meduri, G. U., Abou-Shala, N., Fox, R. C., Jones, C. B., Leeper, K. V., \& Wunderink, R. G. (1991). Noninvasive face mask mechanical ventilation in patients with acute hypercapnic respiratory failure. Chest, 100(2), 445-454.

Millar, J., Lutton, S., \& O'Connor, P. (2014). The use of high-flow nasal oxygen therapy in the management of hypercarbic respiratory failure. Therapeutic Advances in Respiratory Disease, 8(2), 63-64.

Miller, M. J., DiFiore, J. M., Strohl, K. P., \& Martin, R. J. (1990). Effects of nasal CPAP on supraglottic and total pulmonary resistance in preterm infants. Journal of Applied Physiology, 68(1), 141-146.

Miller, M. R., Hankinson, J., Brusasco, V., Burgos, F., Casaburi, R., Coates, A., ... Force, A. E. T. (2005). Standardisation of spirometry. Eur Respir J, 26(2), 319-338.

Miller, T. L., Blackson, T. J., Shaffer, T. H., \& Touch, S. M. (2004). Tracheal gas insufflation-augmented continuous positive airway pressure in a spontaneously breathing model of neonatal respiratory distress. Pediatric Pulmonology, 38(5), 386-395.

Milne, R. J., \& Beasley, R. (2015). Hospital admissions for chronic obstructive pulmonary disease in New Zealand. NZ Med J, 128(1408), 23-35.

Milne, R. J., Hockey, H., \& Rea, H. (2014). Long-term air humidification therapy is cost-effective for patients with moderate or severe chronic obstructive pulmonary disease or bronchiectasis. Value in Health, 17(4), 
320-327.

Moher, D., Liberati, A., Tetzlaff, J., Altman, D. G., Altman, D., Antes, G., ... Tugwell, P. (2009). Preferred reporting items for systematic reviews and meta-analyses: The PRISMA statement. PLoS Medicine. 6(7), e1000097.

Möller, W., Celik, G., Feng, S., Bartenstein, P., Meyer, G., Eickelberg, O., ... Tatkov, S. (2015). Nasal high flow clears anatomical dead space in upper airway models. Journal of Applied Physiology, 118(12), 15251532.

Möller, W., Feng, S., Domanski, U., Franke, K.-J., Celik, G., Bartenstein, P., ... Nilius, G. (2017). Nasal high flow reduces dead space. Journal of Applied Physiology, 122(1), 191-197.

Mundel, T., Feng, S., Tatkov, S., \& Schneider, H. (2013). Mechanisms of nasal high flow on ventilation during wakefulness and sleep. Journal of Applied Physiology. 114; 1058-1065.

Murphy, P. B., Rehal, S., Arbane, G., Bourke, S., Calverley, P. M. A., Crook, A. M., ... Hart, N. (2017). Effect of Home Noninvasive Ventilation With Oxygen Therapy vs Oxygen Therapy Alone on Hospital Readmission or Death After an Acute COPD Exacerbation. JAMA, 317(21), 2177.

Murphy, R., Thethy, S., Raby, S., Beckley, J., Terrace, J., Fiddler, C., ... Robertson, C. (2006). Capillary blood gases in acute exacerbations of COPD. Respiratory Medicine, 100(4), 682-686.

Nagata, K., Kikuchi, T., Horie, T., Shiraki, A., Kitajima, T., Kadowaki, T., ... Tomii, K. (2017). Domiciliary High-Flow Nasal Cannula Oxygen Therapy for Stable Hypercapnic COPD Patients: A Multicenter, Randomized Crossover Trial. Annals of the American Thoracic Society, 15(4), 432439.

Nakos, G., Lachana, A., Prekates, A., Pneumatikos, J., Guillaume, M., Pappas, K., \& Tsagaris, H. (1995). Respiratory effects of traeheal gas insufflation in spontaneously breathing COPD patients. Intensive Care Medicine, 21(11), 904-912.

Nattie, E., \& Li, A. (2012). Central chemoreceptors: Locations and functions. Comprehensive Physiology, 2(1), 221-254.

Nava, S., \& Ceriana, P. (2004). Causes of failure of noninvasive mechanical ventilation. Respiratory Care, 49(3), 295-303.

Nava, S., \& Hill, N. (2002). Non-invasive ventilation in acute respiratory failure. Thorax, 57(3), 192-211.

Nava, S., Navalesi, P., \& Conti, G. (2006). Time of non-invasive ventilation. Intensive Care Medicine, 32(3), 361-370.

Neumann, P., \& Burchardi, H. (1998). Evaluation of respiratory inductive plethysmography in controlled ventilation: Measurement of tidal volume and PEEP-induced changes of end- expiratory lung volume. Chest, 113(2), 443-451.

Nguyen, H. Q., Chu, L., Amy Liu, I.-L., Lee, J. S., Suh, D., Korotzer, B., ... 
Gould, M. K. (2014). Associations between Physical Activity and 30-Day Readmission Risk in Chronic Obstructive Pulmonary Disease. Annals of the American Thoracic Society, 11(5), 695-705.

Nilius, G., Franke, K. J., Domanski, U., Ruhle, K. H., Kirkness, J. P., \& Schneider, H. (2013). Effects of nasal insufflation on arterial gas exchange and breathing pattern in patients with chronic obstructive pulmonary disease and hypercapnic respiratory failure. Adv Exp Med Biol, 755, 27-34.

Nishimura, M. (2015). High-flow nasal cannula oxygen therapy in adults. Journal of Intensive Care, 3(1), 15.

Nishimura, M. (2016). High-Flow Nasal Cannula Oxygen Therapy in Adults: Physiological Benefits, Indication, Clinical Benefits, and Adverse Effects. Respir Care, 61(4), 529-541.

Nishiyama, O., Taniguchi, H., Kondoh, Y., Kimura, T., Ogawa, T., Watanabe, F., \& Arizono, S. (2005). Factors in maintaining long-term improvements in health-related quality of life after pulmonary rehabilitation for COPD. Quality of Life Research, 14(10), 2315-2321.

Nourdine, K., Combes, P., Carton, M. J., Beuret, P., Cannamela, A., \& Ducreux, J. C. (1999). Does noninvasive ventilation reduce the ICU nosocomial infection risk? A prospective clinical survey. Intensive Care Medicine, 25(6), 567-573.

O'Donnell, D. E. (2006). Hyperinflation, dyspnea, and exercise intolerance in chronic obstructive pulmonary disease. Proceedings of the American Thoracic Society, 3(2), 180-184.

O’Donnell, D. E., D’Arsigny, C., Fitzpatrick, M., \& Webb, K. A. (2002). Exercise hypercapnia in advanced chronic obstructive pulmonary disease. American Journal of Respiratory and Critical Care Medicine, 166(5), 663-668.

O'Donnell, D. E., Hernandez, P., Kaplan, A., Aaron, S., Bourbeau, J., Marciniuk, D., ... Voduc, N. (2008). Canadian Thoracic Society recommendations for management of chronic obstructive pulmonary disease - 2008 update - highlights for primary care. Can Respir J., 15 Suppl A(February), 1A-8A.

O'Donnell, D. E., \& Parker, C. M. (2006). COPD exacerbations:

Pathophysiology. Thorax, 61(4), 354-361.

O'Driscoll, B. R., Howard, L. S., Earis, J., \& Mak, V. (2017). BTS guideline for oxygen use in adults in healthcare and emergency settings. Thorax, 72(Suppl 1), ii1-ii90.

O'Reilly Nugent, A., Kelly, P. T., Stanton, J., Swanney, M. P., Graham, B., \& Beckert, L. (2014). Measurement of oxygen concentration delivered via nasal cannulae by tracheal sampling. Respirology, 19(4), 538-543.

On, L. S., Boonyongsunchai, P., Webb, S., Davies, L., Calverley, P. M. A., \& Costello, R. W. (2001). Function of pulmonary neuronal M2 muscarinic receptors in stable chronic obstructive pulmonary disease. American Journal of Respiratory and Critical Care Medicine, 163(6), 1320-1325. 
Osadnik, C. R., Tee, V. S., Carson-Chahhoud, K. V, Picot, J., Wedzicha, J. A., \& Smith, B. J. (2017). Non-invasive ventilation for the management of acute hypercapnic respiratory failure due to exacerbation of chronic obstructive pulmonary disease. Cochrane Database of Systematic Reviews (Online), 7(7), CD004104.

Ospina, M. B., Mrklas, K., Deuchar, L., Rowe, B. H., Leigh, R., Bhutani, M., \& Stickland, M. K. (2017). A systematic review of the effectiveness of discharge care bundles for patients with COPD. Thorax, 72(1), 31-39.

Papachatzakis, I. (2017). High Flow Nasal Cannula with warm humidified air versus Non-Invasive Mechanical Ventilation in Respiratory Failure type II. ERJ, 50(Suppl 61), PA2182.

Paré, P. D., \& Mitzner, W. (2012). Airway-parenchymal interdependence. Comprehensive Physiology, 2(3), 1921-1935.

Parke, R. L., Eccleston, M. L., \& McGuinness, S. P. (2011). The effects of flow on airway pressure during nasal high-flow oxygen therapy. Respir Care, 56(8), 1151-1155.

Parke, R. L., \& McGuinness, S. P. (2013). Pressures delivered by nasal high flow oxygen during all phases of the respiratory cycle. Respir Care, 58(10), 1621-1624.

Parke, R. L., McGuinness, S. P., \& Eccleston, M. L. (2011). A Preliminary Randomized Controlled Trial to Assess Effectiveness of Nasal HighFlow Oxygen in Intensive Care Patients. Respiratory Care, 56(3), 265270.

Parke, R., McGuinness, S., Dixon, R., \& Jull, A. (2013). Open-label, phase II study of routine high-flow nasal oxygen therapy in cardiac surgical patients. British Journal of Anaesthesia, 111(6), 925-931.

Parke, R., McGuinness, S., \& Eccleston, M. (2009). Nasal high-flow therapy delivers low level positive airway pressure. British Journal of Anaesthesia, 103(6), 886-890.

Patil, S. P., Krishnan, J. A., Lechtzin, N., \& Diette, G. B. (2003). In-hospital mortality following acute exacerbations of chronic obstructive pulmonary disease. Archives of Internal Medicine, 163(10), 1180-1186.

Pavlov, I., Plamondon, P., \& Delisle, S. (2017). Nasal high-flow therapy for type II respiratory failure in COPD: A report of four cases. Respiratory Medicine Case Reports, 20; 87-88.

Perera, W. R., Hurst, J. R., Wilkinson, T. M. A., Sapsford, R. J., Müllerova, H., Donaldson, G. C., \& Wedzicha, J. A. (2007). Inflammatory changes, recovery and recurrence at COPD exacerbation. European Respiratory Journal, 29(3), 527-534.

Perrin, K., Wijesinghe, M., Healy, B., Wadsworth, K., Bowditch, R., Bibby, S., ... Beasley, R. (2011). Randomised controlled trial of high concentration versus titrated oxygen therapy in severe exacerbations of asthma. Thorax, 66(11), 937-941.

Perrin, K., Wijesinghe, M., Weatherall, M., \& Beasley, R. (2011). Assessing $\mathrm{PaCO} 2$ in acute respiratory disease: accuracy of a transcutaneous 
carbon dioxide device. Intern Med J, 41(8), 630-633.

Peters, S. G., Holets, S. R., \& Gay, P. C. (2013). High-flow nasal cannula therapy in do-not-intubate patients with hypoxemic respiratory distress. Respiratory Care, 58(4), 597-600.

Petersson, J., \& Glenny, R. W. (2014). Gas exchange and ventilationperfusion relationships in the lung. European Respiratory Journal, 44(4), 1023-1041.

Piatti, G., Ambrosetti, U., Santus, P., \& Allegra, L. (2005). Effects of salmeterol on cilia and mucus in COPD and pneumonia patients. Pharmacological Research, 51(2), 165-168.

Pierson, D. J. (2002). Indications for mechanical ventilation in adults with acute respiratory failure. Respiratory Care, 47(3), 249-62.

Pilcher, J., Cameron, L., Braithwaite, I., Bowles, D., Swain, A., Bailey, M., ... Perrin, K. (2015). Comparative audit of oxygen use in the prehospital setting in acute COPD exacerbation over 5 years. Emerg Med J, 32(3), 234-238.

Pilcher, J., Eastlake, L., Richards, M., Power, S., Cripps, T., Bibby, S., ... Beasley, R. (2017). Physiological effects of titrated oxygen via nasal high-flow cannulae in COPD exacerbations: A randomized controlled cross-over trial. Respirology, 22(6), 1149-1155.

Pilcher J, P. K. and B. R. (2013). The effect of high concentration oxygentherapy on $\mathrm{PaCO} 2$ in acute and chronicrespiratory disorders. Translational Respiratory Medicine, 1, 8-10.

Pisani, L., Fasano, L., Corcione, N., Comellini, V., Musti, M. A., Brandao, M., ... Nava, S. (2017). Change in pulmonary mechanics and the effect on breathing pattern of high flow oxygen therapy in stable hypercapnic COPD. Thorax, 72(4), 373-375.

Pisani, L., \& Vega, M. L. (2017). Use of Nasal High Flow in Stable COPD: Rationale and Physiology. COPD: Journal of Chronic Obstructive Pulmonary Disease, 14(3), 346-350.

Plant, P. K., Owen, J. L., \& Elliott, M. W. (2000). Early use of non-invasive ventilation for acute exacerbations of chronic obstructive pulmonary disease on general respiratory wards: a multicentre randomised controlled trial. Lancet, 355(9219), 1931-1935.

Plant, P., Owen, J., \& Elliot, M. (2001). Non-invasive ventilation in acute exacerbations of chronic obstructive pulmonary disease: long term survival and predictors of in-hospital outcome. Thorax, 59(9), 708-712.

Porter, M. (2010). What is Value in Healthcare? Perspective, 363(1), 1-3.

Press, V. G., Arora, V. M., Trela, K. C., Adhikari, R., Zadravecz, F. J., Liao, C., ... Krishnan, J. A. (2016). Effectiveness of interventions to teach metered-dose and diskus inhaler techniques: A randomized trial. Annals of the American Thoracic Society, 13(6), 816-824.

Price, L. C., Lowe, D., Hosker, H. S., Anstey, K., Pearson, M. G., Roberts, C. M., ... the Royal College of Physicians Clinical Effectiveness Evaluation, 
U. (2006). UK National COPD Audit 2003: Impact of hospital resources and organisation of care on patient outcome following admission for acute COPD exacerbation. Thorax, 61(10), 837-842.

Puhan, M. A., Gimeno-Santos, E., Cates, C. J., \& Troosters, T. (2016). Pulmonary rehabilitation following exacerbations of chronic obstructive pulmonary disease. Cochrane Database of Systematic Reviews, 2016(12).

Rabe, K. F., \& Watz, H. (2017). Chronic obstructive pulmonary disease. The Lancet, 389(10082), 1931-1940.

Rang, L. C. F., Murray, H. E., Wells, G. A., \& Macgougan, C. K. (2002). Can peripheral venous blood gases replace arterial blood gases in emergency department patients? Canadian Journal of Emergency Medical Care, 4(1), 7-15.

Rea, H., McAuley, S., Jayaram, L., Garrett, J., Hockey, H., Storey, L., ... O'Donnell, K. (2010). The clinical utility of long-term humidification therapy in chronic airway disease. Respir Med, 104(4), 525-533.

Rennard, S. I., \& Vestbo, J. (2006). COPD: the dangerous underestimate of 15\%. Lancet, 367(9518), 1216-1219.

Restrepo, R. D., Hirst, K. R., Wittnebel, L., \& Wettstein, R. (2012). AARC clinical practice guideline: transcutaneous monitoring of carbon dioxide and oxygen: 2012. Respir Care, 57(11), 1955-1962.

Ricard, J.-D., Dib, F., Esposito-Farese, M., Messika, J., \& Girault, C. (2018). Comparison of high flow nasal cannula oxygen and conventional oxygen therapy on ventilatory support duration during acute-on-chronic respiratory failure: study protocol of a multicentre, randomised, controlled trial. The 'HIGH-FLOW ACRF' study. BMJ Open, 8(9), e022983.

Riera, J., Pérez, P., Cortés, J., Roca, O., Masclans, J. R., \& Rello, J. (2013). Effect of High Flow Nasal Cannula and Body Position on End-Expiratory Lung Volume. A Cohort Study Using Electrical Impedance Tomography. Respiratory Care, 58(4), 589-596.

Ritchie, J. E., Williams, A. B., Gerard, C., \& Hockey, H. (2011). Evaluation of a humidified nasal high-flow oxygen system, using oxygraphy, capnography and measurement of upper airway pressures. Anaesth Intensive Care, 39(6), 1103-1110.

Rittayamai, N., Tscheikuna, J., Praphruetkit, N., \& Kijpinyochai, S. (2015). Use of High-Flow Nasal Cannula for Acute Dyspnea and Hypoxemia in the Emergency Department. Respiratory Care, 60(10), 1377-1382.

Roberts, C. M., Stone, R. A., Buckingham, R. J., Pursey, N. A., Lowe, D., National Chronic Obstructive Pulmonary Disease, R., \& Outcomes Project implementation, group. (2011). Acidosis, non-invasive ventilation and mortality in hospitalised COPD exacerbations. Thorax, 66(1), 43-48.

Robinson, T. D., Freiberg, D. B., Regnis, J. A., \& Young, I. H. (2000). The role of hypoventilation and ventilation-perfusion redistribution in oxygen- 
induced hypercapnia during acute exacerbations of chronic obstructive pulmonary disease. American Journal of Respiratory and Critical Care Medicine, 161(5), 1524-1529.

Robson B, H. R. (2007). Hauora: Māori Standards of Health IV. A study of the years 2000-2005. (H. R. Robson B, Ed.). Wellington: Te Rōpū Rangahau Hauora a Eru Pōmare.

Roca, O., Pérez-Terán, P., Masclans, J. R., Pérez, L., Galve, E., Evangelista, A., \& Rello, J. (2013). Patients with New York Heart Association class III heart failure may benefit with high flow nasal cannula supportive therapy. High flow nasal cannula in heart failure. Journal of Critical Care, 28(5), 741-746.

Roca, O., Riera, J., Torres, F., \& Masclans, J. R. (2010). High-flow oxygen therapy in acute respiratory failure. Respir Care, 55(4), 408-413.

Rocco, M., Dell'Utri, D., Morelli, A., Spadetta, G., Conti, G., Antonelli, M., \& Pietropaoli, P. (2004). Noninvasive ventilation by helmet or face mask in immunocompromised patients: A case-control study. Chest, 126(5), 1508-1515.

Rochwerg, B., Brochard, L., Elliott, M. W., Hess, D., Hill, N. S., Nava, S., ... Raoof, S. (2017). Official ERS/ATS clinical practice guidelines: Noninvasive ventilation for acute respiratory failure. European Respiratory Journal, 50(4).

Rodriguez, P., Lellouche, F., Aboab, J., Buisson, C. B., \& Brochard, L. (2006). Transcutaneous arterial carbon dioxide pressure monitoring in critically ill adult patients. Intensive Care Med, 32(2), 309-312.

Roediger, R., Beck-Schimmer, B., Theusinger, O. M., Rusch, D., Seifert, B., Spahn, D. R., ... Baulig, W. (2011). The revised digital transcutaneous $\mathrm{PCO} / \mathrm{SpO} 2$ ear sensor is a reliable noninvasive monitoring tool in patients after cardiac surgery. J Cardiothorac Vasc Anesth, 25(2), 243249.

Rossi, A., Ganassini, A., Polese, G., \& Grassi, V. (1997). Pulmonary hyperinflation and ventilator-dependent patients. European Respiratory Journal, 10(7), 1663-1674.

Roussos, C., \& Koutsoukou, A. (2003). Respiratory failure. European Respiratory Journal, 22(Supplement 47), 3s-14s.

Royal College of Physicians of London. (2010). Chronic obstructive disease: Management of chronic obstructive pulmonary disease $n$ adults in primary and secondary care. National Clinical Guideline Centre.

Rudolf, M., Turner, J. A., Harrison, B. D., Riordan, J. F., \& Saunders, K. B. (1979). Changes in arterial blood gases during and after a period of oxygen breathing in patients with chronic hypercapnic respiratory failure and in patients with asthma. Clin Sci (Lond), 57(5), 389-396.

Rutland, J., Griffin, W. M., \& Cole, P. J. (1982). Human ciliary beat frequency in epithelium from intrathoracic and extrathoracic airways. The American Review of Respiratory Disease, 125(1), 100-105.

Saslow, J. G., Aghai, Z. H., Nakhla, T. a, Hart, J. J., Lawrysh, R., Stahl, G. 
E., \& Pyon, K. H. (2006). Work of breathing using high-flow nasal cannula in preterm infants. Journal of Perinatology: Official Journal of the California Perinatal Association, 26(8), 476-480.

Schwabbauer, N., Berg, B., Blumenstock, G., Haap, M., Hetzel, J., \& Riessen, R. (2014). Nasal high-flow oxygen therapy in patients with hypoxic respiratory failure: Effect on functional and subjective respiratory parameters compared to conventional oxygen therapy and non-invasive ventilation (NIV). BMC Anesthesiology, 14, 66.

Seemungal, T. a, Donaldson, G. C., Bhowmik, A., Jeffries, D. J., \& Wedzicha, J. a. (2000). Time course and recovery of exacerbations in patients with chronic obstructive pulmonary disease. American Journal of Respiratory and Critical Care Medicine, 161(5), 1608-1613.

Seneff, M. G., Wagner, D. P., Wagner, R. P., Zimmerman, J. E., \& Knaus, W. A. (1995). Hospital and 1-year survival of patients admitted to intensive care units with acute exacerbation of chronic obstructive pulmonary disease. JAMA, 274(23), 1852-1857.

Senn, O., Clarenbach, C. F., Kaplan, V., Maggiorini, M., \& Bloch, K. E. (2005). Monitoring carbon dioxide tension and arterial oxygen saturation by a single earlobe sensor in patients with critical illness or sleep apnea. Chest, 128(3), 1291-1296.

Shah, T., Churpek, M. M., Perraillon, M. C., \& Konetzka, R. T. (2015). Understanding why patients with COPD get readmitted: A large national study to delineate the medicare population for the readmissions penalty expansion. Chest, 147(5), 1219-1226.

Shah, T., Press, V. G., Huisingh-Scheetz, M., \& White, S. R. (2016). COPD Readmissions. Chest, 150(4), 916-926.

Sharif, R., Parekh, T. M., Pierson, K. S., Kuo, Y. F., \& Sharma, G. (2014). Predictors of early readmission among patients 40 to 64 years of age hospitalized for chronic obstructive pulmonary disease. Annals of the American Thoracic Society, 11(5), 685-694.

Sharma, G., Kuo, Y. F., Freeman, J. L., Zhang, D. D., \& Goodwin, J. S. (2010). Outpatient follow-up visit and 30-day emergency department visit and readmission in patients hospitalized for chronic obstructive pulmonary disease. Archives of Internal Medicine, 170(18), 1664-1670.

Shelly, M. P., Lloyd, G. M., \& Park, G. R. (1988). A review of the mechanisms and methods of humidification of inspired gases. Intensive Care Medicine, 14(1), 1-9.

Shirtcliffe, P., Weatherall, M., Marsh, S., Travers, J., Hansell, A., McNaughton, A., ... Beasley, R. (2007). COPD prevalence in a random population survey: a matter of definition. Eur Respir J, 30(2), 232-239.

Silverman, E. K., Ph, D., Sandhaus, R. A., \& Ph, D. (2009). Alpha 1 Antitrypsin Deficiency. New England Journal of Medicine, 360(26), 2749-2757.

Sim, M. A. B., Dean, P., Kinsella, J., Black, R., Carter, R., \& Hughes, M. (2008). Performance of oxygen delivery devices when the breathing 
pattern of respiratory failure is simulated. Anaesthesia 63 (9), 938-940.

Singh, G., Zhang, W., Kuo, Y. F., \& Sharma, G. (2016). Association of Psychological Disorders With 30-Day Readmission Rates in Patients With COPD. Chest, 149(4), 905-915.

Singh, M. (2006). Heated, humidified air for the common cold. Cochrane Database of Systematic Reviews, May 11(5), CD001728.

Soler-Cataluña, J. J., Martínez-García, M. Á., Sánchez, P. R., Salcedo, E., Navarro, M., \& Ochando, R. (2005). Severe acute exacerbations and mortality in patients with chronic obstructive pulmonary disease. Thorax, 60(11), 925-931.

Soo Hoo Guy W, Santiago Silverio, Williams, A. J. (1994). Nasal mechanical ventilation for hypercapnic respiratory failure in COPD: Determinants of success and failure. Crit Care Med, 22(8), 1253-1261.

Sorheim, I. C., Johannessen, A., Gulsvik, A., Bakke, P. S., Silverman, E. K., \& DeMeo, D. L. (2010). Gender differences in COPD: Are women more susceptible to smoking effects than men? Thorax, 65(6), 480-485.

Soriano, J. B., Abajobir, A. A., Abate, K. H., Abera, S. F., Agrawal, A., Ahmed, M. B., ... Vos, T. (2017). Global, regional, and national deaths, prevalence, disability-adjusted life years, and years lived with disability for chronic obstructive pulmonary disease and asthma, 1990-2015: a systematic analysis for the Global Burden of Disease Study 2015. The Lancet Respiratory Medicine, 5(9), 691-706.

Spoletini, G., Alotaibi, M., Blasi, F., \& Hill, N. S. (2015). Heated humidified high-flow nasal oxygen in adults: Mechanisms of action and clinical implications. Chest, 148(1), 253-261.

Steer, J., Gibson, G. J., \& Bourke, S. C. (2010). Predicting outcomes following hospitalization for acute exacerbations of COPD. QJM, 103(11), 817-829.

Steer, J., Norman, E. M., Afolabi, O. A., Gibson, G. J., \& Bourke, S. C. (2012). Dyspnoea severity and pneumonia as predictors of in-hospital mortality and early readmission in acute exacerbations of COPD. Thorax, 67(2), 117-121.

Stéphan, F., Barrucand, B., Petit, P., Rézaiguia-Delclaux, S., Médard, A., Delannoy, B., ... Ouattara, A. (2015). High-flow nasal oxygen vs noninvasive positive airway pressure in hypoxemic patients after cardiothoracic surgery: A randomized clinical trial. Journal of the American Medical Association (Vol. 313, pp. 2331-2339).

Storgaard, L. H., Hockey, H., Laursen, B. S., \& Weinreich, U. M. (2018). Long-term effects of oxygen-enriched nasal high flow treatment in COPD with chronic hypoxemic respiratory failure. Int J Chron Obstruct Pulmon Dis, 13, 1195-1205.

Storre, J. H., Magnet, F. S., Dreher, M., \& Windisch, W. (2011).

Transcutaneous monitoring as a replacement for arterial $\mathrm{PCO}_{2}$ monitoring during nocturnal non-invasive ventilation. Respir Med, 105(1), 143-150. 
Struik, F. M., Lacasse, Y., Goldstein, R., Kerstjens, H. A. M., \& Wijkstra, P. J. (2013). Nocturnal non-invasive positive pressure ventilation for stable chronic obstructive pulmonary disease. Cochrane Database of Systematic Reviews. Jun 13(6), CD002878.

Struik, F. M., Sprooten, R. T. M., Kerstjens, H. A. M., Bladder, G., Zijnen, M., Sin, J. A., ... Wijkstra, P. J. (2014). Nocturnal non-invasive ventilation in copd patients with prolonged hypercapnia after ventilatory support for acute respiratory failure: A randomised, controlled, parallel-group study. Thorax, 69(9), 826-834.

Sztrymf, B., Messika, J., Bertrand, F., Hurel, D., Leon, R., Dreyfuss, D., \& Ricard, J. D. (2011). Beneficial effects of humidified high flow nasal oxygen in critical care patients: A prospective pilot study. Intensive Care Medicine, 37(11), 1780-1786.

Sztrymf, B., Messika, J., Mayot, T., Lenglet, H., Dreyfuss, D., \& Ricard, J. D. (2012). Impact of high-flow nasal cannula oxygen therapy on intensive care unit patients with acute respiratory failure: A prospective observational study. Journal of Critical Care, 27(3).

The Ministry of Health New Zealand. (2015). Tatau Kahukura Māori Health Chart Book.

Therapeutic Goods Adminstration. (2011). Australian regulatory guidelines for medical devices (ARGMD). v1.1.

Tiep B, B. M. (2002). High flow nasal vs high flow mask oxygen delivery: tracheal gas concentrations through a head extention airway model. Respir Care, 47(9), 1079.

Torres, a, Aznar, R., Gatell, J. M., Jiménez, P., González, J., Ferrer, a, ... Rodriguez-Roisin, R. (1990). Incidence, risk, and prognosis factors of nosocomial pneumonia in mechanically ventilated patients. The American Review of Respiratory Disease, 142, 523-528.

Troyer, A. D. E., \& Estenne, M. (1981). Limitations of measurement of transdiaphragmatic pressure in detecting diaphragmatic weakness. Thorax, 36, 169-174.

Valta, P., Takala, J., Foster, R., Weissman, C., \& Kinney, J. M. (1992). Evaluation of respiratory inductive plethysmography in the measurement of breathing pattern and PEEP-induced changes in lung volume. Chest, 102(1), 234-238.

Vogelmeier, C. F., Criner, G. J., Martinez, F. J., Anzueto, A., Barnes, P. J., Bourbeau, J., ... Agusti, A. (2017). Global Strategy for the Diagnosis, Management, and Prevention of Chronic Obstructive Lung Disease 2017 Report: GOLD Executive Summary. European Respiratory Journal, 49(3), 1700214.

Vogelsinger, H., Halank, M., Braun, S., Wilkens, H., Geiser, T., Ott, S., ... Kaehler, C. M. (2017). Efficacy and safety of nasal high-flow oxygen in COPD patients. BMC Pulmonary Medicine, 17(1), 143.

Volsko, T. A., Fedor, K., Amadei, J., \& Chatburn, R. L. (2011). High flow through a nasal cannula and CPAP effect in a simulated infant model. 
Respiratory Care, 56(12), 1893-1900.

Wagstaff, T. A. J., \& Soni, N. (2007). Performance of six types of oxygen delivery devices at varying respiratory rates. Anaesthesia, 62(5), 492503.

Wang, H., Naghavi, M., Allen, C., Barber, R., Bhutta, Z. A., Carter, C., ... Dandona, H. (2016). Global, regional, and national life expectancy, allcause mortality, and cause-specific mortality for 249 causes of death, 1980-2015: a systematic analysis for the Global Burden of Disease Study 2015. The Lancet, 388(10053), 1459-1544.

Wanner, A., Salathe, M., \& O'Riordan, T. G. (1996). Mucociliary clearance in the airways. American Journal of Respiratory and Critical Care Medicine, 154(6), 1868-1902.

West, J. B. (2012). Respiratory Physiology-The Essentials. Lippincott, Williams \& Wilkins.

West, J. B., \& Wagner, P. D. (1998). Pulmonary gas exchange. American Journal of Respiratory and Critical Care Medicine, 157(4 Pt 2), S82-7.

Wettstein, R. B., Shelledy, D. C., \& Peters, J. I. (2005). Delivered oxygen concentrations using low-flow and high-flow nasal cannulas. Respiratory Care, 50(5), 604-609.

WHO. (2018). Burden of COPD http://www.who.int/respiratory/copd/burden/en/.

Wijesinghe, M., Perrin, K., Healy, B., Weatherall, M., \& Beasley, R. (2012). Randomized controlled trial of high concentration oxygen in suspected community-acquired pneumonia. J R Soc Med, 105(5), 208-216.

Wijesinghe, M., Williams, M., Perrin, K., Weatherall, M., \& Beasley, R. (2011). The effect of supplemental oxygen on hypercapnia in subjects with obesity-associated hypoventilation: a randomized, crossover, clinical study. Chest, 139(5), 1018-1024.

Wilkinson, T., North, M., \& Bourne, S. C. (2014). Reducing hospital admissions and improving the diagnosis of COPD in Southampton City: Methods and results of a 12-month service improvement project. Npj Primary Care Respiratory Medicine, 24 (0), 14035.

Williams, R., Rankin, N., Smith, T., Galler, D., \& Seakins, P. (1996).

Relationship between the humidity and temperature of inspired gas and the function of the airway mucosa. Critical Care Medicine, 24(11), 19201929.

Wood, K. A., Lewis, L., Von Harz, B., \& Kollef, M. H. (1998). The use of noninvasive positive pressure ventilation in the emergency department: results of a randomized clinical trial. Chest, 113(5), 1339-1346.

Woodcock, A., Boucot, I., Leather, D. A., Crawford, J., Collier, S., Bakerly, N. D., ... Vestbo, J. (2018). Effectiveness versus efficacy trials in COPD: how study design influences outcomes and applicability. European Respiratory Journal, 51(2), 1701531.

Xue, Q., Wu, X., Jin, J., Yu, B., \& Zheng, M. (2010). Transcutaneous carbon 
dioxide monitoring accurately predicts arterial carbon dioxide partial pressure in patients undergoing prolonged laparoscopic surgery. Anesthesia and Analgesia, 111(2), 417-420.

Yager, J. A., Ellman, H., \& Dulfano, M. J. (1980). Human ciliary beat frequency at three levels of the tracheobronchial tree. The American Review of Respiratory Disease, 121(4), 661-665.

Yaghi, A., \& Dolovich, M. (2016). Airway Epithelial Cell Cilia and Obstructive Lung Disease. Cells, 5(4), 40.

Yang, I. A., Brown, J. L., George, J., Jenkins, S., McDonald, C. F., McDonald, V. M., ... Dabscheck, E. (2017). COPD-X Australian and New Zealand guidelines for the diagnosis and management of chronic obstructive pulmonary disease: 2017 update. The Medical Journal of Australia, 207(10), 436-442.

Yang IA, Brown JL, George J, Jenkins S, McDonald CF, McDonald V, Smith B, Zwar N, D. E. (2018). The COPD-X Plan: Australian and New Zealand Guidelines for the management of Chronic Obstructive Pulmonary Disease 2018 Version 2.53.

Yildizdaş, D., Yapicioğlu, H., Yilmaz, H. L., \& Sertdemir, Y. (2004). Correlation of simultaneously obtained capillary, venous, and arterial blood gases of patients in a paediatric intensive care unit. Archives of Disease in Childhood, 89(2), 176-180.

Zavorsky, G. S., Cao, J., Mayo, N. E., Gabbay, R., \& Murias, J. M. (2007). Arterial versus capillary blood gases: A meta-analysis. Respiratory Physiology and Neurobiology, 155(3), 268-279.

Zhou, Y., Zou, Y., Li, X., Chen, S., Zhao, Z., He, F., ... Ran, P. (2014). Lung Function and Incidence of Chronic Obstructive Pulmonary Disease after Improved Cooking Fuels and Kitchen Ventilation: A 9-Year Prospective Cohort Study. PLoS Medicine, 11(3).

Zwerink, M., Brusse-Keizer, M., van der Valk, P., Zielhuis, G., Monninkhof, E., van der Palen, J., ... Effing, T. (2014). Self management for patients with chronic obstructive pulmonary disease (review). Cochrane Database of Syst Rev, 3(3), CD002990. 


\section{Appendices}

\subsection{Appendix I - Full description of mechanism of actions studies}

\subsubsection{PEEP EFFECT}

\subsubsection{Evidence of PEEP in healthy volunteers}

Pharyngeal pressures were measured in 10 healthy volunteers to demonstrate a significant flow dependent positive pressure effect of NHF (Groves \& Tobin, 2007). NHF at between $10-60 \mathrm{~L} / \mathrm{min}$ were compared with no flow. A maximum expiratory pharyngeal pressure with mouth closed of $7.4 \mathrm{cmH}_{2} 0$ at $60 \mathrm{~L} / \mathrm{min}$ was recorded compared to mouth open of $2.7 \mathrm{cmH}_{2} \mathrm{O}$, with an overall $p$-value across all of the flows $<0.001$. Inspiratory pharyngeal pressure was no different according to whether the mouth was open or closed and reached a maximum of $1.6 \mathrm{cmH}_{2} \mathrm{O}$. The pressure effect had a positive linear dependence on flow for both inspiratory and expiratory pressure.

The flow-dependent effect was seen in a healthy volunteer study also (Ritchie et al., 2011). At $50 \mathrm{~L} / \mathrm{min}$ there was a mean nasopharyngeal pressure of just over $5 \mathrm{cmH}_{2} \mathrm{O}$ with a maximum of $7.1 \mathrm{cmH}_{2} \mathrm{O}$ with the mouth closed. This was substantially higher than with the mouth open which was below $1 \mathrm{cmH}_{2} \mathrm{O}$.

\subsection{EVIDENCE OF PEEP IN NEONATES}

Oesophageal pressures were measured using a differential pressure transducer to indicate end-expiratory pressure in 15 neonates, demonstrating a flow-dependent increase up to $3 \mathrm{cmH}_{2} \mathrm{O}$ with increasing flows up to $6 \mathrm{~L} / \mathrm{min}$, similar to nasal CPAP (Lampland et al., 2009).

Maximum pressures of $0.6-4.1 \mathrm{cmH}_{2} \mathrm{O}$ at $\mathrm{NHF}$ flows of $1-3 \mathrm{~L} / \mathrm{min}$ were observed in neonates using an oral pressure gauge but with the mouth closed (Kubicka et al., 2008).

Concerns have been raised about the variability of the distending pressure in neonates (Hornik \& Turner, 2011; Volsko, Fedor, Amadei, \& Chatburn, 2011) 
and the diameter of the nasal prongs relates to the positive pressure effect (Volsko et al., 2011).

\subsection{EVIDENCE OF PEEP POST-CARDIAC SURGERY}

NHF at $35 \mathrm{~L} /$ min with mouth closed generated a mean (SD) nasopharyngeal airway pressure of 2.7 (1.04) $\mathrm{cmH}_{2} \mathrm{O}$ in 15 post cardiothoracic surgery patients, compared to $1.2(0.76) \mathrm{cmH}_{2} \mathrm{O}$ in mouth open position, $\mathrm{P}=0.0001$ ( $\mathrm{R}$. Parke et al., 2009) and standard Hudson face mask.

To demonstrate the flow-dependent PEEP effect, nasopharyngeal pressure measurements were repeated in 15 post-cardiac patients ( $R$ L Parke et al., 2011). With the mouth closed, the mean (SD) positive nasopharyngeal pressures at 30,40 , and $50 \mathrm{~L} /$ min were $1.93(1.25) \mathrm{cmH}_{2} \mathrm{O}, 2.58(1.54) \mathrm{cmH}_{2} \mathrm{O}$, and 3.31 (1.05) $\mathrm{cmH}_{2} \mathrm{O}$, respectively. A positive linear relationship of the flowdependence was seen so for every 10L/min increase in NHF rate, mean airway pressure increased by $0.69 \mathrm{~cm} \mathrm{H}_{2} \mathrm{O}$ with mouth closed and $0.35 \mathrm{cmH}_{2} \mathrm{O}$ with mouth open.

While the peak nasopharyngeal pressures were seen during expiration, there was a positive pressure effect throughout the entire respiratory cycle in a repeat trial in 15 post cardiothoracic surgery patients $(R L$ Parke \& McGuinness, 2013). There was again a flow-dependence to the positive pressure effect with a maximum expiratory pressure of $4.86 \mathrm{cmH}_{2} \mathrm{O}+/-1.79$ at $50 \mathrm{~L} / \mathrm{min}$ and the findings suggest an application for NHF post-surgery to prevent atelectasis. The authors followed this trial up with a large RCT in postcardiac surgery patients and in fact found no difference in rates of atelectasis on chest x-ray, ICU length of stay or oxygenation at day 3 with prophylactic NHF at $45 \mathrm{~L} /$ min compared to standard oxygen but did significantly reduce need for NIV or intubation ( $27.8 \%$ vs $45 \%$ of patients, $\mathrm{P}<0.001)$ (R. Parke et al., 2013).

Electrical impedance tomography was used in 20 post-cardiac surgery patients respiratory dysfunction as a measure of EELV change, comparing NHF at 35-50L/min against low flow oxygen (A Corley et al., 2011). Oropharyngeal pressure was significantly higher by $3+/-1.2 \mathrm{cmH}_{2} \mathrm{O}$ with $\mathrm{NHF}$ (95\% Cl 2.4-3.7, paired t-test $\mathrm{P}<0.001)$ and lung impedance, which correlates 
with end expiratory lung volumes hence functional residual capacity, was increased by $25.6 \%(95 \% \mathrm{Cl} 24.3-26.9, \mathrm{P}<0.001)$ with NHF. Respiratory rate was also reduced by 3.4 breaths per minute and $\mathrm{PaO}_{2} / \mathrm{F}_{i} \mathrm{O}_{2}$ ratio was improved by $30.6 \mathrm{mmHg}$. Airway pressure was no different whether the mouth was open or closed, but EELV was significantly higher in patients with higher BMls. The overall results again suggest an improvement in alveolar recruitment and ventilation, supported by a healthy volunteer study also demonstrating and increase in EELV using electrical impedance tomography (Riera et al., 2013).

\subsection{EVIDENCE OF PEEP IN COPD}

NHF increased the mean airway pressure in a positive linear dependent manner, with a maximum of $3.01 \pm 1.03 \mathrm{mbar}$ at $50 \mathrm{~L} / \mathrm{min}$. An increase of each $10 \mathrm{~L} /$ min increment of NHF resulted in a 1.4 to 1.6 fold significant mean pressure increase of (Bräunlich \& Köhler, 2016). However, there was a significantly higher PEEP with nasal CPAP and nasal bi-level compared to NHF.

\subsection{Appendix II - Validation of methods: accuracy of transcutaneous monitoring - a prospective observational cohort study (Study 1a) and post-hoc analyses from an RCT (Study 1b).}

\subsubsection{Background}

Three of the studies presented in this thesis (Studies 2, 5 and 6) used transcutaneous monitoring of the partial pressure of carbon dioxide $\left(\mathrm{P}_{\mathrm{t}} \mathrm{CO}_{2}\right)$ as an outcome measure. It is therefore important to present the background of this technique and to validate its use in the setting it will be used for in this thesis.

ABG sampling is the gold standard measurement to estimate arterial partial pressures of oxygen and carbon dioxide levels (Storre et al, 2011) and is a critical measure of ventilation and acid/base balance as described earlier in Section 1.3.1 (Management of AECOPD) with normal values presented in 
Table 1-1. An ABG is obtained by passing a thin needle into an artery, usually the radial artery in the wrist, to collect a single blood sample of approximately $1 \mathrm{~mL}$ that is subsequently sent to the lab for analysis of gases including $\mathrm{PaO}_{2}$, $\mathrm{PaCO}_{2}$ as well as $\mathrm{pH}$ and $\mathrm{HCO}^{-}$. An arterial cannula may be placed in settings such as ICU or during major operations, in order to allow easy access to multiple arterial blood samples.

An alternative to $A B G$ sampling is with transcutaneous monitoring of the partial pressure of carbon dioxide. The advantages of transcutaneous monitoring over ABG sampling are: 1) a simple, non-invasive measurement using a small clip, usually placed on the earlobe, 2) continuous monitoring of carbon dioxide and oxygen levels (Fruchter et al, 2011; Roediger et al., 2011; Storre et al., 2011; Xue et al, 2010), 3) a painless technique, 4) avoiding the potential complications of an ABG such as haematoma formation, infection, distal ischaemia (tissue damage from a lack of blood supply to the area delivered blood by the artery) and fistula or aneurysm formation (Heidari et al., 2013), 5) no requirement for trained healthcare professionals to do the procedure, and 6) avoiding the additional risk of needle stick injury with an ABG.

Transcutaneous carbon dioxide monitor accuracy has been demonstrated in a number of settings, including healthy subjects (Fuke et al., 2009), and patients with COPD (Cox et al, 2006), sleep disorders, critical illness (Rodriguez et al, 2006; Senn et al, 2005) and a mixed group presenting to an emergency department (McVicar \& Eager, 2009; Perrin et al, 2011).

The SenTec is a transcutaneous monitor that has been used at MRINZ in several studies investigating the effects of oxygen therapy. It is able to measure both $\mathrm{P}_{\mathrm{t}} \mathrm{CO}_{2}$ and $\mathrm{S}_{\mathrm{t}} \mathrm{O}_{2}$ (transcutaneous oxygen saturation - measured by the standard spectrophotometric method of pulse oximetry).

SenTec transcutaneous carbon dioxide measurement relies on the property of carbon dioxide having a high tissue solubility and diffuses through the skin (Cox et al, 2006). The SenTec sensor membrane measures the change in $\mathrm{pH}$ (hence a change in electrical potential) as the diffused carbon dioxide dissolves in the sensor membrane electrolyte layer, and derives the $\mathrm{P}_{\mathrm{tCO}}$ according to an inbuilt algorithm. Figure 9.1 describes the complete method of $\mathrm{P}_{\mathrm{t}} \mathrm{CO}_{2}$ measurement. 
A small sensor is clipped to the skin (usually the earlobe ${ }^{\wedge}$ ) with a drop of

"contact" liquid placed between the sensor and skin to ensure close contact.

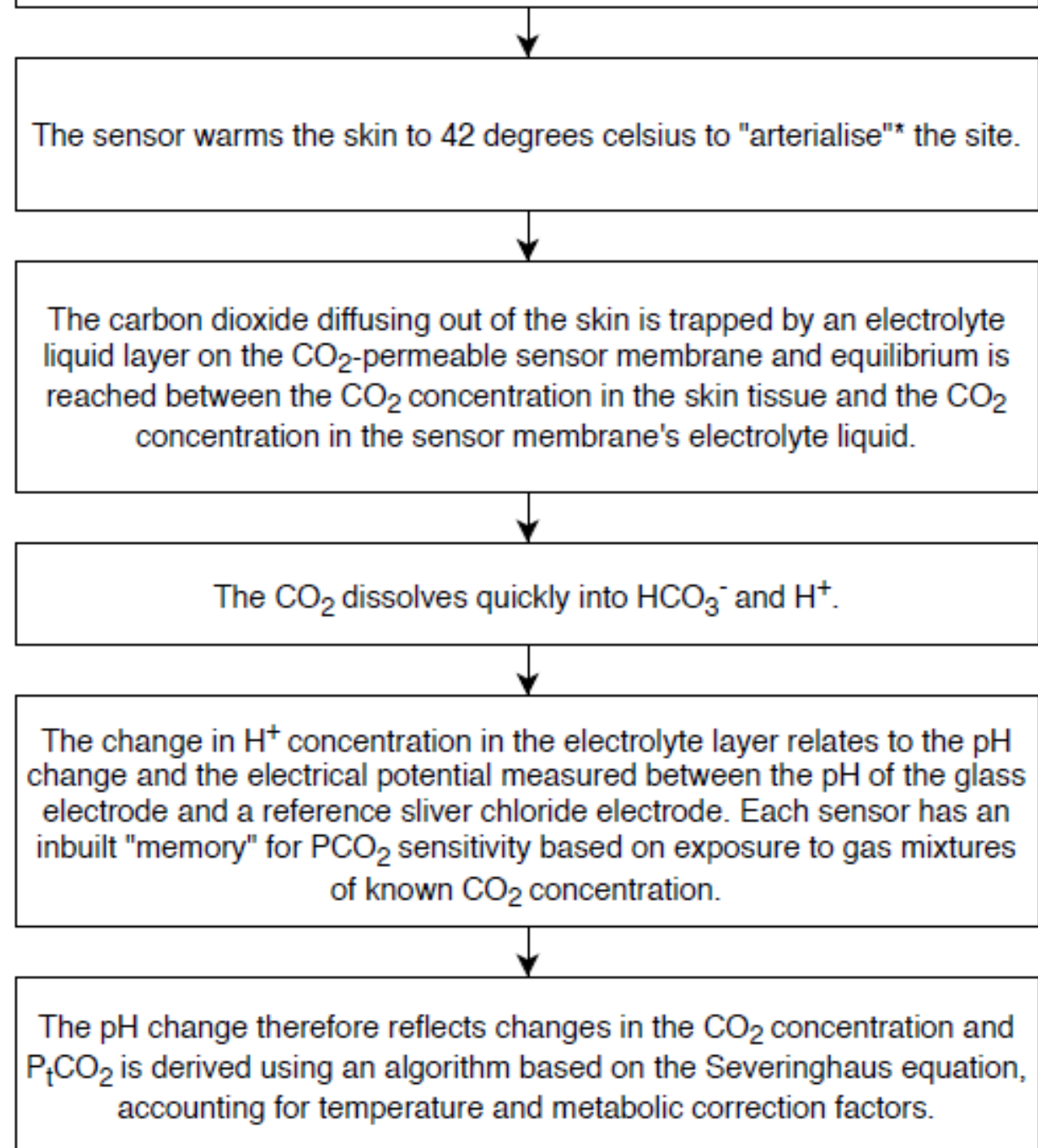

Figure 9-1 Flow chart describing how the SenTec transcutaneous device measures $\mathrm{PtCO}_{2}$.

^ Usually the earlobe is selected as it has a high density of capillaries, although other locations on the body can be used such as the forehead or clavicle which have a thin epidermis and high density of capillaries.

*Arterialisation of the site is important to reduce the arterio-venous carbon dioxide partial pressure difference in the capillary, stabilise metabolism and increase gas diffusion.

A replaceable gas canister containing $8 \%$ carbon dioxide is attached inside the device and serves as the carbon dioxide calibration reference, which the SenTec uses to self-calibrate. The limitations of SenTec $\mathrm{P}_{\mathrm{t}} \mathrm{CO}_{2}$ monitoring are: 
1. Regular maintenance. The sensor membrane requires changing every 28 days. Gas canisters are changed when prompted by the SenTec display. A replaceable gas canister containing $8 \%$ carbon dioxide is attached inside the device and serves as the carbon dioxide calibration reference, which the SenTec uses for selfcalibration.

2. "Drift" of $\mathrm{P}_{\mathrm{tCO}}$ values. A slow deterioration in the sensor membrane electrolyte layer composition (mainly the water drying out) over several hours, affects the measured potential change. The manufacturer estimates the drift equates to $<0.5 \% / \mathrm{hr}$. The Vsign software used to analyse recordings also displays driftcorrected values when data is downloaded. Self-calibration against the $8 \%$ carbon dioxide mixture and regular membrane changes help to offset this effect.

3. "Overshoot" of initial $\mathrm{P}_{\mathrm{t}} \mathrm{CO}_{2}$ values. The sensor requires a period of time to warm the skin and for skin carbon dioxide to reach equilibrium with the sensor carbon dioxide. The manufacturers recommend 2-10 $\mathrm{min}$ for stabilisation, however 20-30 $\mathrm{min}$ is reported by the literature (see Study 1a Study procedures) (Domingo et al., 2010; Kagawa et al., 2004).

4. Consistent over-measurement of $\mathrm{P}_{\mathrm{t}} \mathrm{CO}_{2}$ compared to $\mathrm{P}_{\mathrm{a}} \mathrm{CO}_{2}$. Input of regular $\mathrm{ABG}$ values can be done to make a " $\mathrm{PCO}_{2}$ In-vivo correction", adjusting the metabolic offset component of the inbuilt algorithm.

5. Possible inaccuracies in clinical scenarios such as a hypoperfused state e.g. shock, hypothermia.

6. Inadequate contact with the skin. The diffused carbon dioxide will therefore mix with ambient air and influence readings.

\subsubsection{Rationale}

MRINZ owns two SenTec devices. While the SenTec has been validated for accuracy previously,(Domingo et al., 2006; Rodriguez et al., 2006; Roediger et al., 2011; Storre et al., 2011) the MRINZ devices were validated in the 
setting and under the standard operating procedures that are used in MRINZ trials including those in this thesis.

This chapter comprises two studies undertaken at MRINZ which I was a coinvestigator for. Study 1b naturally followed on from the results of Study 1a. These data have also been presented by the study co-investigators, George Bardsley and Janine Pilcher, as part of their respective theses.

For Study 1a, the aim was to recruit outpatients who were due to receive an $A B G$ as part of their clinical management at Wellington Hospital, and when the $A B G$ sample was taken, the study investigator recorded the carbon dioxide and oxygen saturation levels displayed by the transcutaneous monitor. These values were then compared to the $A B G$ results. Based on the results of Study 1a, Study $1 \mathrm{~b}$ was then required to measure $\mathrm{P}_{\mathrm{t}} \mathrm{CO}_{2}$ change over time, comparing serial $\mathrm{P}_{\mathrm{t}} \mathrm{CO}_{2}$ and $\mathrm{P}_{c a p} \mathrm{CO}_{2}$ measurements.

\subsubsection{Study 1a - Accuracy of the SenTec transcutaneous carbon dioxide monitoring device in assessing $\mathrm{PaCO}_{2}$ : A prospective observational cohort study}

\subsubsection{Aims and hypotheses}

Using the two SenTec devices, the aims were to determine the agreement between $\mathrm{P}_{\mathrm{a}} \mathrm{CO}_{2}$ and $\mathrm{P}_{\mathrm{t}} \mathrm{CO}_{2}$, and $\mathrm{S}_{\mathrm{a}} \mathrm{O}_{2}$ and $\mathrm{S}_{\mathrm{t}} \mathrm{O}_{2}$ measurement in terms of bias and limits of agreement.

The hypothesis was that there would be close agreement between $\mathrm{PaCO}_{2}$ and $\mathrm{P}_{\mathrm{t}} \mathrm{CO}_{2}$, and $\mathrm{S}_{\mathrm{a}} \mathrm{O}_{2}$ and $\mathrm{S}_{\mathrm{t}} \mathrm{O}_{2}$ measurements.

\subsubsection{Methods}

\subsection{DESIGN}

Single centre, prospective, observational study.

\subsection{ELIGIBILITY CRITERIA}

\subsection{INCLUSION CRITERIA}

Any patient requiring an $A B G$ measurement as part of their clinical care at Wellington Hospital Respiratory outpatient department. 


\subsection{EXCLUSION CRITERIA}

1. Diagnosis of sickle cell anaemia, methaemoglobinemia, or carbon monoxide poisoning (all factors known to affect oxygen saturation recordings).

2. Patients previously recruited to the study with paired transcutaneous and arterial values successfully recorded on the same device.

3. Age under 16 years.

4. Poor reading/signal quality from participant's earlobe(s) as per display warnings on transcutaneous device.

5. Any other condition which, at the investigator's discretion, was believed may present a safety risk or impact upon the feasibility of the study or the interpretation of the study results.

\subsection{SAMPLE EXCLUSION CRITERIA}

Measurements paired with blood gas values subsequently identified to be venous or unusable were excluded (for further detail see Study Procedures below).

\subsubsection{Recruitment and consent}

Potential participants were identified in outpatient clinics at the Respiratory Department in Wellington Hospital. Full written informed consent took place prior to study participation. The study received ethics approval by the Southern Health and Disability Ethics Committee (HDEC) of New Zealand (Ref: 15/STH/121) and was prospectively registered with the Australia and New Zealand Clinical Trials Registry (ANZCTR, Trial ID: ACTRN12615001154594).

\subsubsection{Study Procedures}

Demographic data were collected then for each participant, one SenTec was attached to each earlobe while they were seated. For each of the two SenTec devices, there were 16 measurements made, which were individually paired (one device on one participant) or paired simultaneously (both devices on one participant at the same time - one on each earlobe).

\subsubsection{1 $\mathrm{P}_{\mathrm{t}} \mathrm{CO}_{2}$ STABILISATION}

The SenTec sensors were stabilised on the earlobe for at least $30 \mathrm{~min}$, a timeperiod based on previous studies.(Christian Domingo et al., 2010; Kagawa et 
al., 2004) In order to demonstrate SenTec $\mathrm{P}_{\mathrm{t}} \mathrm{CO}_{2}$ measurement stability, the $\mathrm{P}_{\mathrm{t}} \mathrm{CO}_{2}$ at 2 min prior to the end of the stabilisation period had to be within 1 $\mathrm{mmHg}$ of the value at the end of stabilisation, and the probe temperature 42 degrees. If not, the stabilisation period was continued until there was a 2-min period where the $\mathrm{P}_{\mathrm{tCO}}$ at the start was within $1 \mathrm{mmHg}$ of the $\mathrm{P}_{\mathrm{t}} \mathrm{CO}_{2}$ at the end, and the temperature was 42 degrees.

Once $\mathrm{P}_{\mathrm{t}} \mathrm{CO}_{2}$ stabilisation was obtained, either the member of the participant's usual clinical team (such as a doctor or respiratory technician) or the study investigator would perform $A B G$ collection. $\mathrm{P}_{\mathrm{t}} \mathrm{CO}_{2}$ and $\mathrm{S}_{\mathrm{t}} \mathrm{O}_{2}$ values were recorded following visualisation of blood entering the collection vial (heparinised BD Preset ${ }^{\mathrm{TM}}$ Eclipse $^{\mathrm{TM}}$ Arterial Blood Collection Syringe, with 23guage needle). ABG analysis was completed at the Wellington Hospital Laboratory (Radiometer ABL800 FLEX, Copenhagen, Denmark). Once the $A B G$ was analysed, $\mathrm{P}_{\mathrm{a}} \mathrm{CO}_{2}$ and $\mathrm{S}_{2} \mathrm{O}_{2}$ values were recorded from the $\mathrm{ABG}$ print out.

\subsubsection{Sample size and statistical methods}

The sample size of 16 patients per device was based on a one sided test ruling out a carbon dioxide bias difference of more than $1 \mathrm{mmHg}$, with $80 \%$ power and an SD of $1.9 \mathrm{mmHg}$. This was consistent with the previous sample size and SDs from the TOSCA (another transcutaneous monitor) validation performed by the MRINZ in patients with pneumonia and asthma (Perrin et al., 2011). Limits of agreement were estimated for the difference between ABG and individual SenTec devices and between SenTec devices based on the mean paired differences and the SDs of these differences as shown on BlandAltman plots.

Mixed linear models were used with random effects for participants and SenTec devices and fixed effects for ABG versus transcutaneous measurements of $\mathrm{PCO}_{2}$ and $\mathrm{S}_{\mathrm{a}} \mathrm{O}_{2}$, including an interaction term to examine if the slope relationships between the co-variates and either $\mathrm{PCO}_{2}$ or $\mathrm{S}_{\mathrm{a}} \mathrm{O}_{2}$ differed between ABG and transcutaneous device. SAS version 9.3 was used. 


\subsubsection{Results}

Participants were recruited between November 2015 and January 2016. Sixteen pairs of data were collected, except for SenTec device \#1 which had one unreliable reading and had one recording excluded, therefore, 15 paireddata points were collected and analysed from this device.

Baseline characteristics are presented below in Table 9.1 showing a mean (SD) age of 67 (11.9) years and $75 \%$ of participants were female. The mean $\mathrm{P}_{\mathrm{aCO}}$ was 41.7 (9.9) $\mathrm{mmHg}$ and mean $\mathrm{P}_{\mathrm{t}} \mathrm{CO}_{2}$ from both devices was 39.9 (8.7) $\mathrm{mmHg}$. The mean $\mathrm{SaO} 2$ was 92.2 (7.2) \% and mean $\mathrm{StO} 2$ was 91.5 (8.6) $\%$.

Table 9-1 Study 1a baseline participant characteristics.

\begin{tabular}{|c|c|c|c|}
\hline Characteristic & Mean (SD) & Median (IQR) & Min to Max \\
\hline Age (years) & $67.0(11.9)$ & 71.7 (58.2 to 74.6$)$ & 39.9 to 85.3 \\
\hline Carboxyhaemoglobin & $2.2(2.2)$ & $1.6(1.3$ to 1.8$)$ & 0.9 to 8.1 \\
\hline Haemoglobin (g/L) & $133.9(20.3)$ & 136.5 (122 to 145$)$ & 76 to 167 \\
\hline Methhaemoglobin & $0.81(0.31)$ & 0.75 (0.60 to 0.95$)$ & 0.30 to 1.50 \\
\hline $\mathrm{P}_{\mathrm{a}} \mathrm{CO}_{2}(\mathrm{mmHg})$ & $41.7(9.9)$ & 41.0 (36.5 to 44.0$)$ & 27.0 to 71.0 \\
\hline $\mathrm{P}_{\mathrm{a}} \mathrm{O}_{2}(\mathrm{mmHg})$ & $71.5(19.3)$ & 73.5 (60.0 to 80.0$)$ & 38.0 to 116.0 \\
\hline $\mathrm{S}_{\mathrm{aO}} \mathrm{O}_{2}$ & $92.2(7.2)$ & 95.0 (91.0 to 96.5$)$ & 74.0 to 99.0 \\
\hline $\mathrm{P}_{\mathrm{t}} \mathrm{CO}_{2}$ SenTec \# $1^{1}$ & $39.8(8.8)$ & 38.9 (34.1 to 43.6 ) & 27.7 to 65.9 \\
\hline $\mathrm{P}_{\mathrm{t}} \mathrm{CO}_{2}$ SenTec \# 2 & $39.9(8.6)$ & 40.2 (34.7 to 42.2$)$ & 27.2 to 66.0 \\
\hline $\mathrm{S}_{\mathrm{t}} \mathrm{O}_{2}$ SenTec \# 1 & $91.4(8.8)$ & 93.5 (90.5 to 97.0$)$ & 65.0 to 99.0 \\
\hline \multirow[t]{2}{*}{$\mathrm{S}_{\mathrm{t}} \mathrm{O}_{2}$ SenTec \# 2} & $91.6(8.3)$ & 94.5 (90.5 to 96.0$)$ & 67.0 to 100.0 \\
\hline & & $\mathrm{N} / 16(\%)$ & \\
\hline \multicolumn{4}{|l|}{ Ethnicity } \\
\hline \multicolumn{2}{|l|}{ Maori } & $1(6)$ & \\
\hline \multicolumn{2}{|l|}{ NZ European } & $13(81)$ & \\
\hline \multicolumn{2}{|l|}{ Pacific } & $2(13)$ & \\
\hline \multicolumn{2}{|l|}{ Female Sex } & $12(75)$ & \\
\hline \multicolumn{4}{|l|}{ Smoking status } \\
\hline \multicolumn{2}{|l|}{ Current } & $1(6)$ & \\
\hline \multicolumn{2}{|l|}{ Ex } & $10(63)$ & \\
\hline \multicolumn{2}{|l|}{ Never } & $5(31)$ & \\
\hline
\end{tabular}

Derived comparison values between $A B G$ and both SenTec devices, in addition to each device compared against each other are shown below in Table 9.2.

The mean (SD) difference between $\mathrm{ABG}\left(\mathrm{P}_{\mathrm{a}} \mathrm{CO}_{2}\right)$ and both devices $\left(\mathrm{P}_{\mathrm{t}} \mathrm{CO}_{2}\right)$ for carbon dioxide recordings was $2.2(3.1) \mathrm{mmHg}$ higher on arterial sample 
than transcutaneous reading and there was a negligible difference between devices.

Table 9-2 Data description for the differences between each SenTec and ABG values, and between SenTec \#1 and \#2.

\begin{tabular}{|c|c|c|c|}
\hline Variable & Mean (SD) & Median (IQR) & Min to Max \\
\hline \multicolumn{4}{|c|}{ ABG vs SenTec \# 1} \\
\hline $\mathrm{PCO}_{2}$ difference ${ }^{1}$ & $2.2(3.1)$ & $2.1(0.1$ to 5.1$)$ & -4.6 to 7.0 \\
\hline $\mathrm{PCO}_{2}$ average ${ }^{1}$ & $40.9(9.4)$ & 41.2 (34.9 to 42.9 ) & 27.4 to 68.5 \\
\hline StO2 difference & $0.8(2.7)$ & $0(-1$ to 2$)$ & -2 to 9 \\
\hline StO2 average & $91.8(7.9)$ & 94.5 (90.8 to 96.8$)$ & 69.5 to 98.0 \\
\hline \multicolumn{4}{|c|}{ ABG vs SenTec \# 2} \\
\hline $\mathrm{PCO}_{2}$ difference & $2.2(3.1)$ & $2.1(0.1$ to 5.1$)$ & -4.6 to 7.0 \\
\hline $\mathrm{PCO}_{2}$ average & $40.8(9.2)$ & 40.7 (35.5 to 42.5$)$ & 27.1 to 68.5 \\
\hline StO2 difference & $0.6(2.2)$ & $0(0$ to 1$)$ & -3 to 7 \\
\hline $\mathrm{StO} 2$ average & $91.9(7.7)$ & 94.8 (90.8 to 96.5$)$ & 70.5 to 98.5 \\
\hline \multicolumn{4}{|l|}{ SenTec \# 2 vs \# 1} \\
\hline $\mathrm{P}_{\mathrm{t}} \mathrm{OO}_{2}$ difference ${ }^{1}$ & $-0.3(1.3)$ & $-0.1(-1.4$ to 0.8$)$ & -2.5 to 1.9 \\
\hline $\mathrm{PtCO}_{2}$ average $^{1}$ & $39.9(8.8)$ & 39.9 (34.4 to 42.7$)$ & 27.5 to 66.0 \\
\hline StO2 difference & $-0.1(1.2)$ & $0(-1$ to 1$)$ & -2 to 2 \\
\hline StO2 average & $91.5(8.5)$ & 94.0 (90.5 to 96.5$)$ & 66.0 to 99.0 \\
\hline
\end{tabular}

The comparison of $A B G$ and SenTec values for $\mathrm{P}_{\mathrm{t}} \mathrm{CO}_{2}$ and $\mathrm{StO}$, as well as between SenTec \#1 and \#2 are shown in Table 9.2 and the limits of agreement in the following 6 Bland-Altman plots (Fig 9.2 to 9.7).

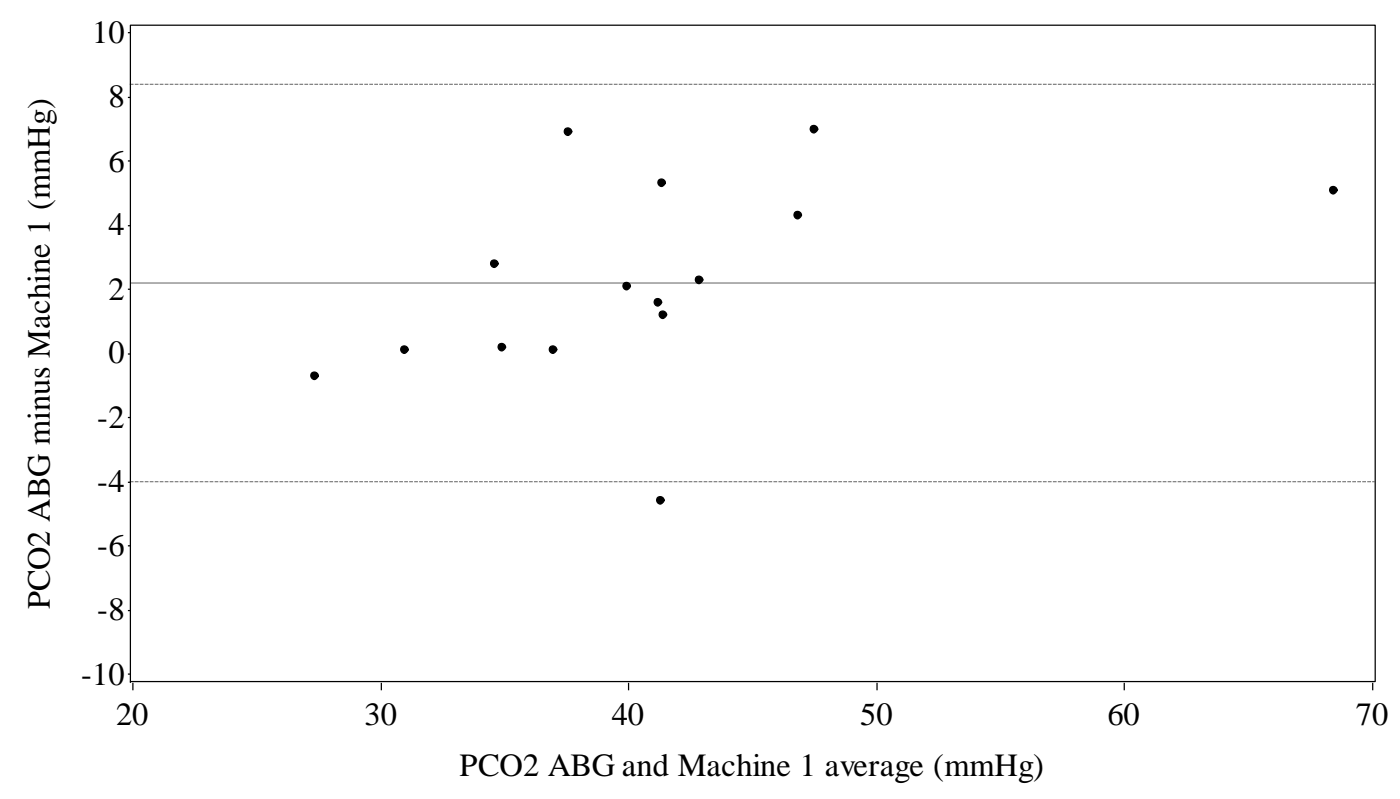


Figure 9-2 Bland-Altman Plots of limits of agreement of $\mathrm{PCO}_{2}$ between $A B G$ and SenTec \#1. The horizontal lines represent two standard deviations of the differences.

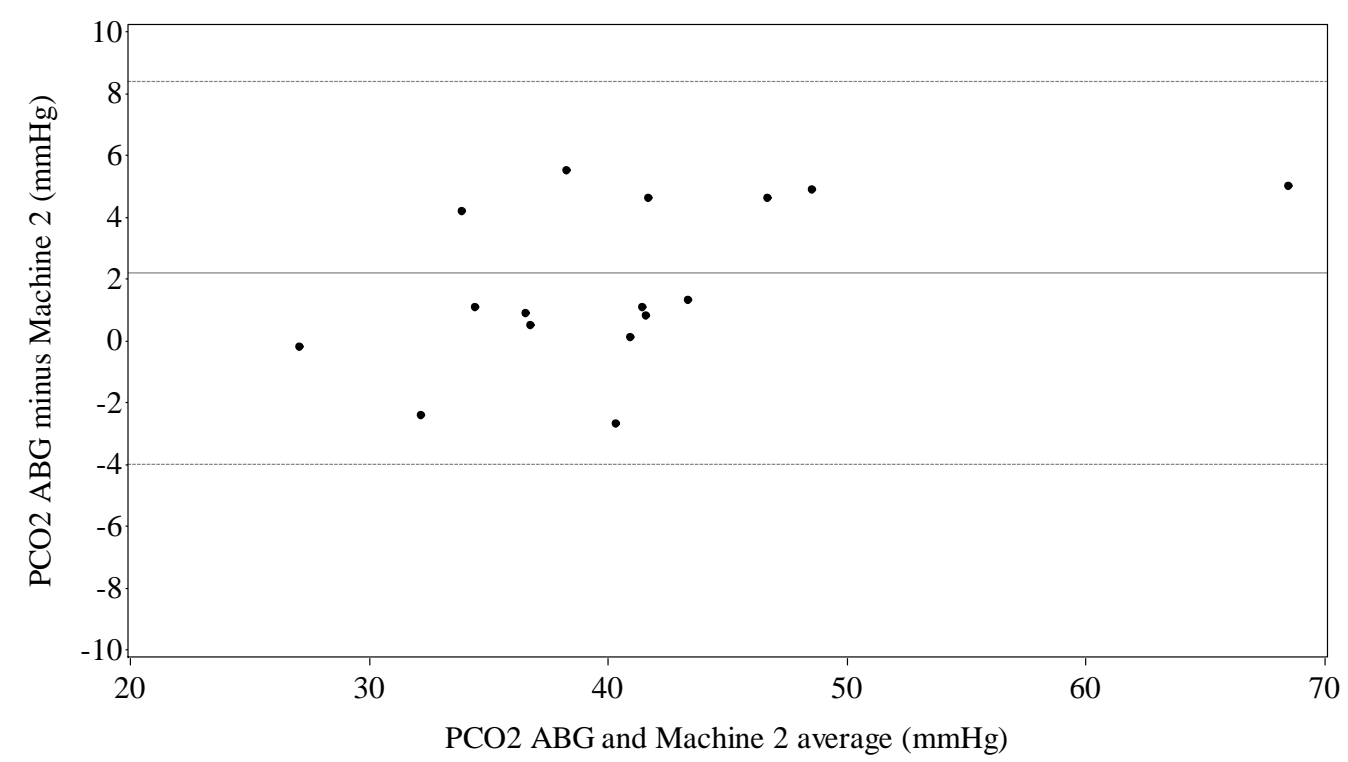

Figure 9-3 Bland-Altman plots of limits of agreement of $\mathrm{PCO}_{2}$ between ABG and SenTec \# 2. The horizontal lines represent two standard deviations of the differences.

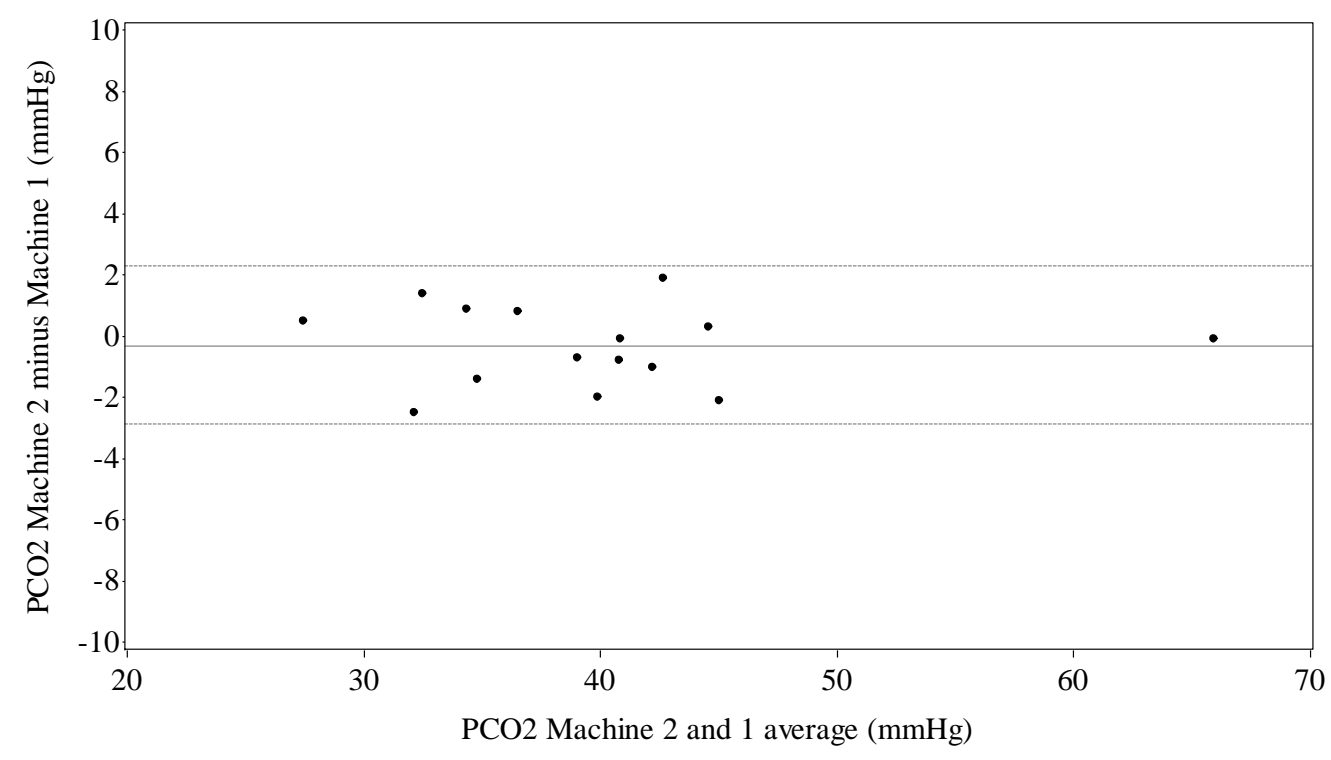

Figure 9-4 Bland-Altman plots of limits of agreement of $\mathrm{PCO}_{2}$ between $A B G$ and SenTec \# 1 and 2. The horizontal lines represent two standard deviations of the differences. 


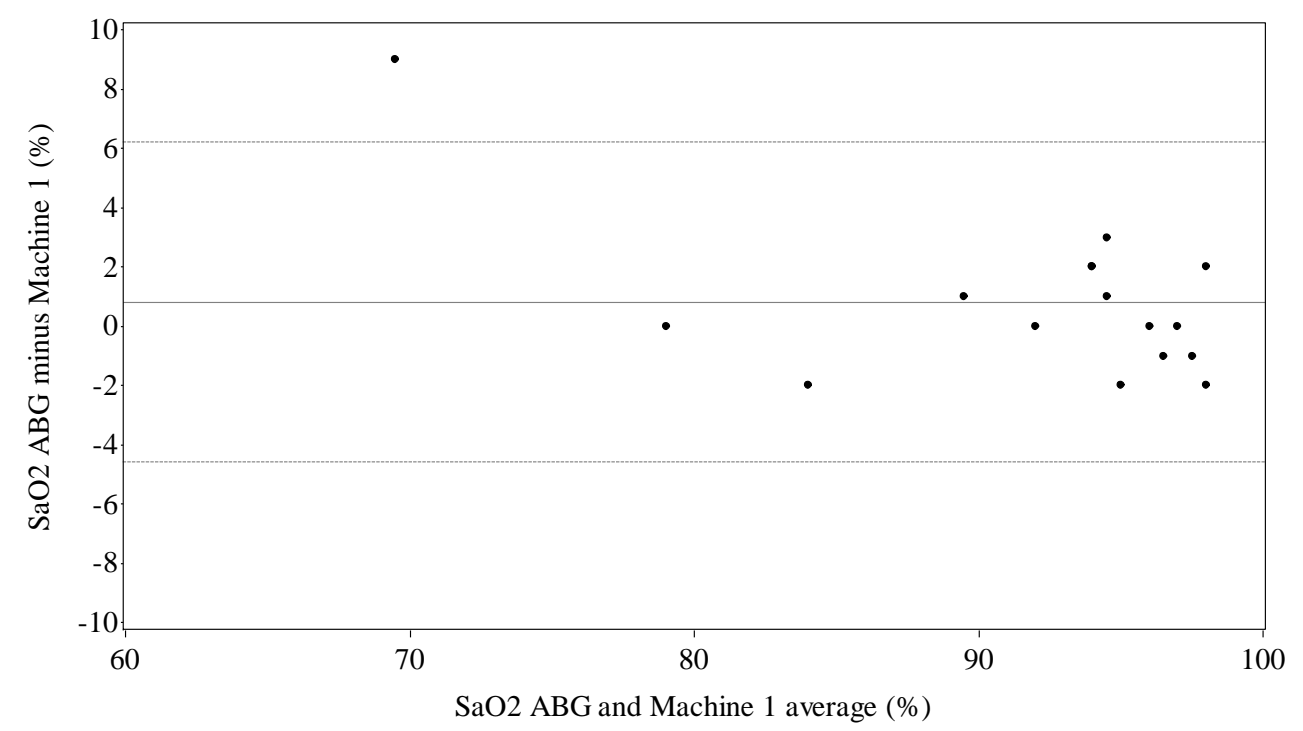

Figure 9-5 Bland-Altman plots of limits of agreement of $\mathrm{S}_{\mathrm{a}} \mathrm{O}_{2}$ between ABG and SenTec \#1. The horizontal lines represent two standard deviations of the differences.

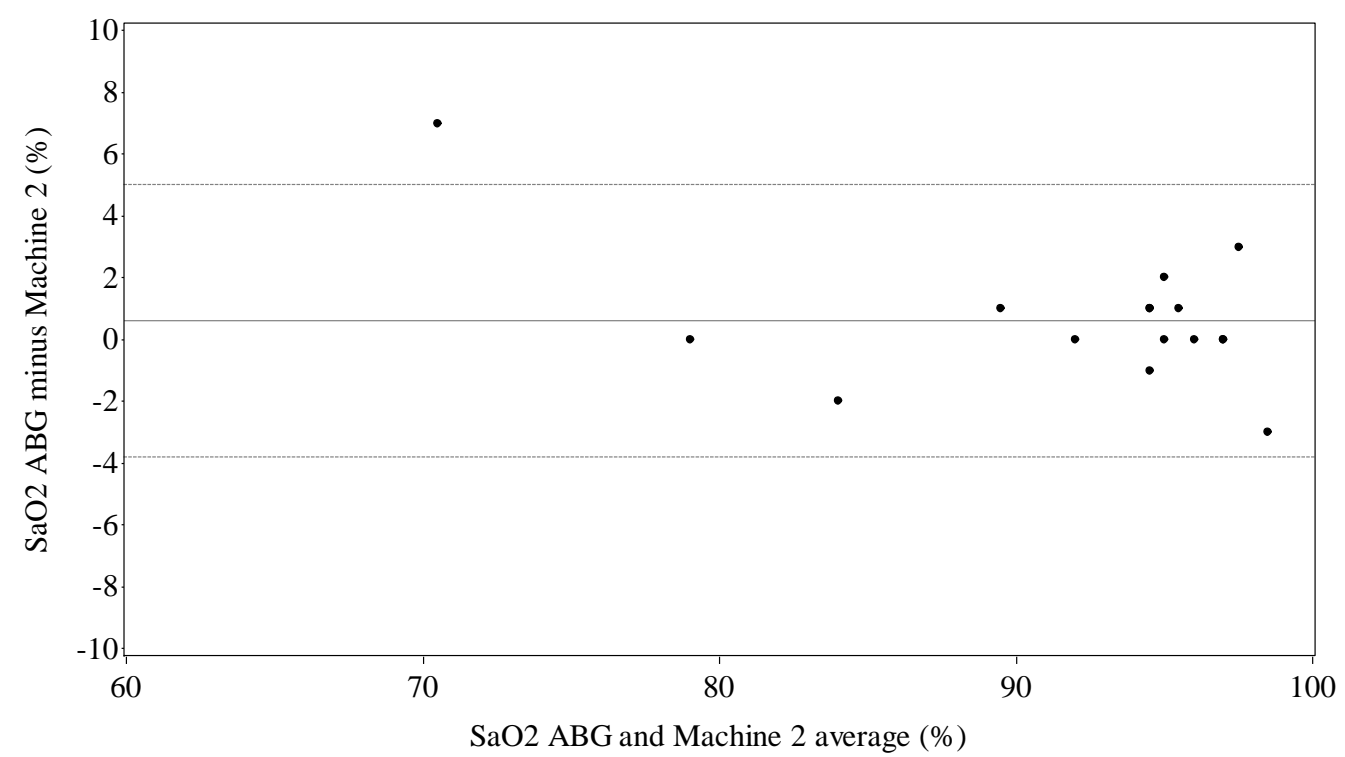

Figure 9-6 Bland-Altman plots of limits of agreement of $\mathrm{S}_{\mathrm{a}} \mathrm{O}_{2}$ between ABG and SenTec \#2. The horizontal lines represent two standard deviations of the differences. 


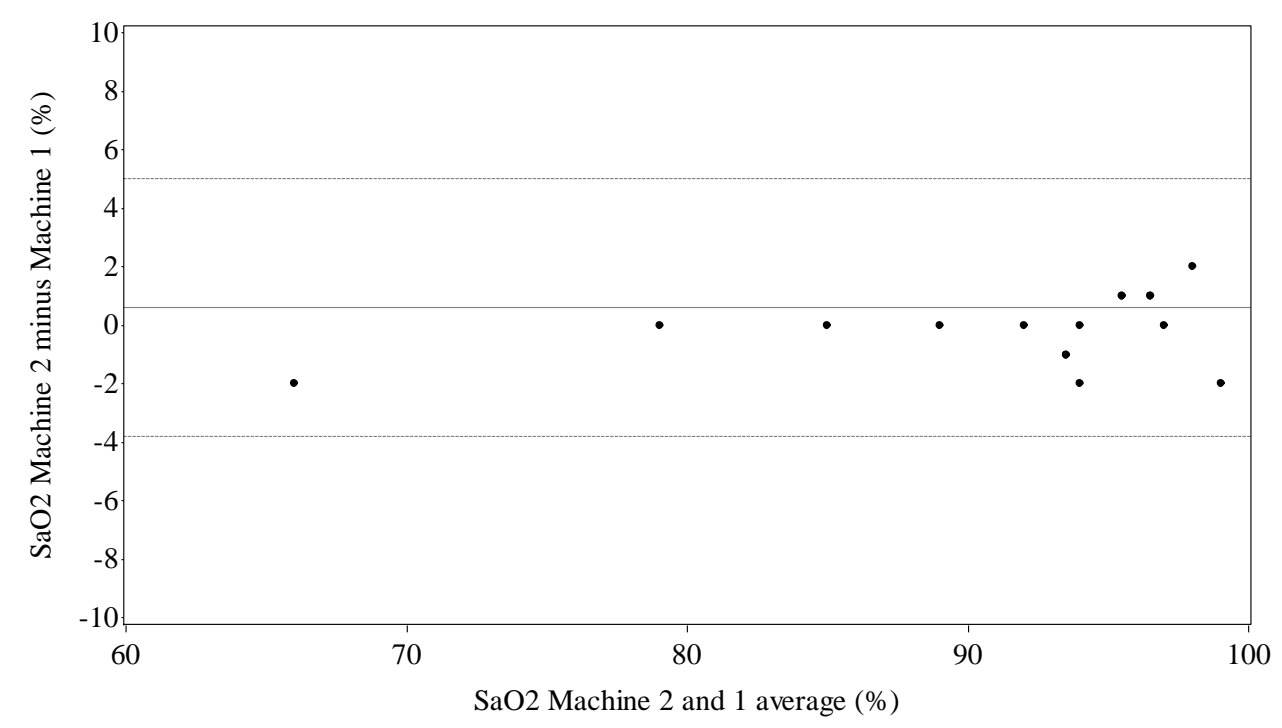

Figure 9-7 Bland-Altman plots of limits of agreement of $\mathrm{SaO} 2$ between ABG and SenTec \# 1 and \#2. The horizontal lines represent two standard deviations of the differences.

Values for limits of agreement are shown below in Table 9.3. Overall, both SenTec devices had a bias of $2.2 \mathrm{mmHg}$ higher than actual arterial values for $\mathrm{PaCO}_{2}$, with wide limits of agreement of $\pm 6.2 \mathrm{mmHg}(-4$ to $8.4 \mathrm{mmHg})$. Between the 2 devices, the limits of agreement were narrow $(-0.3 \mathrm{mmHg})$ indicating a negligible difference in transcutaneous measurements compared to each other.

Bias and limits of agreement for oxygen saturations are also reported for individual devices below with similarly wide limits of agreement.

Table 9-3 Bias and limits of agreement between ABG and SenTec \#1 and SenTec \#2.

\begin{tabular}{lll}
\hline Comparison & Bias & Limits of agreement \\
\hline ABG vs SenTec \# 1 & & \\
\hline $\mathrm{PCO}_{2}$ & 2.2 & -4 to 8.4 \\
$\mathrm{~S}_{2} \mathrm{O}_{2}$ & 0.8 & -4.6 to 6.2 \\
\hline ABG vs SenTec \# 2 & \\
\hline $\mathrm{PCO}_{2}$ & 2.2 & -4 to 8.4 \\
$\mathrm{~S}_{2} O_{2}$ & 0.6 & -3.8 to 5 \\
\hline $\mathrm{SenTeC} \mathrm{\#} \mathrm{2} \mathrm{vs} \mathrm{\#} \mathrm{1}$ & -0.3 & -2.9 to 2.3 \\
\hline $\mathrm{PCO}_{2}$ & -0.1 & -2.5 to 2.3 \\
$\mathrm{SaO}_{2}$ &
\end{tabular}

$A B G$ : arterial blood gas, $\mathrm{SaO2}$ : arterial oxygen saturation, $\mathrm{PCO}_{2}$ : partial pressure of carbon dioxide measured either transcutaneously or via $A B G$. 


\subsubsection{Discussion}

The results indicate that the SenTec monitors were performing in line with reported limits of agreement in other studies (Fuke et al., 2009; McVicar \& Eager, 2009; Rodriguez et al., 2006; Senn et al., 2005), and confirm there are limitations in interpreting single $\mathrm{P}_{\mathrm{tCO}}$ values without $A B G$ validation (Restrepo et al, 2012).

The limitations of this study relate to the methodology. Participants were enrolled almost exclusively from the outpatient department and were in a stable clinical state. They were mostly normocapnic, with a mean $\mathrm{PCO}_{2}$, either arterial or transcutaneous, of around $40 \mathrm{mmHg}$. Testing transcutaneous monitors in in patients with very high or low $\mathrm{PCO}_{2}$, and low $\mathrm{S}_{p} \mathrm{O}_{2}$ has been reported (Bendjelid et al., 2005; Janssens et al, 1998; Cox et al, 2006; Senn et al., 2005) giving some confidence in the reliability of measurements with wide variation; however, there was no evidence from this study to proffer a conclusion about the accuracy of $\mathrm{P}_{\mathrm{t}} \mathrm{CO}_{2}$ with very high or very low values - a similar conclusion attained by a recent systematic review and meta-analysis of transcutaneous monitoring (Conway et al, 2018).

\subsubsection{Conclusion}

The SenTec transcutaneous devices had limitations with accuracy in measuring absolute values of $\mathrm{PaCO}_{2}$, and, if being used in a clinical setting, these limits of agreement may be clinically significant and impact management. These findings suggest it may be useful in the research setting where it is hard to justify multiple ABG samples, given the risks to participants of hand ischaemia and a potentially painful procedure. However, for the clinical trials in this thesis, the wide limits of agreement for absolute $\mathrm{P}_{2} \mathrm{CO}_{2}$ measures found in this observational study resulted in the need for further validation of $\mathrm{P}_{\mathrm{t}} \mathrm{CO}_{2}$ monitoring. 


\subsubsection{Study $1 \mathrm{~b}$ - Post-hoc analyses of $\mathrm{P}_{t} \mathrm{CO}_{2}$ accuracy compared to $\mathrm{P}_{\text {cap }} \mathrm{CO}_{2}$ from an $\mathrm{RCT}$}

\subsubsection{Rationale}

Study 1 a demonstrated that the limits of agreement for $\mathrm{P}_{\mathrm{t}} \mathrm{CO}_{2}$ compared with $\mathrm{PaCO}_{2}$ were too wide for $\mathrm{P}_{\mathrm{t}} \mathrm{CO}_{2}$ to be used as an accurate, surrogate, single measure of $\mathrm{PaCO}_{2}$. The diagnosis of hypercapnic respiratory failure may be incorrect due to the limits of agreement of $\pm 6.2 \mathrm{mmHg}$. A similar conclusion was made from a recent systematic review and meta-analysis comparing $\mathrm{P}_{\mathrm{aCO}}$ and $\mathrm{P}_{\mathrm{t}} \mathrm{CO}_{2}$ measurements (Conwayet al $\mathrm{A}, 2018$ ).

However, the primary outcome measure for the trials in this thesis are the change in $\mathrm{P}_{t} \mathrm{CO}_{2}$ over time, rather than the absolute $\mathrm{PaCO}_{2}$ values. The limits of agreement for change in $\mathrm{P}_{\mathrm{t}} \mathrm{CO}_{2}$ and $\mathrm{P}_{\mathrm{a}} \mathrm{CO}_{2}$ were not investigated in Study $1 \mathrm{a}$ and became the focus of Study $1 \mathrm{~b}$.

$\mathrm{Dr}$ George Bardsley was the main investigator and I was a co-investigator for a 90 participant RCT comparing the effect on $\mathrm{P}_{\mathrm{t}} \mathrm{CO}_{2}$ of air versus oxygen nebulisers in AECOPD. As a by-product of this study, data were collected comparing $\mathrm{P}_{\text {cap }} \mathrm{CO}_{2}$ and $\mathrm{P}_{\mathrm{t}} \mathrm{CO}_{2}$ values which were analysed to draw a conclusion on the accuracy of the SenTec device measurement of $\mathrm{P}_{\mathrm{tCO}}$ change over time. This RCT and post-hoc analysis formed the major part of George Beardsley's MD thesis (completed at MRINZ, submitted to the University of Newcastle upon Tyne in 2018). Janine Pilcher also presented these data in her $\mathrm{PhD}$ thesis submitted to Victoria University of Wellington in 2018.

\subsubsection{Aims and hypotheses}

The aims were:

- To describe the absolute agreement between $\mathrm{P}_{\text {cap }} \mathrm{CO}_{2}$ and $\mathrm{P}_{\mathrm{t}} \mathrm{CO}_{2}$ measurement in terms of bias and limits of agreement and also to compare their agreement with $\mathrm{P}_{c a p} \mathrm{CO}_{2}$ and $\mathrm{P}_{t} \mathrm{CO}_{2}$ change over time.

The hypothesis was:

- the trend in $\mathrm{P}_{\mathrm{t}} \mathrm{CO}_{2}$ change would show more precise agreement with $\mathrm{P}_{\text {cap }} \mathrm{CO}_{2}$ and be sufficiently accurate to guide treatment in hypercapnic respiratory failure. 


\subsubsection{Methods}

Paired $\mathrm{P}_{\mathrm{t}} \mathrm{CO}_{2}$ (SenTec AG, Switzerland) and $\mathrm{P}_{\text {cap }} \mathrm{CO}_{2}$ data were available from 80 adults with COPD who took part in a study investigating the effect of oxygen-driven versus air-driven nebulisers. $\mathrm{P}_{\text {cap }} \mathrm{CO}_{2}$ and $\mathrm{P}_{\mathrm{t}} \mathrm{CO}_{2}$ measurements were taken at the zero time-point and at $35 \mathrm{~min}$.

\subsection{CBG SAMPLING}

CBG sampling for the estimation of $\mathrm{PaCO}_{2}$ and $\mathrm{pH}$ is accurate in both stable and acute settings, including COPD, and across a wide range of values (Murphy et al., 2006; Yildizdaş et al., 2004). The mean difference between $\mathrm{PaCO} 2$ and $\mathrm{PcapCo} 2$ in one study was $0.7 \mathrm{mmHg}(95 \%$ limits of agreement 5.01 to $6.2 \mathrm{mmHg}$ ) and $\mathrm{pH}$ is reported in $\mathrm{H}^{+}$ion concentration, with a difference of $1.04 \mathrm{nmol} / \mathrm{L}$ (95\% limits of agreement -2.18 to 4.27 )( (Murphy et al., 2006). A 187 patient study in ED demonstrated a $\mathrm{PaCO}_{2} / \mathrm{P}_{\mathrm{cap}} \mathrm{CO}_{2}$ relationship of $0.21 \mathrm{mmHg} \pm 5.0 \mathrm{mmHg}$, and $\mathrm{pH}$ relationship of $0.00 \pm 0.05$ units (Heidari et al., 2013), and a 29 study meta-analysis showed a strong correlation between arterial and capillary samples with a mean $\mathrm{PCO}_{2}$ bias of $2.2 \mathrm{mmHg}$ and mean pH bias of 0.02 units (Zavorsky et al., 2007). The differences between earlobe and fingertip collection sites for these values are clinically unimportant (Heidari et al., 2013; Murphy et al., 2006; Zavorsky et al., 2007).

The CBG collection method required 'arterialisation' of the fingertip, which consists of heat application or application of a topical vasodilatory substance to increase the arteriolar component of the capillary bed to more accurately reflect the arterial value (Zavorsky et al, 2007). A sterile lancet was then used to puncture the capillary bed. The first drop of blood was wiped clear, and a heparinised capillary collection tube was placed on the drop of blood that forms over the puncture site. Blood is then propelled into the capillary tube by the combination of surface tension and adhesive forces between the blood and tubing. Once full with $1 \mathrm{~mL}$ of blood, one end of the tube was capped off and a metallic microfilament 'flea' was inserted into the tube before capping off the other end. A magnet was then moved backand-forth along the capillary tube to move the microfilament, mixing the blood 
to heparinise the sample and prevent clotting. The sample was analysed using the Radiometer ABL800 FLEX machine.

\subsubsection{Statistical analyses}

A limits of agreement analysis was performed, with estimation of the bias by paired $\mathrm{t}$-test for the differences in the two measurements.

\subsubsection{Results}

Eighty participants were recruited between May 2015 and June 2016. The limits of agreement between $\mathrm{P}_{\mathrm{t}} \mathrm{CO}_{2}$ and $\mathrm{P}_{\text {cap }} \mathrm{CO}_{2}$ were approximately \pm 3.8 $\mathrm{mmHg}$ for each individual measurement obtained $(n=160)$. This is illustrated in the Bland-Altman plot (Fig 9.8) below. The mean change over $35 \mathrm{~min}$ in $\mathrm{P}_{\mathrm{t}} \mathrm{CO}_{2}$ was $1.7 \mathrm{mmHg}$ (SD 2.2, range -2.5 to 8.0 ), and the mean change in $\mathrm{P}_{\text {cap }} \mathrm{CO}_{2}$ was $1.7 \mathrm{mmHg}$ (SD 2.3, range -3.0 to 9.0 ), revealing an estimate of bias for change in carbon dioxide of minus $0.03(95 \% \mathrm{Cl}-0.44$ to 0.38$) \mathrm{P}=0.89$.

Table 9-4 Comparisons in CBG-measured $\mathrm{PCO}_{2}$ and SenTec-measured $\mathrm{PCO}_{2}$

\begin{tabular}{llll}
\hline Variable & Mean (SD) & Median (IQR) & Min to Max \\
\hline Difference & $-0.03(1.9)$ & $0.0(-0.95$ to 1.15$)$ & -6.6 to 4.5 \\
between $\mathrm{PCO}_{2}$ & & & \\
change & & & \\
$(\mathrm{mmHg})$ & & & \\
\hline
\end{tabular}




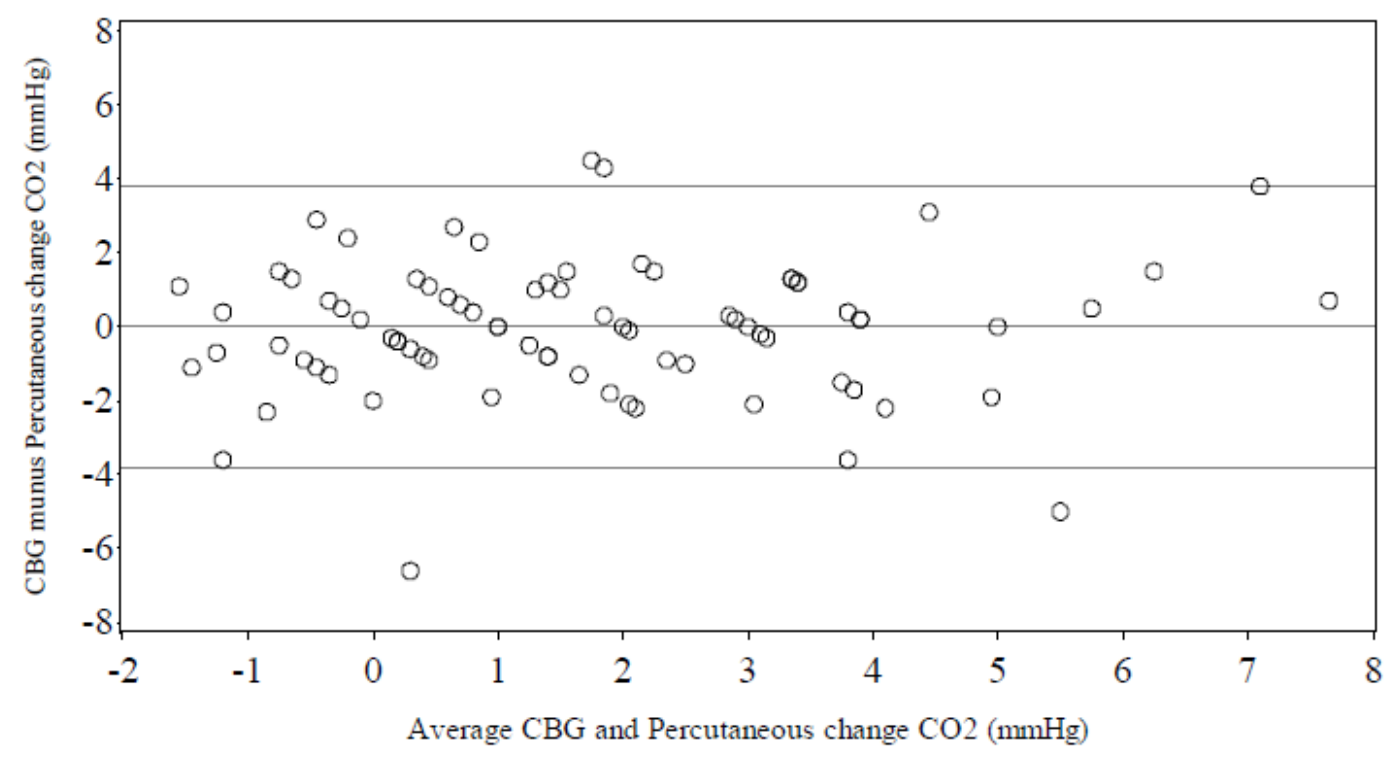

Figure 9-8 Bland altman plot of the mean $\mathrm{CBG}$ and $\mathrm{P}_{\mathrm{t}} \mathrm{CO}_{2}$ change over 35 min compared to the mean difference in CBG minus $\mathrm{PtCO}_{2}$ change over $35 \mathrm{~min}$. The horizontal lines represent $2 \mathrm{SD}$ of the differences.

\subsubsection{Discussion}

Despite reasonably large limits of agreement for individual measurements, there was no statistically significant difference in the $\mathrm{PCO}_{2}$ change from baseline over time measured by either CBG or transcutaneous monitor.

\subsubsection{Conclusion}

The SenTec transcutaneous monitor can be used to accurately measure the change in $\mathrm{PaCO}_{2}$ over time without any significant bias in cardiovascularly stable people.

\subsection{Appendix III - Study 1a}

\subsubsection{Participant Information Sheet}

\section{Participant Information Sheet}




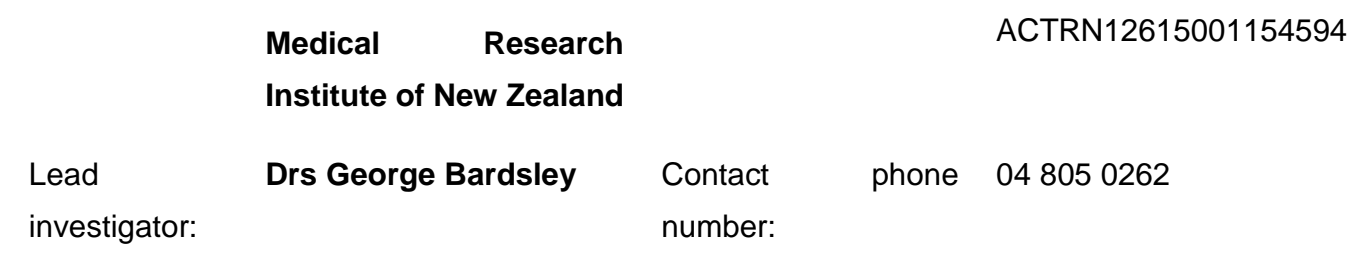

You are invited to take part in a study on the measurement of oxygen levels and carbon dioxide levels. Whether or not you take part is your choice. If you don't want to take part, you don't have to give a reason, and it won't affect the care you receive. If you do want to take part now, but change your mind later, you can pull out of the study at any time.

This Participant Information Sheet will help you decide if you'd like to take part. It sets out why we are doing the study, what your participation would involve, what the benefits and risks to you might be, and what would happen after the study ends. We will go through this information with you and answer any questions you may have. You do not have to decide today whether or not you will participate in this study. Before you decide you may want to talk about the study with other people, such as family, whānau, friends, or healthcare providers. Feel free to do this.

If you agree to take part in this study, you will be asked to sign the Consent Form on the last page of this document. You will be given a copy of both the Participant Information Sheet and the Consent Form to keep.

This document is 6 pages long, including the Consent Form. Please make sure you have read and understood all the pages.

\section{What is the purpose of the study?}

Measuring the amount of oxygen and carbon dioxide in a patient's blood is important for assessing their condition and guiding management. They can be measured by an arterial blood gas sample (ABG) or transcutaneous monitoring. The $A B G$ is considered the most accurate measure of oxygen and carbon dioxide levels and involves a single blood sample taken with a needle. It therefore does not provide a continuous measure of carbon dioxide or oxygen levels.

Transcutaneous (through the skin) monitoring has the advantage of being a simple test which involves the application of a sensor to the earlobe. It is 
painless and can provide continuous information on a patient's carbon dioxide and oxygen levels.

However, it is possible transcutaneous values may not accurately match the values obtained from an ABG. The aim of this study is to compare transcutaneous values to the values measured from your upcoming $A B G$. Up to 32 participants will be recruited.

This study is coordinated and funded through the Medical Research Institute of New Zealand. You can contact the principal investigator, Dr George Bardsley, at the Medical Research Institute of New Zealand on 048050262 or george.bardsley@mrinz.ac.nz.

This study is approved by the HDEC ethics committee, 15/STH/121.

\section{What will my participation in the study involve?}

Patients who are about to have an ABG are eligible to take part in this study. Your participation will involve having either one or two transcutaneous monitors (small clips which measure your carbon dioxide and oxygen levels) painlessly placed on your earlobe(s). They will be put in place at least 30 minutes before your $A B G$ is taken, and removed when the $A B G$ is finished.

There may be the option to have one of the study investigators perform your $A B G$, rather than one of the medical team looking after you. If this is an option we will let you know and ask if you are comfortable with this. We would only perform the ABG for you with your permission.

Occasionally an ABG is taken and the results are not able to be used for this study (for example the blood sample came from a vein, rather than an artery). If the team looking after you decides you need another ABG, we may ask you if we can come back again to measure your carbon dioxide and oxygen levels from the transcutaneous monitor when your next $A B G$ is done.

We will also take a few minutes to collect data from you or your medical record on: your health conditions, age, skin colour, smoking status and ethnicity. Skin colour is measured because it has been demonstrated to affect the accuracy of oxygen levels, and the FDA recommends it is taken into account when 
oxygen monitors are evaluated. Skin colour will be assessed using a standard medical scale called the Fitzpatrick scale.

\section{What are the possible benefits and risks of this study?}

You will remain under the care of your doctor(s) and nurse(s) during the study. Application of the transcutaneous monitor to your earlobe is simple and painless. A benefit of taking part is that you are contributing valuable information to an important study that may change the way we monitor patient carbon dioxide and oxygen levels in clinical trials and in the hospital.

\section{Who pays for the study?}

Participation in the study is free.

\section{What if something goes wrong?}

If you were injured in this study, which is unlikely, you may be eligible for compensation from ACC just as you would be if you were injured in an accident at work or at home. You will have to lodge a claim with ACC, which may take some time to assess. If your claim is accepted, you will receive funding to assist in your recovery. If you have private health or life insurance, you may wish to check with your insurer that taking part in this study won't affect your cover.

\section{What are my rights?}

Your participation in this study is voluntary (your choice). You are free to decline participation or to withdraw from the research at any time, without experiencing any disadvantage. You do not have to provide a reason and your future healthcare will not be affected.

If you decide to participate in the study, the research staff will collect medical and personal information about you as part of doing the study. You have the right to access information collected about you during the study.

By agreeing to take part in this research, you will allow your medical information and results to be seen by people who check that the research was done properly.

No material which could personally identify you will be used in any reports on this study. Your subject data will be identified by a code number. You are able to ask to see your data. Data will be stored by the MRINZ for 10 years.

An interpreter can be provided for you if you require one. 
You may have a friend, family or Whānau member to support and help you understand the risks and/or benefits of this study and any other explanation you may require.

\section{What happens after the study or if I change my mind?}

You can decide not to participate at any stage.

Original data records will be kept in a secure place by the Medical Research Institute of New Zealand for ten years and then destroyed.

If you wish to find out the overall results of the study we can send these to you after completion. We anticipate this will be in 2016.

\section{Who do I contact for more information or if I have concerns?}

If you have any questions, concerns or complaints about the study at any stage, you can contact:

Medical Research Institute of New Zealand

Phone: 048050262

Email: George.Bardsley@mrinz.ac.nz

If you want to talk to someone who isn't involved with the study, you can contact an independent health and disability advocate on:

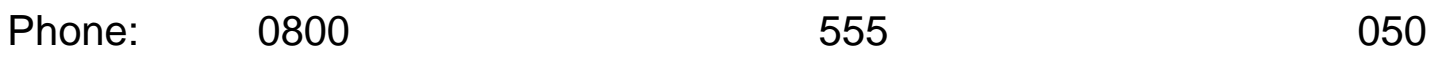

$\begin{array}{llllll}\text { Fax: } & 0800 & 2 & \text { SUPPORT } & (0800 & 2787\end{array}$

Email: $\quad$ advocacy@hdc.org.nz

For Māori health support please contact :

Whānau Care Services (0800 999 442)

You can also contact the health and disability ethics committee (HDEC) that approved this study on:
Phone: $\quad 08004$ ETHICS
Email: $\quad$ hdecs@moh.govt.nz 
If you need an INTERPRETER, please tell us.

Please tick to indicate you consent to the following

I have read, or have had read to me in my first language, and I understand the Participant Information Sheet.

Yes

I have been given sufficient time to consider whether or not to participate in this study.

Yes $\square$

I have had the opportunity to use a legal representative, whānau/ family support or a friend to help me ask Yes $\square$ questions and understand the study.

I am satisfied with the answers I have been given

regarding the study and I have a copy of this consent form Yes and information sheet.

I understand that taking part in this study is voluntary (my choice) and that I may withdraw from the study at any time Yes without this affecting my medical care.

I consent to the research staff collecting and processing my information, including information about my health.

If I decide to withdraw from the study, I agree that the information collected about me up to the point when I Yes withdraw may continue to be processed.

I consent to my GP or current provider being informed about my participation in the study and of any significant Yes abnormal results obtained during the study.

I agree to an approved auditor appointed by the New Zealand Health and Disability Ethic Committees, or any relevant regulatory authority or their approved representative reviewing my relevant medical records for the sole purpose of checking the accuracy of the information recorded for the study. 
I understand that my participation in this study is confidential and that no material, which could identify me Yes personally, will be used in any reports on this study.

I understand the compensation provisions in case of injury during the study.

Yes

I know who to contact if I have any questions about the study in general.

Yes

I understand my responsibilities as a study participant. $\quad$ Yes $\square$

If it is an option, I give permission for one of the study

investigators to perform the ABG, rather than one of the Yes $\square$ No $\square$ medical team looking after me.

I wish to receive a summary of the results from the study. Yes $\square$ No $\square$

If yes, please state address:

\section{Declaration by participant:}

I hereby consent to take part in this study.

Participant's name:

Signature:

Date:

\section{Declaration by member of research team:}

I have given a verbal explanation of the research project to the participant, and have answered the participant's questions about it.

I believe that the participant understands the study and has given informed consent to participate. 


\subsection{Appendix IV - Study 2}

\subsubsection{Participant Information Sheet (Version 3, 12/6/15)}

\section{Investigating the effect of the AIRVO® device on carbon dioxide levels in patients with stable COPD.}

\section{Principal investigator:}

Professor Richard Beasley

Medical Research Institute of New Zealand, Wellington Hospital, Private Bag 7902, Wellington. Phone: +64 48050147

\section{Introduction}

You are invited to take part in a study investigating the effect of high flow nasal cannula therapy (HFNC) in improving outcomes in people with Chronic Obstructive Pulmonary Disease (COPD). HFNC therapy is a method of delivering humidified air under high pressure to the nasal passages, designed to help breathing.

HFNC therapy in this study will be delivered through the AIRVO device developed by Fisher and Paykel Healthcare. Fisher and Paykel Healthcare are partially funding the study, in conjunction with Medical Research Institute of New Zealand.

\section{Participation}

Your participation is entirely voluntary (your choice). Your decision whether or not to participate will not affect your health care in any way or your future relationship with the hospital or your GP. If you do agree to take part in the study, you are free to withdraw at any time, without having to give a reason. 
Participation in this study may also be stopped if the study doctor decides it is not in your best interests to continue.

\section{What is the aim of the study?}

COPD is a common lung condition in New Zealand adults, often requiring admission to hospital. As a disease of the lungs, COPD can affect a person's ability to breathe; in particular to breathe out a waste gas called carbon dioxide. As a result, people with COPD can develop a build-up of carbon dioxide in the lungs which can be dangerous as it causes the blood to become acidic leading to symptoms such as sleepiness, confusion and coma. In these circumstances people may need to come in to hospital and be connected to a machine or mask to help their breathing (ventilator).

The AIRVO device is a method by which warmed and humidified air can be delivered to the lungs via the nose. It is thought that using the AIRVO in people with COPD may help to reduce their level of carbon dioxide, and therefore prevent them from developing the symptoms mentioned above. This may also reduce the likelihood of these people needing to be put on to a ventilation machine in hospital. The AIRVO is pictured below.

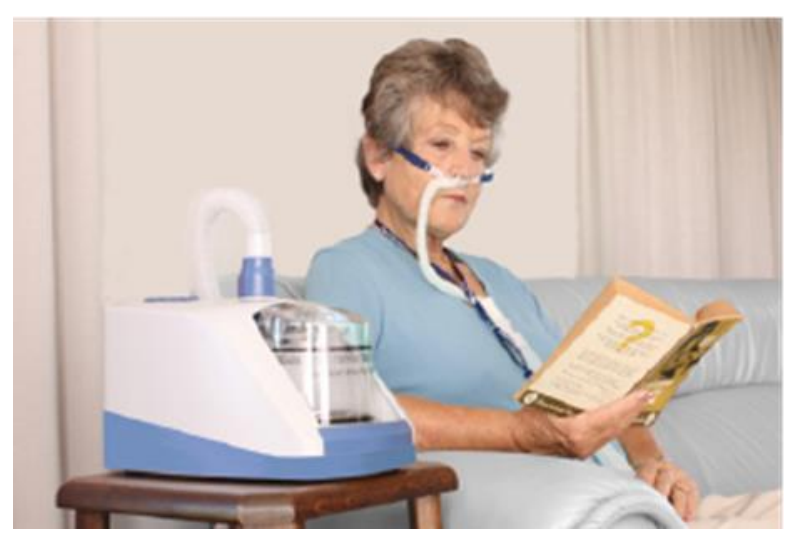

The benefits of using AIRVO in patients with well controlled COPD have not been well studied; we therefore wish to gain more knowledge in this area. We hope to achieve this by giving AIRVO to participants with COPD whilst monitoring the levels of carbon dioxide in their blood to see if the AIRVO is effective in reducing this.

\section{What does the study involve?}


48 people will take part in the study, which will be conducted at the Medical Research Institute of New Zealand situated at Wellington Regional Hospital in Newtown.

If you choose to participate in the study it will involve a single visit lasting for around 3 hours. You will be approached to take part in this study if you have a diagnosis of COPD and your symptoms are currently stable.

On the day of your appointment, you will be asked to come to the Medical Research Institute of New Zealand. One of our researchers will explain the study in full to you and ask you to sign a consent form. You will then be asked some questions about yourself and your medical history. We will also measure your height and weight and perform some lung function tests which involve breathing in to a machine through a mouth piece. The results of these tests will tell us whether it is ok to go ahead with the rest of the study. If this is the case, elasticated fabric bands will be placed around your chest and abdomen to monitor how much you are breathing in and out. These should be comfortable and not restrict your breathing in any way.

You will be seated comfortably for the majority of the study time. Before we start we will attach a small painless clip to your earlobe which will record your carbon dioxide levels, oxygen levels and heart rate throughout the study. There are 4 main parts to the study which last 20 minutes each and will be carried out in a random order (like flipping a coin) decided on the day. You will spend a total of 60 minutes on the AIRVO machine (20 minutes each of low, medium and high flows of air at different times) and 20 minutes of breathing air normally through no device. After each 20 minute session you will be monitored for 15 minutes off of the AIRVO machine and during this time you will be asked to complete a questionnaire about how you found the equipment and experience.

It is important that one of the researchers is not able to see or hear the order in which you receive the different flows or air. They will therefore be sitting behind a screen obscured from view and wearing earplugs. It is also important for the results that you are not aware which flow of air you are receiving during the study so this will also be blocked from view (if you wish to know afterwards we can tell you this at the end). 
The study can be stopped at any point if you feel uncomfortable or unwell and you have the right to ask that your participation in the study be stopped at any point.

At the end of the study visit we will run through the findings with you, to explain how the AIRVO affected you.

\section{Confidentiality and data privacy}

If you decide to participate in the study, the study doctor and research staff will collect medical and personal information about you as part of doing the study.

By agreeing to take part in this research, you allow any medical information and results recorded in the study to be seen by people who check that the research was done properly (not all your medical records).

No material which could personally identify you will be used in any reports on this study. Your information will be identified by a code number. During the study the data will be kept at MRINZ. We will send some data to Fisher and Paykel and the study statistician for analysis, this will be made anonymous by using the code number. Original data records will be kept in a secure place for ten years and then destroyed, as arranged by MRINZ.

\section{What are the risks and safety issues?}

An increase in blood carbon dioxide levels for a long period of time can lead to increased heart rate, drowsiness and headache. It is unlikely that your carbon dioxide levels will increase during the study treatment as you will be breathing room air throughout. You will also be monitored very closely by the study Doctor and treatment will be stopped immediately if your carbon dioxide levels increase above a certain level. This means if you do experience any symptoms they will be short-lived.

In our other studies of AIRVO use, most people tolerated the AIRVO well. However a few participants reported mild headache. You are able to stop using the AIRVO at any stage. Participation in the study is free; however you may have travel expenses to get to Wellington Regional Hospital for which you will be provided reimbursement. 


\section{What are the benefits?}

Measurements of your carbon dioxide levels, oxygen levels, heart rate and breathing rate will be used to understand the effect of AIRVO in patients with COPD. Results from your participation in the study will contribute valuable information to the way we treat people with COPD in the future.

\section{Who is eligible to take part?}

You are eligible to take part in this study if you have a diagnosis of COPD made by a doctor and your condition is currently stable.

You will not be eligible if:

1. You are aged under 40

2. You have not smoked for a long period in your lifetime.

3. You currently are suffering from an exacerbation (worsening) of your COPD, or have been admitted to hospital for this reason in the past 6 weeks.

4. You have history of a disease affecting the nasal passages that will affect the flow of air from the HFNC (such as septal deviation or polyps).

5. Any other condition or problem which the investigator feels would put your health at risk or impact upon the accuracy of study results.

\section{Participant's rights}

An interpreter can be provided for you if you require one. You may have a friend, family or Whānau member to support and help you understand the risks and/or benefits of this study and any other explanation you may require.

If you have any queries or concerns regarding your rights as a participant in this study, you may wish to contact an independent health and disability advocate:

Free phone: 0800555050

Free fax: 08002 SUPPORT $(0800$ 2787

Email: advocacy@hdc.org.nz 
The investigator's own contact details are listed at the beginning of this form.

For Māori health support please contact Whānau care services (Phone: 04 806 0948).

\section{Compensation for injury}

In the unlikely event of a physical injury as a result of your participation in this study, you may be covered by ACC under the Injury Prevention, Rehabilitation, and Compensation Act 2001. ACC cover is not automatic, and your case will need to be assessed by ACC according to the provisions of the Injury Prevention, Rehabilitation, and Compensation Act 2001. If your claim is accepted by ACC, you still might not get any compensation. This depends on a number of factors, such as whether you are an earner or non-earner. ACC usually provides only partial reimbursement of costs and expenses, and there may be no lump sum compensation payable. There is no cover for mental injury unless it is a result of physical injury. If you have ACC cover, generally this will affect your right to sue the investigators.

If you have any questions about ACC, contact your nearest ACC office or the investigator.

If you have private health or life insurance, you may wish to check with your insurer that taking part in this study won't affect your cover

\section{Statement of Approval}

This study has received ethical approval from Northern A Ethics Committee, Reference number 15/NTA/4.

\section{Contact Details}

Dr Steven McKinstry

$\mathrm{Ph}+6448050261$

Steve.McKinstry@mrinz.ac.nz 


\section{Participant Consent Form}

If you need an INTERPRETER, please tell us.

Please tick to indicate you consent to the following:

I have read, or have had read to me in my first Yes No language, and I understand the Participant Information Sheet.

I have been given sufficient time to consider whether Yes No or not to participate in this study.

I have had the opportunity to use a legal Yes No representative, whanau/ family support or a friend to help me ask questions and understand the study.

I am satisfied with the answers I have been given regarding the study and I have a copy of this consent Yes No form and information sheet.

I understand that taking part in this study is voluntary Yes No (my choice) and that I may withdraw from the study at any time without this affecting my medical care.

I consent to the research staff collecting and Yes No processing my information, including information about my health.

If I decide to withdraw from the study, I agree that the information collected about me up to the point when

I withdraw may continue to be processed.

I consent to my GP or current provider being informed about my participation in the study and of Yes No any significant abnormal results obtained during the study. $\square$ 
I agree to an approved auditor appointed by the New Zealand Health and Disability Ethic Committees, or any relevant regulatory authority or their approved Yes No representative reviewing my relevant medical records for the sole purpose of checking the accuracy of the information recorded for the study.

I understand that my participation in this study is confidential and that no material, which could identify Yes No me personally, will be used in any reports on this study.

I understand the compensation provisions in case of injury during the study.

I know who to contact if I have any questions about Yes No the study in general.

I understand my responsibilities as a study Yes No participant.

I wish to receive a summary of the results from the Yes No study.

\section{Declaration by participant:}

I hereby consent to take part in this study.

Participant's name:

Signature: Date:

\section{Declaration by member of research team:}


I have given a verbal explanation of the research project to the participant, and have answered the participant's questions about it.

I believe that the participant understands the study and has given informed consent to participate.

Researcher's name:

Signature: Date:

\subsubsection{Tolerability questionnaire}

\section{TOLERABILITY OF AIRVO IN PARTICIPANTS WITH STABLE COPD}

FIRST INTERVENTION (or N/A if given room air $\boldsymbol{\square}$ )

Please mark on the line scales below with a vertical line (i.e. I) where you feel is appropriate:

1. Overall I found the intervention:

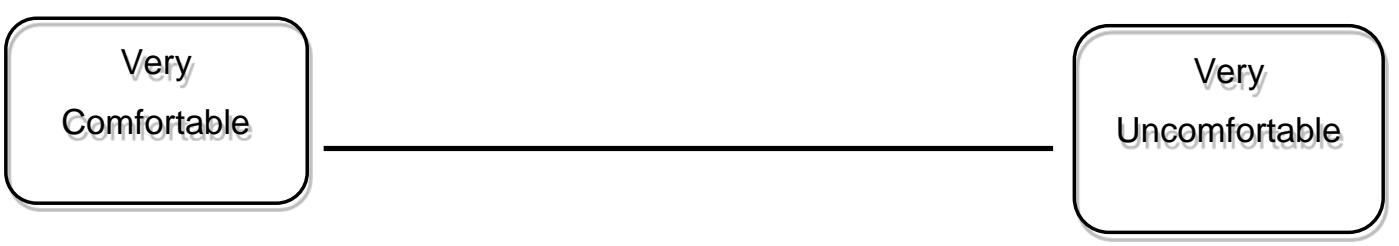

Any comments:

2. My nasal passages felt: 


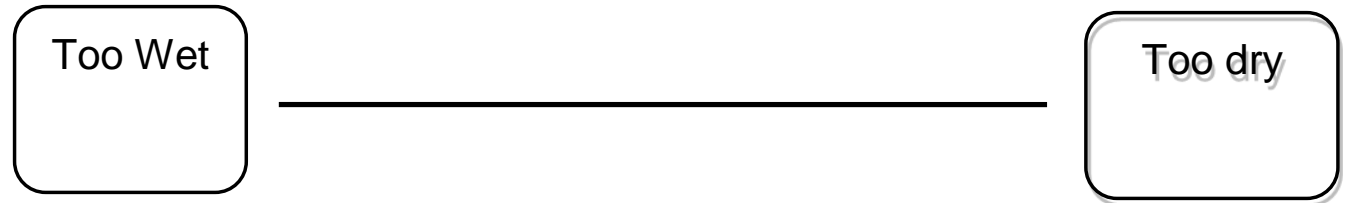

Any comments:

3. The weight of the nasal interface was:

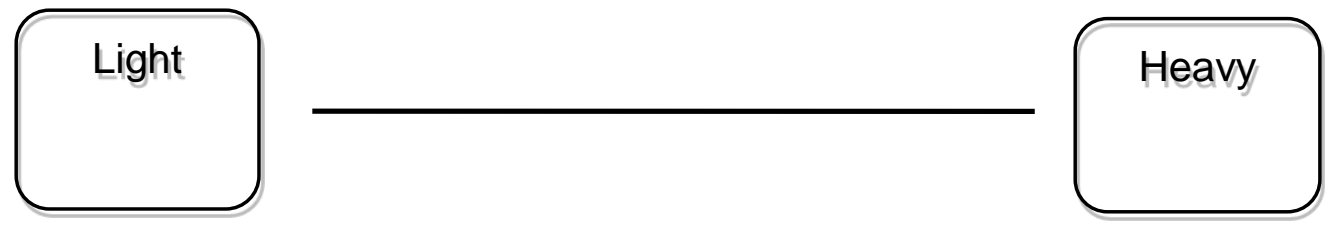

Any comments:

4. The AIRVO equipment was:

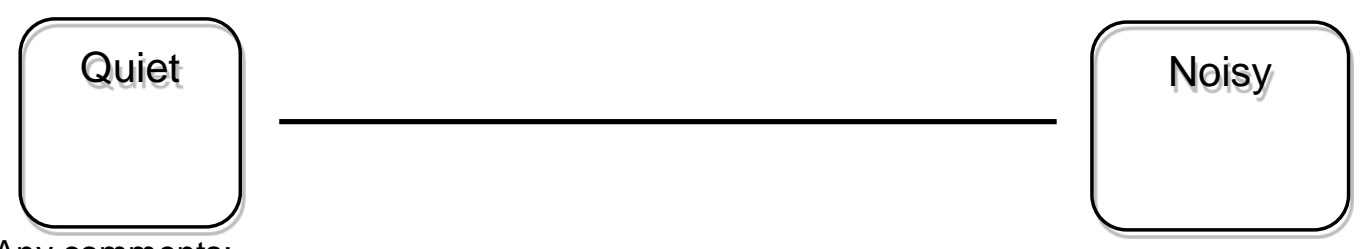

Any comments:

5. I found applying and removing the nasal interface:

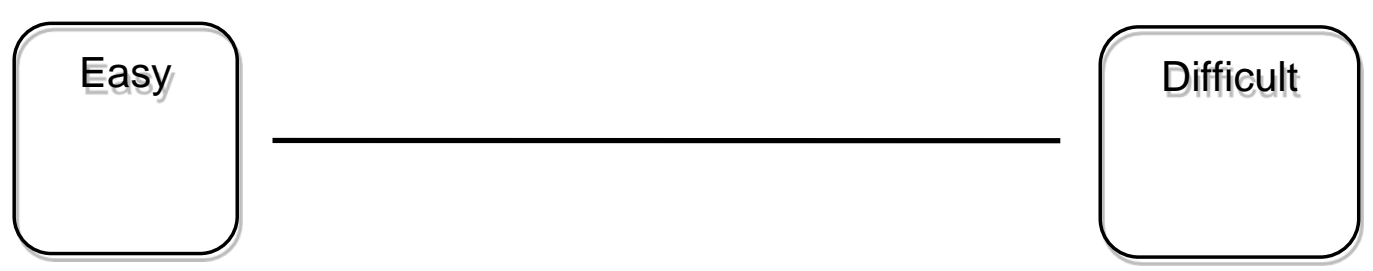

Any comments:

6. If the AIRVO was recommended for future medical care, the likelihood of me using this again would be:

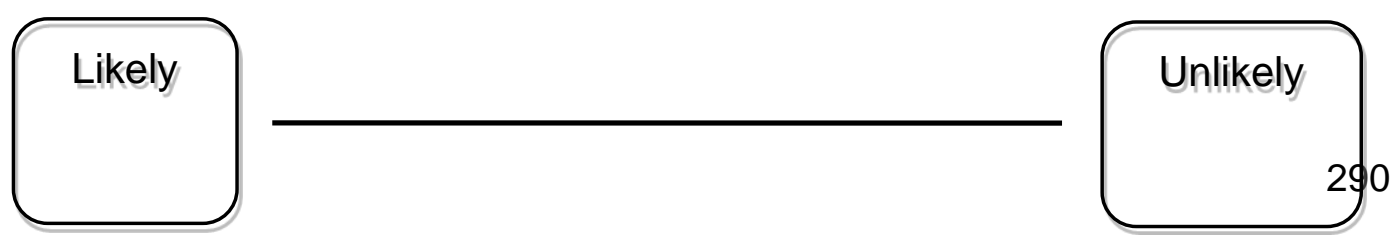




\subsection{Appendix V - Study 3}

\subsubsection{Participant Information Sheet (Version 4, 12/12/15)}

\section{Study title: NASAL HIGH FLOW THERAPY USE FOLLOWING HOSPITALISATION FOR AN EXACERBATION OF COPD}

$\begin{array}{llllll}\text { Locality: } & \text { Wellington Hospital } & \begin{array}{l}\text { Ethics } \\ \text { ref.: }\end{array} & \text { committee } & \text { 15/NTB/92 } \\ & & & & \\ & & & & \\ & & \text { Contact } & \text { phone } & +64 & 4 \\ \text { Lead } & \text { Dr James Fingleton } & & \begin{array}{l}\text { number: } \\ \text { investigator: }\end{array} & & \mathbf{8 0 5 0 2 6 1}\end{array}$

You are invited to take part in a research study investigating the effect of nasal high flow therapy in people with chronic obstructive pulmonary disease (COPD). Specifically, the study will investigate the effect of nasal high flow in people who have suffered an exacerbation (worsening) of their COPD requiring hospital treatment. Nasal high flow in this study will be delivered via the myAIRVO 2 device, developed by Fisher and Paykel Healthcare, who are also financially sponsoring MRINZ to conduct the study.

Whether or not you take part is your choice. If you don't want to take part, you don't have to give a reason, and it won't affect the care you receive. If you do want to take part now, but change your mind later, you can let us know at any time that you no longer want to take part in the study. This will not affect any ongoing care you receive. This Participant Information Sheet should provide information to help you decide if you would like to take part. It tells you why we are doing the study, what your involvement would be, what the benefits and risks to you might be, and what would happen after the study ends. We will go through this information with you and answer 
any questions you may have. You do not have to decide today whether or not you will participate in this study. Before you decide you may want to talk about the study with other people, such as family, whānau, friends, or other healthcare providers.

If you agree to take part in this study, you will be asked to sign the Consent Form on the last page of this document. You will be given a copy of both the Participant Information Sheet and the Consent Form to keep.

This document is 10 pages long, including the Consent Form. Please make sure you have read and understood all the pages.

\section{What is the purpose of the study?}

Chronic Obstructive Pulmonary Disease (COPD) is a common lung condition in adults, often requiring admission to hospital.

Nasal high flow or NHF for short, is a therapy that delivers warmed and humidified air to the lungs via a nasal cannula. Research has shown that using NHF therapy over a 12 month period in patients with COPD reduced exacerbation days and improved lung function and quality of life.

A picture of the myAIRVO 2 device is shown below:

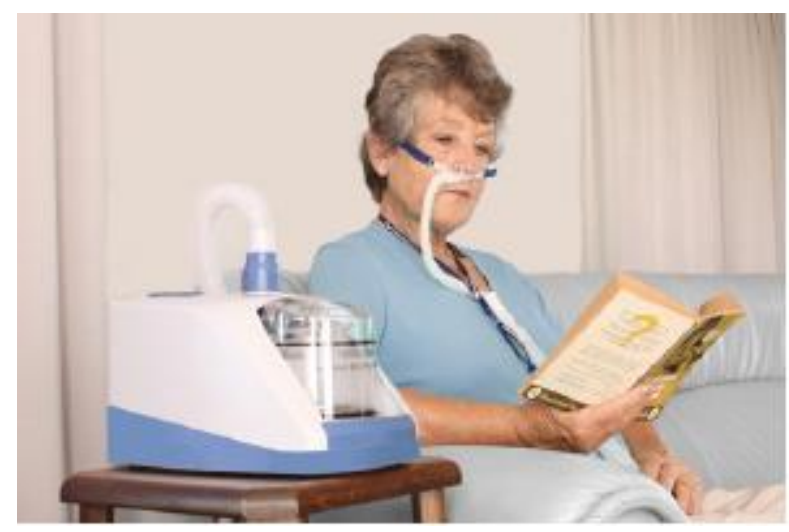

All the benefits of using NHF therapy in patients with COPD at home have not been fully studied; we therefore wish to gain more knowledge in this area, which will help us design a larger study to be carried out in the future. To understand how patients use the therapy we want to give NHF therapy at home to patients with COPD for 1 month. After an initial introduction to the therapy in hospital and a home visit to make sure the device is set up correctly, people on the study will be contacted by phone every week to check their progress and make sure everything is OK. They will also be asked to complete a daily diary during and a short questionnaire at the end of the study. The study coordinators will also be available by phone during business hours for anyone on the study if any questions or concerns arise during the study period. 
In NZ, the study is carried out by MRINZ with funding from Fisher and Paykel Healthcare who designed and manufacture the myAIRVO 2 device. The investigator is $\mathrm{Dr}$ James Fingleton who can be contacted on the phone number on page one if you would like to speak with him directly.

This study has been approved by the Northern B HDEC and is registered on https://clinicaltrials.gov (NCT02552732).

\section{What will my participation in the study involve?}

80 people from the US and 20 people from New Zealand will take part in the study. You have been asked to take part in this study because you have been admitted to hospital for your COPD.

What the study involves is outlined below:

1. Once the study has been explained and we have your permission to go ahead you will be visited on the ward before you go home and asked some questions about yourself and your medical history. With your permission, we will also access your medical records to check aspects of your medical history, such as previously recorded lung function results. We will bring a myAIRVO 2 device to the ward for you to try and give a basic explanation of how to use it. We will also teach you how to use the machine and ask you to try it while you are in hospital so you can get used to the therapy while there is someone around to answer any questions that you have when you start to use the therapy. We may send you home with the equipment or we may bring the equipment to your home depending on what is best for you.

2. On the day of discharge or the day after, we will arrange for our staff to visit you at home. During this visit, we will check you are happy with setting up and operating the device. We will also show you how to maintain the device. We will also ask you to perform some blowing tests (spirometry) which involve breathing in to a machine through a mouth piece to see how much air you are able to blow out. This tells us how your lungs are working. The visit may take up to one and a half hours.

3. For the next 30 days you will be asked to use the therapy as much as possible/practical every day. We will ask you to record the date of any visits to health care professionals or changes to your medication. You will be given contact details if you have any problems with the equipment.

Please note: You will be expected to contact your usual GP or the Emergency Services if you have any medical problems, just as you would if you were not taking part in the study.

4. Each week we will contact you by telephone to check that you have not had any problems using the myAIRVO 2 device. We will also collect some information about your health and any hospital/GP visits and ask you if you have any questions or feedback you may wish to share with us about using myAIRVO 2. 
5. You will only be required to use myAIRVO 2 for the first 30 days after you receive it. After this time we will collect the device. This can either be done by you attending MRINZ (situated at Wellington Regional Hospital) or we can arrange a home visit. During this visit you will need to spend 30-60 minutes to as we will ask you about your symptoms or any hospital/GP visits. You will also be asked to repeat the blowing test and complete a questionnaire relating to your experience of using the myAIRVO 2 device.

The time you use myAIRVO 2 will be recorded. If you wish to adjust the flow of air or the temperature you will be asked to record this in your daily diary. If you have any concerns about using myAIRVO 2 please stop using it until you have been able to speak with the study investigator.

If you report that you have been in hospital we will check your clinical records at that hospital to confirm the details of your hospital attendance.

\section{What will happen if I don't take part?}

If you decide not to take part or withdraw from the study you don't have to give a reason, and it won't affect the ongoing care you receive. 


\section{What are the possible risks and benefits of this study?}

\section{Risks}

MyAIRVO 2 contains a plate that heats up which warms water in a chamber, care must be taken if you are inserting or removing the chamber immediately after use as the heater plate will still be hot. The risks of using the electrically powered myAIRVO 2 device to humidify the air are: electric shocks, burns and tubing melt down. The myAIRVO 2 device should always be used as per the instruction manual. You are responsible for cleaning your myAIRVO 2 device regularly to reduce the risk of infection. You will be given a user manual and training which will provide information about how and when to clean the device.

You will be asked to perform a blowing test called spirometry as part of the study. This involves breathing hard and fast through a tube to measure how your lungs are functioning. In some people it can make them feel light headed and cause them to cough.

\section{Benefits}

NHF therapy may improve health outcomes following discharge from hospital, which is why this study has been developed. Knowledge of your experience using myAIRVO 2 , your symptoms and your need for further medical care during the study period will be used to inform the investigators if NHF therapy should be studied in more people. The data from this study is expected to be published. This data and data from other studies may be used by doctors to help guide how we treat patients with COPD.

\section{Who pays for the study?}

The study is funded by Fisher and Paykel Healthcare. You do not need to pay to take part in this study. If you travel to Wellington Regional Hospital for the last study visit, we will cover your travel costs.

\section{What if something goes wrong?}

If you are injured or harmed as a result of treatment given during this study, which is highly unlikely, you won't be eligible for compensation from ACC. However, compensation will be available from the study's sponsor, Fisher and Paykel Healthcare, in line with industry guidelines. We can give you a copy of these guidelines if you wish. You have the right to take action through the courts if you disagree with the amount of compensation awarded.

If you have private health or life insurance, you may wish to check with your insurer that taking part in this study will not affect your cover.

\section{What are my rights?}


Taking part in this study is entirely voluntary (your choice). Your decision whether or not to take part will not affect your healthcare in any way or your future relationship with the hospital or your GP. If you do take part in the study and change your mind, you are free to withdraw at any time without having to give a reason. The study investigator or your doctor may also stop you from continuing with the study if they decide it is not in your best interest to continue.

If you take part in the study, we will be happy to inform you of the overall results. We will also provide you with your personal results on request. If we identify any effects from the treatment that may affect your health during the study we will inform you immediately.

Your study information will be labeled with a code. The link between the code and your name and address (identifying information) will only be held by the study investigator and the research staff. Only unidentified data will be sent to the study sponsor, Fisher and Paykel Healthcare (or a third party they have nominated). The sponsor (or third party) will analyze the data so that the results may be published. No material which could personally identify you will be used in any reports.

The data may be used or sent outside of NZ. The sponsor will make sure that your data is secure. Data collected will be kept in a secure location by MRINZ for at least 10 years following study completion.

The Sponsor, Northern B Health and Disability Ethics Committee or regulator may check your health information, if the study is audited, or for study monitoring purposes. This is to make sure the study is being run properly.

An interpreter to translate this information can be provided for you if you require.

You may have a friend, family, or whānau member to support and help you understand the risks and/or benefits of this study and any other explanation you may require.

If you have any queries or concerns regarding your rights to take in this study, you may wish to contact an independent health and disability advocate:

$\begin{array}{llrr}\text { Free } & \text { phone: } & 0800 & 555\end{array}$

$\begin{array}{llllll}\text { Free } \quad \text { fax: } & 0800 & 2 & \text { SUPPORT } & (0800 & 2787\end{array}$

Email: advocacy@hdc.org.nz

\section{What happens after the study or if $i$ change my mind?}


At the end of the study we can give you a summary of the results. These results can be e-mailed or posted to you. There may be some delay between taking part in the study and receiving the results as the whole study needs to be finished before the results can be reviewed. If you wish to have a myAIRVO 2 device for home use after the study has finished you will need to discuss this with your GP.

If you withdraw from the study or after 30 days of treatment we will collect the myAIRVO 2 device from you.

If you withdraw from the study we will keep the data collected up to the time you withdraw, unless you specifically ask us not to. Original data records will be kept in a secure place for ten years and then destroyed.

Once again, if you do agree to take part in the study and then change your mind, you can stop at any time without having to give a reason.

\section{Who do I contact for more information or if I have concerns?}

If you have any questions, concerns or complaints about the study at any stage, you can contact one of the study Investigators / Co-Investigators:

Dr James Fingleton

Dr Steven McKinstry

Phone: +64 48050261

Phone: +64 48050261

E-mail: James.Fingleton@mrinz.ac.nz

E-mail:

steve.mckinstry@mrinz.ac.nz

If you want to talk to someone who isn't involved with the study, you can contact an independent health and disability advocate on:

Phone: $\quad 0800 \quad 555 \quad 050$

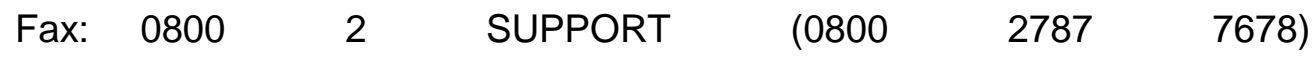

Email: advocacy@hdc.org.nz

For Māori Heath support please contact Whānau Care Services:

Phone: $\quad+64 \quad 4 \quad 806$

0948

Email: wcs@ccdhb.org.nz

You can also contact the health and disability ethics committee (HDEC) that approved this study on:

Phone:0800 4 ETHICS

Email: hdecs@moh.govt.nz 


\section{Consent Form}

If you need an INTERPRETER, please tell us.

\section{Please tick to indicate you consent to the following:}

I have read, or I have had read to me in my first language, and I Yes understand the Participant Information Sheet.

$\square$

I have been given sufficient time to consider whether or not to Yes participate in this study.

I have had the opportunity to use a legal representative, whānau/family support or a friend to help me ask questions and understand the study.

I am satisfied with the answers I have been given regarding the study Yes and I have a copy of this consent form and information sheet.

I understand that taking part in this study is voluntary (my choice) and that I may withdraw from the study at any time without this affecting my medical care.

I consent to the research staff collecting and processing my Yes information, including information about my health.

I consent to the research staff collecting information about my health Yes from my medical records, such as my previous spirometry results.

I consent to my GP or current provider being informed about my participation in the study and of any significant abnormal results obtained during the study.

I agree to have an approved auditor appointed by the New Zealand Health and Disability Ethic Committees, any relevant regulatory authority or their approved representative, or a sponsor appointed study monitor reviewing my relevant medical records for the sole purpose of checking the accuracy of the information recorded for the study.

I understand that my participation in this study is confidential and that no material, which could identify me personally, will be used in any reports on this study.

I understand the compensation provisions in case of injury during the Yes study.

I know who to contact if I have any questions about the study in Yes general.

I understand my responsibilities as a study participant. 
If I decide to withdraw from the study, I agree that the information collected about me up to the point when I withdraw may continue to be processed.

I wish to receive a summary of the results from the study.

\section{Declaration by participant:}

I hereby consent to take part in this study.

Participant's name:

Signature:

Date:

Declaration by member of research team:

I have given a verbal explanation of the research project to the participant, and have answered the participant's questions about it.

I believe that the participant understands the study and has given informed consent to participate.

Researcher's name:

Signature:

Date:

\subsubsection{Tolerability questionnaire}

NASAL HIGH FLOW THERAPY USE FOLLOWING HOSPITALISATION FOR AN EXACERBATION OF COPD: A FEASIBILITY STUDY

\section{NHF THERAPY QUESTIONNAIRE}

Please Circle the most appropriate choice for each question

1. Was myAIRVO 2 easy to use at home? 


$\begin{array}{llllllllll}1 & 2 & 3 & 4 & 5 & 6 & 7 & 8 & 9 & 10\end{array}$

Agree

Disagree

Any comments:-

2. Were the cannula, breathing tube, water chamber easy to use?

$\begin{array}{llllllllll}1 & 2 & 3 & 4 & 5 & 6 & 7 & 8 & 9 & 10\end{array}$

Agree

Disagree

Any comments:-

3. The process of cleaning the myAIRVO 2 device and accessories was:

$\begin{array}{llllllllll}1 & 2 & 3 & 4 & 5 & 6 & 7 & 8 & 9 & 10\end{array}$

Straightforward

Complicated

Any comments:-

4. Overall I found the intervention:

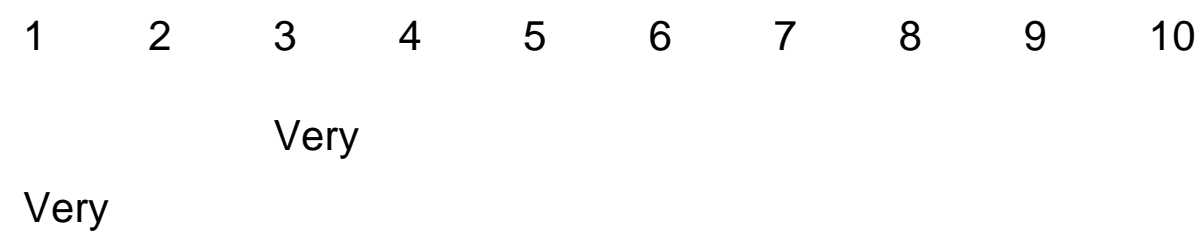

Comfortable

Uncomfortable

Any comments:- 
5. A. Did you alter the temperature setting? (circle one) Yes No

B. If you answered yes above, please answer the following:

Which temperature setting did you find more comfortable?

37 degrees

34 degrees

6. A. Did you alter the flow setting? (circle one)

Yes No

B. If you answered yes above, please answer the following:

Which flow setting did you find more comfortable?

$\square 20 \mathrm{~L} / \mathrm{min}$

$\square 25 \mathrm{~L} / \mathrm{min}$

$\square 30 \mathrm{~L} / \mathrm{min}$

7. If you were asked to continue using myAIRVO 2 daily as part of your clinical management how much longer would you be willing to use it for?

Not at all

$\square 3$ months

$\square 6$ months

$\square 12$ months

$\square$ Indefinitely

Do you have any further comments? 


\subsubsection{Summary sheet}

\section{Participant summary sheet regarding use of the AIRVO device:}

\section{For participants who do NOT use home oxygen}

- Please make sure you are familiar with the myAIRVO 2 manual.

- Please ensure that you switch off myAIRVO 2 when you are not using it.

- myAIRVO 2 must only be used by yourself: please do not allow friends or family to use it.

- You may adjust the temperature settings on myAIRVO 2. Whenever you adjust the temperature please record the changes in your diary.

- You may adjust the air flow rate on myAIRVO 2. You may set it as low as $20 \mathrm{~L} / \mathrm{min}$ or as high as $30 \mathrm{~L} / \mathrm{min}$. Whenever you adjust the flow rate please record the changes in your diary.

- Only the nasal cannula, tubing and water chamber provided may be used with myAIRVO 2. You shouldn't need to change the nasal cannula during the study period. If you break the nasal cannula, tubing and water chamber please contact the investigators who can arrange for them to be replaced.

- If you have any concerns about using myAIRVO 2 please do not to use it and stop using it until you have been able to speak with the study investigator.

\section{Please use myAIRVO 2 as much as possible/practical each day for the next 30 days}

\section{See over for your scheduled calls and home visit}

Study investigators are available during business hours to answer any questions. Please contact:

\section{Dr Steven McKinstry}

Medical Research Institute of New Zealand, Level 7, Clinical Services Building, Wellington Regional Hospital, Newtown, New Zealand

Email: steve.mckinstry@mrinz.ac.nz 
Please contact your GP or the Emergency Services if you have any medical concerns, just as you would if you were not on the trial

In a medical emergency please call 111 immediately

If you need to see your GP, are admitted to hospital or contact

Emergency Services please contact the investigator to let them

know as soon as you are able.

9.6 Appendix VI - Study 4

9.6.1 Participant/relative Information Sheet and Deferred consent form

\section{A Study to assess the treatment of respiratory failure in COPD}

\section{PLEASE NOTE: If you wish to have an interpreter please tell us}

What is this study for and what are the benefits?

- The standard treatment for the first 1 hour in patients with COPD who come to hospital with acute respiratory failure, is giving oxygen treatment through nasal prongs or a mask along with the usual nebulisers, steroid medication and possibly antibiotics.

- After 1 hour, patients are reassessed and may need to progress onto more invasive treatment eg with a tight-fitting mask (BiPAP) or intubation.

- We intend to do a large "full" study in the future investigating whether oxygen treatment through the "AIRVO" device is better than standard nasal prongs or a mask. The "AIRVO" is able to deliver high flows of heated, humidified oxygen through slightly larger than usual nasal prongs.

- The aim of this "feasibility" study is to gather information about how well we currently follow protocols in treatment during this first 1 hour and some parts of the design of our "full" study.

- This is in order to make the "full" study run smoothly and show more accurate results.

How is the "feasibility" study carried out?

- The aim for your treatment in this first 1 hour is as stated above, according to the Hospital protocol. This included taking an arterial blood gas sample initially and then 1 hour after treatment. 
- We would collect the results of your arterial blood gas sample to determine your response to standard oxygen treatment. We would follow your progress through hospital to see if you need more invasive treatment.

- We would collect personal and medical data which will be seen by research doctors and staff however no material which could personally identify you (or your relative) will be used in any reports on this study.

- Participation in this "feasibility" study will not change the care you would have received anyway.

\section{Questions?}

- If you have any questions regarding this study, please contact us using the phone number below or ask a member of staff.

- If you wish to remove your (or your relatives) data from this study, please complete the 'declining participation' form overleaf and hand it to a member of staff.

- Thank you for to taking the time to read this.

\section{Contact details:}

James Fingleton (Lead Investigator) Steven

McKinstry (Co-investigator)

Telephone: +64-4-805 0247

$+64-4-805-0233$

Email: James.Fingleton@mrinz.ac.nz

Steve.mckinstry@mrinz.ac.nz

Medical Research Institute of New Zealand (MRINZ)

Local Māori health advisor: Whanau Care Services, Wellington Hospital, CCDHB

Phone: 043855956

ETHICS HDEC approval number: TBC Contact: 08004 ETHICS

\section{Declining Participation}

I have read, or have had read to me in my first language, and I understand the Participant Information Sheet.

Yes

No

I DO NOT want information about me or my relative included in the study (if it is a relative please state your name and theirs below). Completing this form will mean all data linked to you (or your relative) will be removed from study records.

\section{Participant Name}

Date of Birth

Signature

Date

\section{Relatives Only (if applicable):}

\section{Name}




\section{Signature}

Date

\subsubsection{CCDHB guidelines for assessment and management of AECOPD}

An exacerbation of COPD is defined as an acute event characterised by a worsening of the patient's respiratory symptoms that is beyond normal day-today variations and leads to a change in medication.

- An exacerbation of COPD is most often precipitated by infection (viral or bacterial).

- The common symptoms are breathlessness, cough (with or without sputum), and wheeze.

In This Section

Assessment

Management of an Acute Exacerbation of COPD

Monitoring and Discharge

\section{Assessment}

An acute exacerbation of COPD usually includes an acute change in one or more of the following:

- Cough - increase in frequency and severity

- Sputum - change in volume or colour

- Dyspnoea - increases

For patients with a severe exacerbation, the evaluation should generally include the following:

- Assessment of pulse oxygen saturation

- A chest radiograph (to exclude pneumonia, pneumothorax, pulmonary edema, pleural effusion)

- Laboratory studies (eg, complete blood count and differential, serum electrolytes and glucose)

- Arterial blood gas analysis, if acute or acute-on-chronic respiratory acidosis is suspected or if ventilatory support is anticipated.

Differential diagnosis can include acute decompensated heart failure, pulmonary embolism (PE), pneumonia, or pneumothorax.

\section{Management of an Acute Exacerbation of COPD}

- Short acting bronchodilators 
- The doses below are for management of acute symptoms:

- Salbutamol 600 micrograms via metered dose inhaler and spacer (6 inhalations, each inhaled separately) repeated at 10 to 20 minute intervals (if necessary) up to 6 times or $2.5 \mathrm{mg}$ up to hourly by jet nebulisation (maximum $40 \mathrm{mg}$ daily)

- If patients are thought to require nebulised salbutamol more than hourly after initial treatment and stabilisation, seek senior assistance

- Salbutamol dose intervals are titrated to response

and

- Anticholinergic agent - ipratropium 40 to 80 micrograms (2 to 4 inhalations) can be given by pressurised metered dose inhaler and spacer (maximum 240 micrograms daily), or 500 micrograms (up to 6 hourly - maximum dose) by jet nebulisation.

- Glucocorticoids: oral steroids hasten resolution and reduce the likelihood of relapse. Prednisone 20 to $40 \mathrm{mg}$ daily for 5 to 10 days is adequate. Longer courses add no further benefit and have a higher risk of side effects.

- Antibiotics: Many exacerbations are viral or have non-infective causes. Antibiotics have been shown to be effective when all of the following are present:

- increased dyspnoea

- increased sputum

- purulent sputum.

See Antibiotic Guidelines - Exacerbations of COPD and Chronic Bronchitis.

- Controlled oxygen therapy: This is indicated in patients with hypoxia, with the aim of improving oxygen saturation to about 88 to $90 \%\left(\mathrm{PaO}_{2}\right.$ $>60 \mathrm{mmHg}$ ).

- In all COPD patients, even those with no history of $\mathrm{CO}_{2}$ retention, oxygen therapy should be started at a low dose and titrated up. Start with 1 to $2 \mathrm{~L} / \mathrm{min}$ via nasal prongs. If the patient requires more than 4 L/min, either switch to a Hudson mask or consider the use of high flow nasal prongs. All patients requiring more than $4 \mathrm{~L} / \mathrm{min}$ NP require an $A B G$ and consideration of senior review.

- Ensure oxygen is charted on the patient's medication chart.

- Once oxygen therapy is started, an ABG should be checked within an hour to ensure satisfactory oxygenation without $\mathrm{CO}_{2}$ retention or acidosis.

- Ventilatory assistance: Patients with increasing $\mathrm{CO}_{2}$ and worsening acidosis despite optimal medical treatment and titrated oxygen therapy may require invasive or non-invasive ventilation.

Monitoring and Discharge 
The aim is to relieve hypoxaemia and obtain improvement in clinical signs and symptoms.

- Clinical examination: Reduction in wheeze, accessory muscle use, respiratory rate, distress.

- Gas exchange: Arterial blood gas levels and/or pulse oximetry levels should be monitored until the patient's condition is stable (SpO2 88\%$92 \%)$.

- Discharge planning: Discharge planning should be commenced within 24-48 hours of admission.

- For information on primary care management of COPD, see HealthPathways COPD guidance.

\subsection{Appendix VII Study 5}

\subsubsection{Participant Information Sheet (Version 2, 4/11/16)}

\section{Study title: $\quad$ Nasal High Flow (NHF) vs Non-Invasive Ventilation (NIV) in COPD patients with chronic respiratory failure}

$\begin{array}{llllll}\text { Locality: } & \text { Capital and Coast DHB } & \begin{array}{l}\text { Ethics } \\ \text { ref: }\end{array} & \text { committee } & \text { 16/NTB/207 } \\ & \text { Dr Steven McKinstry } & \begin{array}{l}\text { Contact } \\ \text { number: }\end{array} & \text { phone } & (04) & 805 \\ \begin{array}{l}\text { Lead } \\ \text { investigator: }\end{array} & & 0147 & \end{array}$

You are invited to take part in a study comparing two types of masks used for breathing therapy in Chronic Obstructive Pulmonary Disease. Whether or not you take part is your choice. If you don't want to take part, you don't have to give a reason, and it won't affect the care you receive. If you do want to take part now, but change your mind later, you can pull out of the study at any time.

This Participant Information Sheet will help you decide if you'd like to take part. It sets out why we are doing the study, what your participation would involve, what the benefits and risks to you might be, and what would happen after the study ends. We will go through this information with you and answer any questions you may have. You do not have to decide today whether or not you will participate in this study. Before you decide, you may want to talk about 
the study with other people, such as family, whānau, friends, or healthcare providers. Feel free to do this.

If you agree to take part in this study, you will be asked to sign the Consent Form on the last page of this document. You will be given a copy of both the Participant Information Sheet and the Consent Form to keep.

This document is 9 pages long, including the Consent Form. Please make sure you have read and understood all the pages.

\section{What is the purpose of the study?}

COPD is a common lung condition in New Zealand adults, often requiring admission to hospital. It is a disease of the lungs so COPD can affect a person's ability to breathe; in particular, to breathe out a waste gas called carbon dioxide. As a result, people with COPD can develop a build-up of carbon dioxide in the lungs, which can be dangerous as it dissolves in the blood to make it more acidic. This can cause someone to become very unwell as their lungs "fail", leading to symptoms such as sleepiness, confusion and coma. In these cases people would need to come in to hospital and be connected to a mask to help their breathing, called Non-invasive Ventilation (NIV). The NIV mask fits tightly to the face from the bridge of the nose to the chin, covering the mouth and nose. This is a way of forcing air and oxygen into the lungs and flushing out the carbon dioxide more efficiently and has been proven to be very effective.

The NIV mask is pictured below. 


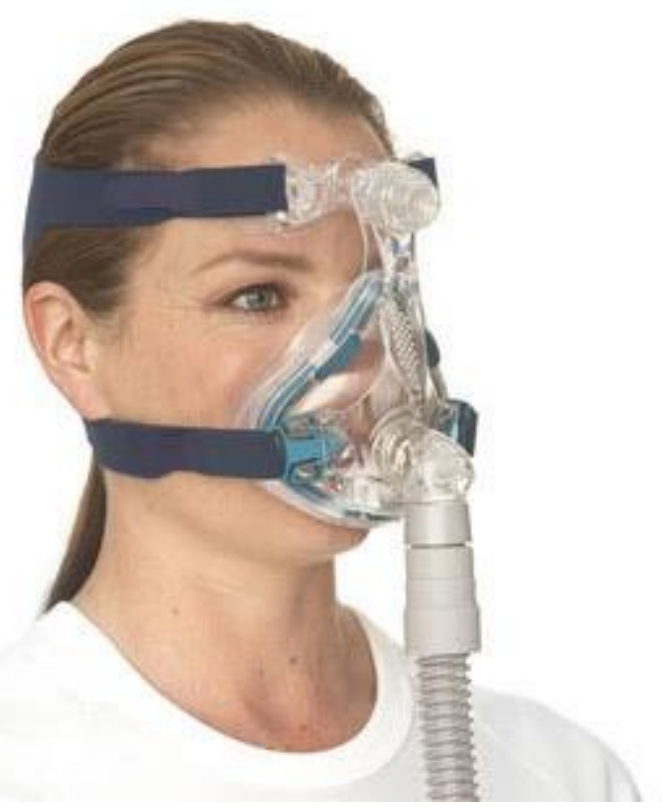

The Nasal High Flow device, called "AIRVO", is another, newer treatment, by which warmed and humidified air and oxygen can be delivered to the lungs through the nose rather than a face mask. It is thought that using the AIRVO in people with COPD may help to reduce their level of carbon dioxide, and therefore prevent them from developing the problems mentioned above. We have finished a study showing this to be true but we need to test it against the current standard treatment which is NIV. The AIRVO is pictured below.

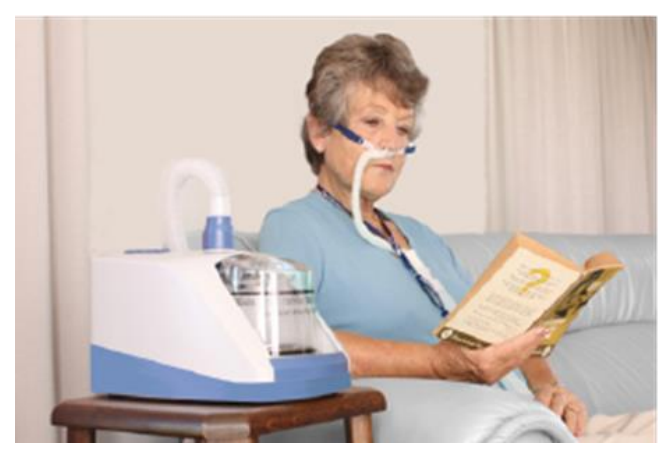

The benefits of using AIRVO in patients with COPD, compared to NIV, have not been well studied; we therefore wish to gain more knowledge in this area. We hope to achieve this by giving both AIRVO and NIV to participants with COPD whilst monitoring the levels of carbon dioxide in their blood to see if the AIRVO is as effective as NIV in reducing this.

The study is sponsored and carried out by Medical Researchers from Medical Research Institute of New Zealand (MRINZ). Fisher and Paykel Healthcare, 
who design and make the AIRVO, provide devices and equipment for us to use. The Lead Investigator is Dr James Fingleton who can be contacted on the phone number on page one if you would like to speak with him directly.

This study has been approved by the Northern B Health and Disability Ethics Committee (Ref 16/NTB/207) and is registered on ANZCTR with the title as above.

\section{What will my participation in the study involve?}

24 people will take part in the study, which will be conducted at the Medical Research Institute of New Zealand situated at Wellington Regional Hospital in Newtown.

If you choose to participate in the study it will involve a single visit lasting for around 3 hours. You will be approached to take part in this study if you are over 40 years of age, have a diagnosis of COPD, have smoked for more than 10 years, have previously been known to have high carbon dioxide levels in a previous study or hospital blood test (called hypercapnia) and your chest symptoms are currently stable. You will not be eligible for the study if you're currently taking a new course of antibiotics or steroid medications for your chest, have a diagnosis of Obesity Hypoventilation syndrome and/or Obstructive Sleep Apnoea, or have a problem with your nose such that wearing the AIRVO would be too uncomfortable. The study investigator will review these things again on the day, along with checking you don't require too much extra oxygen, through standard plastic nasal prongs, prior to starting the study.

On the day of your appointment, you will be asked to come to the Medical Research Institute of New Zealand. One of our researchers will explain the study in full to you and ask you to sign a consent form. You will then be asked some questions about yourself and your medical history. We will also measure your height and weight and perform some breathing tests which involve breathing in to a machine through a mouth piece. The results of these tests will tell us whether it is ok to go ahead with the rest of the study.

You will be seated comfortably for the majority of the study time. Before we start we will attach a small painless clip to your earlobe which will record your 
carbon dioxide levels, oxygen levels and heart rate throughout the study. This takes 30 minutes to warm up and give us an accurate reading of your blood carbon dioxide level. If it is within normal range then you wouldn't be eligible for the study and would not need to do any further tests. If it is high (hypercapnia), then we will do a small finger prick test to confirm this and you will then go on to the rest of the study.

There are 2 treatments in the study which each last 1 hour and will be carried out in a random order (like flipping a coin) decided on the day. You will spend a total of 60 minutes on the AIRVO machine and 60 minutes on the NIV machine. Between the sessions, you will be monitored for 15 minutes off the machines and during this time, you will be asked to complete a questionnaire about how you found the equipment and experience.

It is important that one of the researchers is not able to see or hear the order in which you receive the different machines. They will therefore be sitting behind a screen obscured from view and wearing earplugs. You will be able to tell the difference between the machines as the AIRVO nasal prongs sit just inside the nostrils whereas the NIV is a tight-fitting face mask.

The study can be stopped at any point if you feel uncomfortable or unwell and you have the right to ask that your participation in the study be stopped at any point.

\section{What are the possible benefits and risks of this study?}

\section{What are the possible benefits?}

Measurements of your carbon dioxide levels, oxygen levels, heart rate and breathing rate will be used to understand the effect of AIRVO and NIV in patients with COPD. Results from your participation in the study will contribute valuable information to the way we treat people with COPD in the future. At the end of the visit you will be offered an up to date record of your lung function which you can share with your usual doctor. There will be no lasting benefit for you otherwise from using either the AIRVO and NIV for such a short period of time. 


\section{What are the possible risks?}

NIV has been used for lung conditions for over 15 years now but there are some side effects which have been reported when used in hospital, during which it's used for long periods of time - far longer than the 1 hour you will receive in this trial.

The most common side effect is simply people finding it too uncomfortable to wear, others feeling claustrophobic. Other risks from the mask, which are very unlikely to happen during this study, include developing an ulcer across the "bridge" of your nose from the mask or a skin rash. NIV delivers air and oxygen at a certain pressure so other risks are ear or sinus pain, and (with higher pressures) air filling your stomach so you would feel bloated and a bit sick. We can take off the mask simply to stop these side effects, but you would not be able to continue in the trial. The most serious pressure effects may be pneumothorax (air in the space around the lungs) and pneumocephalus (air in the space around the brain), which have been reported very rarely. Other side effects include dryness or blocking of the nasal passages.

In our other studies of AIRVO use, most people tolerated the AIRVO well. However a few participants reported mild headache. Some people find the rush of warm, humidified air uncomfortable but most get used to it shortly after starting. You are able to stop using the AIRVO at any stage. You will also be monitored very closely by the study Doctor and treatment will be stopped immediately if there is any concern about your condition. This means if you do experience any symptoms they will be short-lived.

\section{Who pays for the study?}

Participation in the study is free for you. You may have travel expenses to get to Wellington Regional Hospital for which you will be provided reimbursement.

\section{What if something goes wrong?}

If you were injured in this study, which is unlikely, you would be eligible to apply for compensation from ACC just as you would be if you were injured in an accident at work or at home. This does not mean that your claim will automatically be accepted. You will have to lodge a claim with ACC, which may take some time to assess. If your claim is accepted, you will receive 
funding

If you have private health or life insurance, you may wish to check with your insurer that taking part in this study will not affect your cover.

What are my rights?

Taking part in this study is entirely voluntary (your choice). Your decision whether or not to take part will not affect your healthcare in any way or your future relationship with the hospital or your GP. If you wish to take part in the study, you are free to withdraw at any time, without having to give a reason. The doctor overseeing the study may also stop you from continuing in the study if they decide it is not in your best interests to continue.

If you want to take part, we will also ask you if you would like to be informed of the overall results of this study. You will also be able to look at the results of your lung function tests if you want to. We will tell you if we find out about good or bad effects that may affect your health during the study.

Your study information will be labeled with a code. The link between the code and your name and address (identifying information) and coded study data (de-identified) will only be held by the study doctor and the research staff. No material which could personally identify you will be used in any reports on this study.

The Health and Disability Ethics Committee or regulator may check your health information, if the study is audited, or for study monitoring purposes. This is to make sure the study is being run properly.

Data collected will be kept in a secure location by the MRINZ for at least 10 years following study completion.

An interpreter to translate information for you can be provided for you if you require one.

You may have a friend, family or Whānau member to support and help you understand the risks and/or benefits of this study and any other explanation you may require. 
If you have any queries or concerns regarding your rights as a participant in this study, you may wish to contact an independent health and disability advocate:

$\begin{array}{llll}\text { Free } & \text { phone: } & 0800 & 555\end{array}$ $\left.\begin{array}{lllllll}\text { Free } \quad \text { fax: } & 0800 & 2 & \text { SUPPORT } & (0800 & 2787 & 7678\end{array}\right)$ Email: advocacy@hdc.org.nz

\section{What happens after the study or if I change my mind?}

At the end of the study, we can give you a summary of the results. These results can be e-mailed or posted to you. There may be some delay between taking part in the study and receiving the results as the whole study needs to be finished before the results can be analysed. We are unable to offer you the use of the AIRVO or NIV after the study has finished. This will be a decision for your GP and/or Respiratory specialist.

If you withdraw from the study, we will keep the data collected up to the time you withdraw, unless you specifically ask us not to when you withdraw.

Original data records are kept securely for fifteen years and then appropriately destroyed.

Once again, if you do agree to take part in the study initially and you change your mind, you can stop at any time, without having to give a reason.

\section{Who do I contact for more information or if I have concerns?}

If you have any questions, concerns or complaints about the study at any stage, you can contact:

Dr Steven McKinstry Dr James Fingleton

Phone: $048050261 \quad$ Phone: 048050247

E-mail: steve.mckinstry@mrinz.ac.nz E-mail:

james.fingleton@mrinz.ac.nz 
If you want to talk to someone who isn't involved with the study, you can contact an independent health and disability advocate on:

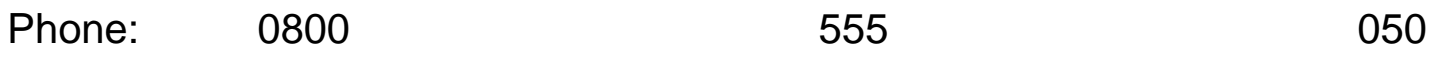

$\begin{array}{lllllll}\text { Fax: } & 0800 & 2 & \text { SUPPORT } & (0800 & 2787 & 7678\end{array}$

Email: $\quad$ advocacy@hdc.org.nz

For Maori health support please contact :

Phone: $\quad(04) \quad 806$

Email:_wcs@ccdhb.org.nz

You can also contact the health and disability ethics committee (HDEC) that approved this study on:

Phone: $\quad 08004$ ETHICS

Email:_hdecs@moh.govt.nz 


\section{CONSENT FORM}

If you need an INTERPRETER, please tell us.

Please read the statements below, tick where there are boxes and sign at the end to indicate you consent to the following:

I have read, or have had read to me in my first language, and I understand the Participant Information Sheet.

I have been given sufficient time to consider whether or not to participate in this study.

I have had the opportunity to use a legal representative, whanau/ family support or a friend to help me ask questions and understand the study.

I am satisfied with the answers I have been given regarding the study and I have a copy of this consent form and information sheet.

I understand that taking part in this study is voluntary (my choice) and that I may withdraw from the study at any time without this affecting my medical care.

I consent to the research staff collecting and processing my information, including information about my health.

If I decide to withdraw from the study, I agree that the information collected about me up to the point when I Yes No withdraw may continue to be processed. 
I consent to my GP or current provider being informed about Yes No my participation in the study and of any significant abnormal results obtained during the study.

I agree to an approved auditor appointed by the New Zealand Health and Disability Ethic Committees, or any relevant regulatory authority or their approved representative reviewing my relevant medical records for the sole purpose of checking the accuracy of the information recorded for the study.

I understand that my participation in this study is confidential and that no material, which could identify me personally, will be used in any reports on this study.

I understand the compensation provisions in case of injury during the study.

I know who to contact if I have any questions about the study in general.

I understand my responsibilities as a study participant.

I wish to receive a summary of the results from the study.

Yes No

\section{Declaration by participant:}

I hereby consent to take part in this study.

Participant's name:

Signature:

Date:

Declaration by member of research team: 
I have given a verbal explanation of the research project to the participant, and have answered the participant's questions about it.

I believe that the participant understands the study and has given informed consent to participate.

Researcher's name:

Signature:

Date:

9.7.2 Tolerability questionnaire (Version $1, \mathbf{1 3} / \mathbf{1 0} / \mathbf{1 6}$ )

\section{TOLERABILITY OF DEVICE QUESTIONNAIRE}

Please rate the following aspects of your device use by marking a vertical line on the scales below i.e.

1. Overall, I found using the device:

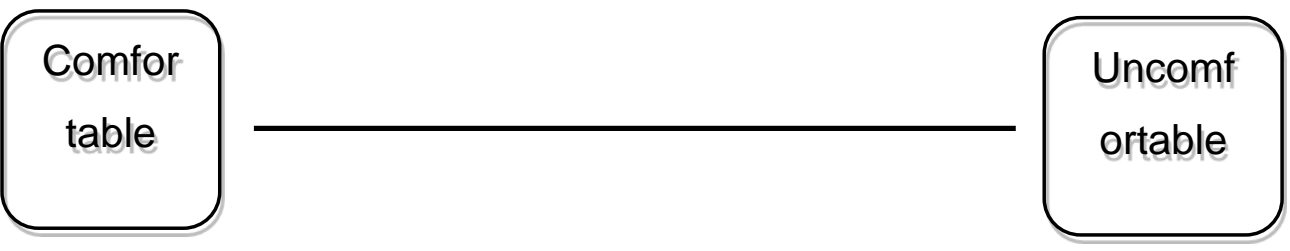

Any comments:

2. The amount of moisture in my nasal passages/mouth felt:

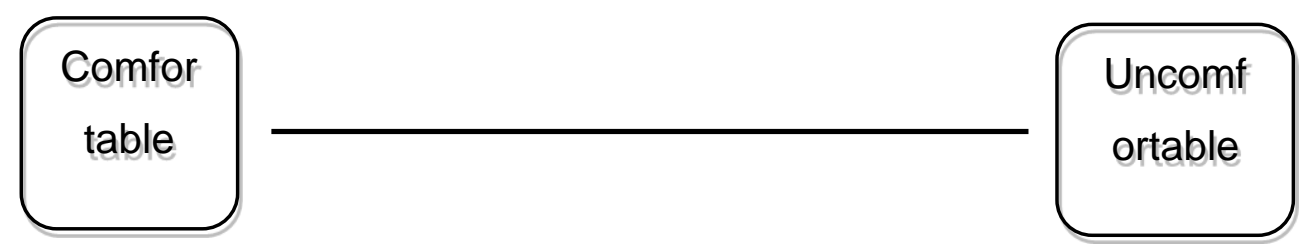

Any comments:

3. I found the fit of the nasal prongs/mask/straps on my face/head : 


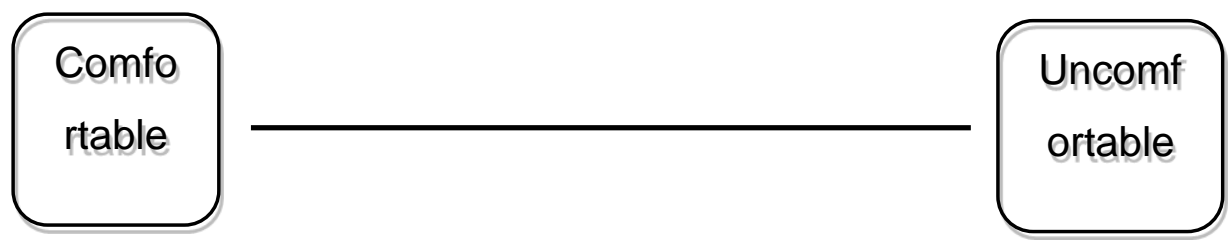

Any comments:

4. The device was:

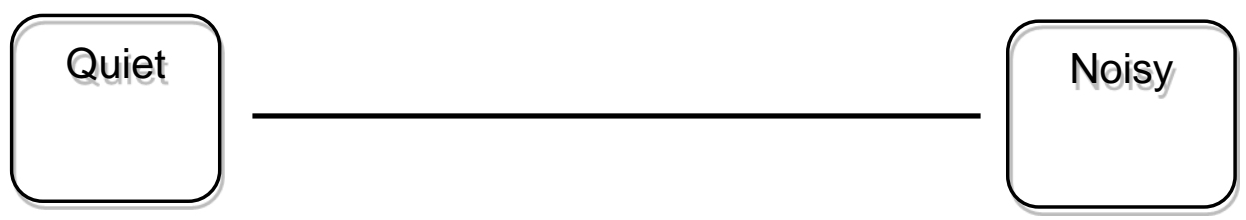

Any comments:

5. I found applying and removing the nasal prongs/mask:

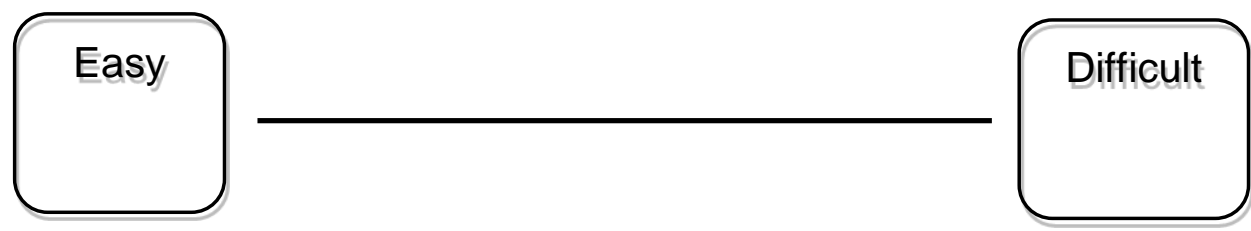

Any

comments:

6. If the device was recommended for future medical care, the likelihood of me using this again would be:

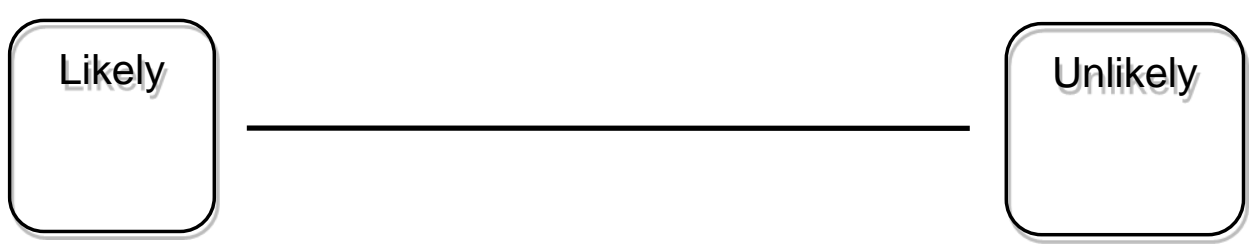

Please

give

a

reason

for

this: 


Do you have any further comments:

\subsubsection{Modified BORG questionnaire}

\section{Modified Borg Dyspnoea ("Breathlessness") Scale Patient Instructions for Modified Borg Dyspnoea Scale}

"This is a scale that asks you to rate the difficulty of your breathing. It starts at number 0 where your breathing is causing you no difficulty at all and progresses through to number 10 where your breathing difficulty is maximal. How much difficulty is your breathing causing you right now?" Circle the number that corresponds to your feeling right now.

$\begin{array}{ll}0 & \text { Nothing at all } \\ 0.5 & \text { Very, very slight (just noticeable) } \\ 1 & \text { Very slight } \\ 2 & \text { Slight } \\ 3 & \text { Moderate } \\ 4 & \text { Somewhat severe } \\ 5 & \text { Severe } \\ 6 & \\ 7 & \\ 8 & \\ 9 & \text { Very severe } \\ 10 & \text { Maximal }\end{array}$




\subsubsection{Supplementary tables}

9.7.4.1 Table a1: Description of supplemental oxygen administered at the end of the wash-in (baseline) and during each of the 2 interventions

\begin{tabular}{|c|c|c|c|c|c|c|c|c|c|}
\hline \multirow[b]{2}{*}{$\begin{array}{l}\text { Time point } \\
\text { (min) }\end{array}$} & \multicolumn{3}{|c|}{ Baseline (end of wash-in) } & \multicolumn{3}{|c|}{ NHF } & \multicolumn{3}{|c|}{ NIV } \\
\hline & $\begin{array}{l}\text { No. of } \\
\text { participants } \\
\text { with oxygen } \\
\text { requirement }\end{array}$ & $\begin{array}{c}\text { Median } \\
\text { flow rate } \\
\text { (L/min) }\end{array}$ & $\begin{array}{l}\text { Min to } \\
\text { Max } \\
\text { (L/min) }\end{array}$ & $\begin{array}{c}\text { No. of } \\
\text { participants } \\
\text { with oxygen } \\
\text { requirement }\end{array}$ & $\begin{array}{l}\text { Median } \\
\text { flow rate } \\
\text { (L/min }\end{array}$ & $\begin{array}{l}\text { Min to } \\
\text { Max } \\
\text { (L/min) }\end{array}$ & $\begin{array}{l}\text { No. of } \\
\text { participants } \\
\text { with oxygen } \\
\text { requirement }\end{array}$ & $\begin{array}{c}\text { Median } \\
\text { flow rate } \\
\text { (L/min) }\end{array}$ & $\begin{array}{l}\text { Min to } \\
\text { Max } \\
\text { (L/min) }\end{array}$ \\
\hline Baseline & 5 & 1.0 & 0.25 to 2 & & & & & & \\
\hline 5 & & & & 2 & 1.125 & 0.25 to 2 & 1 & 2.00 & 2 to 2 \\
\hline 10 & & & & 3 & 1.00 & 0.25 to 2 & 1 & 2.00 & 2 to 2 \\
\hline 15 & & & & 3 & 1.00 & 0.25 to 2 & 1 & 2.00 & 2 to 2 \\
\hline 20 & & & & 3 & 0.50 & 0.25 to 3 & 1 & 2.00 & 2 to 2 \\
\hline 25 & & & & 3 & 1.00 & 0.25 to 3 & 2 & 1.25 & 0.5 to 2 \\
\hline 35 & & & & 3 & 3.00 & 1.0 to 3 & 2 & 1.50 & 1 to 2 \\
\hline 40 & & & & 3 & 2.00 & 1.0 to 3 & 2 & 0.75 & 0.5 to 1 \\
\hline 45 & & & & 2 & 1.50 & 1 to 2 & 2 & 0.75 & 0.5 to 1 \\
\hline 50 & & & & 2 & 0.75 & 0.5 to 1 & 2 & 0.75 & 0.5 to 1 \\
\hline 55 & & & & 2 & 1.50 & 1 to 2 & 1 & 0.50 & 0.5 to 0.5 \\
\hline 60 & & & & 2 & 1.50 & 1 to 2 & 1 & 1.00 & 1 to 1 \\
\hline
\end{tabular}




\subsubsection{Table a2: Data description for respiratory rate}

\begin{tabular}{|c|c|c|c|c|c|c|c|c|c|}
\hline \multirow{2}{*}{$\begin{array}{l}\text { Time point } \\
\text { (mins) }\end{array}$} & \multicolumn{3}{|c|}{ NIV } & \multicolumn{3}{|c|}{ NHF } & \multicolumn{3}{|c|}{$\begin{array}{l}\text { NIV change from baseline minus } \\
\text { NHF change from baseline }\end{array}$} \\
\hline & Mean (SD) & Median (IQR) & Min to Max & Mean (SD) & Median (IQR) & Min to Max & Mean (SD) & Median (IQR) & Min to Max \\
\hline 0 & $18.3(4.3)$ & 19 (16 to 20.5$)$ & 10 to 27 & $16.9(4.4)$ & 16 (13 to 20$)$ & 11 to 29 & & & \\
\hline 5 & $16.3(4.4)$ & 16 (12 to 20$)$ & 10 to 25 & $14.8(4.3)$ & 15.5 (11.5 to 18$)$ & 7 to 22 & $0.2(3.9)$ & $0(-3$ to 4$)$ & -5 to 6 \\
\hline 10 & $16.8(4.5)$ & 16.5 (13 to 20$)$ & 10 to 26 & $15.5(4)$ & 15.5 (14 to 18$)$ & 7 to 24 & $-0.1(5.4)$ & $-0.5(-3.5$ to 2.5$)$ & -14 to 11 \\
\hline 15 & $16(3.9)$ & 15.5 (13 to 18.5$)$ & 9 to 26 & $15.2(4.5)$ & 15 (12.5 to 17.5$)$ & 8 to 24 & $-0.6(5.9)$ & $-1.5(-4.5$ to 4$)$ & -12 to 9 \\
\hline 20 & $16.7(4.3)$ & 16.5 (14.5 to 19.5$)$ & 8 to 25 & $15.3(4.9)$ & 15 (11.5 to 18$)$ & 8 to 25 & $0(5.8)$ & $-0.5(-4.5$ to 3.5$)$ & -10 to 13 \\
\hline 25 & $16.7(3.5)$ & 16.5 (14 to 19.5$)$ & 10 to 24 & $15.8(4.3)$ & 16 (14 to 18$)$ & 8 to 27 & $-0.5(4.3)$ & $-1(-4$ to 2.5$)$ & -9 to 8 \\
\hline 30 & $15.8(3.6)$ & 14.5 (13 to 19$)$ & 11 to 23 & $16.4(4.8)$ & $16(13.5$ to 19$)$ & 8 to 28 & $-2(5.8)$ & $-1.5(-6.5$ to 2$)$ & -15 to 8 \\
\hline 35 & $16(5)$ & 14.5 (12.5 to 19.5$)$ & 9 to 26 & $16.2(5.1)$ & 16 (13 to 19$)$ & 5 to 26 & $-1.6(5.7)$ & $-2(-5$ to 2$)$ & -16 to 10 \\
\hline 40 & $15.5(4.2)$ & 14 (12.5 to 18.5$)$ & 7 to 24 & $16.2(4.7)$ & 16 (13.5 to 19$)$ & 5 to 26 & $-2(6.1)$ & $-2.5(-7$ to 2.5$)$ & -14 to 8 \\
\hline 45 & $16.9(4)$ & 16.5 (14 to 20$)$ & 10 to 26 & $15.7(4.5)$ & $16(13.5$ to 18$)$ & 6 to 26 & $-0.2(5.5)$ & $0(-4.5$ to 3.5$)$ & -13 to 9 \\
\hline 50 & $16.5(4.8)$ & 16 (13 to 20.5$)$ & 8 to 24 & $16.3(4.5)$ & 15.5 (14 to 19.5$)$ & 8 to 26 & $-1.2(4.1)$ & $-0.5(-3$ to 1$)$ & -11 to 7 \\
\hline 55 & $16.5(3.9)$ & 16 (13 to 19.5$)$ & 10 to 24 & $14.3(4)$ & 15 (11 to 17$)$ & 6 to 21 & $0.8(3.8)$ & $0.5(-3$ to 3.5$)$ & -5 to 11 \\
\hline 60 & $17(4.1)$ & 17 (14 to 20.5$)$ & 9 to 25 & $14.8(4.5)$ & 15 (12.5 to 18$)$ & 6 to 24 & $0.8(5.8)$ & $2(-4.5$ to 6$)$ & -9 to 10 \\
\hline
\end{tabular}




\subsubsection{Table a3: Data description for Tolerability questionnaire}

\begin{tabular}{|c|c|c|c|c|c|c|c|c|c|}
\hline \multirow[b]{2}{*}{$\begin{array}{l}\text { Variable } \\
\text { ( } \mathrm{N}=24 \text { for all) }\end{array}$} & \multicolumn{3}{|c|}{ NIV } & \multicolumn{3}{|c|}{ NHF } & \multicolumn{3}{|c|}{ Difference (NIV minus NHF) } \\
\hline & Mean (SD) & Median (IQR) & $\begin{array}{l}\text { Min to } \\
\operatorname{Max}\end{array}$ & $\begin{array}{l}\text { Mean } \\
\text { (SD) }\end{array}$ & Median (IQR) & $\begin{array}{l}\text { Min to } \\
\operatorname{Max}\end{array}$ & $\begin{array}{l}\text { Mean } \\
\text { (SD) }\end{array}$ & Median (IQR) & Min to Max \\
\hline $\begin{array}{l}\text { Ease of } \\
\text { Application }\end{array}$ & $33.1(31.7)$ & 22 (2.5 to 63$)$ & 0 to 100 & $\begin{array}{c}13.8 \\
(18.8)\end{array}$ & 4 (1.5 to 21$)$ & 0 to 66 & $\begin{array}{c}19.3 \\
(29.8)\end{array}$ & $5(0$ to 38$)$ & -20 to 98 \\
\hline Overall Comfort & $41.3(33.0)$ & 45 (8.5 to 74$)$ & 0 to 97 & $\begin{array}{c}16.3 \\
(21.9)\end{array}$ & 8.5 (2 to 20$)$ & 0 to 89 & $\begin{array}{l}25.1 \\
(31.7)\end{array}$ & $16(0$ to 55.5$)$ & -23 to 92 \\
\hline Comfort of fit & $44.5(33.0)$ & $\begin{array}{l}44(16 \text { to } \\
76.5)\end{array}$ & 0 to 97 & $18.3(22)$ & 8.5 (2 to 32 ) & 0 to 69 & $\begin{array}{l}26.3 \\
(23.9)\end{array}$ & 26 (3 to 37.5$)$ & 0 to 90 \\
\hline $\begin{array}{l}\text { Moisture in nasal } \\
\text { passages/mouth }\end{array}$ & $21.1(22.7)$ & $\begin{array}{c}12.5(2 \text { to } \\
37.5)\end{array}$ & 0 to 75 & $\begin{array}{l}23.0 \\
(25.2)\end{array}$ & $\begin{array}{c}12.5(1.5 \text { to } \\
37.5)\end{array}$ & 0 to 80 & $\begin{array}{c}-1.9 \\
(27.9)\end{array}$ & $\begin{array}{c}0(-13.5 \text { to } \\
5.5)\end{array}$ & -68 to 56 \\
\hline Noisiness & $38.6(29.7)$ & $\begin{array}{l}42.5(10 \text { to } \\
62)\end{array}$ & 0 to 100 & $\begin{array}{c}30.4 \\
(26.1)\end{array}$ & 27 (7 to 48.5$)$ & 0 to 100 & $8.2(34.4)$ & $\begin{array}{c}13.5(-1.5 \text { to } \\
31)\end{array}$ & -100 to 76 \\
\hline $\begin{array}{l}\text { Likelihood of } \\
\text { reusing device }\end{array}$ & $30.0(38.4)$ & $\begin{array}{c}9.5 \text { (0.5 to } \\
79)\end{array}$ & 0 to 100 & $\begin{array}{c}15.1 \\
(23.7)\end{array}$ & 3 (1 to 20.5$)$ & 0 to 93 & $\begin{array}{l}15.0 \\
(36.7)\end{array}$ & $1(-0.5$ to 25$)$ & -44 to 100 \\
\hline
\end{tabular}




\subsection{Appendix VIII Study 6}

\subsubsection{Participant Information Sheet (Version 1.1, 20/11/17)}

Study title: $\quad$ Comfort comparison of $2 \mathrm{Bi}-$ level devices in patients with chronic lung diseases.

Locality: $\quad$ Capital and Coast DHB Ethics committee ref: 17CEN225

Hutt Valley DHB

Lead Dr Steven McKinstry

Contact

phone (04) 8050261

investigator:

number:

You are invited to take part in a study comparing two types of masks and machines used for breathing therapy in chronic lung conditions. Whether or not you take part is your choice. If you don't want to take part, you don't have to give a reason, and it won't affect the care you receive. If you do want to take part now, but change your mind later, you can pull out of the study at any time.

This Participant Information Sheet will help you decide if you'd like to take part. It sets out why we are doing the study, what your participation would involve, what the benefits and risks to you might be, and what would happen after the study ends. We will go through this information with you and answer any questions you may have. You do not have to decide today whether or not you will participate in this study. Before you decide, you may want to talk about the study with other people, such as family, whānau, friends, or healthcare providers. Feel free to do this.

If you agree to take part in this study, you will be asked to sign the Consent Form on the last page of this document. You will be given a copy of both the Participant Information Sheet and the Consent Form to keep.

This document is 9 pages long, including the Consent Form. Please make sure you have read and understood all the pages.

What is the purpose of the study? 
Chronic lung conditions are common in New Zealand adults, often requiring admission to hospital. They include lung problems such as chronic obstructive pulmonary disease (COPD) and bronchiectasis and can affect a person's ability to breathe; in particular, to breathe out a waste gas called carbon dioxide. As a result, people with chronic lung conditions can develop a build-up of carbon dioxide in the lungs, which can be dangerous as it dissolves in the blood to make it more acidic. This can cause someone to become very unwell as their lungs "fail", leading to symptoms such as sleepiness, confusion and coma. In these cases people would need to come in to hospital and be connected to a mask and machine to help their breathing, called Bi-level. The Bi-level mask fits tightly to the face from the bridge of the nose to the chin, covering the mouth and nose. This is a way of forcing air and oxygen into the lungs and flushing out the carbon dioxide more efficiently and has been proven to be very effective.

A Bi-level device mask is pictured below.

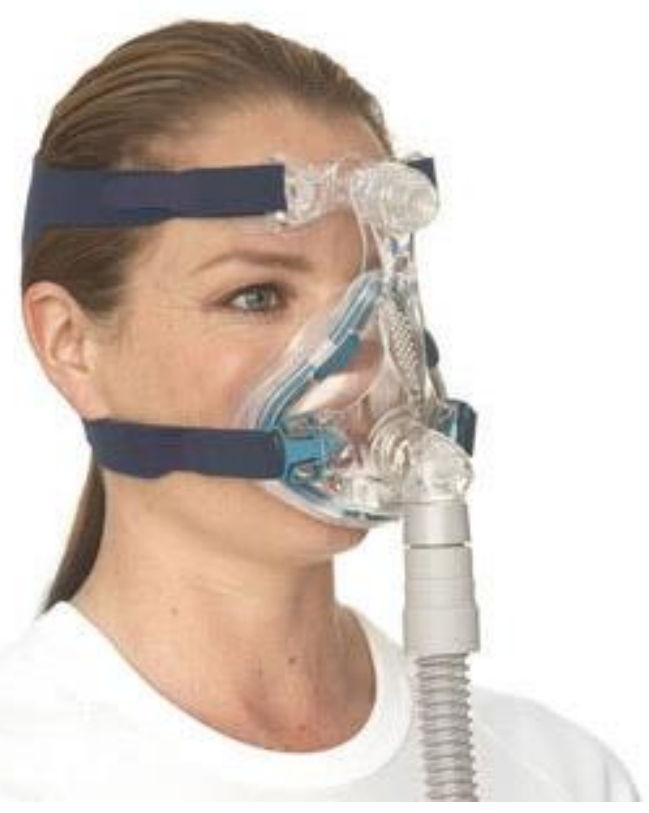

Comfort of the mask and machine has been a problem with Bi-level over the 20 years it has been in use meaning some people need to stop treatment early. Fisher and Paykel Healthcare is a New Zealand company who design and make Bi-level machines and masks and would like to test their new machine against a standard machine used in hospital to compare comfort levels as well as making sure it effectively reduces carbon dioxide. These 2 machines have not been compared against each other before; we therefore wish to gain more knowledge in this area. We hope to achieve this by giving both of the $2 \mathrm{Bi}$-level devices to participants with chronic 
lung conditions. We will monitor the levels of carbon dioxide in the blood to see if they are both as effective, and as comfortable, as each other.

The study is sponsored by Fisher and Paykel Healthcare and carried out by medical researchers from Medical Research Institute of New Zealand (MRINZ). Fisher and Paykel Healthcare, who design and make the machine, provide the devices and equipment for us to use. The Lead Investigator is Dr Steven McKinstry who can be contacted on the phone number on page one if you would like to speak with him directly.

This study has been approved by the Central New Zealand Ethics Committee and is registered on ANZCTR (ACTRN12617001600336) with the title as above.

\section{What will my participation in the study involve?}

30 people will take part in the study, which will be conducted at the Medical Research Institute of New Zealand situated at Wellington Regional Hospital in Newtown, Wellington.

If you choose to participate in the study it will involve a single visit lasting for around 3 hours. You will be approached to take part in this study if you are over 40 years of age, have a diagnosis of a chronic lung condition, and your chest symptoms are currently stable. You will not be eligible for the study if you're currently taking a new course of antibiotics or steroid medications for your chest, have low oxygen levels immediately prior to starting the study, have an implantable medical device or notifiable disease as well as other conditions which make the machines more high-risk for you and the investigators would check this beforehand. The study investigator will review these things again on the day.

On the day of your appointment, you will be asked to come to the Medical Research Institute of New Zealand. One of our researchers will explain the study in full to you and ask you to sign a consent form. You will then be asked some questions about yourself and your medical history. We will also measure your height and weight and perform some breathing tests which involve breathing in to a machine through a mouth piece. 
You will be seated comfortably for the majority of the study time. Before we start we will attach a small painless plastic clip to your earlobe which will record your carbon dioxide levels, oxygen levels and heart rate throughout the study. This takes 20 minutes to warm up and gives us an accurate reading of your blood carbon dioxide levels.

There are 2 treatments in the study which each last 30 minutes and will be carried out in a random order (like flipping a coin) decided on the day. Between the sessions, you will have at least 15 minutes off the machines and during this time, you will be asked to complete a questionnaire about how you found the equipment and experience.

The study can be stopped at any point if you feel uncomfortable or unwell and you have the right to ask that your participation in the study be completely stopped at any point.

\section{What are the possible benefits and risks of this study?}

\section{What are the possible benefits?}

Measurements of your carbon dioxide levels, oxygen levels, heart rate and breathing rate will be used to understand the effect of this new Bi-level machine in patients with chronic lung conditions. Results from your participation in the study will contribute valuable information to the way we treat people with chronic lung conditions in the future. At the end of the visit you will be offered an up to date record of your lung function which you can share with your usual doctor. There will be no lasting benefit for you otherwise from using either of the Bi-level machines for such a short period of time.

\section{What are the possible risks?}

Bi-level has been used for lung conditions for about 20 years now but there are some side effects which have been reported when used in hospital, during which it's used for long periods of time - far longer than the 1 hour you will receive in this trial.

The most common side effect is simply people finding it too uncomfortable to wear, others feeling claustrophobic. Other risks from the mask, which are very unlikely to happen during this study, include developing an ulcer across the "bridge" of your nose from the mask or a skin rash. Bi-level delivers air at a certain pressure so other risks are ear or sinus pain, and (with higher pressures) air filling your stomach so you would 
feel bloated and a bit sick. We can take off the mask simply to stop these side effects, but you would not be able to continue in the trial. The most serious pressure effects, which are very rare, may be pneumothorax (air in the space around the lungs) and pneumocephalus (air in the space around the brain). Other side effects include dryness or blocking of the nasal passages.

You will also be monitored very closely by the study doctor and treatment will be stopped immediately if there is any concern about your condition. This means if you do experience any symptoms they will be short-lived.

\section{Who pays for the study?}

Participation in the study is free for you. The study is sponsored by Fisher and Paykel Healthcare, You may have travel expenses to get to Wellington Regional Hospital for which you will be provided reimbursement.

\section{What if something goes wrong?}

If you are injured as a result of treatment given as part of this study, which is unlikely, you will not be eligible for compensation through ACC. However, compensation will be available from the study sponsor, Fisher and Paykel Healthcare, in line with industry guidelines. We can give you a copy of these guidelines if you wish. You will be able to take action through the courts should you disagree with the amount of compensation offered.

If you have private health or life insurance, you may wish to check with your insurer that taking part in this study will not affect your cover.

\section{What are my rights?}

Taking part in this study is entirely voluntary (your choice). Your decision whether or not to take part will not affect your healthcare in any way or your future relationship with the hospital or your GP. If you wish to take part in the study, you are free to withdraw at any time, without having to give a reason. The doctor overseeing the study may also stop you from continuing in the study if they decide it is not in your best interests to continue.

If you want to take part, we will also ask you if you would like to be informed of the overall results of this study. You will also be able to look at the results of your lung 
function tests if you want to. We will tell you if we find out about good or bad effects that may affect your health during the study.

Your study information will be labelled with a code. The link between the code and your name and address (identifying information) and coded study data (de-identified) will only be held by the study doctor and the research staff. No material which could personally identify you will be used in any reports on this study.

The Health and Disability Ethics Committee or regulator may check your health information, if the study is audited, or for study monitoring purposes. This is to make sure the study is being run properly.

Data collected will be kept in a secure location by the MRINZ for at least 10 years following study completion.

An interpreter to translate information for you can be provided for you if you require one.

You may have a friend, family or Whānau member to support and help you understand the risks and/or benefits of this study and any other explanation you may require.

If you have any queries or concerns regarding your rights as a participant in this study, you may wish to contact an independent health and disability advocate:

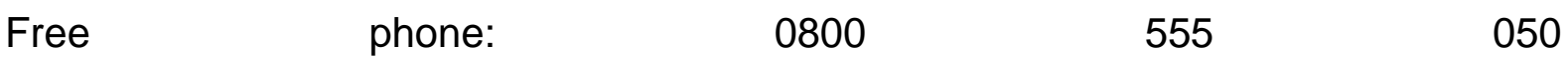

$\begin{array}{lllllll}\text { Free } & \text { fax: } & 0800 & 2 & \text { SUPPORT } & (0800 & 2787\end{array}$

Email: advocacy@hdc.org.nz

\section{What are my responsibilities as a study participant?}

We ask that you make your best effort to follow the study procedures.

As the FPH Bi-level device is still under commercial development with Fisher and Paykel Healthcare, we will be asking you to sign a confidentiality statement in the consent form in which you agree to keep any information relating to this study confidential.

\section{What happens after the study or if I change my mind?}

At the end of the study, we can give you a summary of the results. These results can be e-mailed or posted to you. There may be some delay between taking part in the study and receiving the results as the whole study needs to be finished before the 
results can be analysed. We are unable to offer you the use of the new Bi-level machine at home after the study has finished but it may be used in hospital in the future. If you withdraw from the study, we will keep the data collected up to the time you withdraw, unless you specifically ask us not to when you withdraw.

Original data records are kept securely for fifteen years and then appropriately destroyed.

Once again, if you do agree to take part in the study initially and you change your mind, you can stop at any time, without having to give a reason.

Who do I contact for more information or if I have concerns?

If you have any questions, concerns or complaints about the study at any stage, you can contact:

Dr Steven McKinstry

Phone: 048050261

E-mail: steve.mckinstry@mrinz.ac.nz

If you want to talk to someone who is not involved with the study, you can contact an independent health and disability advocate on:

Phone: $\quad 0800$

555

Fax:

0800

2 SUPPORT

$(0800$

2787

Email: $\quad$ advocacy@hdc.org.nz

For Maori health support please contact :

Phone:

806

0948

Email: wcs@ccdhb.org.nz

You can also contact the health and disability ethics committee (HDEC) that approved this study on:

Phone: $\quad 08004$ ETHICS

Email: $\quad$ hdecs@moh.govt.nz 


\section{CONSENT FORM}

If you need an INTERPRETER, please tell us.

Please read the statements below, tick where there are boxes and sign at the end to indicate you consent to the following:

I have read, or have had read to me in my first language, and I understand the Participant Information Sheet.

I have been given sufficient time to consider whether or not to participate in this study.

I have had the opportunity to use a legal representative, whanau/ family support or a friend to help me ask questions and understand the study.

I am satisfied with the answers I have been given regarding the study and I have a copy of this consent form and information sheet.

I understand that taking part in this study is voluntary (my choice) and that I may withdraw from the study at any time without this affecting my medical care.

I consent to the research staff collecting and processing my information, including information about my health.

If I decide to withdraw from the study, I agree that the Yes No information collected about me up to the point when I withdraw may continue to be processed.

I consent to my GP or current provider being informed about my participation in the study and of any significant abnormal Yes No results obtained during the study. 
I agree to an approved auditor appointed by the New Zealand Health and Disability Ethic Committees, or any relevant regulatory authority or their approved representative reviewing my relevant medical records for the sole purpose of checking the accuracy of the information recorded for the study.

I understand that my participation in this study is confidential and that no material, which could identify me personally, will be used in any reports on this study.

I understand the compensation provisions in case of injury during the study.

I know who to contact if I have any questions about the study in general.

I understand my responsibilities as a study participant.

I wish to receive a summary of the results from the study.

\section{Declaration by participant:}

I hereby consent to take part in this study.

Participant's name:

Signature: Date:

\section{Declaration by member of research team:}

I have given a verbal explanation of the research project to the participant, and have answered the participant's questions about it. 
I believe that the participant understands the study and has given informed consent to participate.

Researcher's name:

Signature:

Date:

\section{Confidentiality statement}

This FPH Bi-level is a new device which has not yet been released for general use. Because it is still being developed, Fisher and Paykel ask that you do not discuss the details of it with anyone. You are free to tell people you are taking part in a study using a new breathing device but Fisher and Paykel ask you do not go into more detail than this. They also ask you do not take any photos or videos of the device. We ask you to sign the statement below from Fisher and Paykel to make sure you agree to this.

The concept of this study, the device, accompany information and anything you may learn or become aware of within this study is commercially sensitive. You must keep any information relating to this study confidential. You must not take any photographs or recordings of the device or your use of it during the study.

I understand my obligations to keep this information confidential.

Participant's name:

Signature:

Date:

9.8.2 Tolerability/comfort

questionnaires

(completed electronically on RedCap) 


\section{Resmed Bipap Tolerability Questionnaire}

Record ID
$\begin{aligned} & \text { Mark on the scale below the breathing comfort of the } \\ & \text { device: }\end{aligned}$

Comments:

How well did the changes in pressure coordinate with

your breathing? i.e. pressure increase during

breathing in and pressure decrease during breathing

out.

Very poorly

Dוm

(Place a mark on the scale above)

Comments:

\begin{tabular}{ll}
\hline How comfortable was the pressure when breathing in? & $\begin{array}{l}\text { Very } \\
\text { uncomfortable }\end{array}$ \\
\hline Comments: & (Place a mark on the scale above) \\
\hline
\end{tabular}

Comments:

How comfortable was the pressure when breathing out?

Very

uncomfortable Very comfortable

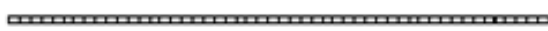

(Place a mark on the scale above)

Comments: 


\section{AIRVO Bipap Tolerability Questionnaire}

Record ID
$\begin{aligned} & \text { Mark on the scale below the breathing comfort of the } \\ & \text { device: }\end{aligned}$

Comments:

How well did the changes in pressure coordinate with

your breathing? i.e. pressure increase during

breathing in and pressure decrease during breathing

out.

Very poorly Excellent

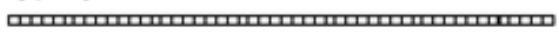

(Place a mark on the scale above)

Comments:

How comfortable was the pressure when breathing in?

Very

uncomfortable Very comfortable

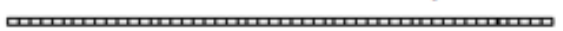

(Place a mark on the scale above)

Comments:

How comfortable was the pressure when breathing out?

Very

uncomfortable

Very comfortable

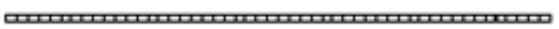

(Place a mark on the scale above)

Comments: 
Overall Tolerability Questionnaire

Record ID

Did you notice any difference in the temperature of

the delivered air between devices?

Yes

No

$\begin{array}{ll}\text { Which one was warmer? } & \bigcirc \text { 1st } \\ & \bigcirc \text { 2nd }\end{array}$

\begin{tabular}{ll}
\hline Did it affect comfort? & $\bigcirc$ Yes \\
& $\bigcirc$ No
\end{tabular}

Did you notice any difference in the noise between

devices?

Yes

Please elaborate:

No

\begin{tabular}{ll}
\hline Which device did you prefer in terms of noise? & $\bigcirc$ 1st device \\
& $\bigcirc$ 2nd device \\
\hline Overall, did you prefer the 1st device or 2nd device? & $\bigcirc$ 1st device \\
& $\bigcirc$ 2nd device \\
& $\bigcirc$ no preference
\end{tabular}

Why? 